\section{Pacific Northwest}

National Laboratory

Operated by Battelle for the

U.S. Department of Energy

\title{
Hanford Site Climatological Data Summary 2003 with Historical Data
}

\author{
D. J. Hoitink \\ J. V. Ramsdell \\ K. W. Burk \\ W. J. Shaw
}

May 2004

Prepared for the U.S. Department of Energy under Contract DE-AC06-76RL01830 


\title{
DISCLAIMER
}

This report was prepared as an account of work sponsored by an agency of the United States Government. Neither the United States Government nor any agency thereof, nor Battelle Memorial Institute, nor any of their employees, makes any warranty, express or implied, or assumes any legal liability or responsibility for the accuracy, completeness, or usefulness of any information, apparatus, product, or process disclosed, or represents that its use would not infringe privately owned rights. Reference herein to any specific commercial product, process, or service by trade name, trademark, manufacturer, or otherwise does not necessarily constitute or imply its endorsement, recommendation, or favoring by the United States Government or any agency thereof, or Battelle Memorial Institute. The views and opinions of authors expressed herein do not necessarily state or reflect those of the United States Government or any agency thereof.

\author{
PACIFIC NORTHWEST NATIONAL LABORATORY \\ operated by \\ BATTELLE \\ for the \\ UNITED STATES DEPARTMENT OF ENERGY \\ under Contract DE-AC06-76RL01830
}

Printed in the United States of America

$$
\begin{aligned}
& \text { Available to DOE and DOE contractors from the } \\
& \text { Office of Scientific and Technical Information, } \\
& \text { P.O. Box 62, Oak Ridge, TN 37831-0062; } \\
& \text { ph: }(865) 576-8401 \\
& \text { fax: (865) 576-5728 } \\
& \text { email: reports@adonis.osti.gov }
\end{aligned}
$$

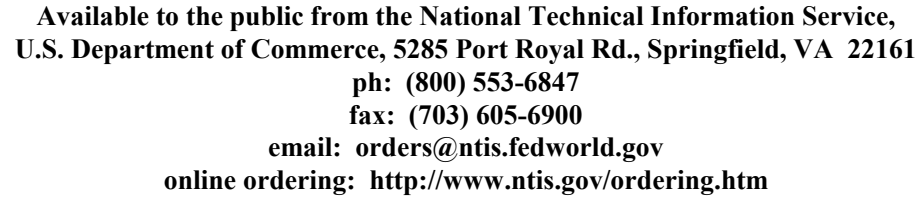

The cover photo (TPO7141-10cn) taken on the Hanford Site is from Lockheed Martin Information Technology, Richland, Washington. The cover design is by S. B. Neely, Pacific Northwest National Laboratory, Richland, Washington.

This document was printed on recycled paper. 


\title{
Hanford Site Climatological Data Summary 2003 with Historical Data
}

\author{
D. J. Hoitink \\ K. W. Burk \\ J. V. Ramsdell \\ W. J. Shaw
}

May 2004

Prepared for

the U.S. Department of Energy

under Contract DE-AC06-76RL01830

Pacific Northwest National Laboratory

Richland, Washington 99352 


\section{Summary}

This document presents the climatological data measured at the U.S. Department of Energy's Hanford Site for calendar year 2003. Pacific Northwest National Laboratory ${ }^{1}$ operates the Hanford Meteorology Station and the Hanford Meteorological Monitoring Network from which these data were collected. This report contains updated historical information for temperature, precipitation, normal and extreme values of temperature and precipitation, and other miscellaneous meteorological parameters. Further, the data are adjunct to and update Hoitink et al. (1999, 2000, 2001, 2002, 2003) and Hoitink and Burk (1994, 1995, 1996, 1997, 1998); however, data from Appendix B - Wind Climatology (Hoitink and Burk 1994) are excluded.

Calendar year 2003 was warmer than normal ${ }^{2}$ at the Hanford Meteorology Station with an average temperature of $55.6^{\circ} \mathrm{F}, 2.0^{\circ} \mathrm{F}$ above normal $\left(53.6^{\circ} \mathrm{F}\right)$. The hottest temperature was $108^{\circ} \mathrm{F}$ on July 28,29 , and 30 , while the coldest was $8^{\circ} \mathrm{F}$ on December 30. For the 12-month period, 9 months were warmer than normal, and 3 months were cooler than normal.

Precipitation for 2003 totaled 8.14 inches, $117 \%$ of normal (6.98 inches); calendar year snowfall totaled 8.7 inches (compared to the normal of 15.4 inches). April 2003 was the wettest April on record with 2.23 inches of precipitation (previous record 1.54 inches in 1995). July 2003 was the driest July on record with no precipitation recorded (previous record was a trace in 1980 and earlier years).

Calendar year 2003 had an average wind speed of $7.8 \mathrm{mph}, 0.2 \mathrm{mph}$ above normal (7.6 mph). There were 36 days with peak gusts $\geq 40 \mathrm{mph}$, compared to a yearly average of 27 days. The peak gust during the year was $60 \mathrm{mph}$ on October 28.

The heating-degree days for 2002-2003 were 4,706 (9\% below the 5,160 normal). Cooling-degree days for 2003 were 1,331 ( $31 \%$ above the 1,014 normal).

For inquiries regarding this report, contact Mr. D. J. Hoitink at Pacific Northwest National Laboratory, P.O. Box 999, Richland, Washington 99352, or by electronic mail at dana.j.hoitink@pnl.gov.

\footnotetext{
${ }^{1}$ Pacific Northwest National Laboratory is operated by Battelle for the U.S. Department of Energy.

${ }^{2}$ Normals for the 2003 summary are 30-year averages based on the period 1971-2000.
} 


\section{Notes on Units of Measure}

This document mainly uses English units (e.g., miles per hour [mph], inches, feet, or degrees Fahrenheit $\left.\left[{ }^{\circ} \mathrm{F}\right]\right)$ when presenting all information. This decision to use English units was based on the fact that English units are still the standard in National Oceanic and Atmospheric Administration (specifically, the National Climatic Data Center and National Weather Service) reporting and publications.

Throughout this document the term "normal" is used to indicate climatological normal, defined as an average value over a period of years of any meteorological element such as temperature, pressure, and rainfall. The convention uses a 30-year time period, ending with the first year of each new decade (such as 1951-1980, 1961-1990, 1971-2000). The time period used for climatological normals for comparative purposes in this document is 1971-2000.

Some useful conversions between English units and metric equivalents are:

$$
\begin{aligned}
& 1 \text { foot }(\mathrm{ft})=0.3048 \text { meter }(\mathrm{m}) \\
& 1 \text { mile }(\mathrm{mi})=1.609 \text { kilometers }(\mathrm{km}) \\
& 1 \text { inch }(\text { in. })=2.54 \text { centimeters }(\mathrm{cm}) \\
& 1 \text { mile per hour }(\mathrm{mph})=0.447 \text { meter } / \text { second }(\mathrm{m} / \mathrm{s}) \\
& \text { degrees Fahrenheit }\left({ }^{\circ} \mathrm{F}\right)=\left(9 / 5 \times{ }^{\circ} \mathrm{C}\right)+32 \\
& \text { degrees Celsius }\left({ }^{\circ} \mathrm{C}\right)=5 / 9 \times\left({ }^{\circ} \mathrm{F}-32\right) \\
& 1 \text { langley }=1 \mathrm{gm}-\mathrm{cal} / \mathrm{cm}^{2}
\end{aligned}
$$




\section{Contents}

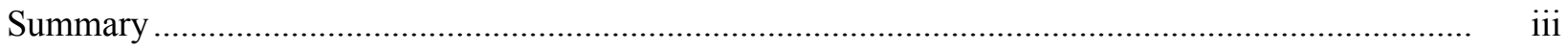

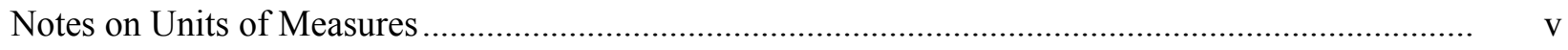

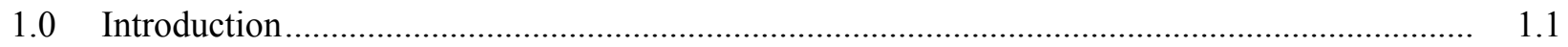

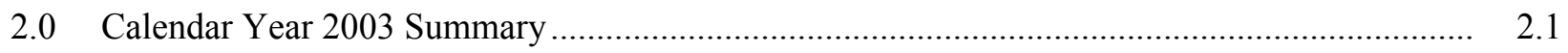

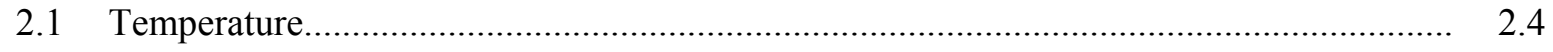

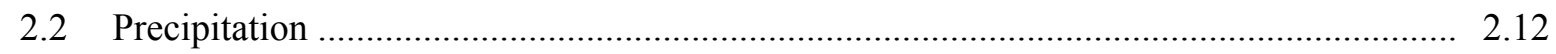

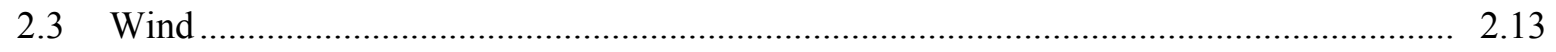

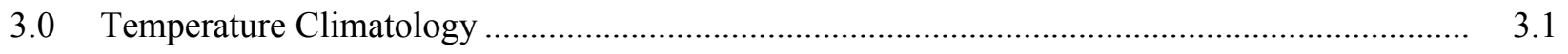

3.1 Monthly, Seasonal, and Annual Average ............................................................ 3.1

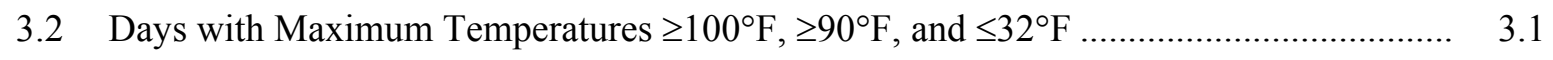

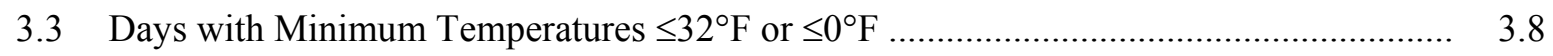

3.4 Monthly Extremes of Daily Maximum and Minimum Temperatures ............................. 3.13

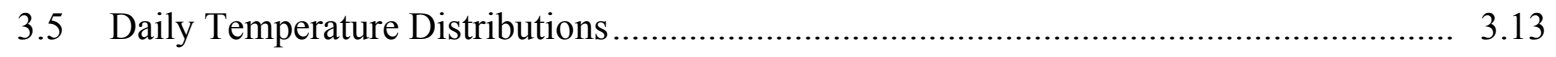

3.6 Average Daily Temperature Range .................................................................. 3.13

3.7 Normal and Extreme Daily Temperatures.......................................................... 3.13

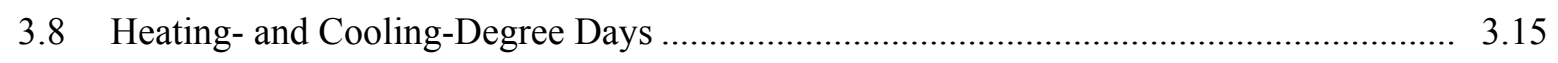

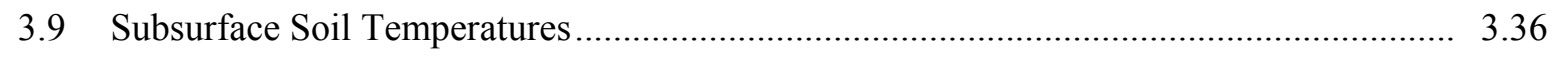

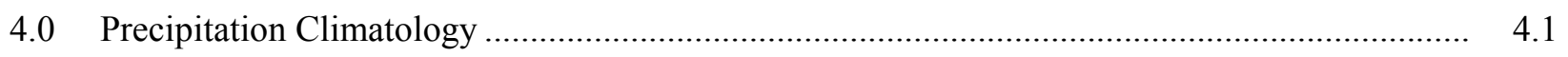

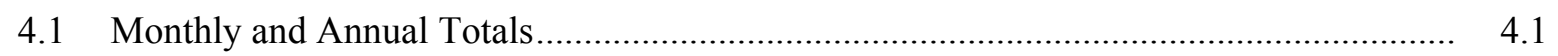

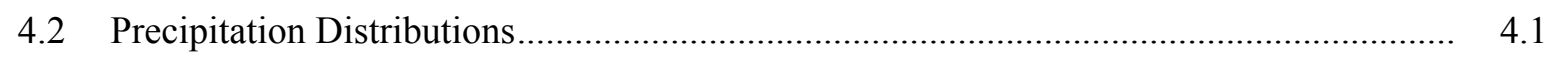

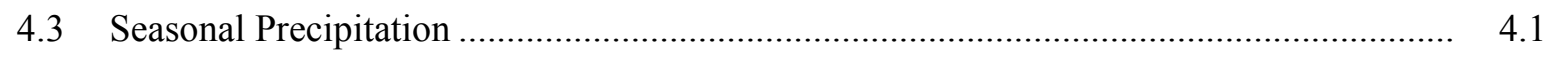

4.4 Average Number of Days with Specified Amounts of Precipitation .............................. 4.1

4.5 Total Time with Precipitation Observed …............................................................ 4.5

4.6 Notable Wet Periods.............................................................................................. 4.6 


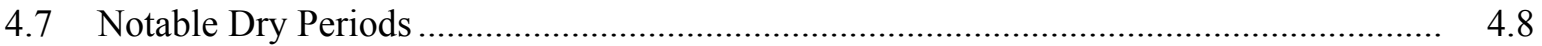

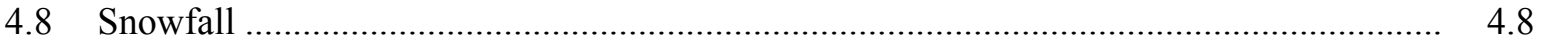

4.9 Normal and Maximum Daily Precipitation ................................................................. 4.11

$5.0 \quad$ Wind Climatology .........................................................................................................

5.1 Monthly and Annual Prevailing Wind Directions, Average Speeds, and Peak Gusts....... $\quad 5.1$

5.2 Days with Peak Gusts Above or Below Specific Thresholds ........................................... 5.1

5.3 Frequency of Monthly and Annual Wind Direction and Speed at 50-Foot Level............. 5.2

5.4 Composite Wind Roses and Joint Frequency Distributions for the Hanford Meteorological Monitoring Network ..................................................................... 5.2

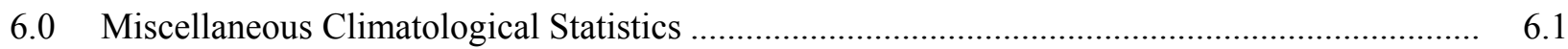

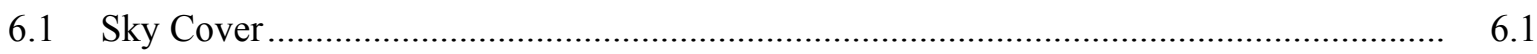

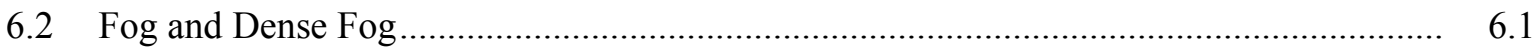

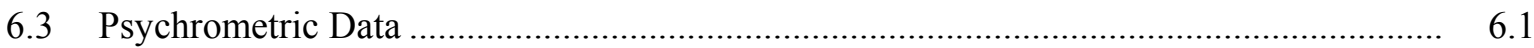

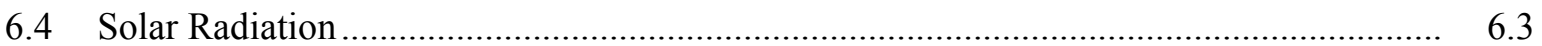

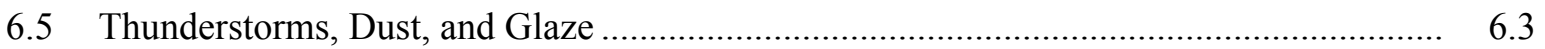

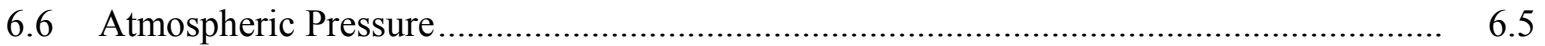

6.7 Sunrise and Sunset Times for the Hanford Meteorological Station ............................... 6.7

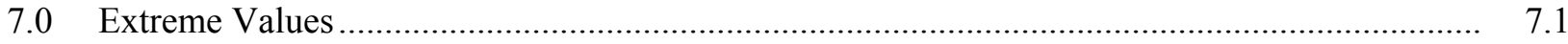

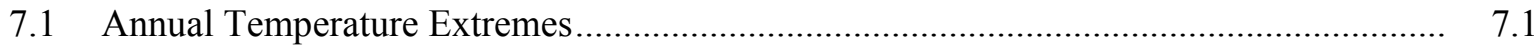

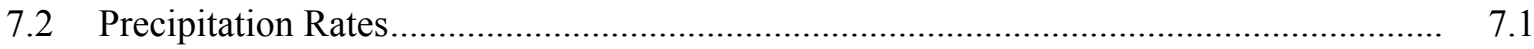

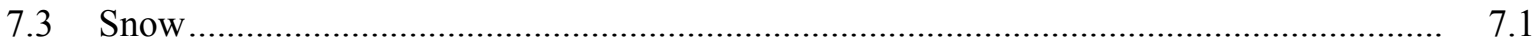

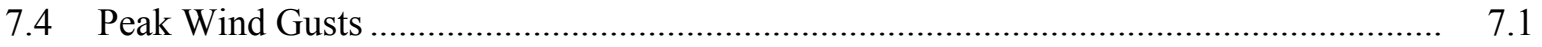

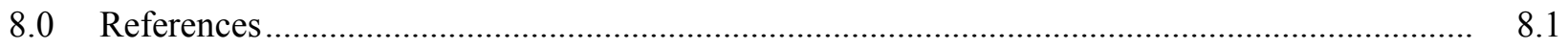

Appendix A - Climate Classification of the Mid-Columbia Region ................................................ A.1

Appendix B - 2003 Wind Climatology ……............................................................................ B.1 


\section{Figures}

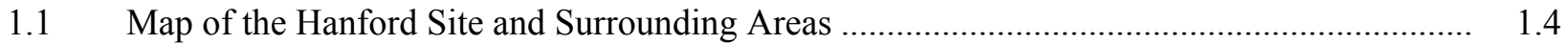

2.1 2003 Observed Daily Temperatures from the Hanford Meteorology Station........................ 2.11

$2.2 \quad 2003$ Hanford Meteorological Monitoring Network Wind Roses at 30 Feet......................... 2.16

2.3 2003 Hanford Meteorological Monitoring Network Wind Roses at 60-Meter Level.............. 2.17

3.1 Graphical Presentation of Daily Maximum Temperatures …............................................. 3.15

3.2 Daily Maximum Temperatures, January 2003 ......................................................... 3.16

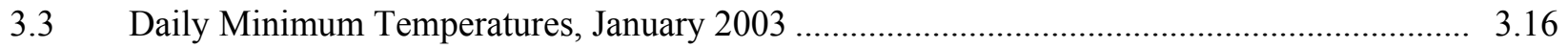

3.4 Daily Maximum Temperatures, February 2003 ............................................................. 3.17

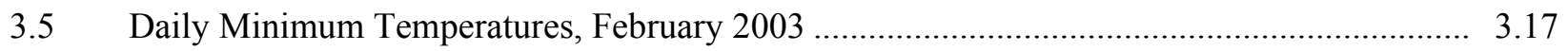

3.6 Daily Maximum Temperatures, March 2003 …......................................................... 3.18

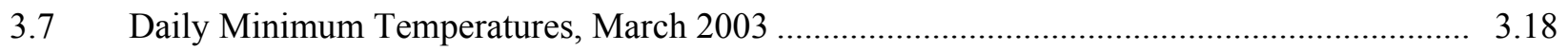

3.8 Daily Maximum Temperatures, April 2003 ….................................................................... 3.19

3.9 Daily Minimum Temperatures, April 2003 .............................................................. 3.19

3.10 Daily Maximum Temperatures, May 2003 f..................................................................... 3.20

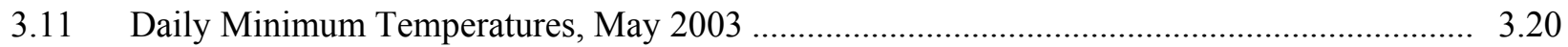

3.12 Daily Maximum Temperatures, June 2003 ….............................................................. 3.21

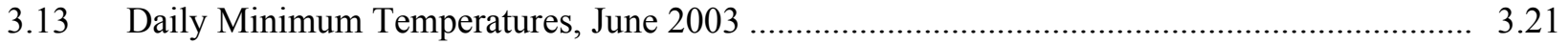

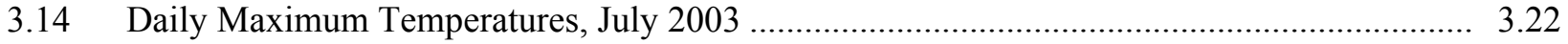

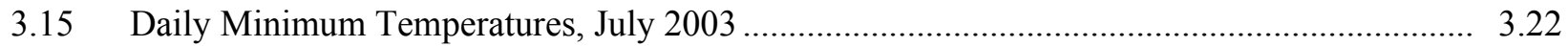

3.16 Daily Maximum Temperatures, August 2003 …............................................................. 3.23

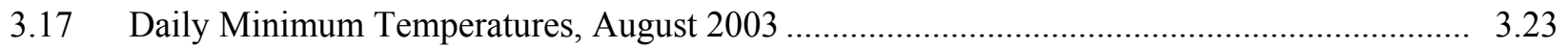

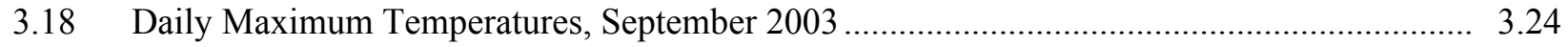

3.19 Daily Minimum Temperatures, September 2003 …......................................................... 3.24

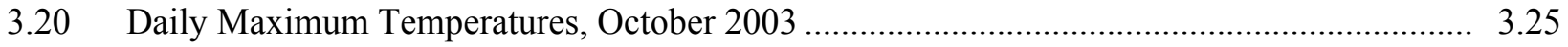


3.21 Daily Minimum Temperatures, October 2003 ............................................................ 3.25

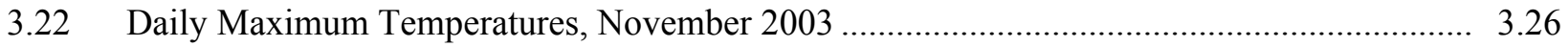

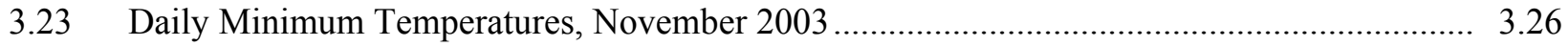

3.24 Daily Maximum Temperatures, December 2003 …....................................................... 3.27

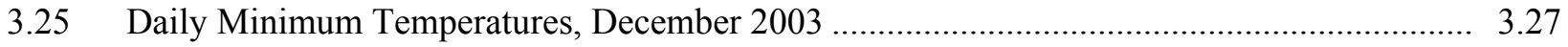

3.26 Climatological Statistics on Heating-Degree Days with Data for the 2002-2003 Heating

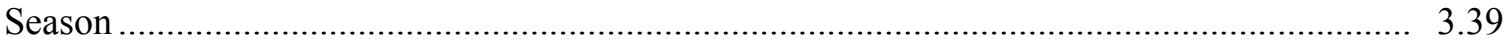

3.27 Climatological Statistics on Cooling-Degree Days with Data for the 2003 Cooling

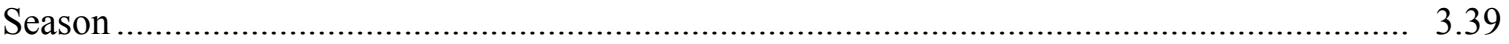

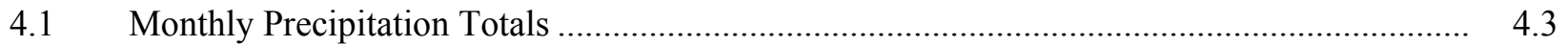

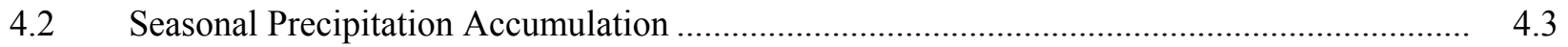

5.1 Hanford Meteorological Monitoring Network Wind Roses at 30 Feet, 1982

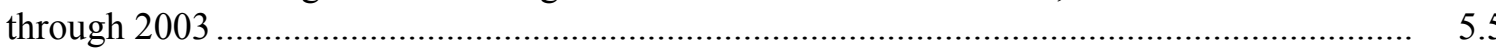

5.2 Hanford Meteorological Monitoring Network Wind Roses at 60-Meter Level, 1986

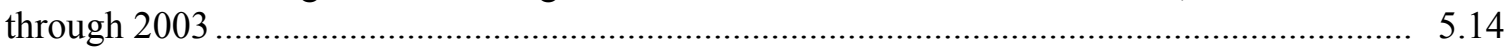

7.1 Probability of an Annual Maximum Temperature Exceeding a Given Value ........................ $\quad 7.2$

7.2 Probability of an Annual Minimum Temperature Being Less Than a Given Value................ $\quad 7.3$

7.3 Probability of Precipitation Rate Exceeding Given Values by Duration ............................... 7.4

7.4 Probability of Exceeding a Given Seasonal Snowfall .....................................................

7.5 Probability of Exceeding a Given Snowfall in a Single Storm ............................................

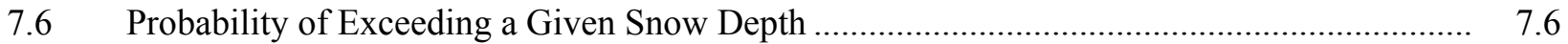

7.7 Probabilities of Peak Wind Gusts Exceeding Given Values................................................. 7.7 


\section{Tables}

1.1 Station Numbers, Names, and Codes for the Hanford Meteorological Monitoring Network

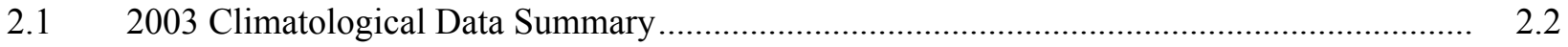

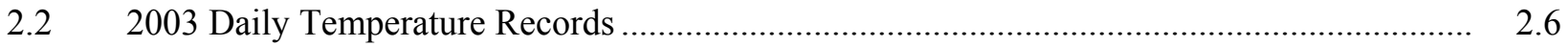

$2.3 \quad 2003$ Monthly and Seasonal Temperature and Precipitation .............................................. 2.7

2.4 2003 Monthly and Annual Average Temperatures from the Hanford Meteorological Monitoring Network

2.5 2003 Monthly and Annual Precipitation from the Hanford Meteorological Monitoring Network

2.6 2003 Monthly and Annual Average Wind Speed from the Hanford Meteorological Monitoring Network

3.1 Monthly and Annual Average Temperatures .......................................................... 3.2

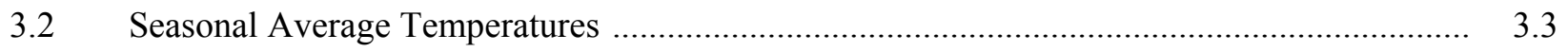

3.3 Monthly and Seasonal Number of Days with Maximum Temperatures Above or Below Certain Thresholds

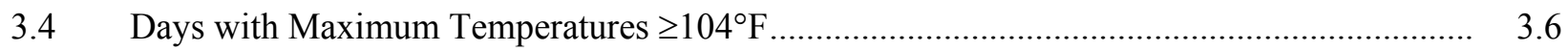

3.5 Record of Annual First and Last Dates with Maximum Temperatures $\geq 90^{\circ} \mathrm{F}$ and Minimum Temperatures $\leq 32^{\circ} \mathrm{F}$.

3.6 Monthly and Annual Maximum Temperatures.............................................................. 3.9

3.7 Monthly and Seasonal Number of Days with Minimum Temperatures at or below $32^{\circ} \mathrm{F}$ or $0^{\circ} \mathrm{F}$

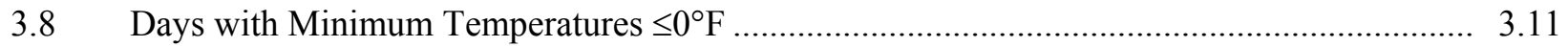

3.9 Monthly and Annual Minimum Temperatures ........................................................... 3.12

3.10 Monthly Normal Temperature and Monthly Extremes of Maximum and Minimum

Temperatures

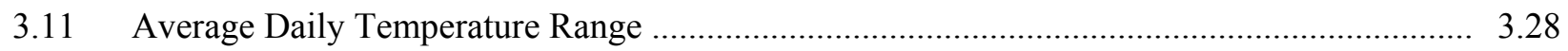

3.12 Normal and Extreme Daily Maximum and Minimum Temperatures .................................. 3.29

3.13 Monthly and Seasonal Heating-Degree Days ........................................................... 3.37 
3.14 Monthly and Annual Cooling-Degree Days …............................................................ 3.38

3.15 Subsurface Soil Temperatures at Depths of 0.5, 15, and 36 Inches................................... 3.40

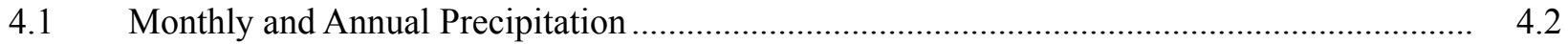

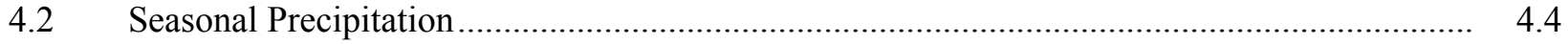

4.3 Average Number of Days with Precipitation of Specified Amount ....................................... 4.5

4.4 Monthly and Annual Averages and Extremes in Total Time with Precipitation Observed:

July 1946 through June 1971, July 1974 through December 2003 ….................................... 4.6

4.5 Total Duration of Precipitation by Month and Year ......................................................... 4.7

4.6 Monthly and Seasonal Snowfall, Including First and Last Dates of Both Trace and

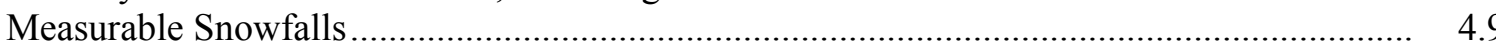

4.7 Snowfall - Greatest Amount from a Single Storm ............................................................ 4.12

4.8 Miscellaneous Snowfall Statistics, 1946 through 2003 ..................................................... 4.13

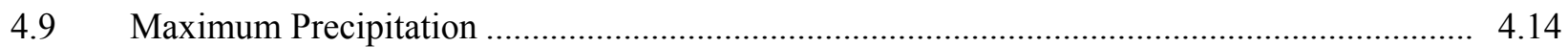

4.10 Normal and Maximum Daily Precipitation....................................................................... 4.15

5.1 Monthly and Annual Prevailing Wind Directions, Average Speeds, and Peak Gusts at 50-Foot Level, 1945 through 2003

5.2 Number of Days with Peak Gusts Above or Below Specific Thresholds at 50-Foot Level, 1945 through 2003

5.3 Frequency of Monthly and Annual Wind Direction and Speed at 50-Foot Level, 1955 through 2003

5.4 Joint Frequency Distributions for Hanford Meteorological Monitoring Network Wind Stations at 30 Feet, 1982 through 2003

5.5 Joint Frequency Distributions for Hanford Meteorological Monitoring Network Wind Stations at 60-Meter Level, 1986 through 2003.

6.1 Average Sky Cover, 1946 through 2003, and Number of Days Clear, Partly Cloudy, and Cloudy, 1954 through 2003.

6.2 Monthly and Annual Number of Days with Fog and Dense Fog, 1945 through 2003 ............. 6.3

6.3 Monthly Averages and Extremes of Psychrometric Data, 1950 through 2003 ...................... 6.4

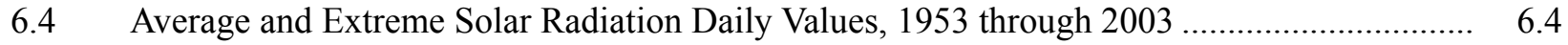

6.5 Average Number of Days of Various Meteorological Phenomena, 1945 through 2003 ........... $\quad 6.5$ 
6.6 Average and Extreme Station and Sea-Level Pressure Data, 1955 through 2003 ................. 6.6

6.7 Sunrise and Sunset Times at the Hanford Meteorological Station ..................................... 6.8

7.1 Return Periods for Annual Maximum and Minimum Temperatures .................................... 7.2

7.2 Precipitation Rates for 1 to 24 Hours Duration and Return Periods from 2 to 1,000 Years

7.3 Precipitation Amounts for 1 to 24 Hours in Periods and Return Periods from 2 to 1,000 Years

7.4 Snowfall Extremes for Return Periods from 2 to 1,000 Years ............................................. 7.5

7.5 Peak Wind Gusts for Return Periods from 2 to 10,000 Years …......................................... 7.7 


\subsection{Introduction}

The U.S. Department of Energy's Hanford Site lies within the semiarid shrub-steppe (see Appendix A) Pasco Basin of the Columbia Plateau in southeastern Washington State. The Hanford Site occupies an area of 586 square miles north of the confluence of the Snake and Yakima Rivers with the Columbia River (DOE 1999). The Columbia River flows through the northern part of the Hanford Site and, turning south, forms part of the site's eastern boundary. The Yakima River runs along part of the southern boundary and joins the Columbia River at the city of Richland, which bounds the Hanford Site on the southeast. Rattlesnake Mountain, Yakima Ridge, and Umtanum Ridge form the southwestern and western boundaries. The Saddle Mountains form the northern boundary of the Hanford Site.

The regional temperatures, precipitation, and winds are greatly affected by the presence of mountain barriers. The Cascade Range, beyond Yakima to the west, influences the climate of the Hanford Site area by its rain shadow effect. The Rocky Mountains and ranges in southern British Columbia protect the inland basin from the more severe cold polar air masses moving south across Canada and from the winter storms associated with them.

This document presents the calendar year 2003 climatological data summary for the Hanford Meteorology Station and additional information for temperature, wind, precipitation, and other meteorological parameters for the Hanford Meteorology Station and the automated stations of the Hanford Meteorological Monitoring Network. Climatological normal and extreme values for temperature and precipitation are also presented. Thirty monitoring stations are located within and near the Hanford Site (Table 1.1, Figure 1.1).

Operation of the Hanford Meteorology Station is a function of the Meteorological and Climatological Services Project funded by the U.S. Department of Energy. This project, managed by the Pacific Northwest National Laboratory, is responsible for providing ongoing meteorological and climatological services to the U.S. Department of Energy and Hanford Site contractors, primarily for emergency response activities, Hanford Site work scheduling, and general site safety. Detailed, real-time meteorological data are needed in the event of a release of hazardous material to the atmosphere from any of the Hanford Site facilities. These data can be used to model atmospheric dispersion and to estimate the environmental impact of the release. Meteorological data and weather forecasts also are necessary to ensure that operations and activities on the Hanford Site are conducted safely, particularly where specific weather conditions might affect those operations or activities. The climatological database also is used in environmental studies, environmental impact reports, facility design, and planning operations.

During the period April 1912 through March 1943, cooperative observers for the U.S. Weather Bureau (now the National Weather Service) recorded daily maximum and minimum temperatures and precipitation, including measurements of unmelted snow at the Hanford town site $\sim 10$ miles eastnortheast of the present Hanford Meteorology Station. From late 1943 until mid-1944, the U.S. Weather 
Table 1.1. Station Numbers, Names, and Codes for the Hanford Meteorological Monitoring Network

\begin{tabular}{|c|c|c|c|c|c|c|}
\hline $\begin{array}{c}\text { Station } \\
\text { Number }\end{array}$ & Station Name & $\begin{array}{c}\text { Station } \\
\text { Code }\end{array}$ & $\begin{array}{c}\text { Station } \\
\text { Elevation }(\mathrm{ft}) \\
\end{array}$ & $\begin{array}{c}\text { Longitude } \\
\text { Degrees }\end{array}$ & $\begin{array}{l}\text { Latitude } \\
\text { Degrees } \\
\end{array}$ & $\begin{array}{l}\text { Period of } \\
\text { Operation } \\
\end{array}$ \\
\hline 1 & Prosser Barricade & PROS & 480 & 119.412 & 46.392 & 01/82 - Present \\
\hline 2 & Emergency Operations Center & EOC & 1,240 & 119.537 & 46.392 & $01 / 82$ - Present \\
\hline 3 & Army Loop Road & ARMY & 565 & 119.551 & 46.489 & 01/82 - Present \\
\hline 4 & Rattlesnake Springs & RSPG & 680 & 119.700 & 46.506 & 01/82 - Present \\
\hline 5 & Edna & EDNA & 410 & 119.397 & 46.587 & 01/82 - Present \\
\hline 6 & 200 East & $200 \mathrm{E}$ & 680 & 119.521 & 46.556 & $01 / 82$ - Present \\
\hline 7 & 200 West & $200 \mathrm{~W}$ & 650 & 119.663 & 46.543 & 01/82 - Present \\
\hline 8 & Beverly & BVLY & 555 & 119.944 & 46.752 & 08/91 - Present \\
\hline 9 & Fast Flux Test Facility & FFTF & 570 & 119.360 & 46.430 & 01/82 - Present \\
\hline 10 & Yakima Barricade & YAKB & 795 & 119.726 & 46.578 & 01/82 - Present \\
\hline 11 & 300 Area & $300 \mathrm{~A}$ & 390 & 119.286 & 46.364 & 01/82 - Present \\
\hline 12 & Wye Barricade & WYEB & 550 & 119.391 & 46.482 & 01/82 - Present \\
\hline 13 & $100-\mathrm{N}$ & $100 \mathrm{~N}$ & 460 & 119.551 & 46.689 & 01/82 - Present \\
\hline 14 & WNP-2 & WPPS & 450 & 119.345 & 46.470 & 01/82 - Present \\
\hline 15 & Franklin County & FRNK & 875 & 119.238 & 46.417 & 01/82 - Present \\
\hline 16 & Gable Mountain & GABL & 1,085 & 119.460 & 46.598 & 01/82 - Present \\
\hline 17 & Ringold & RING & 620 & 119.238 & 46.545 & 01/82 - Present \\
\hline 18 & Richland Airport & $\mathrm{RICH}$ & 390 & 119.301 & 46.301 & 01/82 - Present \\
\hline 19 & Plutonium Finishing Plant-200W & PFP & 675 & 119.633 & 46.545 & 02/94 - Present \\
\hline 20 & Rattlesnake Mountain & RMTN & 3,560 & 119.593 & 46.394 & 01/82 - Present \\
\hline 21 & Hanford Meteorology Station & HMS & 733 & 119.599 & 46.563 & 01/82 - Present \\
\hline 22 & Pasco Airport & PASC & 410 & 119.114 & 46.257 & 10/87 - Present \\
\hline 23 & Gable West & GABW & 490 & 119.558 & 46.612 & 03/86 - Present \\
\hline 24 & $100-\mathrm{F}$ & $100 \mathrm{~F}$ & 410 & 119.452 & 46.635 & 03/86 - Present \\
\hline 25 & Vernita Bridge & VERN & 430 & 119.728 & 46.641 & 02/88 - Present \\
\hline 26 & Benton City & BENT & 1,055 & 119.608 & 46.290 & 02/95 - Present \\
\hline 27 & Tri-City Vocational Skills Center & VSTA & 505 & 119.201 & 46.218 & 02/91 - Present \\
\hline 28 & Roosevelt, WA & SURF & 350 & 120.218 & 45.744 & 09/94 - Present \\
\hline 29 & $100-\mathrm{K}$ & $100 \mathrm{~K}$ & 450 & 119.578 & 46.657 & 03/96 - Present \\
\hline 30 & HAMMER & HAMR & 450 & 119.326 & 46.356 & 01/98 - Present \\
\hline
\end{tabular}

Bureau recorded some meteorological operations in Richland. Then, in 1944 as part of the Manhattan Project, the Hanford Meteorology Station was established. Hourly observations began on December 7, 1944.

The Hanford Meteorology Station and its 408-foot instrument tower are located near the center of the Hanford Site between the 200 West and 200 East Areas (Figure 1.1). Hourly observations of wind direction, wind speed, and air temperature are made at multiple levels on the 408 -foot tower. Throughout this document, wind measurements from the Hanford Meteorology Station are reported from the 50-foot level and temperature measurements are reported from the 3-foot level. A variety of other meteorological variables also are measured or observed, including current weather, dew point temperature, relative humidity, 
precipitation, atmospheric pressure, cloud cover, visibility, and solar radiation. Several climatological summaries of data collected at the Hanford Meteorology Station, at the Hanford town site, and Richland monitoring locations were published over the past 30 years (Jenne and Kerns 1959; Stone et al. 1972, 1983; Hoitink and Burk 1994, 1995, 1996, 1997, 1998; Hoitink et al. 1999, 2000, 2001, 2002, 2003).

This document is composed of the following information. The 2003 calendar year summary of climatological data for the Hanford Site is contained in Section 2.0. Temperature, precipitation, wind, and miscellaneous climatological statistics are contained in Sections 3.0 through 6.0, respectively. Section 7.0 contains information on extreme value analysis. Section 8.0 lists the references cited in the document, and Section 9.0 provides a bibliography of database, computer code, and other pertinent reports. Appendix A describes in detail the climate classification of the Mid-Columbia region. Appendix B gives the station-specific wind roses and joint frequency distributions for 2003. 


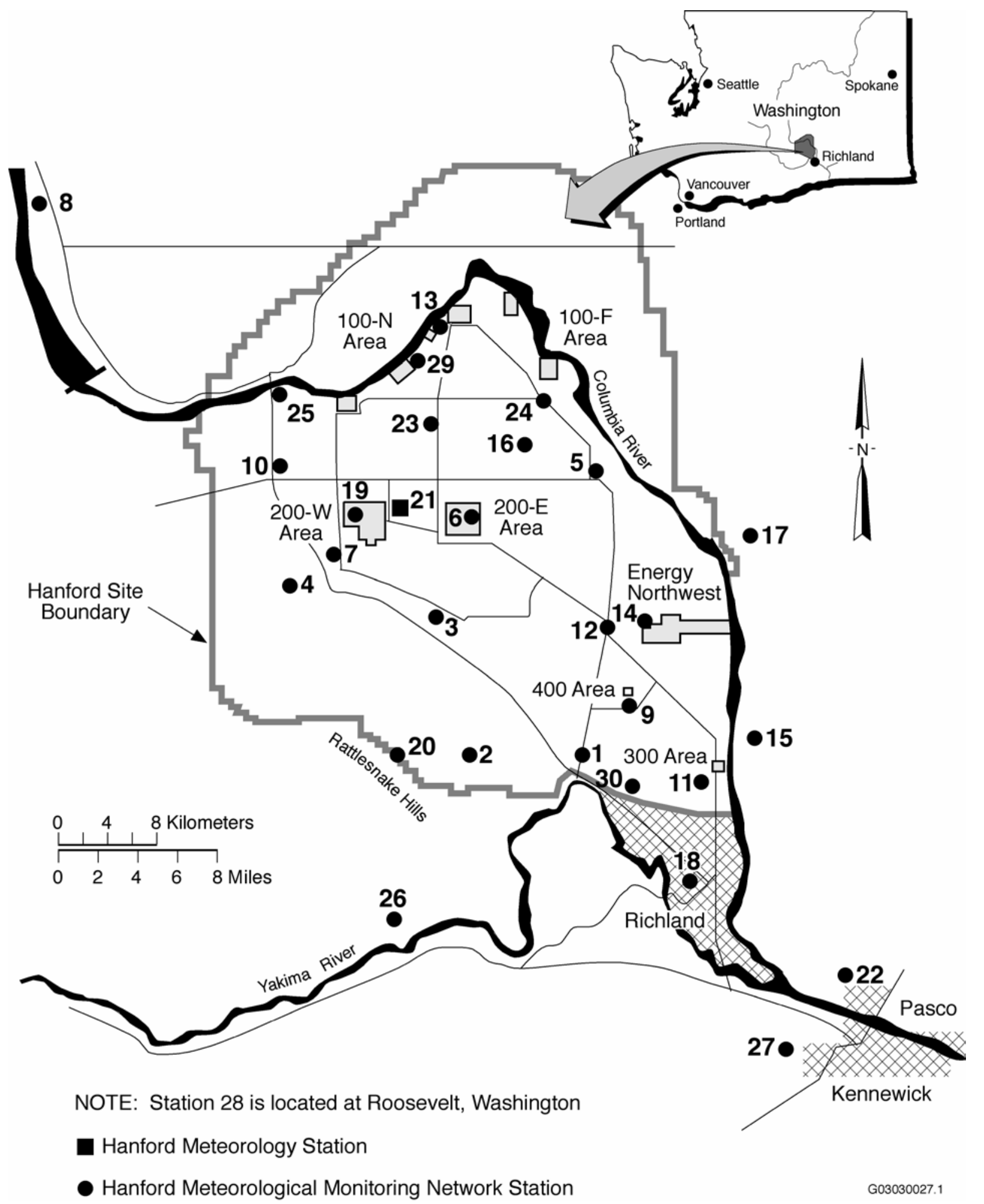

Figure 1.1. Map of the Hanford Site and Surrounding Areas (Refer to Table 1.1 for numbered locations on this map.) 


\subsection{Calendar Year 2003 Summary}

This section summarizes weather conditions for calendar year 2003 (Table 2.1). More detailed information can be found in Section 3.0 - Temperature Climatology, Section 4.0 - Precipitation Climatology, and Section 5.0 - Wind Climatology.

The 2002-2003-winter season (December 2002, January, and February 2003) was much warmer than normal, averaging $38.4^{\circ} \mathrm{F}, 4.6^{\circ}$ above normal $\left(33.8^{\circ} \mathrm{F}\right)$. The warmest winter (1966-67) averaged $40.6^{\circ} \mathrm{F}$, while the coldest (1948-49) averaged only $24.2^{\circ} \mathrm{F}$. Winter season precipitation totaled 5.05 inches, $190 \%$ of normal (2.66 inches). The wettest winter (1996-97) received 5.45 inches, while the driest (1946-47) received 0.70 inch.

The 2003 spring season (March, April, and May) was slightly warmer than normal, averaging $54.1^{\circ} \mathrm{F}$, $0.3^{\circ} \mathrm{F}$ above normal $\left(53.8^{\circ} \mathrm{F}\right)$. The warmest spring (1992) averaged $58.2^{\circ} \mathrm{F}$, while the coolest (1955) averaged $48.0^{\circ} \mathrm{F}$. Spring season 2003 precipitation totaled 2.57 inches, $163 \%$ of normal and was the sixth wettest spring on record. The wettest (1995) received 3.28 inches, while the driest (1968) received only 0.09 inch.

The 2003 summer season (June, July, and August) averaged $76.4^{\circ} \mathrm{F}, 2.7^{\circ}$ above normal $\left(73.7^{\circ} \mathrm{F}\right)$, and was the sixth warmest summer on record. The warmest summer (1958) averaged $78.2^{\circ} \mathrm{F}$, while the coolest (1980) averaged $70.2^{\circ} \mathrm{F}$. The hottest temperature was $108^{\circ} \mathrm{F}$ on July 28,29 , and 30 . Summer precipitation totaled $0.46 \mathrm{inch}, 48$ percent of normal ( $0.95 \mathrm{inch})$. The wettest summer (1950) received 2.99 inches, while the driest (1973) received 0.03 inch.

The 2003 autumn season (September, October, and November) averaged $54.8^{\circ} \mathrm{F}, 1.8^{\circ}$ above normal $\left(53.0^{\circ} \mathrm{F}\right)$. The warmest autumn $(1990)$ averaged $57.1^{\circ} \mathrm{F}$, while the coolest $(1985)$ averaged $44.5^{\circ} \mathrm{F}$. Precipitation for autumn 2003 totaled 0.46 inch, 26\% of normal (1.80 inches). The wettest autumn (1973) received 4.79 inches, while the driest (1976) received only 0.04 inch.

The following are some additional statistics for 2003:

\begin{tabular}{|c|c|c|c|c|}
\hline \multirow[b]{2}{*}{ Category } & \multirow{2}{*}{$\begin{array}{l}\text { Number } \\
\text { of Days }\end{array}$} & \multirow[b]{2}{*}{ Normal } & \multicolumn{2}{|c|}{ Record } \\
\hline & & & Maximum & Minimum \\
\hline Maximum temperatures $\geq 100^{\circ} \mathrm{F}$ & 19 & 12 & $28(1958)$ & $1(1954)$ \\
\hline Maximum temperatures $\geq 90^{\circ} \mathrm{F}$ & 72 & 52 & 79 (1967) & 29 (1980) \\
\hline Maximum temperatures $\leq 32^{\circ} \mathrm{F}$ & 5 & 23 & $58(1984-85)$ & $2(1966-67)$ \\
\hline Minimum temperatures $\geq 70^{\circ} \mathrm{F}$ & 10 & 7 & $21(1958)$ & $0(1954)$ \\
\hline Minimum temperatures $\leq 32^{\circ} \mathrm{F}$ & 98 & 105 & $143(1984-85)$ & $70(1991-92)$ \\
\hline Minimum temperatures $\leq 0^{\circ} \mathrm{F}$ & 0 & 3 & $18(1949-50)$ & $0(2001-02)$ \\
\hline Thunderstorms & 5 & 10 & $23(1948)$ & 3 (1949) \\
\hline Fog (visibility $\leq 6 \mathrm{mi})$ & 45 & 47 & $84(1985-86)$ & $22(1948-49)$ \\
\hline Dense fog (visibility $\leq 0.25 \mathrm{mi}$ ) & 25 & 24 & $42(1950-51)$ & $9(1948-49)$ \\
\hline Peak wind gusts $\leq 12 \mathrm{mph}$ & 33 & 50 & $87(1952)$ & $28(1973)$ \\
\hline Peak wind gusts $\geq 25 \mathrm{mph}$ & 173 & 156 & $192(1999)$ & $123(1952)$ \\
\hline Peak wind gusts $\geq 40 \mathrm{mph}$ & 36 & 27 & $57(1990)$ & $10(1978)$ \\
\hline Peak wind gusts $\geq 50 \mathrm{mph}$ & 6 & 6 & $18(1990)$ & $0(1985)$ \\
\hline
\end{tabular}


Table 2.1. $\quad 2003$ Climatological Data Summary

\begin{tabular}{|c|c|c|c|c|c|c|c|c|c|c|c|c|c|c|c|c|c|c|c|c|c|c|}
\hline \multirow[b]{3}{*}{ 咅 } & \multicolumn{8}{|c|}{ Temperatures, ${ }^{\circ} \mathrm{F}$} & & & & & \multicolumn{8}{|c|}{ Precipitation, inches } & \multirow{2}{*}{\multicolumn{2}{|c|}{$\begin{array}{c}\text { Relative } \\
\text { Humidity, \% }\end{array}$}} \\
\hline & \multicolumn{4}{|c|}{ Averages } & \multicolumn{4}{|c|}{ Extremes } & \multicolumn{4}{|c|}{$\begin{array}{c}\text { Degree Days } \\
\text { Base } 65,{ }^{\circ} \mathrm{F}\end{array}$} & \multirow[b]{2}{*}{$\stackrel{\sqrt[5]{0}}{\stackrel{0}{\ominus}}$} & \multirow[b]{2}{*}{ 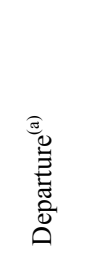 } & \multirow[b]{2}{*}{ 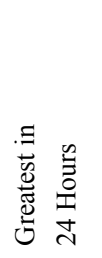 } & \multirow[b]{2}{*}{ ڤัँ } & \multicolumn{4}{|c|}{ Snow, Ice Pellets, inches } & & \\
\hline & 竞 & 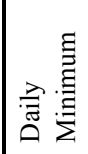 & 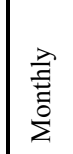 & 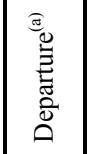 & 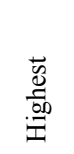 & $\stackrel{\Xi}{\tilde{\Xi}}$ & 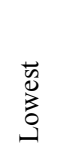 & 气̆ & 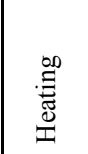 & 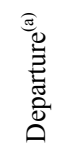 & $\begin{array}{l}.00 \\
. \\
8 \\
8\end{array}$ & 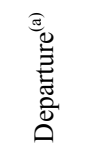 & & & & & 輥 & 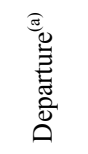 & 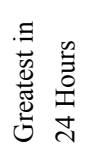 & 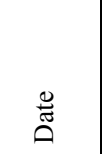 & 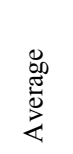 & 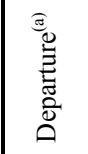 \\
\hline $\mathrm{J}$ & 43.2 & 32.7 & 38.0 & +6.2 & 67 & 26 & 22 & 10 & 838 & -190 & 0 & 0 & 1.87 & +1.00 & 0.42 & $11-12$ & 0.7 & -3.5 & 0.7 & $21-22$ & 86.5 & +9.2 \\
\hline $\mathrm{F}$ & 50.3 & 29.6 & 39.9 & +2.0 & 62 & 21 & 15 & 25 & 705 & -62 & 0 & 0 & 0.82 & +0.14 & 0.38 & $15-16$ & 0 & -2.6 & 0 & - & 66.3 & -4.2 \\
\hline M & 60.7 & 37.4 & 49.0 & +2.9 & 77 & 30 & 27 & $24^{(b)}$ & 500 & -87 & 0 & 0 & 0.26 & -0.32 & 0.10 & 26 & 0 & -0.4 & 0 & - & 55.6 & -1.0 \\
\hline A & 64.3 & 40.1 & 52.2 & -1.3 & 78 & 8 & 24 & 4 & 381 & +31 & 0 & -5 & 2.23 & +1.79 & 1.24 & $13-14$ & 0 & $-\mathrm{T}^{(\mathrm{c})}$ & 0 & - & 55.5 & -8.2 \\
\hline M & 74.4 & 47.8 & 61.1 & $|-0.7|$ & 93 & 28 & 34 & 19 & 181 & +25 & 58 & +1 & 0.08 & -0.47 & 0.06 & 12 & 0 & 0 & 0 & - & 44.4 & +1.4 \\
\hline $\mathrm{J}$ & 87.6 & 57.4 & 72.5 & +3.2 & 100 & 28 & 45 & 21 & 12 & -21 & 234 & +71 & $\mathrm{~T}$ & -0.41 & $\mathrm{~T}$ & 1 & 0 & 0 & 0 & - & 33.3 & -6.3 \\
\hline $\mathrm{J}$ & 97.3 & 63.1 & 80.2 & +3.9 & 108 & $30^{(b)}$ & 52 & 9 & 0 & -4 & 475 & +120 & 0 & -0.27 & 0 & - & 0 & 0 & 0 & - & 28.4 & -5.0 \\
\hline A & 92.2 & 60.7 & 76.5 & +1.1 & 104 & 1 & 53 & 24 & 0 & -5 & 358 & +32 & 0.46 & +0.19 & 0.36 & 7 & 0 & 0 & 0 & - & 36.6 & +1.0 \\
\hline $\mathrm{S}$ & 84.4 & 53.9 & 69.2 & +3.3 & 102 & 4 & 41 & 14 & 47 & -28 & 174 & +71 & 0.24 & -0.09 & 0.24 & $7-8$ & 0 & 0 & 0 & - & 38.8 & -3.5 \\
\hline $\mathrm{O}$ & 71.0 & 43.8 & 57.4 & +4.4 & 89 & 21 & 19 & 31 & 271 & -105 & 32 & +28 & 0.07 & -0.42 & 0.06 & $15-16$ & 0 & -0.1 & 0 & - & 49.1 & -7.3 \\
\hline $\mathrm{N}$ & 48.4 & 27.1 & 37.8 & -2.3 & 68 & 18 & 13 & 22 & 814 & +67 & 0 & 0 & 0.15 & -0.83 & 0.06 & $28-29^{(b)}$ & $\mathrm{T}$ & -2.3 & 0 & - & 62.9 & -10.8 \\
\hline $\mathrm{D}$ & 38.7 & 27.1 & 32.9 & +1.2 & 51 & 6 & 8 & 30 & 993 & -39 & 0 & 0 & 1.96 & +0.85 & 0.56 & 13 & 8.0 & +2.2 & 3.2 & $9-10$ & 87.0 & +6.9 \\
\hline $\begin{array}{c}\text { Year } \\
\text { Total }\end{array}$ & 67.7 & 43.4 & 55.6 & +2.0 & 108 & $\begin{array}{c}\mathrm{Jul} \\
30^{(\mathrm{b})}\end{array}$ & 8 & $\begin{array}{c}\text { Dec } \\
30\end{array}$ & 4,742 & -418 & 1,331 & +316 & 8.14 & +1.16 & 1.24 & $\begin{array}{c}\text { Apr } \\
13-14\end{array}$ & 8.7 & -6.7 & 3.2 & $\begin{array}{l}\text { Dec } \\
9-10\end{array}$ & 53.7 & -0.9 \\
\hline
\end{tabular}


Table 2.1. (contd)

\begin{tabular}{|c|c|c|c|c|c|c|c|c|c|c|c|c|c|c|c|c|c|c|c|c|c|}
\hline \multirow[b]{3}{*}{ 营 } & & & \multicolumn{6}{|c|}{ Solar Radiation, Langleys } & \multicolumn{5}{|c|}{$50-\mathrm{ft}$ Wind } & \multicolumn{8}{|c|}{ Number of Days } \\
\hline & \multicolumn{2}{|c|}{$\begin{array}{c}\text { Mean Sky } \\
\text { Cover, } \\
\text { Tenths }\end{array}$} & \multirow[b]{2}{*}{ 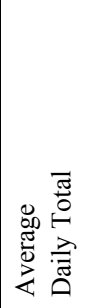 } & \multirow[b]{2}{*}{ 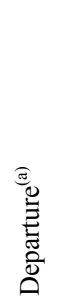 } & \multirow[b]{2}{*}{ 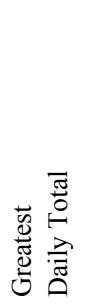 } & \multirow[b]{2}{*}{$\stackrel{\mathscr{\Xi}}{\tilde{\Xi}}$} & \multirow[b]{2}{*}{ 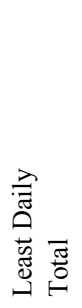 } & \multirow[b]{2}{*}{ ڤึँ } & \multirow{2}{*}{ 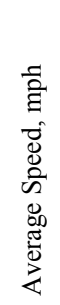 } & \multirow[b]{2}{*}{ 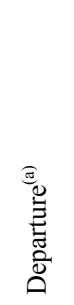 } & \multicolumn{3}{|c|}{ Peak Gusts } & \multirow[b]{2}{*}{ 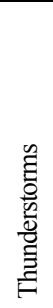 } & \multirow[b]{2}{*}{ 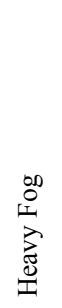 } & \multirow[b]{2}{*}{ 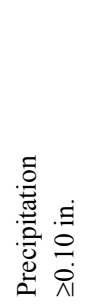 } & \multirow[b]{2}{*}{ 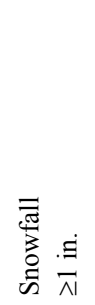 } & \multicolumn{2}{|c|}{$\begin{array}{c}\text { Maximum } \\
\text { Temperature, } \\
{ }^{\circ} \mathrm{F}\end{array}$} & \multicolumn{2}{|c|}{$\begin{array}{c}\text { Minimum } \\
\text { Temperature, }{ }^{\circ} \mathrm{F}\end{array}$} \\
\hline & 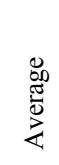 & 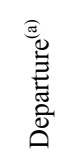 & & & & & & & & & 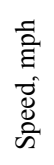 & 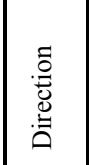 & ڤ̆ & & & & & \begin{tabular}{l}
1 \\
\multirow{2}{1}{} \\
0 \\
0
\end{tabular} & $\begin{array}{l}\stackrel{5}{\mathrm{I}} \\
\stackrel{\mathrm{V}}{\mathrm{V}}\end{array}$ & 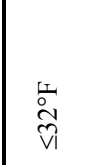 & $\begin{array}{l}\mathrm{I} \\
\stackrel{5}{5} \\
\mathrm{~V} / 1\end{array}$ \\
\hline $\mathrm{J}$ & 9.2 & +1.3 & 57 & -50 & 156 & 28 & 12 & 8 & 5.2 & -1.1 & 39 & $\mathrm{~S}$ & 2 & 0 & 7 & 8 & 0 & 0 & 0 & 14 & 0 \\
\hline $\mathrm{F}$ & 4.1 & -3.4 & 196 & +12 & 293 & 24 & 49 & 13 & 7.1 & 0 & 52 & SW & 20 & 0 & 4 & 3 & 0 & 0 & 0 & 17 & 0 \\
\hline M & 7.0 & +0.2 & 261 & -59 & 397 & 28 & 80 & 12 & 9.3 & +1.3 & 53 & W & 5 & 0 & 0 & 1 & 0 & 0 & 0 & 9 & 0 \\
\hline A & 7.3 & +0.9 & 395 & -55 & 586 & 27 & 138 & 24 & 7.8 & -1.0 & 48 & SW & 9 & 2 & 1 & 3 & 0 & 0 & 0 & 4 & 0 \\
\hline M & 6.7 & +0.8 & 482 & -67 & 635 & 29 & 239 & 30 & 8.2 & -0.7 & 41 & W & 14 & 1 & 0 & 0 & 0 & 2 & 0 & 0 & 0 \\
\hline $\mathrm{J}$ & 2.4 & -2.8 & 610 & +13 & 689 & 30 & 343 & 18 & 9.1 & +0.1 & 45 & WNW & 18 & 0 & 0 & 0 & 0 & 12 & 0 & 0 & 0 \\
\hline A & 2.5 & -0.8 & 484 & -54 & 578 & 1 & 82 & 22 & 7.6 & -0.4 & 45 & WNW & 19 & 2 & 0 & 1 & 0 & 23 & 0 & 0 & 0 \\
\hline S & 3.6 & -0.3 & 356 & -48 & 482 & 1 & 137 & 8 & 7.4 & +0.1 & 44 & WNW & 12 & 0 & 0 & 1 & 0 & 9 & 0 & 0 & 0 \\
\hline $\mathrm{O}$ & 5.5 & -0.1 & 212 & -42 & 314 & 3 & 35 & 16 & 8.6 & +2.1 & 60 & SW & 28 & 0 & 0 & 0 & 0 & 0 & 0 & 3 & 0 \\
\hline $\mathrm{N}$ & 6.1 & -1.4 & 116 & -8 & 217 & 5 & 25 & 19 & 9.9 & +3.4 & 54 & SW & 18 & 0 & 2 & 0 & 0 & 0 & 1 & 25 & 0 \\
\hline $\mathrm{D}$ & 8.0 & -0.1 & 60 & -24 & 133 & 3 & 14 & 2 & 5.5 & -0.5 & 36 & SSW & 6 & 0 & 11 & 5 & 2 & 0 & 4 & 26 & 0 \\
\hline $\begin{array}{l}\text { Year } \\
\text { Total }\end{array}$ & 5.3 & -0.6 & 322 & -32 & 689 & $\begin{array}{c}\text { Jun } \\
30\end{array}$ & 12 & $\begin{array}{c}\text { Jan } \\
8\end{array}$ & 7.8 & +0.2 & 60 & SW & $\begin{array}{l}\text { Oct } \\
28\end{array}$ & 5 & 25 & 22 & 2 & 72 & 5 & 98 & 0 \\
\hline $\begin{array}{l}\text { (b) } \mathrm{L} \\
\text { (c) } \mathrm{T} \\
\mathrm{T}=\mathrm{Tr}\end{array}$ & & & te & es. & & il. & & & & & & & & & & & & & & & \\
\hline
\end{tabular}




\subsection{Temperature}

Calendar year 2003 was warmer than normal at the Hanford Meteorology Station. The average temperature was $55.6^{\circ} \mathrm{F}, 2.0^{\circ}$ above normal $\left(53.6^{\circ} \mathrm{F}\right)$. The warmest years on record are 1992 and 1998 , which averaged $56.4^{\circ} \mathrm{F}$; the coldest year on record is 1985 , which averaged $49.6^{\circ} \mathrm{F}$. The hottest temperature of 2003 was $108^{\circ} \mathrm{F}$ on July 28,29 , and 30 ; the coldest was $8^{\circ} \mathrm{F}$ on December 30 . Calendar year 2003 recorded 72 days with maximum temperatures $\geq 90^{\circ} \mathrm{F}$ compared to a normal of 52 days, a maximum of 79 days in 1967, and a minimum of 29 days in 1980. There were 19 days with maximum temperatures $\geq 100^{\circ} \mathrm{F}$ compared to a normal of 12 days, a maximum of 28 days in 1958, and a minimum of 1 day in 1954.

Nine months during the year were warmer than normal, and 3 months cooler than normal. Five months departed from normal by more than $3^{\circ}$, with January $6.2^{\circ}$ above normal. November 2003 was $2.3^{\circ}$ below normal.

The average temperature for January 2003 was much warmer than normal, averaging $38.0^{\circ} \mathrm{F}, 6.2^{\circ}$ above normal $\left(31.8^{\circ} \mathrm{F}\right)$. The warmest January occurred in 1953 and averaged $42.5^{\circ} \mathrm{F}$, while the coldest, in 1950 , averaged $12.1^{\circ} \mathrm{F}$. Twenty-four days during January 2003 had average temperatures that were normal or above normal.

The average temperature for February 2003 was much warmer than normal, averaging $39.9^{\circ} \mathrm{F}, 2.0^{\circ}$ above normal $\left(37.9^{\circ} \mathrm{F}\right)$. The warmest February occurred in 1958 and averaged $44.5^{\circ} \mathrm{F}$, while the coldest, in 1956 , averaged $25.6^{\circ} \mathrm{F}$. Twenty-one days during February 2003 had average temperatures that were normal or above normal.

The 2002-2003-winter season (December 2002, January, and February 2003) was much warmer than normal, averaging $38.4^{\circ} \mathrm{F}, 4.6^{\circ}$ above normal $\left(33.8^{\circ} \mathrm{F}\right)$. The warmest winter $(1966-67)$ averaged $40.6^{\circ} \mathrm{F}$, while the coldest (1948-49) averaged only $24.2^{\circ} \mathrm{F}$.

The average temperature for March 2003 was warmer than normal, averaging $49.0^{\circ} \mathrm{F}, 2.9^{\circ}$ above normal $\left(46.1^{\circ} \mathrm{F}\right)$. The warmest March occurred in 1992 and averaged $51.5^{\circ} \mathrm{F}$, while the coldest, in 1955, averaged $39.4^{\circ} \mathrm{F}$.

The average temperature for April 2003 was cooler than normal, averaging $52.2^{\circ} \mathrm{F}, 1.3^{\circ}$ below normal $\left(53.5^{\circ} \mathrm{F}\right)$. The warmest April occurred in 1994 and averaged $58.2^{\circ} \mathrm{F}$, while the coldest, in 1955 , averaged $47.5^{\circ} \mathrm{F}$.

The average temperature for May 2003 was cooler than normal, averaging $61.1^{\circ} \mathrm{F}, 0.7^{\circ}$ below normal $\left(61.8^{\circ} \mathrm{F}\right)$. The warmest May occurred in 1947 and averaged $68.7^{\circ} \mathrm{F}$, while the coldest, in 1984 , averaged $56.0^{\circ} \mathrm{F}$. The first day of 2003 with a maximum of $80^{\circ} \mathrm{F}$ or greater occurred on May 22, the second latest in any year to reach that temperature.

The 2003 spring season (March, April, and May) was slightly warmer than normal, averaging $54.1^{\circ} \mathrm{F}$, $0.3^{\circ} \mathrm{F}$ above normal $\left(53.8^{\circ} \mathrm{F}\right)$. The warmest spring $(1992)$ averaged $58.2^{\circ} \mathrm{F}$, while the coolest (1955) averaged $48.0^{\circ} \mathrm{F}$. 
The average temperature for June 2003 was warmer than normal, averaging $72.5^{\circ} \mathrm{F}, 3.2^{\circ}$ above normal $\left(69.3^{\circ} \mathrm{F}\right)$. The warmest June occurred in 1992 and averaged $76.8^{\circ} \mathrm{F}$, while the coolest, in 1953 , averaged $63.0^{\circ} \mathrm{F}$. There were been 14 days with maximum temperatures $\geq 90^{\circ} \mathrm{F}$ through June 30 (normal is 11 ), and one day above $100^{\circ} \mathrm{F}$ (normal through June is 1 ).

The average temperature for July 2003 was much warmer than normal, averaging $80.2^{\circ} \mathrm{F}, 3.9^{\circ}$ above normal $\left(76.3^{\circ} \mathrm{F}\right)$. It was the seventh warmest July on record and the tenth warmest month of all time. The warmest July occurred in 1985 and averaged $82.2^{\circ} \mathrm{F}$, while the coolest, in 1993 , averaged $70.5^{\circ} \mathrm{F}$. The average maximum temperature for the last two weeks of July was $103.5^{\circ} \mathrm{F}$. There were 40 days with maximum temperatures $\geq 90^{\circ} \mathrm{F}$ through July 31 (normal is 30 ), and 13 days above $100^{\circ} \mathrm{F}$ (normal through July is 7).

The average temperature for August 2003 was warmer than normal, averaging $76.5^{\circ} \mathrm{F}, 1.1^{\circ}$ above normal $\left(75.4^{\circ} \mathrm{F}\right)$. The warmest August occurred in 1967 and averaged $81.5^{\circ} \mathrm{F}$, while the coolest, in 1964 , averaged $69.8^{\circ} \mathrm{F}$. There were 63 days with maximum temperatures $\geq 90^{\circ} \mathrm{F}$ through August 31 (normal is 47), and 16 days above $100^{\circ} \mathrm{F}$ (normal through August and for the summer is 12).

The 2003 summer season (June, July, and August) averaged $76.4^{\circ} \mathrm{F}, 2.7^{\circ}$ above normal $\left(73.7^{\circ} \mathrm{F}\right)$, and was the $6^{\text {th }}$ warmest summer on record. The warmest summer $(1958)$ averaged $78.2^{\circ} \mathrm{F}$; while the coolest (1980) averaged $70.2^{\circ} \mathrm{F}$. The hottest temperature was $108^{\circ} \mathrm{F}$ on July 28,29 , and 30.

The average temperature for September 2003 was much warmer than normal, averaging $69.2^{\circ} \mathrm{F}, 3.3^{\circ}$ above normal $\left(65.9^{\circ} \mathrm{F}\right)$. The warmest September occurred in 1990 and averaged $72.4^{\circ} \mathrm{F}$, while the coolest, in 1985 , averaged $58.8^{\circ} \mathrm{F}$. There were 72 days during 2003 with maximum temperatures $\geq 90^{\circ} \mathrm{F}$, the fourth greatest number on record (normal is 52, the most was 79 in 1967 and least was 29 in 1980). There were 19 days during this calendar year with maximum temperatures $\geq 100^{\circ} \mathrm{F}$, compared to a normal of 12 (the most was 28 days in 1958 and least was 1 day in 1954).

The average temperature for October 2003 was much warmer than normal, averaging $57.4^{\circ} \mathrm{F}, 4.4^{\circ}$ above normal $\left(53.0^{\circ} \mathrm{F}\right)$, and the third warmest on record. The warmest October occurred in 1988 and averaged $59.6^{\circ} \mathrm{F}$, while the coolest, in 1984 , averaged $47.9^{\circ} \mathrm{F}$. The period from October $16-28$ averaged $10.2^{\circ}$ above normal.

The average temperature for November 2003 was cooler than normal, averaging $37.8^{\circ} \mathrm{F}, 2.3^{\circ}$ below normal $\left(40.1^{\circ} \mathrm{F}\right)$. The warmest November occurred in 1990 and averaged $46.5^{\circ} \mathrm{F}$, while the coldest, in 1985 , averaged $24.8^{\circ} \mathrm{F}$. The period from November $1-7$ averaged $12.1^{\circ}$ below normal.

The 2003 autumn season (September, October, and November) averaged $54.8^{\circ} \mathrm{F}, 1.8^{\circ}$ above normal $\left(53.0^{\circ} \mathrm{F}\right)$. The warmest autumn $(1990)$ averaged $57.1^{\circ} \mathrm{F}$, while the coolest $(1985)$ averaged $44.5^{\circ} \mathrm{F}$.

The average temperature for December 2003 was warmer than normal, averaging $32.9^{\circ} \mathrm{F}, 1.2^{\circ}$ above normal $\left(31.7^{\circ} \mathrm{F}\right)$. The warmest December occurred in 1957 and averaged $38.5^{\circ} \mathrm{F}$, while the coldest, in 1985 , averaged $21.0^{\circ} \mathrm{F}$. 
Table 2.2 lists the daily temperature records for 2003 along with the previous record and year of occurrence. Table 2.1 lists the monthly and annual totals for numerous meteorological variables for 2003. Table 2.3 lists the 2003 monthly and seasonal temperature and precipitation compared to normals and extremes. Table 2.4, Table 2.5, and Table 2.6 list the 2003 monthly and annual average temperature, precipitation, and wind speed, respectively, from the Hanford Meteorological Monitoring Network.

Figure 2.1 depicts the 2003 observed daily maximum and minimum temperatures and the normal maximum, minimum, and mean daily temperatures for the Hanford Meteorology Station.

Table 2.2. $\quad 2003$ Daily Temperature Records (previous record and year of occurrence in parentheses)

\begin{tabular}{|c|c|c|c|c|}
\hline \multirow[b]{2}{*}{ Date } & \multicolumn{2}{|c|}{ Maximum $\left({ }^{\circ} \mathrm{F}\right)$} & \multicolumn{2}{|c|}{ Minimum $\left({ }^{\circ} \mathrm{F}\right)$} \\
\hline & High & Low & High & Low \\
\hline Jan 26 & $67(61,1971)$ & & & \\
\hline Mar 11 & & & $44^{(\mathrm{a})}\left(44,2002^{(\mathrm{b})}\right)$ & \\
\hline Mar 13 & & & $49\left(44,1998^{(\mathrm{b})}\right)$ & \\
\hline Mar 14 & & & $48(45,1961)$ & \\
\hline Mar 21 & & & $47\left(46,1998^{(\mathrm{b})}\right)$ & \\
\hline Mar 30 & $77(75,1992)$ & & & \\
\hline Mar 31 & & & $49(47,1961)$ & \\
\hline Apr 4 & & & & $24(27,1950)$ \\
\hline May 17 & & $58(61,1974)$ & & $36\left(38,1988^{(b)}\right)$ \\
\hline Jun 21 & & & & $45^{(\mathrm{a})}(45,1956)$ \\
\hline Jun 28 & & & $68^{(\mathrm{a})}(68,1987)$ & \\
\hline Jul 28 & $108^{(\mathrm{a})}\left(108,1998^{(\mathrm{b})}\right)$ & & & \\
\hline Jul 29 & $108(107,1982)$ & & & \\
\hline Jul 30 & $108(107,1971)$ & & & \\
\hline Sep 4 & $102^{(a)}(102,1988)$ & & & \\
\hline Sep 5 & $100^{(\mathrm{a})}(102,1955)$ & & & \\
\hline Sep 6 & & & $68\left(65,1957^{(\mathrm{b})}\right)$ & \\
\hline Sep 27 & & & $60^{(\mathrm{a})}(60,1949)$ & \\
\hline Sep 29 & & & $61^{\text {(a) }}(61,1989)$ & \\
\hline Oct 5 & & & $56\left(52,1988^{(\mathrm{b})}\right)$ & \\
\hline Oct 6 & $87(85,1980)$ & & & \\
\hline Oct 17 & $79(77,1997)$ & & $49^{(\mathrm{a})}(49,1955)$ & \\
\hline Oct 18 & $77(76,1973)$ & & & \\
\hline Oct 19 & & & $54(52,1992)$ & \\
\hline Oct 20 & $80(74,1978)$ & & $56(54,1973)$ & \\
\hline Oct 21 & $89^{(\mathrm{c})}\left(73,1991^{(\mathrm{b})}\right)$ & & $65^{(\mathrm{d})}(54,1963)$ & \\
\hline Oct 22 & $81(74,1959)$ & & $57(51,1952)$ & \\
\hline Oct 28 & $80\left(68,1965^{(b)}\right)$ & & & \\
\hline Nov 2 & & $37(38,1991)$ & & \\
\hline Nov 5 & & & & $18\left(20,1973^{(\mathrm{b})}\right)$ \\
\hline Nov 18 & $68(64,1946)$ & & $52(47,1954)$ & \\
\hline (a) $\mathrm{Tic}$ & & & & \\
\hline (b) $\mathrm{Mo}$ & at of several occurre & & & \\
\hline (c) Tie & me record for Octob & viously recorde & ober 3, 1958 and C & er 4, 1980). \\
\hline (d) $\mathrm{All}$ & record for October & is record $60^{\circ} \mathrm{F}$ or & ober 25,1945 , and & ber 2 and 15 , \\
\hline
\end{tabular}


Table 2.3. 2003 Monthly and Seasonal Temperature and Precipitation

\begin{tabular}{|c|c|c|c|c|c|c|c|c|c|c|c|c|c|c|}
\hline $\begin{array}{l}\text { Month/ } \\
\text { Season }\end{array}$ & $\begin{array}{c}\text { Average } \\
\text { Temperature, } \\
{ }^{\circ} \mathrm{F} \\
\end{array}$ & Departure $^{(a)}$ & $\begin{array}{c}\text { Normal, } \\
{ }^{\circ} \mathrm{F}\end{array}$ & $\begin{array}{c}\text { Warmest } \\
\text { of Record, } \\
{ }^{\circ} \mathrm{F} \\
\end{array}$ & Year & $\begin{array}{l}\text { Coolest of } \\
\text { Record, }{ }^{\circ} \mathrm{F} \\
\end{array}$ & Year & $\begin{array}{l}\text { Precipitation, } \\
\text { in. } \\
\end{array}$ & $\begin{array}{l}\text { Percent } \\
\text { of } \\
\text { Normal } \\
\end{array}$ & Normal & $\begin{array}{l}\text { Wettest of } \\
\text { Record, in. }\end{array}$ & Year & $\begin{array}{c}\text { Driest of } \\
\text { Record, } \\
\text { in. } \\
\end{array}$ & Year \\
\hline Jan & 38.0 & +6.2 & 31.8 & 42.5 & 1953 & 12.1 & 1950 & 1.87 & 215 & 0.87 & 2.47 & 1970 & 0.08 & 1977 \\
\hline Feb & 39.9 & +2.0 & 37.9 & 44.5 & 1958 & 25.6 & 1956 & 0.82 & 120 & 0.68 & 2.10 & 1961 & $\mathrm{~T}$ & $1988^{(\mathrm{b})}$ \\
\hline Mar & 49.0 & +2.9 & 46.1 & 51.5 & 1992 & 39.4 & 1955 & 0.26 & 45 & 0.58 & 1.86 & 1957 & 0.02 & 1968 \\
\hline Apr & 52.2 & -1.3 & 53.5 & 58.2 & 1994 & 47.5 & 1955 & 2.23 & 507 & 0.44 & 2.23 & 2003 & $\mathrm{~T}$ & $1999^{(\mathrm{b})}$ \\
\hline May & 61.1 & -0.7 & 61.8 & 68.7 & 1947 & 56.0 & 1984 & 0.08 & 14 & 0.55 & 2.03 & 1972 & $\mathrm{~T}$ & $1992^{(\mathrm{b})}$ \\
\hline Jun & 72.5 & +3.2 & 69.3 & 76.8 & 1992 & 63.0 & 1953 & $\mathrm{~T}$ & 0 & 0.41 & 2.92 & 1950 & $\mathrm{~T}$ & $2003^{(\mathrm{b})}$ \\
\hline Jul & 80.2 & +3.9 & 76.3 & 82.2 & 1985 & 70.5 & 1993 & 0 & 0 & 0.27 & 1.76 & 1993 & 0 & 2003 \\
\hline Aug & 76.5 & +1.1 & 75.4 & 81.5 & 1967 & 69.8 & 1964 & 0.46 & 170 & 0.27 & 1.36 & 1977 & 0 & $1988^{(\mathrm{b})}$ \\
\hline Sep & 69.2 & +3.3 & 65.9 & 72.4 & 1990 & 58.8 & 1985 & 0.24 & 73 & 0.33 & 1.34 & 1947 & 0 & $1999^{(\mathrm{b})}$ \\
\hline Oct & 57.4 & +4.4 & 53.0 & 59.6 & 1988 & 47.9 & 1984 & 0.07 & 14 & 0.49 & 2.72 & 1957 & $\mathrm{~T}$ & $1987^{(\mathrm{b})}$ \\
\hline Nov & 37.8 & -2.3 & 40.1 & 46.5 & 1990 & 24.8 & 1985 & 0.15 & 15 & 0.98 & 2.67 & 1996 & $\mathrm{~T}$ & 1976 \\
\hline Dec & 32.9 & +1.2 & 31.7 & 38.5 & 1957 & 21.0 & 1985 & 1.96 & 177 & 1.11 & 3.69 & 1996 & 0.07 & 1999 \\
\hline Winter $^{(\mathrm{c})}$ & 38.4 & +4.6 & 33.8 & 40.6 & $1966-67$ & 24.2 & $1948-49$ & 5.05 & 190 & 2.66 & 5.45 & $1996-97$ & 0.70 & $1946-47$ \\
\hline Spring & 54.1 & +0.3 & 53.8 & 58.2 & 1992 & 48.0 & 1955 & 2.57 & 163 & 1.58 & 3.28 & 1995 & 0.09 & 1968 \\
\hline Summer & 76.4 & +2.7 & 73.7 & 78.2 & 1958 & 70.2 & 1980 & 0.46 & 48 & 0.95 & 2.99 & 1950 & 0.03 & 1973 \\
\hline Autumn & 54.8 & +1.8 & 53.0 & 57.1 & 1990 & 44.5 & 1985 & 0.46 & 26 & 1.80 & 4.79 & 1973 & 0.04 & 1976 \\
\hline $\begin{array}{l}\text { Calendar } \\
\text { Year } \\
\text { Total }\end{array}$ & 55.6 & +2.0 & 53.6 & 56.4 & $1998^{(\mathrm{b})}$ & 49.6 & 1985 & 8.14 & 117 & 6.98 & 12.31 & 1995 & 2.99 & 1976 \\
\hline $\begin{array}{l}\text { (a) Dep } \\
\text { (b) Late } \\
\text { (c) Win } \\
\mathrm{T}=\text { Trace }\end{array}$ & $\begin{array}{l}\text { arture indicates } \\
\text { st of multiple oc } \\
\text { ter is December }\end{array}$ & $\begin{array}{l}\text { positive or neg } \\
\text { currences. } \\
\text { 2002, January }\end{array}$ & $\begin{array}{l}\text { ative depart } \\
\text { and Februa }\end{array}$ & $\begin{array}{l}\text { ure from } 30- \\
\text { ry } 2003 \text {. }\end{array}$ & -year (1971 & 1-2000) clima & atological $n$ & ormals. & & & & & & \\
\hline
\end{tabular}


Table 2.4. 2003 Monthly and Annual Average Temperatures $\left({ }^{\circ} \mathrm{F}\right)$ from the Hanford Meteorological Monitoring Network

\begin{tabular}{|c|c|c|c|c|c|c|c|c|c|c|c|c|c|}
\hline Station & Jan & Feb & Mar & Apr & May & Jun & Jul & Aug & Sep & Oct & Nov & Dec & Annual \\
\hline 1 PROS & 38.6 & 39.4 & 49.5 & 53.1 & 61.6 & 72.8 & 80.1 & 76.1 & 67.6 & 56.3 & 37.9 & 33.8 & 55.7 \\
\hline $2 \mathrm{EOC}$ & 38.6 & 40.2 & 49.3 & 51.8 & 60.4 & 71.9 & 79.9 & 76.6 & 69.9 & 58.2 & 39.4 & 34.2 & 55.9 \\
\hline 3 ARMY & 38.8 & 39.9 & 49.9 & 53.2 & 61.7 & 73.4 & 81.0 & 77.0 & 69.4 & 57.9 & 38.7 & 34.0 & 56.4 \\
\hline 4 RSPG & 38.6 & 39.6 & 49.5 & 52.3 & 60.5 & 72.3 & 80.0 & 76.4 & 68.5 & 57.0 & 38.2 & 33.6 & 55.6 \\
\hline 5 EDNA & 38.3 & 39.4 & 48.7 & 52.7 & 61.0 & 72.5 & 79.8 & 75.7 & 67.7 & 56.5 & 37.5 & 33.9 & 55.5 \\
\hline $6200 \mathrm{E}$ & 38.6 & 40.7 & 50.2 & 53.7 & 62.5 & 74.2 & 82.1 & 78.1 & 70.7 & 58.8 & 39.4 & 34.3 & 57.0 \\
\hline $7200 \mathrm{~W}$ & 38.0 & 38.9 & 49.5 & 52.8 & 61.0 & 72.7 & 80.6 & 76.3 & 68.5 & 56.3 & 37.2 & 33.2 & 55.5 \\
\hline 8 BVLY & 38.1 & 40.3 & 48.7 & 53.1 & 61.5 & 72.5 & 79.8 & 75.5 & 68.2 & 56.9 & 39.2 & 34.5 & 55.8 \\
\hline 9 FFTF & 38.4 & 39.8 & 49.6 & 52.9 & 61.4 & 72.6 & 80.3 & 76.5 & 68.8 & 57.3 & 38.6 & 34.0 & 55.9 \\
\hline 10 YAKB & 38.0 & 39.9 & 49.0 & 52.3 & 61.4 & 73.6 & 83.3 & 77.7 & 70.5 & 57.8 & 38.6 & 33.4 & 55.9 \\
\hline $11300 \mathrm{~A}$ & 39.4 & 39.7 & 50.1 & 53.5 & 61.4 & 72.0 & 79.3 & 76.1 & 67.7 & 56.9 & 38.7 & 34.9 & 55.8 \\
\hline 12 WYEB & 38.5 & 39.9 & 49.8 & 53.2 & 61.8 & 73.2 & 80.9 & 76.8 & 69.0 & 57.4 & 38.4 & 34.0 & 56.2 \\
\hline $13100 \mathrm{~N}$ & 37.7 & 39.3 & 47.9 & 52.2 & 60.7 & 72.3 & 79.2 & 75.6 & 68.7 & 57.1 & 38.0 & 33.9 & 55.2 \\
\hline 14 WPPS & 38.6 & 39.0 & 49.3 & 52.8 & 61.1 & 72.7 & 80.4 & 76.1 & 68.3 & 56.9 & 37.9 & 34.3 & 55.7 \\
\hline 15 FRNK & 38.2 & 39.5 & 48.9 & 51.4 & 59.3 & 69.4 & 76.1 & 72.3 & 65.7 & 55.3 & 38.1 & 33.6 & 54.1 \\
\hline $16 \mathrm{GABL}$ & 37.9 & 40.6 & 48.9 & 52.1 & 60.8 & 72.5 & 80.6 & 77.0 & 70.2 & 58.7 & 39.1 & 33.7 & 56.1 \\
\hline 17 RING & 38.5 & 39.2 & 48.5 & 51.9 & 59.5 & 69.7 & 75.6 & 72.6 & 65.5 & 55.2 & 37.9 & 34.2 & 54.1 \\
\hline $18 \mathrm{RICH}$ & 40.0 & 40.9 & 51.4 & 54.1 & 61.8 & 72.5 & 80.2 & 76.8 & 68.6 & 57.9 & 40.0 & 35.4 & 56.7 \\
\hline 19 PFP & 38.0 & 39.8 & 49.6 & 52.9 & 61.5 & 73.4 & 81.1 & 77.2 & 69.8 & 57.9 & 38.4 & 33.5 & 56.2 \\
\hline 20 RMTN & 32.8 & 32.5 & 39.4 & 41.9 & 50.8 & 62.9 & 72.3 & 68.6 & 61.7 & 50.6 & 31.1 & 29.1 & 47.9 \\
\hline $21 \mathrm{HMS}$ & 38.0 & 39.9 & 49.0 & 52.2 & 61.1 & 72.5 & 80.2 & 76.5 & 69.2 & 57.4 & 37.8 & 32.9 & 55.6 \\
\hline 22 PASC & 39.9 & 39.8 & 51.3 & 54.3 & 62.0 & 72.5 & 80.2 & 76.6 & 67.6 & 57.0 & 39.4 & 35.0 & 56.4 \\
\hline 23 GABW & 37.6 & 38.6 & 48.1 & 52.1 & 60.8 & 72.7 & 80.4 & 75.9 & 68.0 & 56.0 & 36.5 & 33.2 & 55.1 \\
\hline $24100 \mathrm{~F}$ & 38.1 & 39.1 & 48.5 & 52.7 & 61.2 & 72.7 & 79.9 & 75.8 & 68.0 & 56.6 & 37.5 & 33.9 & 55.4 \\
\hline 25 VERN & 38.9 & 40.7 & 49.2 & 53.2 & 61.9 & 73.4 & 81.2 & 77.7 & 70.5 & 58.6 & 40.0 & 35.1 & 56.8 \\
\hline 26 BENT & 38.8 & 39.6 & 48.6 & 51.8 & 60.8 & 70.3 & 77.7 & 73.9 & 67.5 & 56.1 & 38.0 & 33.6 & 54.8 \\
\hline 27 VSTA & 40.7 & 41.4 & 51.8 & 54.5 & 61.2 & 72.5 & 80.7 & 77.0 & 68.9 & 58.3 & 40.7 & 36.2 & 56.7 \\
\hline 28 SURF & 40.7 & 40.8 & 49.6 & 52.5 & 60.7 & 71.2 & 80.1 & 75.7 & 68.5 & 57.3 & 40.5 & 37.4 & 56.3 \\
\hline $29100 \mathrm{~K}$ & 38.3 & 39.8 & 48.6 & 52.8 & 61.7 & 73.1 & 80.3 & 76.5 & 69.3 & 57.4 & 38.2 & 34.1 & 55.9 \\
\hline 30 HAMR & 39.3 & 40.0 & 50.5 & 53.6 & 61.6 & 72.2 & 80.0 & 76.4 & 68.3 & 57.3 & 39.1 & 34.7 & 56.2 \\
\hline
\end{tabular}


Table 2.5. 2003 Monthly and Annual Precipitation (inches) from the Hanford Meteorological Monitoring Network ${ }^{(a)}$

\begin{tabular}{|c|c|c|c|c|c|c|c|c|c|c|c|c|c|}
\hline Station & Jan & Feb & Mar & Apr & May & Jun & Jul & Aug & Sep & Oct & Nov & Dec & Annual \\
\hline 1 PROS & 1.56 & 0.69 & 0.28 & 0.95 & 0.20 & 0.00 & 0.00 & 0.58 & 0.29 & 0.06 & 0.16 & 1.27 & 6.04 \\
\hline $2 \mathrm{EOC}$ & 1.98 & 0.73 & 0.63 & 1.12 & 0.35 & 0.00 & 0.00 & 0.89 & 0.02 & 0.15 & 0.37 & 1.74 & 7.98 \\
\hline 3 ARMY & 0.59 & 0.50 & 0.20 & 1.32 & 0.33 & 0.00 & 0.00 & 0.53 & 0.24 & 0.20 & 0.14 & 1.38 & 5.43 \\
\hline 4 RSPG & 1.51 & 0.62 & 0.25 & 1.57 & 0.40 & 0.00 & 0.00 & 0.34 & 0.13 & 0.10 & 0.16 & 1.75 & 6.83 \\
\hline $6200 \mathrm{E}$ & 1.57 & 0.61 & 0.27 & 1.89 & 0.08 & 0.00 & 0.00 & 0.35 & 0.19 & 0.05 & 0.10 & 1.22 & 6.33 \\
\hline $7200 \mathrm{~W}$ & 1.73 & 0.78 & 0.21 & 1.93 & 0.25 & 0.00 & 0.00 & 0.30 & 0.08 & 0.04 & 0.11 & 1.41 & 6.84 \\
\hline 8 BVLY & 1.02 & 0.31 & 0.11 & 1.53 & 0.09 & 0.00 & 0.00 & 0.21 & 0.04 & 0.02 & 0.01 & 1.07 & 4.41 \\
\hline 9 FFTF & 1.08 & 0.54 & 0.19 & 1.16 & 0.34 & 0.00 & 0.00 & 0.40 & 0.22 & 0.08 & 0.17 & 0.65 & 4.83 \\
\hline 10 YAKB & 1.76 & 0.85 & 0.27 & 2.07 & 0.15 & 0.00 & 0.00 & 0.32 & 0.09 & 0.04 & 0.04 & 1.70 & 7.29 \\
\hline $11300 \mathrm{~A}$ & 1.31 & 0.55 & 0.48 & 1.13 & 0.14 & 0.00 & 0.00 & 0.10 & 0.29 & 0.06 & 0.29 & 1.36 & 5.71 \\
\hline 12 WYEB & 1.73 & 0.70 & 0.29 & 1.25 & 0.39 & 0.00 & 0.00 & 0.49 & 0.13 & 0.14 & 0.28 & 1.58 & 6.98 \\
\hline $13100 \mathrm{~N}$ & 1.79 & 0.49 & 0.21 & 1.56 & 0.00 & 0.00 & 0.00 & 0.37 & 0.05 & 0.05 & 0.14 & 1.37 & 6.03 \\
\hline 14 WPPS & 1.69 & 0.72 & 0.28 & 1.43 & 0.29 & 0.00 & 0.00 & 0.47 & 0.17 & 0.14 & 0.30 & 1.54 & 7.03 \\
\hline 17 RING & 1.83 & 0.62 & 0.53 & 1.47 & 0.05 & 0.00 & 0.00 & 0.25 & 0.09 & 0.11 & 0.48 & 1.51 & 6.94 \\
\hline $18 \mathrm{RICH}$ & 1.17 & 0.52 & 0.22 & 0.54 & 0.18 & 0.00 & 0.00 & 0.17 & 0.20 & 0.10 & 0.22 & 1.15 & 4.47 \\
\hline 20 RMTN & 1.88 & 0.70 & 0.36 & 0.85 & 0.61 & 0.00 & 0.00 & 0.84 & 0.15 & 0.18 & 0.15 & 0.87 & 6.59 \\
\hline $21 \mathrm{HMS}$ & 1.87 & 0.82 & 0.26 & 2.23 & 0.08 & 0.00 & 0.00 & 0.46 & 0.24 & 0.07 & 0.15 & 1.96 & 8.14 \\
\hline 22 PASC & 1.81 & 0.84 & 0.31 & 0.86 & 0.51 & 0.00 & 0.00 & 0.02 & 0.23 & 0.21 & 0.30 & 1.41 & 6.50 \\
\hline $24100 \mathrm{~F}$ & 1.65 & 0.56 & 0.44 & 1.79 & 0.00 & 0.00 & 0.00 & 0.42 & 0.11 & 0.14 & 0.16 & 1.97 & 7.24 \\
\hline 26 BENT & 2.03 & 0.97 & 0.64 & 1.40 & 0.12 & 0.00 & 0.00 & 0.56 & 0.19 & 0.24 & 0.31 & 1.89 & 8.35 \\
\hline 27 VSTA & 1.47 & 0.67 & 0.24 & 0.43 & 0.43 & 0.00 & 0.00 & 0.10 & 0.16 & 0.22 & 0.28 & 1.41 & 5.41 \\
\hline 28 SURF & 2.42 & 0.72 & 0.82 & 0.89 & 0.20 & 0.00 & 0.00 & 0.44 & 0.05 & 0.45 & 0.63 & 1.93 & 8.55 \\
\hline $29100 \mathrm{~K}$ & -- & -- & 0.26 & 2.05 & 0.01 & 0.00 & 0.00 & 0.30 & 0.10 & 0.15 & 0.16 & 1.74 & (b) \\
\hline
\end{tabular}

(a) Stations 5, 15, 16, 19, 23, and 25 are solar powered; therefore, insufficient power is available to operate the heated tippingbucket precipitation gauges.

(b) Incomplete data.

NOTE: Dashes indicate no data are available. 
Table 2.6. $\quad 2003$ Monthly and Annual Average Wind Speed (mph) from the Hanford Meteorological Monitoring Network

\begin{tabular}{|c|c|c|c|c|c|c|c|c|c|c|c|c|c|}
\hline Station & Jan & Feb & Mar & Apr & May & Jun & Jul & Aug & Sep & Oct & Nov & Dec & Annual \\
\hline 1 PROS & 5.3 & 6.8 & 9.2 & 7.9 & 8.0 & 7.7 & 6.7 & 6.4 & 6.3 & 7.6 & 8.8 & 6.8 & 7.3 \\
\hline $2 \mathrm{EOC}$ & 6.9 & 9.9 & 12.5 & 9.8 & 9.0 & 8.2 & 7.0 & 7.5 & 9.0 & 10.9 & 12.8 & 8.0 & 9.3 \\
\hline 3 ARMY & 3.7 & 5.3 & 7.4 & 7.1 & 7.2 & 7.2 & 5.3 & 5.6 & 6.1 & 7.8 & 7.5 & 5.1 & 6.3 \\
\hline 4 RSPG & 4.8 & 7.8 & 9.3 & 8.1 & 8.0 & 8.0 & 7.5 & 7.6 & 7.7 & 8.8 & 8.6 & 5.4 & 7.6 \\
\hline 5 EDNA & 4.3 & 5.6 & 7.3 & 6.6 & 6.5 & 7.3 & 6.6 & 6.0 & 5.9 & 7.2 & 6.7 & 4.9 & 6.2 \\
\hline $6200 \mathrm{E}$ & 4.4 & 6.5 & 8.7 & 7.7 & 8.1 & 9.3 & 8.4 & 7.6 & 7.5 & 8.2 & 7.6 & 5.4 & 7.5 \\
\hline $7200 \mathrm{~W}$ & 3.9 & 5.5 & 8.1 & 6.9 & 6.9 & 7.8 & 6.8 & 6.1 & 5.8 & 7.1 & 6.7 & 4.4 & 6.3 \\
\hline 8 BVLY & 4.5 & 5.5 & 6.5 & 5.9 & 6.1 & 7.4 & 6.4 & 5.8 & 5.3 & 5.9 & 5.9 & 4.9 & 5.8 \\
\hline 9 FFTF & 5.8 & 7.9 & 10.0 & 8.6 & 8.5 & 8.6 & 7.3 & 7.3 & 7.5 & 9.1 & 9.5 & 6.8 & 8.1 \\
\hline 10 YAKB & 4.9 & 7.1 & 8.9 & 7.9 & 8.6 & 9.8 & 8.7 & 7.9 & 7.4 & 8.4 & 8.0 & 5.6 & 7.8 \\
\hline $11300 \mathrm{~A}$ & 5.6 & 7.3 & 9.4 & 8.2 & 8.3 & 7.9 & 7.0 & 6.8 & 6.3 & 8.1 & 9.1 & 6.6 & 7.5 \\
\hline 12 WYEB & 5.0 & 6.9 & 8.6 & 7.8 & 7.8 & 8.1 & 7.0 & 6.7 & 6.9 & 8.5 & 8.2 & 6.0 & 7.3 \\
\hline $13100 \mathrm{~N}$ & 3.7 & 5.6 & 6.7 & 6.3 & 6.7 & 7.6 & 6.5 & 5.6 & 5.8 & 6.6 & 5.9 & 4.0 & 5.9 \\
\hline 14 WPPS & 5.0 & 6.4 & 8.4 & 7.6 & 7.1 & 7.4 & 6.4 & 6.2 & 6.3 & 7.7 & 7.9 & 5.8 & 6.8 \\
\hline 15 FRNK & 5.3 & 7.0 & 8.6 & 7.2 & 6.6 & 6.4 & 5.6 & 5.4 & 5.7 & 7.0 & 7.9 & 5.8 & 6.5 \\
\hline 16 GABL & 7.0 & 9.8 & 13.1 & 11.5 & 11.5 & 12.8 & 11.7 & 10.7 & 11.2 & 12.5 & 12.1 & 8.2 & 11.0 \\
\hline 17 RING & 4.7 & 6.3 & 7.8 & 6.5 & 6.4 & 6.2 & 5.4 & 5.3 & 5.7 & 7.3 & 7.7 & 5.2 & 6.2 \\
\hline $18 \mathrm{RICH}$ & 4.5 & 6.0 & 8.1 & 6.9 & 6.8 & 6.2 & 5.2 & 5.2 & 5.1 & 6.5 & 7.4 & 5.6 & 6.1 \\
\hline 19 PFP & 3.3 & 4.3 & 6.6 & 5.5 & 5.5 & 6.0 & 5.1 & 4.7 & 4.5 & 5.9 & 5.1 & 3.4 & 5.0 \\
\hline 20 RMTN & 15.9 & 19.3 & 24.2 & 18.3 & 15.6 & 13.6 & 11.0 & 12.8 & 16.8 & 22.8 & 22.9 & 17.9 & 17.6 \\
\hline $21 \mathrm{HMS}$ & 5.2 & 7.1 & 9.3 & 7.8 & 8.2 & 9.1 & 8.4 & 7.6 & 7.4 & 8.6 & 9.9 & 5.5 & 7.8 \\
\hline 22 PASC & 3.9 & 4.9 & 7.6 & 6.4 & 6.1 & 5.6 & 4.6 & 4.5 & 4.3 & 5.7 & 6.4 & 4.3 & 5.4 \\
\hline $23 \mathrm{GABW}$ & 4.1 & 5.7 & 7.3 & 6.7 & 7.1 & 8.5 & 7.6 & 6.5 & 6.2 & 6.9 & 6.1 & 4.3 & 6.4 \\
\hline $24100 \mathrm{~F}$ & 3.6 & 5.4 & 6.9 & 6.5 & 6.4 & 7.4 & 6.3 & 5.8 & 5.7 & 7.0 & 6.2 & 4.0 & 5.9 \\
\hline 25 VERN & 5.3 & 6.8 & 7.6 & 7.3 & 8.3 & 9.7 & 8.8 & 7.9 & 7.3 & 7.5 & 7.0 & 5.9 & 7.4 \\
\hline 26 BENT & 4.7 & 6.9 & 7.6 & 6.5 & 6.1 & 6.1 & 5.6 & 5.6 & 5.8 & 6.7 & 6.4 & 4.9 & 6.1 \\
\hline 27 VSTA & 4.2 & 5.2 & 8.4 & 7.0 & 6.5 & 6.3 & 5.3 & 5.3 & 5.0 & 6.0 & 7.0 & 4.8 & 5.9 \\
\hline $28 \mathrm{SURF}$ & 5.1 & 9.1 & 11.1 & 10.3 & 11.6 & 12.6 & 12.1 & 10.8 & 9.3 & 9.1 & 8.9 & 6.9 & 9.7 \\
\hline $29100 \mathrm{~K}$ & 3.8 & 5.4 & 7.1 & 6.6 & 7.1 & 8.1 & 6.8 & 6.0 & 5.8 & 6.7 & 5.7 & 4.1 & 6.1 \\
\hline 30 HAMR & 4.9 & 6.6 & 8.4 & 7.4 & 7.4 & 7.2 & 5.9 & 6.0 & 5.9 & 7.2 & 8.4 & 6.2 & 6.8 \\
\hline
\end{tabular}


Daily Temperatures - 2003

Hanford Meteorological Station

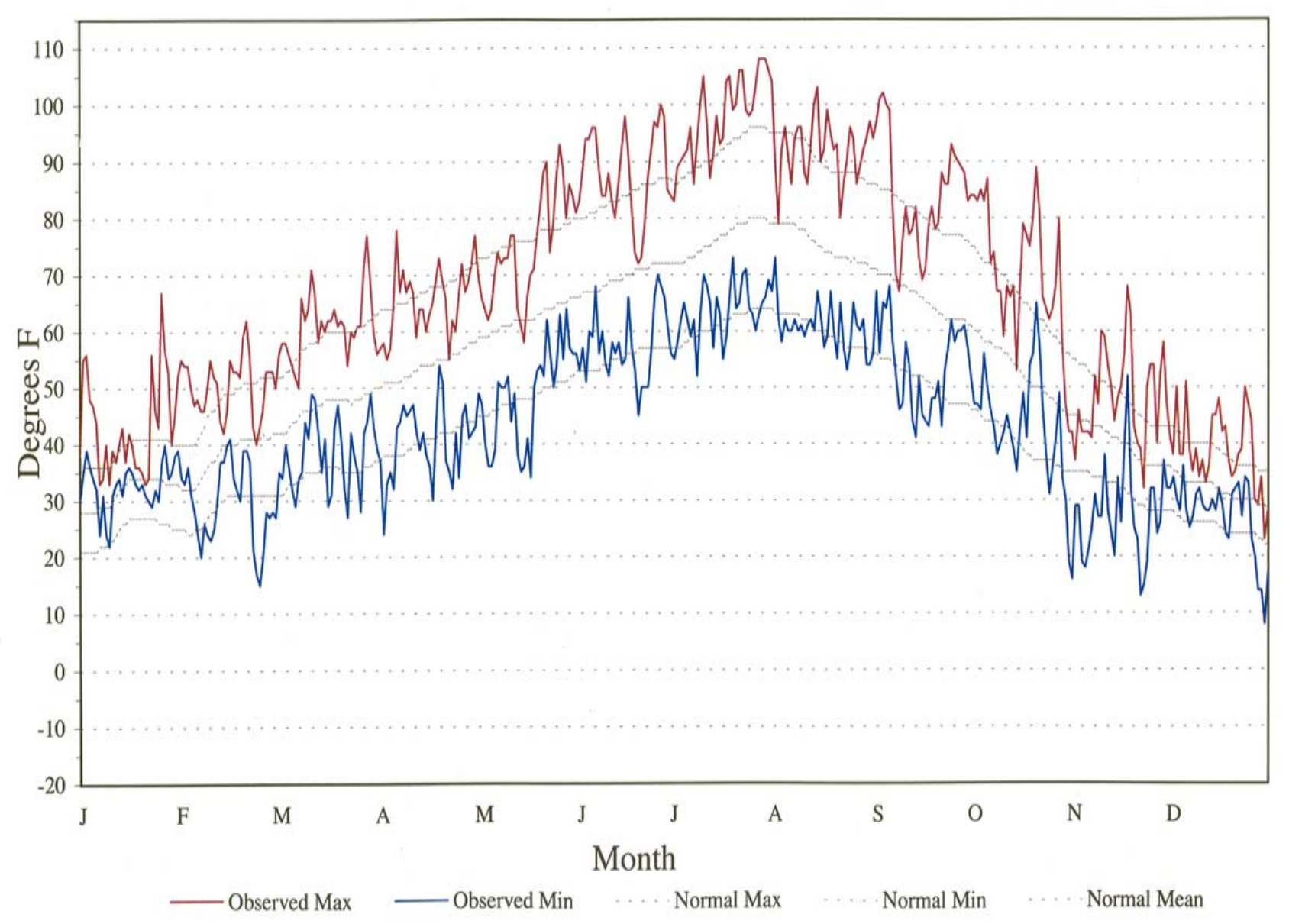

Figure 2.1. 2003 Observed Daily Temperatures from the Hanford Meteorology Station 


\subsection{Precipitation}

Precipitation for 2003 totaled 8.14 inches, $117 \%$ of normal ( 6.98 inches). The wettest year was 1995 with 12.31 inches; the driest was 1976 with only 2.99 inches. Calendar year snowfall totaled 8.7 inches, compared to an annual normal snowfall of 15.4 inches. The greatest calendar year snowfall was 57.5 inches (1996); the least was 0.6 inch (1999).

Precipitation for January 2003 was 1.87 inches, $215 \%$ of normal $(0.87$ inch). This was the fifth wettest January on record. The wettest, in 1970, received 2.47 inches, while the driest, in 1977, received $0.08 \mathrm{inch}$. Snowfall for January 2003 totaled $0.7 \mathrm{inch}$, compared to a normal of 4.2 inches. Total snow season snowfall is only 1.3 inches, compared to a normal through January of 12.4 inches.

Precipitation for February 2003 was 0.82 inch, $120 \%$ of normal ( 0.68 inch). The wettest February occurred in 1961 and received 2.10 inches, while the driest, in 1988 and earlier years, received only a trace. There was no snow recorded in February 2003, compared to a normal of 2.6 inches. Total snow season snowfall is only 1.3 inches, compared to a normal through February of 15.0 inches.

Winter season precipitation totaled 5.05 inches, $190 \%$ of normal (2.66 inches). The wettest winter (1996-97) received 5.45 inches, while the driest (1946-47) received $0.70 \mathrm{inch}$.

Precipitation for March 2003 was 0.26 inches, $45 \%$ of normal ( 0.58 inch). The wettest March occurred in 1957 and received 1.86 inches, while the driest, in 1968, received 0.02 inch. There was no snow recorded in March 2003, compared to a normal of 0.4 inch. Total snow season snowfall was only 1.3 inches, compared to a normal of 15.4 inches.

April 2003 established several precipitation records. Precipitation for the month totaled 2.23 inches, $507 \%$ of normal ( 0.44 inches), the wettest April on record. The previous wettest April occurred in 1995 and received 1.54 inches, while the driest, in 1999 and earlier years, received only a trace. It was also the thirteenth wettest month at the Hanford Meteorological Station since record keeping began in December 1944. The wettest month ever was December 1996 with 3.69 inches. There were two days in April 2003 with precipitation totals $>0.50$ inch (an April record), with one 24-hour period receiving 1.24 inches, a record 24-hour precipitation total for any April, and the fifth greatest 24-hour amount in the history of the Hanford Meteorological Station. The greatest 24-hour precipitation amount was 1.91 inches on October 1-2, 1957.

Precipitation for May 2003 was 0.08 inch, 14\% of normal ( 0.55 inch). The wettest May occurred in 1972 and received 2.03 inches, while the driest May, in 1992 and earlier years, received only a trace.

Spring season 2003 precipitation totaled 2.57 inches, $163 \%$ of normal and was the sixth wettest spring on record. The wettest (1995) received 3.28 inches, while the driest (1968) received only 0.09 inch.

Precipitation for June 2003 totaled only a trace, compared to a June normal of 0.41 inch. June 1979 and 1986 also received only a trace of precipitation. The wettest June occurred in 1950 and received 2.92 inches. 
There was no precipitation recorded during July 2003, compared to a July normal of $0.27 \mathrm{inch}$. This is the first time that any July has been totally without rainfall; numerous previous months of July have received only a trace. This was also only the fifth month not to receive any precipitation - that also happened in August 1955, August 1988, September 1991, and September 1999. The last July to record only a trace was in 1980. The wettest July on record, in 1993, received 1.73 inches. The last measurable precipitation at the Hanford Meteorology Station occurred on May 16 (a period of 76 days, and currently the third longest period on record without measurable precipitation).

Precipitation for August 2003 totaled 0.46 inch, 170\% of normal ( 0.27 inch). The wettest August on record, in 1977, received 1.36 inches; and the driest, in 1988 and 1955, received no precipitation. The period from June 2 through August 1 (61 days) was the second longest period on record with no precipitation (the longest period was 66 days, from July 14, 1988 through September 17, 1988).

Precipitation for September 2003 totaled 0.24 inch, 73\% of normal ( 0.33 inch). The wettest September on record, in 1947, received 1.34 inches; and the driest, in 1991 and 1999, received no precipitation.

Precipitation for October 2003 totaled 0.07 inch, 14\% of normal ( 0.49 inch). The wettest October on record, in 1957, received 2.72 inches; and the driest $(1972,1978$, and 1987) received only a trace.

Precipitation for November 2003 totaled 0.15 inch, 15\% of normal ( 0.98 inch). The wettest November on record, in 1996, received 2.67 inches; and the driest (1976) received only a trace. A trace of snow was received in November 2003 (normal for November is 2.3 inches; the greatest November total was 18.3 inches in 1985).

Precipitation for autumn 2003 totaled 0.46 inch, 26\% of normal (1.80 inches). The wettest autumn (1973) received 4.79 inches, while the driest (1976) received only 0.04 inch.

Precipitation for December 2003 totaled 1.96 inches, 177\% of normal (1.11 inches). The wettest December on record, in 1996, received 3.69 inches; and the driest (1999) received 0.07 inch. The period from November 28 through December 14 (17 days) established a new record for consecutive days with precipitation. The previous record was 15 days from December 19, 1985 through January 2, 1986 (and three previous occasions). Snowfall for December 2003 totaled 8.0 inches, compared to a normal of 5.8 inches. Total snowfall for the 2003-2004 snow season (through December) was 8.0 inches, compared to a normal of 8.2 inches.

\subsection{Wind}

The average wind speed for 2003 was 7.8 miles per hour ( $\mathrm{mph}$ ), which was $0.2 \mathrm{mph}$ above normal (7.6 mph). The windiest year was 1999, which averaged $8.8 \mathrm{mph}$, while 1957 was the year with the lightest winds, averaging $6.3 \mathrm{mph}$. The peak gust for 2003 was $60 \mathrm{mph}$ on October 28 .

The average wind speed for January 2003 was $5.2 \mathrm{mph}, 1.1 \mathrm{mph}$ below normal (6.3 $\mathrm{mph}$ ). The windiest January on record averaged $10.3 \mathrm{mph}$ (1972), while the January with the lightest winds (1985) averaged $2.9 \mathrm{mph}$. The peak gust for the month was $39 \mathrm{mph}$ on January 2. The record wind gust for January was $80 \mathrm{mph}$ in 1972. 
The average wind speed for February 2003 was $7.1 \mathrm{mph}$, right at normal $(7.1 \mathrm{mph})$. The windiest February on record averaged $11.1 \mathrm{mph}$ (1999), while the February with the lightest winds (1963) averaged $4.6 \mathrm{mph}$. The peak gust for the month was $52 \mathrm{mph}$ on February 20. The record wind gust for February was $65 \mathrm{mph}$ in 1971.

The average wind speed for March 2003 was $9.3 \mathrm{mph}, 1.3 \mathrm{mph}$ above normal ( $8.0 \mathrm{mph}$ ). The windiest March on record averaged $10.7 \mathrm{mph}$ (1977 and earlier years), while the March with the lightest winds (1958) averaged $5.9 \mathrm{mph}$. The peak gust for the month was $53 \mathrm{mph}$ on March 5. The record wind gust for March was $70 \mathrm{mph}$ in 1956.

The average wind speed for April 2003 was $7.8 \mathrm{mph}, 1.0 \mathrm{mph}$ below normal ( $8.8 \mathrm{mph})$. The windiest April on record averaged $11.1 \mathrm{mph}$ (1972 and earlier years), while the April with the lightest winds (1958 and earlier years) averaged $7.4 \mathrm{mph}$. The peak gust for the month was $48 \mathrm{mph}$ on April 9. The record wind gust for April was 73 mph in 1972.

The average wind speed for May 2003 was $8.2 \mathrm{mph}, 0.7 \mathrm{mph}$ below normal $(8.9 \mathrm{mph})$. The windiest May on record averaged $10.7 \mathrm{mph}$ (1983), while the May with the lightest winds (1957) averaged $5.8 \mathrm{mph}$. The peak gust for the month was $41 \mathrm{mph}$ on May 14. The record wind gust for May was $71 \mathrm{mph}$ in 1948 .

The average wind speed for June 2003 was $9.1 \mathrm{mph}, 0.1 \mathrm{mph}$ above normal (9.0 mph). The windiest June on record averaged $10.7 \mathrm{mph}$ (1983), while the June with the lightest winds (1950) averaged $7.7 \mathrm{mph}$. The peak gust for the month was $45 \mathrm{mph}$ on June 18. The record wind gust for June was $72 \mathrm{mph}$ in 1957.

The average wind speed for July 2003 was $8.4 \mathrm{mph}, 0.2 \mathrm{mph}$ below normal ( $8.6 \mathrm{mph}$ ). The windiest July on record averaged $10.7 \mathrm{mph}$ (1983), while the July with the lightest winds (1955) averaged $6.8 \mathrm{mph}$. The peak gust for the month was $41 \mathrm{mph}$ on July 12. The record wind gust for July was $69 \mathrm{mph}$ in 1979.

The average wind speed for August 2003 was $7.6 \mathrm{mph}, 0.4 \mathrm{mph}$ below normal $(8.0 \mathrm{mph})$. The windiest August on record averaged $9.5 \mathrm{mph}$ (1996), while the August with the lightest winds (1956) averaged $6.0 \mathrm{mph}$. The peak gust for the month was $45 \mathrm{mph}$ on August 19. The record wind gust for July was $66 \mathrm{mph}$ in 1961.

The average wind speed for September 2003 was $7.4 \mathrm{mph}, 0.1 \mathrm{mph}$ above normal (7.3 $\mathrm{mph})$. The windiest September on record averaged $9.2 \mathrm{mph}$ (1961), while the September with the lightest winds (1957) averaged $5.4 \mathrm{mph}$. The peak gust for the month was $44 \mathrm{mph}$ on September 12 and 19. The record wind gust for September was $65 \mathrm{mph}$ in 1953.

The average wind speed for October 2003 was $8.6 \mathrm{mph}, 2.1 \mathrm{mph}$ above normal ( $6.5 \mathrm{mph})$. The windiest October on record averaged $9.1 \mathrm{mph}$ (1946), while the October with the lightest winds (1952) averaged $4.4 \mathrm{mph}$. The peak gust for the month was $60 \mathrm{mph}$ on October 28. The 8 days with peak gusts $\geq 40 \mathrm{mph}$ tied the record for that category, also occurring in 1967. The record wind gust for October was $72 \mathrm{mph}$ in 1997. 
The average wind speed for November 2003 was $9.9 \mathrm{mph}, 3.4 \mathrm{mph}$ above normal ( $6.5 \mathrm{mph})$. The windiest November on record averaged $10.0 \mathrm{mph}$ (1990), while the November with the lightest winds (1956) averaged $2.9 \mathrm{mph}$. The peak gust for the month was $54 \mathrm{mph}$ on November 18. The record wind gust for November was $67 \mathrm{mph}$ in 1993.

The average wind speed for December 2003 was $5.6 \mathrm{mph}, 0.4 \mathrm{mph}$ below normal (6.0 mph). The windiest December on record averaged $8.3 \mathrm{mph}$ (1968), while the December with the lightest winds (1985) averaged $3.3 \mathrm{mph}$. The peak gust for the month was $36 \mathrm{mph}$ on December 6 . The record wind gust for December was $71 \mathrm{mph}$ in 1955.

Figure 2.2 and Figure 2.3 give a composite of the wind roses (at the 30-foot and 60-meter levels, respectively) from the Hanford Meteorological Monitoring Network for 2003. The Appendix B gives the individual 2003 wind roses from the Hanford Meteorological Monitoring Network stations. 


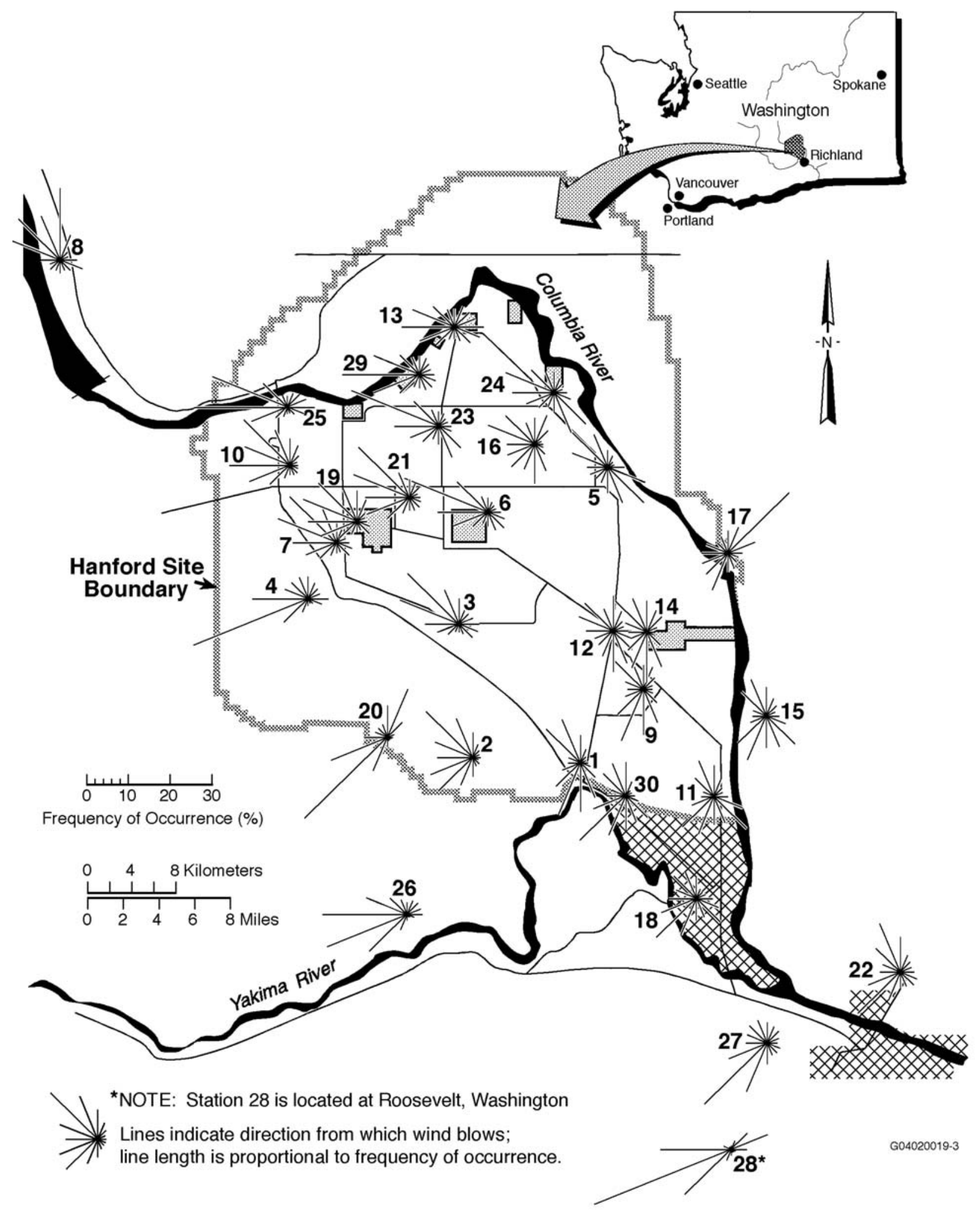

Figure 2.2. 2003 Hanford Meteorological Monitoring Network Wind Roses at 30 Feet (Refer to Table 1.1 for the names of the numbered locations on this map; see Appendix $B$ for station-specific wind rose.) 


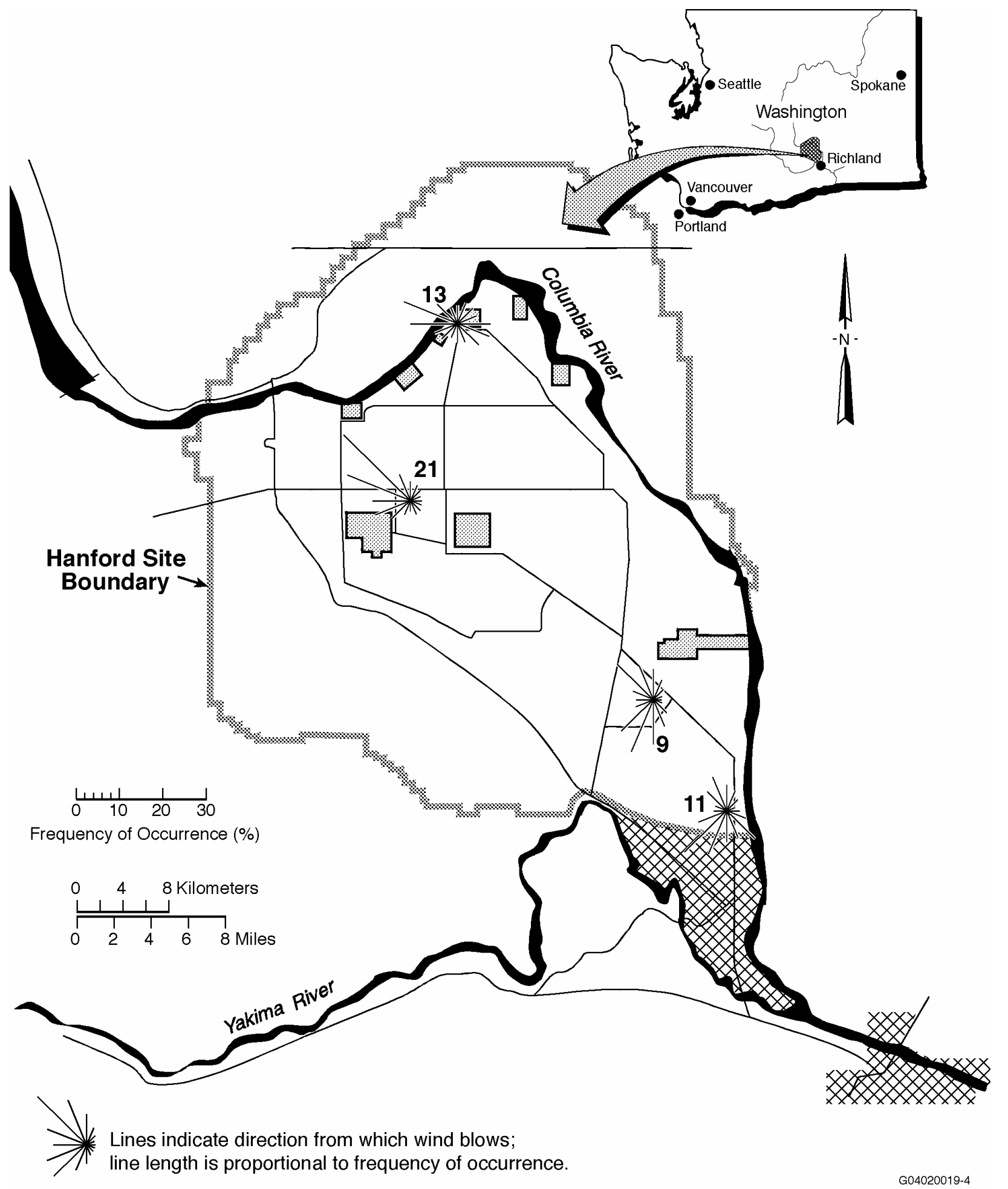

Figure 2.3. 2003 Hanford Meteorological Monitoring Network Wind Roses at 60-Meter Level (Refer to Table 1.1 for the names of the numbered locations on this map; see Appendix B for station-specific wind rose.) 


\subsection{Temperature Climatology}

\subsection{Monthly, Seasonal, and Annual Average}

Monthly, seasonal, and annual average temperatures, computed from observed daily maximum and minimum temperatures for the period 1945 through 2003, are presented in Table 3.1 and Table 3.2. In these tables, the highest and lowest values representing the warmest and coldest month, season, or year, are noted. Averages are based on the entire period of record, and climatological normal temperatures are based on the period 1971 through 2000.

As indicated in Table 3.1, much wider ranges and variabilities in temperatures are found during the late autumn and winter months (November through February) than during the rest of the year. The range of average monthly temperatures for January is from $12.1^{\circ} \mathrm{F}(1950)$ to $42.5^{\circ} \mathrm{F}(1953)$, a span of $30.4^{\circ} \mathrm{F}$; for November, $21.7^{\circ} \mathrm{F}$; February, $18.9^{\circ} \mathrm{F}$; and December, $17.5^{\circ} \mathrm{F}$; whereas for the rest of the year, the monthly temperature span is from a low of $10.5^{\circ} \mathrm{F}$ in April to a high of $13.8^{\circ} \mathrm{F}$ in June. The coldest month recorded was January $1950\left(12.1^{\circ} \mathrm{F}\right)$; the hottest month recorded was July $1985\left(82.2^{\circ} \mathrm{F}\right)$. As shown in Table 3.2, the seasonal range is from $8.0^{\circ} \mathrm{F}$ during the summer (June, July, and August) to $16.4^{\circ} \mathrm{F}$ in winter (December, January, and February). The coldest season was the winter of $1948-1949\left(24.2^{\circ} \mathrm{F}\right)$; the hottest was the summer of $1958\left(78.2^{\circ} \mathrm{F}\right)$.

\subsection{Days with Maximum Temperatures $\geq 100^{\circ} \mathrm{F}, \geq 90^{\circ} \mathrm{F}$, and $\leq 32^{\circ} \mathrm{F}$}

Table 3.3 contains the number of days each year with maximum temperatures in the categories $\geq 100^{\circ} \mathrm{F}, \geq 90^{\circ} \mathrm{F}$, and $\leq 32^{\circ} \mathrm{F}$.

Maximum temperatures $\geq 100^{\circ} \mathrm{F}$ have occurred as early as May 5 (1966) and as late as September 6 (1955). The annual number of days with maximum temperatures in this category ranged from 1 to 28 (1954 and 1958, respectively). The greatest number of consecutive days with maximum temperatures $\geq 100^{\circ} \mathrm{F}$ is 11, which occurred 3 times: July 22 through August 1, 1962; August 10 through 20, 1967; and August 6 through 16, 1981.

One particularly notable period of above normal temperatures occurred July 15 through August 13, 1971. This 30-day period included 27 days with maximum temperatures $\geq 100^{\circ} \mathrm{F}$ in 3 separate periods of 9 consecutive days each. The lowest maximum temperature during the 30 -day period was $98^{\circ} \mathrm{F}$; the highest was $112^{\circ} \mathrm{F}$. The average maximum temperature during this period was $104.7^{\circ} \mathrm{F}$.

Table 3.4 lists the dates of all occurrences of maximum temperatures $\geq 104^{\circ} \mathrm{F}$. 
Table 3.1. Monthly and Annual Average Temperatures $\left({ }^{\circ} \mathrm{F}\right)$

\begin{tabular}{|c|c|c|c|c|c|c|c|c|c|c|c|c|c|}
\hline Year & Jan & Feb & Mar & Apr & May & Jun & Jul & Aug & Sep & Oct & Nov & Dec & Annual \\
\hline 1945 & 33.9 & 38.6 & 42.1 & 50.3 & 61.7 & 67.5 & 78.0 & 77.5 & 64.6 & 56.4 & 40.6 & 32.7 & 53.7 \\
\hline 1946 & 34.4 & 39.6 & 45.5 & 53.7 & 64.2 & 66.9 & 76.1 & 76.6 & 63.5 & 49.5 & 35.8 & 34.8 & 53.4 \\
\hline 1947 & 27.4 & 40.0 & 49.6 & 56.1 & $68.7^{(\mathrm{a})}$ & 67.8 & 75.3 & 71.8 & 65.4 & 53.4 & 41.2 & 33.1 & 54.2 \\
\hline 1948 & 32.0 & 31.8 & 42.1 & 49.4 & 58.3 & 72.4 & 72.8 & 71.8 & 64.4 & 51.0 & 40.8 & 26.9 & 51.1 \\
\hline 1949 & 13.9 & 31.8 & 45.2 & 55.5 & 67.0 & 69.3 & 74.9 & 74.8 & 68.3 & 50.2 & 45.2 & 35.1 & 52.6 \\
\hline 1950 & $12.1^{(a)}$ & 30.7 & 42.3 & 49.9 & 59.0 & 66.5 & 75.4 & 76.4 & 67.5 & 51.1 & 40.7 & 36.2 & 50.6 \\
\hline 1951 & 33.0 & 36.9 & 40.1 & 54.1 & 61.1 & 69.4 & 76.7 & 74.2 & 66.8 & 51.5 & 39.5 & 27.4 & 52.6 \\
\hline 1952 & 25.2 & 36.7 & 44.1 & 55.2 & 62.7 & 67.1 & 77.0 & 74.0 & 69.0 & 59.0 & 34.0 & 34.8 & 53.2 \\
\hline 1953 & $42.5^{(a)}$ & 41.2 & 46.2 & 51.0 & 58.0 & $63.0^{(\mathrm{a})}$ & 75.8 & 74.0 & 67.8 & 55.4 & 43.4 & 37.6 & 54.7 \\
\hline 1954 & 28.9 & 39.3 & 41.5 & 51.4 & 62.9 & 65.5 & 73.9 & 71.4 & 65.1 & 51.4 & 46.0 & 34.0 & 52.6 \\
\hline 1955 & 30.0 & 35.3 & $39.4^{(\mathrm{a})}$ & $47.5^{(a)}$ & 57.0 & 70.2 & 73.0 & 75.5 & 66.4 & 53.3 & 31.3 & 29.4 & 50.7 \\
\hline 1956 & 31.8 & $25.6^{\text {(a) }}$ & 43.8 & 56.2 & 65.3 & 65.7 & 78.9 & 75.3 & 67.3 & 52.1 & 36.6 & 34.6 & 52.8 \\
\hline 1957 & 16.5 & 34.1 & 44.0 & 55.2 & 65.9 & 70.8 & 74.3 & 72.9 & 69.0 & 50.7 & 40.4 & $38.5^{(a)}$ & 52.7 \\
\hline 1958 & 37.1 & $44.5^{(\mathrm{a})}$ & 43.5 & 51.3 & 68.1 & 73.9 & 81.2 & 79.4 & 65.6 & 54.4 & 40.6 & 35.2 & 56.2 \\
\hline 1959 & 32.0 & 35.5 & 45.1 & 54.2 & 57.5 & 68.6 & 77.7 & 71.8 & 62.6 & 53.4 & 36.5 & 33.1 & 52.3 \\
\hline 1960 & 23.3 & 37.4 & 45.1 & 52.6 & 58.5 & 70.1 & 81.8 & 71.4 & 67.7 & 54.5 & 41.2 & 29.0 & 52.7 \\
\hline 1961 & 35.0 & 43.7 & 46.1 & 52.3 & 60.0 & 74.0 & 79.4 & 80.2 & 63.8 & 51.6 & 35.3 & 33.7 & 54.6 \\
\hline 1962 & 29.8 & 36.6 & 42.6 & 55.6 & 56.9 & 68.3 & 76.0 & 71.9 & 67.1 & 52.6 & 43.2 & 36.8 & 53.1 \\
\hline 1963 & 25.4 & 38.3 & 46.4 & 49.8 & 61.7 & 69.4 & 72.4 & 75.7 & 71.1 & 56.0 & 42.8 & 30.2 & 53.3 \\
\hline 1964 & 35.6 & 38.1 & 43.8 & 50.2 & 59.7 & 67.7 & 74.5 & $69.8^{(\mathrm{a})}$ & 63.0 & 53.3 & 38.2 & 25.5 & 51.6 \\
\hline 1965 & 32.3 & 40.5 & 42.9 & 54.8 & 60.5 & 69.3 & 76.5 & 74.7 & 62.4 & 57.1 & 43.1 & 33.0 & 53.9 \\
\hline 1966 & 34.0 & 39.9 & 45.4 & 54.6 & 63.2 & 66.9 & 73.3 & 75.6 & 68.8 & 53.4 & 43.7 & 38.2 & 54.8 \\
\hline 1967 & 39.8 & 43.7 & 44.3 & 47.6 & 60.5 & 72.5 & 78.6 & $81.5^{(\mathrm{a})}$ & 71.8 & 55.1 & 41.5 & 33.1 & 55.8 \\
\hline 1968 & 35.7 & 41.8 & 49.0 & 51.3 & 62.4 & 69.8 & 79.7 & 71.5 & 66.8 & 50.3 & 41.7 & 30.6 & 54.2 \\
\hline 1969 & 19.8 & 31.7 & 45.8 & 52.2 & 64.6 & 75.1 & 76.0 & 72.8 & 67.4 & 51.0 & 40.2 & 34.6 & 52.6 \\
\hline 1970 & 30.7 & 40.6 & 45.0 & 49.0 & 61.5 & 73.6 & 78.6 & 76.3 & 61.8 & 50.9 & 39.7 & 30.8 & 53.2 \\
\hline 1971 & 35.8 & 39.1 & 40.7 & 52.0 & 64.0 & 65.3 & 78.7 & 80.5 & 61.5 & 51.7 & 40.4 & 30.6 & 53.4 \\
\hline 1972 & 30.5 & 34.8 & 47.0 & 49.6 & 64.3 & 69.7 & 76.2 & 77.6 & 61.4 & 52.3 & 39.9 & 27.3 & 52.6 \\
\hline 1973 & 29.1 & 38.5 & 47.4 & 53.6 & 63.1 & 68.7 & 78.2 & 73.9 & 65.7 & 52.4 & 38.4 & 38.1 & 53.9 \\
\hline 1974 & 29.4 & 40.9 & 45.2 & 52.9 & 57.9 & 72.6 & 74.5 & 75.5 & 68.0 & 52.5 & 41.6 & 36.2 & 53.9 \\
\hline 1975 & 32.5 & 33.7 & 42.5 & 48.2 & 60.2 & 67.2 & 79.5 & 71.0 & 68.0 & 52.5 & 39.5 & 34.5 & 52.4 \\
\hline 1976 & 32.0 & 37.6 & 41.4 & 50.8 & 60.5 & 65.6 & 75.1 & 70.8 & 69.0 & 52.4 & 40.6 & 30.7 & 52.2 \\
\hline 1977 & 25.2 & 40.5 & 45.4 & 57.3 & 56.9 & 72.6 & 73.7 & 79.2 & 61.5 & 52.0 & 38.9 & 33.8 & 53.1 \\
\hline 1978 & 32.5 & 37.9 & 47.5 & 51.9 & 58.6 & 70.3 & 75.7 & 72.7 & 63.8 & 52.2 & 32.3 & 27.5 & 51.9 \\
\hline 1979 & 13.9 & 34.2 & 46.5 & 52.8 & 64.1 & 70.8 & 77.2 & 74.6 & 69.2 & 56.5 & 34.2 & 36.4 & 52.5 \\
\hline 1980 & 23.7 & 34.6 & 44.5 & 55.2 & 61.4 & 64.7 & 74.7 & 71.2 & 66.0 & 52.6 & 41.0 & 36.6 & 52.2 \\
\hline 1981 & 38.0 & 39.7 & 48.7 & 54.0 & 60.5 & 66.0 & 73.9 & 79.0 & 66.3 & 52.0 & 42.7 & 32.8 & 54.5 \\
\hline 1982 & 29.8 & 38.1 & 45.9 & 49.4 & 60.4 & 73.1 & 74.9 & 75.8 & 65.4 & 51.4 & 36.9 & 32.0 & 52.8 \\
\hline 1983 & 37.5 & 40.9 & 48.5 & 51.1 & 63.8 & 65.4 & 71.3 & 74.4 & 61.7 & 52.6 & 43.6 & 21.2 & 52.7 \\
\hline 1984 & 31.6 & 38.7 & 47.2 & 50.5 & $56.0^{(\mathrm{a})}$ & 65.7 & 76.1 & 74.0 & 62.1 & $47.9^{(a)}$ & 39.4 & 23.6 & 51.1 \\
\hline 1985 & 25.0 & 29.9 & 44.0 & 55.5 & 63.2 & 70.2 & $82.2^{(\mathrm{a})}$ & 70.5 & $58.8^{(\mathrm{a})}$ & 49.8 & $24.8^{(a)}$ & $21.0^{(a)}$ & $49.6^{(\mathrm{a})}$ \\
\hline 1986 & 34.0 & 39.1 & 48.6 & 50.9 & 62.3 & 73.0 & 70.6 & 79.2 & 62.2 & 54.7 & 42.3 & 32.4 & 54.1 \\
\hline 1987 & 30.7 & 40.1 & 48.3 & 58.0 & 66.2 & 73.4 & 74.3 & 76.6 & 69.9 & 55.5 & 43.6 & 31.5 & 55.7 \\
\hline 1988 & 31.9 & 41.0 & 45.9 & 55.2 & 61.1 & 69.2 & 77.3 & 75.2 & 65.6 & $59.6^{(\mathrm{a})}$ & 44.2 & 31.8 & 54.8 \\
\hline 1989 & 37.2 & 27.3 & 43.8 & 56.6 & 61.5 & 72.0 & 75.5 & 73.4 & 67.4 & 54.0 & 44.3 & 33.3 & 53.9 \\
\hline 1990 & 40.4 & 37.6 & 48.0 & 57.9 & 60.7 & 70.1 & 80.8 & 76.8 & $72.4^{(a)}$ & 52.3 & $46.5^{(a)}$ & 24.1 & 55.6 \\
\hline 1991 & 28.7 & $44.5^{(\mathrm{a})}$ & 44.1 & 54.0 & 60.4 & 65.6 & 78.0 & 78.9 & 69.7 & 52.9 & 41.3 & 37.8 & 54.7 \\
\hline 1992 & 37.5 & 42.6 & $51.5^{(\mathrm{a})}$ & 56.0 & 67.2 & $76.8^{(a)}$ & 76.6 & 76.9 & 64.5 & 55.7 & 41.2 & 30.0 & $56.4^{(a)}$ \\
\hline 1993 & 24.8 & 30.8 & 43.2 & 52.5 & 66.5 & 68.4 & $70.5^{\text {(a) }}$ & 73.1 & 66.4 & 55.4 & 34.6 & 35.4 & 51.8 \\
\hline 1994 & 38.6 & 36.0 & 49.2 & $58.2^{(\mathrm{a})}$ & 64.9 & 69.8 & 81.0 & 76.6 & 70.5 & 54.4 & 39.6 & 35.1 & 56.2 \\
\hline 1995 & 34.2 & 43.1 & 46.1 & 52.6 & 64.5 & 68.1 & 77.1 & 72.0 & 69.9 & 52.1 & 44.1 & 32.6 & 54.7 \\
\hline 1996 & 28.8 & 32.8 & 44.8 & 55.0 & 58.1 & 69.0 & 79.5 & 75.6 & 64.4 & 52.4 & 38.4 & 29.8 & 52.4 \\
\hline 1997 & 33.6 & 40.2 & 47.4 & 51.8 & 65.0 & 68.5 & 75.3 & 78.0 & 66.8 & 53.2 & 43.2 & 34.7 & 54.8 \\
\hline 1998 & 36.2 & 42.2 & 48.4 & 54.4 & 62.4 & 71.0 & 82.0 & 77.9 & 71.0 & 52.4 & 45.6 & 33.0 & $56.4^{(\mathrm{a})}$ \\
\hline 1999 & 38.3 & 41.7 & 46.3 & 50.9 & 57.9 & 67.4 & 73.8 & 76.2 & 65.0 & 51.8 & 45.8 & 37.7 & 54.4 \\
\hline 2000 & 32.9 & 38.7 & 44.7 & 55.4 & 61.2 & 69.9 & 75.5 & 74.0 & 63.6 & 52.1 & 34.0 & 29.8 & 52.6 \\
\hline 2001 & 33.4 & 35.7 & 46.8 & 51.4 & 63.7 & 66.5 & 76.0 & 77.7 & 69.0 & 53.5 & 42.8 & 34.9 & 54.3 \\
\hline 2002 & 37.6 & 38.4 & 42.4 & 53.2 & 60.1 & 71.6 & 79.6 & 75.5 & 66.3 & 50.4 & 41.0 & 37.2 & 54.4 \\
\hline 2003 & 38.0 & 39.9 & 49.0 & 52.2 & 61.1 & 72.5 & 80.2 & 76.5 & 69.2 & 57.4 & 37.8 & 32.9 & 55.6 \\
\hline Average $^{(\mathrm{b})}$ & 31.1 & 37.7 & 45.3 & 52.9 & 61.8 & 69.4 & 76.5 & 75.1 & 66.2 & 53.0 & 40.1 & 32.5 & 53.5 \\
\hline Normal & 31.8 & 37.9 & 46.1 & 53.5 & 61.8 & 69.3 & 76.3 & 75.4 & 65.9 & 53.0 & 40.1 & 31.7 & 53.6 \\
\hline
\end{tabular}

(a) Highest and lowest averages.

(b) Based on entire period of record, 1945 through 2003. 
Table 3.2. Seasonal Average Temperatures $\left({ }^{\circ} \mathbf{F}\right)$

\begin{tabular}{|c|c|c|c|c|}
\hline Year & $\begin{array}{c}\text { Winter } \\
\text { Dec-Feb }\end{array}$ & $\begin{array}{c}\text { Spring } \\
\text { Mar-May }\end{array}$ & $\begin{array}{l}\text { Summer } \\
\text { Jun-Aug }\end{array}$ & $\begin{array}{l}\text { Autumn } \\
\text { Sep-Nov }\end{array}$ \\
\hline 1945 & -- & 51.4 & 74.3 & 53.9 \\
\hline 1946 & 35.6 & 54.5 & 73.2 & 49.6 \\
\hline 1947 & 34.1 & 58.1 & 71.6 & 53.3 \\
\hline 1948 & 32.3 & 49.9 & 72.3 & 52.1 \\
\hline 1949 & $24.2^{(\mathrm{a})}$ & 55.9 & 73.0 & 54.6 \\
\hline 1950 & 26.0 & 50.4 & 72.8 & 53.1 \\
\hline 1951 & 35.4 & 51.8 & 73.4 & 52.6 \\
\hline 1952 & 29.8 & 54.0 & 72.7 & 54.0 \\
\hline 1953 & 39.5 & 51.7 & 70.9 & 55.5 \\
\hline 1954 & 35.3 & 51.9 & 70.3 & 54.2 \\
\hline 1955 & 33.1 & $48.0^{(a)}$ & 72.9 & 50.3 \\
\hline 1956 & 28.9 & 55.1 & 73.3 & 52.0 \\
\hline 1957 & 28.4 & 55.0 & 72.7 & 53.4 \\
\hline 1958 & 40.0 & 54.3 & $78.2^{(\mathrm{a})}$ & 53.5 \\
\hline 1959 & 34.2 & 52.3 & 72.7 & 50.8 \\
\hline 1960 & 31.3 & 52.1 & 74.4 & 54.5 \\
\hline 1961 & 35.9 & 52.8 & 77.9 & 50.2 \\
\hline 1962 & 33.4 & 51.7 & 72.1 & 54.3 \\
\hline 1963 & 33.5 & 52.6 & 72.5 & 56.6 \\
\hline 1964 & 34.6 & 51.2 & 70.7 & 51.5 \\
\hline 1965 & 32.8 & 52.7 & 73.5 & 54.2 \\
\hline 1966 & 35.6 & 54.4 & 71.9 & 55.3 \\
\hline 1967 & $40.6^{\text {(a) }}$ & 50.8 & 77.5 & 56.1 \\
\hline 1968 & 36.9 & 54.2 & 73.7 & 52.9 \\
\hline 1969 & 27.4 & 54.2 & 74.6 & 52.9 \\
\hline 1970 & 35.3 & 51.8 & 76.2 & 50.8 \\
\hline 1971 & 35.2 & 52.2 & 74.8 & 51.2 \\
\hline 1972 & 32.0 & 53.6 & 74.5 & 51.2 \\
\hline 1973 & 31.6 & 54.7 & 73.6 & 52.2 \\
\hline 1974 & 36.1 & 52.0 & 74.2 & 54.0 \\
\hline 1975 & 34.1 & 50.3 & 72.6 & 53.3 \\
\hline 1976 & 34.7 & 50.9 & 70.5 & 54.0 \\
\hline 1977 & 32.1 & 53.2 & 75.2 & 50.8 \\
\hline 1978 & 34.7 & 52.7 & 72.9 & 49.4 \\
\hline 1979 & 25.2 & 54.5 & 74.2 & 53.3 \\
\hline 1980 & 31.6 & 53.7 & $\mathbf{7 0 . 2 ^ { ( \mathrm { a } ) }}$ & 53.2 \\
\hline 1981 & 38.1 & 54.4 & 73.0 & 53.7 \\
\hline 1982 & 33.6 & 51.9 & 74.6 & 51.2 \\
\hline 1983 & 36.8 & 54.5 & 70.4 & 52.6 \\
\hline 1984 & 30.5 & 51.2 & 71.9 & 49.8 \\
\hline 1985 & 26.2 & 54.2 & 74.3 & $44.5^{\text {(a) }}$ \\
\hline 1986 & 31.4 & 53.9 & 74.3 & 53.1 \\
\hline 1987 & 34.4 & 57.5 & 74.8 & 56.3 \\
\hline 1988 & 34.8 & 54.1 & 73.9 & 56.5 \\
\hline 1989 & 32.1 & 54.0 & 73.6 & 55.2 \\
\hline 1990 & 37.1 & 55.5 & 75.9 & $\mathbf{5 7 . 1}^{\text {(a) }}$ \\
\hline 1991 & 32.4 & 52.8 & 74.2 & 54.6 \\
\hline 1992 & 39.3 & $58.2^{(\mathrm{a})}$ & 76.8 & 53.8 \\
\hline 1993 & 28.5 & 54.1 & 70.7 & 52.1 \\
\hline 1994 & 36.7 & 57.4 & 75.8 & 54.8 \\
\hline 1995 & 37.5 & 54.4 & 72.4 & 55.4 \\
\hline 1996 & 31.4 & 52.6 & 74.7 & 51.7 \\
\hline 1997 & 34.5 & 54.7 & 73.9 & 54.4 \\
\hline 1998 & 37.7 & 55.1 & 77.0 & 56.3 \\
\hline 1999 & 37.7 & 51.7 & 72.5 & 54.2 \\
\hline 2000 & 36.4 & 53.8 & 73.1 & 49.9 \\
\hline 2001 & 32.9 & 54.0 & 73.4 & 55.1 \\
\hline 2002 & 37.0 & 51.9 & 75.7 & 52.6 \\
\hline 2003 & 38.4 & 54.1 & 76.4 & 54.8 \\
\hline Average $^{(b)}$ & 33.7 & 53.3 & 73.6 & 53.1 \\
\hline Normal & 33.8 & 53.8 & 73.7 & 53.0 \\
\hline
\end{tabular}

(a) Highest and lowest averages.

(b) Based on entire period of record, 1945 through 2003. 
Table 3.3. Monthly and Seasonal Number of Days with Maximum Temperatures $\left({ }^{\circ} \mathbf{F}\right)$ Above or Below Certain Thresholds

\begin{tabular}{|c|c|c|c|c|c|c|}
\hline \multirow[b]{2}{*}{ Year } & \multicolumn{6}{|c|}{$100^{\circ} \mathrm{F}$ or Above } \\
\hline & May & Jun & Jul & Aug & Sep & Total \\
\hline 1945 & 0 & 0 & 8 & 4 & 0 & 12 \\
\hline 1946 & 0 & 0 & 7 & 6 & 0 & 13 \\
\hline 1947 & 1 & 0 & 2 & 0 & 0 & 3 \\
\hline 1948 & 0 & 2 & 0 & 0 & 0 & 2 \\
\hline 1949 & 0 & 1 & 6 & 2 & 1 & 10 \\
\hline 1950 & 0 & 0 & 2 & 3 & 2 & 7 \\
\hline 1951 & 0 & 0 & 8 & 3 & 0 & 11 \\
\hline 1952 & 0 & 0 & 9 & 4 & 0 & 13 \\
\hline 1953 & 0 & 0 & 4 & 4 & 0 & 8 \\
\hline 1954 & 0 & 0 & 1 & 0 & 0 & $\mathbf{1}^{\text {(a) }}$ \\
\hline 1955 & 0 & 2 & 5 & 2 & 2 & 11 \\
\hline 1956 & 0 & 0 & 10 & 5 & 0 & 15 \\
\hline 1957 & 0 & 1 & 1 & 0 & 0 & 2 \\
\hline 1958 & 1 & 6 & 10 & 11 & 0 & $28^{(a)}$ \\
\hline 1959 & 0 & 0 & 8 & 1 & 0 & 9 \\
\hline 1960 & 0 & 0 & 16 & 5 & 0 & 21 \\
\hline 1961 & 0 & 7 & 8 & 10 & 0 & 25 \\
\hline 1962 & 0 & 0 & 10 & 1 & 0 & 11 \\
\hline 1963 & 0 & 3 & 0 & 3 & 0 & 6 \\
\hline 1964 & 0 & 0 & 6 & 0 & 0 & 6 \\
\hline 1965 & 0 & 0 & 6 & 5 & 0 & 11 \\
\hline 1966 & 1 & 0 & 2 & 4 & 0 & 7 \\
\hline 1967 & 0 & 2 & 6 & 15 & 0 & 23 \\
\hline 1968 & 0 & 0 & 10 & 3 & 0 & 13 \\
\hline 1969 & 0 & 3 & 4 & 2 & 0 & 9 \\
\hline 1970 & 0 & 9 & 11 & 5 & 0 & 25 \\
\hline 1971 & 0 & 0 & 16 & 11 & 0 & 27 \\
\hline 1972 & 0 & 0 & 5 & 10 & 0 & 15 \\
\hline 1973 & 0 & 2 & 10 & 5 & 0 & 17 \\
\hline 1974 & 0 & 6 & 5 & 3 & 0 & 14 \\
\hline 1975 & 0 & 0 & 9 & 0 & 0 & 9 \\
\hline 1976 & 0 & 1 & 2 & 0 & 1 & 4 \\
\hline 1977 & 0 & 1 & 2 & 13 & 0 & 16 \\
\hline 1978 & 0 & 1 & 6 & 6 & 0 & 13 \\
\hline 1979 & 0 & 2 & 7 & 1 & 0 & 10 \\
\hline 1980 & 0 & 0 & 3 & 0 & 0 & 3 \\
\hline
\end{tabular}

\begin{tabular}{|c|c|c|c|c|c|c|c|}
\hline \multicolumn{8}{|c|}{$90^{\circ} \mathrm{F}$ or Above } \\
\hline Apr & May & Jun & Jul & Aug & Sep & Oct & Total \\
\hline 0 & 1 & 7 & 22 & 21 & 5 & 0 & 56 \\
\hline 1 & 0 & 4 & 15 & 18 & 0 & 0 & 38 \\
\hline 0 & 8 & 4 & 17 & 11 & 2 & 0 & 42 \\
\hline 0 & 1 & 9 & 14 & 7 & 7 & 0 & 38 \\
\hline 0 & 8 & 8 & 15 & 18 & 8 & 0 & 57 \\
\hline 0 & 1 & 5 & 20 & 22 & 8 & 0 & 56 \\
\hline 0 & 1 & 8 & 23 & 19 & 5 & 0 & 56 \\
\hline 0 & 2 & 5 & 21 & 17 & 12 & 0 & 57 \\
\hline 0 & 0 & 0 & 21 & 13 & 11 & 0 & 45 \\
\hline 0 & 2 & 3 & 20 & 9 & 3 & 0 & 37 \\
\hline 0 & 0 & 9 & 12 & 19 & 8 & 0 & 48 \\
\hline 0 & 7 & 2 & 22 & 16 & 7 & 0 & 54 \\
\hline 0 & 3 & 8 & 14 & 8 & 6 & 0 & 39 \\
\hline 0 & 8 & 11 & 28 & 25 & 5 & 0 & 77 \\
\hline 0 & 1 & 7 & 21 & 12 & 3 & 0 & 44 \\
\hline 0 & 1 & 12 & 28 & 12 & 5 & 0 & 58 \\
\hline 0 & 1 & 15 & 26 & 24 & 1 & 0 & 67 \\
\hline 0 & 0 & 11 & 17 & 10 & 8 & 0 & 46 \\
\hline 0 & 4 & 7 & 8 & 18 & 11 & 0 & 48 \\
\hline 0 & 0 & 5 & 14 & 10 & 2 & 0 & 31 \\
\hline 0 & 1 & 7 & 20 & 12 & 1 & 0 & 41 \\
\hline 0 & 5 & 2 & 15 & 21 & 7 & 0 & 50 \\
\hline 0 & 2 & 13 & 25 & 27 & 12 & 0 & $79^{(\mathrm{a})}$ \\
\hline 1 & 1 & 5 & 22 & 12 & 4 & 0 & 45 \\
\hline 0 & 6 & 17 & 20 & 15 & 7 & 0 & 65 \\
\hline 0 & 2 & 15 & 22 & 19 & 0 & 0 & 58 \\
\hline 0 & 2 & 2 & 20 & 26 & 2 & 0 & 52 \\
\hline 0 & 5 & 8 & 21 & 19 & 5 & 0 & 58 \\
\hline 0 & 6 & 7 & 21 & 18 & 4 & 0 & 56 \\
\hline 0 & 0 & 18 & 16 & 18 & 6 & 0 & 58 \\
\hline 0 & 2 & 4 & 22 & 12 & 8 & 0 & 48 \\
\hline 0 & 1 & 4 & 17 & 9 & 4 & 0 & 35 \\
\hline 1 & 0 & 13 & 16 & 22 & 0 & 0 & 52 \\
\hline 0 & 0 & 12 & 17 & 11 & 2 & 0 & 42 \\
\hline 0 & 1 & 13 & 23 & 20 & 7 & 0 & 64 \\
\hline 0 & 0 & 0 & 18 & 9 & 2 & 0 & $29^{(a)}$ \\
\hline
\end{tabular}

\begin{tabular}{ccrrrrrr}
\multicolumn{7}{c}{$32^{\circ}$ F or Below } \\
\hline Season & Oct & Nov & Dec & Jan & Feb & Mar & Total \\
\hline $1944-45$ & -- & -- & -- & 12 & 1 & 1 & 14 \\
$1945-46$ & 0 & 2 & 9 & 0 & 0 & 0 & 11 \\
$1946-47$ & 0 & 4 & 4 & 14 & 0 & 0 & 22 \\
$1947-48$ & 0 & 0 & 6 & 8 & 9 & 0 & 23 \\
$1948-49$ & 0 & 0 & 13 & 28 & 8 & 0 & 49 \\
$1949-50$ & 0 & 0 & 5 & 24 & 5 & 1 & 35 \\
$1950-51$ & 0 & 0 & 2 & 8 & 2 & 0 & 12 \\
$1951-52$ & 0 & 0 & 16 & 19 & 0 & 0 & 35 \\
$1952-53$ & 0 & 9 & 6 & 1 & 0 & 0 & 16 \\
$1953-54$ & 0 & 0 & 2 & 12 & 4 & 0 & 18 \\
$1954-55$ & 0 & 0 & 5 & 13 & 2 & 1 & 21 \\
$1955-56$ & 0 & 15 & 16 & 7 & 15 & 0 & 53 \\
$1956-57$ & 0 & 7 & 10 & 22 & 7 & 0 & 46 \\
$1957-58$ & 0 & 0 & 2 & 2 & 0 & 0 & 4 \\
$1958-59$ & 0 & 3 & 5 & 8 & 2 & 0 & 18 \\
$1959-60$ & 0 & 5 & 7 & 23 & 1 & 2 & 38 \\
$1960-61$ & 0 & 0 & 14 & 10 & 0 & 0 & 24 \\
$1961-62$ & 0 & 0 & 7 & 12 & 2 & 0 & 21 \\
$1962-63$ & 0 & 0 & 3 & 14 & 3 & 0 & 20 \\
$1963-64$ & 0 & 1 & 11 & 3 & 0 & 0 & 15 \\
$1964-65$ & 0 & 0 & 14 & 5 & 0 & 0 & 19 \\
$1965-66$ & 0 & 1 & 8 & 3 & 0 & 0 & 12 \\
$1966-67$ & 0 & 0 & 2 & 0 & 0 & 0 & $2^{(\mathrm{a})}$ \\
$1967-68$ & 0 & 0 & 10 & 4 & 0 & 0 & 14 \\
$1968-69$ & 0 & 0 & 7 & 20 & 4 & 0 & 31 \\
$1969-70$ & 0 & 3 & 9 & 15 & 0 & 0 & 27 \\
$1970-71$ & 0 & 3 & 11 & 9 & 1 & 0 & 24 \\
$1971-72$ & 1 & 0 & 10 & 9 & 7 & 0 & 27 \\
$1972-73$ & 0 & 0 & 14 & 10 & 0 & 0 & 24 \\
$1973-74$ & 0 & 4 & 1 & 12 & 0 & 0 & 17 \\
$1974-75$ & 0 & 0 & 0 & 6 & 6 & 0 & 12 \\
$1975-76$ & 0 & 3 & 5 & 7 & 0 & 0 & 15 \\
$1976-77$ & 0 & 0 & 12 & 20 & 3 & 0 & 35 \\
$1977-78$ & 0 & 5 & 9 & 6 & 2 & 0 & 22 \\
$1978-79$ & 0 & 7 & 11 & 30 & 4 & 0 & 52 \\
$1979-80$ & 0 & 7 & 3 & 16 & 6 & 0 & 32
\end{tabular}


Table 3.3. (contd)

\begin{tabular}{|c|c|c|c|c|c|c|}
\hline \multirow[b]{2}{*}{ Year } & \multicolumn{6}{|c|}{$100^{\circ} \mathrm{F}$ or Above } \\
\hline & May & Jun & Jul & Aug & Sep & Total \\
\hline 1981 & 0 & 0 & 3 & 13 & 0 & 16 \\
\hline 1982 & 0 & 2 & 5 & 3 & 0 & 10 \\
\hline 1983 & 1 & 0 & 1 & 0 & 0 & 2 \\
\hline 1984 & 0 & 0 & 3 & 3 & 0 & 6 \\
\hline 1985 & 0 & 1 & 15 & 0 & 0 & 16 \\
\hline 1986 & 3 & 1 & 0 & 6 & 0 & 10 \\
\hline 1987 & 1 & 5 & 3 & 4 & 1 & 14 \\
\hline 1988 & 0 & 0 & 8 & 3 & 3 & 14 \\
\hline 1989 & 0 & 0 & 2 & 2 & 0 & 4 \\
\hline 1990 & 0 & 0 & 11 & 9 & 0 & 20 \\
\hline 1991 & 0 & 0 & 4 & 8 & 0 & 12 \\
\hline 1992 & 0 & 7 & 5 & 9 & 0 & 21 \\
\hline 1993 & 1 & 0 & 0 & 2 & 0 & 3 \\
\hline 1994 & 0 & 1 & 13 & 7 & 0 & 21 \\
\hline 1995 & 0 & 0 & 5 & 3 & 1 & 9 \\
\hline 1996 & 0 & 0 & 13 & 6 & 0 & 19 \\
\hline 1997 & 0 & 0 & 3 & 7 & 0 & 10 \\
\hline 1998 & 0 & 0 & 14 & 9 & 3 & 26 \\
\hline 1999 & 0 & 1 & 4 & 2 & 0 & 7 \\
\hline 2000 & 0 & 1 & 5 & 1 & 0 & 7 \\
\hline 2001 & 1 & 1 & 4 & 8 & 0 & 14 \\
\hline 2002 & 0 & 3 & 11 & 2 & 0 & 16 \\
\hline 2003 & 0 & 1 & 12 & 3 & 3 & 19 \\
\hline Average $^{(b)}$ & $<1$ & 1 & 6 & 4 & $<1$ & 12 \\
\hline Normal & $<1$ & 1 & 6 & 5 & $<1$ & 12 \\
\hline
\end{tabular}

\begin{tabular}{rccrrrrr}
\multicolumn{8}{c}{$90^{\circ}$ or Above } \\
\hline Apr & May & Jun & Jul & Aug & Sep & Oct & Total \\
\hline 1 & 0 & 4 & 19 & 22 & 11 & 0 & 57 \\
0 & 0 & 15 & 16 & 17 & 5 & 0 & 53 \\
0 & 8 & 2 & 9 & 13 & 0 & 0 & 32 \\
0 & 1 & 4 & 21 & 16 & 4 & 0 & 46 \\
0 & 3 & 10 & 30 & 7 & 0 & 0 & 50 \\
0 & 6 & 11 & 9 & 27 & 3 & 0 & 56 \\
2 & 6 & 15 & 14 & 19 & 12 & 0 & 68 \\
0 & 4 & 11 & 19 & 20 & 7 & 0 & 61 \\
0 & 0 & 13 & 20 & 9 & 3 & 0 & 45 \\
0 & 1 & 8 & 24 & 15 & 12 & 0 & 60 \\
0 & 0 & 1 & 25 & 23 & 5 & 0 & 54 \\
0 & 8 & 16 & 15 & 17 & 3 & 0 & 59 \\
0 & 7 & 6 & 4 & 15 & 11 & 0 & 43 \\
0 & 5 & 8 & 25 & 18 & 12 & 0 & 68 \\
0 & 4 & 7 & 17 & 11 & 12 & 0 & 51 \\
0 & 0 & 8 & 25 & 18 & 5 & 0 & 56 \\
0 & 5 & 3 & 18 & 22 & 5 & 0 & 53 \\
1 & 3 & 7 & 26 & 24 & 12 & 0 & 73 \\
0 & 2 & 5 & 17 & 21 & 4 & 0 & 49 \\
0 & 0 & 9 & 19 & 16 & 1 & 0 & 45 \\
0 & 7 & 3 & 20 & 21 & 8 & 0 & 59 \\
0 & 0 & 11 & 24 & 17 & 7 & 0 & 59 \\
0 & 2 & 12 & 26 & 23 & 9 & 0 & 72 \\
& & & & & & & \\
$<1$ & 3 & 8 & 19 & 17 & 6 & 0 & 52 \\
$<1$ & 3 & 8 & 19 & 17 & 6 & 0 & 52 \\
& & & & & & &
\end{tabular}

\begin{tabular}{ccrrrccc}
\multicolumn{8}{c}{$32^{\circ} \mathrm{F}$ or Below } \\
\hline Season & Oct & Nov & Dec & Jan & Feb & Mar & Total \\
\hline $1980-81$ & 0 & 1 & 6 & 0 & 2 & 0 & 9 \\
$1981-82$ & 0 & 0 & 8 & 10 & 2 & 0 & 20 \\
$1982-83$ & 0 & 3 & 10 & 5 & 0 & 0 & 18 \\
$1983-84$ & 0 & 0 & 25 & 12 & 1 & 0 & 38 \\
$1984-85$ & 0 & 2 & 18 & 29 & 9 & 0 & $\mathbf{5 8}^{(\mathrm{a})}$ \\
$1985-86$ & 0 & 15 & 25 & 5 & 1 & 0 & 46 \\
$1986-87$ & 0 & 0 & 7 & 9 & 0 & 0 & 16 \\
$1987-88$ & 0 & 0 & 16 & 11 & 1 & 0 & 28 \\
$1988-89$ & 0 & 0 & 11 & 2 & 8 & 1 & 22 \\
$1989-90$ & 0 & 2 & 6 & 0 & 1 & 0 & 9 \\
$1990-91$ & 0 & 0 & 15 & 13 & 0 & 0 & 28 \\
$1991-92$ & 0 & 0 & 3 & 0 & 0 & 0 & 3 \\
$1992-93$ & 0 & 1 & 11 & 20 & 8 & 2 & 42 \\
$1993-94$ & 0 & 6 & 4 & 1 & 8 & 0 & 19 \\
$1994-95$ & 0 & 0 & 5 & 6 & 2 & 0 & 13 \\
$1995-96$ & 0 & 0 & 8 & 9 & 5 & 0 & 22 \\
$1996-97$ & 0 & 5 & 12 & 8 & 2 & 0 & 27 \\
$1997-98$ & 0 & 0 & 2 & 5 & 0 & 0 & 7 \\
$1998-99$ & 0 & 0 & 7 & 3 & 0 & 0 & 10 \\
$1999-2000$ & 0 & 0 & 4 & 4 & 0 & 0 & 8 \\
$2000-2001$ & 0 & 5 & 10 & 6 & 2 & 0 & 23 \\
$2001-2002$ & 0 & 2 & 5 & 0 & 0 & 0 & 7 \\
$2002-2003$ & 0 & 4 & 3 & 0 & 0 & 0 & 7 \\
$2003-2004$ & 0 & 1 & 4 & -- & -- & -- & 5 \\
Average & $<1$ & 2 & 8 & 10 & 2 & $<1$ & 23 \\
Normal & $<1$ & 2 & 9 & 9 & 3 & $<1$ & 23
\end{tabular}

(a) Greatest and least seasonal totals.

(b) Based on entire period of record, 1945 through 2003.

Note: Dashes indicate no data are available. 
Table 3.4. Days with Maximum Temperatures $\geq 104^{\circ} \mathrm{F}$

\begin{tabular}{|c|c|c|c|c|c|c|}
\hline $\begin{array}{c}\text { Temperature } \\
\left({ }^{\circ} \mathrm{F}\right)\end{array}$ & & & Date(s)o & ccurrence & & \\
\hline 113 & $07 / 13 / 2002$ & 08/04/1961 & & & & \\
\hline 112 & $07 / 12 / 2002$ & $07 / 27 / 1998$ & 08/09/1971 & & & \\
\hline 111 & $07 / 22 / 1994$ & 06/23/1992 & $07 / 31 / 1971$ & & & \\
\hline 110 & $\begin{array}{l}08 / 04 / 1998 \\
07 / 18 / 1960\end{array}$ & $\begin{array}{l}07 / 23 / 1990 \\
07 / 17 / 1960\end{array}$ & 07/20/1979 & 07/09/1975 & 08/08/1972 & $07 / 06 / 1968$ \\
\hline 109 & $\begin{array}{l}07 / 11 / 2002 \\
07 / 11 / 1990\end{array}$ & $\begin{array}{l}08 / 10 / 1996 \\
07 / 19 / 1979\end{array}$ & $\begin{array}{l}07 / 24 / 1994 \\
08 / 07 / 1972\end{array}$ & $\begin{array}{l}07 / 23 / 1994 \\
08 / 10 / 1971\end{array}$ & $\begin{array}{l}07 / 21 / 1994 \\
08 / 01 / 1971\end{array}$ & $08 / 14 / 1992$ \\
\hline 108 & $\begin{array}{l}07 / 30 / 2003 \\
07 / 15 / 1996 \\
08 / 12 / 1971 \\
07 / 04 / 1968 \\
06 / 17 / 1961\end{array}$ & $\begin{array}{l}07 / 29 / 2003 \\
06 / 24 / 1992 \\
08 / 11 / 1971 \\
08 / 18 / 1967\end{array}$ & $\begin{array}{l}07 / 28 / 2003 \\
08 / 05 / 1990 \\
07 / 27 / 1971 \\
08 / 17 / 1967\end{array}$ & $\begin{array}{l}07 / 23 / 1998 \\
07 / 18 / 1979 \\
07 / 19 / 1971 \\
08 / 16 / 1967\end{array}$ & $\begin{array}{l}07 / 26 / 1998 \\
07 / 27 / 1975 \\
07 / 28 / 1968 \\
07 / 31 / 1965\end{array}$ & $\begin{array}{l}07 / 26 / 1996 \\
07 / 05 / 1975 \\
07 / 08 / 1968 \\
07 / 13 / 1961\end{array}$ \\
\hline 107 & $\begin{array}{l}07 / 31 / 2000 \\
07 / 29 / 1982 \\
07 / 30 / 1971 \\
07 / 19 / 1959\end{array}$ & $\begin{array}{l}08 / 13 / 1992 \\
07 / 28 / 1982 \\
07 / 28 / 1971 \\
07 / 28 / 1958\end{array}$ & $\begin{array}{l}08 / 01 / 1992 \\
08 / 08 / 1981 \\
07 / 05 / 1968 \\
07 / 14 / 1955\end{array}$ & $\begin{array}{l}07 / 31 / 1992 \\
07 / 17 / 1979 \\
08 / 03 / 1961\end{array}$ & $\begin{array}{l}06 / 25 / 1992 \\
08 / 18 / 1977 \\
07 / 22 / 1959\end{array}$ & $\begin{array}{l}07 / 14 / 1987 \\
08 / 08 / 1971 \\
07 / 20 / 1959\end{array}$ \\
\hline 106 & $\begin{array}{l}07 / 31 / 2003 \\
08 / 05 / 1998 \\
08 / 18 / 1992 \\
07 / 25 / 1984 \\
07 / 10 / 1975 \\
08 / 01 / 1965 \\
07 / 09 / 1952\end{array}$ & $\begin{array}{l}07 / 23 / 2003 \\
08 / 14 / 1997 \\
07 / 18 / 1992 \\
07 / 22 / 1980 \\
07 / 29 / 1973 \\
07 / 12 / 1964\end{array}$ & $\begin{array}{l}07 / 22 / 2003 \\
08 / 04 / 1997 \\
06 / 22 / 1992 \\
08 / 09 / 1978 \\
07 / 15 / 1973 \\
07 / 24 / 1962\end{array}$ & $\begin{array}{l}07 / 24 / 2002 \\
07 / 27 / 1996 \\
09 / 01 / 1987 \\
07 / 23 / 1978 \\
08 / 06 / 1972 \\
06 / 16 / 1961\end{array}$ & $\begin{array}{l}07 / 04 / 2001 \\
07 / 14 / 1996 \\
06 / 30 / 1987 \\
08 / 17 / 1977 \\
07 / 20 / 1971 \\
06 / 22 / 1958\end{array}$ & $\begin{array}{l}08 / 14 / 1998 \\
08 / 02 / 1994 \\
07 / 09 / 1985 \\
08 / 13 / 1977 \\
07 / 04 / 1970 \\
07 / 19 / 1956\end{array}$ \\
\hline 105 & $\begin{array}{l}07 / 19 / 2003 \\
08 / 06 / 1997 \\
07 / 17 / 1992 \\
07 / 20 / 1985 \\
08 / 04 / 1978 \\
07 / 19 / 1970 \\
08 / 30 / 1967 \\
08 / 02 / 1961 \\
07 / 30 / 1952\end{array}$ & $\begin{array}{c}07 / 11 / 2003 \\
08 / 05 / 1997 \\
07 / 03 / 1991 \\
07 / 27 / 1982 \\
08 / 03 / 1978 \\
07 / 16 / 1970 \\
08 / 20 / 1967 \\
08 / 11 / 1960 \\
07 / 10 / 1952\end{array}$ & $\begin{array}{l}08 / 12 / 2001 \\
08 / 25 / 1996 \\
07 / 22 / 1990 \\
07 / 26 / 1982 \\
07 / 04 / 1975 \\
07 / 08 / 1970 \\
08 / 19 / 1967 \\
07 / 19 / 1960 \\
07 / 20 / 1946\end{array}$ & $\begin{array}{l}07 / 28 / 1999 \\
07 / 28 / 1996 \\
07 / 15 / 1990 \\
08 / 16 / 1981 \\
07 / 21 / 1971 \\
07 / 27 / 1968 \\
08 / 15 / 1967 \\
07 / 07 / 1960 \\
08 / 16 / 1945\end{array}$ & $\begin{array}{l}08 / 13 / 1998 \\
07 / 24 / 1996 \\
07 / 26 / 1988 \\
08 / 13 / 1981 \\
07 / 18 / 1971 \\
07 / 07 / 1968 \\
08 / 13 / 1967 \\
07 / 13 / 1955\end{array}$ & $\begin{array}{l}07 / 22 / 1998 \\
07 / 19 / 1995 \\
08 / 09 / 1987 \\
08 / 12 / 1981 \\
08 / 23 / 1970 \\
07 / 03 / 1968 \\
07 / 03 / 1967 \\
08 / 04 / 1952\end{array}$ \\
\hline 104 & $\begin{array}{l}08 / 01 / 2003 \\
07 / 25 / 1998 \\
07 / 18 / 1995 \\
07 / 20 / 1988 \\
07 / 04 / 1985 \\
07 / 04 / 1981 \\
07 / 28 / 1974 \\
08 / 09 / 1972 \\
06 / 23 / 1970 \\
07 / 25 / 1962 \\
07 / 18 / 1959 \\
08 / 22 / 1956 \\
07 / 23 / 1951 \\
06 / 29 / 1948 \\
07 / 10 / 1945\end{array}$ & $\begin{array}{c}07 / 18 / 2003 \\
07 / 17 / 1998 \\
07 / 20 / 1994 \\
05 / 31 / 1986 \\
08 / 07 / 1982 \\
08 / 08 / 1978 \\
08 / 01 / 1973 \\
08 / 13 / 1971 \\
06 / 21 / 1970 \\
07 / 23 / 1962 \\
08 / 25 / 1958 \\
07 / 24 / 1956 \\
07 / 17 / 1951 \\
08 / 22 / 1946\end{array}$ & $\begin{array}{c}06 / 26 / 2002 \\
08 / 09 / 1996 \\
07 / 17 / 1994 \\
05 / 30 / 1986 \\
08 / 11 / 1981 \\
07 / 25 / 1978 \\
07 / 27 / 1973 \\
08 / 07 / 1971 \\
08 / 31 / 1967 \\
08 / 14 / 1961 \\
08 / 24 / 1958 \\
07 / 23 / 1956 \\
08 / 01 / 1949 \\
08 / 21 / 1946\end{array}$ & $\begin{array}{l}08 / 15 / 2001 \\
07 / 25 / 1996 \\
07 / 10 / 1990 \\
07 / 29 / 1985 \\
08 / 10 / 1981 \\
08 / 20 / 1977 \\
07 / 19 / 1973 \\
07 / 16 / 1971 \\
08 / 11 / 1967 \\
06 / 18 / 1961 \\
08 / 11 / 1958 \\
07 / 21 / 1956 \\
07 / 31 / 1949 \\
07 / 28 / 1946\end{array}$ & $\begin{array}{l}08 / 09 / 2000 \\
07 / 23 / 1996 \\
07 / 25 / 1988 \\
07 / 21 / 1985 \\
08 / 07 / 1981 \\
08 / 12 / 1977 \\
06 / 22 / 1973 \\
07 / 09 / 1970 \\
07 / 12 / 1967 \\
08 / 10 / 1960 \\
07 / 17 / 1958 \\
07 / 22 / 1955 \\
07 / 30 / 1949 \\
07 / 21 / 1946\end{array}$ & $\begin{array}{l}08 / 03 / 1998 \\
07 / 13 / 1996 \\
07 / 21 / 1988 \\
07 / 08 / 1985 \\
07 / 27 / 1981 \\
07 / 30 / 1974 \\
08 / 28 / 1972 \\
07 / 03 / 1970 \\
07 / 30 / 1965 \\
07 / 16 / 1960 \\
07 / 11 / 1958 \\
08 / 15 / 1953 \\
07 / 15 / 1949 \\
07 / 11 / 1945\end{array}$ \\
\hline
\end{tabular}

Maximum temperatures $\geq 90^{\circ} \mathrm{F}$ occur an average of 52 times per year and vary from a low of 29 times in 1980 to a high of 79 times in 1967. The earliest occurrences varied from early in the year (April 24, 1977) to late in the year (July 2, 1953), with an average annual occurrence of May 21 (Table 3.5). The latest annual occurrence of maximum temperatures $\geq 90^{\circ} \mathrm{F}$ varied from August 17 (1983) to September 29 
Table 3.5. Record of Annual First and Last Dates with Maximum Temperatures $\geq 90^{\circ} \mathrm{F}$ and Minimum Temperatures $\leq 32^{\circ} \mathrm{F}$

\begin{tabular}{|c|c|c|c|c|c|}
\hline \multirow[b]{2}{*}{ Year } & \multicolumn{2}{|c|}{ Maximum Temperature $\geq 90^{\circ} \mathrm{F}$} & \multicolumn{2}{|c|}{ Minimum Temperature $\leq 32^{\circ} \mathrm{F}$} & \multirow[b]{2}{*}{ Growing Days $^{(\mathrm{a})}$} \\
\hline & First in Spring & Last in Summer & Last in Spring & First in Autumn & \\
\hline 1945 & May 30 & Sep 14 & Apr 10 & Oct 18 & 190 \\
\hline 1946 & Apr 25 & Aug 24 & Apr 07 & Oct 11 & 186 \\
\hline 1947 & May 06 & Sep 12 & Apr 07 & Nov 04 & 210 \\
\hline 1948 & May 26 & Sep 13 & May 02 & Oct 17 & 167 \\
\hline 1949 & May 08 & Sep 27 & May 03 & Oct 08 & 157 \\
\hline 1950 & May 26 & Sep 23 & Apr 27 & Nov 08 & 194 \\
\hline 1951 & May 22 & Sep 19 & Apr 21 & Oct 15 & 176 \\
\hline 1952 & May 24 & Sep 26 & Apr 29 & Nov 01 & 185 \\
\hline 1953 & Jul 02 $2^{(\mathrm{b})}$ & Sep 15 & Apr 15 & Oct 24 & 191 \\
\hline 1954 & May 17 & Sep 10 & May 01 & Oct 01 & 152 \\
\hline 1955 & Jun 06 & Sep 10 & May 14 & Oct 31 & 169 \\
\hline 1956 & May 16 & Sep 19 & Apr 06 & Oct 22 & 198 \\
\hline 1957 & May 29 & Sep 15 & Mar 26 & Oct 22 & 209 \\
\hline 1958 & May 18 & Sep 10 & $\operatorname{Mar} 19^{(\mathrm{b})}$ & Oct 21 & 215 \\
\hline 1959 & May 13 & Sep 13 & May 05 & Oct 30 & 177 \\
\hline 1960 & May 10 & Sep 18 & Apr 21 & Oct 11 & 172 \\
\hline 1961 & May 25 & Sep 04 & Apr 19 & Oct 20 & 183 \\
\hline 1962 & Jun 08 & Sep 26 & May 04 & $\operatorname{Nov} 12^{(b)}$ & 191 \\
\hline 1963 & May 20 & Sep 29 & Apr 16 & Oct 25 & 191 \\
\hline 1964 & Jun 23 & Sep 24 & Apr 19 & Oct 16 & 179 \\
\hline 1965 & May 28 & Sep 01 & May 05 & Oct 16 & 163 \\
\hline 1966 & May 03 & Sep 22 & Apr 19 & Oct 14 & 177 \\
\hline 1967 & May 20 & Sep 28 & Apr 28 & Oct 26 & 180 \\
\hline 1968 & Apr 29 & Sep 09 & Apr 22 & Oct 21 & 181 \\
\hline 1969 & May 09 & Sep 12 & Apr 26 & Oct 13 & 169 \\
\hline 1970 & May 16 & Aug 31 & May 11 & Oct 07 & 148 \\
\hline 1971 & May 11 & Sep 10 & Apr 22 & Oct 16 & 176 \\
\hline 1972 & May 13 & Sep 16 & Apr 30 & Sep 25 & 147 \\
\hline 1973 & May 13 & Sep 11 & Apr 08 & Oct 04 & 178 \\
\hline 1974 & Jun 10 & Sep 25 & May $16^{(b)}$ & Oct 06 & 142 \\
\hline 1975 & May 30 & Sep 15 & Apr 29 & Oct 23 & 176 \\
\hline 1976 & May 16 & Sep 29 & Apr 23 & Oct 19 & 178 \\
\hline 1977 & Apr 24 $4^{(b)}$ & Aug 22 & Apr 14 & Oct 27 & 195 \\
\hline 1978 & Jun 02 & Sep 03 & Apr 23 & Oct 07 & 166 \\
\hline 1979 & May 22 & Sep 20 & Apr 19 & Oct 31 & 194 \\
\hline 1980 & Jun 01 & Sep 06 & Apr 11 & Oct 22 & 193 \\
\hline 1981 & Apr 30 & Sep 18 & Apr 13 & Oct 14 & 183 \\
\hline 1982 & Jun 10 & Sep 08 & Apr 21 & Oct 18 & 179 \\
\hline 1983 & May 23 & Aug $17^{(\mathrm{b})}$ & Apr 16 & Oct 11 & 177 \\
\hline 1984 & May 29 & Sep 18 & Apr 13 & Oct 14 & 183 \\
\hline 1985 & May 18 & Aug 29 & Apr 21 & Oct 07 & 168 \\
\hline 1986 & May 25 & Sep 04 & Apr 30 & Nov 09 & 192 \\
\hline 1987 & Apr 27 & Sep 23 & Apr 20 & Oct 16 & 178 \\
\hline 1988 & May 11 & Sep 14 & Apr 09 & Oct 27 & 200 \\
\hline 1989 & Jun 01 & Sep 24 & Mar 30 & Oct 29 & 212 \\
\hline 1990 & May 05 & Sep 29 & Mar 27 & Oct 17 & 203 \\
\hline 1991 & Jun 10 & Sep 26 & Apr 08 & Oct 22 & 196 \\
\hline 1992 & May 04 & Sep 03 & Apr 08 & Oct 15 & 189 \\
\hline 1993 & May 10 & Sep $29^{(b, c)}$ & Apr 06 & Oct 20 & 196 \\
\hline 1994 & May 07 & Sep 28 & Mar 26 & Oct 29 & 216 \\
\hline 1995 & May 28 & Sep 17 & Apr 15 & Oct 29 & 196 \\
\hline 1996 & Jun 02 & Sep 15 & May 08 & Oct 17 & 161 \\
\hline 1997 & May 12 & Sep 25 & May 02 & Oct 08 & 158 \\
\hline 1998 & Apr 30 & Sep 17 & Apr 13 & Oct 19 & 188 \\
\hline 1999 & May 23 & Sep 22 & May 08 & Oct 17 & 163 \\
\hline 2000 & Jun 04 & Sep 14 & Apr 07 & Sep $23^{(b)}$ & 168 \\
\hline 2001 & May 12 & Sep 24 & Apr 14 & Oct 28 & 196 \\
\hline 2002 & Jun 12 & Sep 15 & May 8 & Oct 12 & 156 \\
\hline 2003 & May 24 & Sep 27 & Apr 25 & Oct 25 & 182 \\
\hline Average $^{(\mathrm{d})}$ & May 21 & Sep 16 & Apr 20 & Oct 19 & 181 \\
\hline Normal & May 21 & Sep 16 & Apr 18 & Oct 17 & 181 \\
\hline
\end{tabular}

(a) Days between last freezing temperature in spring and first freezing temperature in autumn.

(b) Earliest and latest dates.

(c) Also in previous years.

(d) Based on entire period of record, 1945 through 2003. 
(1993 and earlier years). The average date for maximum temperatures $\geq 90^{\circ} \mathrm{F}$ for the period 1946 through 2003 is September 16. The longest period of consecutive maximum temperatures $\geq 90^{\circ} \mathrm{F}$ is 32 days from July 13 through August 13, 1971.

The average seasonal number of days with maximum temperatures $\leq 32^{\circ} \mathrm{F}$ is 23 . The earliest seasonal occurrence of a day with a maximum temperature $\leq 32^{\circ} \mathrm{F}$ was October 30 (1971) and the latest was March 11 (1950). The number of winter days with maximum temperatures $\leq 32^{\circ} \mathrm{F}$ varied from 2 to 58 days (winters of 1966-1967 and 1984-1985, respectively). The greatest consecutive number of days with maximum temperatures $\leq 32^{\circ} \mathrm{F}$ is 29 days, from December 30, 1984, through January 27, 1985 . During the period December 27, 1978, through February 4, 1979 (40 days), only 1 maximum temperature greater than $32^{\circ} \mathrm{F}$ occurred. The average maximum temperature for that period was $21^{\circ} \mathrm{F}$.

Table 3.6 lists the monthly and annual maximum temperatures. Only 6 days were recorded when the daily maximum temperature was $\leq 0^{\circ} \mathrm{F}$. These were:

\begin{tabular}{lc}
\multicolumn{1}{c}{ Maximum Date } & Temperature \\
\cline { 1 - 1 } January 31, 1950 & $-2^{\circ} \mathrm{F}$ \\
February 1, 1950 & $-3^{\circ} \mathrm{F}$ \\
February 2, 1950 & $-3^{\circ} \mathrm{F}$ \\
January 27, 1957 & $0^{\circ} \mathrm{F}$ \\
December 29, 1968 & $-2^{\circ} \mathrm{F}$ \\
December 30, 1968 & $-2^{\circ} \mathrm{F}$
\end{tabular}

\subsection{Days with Minimum Temperatures $\leq 32^{\circ} \mathrm{F}$ or $\leq 0^{\circ} \mathrm{F}$}

The monthly and seasonal number of days with minimum temperatures at or below $32^{\circ} \mathrm{F}$ or $0^{\circ} \mathrm{F}$ are listed in Table 3.7.

The seasonal average number of days with minimum temperatures $\leq 32^{\circ} \mathrm{F}$ is 106 ; however, the number ranges from 70 to 143 days (winters of 1991-1992 and 1984-1985, respectively). The greatest consecutive number of days with minimum temperatures of $\leq 32^{\circ} \mathrm{F}$ is 93 , from November 9,1978 , through February 9, 1979.

The first autumn temperature $\leq 32^{\circ} \mathrm{F}$ occurred as early as September 23 (2000) and as late as November 12 (1962). The average date is October 19 (Table 3.5). The last date in spring for minimum temperatures $\leq 32^{\circ} \mathrm{F}$ varied from March 19 (1958) to May 16 (1974), with an average date of April 20. The average number of days between last freezing temperature in the spring and first freezing temperature in the autumn is 181 days.

On average, 3 days per winter season have a minimum temperature $\leq 0^{\circ} \mathrm{F}$; however, nearly half of all winters have no minimum temperatures in this category (Table 3.7). The greatest number of these days in any season was 18 (winter of 1949-1950) and the least number of these days was 0 (as recently as the winter of 2002-2003). The greatest number of consecutive days with minimum temperatures $\leq 0^{\circ} \mathrm{F}$ is 11 days, from January 25 through February 4, 1950. During this same period, 4 consecutive days had minimum temperatures $\leq-20^{\circ} \mathrm{F}$. Table 3.8 lists all days with minimum temperatures $\leq 0^{\circ} \mathrm{F}$. Table 3.9 lists monthly and annual minimum temperatures. 
Table 3.6. Monthly and Annual Maximum Temperatures $\left({ }^{\circ} \mathrm{F}\right)$

\begin{tabular}{|c|c|c|c|c|c|c|c|c|c|c|c|c|c|}
\hline Year & Jan & Feb & Mar & Apr & May & Jun & Jul & Aug & Sep & Oct & Nov & $\underline{\text { Dec }}$ & Annual \\
\hline 1945 & 61 & 65 & 72 & 76 & 90 & 98 & 104 & 105 & 93 & 84 & 72 & 57 & 105 \\
\hline 1946 & 57 & 60 & 76 & 91 & 89 & 98 & 105 & 104 & 89 & 75 & 64 & 64 & 105 \\
\hline 1947 & 57 & 68 & 76 & 88 & 101 & 97 & 103 & 98 & 94 & 83 & 63 & 55 & 103 \\
\hline 1948 & 60 & 64 & 73 & 76 & 91 & 104 & 98 & 97 & 98 & 78 & 57 & 52 & 104 \\
\hline 1949 & 48 & 56 & 64 & 84 & 98 & 102 & 104 & 104 & 100 & 74 & 65 & 60 & 104 \\
\hline 1950 & 50 & 63 & 64 & 78 & 90 & 99 & 102 & 103 & 102 & 76 & 62 & 55 & 103 \\
\hline 1951 & 55 & 65 & 65 & 82 & 94 & 97 & 104 & 101 & 97 & 79 & 60 & 58 & 104 \\
\hline 1952 & 50 & 55 & 70 & 89 & 92 & 94 & 106 & 105 & 97 & 85 & 62 & 54 & 106 \\
\hline 1953 & 63 & 65 & 69 & 78 & 88 & 86 & 103 & 104 & 97 & 81 & 65 & 59 & 104 \\
\hline 1954 & 59 & 63 & 65 & 83 & 98 & 94 & 100 & 99 & 92 & 73 & 62 & 54 & 100 \\
\hline 1955 & 50 & 58 & 63 & 77 & 86 & 102 & 107 & 101 & 101 & 75 & 66 & 56 & 107 \\
\hline 1956 & 59 & 56 & 64 & 85 & 96 & 95 & 106 & 104 & 94 & 79 & 64 & 59 & 106 \\
\hline 1957 & 48 & 65 & 66 & 89 & 97 & 100 & 102 & 96 & 98 & 73 & 60 & 59 & 102 \\
\hline 1958 & 60 & 63 & 63 & 78 & 101 & 106 & 107 & 104 & 97 & 89 & 67 & 60 & 107 \\
\hline 1959 & 59 & 60 & 65 & 79 & 91 & 97 & 107 & 103 & 92 & 77 & 70 & 64 & 107 \\
\hline 1960 & 55 & 55 & 83 & 82 & 90 & 96 & 110 & 105 & 94 & 82 & 63 & 52 & 110 \\
\hline 1961 & 60 & 64 & 68 & 75 & 94 & 108 & 108 & 113 & 90 & 81 & 58 & 56 & 113 \\
\hline 1962 & 63 & 60 & 70 & 85 & 81 & 98 & 106 & 100 & 97 & 76 & 67 & 56 & 106 \\
\hline 1963 & 56 & 64 & 70 & 72 & 93 & 102 & 96 & 101 & 98 & 83 & 61 & 57 & 102 \\
\hline 1964 & 57 & 60 & 74 & 73 & 88 & 95 & 106 & 97 & 90 & 80 & 60 & 57 & 106 \\
\hline 1965 & 60 & 67 & 71 & 82 & 91 & 96 & 108 & 106 & 91 & 84 & 64 & 56 & 108 \\
\hline 1966 & 56 & 59 & 78 & 81 & 100 & 95 & 100 & 102 & 99 & 82 & 64 & 56 & 102 \\
\hline 1967 & 62 & 67 & 65 & 71 & 92 & 101 & 105 & 108 & 98 & 78 & 65 & 62 & 108 \\
\hline 1968 & 66 & 64 & 68 & 90 & 90 & 99 & 110 & 102 & 97 & 73 & 60 & 59 & 110 \\
\hline 1969 & 44 & 46 & 74 & 80 & 95 & 103 & 101 & 102 & 96 & 74 & 63 & 54 & 103 \\
\hline 1970 & 56 & 60 & 67 & 71 & 92 & 104 & 106 & 105 & 89 & 86 & 63 & 58 & 106 \\
\hline 1971 & 72 & 66 & 65 & 76 & 92 & 99 & 111 & 112 & 91 & 85 & 64 & 50 & 112 \\
\hline 1972 & 59 & 68 & 76 & 78 & 96 & 98 & 103 & 110 & 95 & 83 & 58 & 65 & 110 \\
\hline 1973 & 51 & 61 & 68 & 80 & 98 & 104 & 106 & 104 & 98 & 76 & 62 & 58 & 106 \\
\hline 1974 & 61 & 59 & 69 & 77 & 86 & 103 & 104 & 103 & 92 & 80 & 64 & 60 & 104 \\
\hline 1975 & 56 & 58 & 65 & 75 & 90 & 95 & 110 & 98 & 96 & 82 & 75 & 62 & 110 \\
\hline 1976 & 59 & 59 & 69 & 80 & 90 & 100 & 102 & 98 & 102 & 84 & 71 & 57 & 102 \\
\hline 1977 & 61 & 70 & 73 & 94 & 82 & 100 & 101 & 107 & 87 & 75 & 68 & 64 & 107 \\
\hline 1978 & 51 & 57 & 74 & 76 & 87 & 101 & 106 & 106 & 90 & 81 & 69 & 54 & 106 \\
\hline 1979 & 37 & 62 & 76 & 83 & 94 & 102 & 110 & 101 & 96 & 84 & 59 & 59 & 110 \\
\hline 1980 & 51 & 59 & 68 & 87 & 87 & 88 & 106 & 98 & 95 & 89 & 65 & 69 & 106 \\
\hline 1981 & 55 & 66 & 70 & 91 & 89 & 96 & 104 & 107 & 99 & 83 & 65 & 58 & 107 \\
\hline 1982 & 57 & 68 & 71 & 81 & 88 & 102 & 107 & 104 & 94 & 75 & 63 & 62 & 107 \\
\hline 1983 & 61 & 62 & 64 & 77 & 103 & 92 & 100 & 99 & 87 & 78 & 67 & 46 & 103 \\
\hline 1984 & 60 & 62 & 67 & 79 & 94 & 96 & 106 & 103 & 92 & 81 & 61 & 52 & 106 \\
\hline 1985 & 36 & 60 & 68 & 82 & 95 & 102 & 106 & 97 & 86 & 74 & 66 & 39 & 106 \\
\hline 1986 & 57 & 72 & 74 & 84 & 104 & 103 & 99 & 103 & 95 & 84 & 63 & 52 & 104 \\
\hline 1987 & 55 & 60 & 70 & 93 & 102 & 106 & 107 & 105 & 106 & 87 & 66 & 59 & 107 \\
\hline 1988 & 54 & 71 & 71 & 83 & 94 & 99 & 105 & 102 & 102 & 88 & 69 & 57 & 105 \\
\hline 1989 & 67 & 53 & 67 & 80 & 88 & 97 & 101 & 103 & 94 & 80 & 73 & 58 & 103 \\
\hline 1990 & 60 & 64 & 76 & 81 & 94 & 96 & 110 & 108 & 98 & 80 & 68 & 57 & 110 \\
\hline 1991 & 59 & 66 & 69 & 82 & 83 & 93 & 105 & 103 & 95 & 88 & 65 & 59 & 105 \\
\hline 1992 & 60 & 62 & 78 & 85 & 98 & 111 & 107 & 109 & 91 & 87 & 62 & 53 & 111 \\
\hline 1993 & 56 & 52 & 66 & 73 & 100 & 98 & 96 & 100 & 98 & 86 & 65 & 67 & 100 \\
\hline 1994 & 61 & 63 & 79 & 88 & 95 & 101 & 111 & 106 & 94 & 84 & 62 & 64 & 111 \\
\hline 1995 & 67 & 68 & 69 & 80 & 95 & 98 & 105 & 102 & 101 & 74 & 69 & 57 & 105 \\
\hline 1996 & 58 & 63 & 68 & 82 & 86 & 98 & 108 & 109 & 94 & 86 & 66 & 52 & 109 \\
\hline 1997 & 57 & 64 & 76 & 75 & 94 & 98 & 101 & 106 & 95 & 77 & 63 & 52 & 106 \\
\hline 1998 & 57 & 58 & 72 & 92 & 93 & 99 & 112 & 110 & 103 & 84 & 67 & 60 & 112 \\
\hline 1999 & 62 & 62 & 75 & 82 & 97 & 102 & 105 & 101 & 91 & 81 & 76 & 62 & 105 \\
\hline 2000 & 55 & 54 & 68 & 82 & 87 & 100 & 107 & 104 & 92 & 76 & 58 & 49 & 107 \\
\hline 2001 & 56 & 54 & 70 & 83 & 101 & 100 & 106 & 105 & 95 & 83 & 68 & 58 & 106 \\
\hline 2002 & 63 & 68 & 70 & 80 & 86 & 104 & 113 & 103 & 96 & 81 & 67 & 56 & 113 \\
\hline 2003 & 67 & 62 & 77 & 78 & 93 & 100 & 108 & 104 & 102 & 89 & 68 & 51 & 108 \\
\hline
\end{tabular}


Table 3.7. Monthly and Seasonal Number of Days with Minimum Temperatures $\left({ }^{\circ} F\right)$ at or below $32^{\circ} \mathrm{F}$ or $0^{\circ} \mathrm{F}$

\begin{tabular}{|c|c|c|c|c|c|c|c|c|c|c|c|c|c|c|c|}
\hline \multirow[b]{2}{*}{ Season } & \multicolumn{10}{|c|}{ Minimum Temperature $\leq 32^{\circ} \mathrm{F}$} & \multicolumn{5}{|c|}{ Minimum Temperature $\leq 0^{\circ} \mathrm{F}$} \\
\hline & Sep & Oct & Nov & Dec & Jan & Feb & Mar & Apr & May & Total & Nov & Dec & Jan & Feb & Total \\
\hline 1944-1945 & -- & -- & -- & -- & 27 & 18 & 12 & 6 & 0 & 63 & -- & -- & 0 & 0 & 0 \\
\hline 1945-1946 & 0 & 5 & 14 & 25 & 27 & 20 & 10 & 2 & 0 & 103 & 0 & 0 & 0 & 0 & 0 \\
\hline 1946-1947 & 0 & 8 & 23 & 24 & 27 & 19 & 6 & 1 & 0 & 108 & 0 & 0 & 1 & 0 & 1 \\
\hline 1947-1948 & 0 & 0 & 11 & 26 & 25 & 24 & 21 & 7 & 1 & 115 & 0 & 0 & 0 & 0 & 0 \\
\hline 1948-1949 & 0 & 8 & 15 & 30 & 31 & 25 & 11 & 4 & 1 & 125 & 0 & 2 & 9 & 0 & 11 \\
\hline $1949-1950$ & 0 & 10 & 4 & 25 & 30 & 22 & 18 & 4 & 0 & 113 & 0 & 0 & 14 & 4 & $18^{(\mathrm{a})}$ \\
\hline $1950-1951$ & 0 & 0 & 13 & 19 & 26 & 25 & 21 & 2 & 0 & 106 & 0 & 0 & 0 & 0 & 0 \\
\hline $1951-1952$ & 0 & 6 & 19 & 26 & 31 & 24 & 20 & 6 & 0 & 132 & 0 & 0 & 0 & 0 & 0 \\
\hline $1952-1953$ & 0 & 0 & 25 & 19 & 9 & 15 & 12 & 4 & 0 & 84 & 0 & 0 & 0 & 0 & 0 \\
\hline 1953-1954 & 0 & 1 & 14 & 22 & 23 & 16 & 19 & 4 & 1 & 100 & 0 & 0 & 2 & 0 & 2 \\
\hline 1954-1955 & 0 & 6 & 6 & 26 & 30 & 25 & 22 & 10 & 1 & 126 & 0 & 0 & 0 & 0 & 0 \\
\hline $1955-1956$ & 0 & 1 & 22 & 28 & 25 & 26 & 14 & 2 & 0 & 118 & 1 & 0 & 2 & 3 & 6 \\
\hline $1956-1957$ & 0 & 3 & 18 & 21 & 31 & 23 & 11 & 0 & 0 & 107 & 0 & 0 & 12 & 1 & 13 \\
\hline $1957-1958$ & 0 & 2 & 17 & 16 & 19 & 5 & 16 & 0 & 0 & 75 & 0 & 0 & 0 & 0 & 0 \\
\hline 1958-1959 & 0 & 4 & 14 & 24 & 25 & 24 & 14 & 2 & 1 & 108 & 0 & 0 & 2 & 0 & 2 \\
\hline $1959-1960$ & 0 & 2 & 24 & 26 & 31 & 21 & 10 & 4 & 0 & 118 & 0 & 0 & 1 & 0 & 1 \\
\hline 1960-1961 & 0 & 4 & 15 & 29 & 23 & 10 & 7 & 5 & 0 & 93 & 0 & 0 & 0 & 0 & 0 \\
\hline 1961-1962 & 0 & 7 & 28 & 26 & 27 & 17 & 19 & 0 & 1 & 125 & 0 & 0 & 1 & 0 & 1 \\
\hline $1962-1963$ & 0 & 0 & 13 & 17 & 27 & 17 & 11 & 2 & 0 & 87 & 0 & 0 & 2 & 0 & 2 \\
\hline 1963-1964 & 0 & 5 & 8 & 31 & 26 & 26 & 16 & 4 & 0 & 116 & 0 & 0 & 0 & 0 & 0 \\
\hline 1964-1965 & 0 & 5 & 13 & 29 & 25 & 18 & 19 & 1 & 1 & 111 & 0 & 2 & 0 & 0 & 2 \\
\hline $1965-1966$ & 0 & 1 & 8 & 25 & 26 & 22 & 13 & 3 & 0 & 98 & 0 & 0 & 0 & 0 & 0 \\
\hline 1966-1967 & 0 & 3 & 11 & 18 & 20 & 17 & 18 & 9 & 0 & 96 & 0 & 0 & 0 & 0 & 0 \\
\hline $1967-1968$ & 0 & 1 & 17 & 25 & 23 & 13 & 6 & 5 & 0 & 90 & 0 & 0 & 0 & 0 & 0 \\
\hline 1968-1969 & 0 & 4 & 8 & 24 & 30 & 25 & 15 & 1 & 0 & 107 & 0 & 4 & 5 & 1 & 10 \\
\hline $1969-1970$ & 0 & 5 & 19 & 21 & 28 & 13 & 16 & 7 & 1 & 110 & 0 & 0 & 0 & 0 & 0 \\
\hline 1970-1971 & 0 & 8 & 14 & 28 & 24 & 19 & 20 & 7 & 0 & 120 & 0 & 0 & 0 & 0 & 0 \\
\hline $1971-1972$ & 0 & 9 & 18 & 27 & 25 & 23 & 13 & 6 & 0 & 121 & 0 & 0 & 3 & 1 & 4 \\
\hline $1972-1973$ & 3 & 6 & 13 & 23 & 30 & 23 & 10 & 4 & 0 & 112 & 0 & 7 & 1 & 0 & 8 \\
\hline 1973-1974 & 0 & 4 & 14 & 16 & 19 & 15 & 12 & 0 & 1 & 81 & 0 & 0 & 8 & 0 & 8 \\
\hline 1974-1975 & 0 & 4 & 12 & 26 & 29 & 24 & 17 & 7 & 0 & 119 & 0 & 0 & 0 & 0 & 0 \\
\hline $1975-1976$ & 0 & 2 & 23 & 28 & 30 & 22 & 19 & 6 & 0 & 130 & 0 & 0 & 0 & 0 & 0 \\
\hline 1976-1977 & 0 & 8 & 17 & 30 & 30 & 19 & 14 & 1 & 0 & 119 & 0 & 0 & 0 & 0 & 0 \\
\hline $1977-1978$ & 0 & 3 & 18 & 25 & 22 & 17 & 11 & 4 & 0 & 100 & 0 & 1 & 2 & 0 & 3 \\
\hline 1978-1979 & 0 & 7 & 26 & 31 & 31 & 21 & 13 & 2 & 0 & 131 & 0 & 3 & 8 & 2 & 13 \\
\hline $1979-1980$ & 0 & 1 & 23 & 22 & 31 & 22 & 13 & 3 & 0 & 115 & 0 & 0 & 1 & 0 & 1 \\
\hline 1980-1981 & 0 & 4 & 16 & 16 & 17 & 17 & 11 & 6 & 0 & 87 & 0 & 0 & 0 & 0 & 0 \\
\hline 1981-1982 & 0 & 5 & 13 & 23 & 27 & 17 & 12 & 12 & 0 & 109 & 0 & 0 & 2 & 0 & 2 \\
\hline $1982-1983$ & 0 & 4 & 21 & 26 & 20 & 13 & 4 & 9 & 0 & 97 & 0 & 0 & 0 & 0 & 0 \\
\hline 1983-1984 & 0 & 3 & 11 & 31 & 26 & 17 & 5 & 2 & 0 & 95 & 0 & 4 & 0 & 0 & 4 \\
\hline 1984-1985 & 0 & 14 & 20 & 31 & 31 & 25 & 20 & 2 & 0 & $143^{(a)}$ & 0 & 4 & 0 & 3 & 7 \\
\hline 1985-1986 & 0 & 7 & 23 & 31 & 23 & 17 & 8 & 4 & 0 & 113 & 5 & 1 & 0 & 0 & 6 \\
\hline 1986-1987 & 0 & 0 & 11 & 29 & 25 & 17 & 9 & 2 & 0 & 93 & 0 & 0 & 0 & 0 & 0 \\
\hline $1987-1988$ & 0 & 3 & 11 & 25 & 29 & 22 & 13 & 2 & 0 & 105 & 0 & 0 & 0 & 0 & 0 \\
\hline 1988-1989 & 0 & 1 & 12 & 23 & 24 & 25 & 11 & 0 & 0 & 96 & 0 & 0 & 0 & 4 & 4 \\
\hline $1989-1990$ & 0 & 2 & 11 & 25 & 18 & 20 & 11 & 0 & 0 & 87 & 0 & 0 & 0 & 0 & 0 \\
\hline 1990-1991 & 0 & 2 & 11 & 27 & 27 & 14 & 14 & 2 & 0 & 97 & 0 & 8 & 0 & 0 & 8 \\
\hline 1991-1992 & 0 & 6 & 8 & 18 & 22 & 11 & 3 & 2 & 0 & $70^{(a)}$ & 0 & 0 & 0 & 0 & 0 \\
\hline 1992-1993 & 0 & 1 & 9 & 29 & 27 & 23 & 10 & 1 & 0 & 100 & 0 & 0 & 2 & 0 & 2 \\
\hline 1993-1994 & 0 & 5 & 26 & 22 & 21 & 21 & 12 & 0 & 0 & 107 & 1 & 0 & 0 & 0 & 1 \\
\hline 1994-1995 & 0 & 2 & 20 & 24 & 20 & 11 & 13 & 2 & 0 & 92 & 0 & 0 & 0 & 0 & 0 \\
\hline 1995-1996 & 0 & 3 & 12 & 25 & 28 & 22 & 13 & 5 & 1 & 109 & 0 & 0 & 2 & 3 & 5 \\
\hline 1996-1997 & 0 & 7 & 19 & 26 & 24 & 18 & 10 & 7 & 1 & 112 & 0 & 1 & 0 & 0 & 1 \\
\hline 1997-1998 & 0 & 4 & 12 & 25 & 21 & 14 & 9 & 3 & 0 & 88 & 0 & 0 & 0 & 0 & 0 \\
\hline 1998-1999 & 0 & 5 & 10 & 23 & 20 & 15 & 13 & 7 & 2 & 95 & 0 & 1 & 0 & 0 & 1 \\
\hline $1999-2000$ & 0 & 6 & 10 & 22 & 29 & 21 & 14 & 2 & 0 & 104 & 0 & 0 & 0 & 0 & 0 \\
\hline 2000-2001 & 1 & 5 & 27 & 30 & 28 & 26 & 12 & 4 & 0 & 133 & 0 & 0 & 0 & 0 & 0 \\
\hline 2001-2002 & 0 & 1 & 14 & 26 & 19 & 22 & 20 & 7 & 1 & 110 & 0 & 0 & 0 & 0 & 0 \\
\hline $2002-2003$ & 0 & 10 & 14 & 15 & 14 & 17 & 9 & 4 & 0 & 83 & 0 & 0 & 0 & 0 & $0^{(a, b)}$ \\
\hline Average $^{(\mathrm{c})}$ & $<1$ & 4 & 15 & 25 & 25 & 19 & 13 & 4 & $<1$ & 106 & $<1$ & 1 & 1 & $<1$ & 3 \\
\hline Normal & $<1$ & 4 & 16 & 25 & 25 & 19 & 12 & 4 & $<1$ & 105 & $<1$ & 1 & 1 & $<1$ & 3 \\
\hline
\end{tabular}

(a) Greatest and least seasonal totals.

(b) Most recent of numerous occurrences.

(c) Based on entire period of record, 1945 through 2003. 
Table 3.8. Days with Minimum Temperatures $\leq 0^{\circ} \mathrm{F}$

\begin{tabular}{|c|c|c|c|c|c|c|}
\hline $\begin{array}{c}\text { Temperature } \\
\left({ }^{\circ} \mathrm{F}\right) \\
\end{array}$ & & & Date(s) & ccurrence & & \\
\hline-23 & $02 / 03 / 50$ & $02 / 01 / 50$ & & & & \\
\hline-22 & $01 / 26 / 57$ & & & & & \\
\hline-21 & $01 / 27 / 57$ & $02 / 02 / 50$ & $01 / 31 / 50$ & & & \\
\hline-18 & $02 / 01 / 96$ & $01 / 31 / 96$ & $01 / 29 / 50$ & & & \\
\hline-15 & $02 / 03 / 96$ & & & & & \\
\hline-14 & $02 / 02 / 96$ & $12 / 30 / 68$ & $01 / 29 / 57$ & $01 / 28 / 57$ & & \\
\hline-13 & $11 / 23 / 85$ & $12 / 22 / 83$ & $01 / 09 / 74$ & $12 / 16 / 64$ & $01 / 30 / 50$ & \\
\hline-12 & $12 / 22 / 90$ & $11 / 24 / 85$ & $02 / 01 / 79$ & $12 / 17 / 64$ & $01 / 25 / 57$ & \\
\hline-11 & $01 / 30 / 96$ & $01 / 01 / 79$ & $01 / 17 / 50$ & $01 / 14 / 50$ & $01 / 25 / 49$ & \\
\hline-10 & $\begin{array}{l}12 / 29 / 90 \\
02 / 02 / 56\end{array}$ & $\begin{array}{l}12 / 21 / 90 \\
02 / 01 / 56\end{array}$ & $02 / 02 / 79$ & $12 / 30 / 78$ & $01 / 06 / 74$ & $12 / 29 / 68$ \\
\hline-9 & $12 / 23 / 83$ & $01 / 06 / 79$ & $12 / 31 / 78$ & $01 / 02 / 78$ & $01 / 08 / 74$ & \\
\hline-8 & $\begin{array}{l}12 / 01 / 85 \\
01 / 16 / 50\end{array}$ & $01 / 06 / 82$ & $01 / 07 / 74$ & $12 / 10 / 72$ & $01 / 23 / 69$ & $01 / 30 / 57$ \\
\hline-7 & $01 / 07 / 79$ & $01 / 31 / 56$ & $01 / 28 / 50$ & $01 / 05 / 50$ & & \\
\hline-6 & $\begin{array}{l}12 / 28 / 96 \\
01 / 29 / 69 \\
01 / 11 / 49\end{array}$ & $\begin{array}{l}11 / 22 / 85 \\
01 / 28 / 69\end{array}$ & $\begin{array}{l}01 / 31 / 79 \\
01 / 18 / 57\end{array}$ & $\begin{array}{l}01 / 05 / 74 \\
01 / 20 / 54\end{array}$ & $\begin{array}{l}12 / 13 / 72 \\
01 / 04 / 50\end{array}$ & $\begin{array}{l}12 / 08 / 72 \\
01 / 24 / 49\end{array}$ \\
\hline-5 & $\begin{array}{l}02 / 05 / 89 \\
01 / 15 / 50\end{array}$ & $02 / 04 / 85$ & $01 / 01 / 78$ & $1 / 10 / 74$ & $12 / 12 / 72$ & $12 / 09 / 72$ \\
\hline-4 & $\begin{array}{l}01 / 13 / 93 \\
01 / 11 / 74 \\
01 / 12 / 49\end{array}$ & $\begin{array}{l}12 / 23 / 90 \\
12 / 11 / 72\end{array}$ & $\begin{array}{l}02 / 04 / 89 \\
01 / 28 / 72\end{array}$ & $\begin{array}{l}12 / 19 / 84 \\
01 / 12 / 63\end{array}$ & $\begin{array}{l}12 / 21 / 83 \\
01 / 28 / 49\end{array}$ & $\begin{array}{l}01 / 27 / 79 \\
01 / 13 / 49\end{array}$ \\
\hline-3 & $\begin{array}{l}02 / 06 / 89 \\
12 / 29 / 78 \\
01 / 11 / 63\end{array}$ & $\begin{array}{l}11 / 25 / 85 \\
12 / 31 / 77 \\
01 / 17 / 57\end{array}$ & $\begin{array}{l}02 / 03 / 85 \\
01 / 31 / 69\end{array}$ & $\begin{array}{l}12 / 18 / 84 \\
01 / 30 / 69\end{array}$ & $\begin{array}{l}01 / 10 / 80 \\
12 / 31 / 68\end{array}$ & $\begin{array}{l}01 / 08 / 79 \\
12 / 28 / 68\end{array}$ \\
\hline-2 & $\begin{array}{l}12 / 31 / 90 \\
01 / 04 / 74 \\
01 / 10 / 49\end{array}$ & $\begin{array}{l}12 / 30 / 90 \\
12 / 14 / 72 \\
12 / 27 / 48\end{array}$ & $\begin{array}{l}12 / 20 / 90 \\
01 / 22 / 62\end{array}$ & $\begin{array}{l}12 / 21 / 84 \\
01 / 31 / 57\end{array}$ & $\begin{array}{l}12 / 20 / 84 \\
01 / 19 / 57\end{array}$ & $\begin{array}{l}01 / 05 / 79 \\
01 / 20 / 49\end{array}$ \\
\hline-1 & $\begin{array}{l}12 / 21 / 98 \\
02 / 01 / 69 \\
01 / 30 / 56\end{array}$ & $\begin{array}{l}11 / 24 / 93 \\
01 / 18 / 60 \\
11 / 14 / 55\end{array}$ & $\begin{array}{l}11 / 26 / 85 \\
01 / 04 / 59 \\
02 / 04 / 50\end{array}$ & $\begin{array}{l}01 / 08 / 73 \\
02 / 02 / 57 \\
01 / 25 / 50\end{array}$ & $\begin{array}{l}02 / 03 / 72 \\
01 / 16 / 57 \\
01 / 13 / 50\end{array}$ & $\begin{array}{l}01 / 26 / 72 \\
02 / 03 / 56\end{array}$ \\
\hline 0 & $\begin{array}{l}01 / 11 / 93 \\
01 / 28 / 79 \\
01 / 26 / 50\end{array}$ & $\begin{array}{l}12 / 24 / 90 \\
01 / 27 / 72 \\
01 / 04 / 49\end{array}$ & $\begin{array}{l}02 / 02 / 89 \\
01 / 03 / 59 \\
12 / 26 / 48\end{array}$ & $\begin{array}{l}02 / 06 / 85 \\
01 / 24 / 57 \\
01 / 15 / 47\end{array}$ & $\begin{array}{l}12 / 27 / 83 \\
01 / 21 / 54\end{array}$ & $\begin{array}{l}01 / 07 / 82 \\
01 / 27 / 50\end{array}$ \\
\hline
\end{tabular}


Table 3.9. Monthly and Annual Minimum Temperatures $\left({ }^{\circ} \mathbf{F}\right)$

\begin{tabular}{|c|c|c|c|c|c|c|c|c|c|c|c|c|c|}
\hline Year & Jan & Feb & $\underline{\text { Mar }}$ & Apr & May & Jun & Jul & Aug & Sep & Oct & Nov & Dec & Annual \\
\hline 1945 & 21 & 14 & 10 & 28 & 38 & 46 & 53 & 47 & 35 & 26 & 16 & 13 & 10 \\
\hline 1946 & 18 & 18 & 25 & 30 & 33 & 44 & 50 & 49 & 35 & 21 & 16 & 6 & 6 \\
\hline 1947 & 0 & 11 & 23 & 32 & 42 & 45 & 53 & 50 & 40 & 34 & 22 & 16 & 0 \\
\hline 1948 & 14 & 1 & 13 & 28 & 32 & 51 & 49 & 47 & 34 & 22 & 20 & -2 & -2 \\
\hline 1949 & -11 & 3 & 27 & 30 & 31 & 42 & 49 & 47 & 38 & 23 & 28 & 10 & -11 \\
\hline 1950 & -21 & -23 & 20 & 27 & 38 & 44 & 49 & 51 & 38 & 34 & 21 & 22 & -23 \\
\hline 1951 & 6 & 18 & 22 & 26 & 37 & 41 & 51 & 47 & 39 & 27 & 23 & 4 & 4 \\
\hline 1952 & 5 & 16 & 24 & 27 & 37 & 42 & 49 & 46 & 42 & 34 & 7 & 17 & 5 \\
\hline 1953 & 24 & 20 & 23 & 27 & 36 & 40 & 52 & 51 & 37 & 30 & 24 & 20 & 20 \\
\hline 1954 & -6 & 17 & 18 & 26 & 28 & 41 & 45 & 48 & 36 & 26 & 23 & 14 & -6 \\
\hline 1955 & 18 & 15 & 6 & 26 & 31 & 42 & 43 & 48 & 37 & 32 & -1 & 9 & -1 \\
\hline 1956 & -7 & -10 & 15 & 28 & 38 & 40 & 54 & 49 & 39 & 31 & 15 & 2 & -10 \\
\hline 1957 & -22 & -1 & 28 & 34 & 48 & 48 & 51 & 52 & 36 & 32 & 20 & 23 & -22 \\
\hline 1958 & 16 & 29 & 23 & 34 & 38 & 47 & 49 & 53 & 34 & 30 & 9 & 21 & 9 \\
\hline 1959 & -1 & 19 & 25 & 30 & 30 & 41 & 49 & 49 & 41 & 26 & 6 & 14 & -1 \\
\hline 1960 & -1 & 10 & 13 & 30 & 33 & 46 & 52 & 41 & 40 & 30 & 22 & 14 & -1 \\
\hline 1961 & 16 & 27 & 25 & 31 & 38 & 44 & 50 & 56 & 36 & 26 & 10 & 3 & 3 \\
\hline 1962 & -2 & 7 & 15 & 33 & 31 & 37 & 42 & 49 & 40 & 34 & 16 & 16 & -2 \\
\hline 1963 & -4 & 8 & 22 & 28 & 36 & 45 & 49 & 49 & 45 & 23 & 17 & 7 & -4 \\
\hline 1964 & 15 & 19 & 15 & 30 & 35 & 45 & 50 & 44 & 39 & 30 & 20 & -13 & -13 \\
\hline 1965 & 10 & 18 & 14 & 32 & 32 & 48 & 50 & 42 & 33 & 30 & 26 & 10 & 10 \\
\hline 1966 & 17 & 19 & 19 & 26 & 37 & 38 & 48 & 50 & 43 & 29 & 22 & 22 & 17 \\
\hline 1967 & 23 & 20 & 20 & 27 & 34 & 47 & 52 & 56 & 43 & 30 & 17 & 6 & 6 \\
\hline 1968 & 10 & 15 & 25 & 23 & 33 & 42 & 51 & 47 & 39 & 30 & 23 & -14 & -14 \\
\hline 1969 & -8 & -1 & 22 & 31 & 38 & 52 & 53 & 45 & 41 & 29 & 19 & 19 & -8 \\
\hline 1970 & 8 & 21 & 24 & 26 & 30 & 46 & 50 & 52 & 34 & 23 & 11 & 8 & 8 \\
\hline 1971 & 8 & 15 & 15 & 27 & 36 & 44 & 44 & 51 & 38 & 13 & 21 & 5 & 5 \\
\hline 1972 & -4 & -1 & 24 & 26 & 36 & 45 & 50 & 49 & 30 & 20 & 24 & -8 & -8 \\
\hline 1973 & -1 & 21 & 26 & 27 & 34 & 45 & 46 & 46 & 43 & 31 & 16 & 14 & -1 \\
\hline 1974 & -13 & 23 & 21 & 33 & 32 & 41 & 48 & 48 & 40 & 29 & 24 & 17 & -13 \\
\hline 1975 & 14 & 10 & 19 & 21 & 33 & 38 & 53 & 46 & 44 & 26 & 15 & 14 & 10 \\
\hline 1976 & 16 & 10 & 11 & 25 & 35 & 37 & 47 & 44 & 42 & 28 & 13 & 12 & 10 \\
\hline 1977 & 4 & 21 & 24 & 31 & 34 & 39 & 49 & 48 & 36 & 28 & 9 & -3 & -3 \\
\hline 1978 & -9 & 17 & 25 & 30 & 37 & 44 & 50 & 47 & 41 & 21 & 7 & -10 & -10 \\
\hline 1979 & -11 & -12 & 20 & 29 & 38 & 45 & 39 & 53 & 42 & 32 & 13 & 19 & -12 \\
\hline 1980 & -3 & 19 & 25 & 28 & 38 & 40 & 47 & 42 & 41 & 30 & 18 & 9 & -3 \\
\hline 1981 & 23 & 8 & 24 & 24 & 35 & 40 & 45 & 48 & 34 & 27 & 19 & 8 & 8 \\
\hline 1982 & -8 & 9 & 24 & 24 & 33 & 47 & 45 & 51 & 41 & 26 & 18 & 13 & -8 \\
\hline 1983 & 12 & 15 & 29 & 27 & 37 & 40 & 49 & 50 & 35 & 29 & 22 & -13 & -13 \\
\hline 1984 & 10 & 24 & 25 & 30 & 33 & 37 & 51 & 47 & 36 & 12 & 25 & -4 & -4 \\
\hline 1985 & 5 & -5 & 21 & 26 & 33 & 44 & 56 & 46 & 33 & 26 & -13 & -8 & -13 \\
\hline 1986 & 12 & 15 & 29 & 28 & 37 & 43 & 48 & 54 & 38 & 33 & 16 & 18 & 12 \\
\hline 1987 & 9 & 18 & 24 & 30 & 38 & 43 & 49 & 51 & 41 & 31 & 17 & 9 & 9 \\
\hline 1988 & 14 & 9 & 24 & 31 & 35 & 42 & 47 & 52 & 38 & 32 & 28 & 8 & 8 \\
\hline 1989 & 15 & -5 & 14 & 35 & 39 & 46 & 49 & 52 & 44 & 27 & 21 & 19 & -5 \\
\hline 1990 & 22 & 9 & 24 & 37 & 39 & 47 & 46 & 52 & 48 & 31 & 28 & -12 & -12 \\
\hline 1991 & 5 & 26 & 22 & 31 & 38 & 44 & 55 & 47 & 42 & 23 & 23 & 20 & 5 \\
\hline 1992 & 19 & 22 & 32 & 27 & 37 & 49 & 54 & 43 & 40 & 30 & 17 & 12 & 12 \\
\hline 1993 & -4 & 3 & 17 & 32 & 35 & 46 & 50 & 43 & 37 & 29 & -1 & 21 & -4 \\
\hline 1994 & 20 & 5 & 19 & 35 & 36 & 44 & 50 & 53 & 47 & 30 & 19 & 8 & 5 \\
\hline 1995 & 8 & 8 & 21 & 28 & 39 & 47 & 52 & 45 & 42 & 16 & 17 & 16 & 8 \\
\hline 1996 & -18 & -18 & 18 & 30 & 29 & 45 & 49 & 48 & 34 & 34 & 17 & -6 & -18 \\
\hline 1997 & 8 & 20 & 28 & 25 & 30 & 46 & 49 & 52 & 44 & 29 & 23 & 19 & 8 \\
\hline 1998 & 7 & 22 & 23 & 29 & 39 & 47 & 58 & 50 & 43 & 25 & 27 & -1 & -1 \\
\hline 1999 & 18 & 20 & 25 & 25 & 30 & 38 & 45 & 43 & 36 & 27 & 26 & 20 & 18 \\
\hline 2000 & 18 & 21 & 24 & 29 & 33 & 43 & 44 & 49 & 32 & 30 & 18 & 13 & 13 \\
\hline 2001 & 20 & 17 & 23 & 28 & 34 & 44 & 53 & 50 & 38 & 31 & 26 & 16 & 16 \\
\hline 2002 & 13 & 16 & 18 & 29 & 32 & 46 & 50 & 50 & 38 & 7 & 11 & 23 & 7 \\
\hline 2003 & 22 & 15 & 27 & 24 & 34 & 45 & 52 & 53 & 41 & 19 & 13 & 8 & 8 \\
\hline
\end{tabular}




\subsection{Monthly Extremes of Daily Maximum and Minimum Temperatures}

Monthly extremes of daily maximum and minimum temperatures are presented in Table 3.10. Note that ranges are comparable in the winter and in the summer. February temperatures ranged from $72^{\circ} \mathrm{F}$ to $-23^{\circ} \mathrm{F}$, a range of $95^{\circ} \mathrm{F}$. July temperatures ranged from $112^{\circ} \mathrm{F}$ to $39^{\circ} \mathrm{F}$, a range of $83^{\circ} \mathrm{F}$.

\subsection{Daily Temperature Distributions}

Daily temperatures are generally described relative to a long-term average temperature or to a record high or low temperature. For example, the daily maximum temperature may be described as above average or near the record for the day. However, this type of description does not provide information about whether the temperature is in the range of temperatures that is typical for the day. Figure 3.1 shows an example of a different way of presenting temperature information that places the temperatures in the context of the climatological records. The figure shows the record low and high daily maximum temperatures at the Hanford Meteorology Station for January 7 based on climatological records from 1945 through 2003. Between the record low and high temperatures, the figure has a bar that shows the range of daily maximum temperatures that have occurred $70 \%$ of the time centered on the median. This range can be considered the range of typical daily maximum temperatures for the date. In $15 \%$ of the years, the daily maximum temperature has been above the range, and in $15 \%$ of the years, it has been below the range. On the bar there is a horizontal mark that indicates the median daily maximum temperature. The median temperature is the daily maximum temperature that has been exceeded in $50 \%$ of the years of record. For the Hanford Meteorology Station, the median daily maximum temperature is generally quite close to the long-term average daily maximum temperature. Finally, there is a solid square on the bar. The solid square shows the daily maximum temperature for January 7, 2002. A similar presentation can be prepared for daily minimum temperatures. Figure 3.2 through 3.25 show daily maximum and daily minimum temperature data by month and the data for 2003 .

\subsection{Average Daily Temperature Range}

Table 3.11 represents the average daily temperature range by month and year for the period 1945 through 2003. This statistic is compiled by determining each daily temperature range (the difference between the maximum and minimum temperature), totaling for every day of the month, and dividing by the number of days in the month. As can be seen from the table, the average daily temperature ranges for July and August $\left(>30^{\circ} \mathrm{F}\right)$ are more than double the ranges for December and January $\left(<15^{\circ} \mathrm{F}\right)$. The lowest average daily temperature range was $6.8^{\circ} \mathrm{F}$ in January 1985 ; the greatest was $34.5^{\circ} \mathrm{F}$ in August 1967 . The greatest range for any single day was $48^{\circ} \mathrm{F}$ on August 14,1995 (high of $93^{\circ} \mathrm{F}$, low of $45^{\circ} \mathrm{F}$ ) and also on May 11,1946 (high of $86^{\circ} \mathrm{F}$, low of $38^{\circ} \mathrm{F}$ ). The smallest range for any single day was $1^{\circ} \mathrm{F}$ as recently as December 25,2000 (high of $31^{\circ} \mathrm{F}$, low of $30^{\circ} \mathrm{F}$ ).

\subsection{Normal and Extreme Daily Temperatures}

Table 3.12 lists the normal and extreme daily maximum and minimum temperatures. Climatological normals are computed every 10 years and are based on a 30-year period, ending with the first year of each new decade. This table is using normals based on the period 1971 through 2000. The normal temperatures in Table 3.12 are computed using a 7-day running mean, centered on each day. 


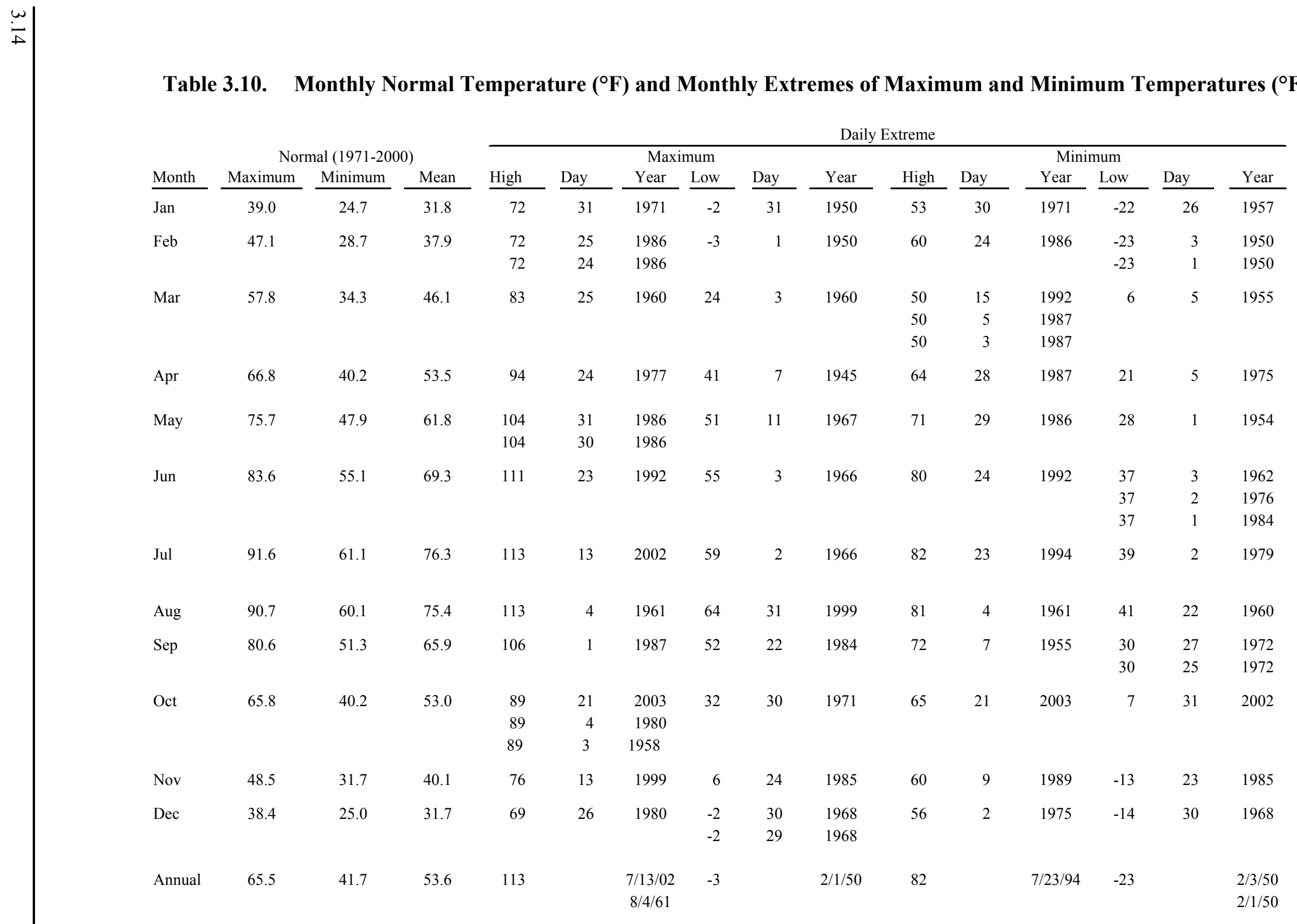




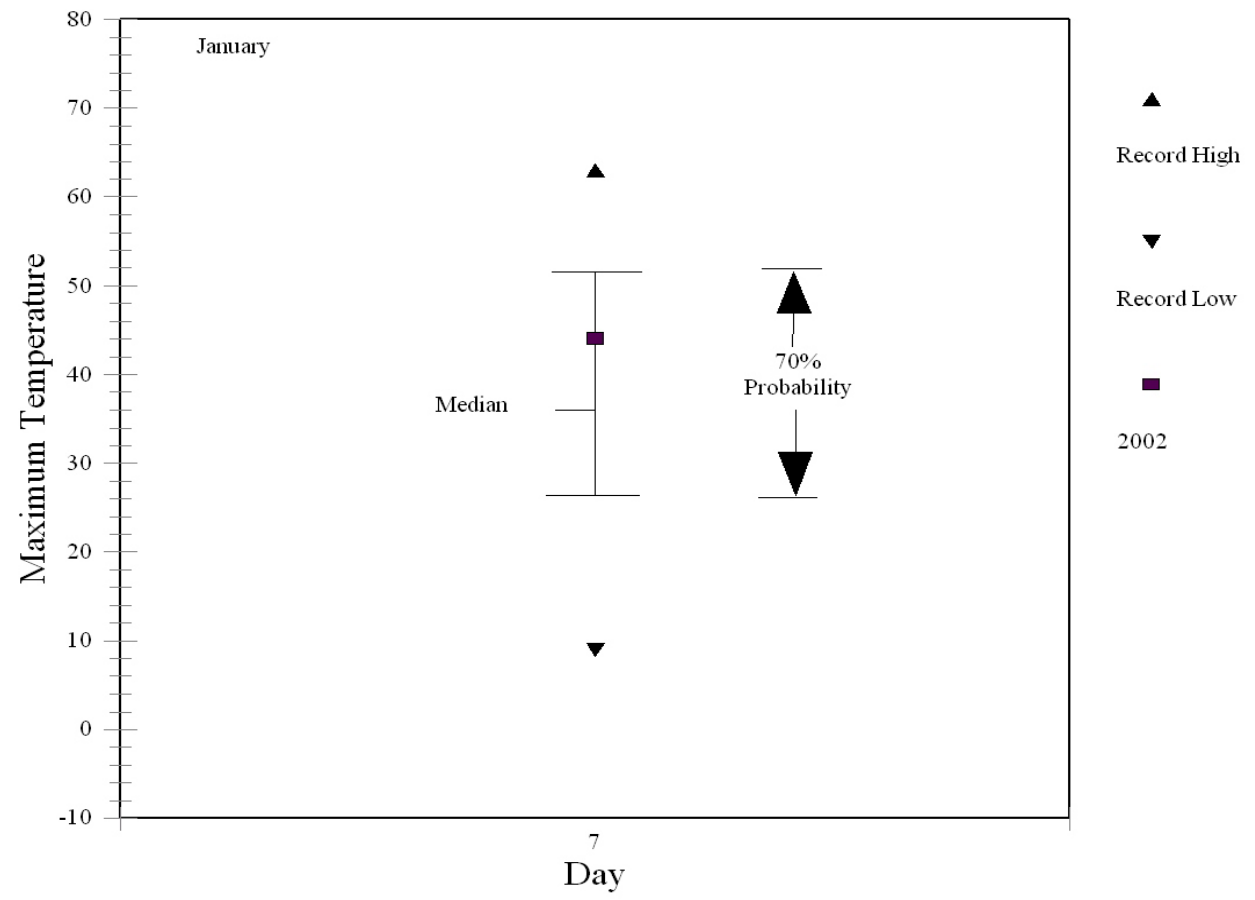

Figure 3.1. Graphical Presentation of Daily Maximum Temperatures

Four possible temperature extremes are presented for each day, a record high and low maximum and a record high and low minimum. These daily records, plus the year of occurrence for the period 1945 through 2002, also are indicated in Table 3.12.

\subsection{Heating- and Cooling-Degree Days}

Data about heating- and cooling-degree days are generally used by the utility industry and those involved in building design to assess heating and cooling energy requirements. A temperature of $65^{\circ} \mathrm{F}$ is generally used as the basis for this calculation. To determine whether a day has either heating-degree days or cooling-degree days, 65 is subtracted from the daily average temperature (computed by adding the daily maximum and minimum temperatures and dividing by two). If the difference is positive, the day has cooling-degree days. If the difference is negative, the day has heating-degree days.

Example Calculations

\begin{tabular}{llll}
\hline & \multicolumn{1}{c}{ Summer Day } & & Winter Day \\
\cline { 2 - 2 } Daily high temperature & 90 & & 42 \\
Daily low temperature & 60 & & 20 \\
Daily average temperature & $75(150 \div 2)$ & & $31(62 \div 2)$ \\
Threshold temperature & -65 & & -65 \\
Difference & $10(10 \mathrm{CDDs})^{*}$ & & $-34(34 \mathrm{HDDs}) *$ \\
& &
\end{tabular}




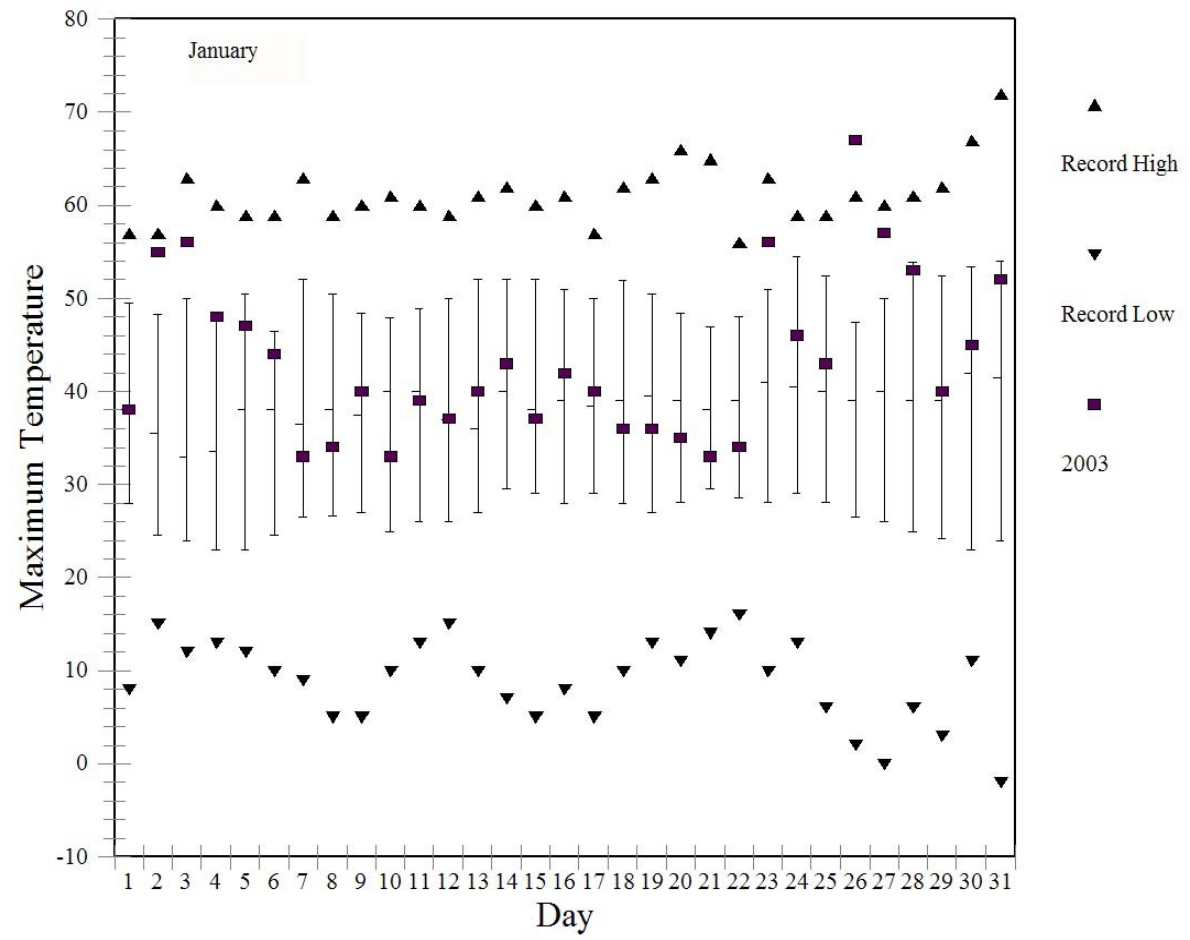

Figure 3.2. Daily Maximum Temperatures $\left({ }^{\circ} \mathrm{F}\right)$, January 2003

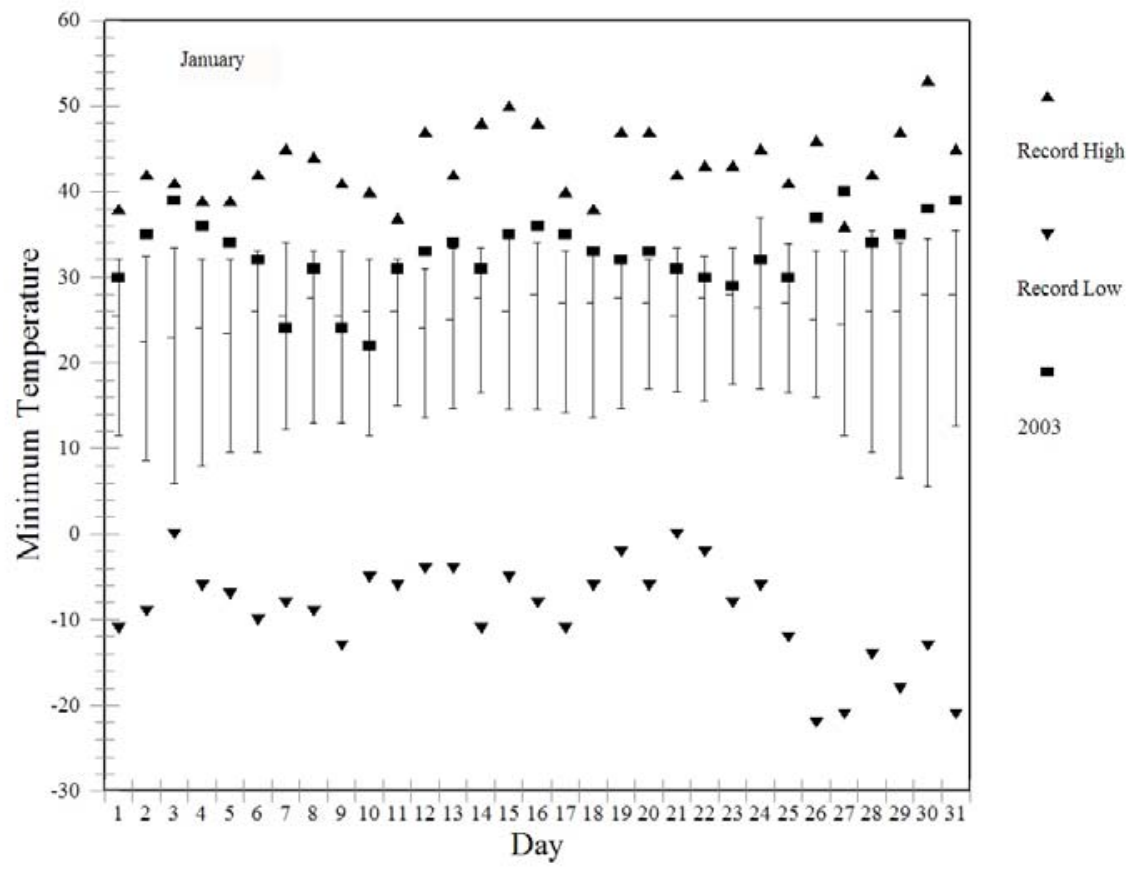

Figure 3.3. Daily Minimum Temperatures $\left({ }^{\circ}\right.$ F), January 2003 


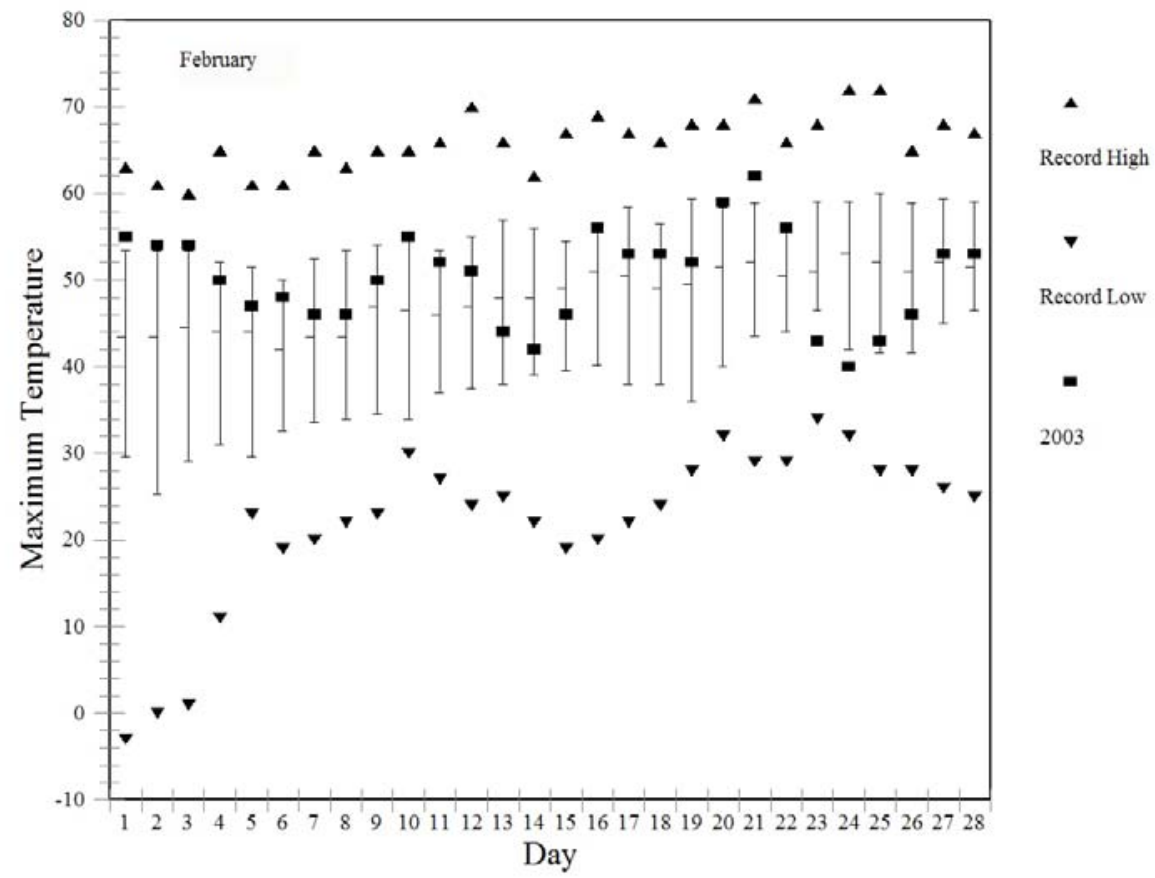

Figure 3.4. Daily Maximum Temperatures $\left({ }^{\circ}\right.$ F), February 2003

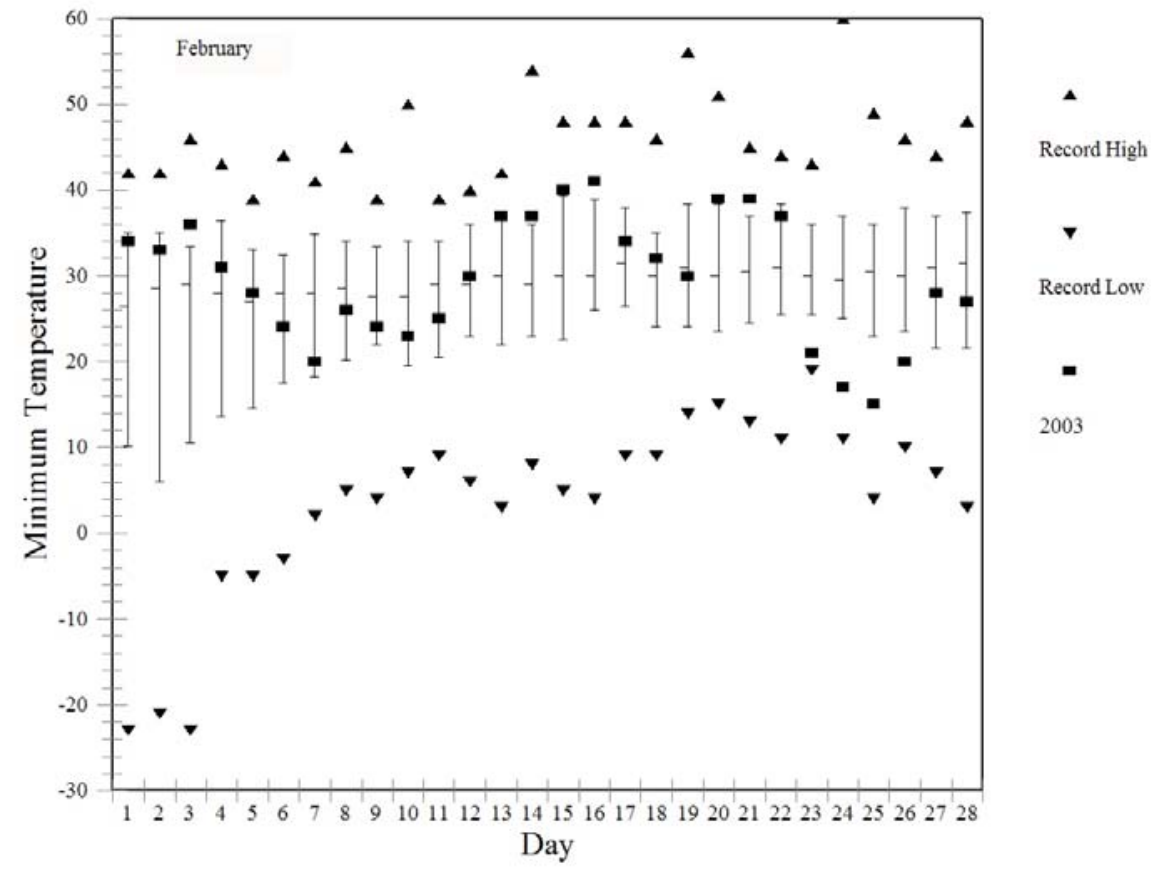

Figure 3.5. Daily Minimum Temperatures $\left({ }^{\circ}\right.$ F), February 2003 


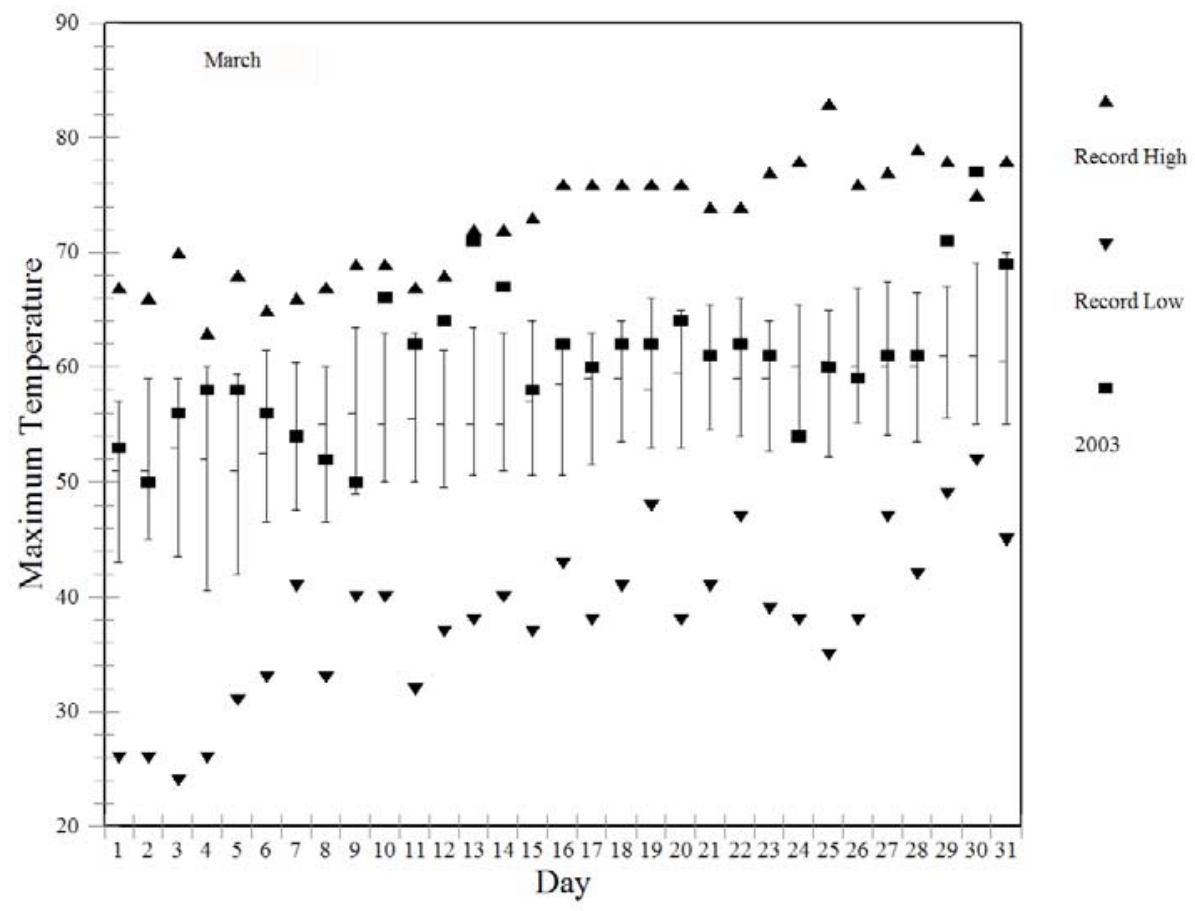

Figure 3.6. Daily Maximum Temperatures $\left({ }^{\circ}\right.$ F), March 2003

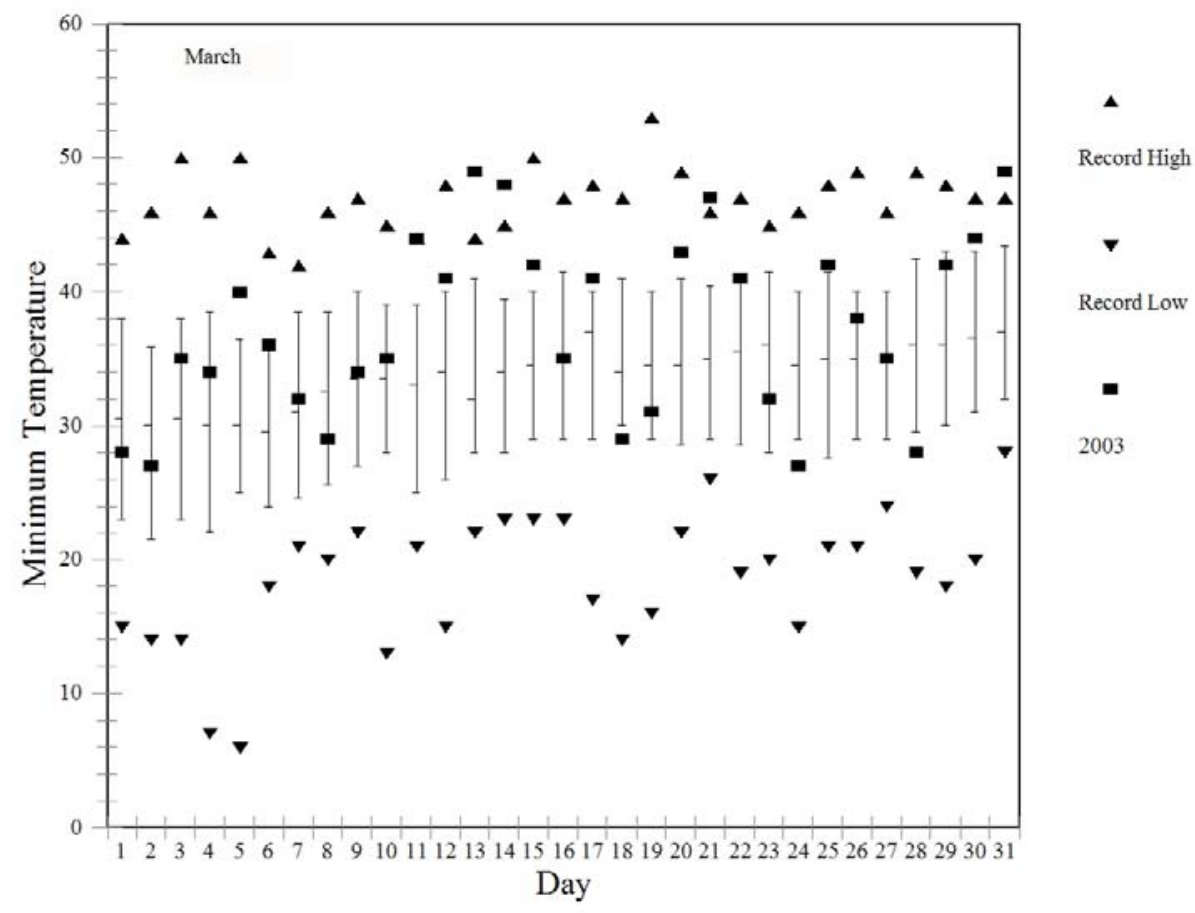

Figure 3.7. Daily Minimum Temperatures $\left({ }^{\circ}\right.$ F), March 2003 


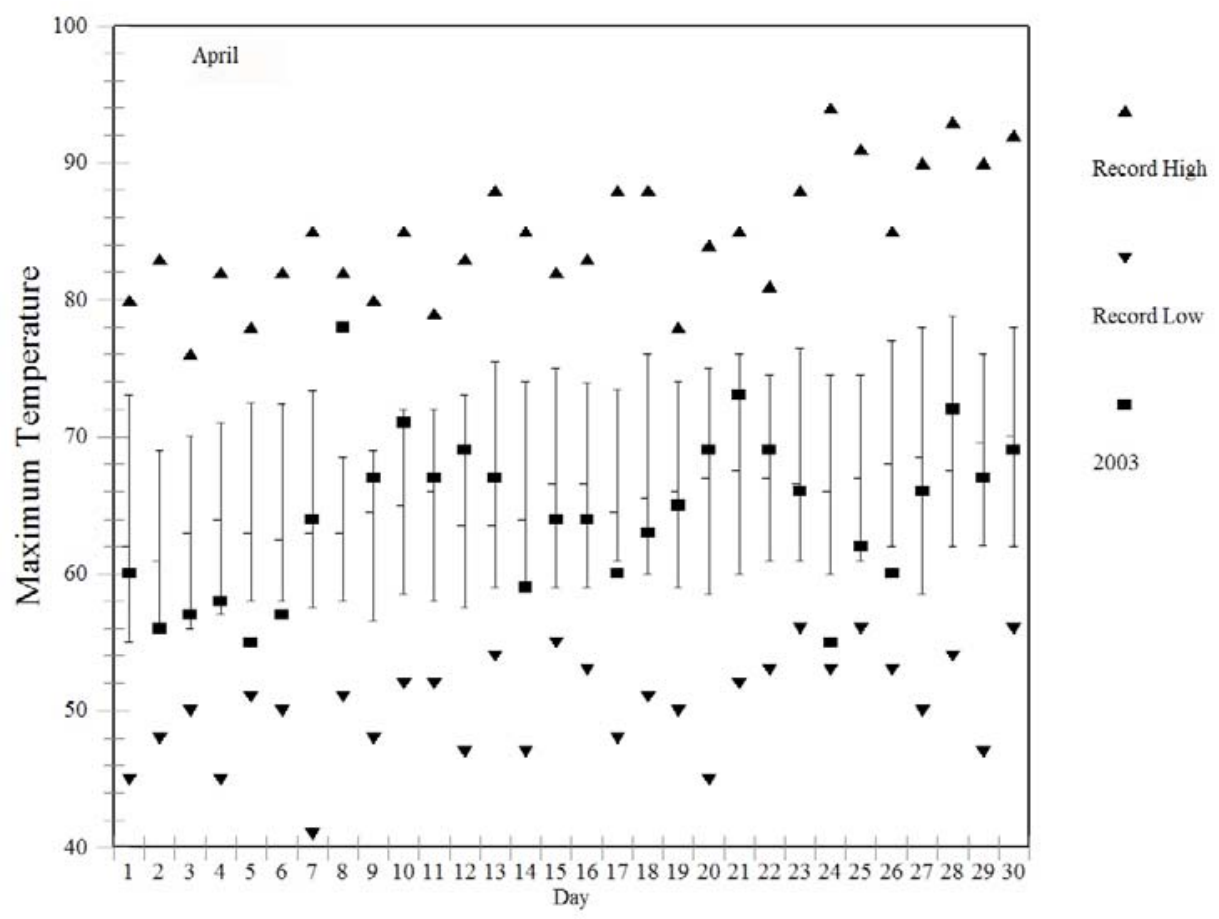

Figure 3.8. Daily Maximum Temperatures $\left({ }^{\circ}\right.$ F), April 2003

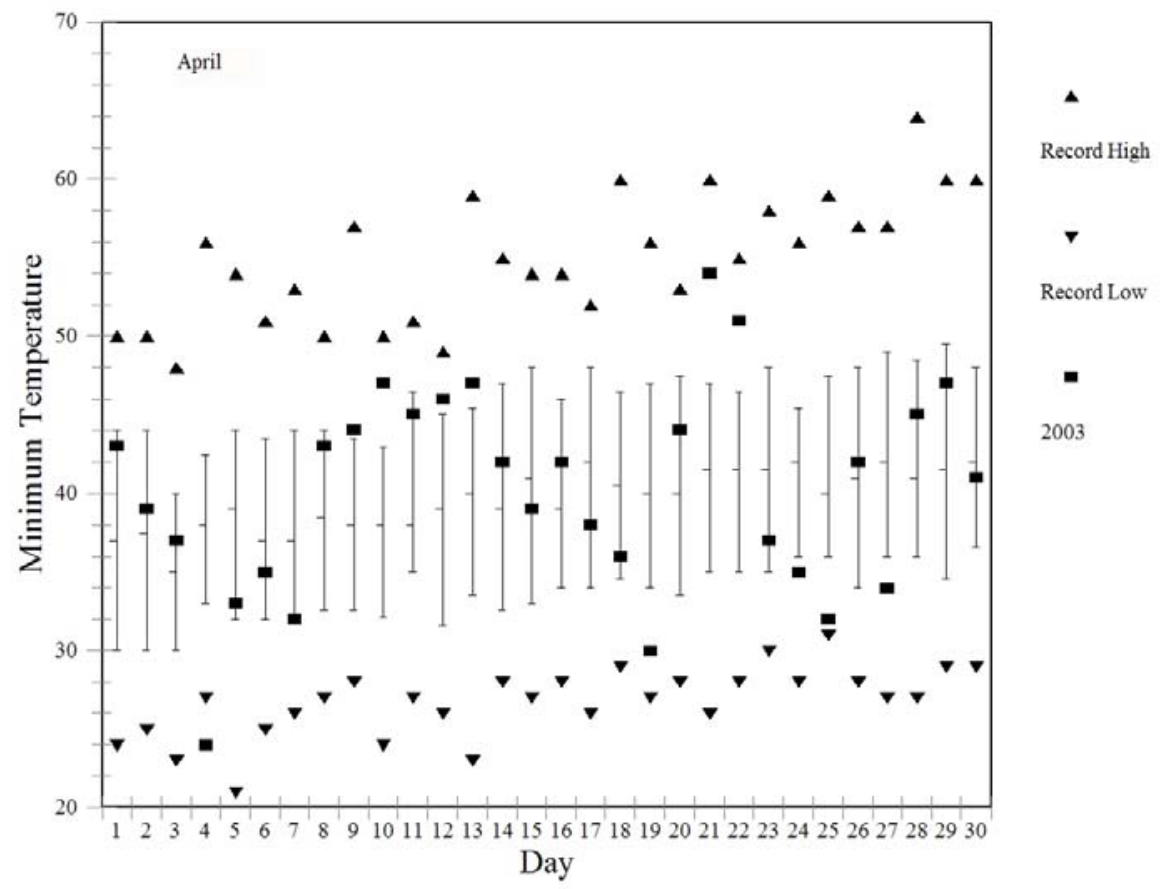

Figure 3.9. Daily Minimum Temperatures $\left({ }^{\circ} \mathbf{F}\right)$, April 2003 


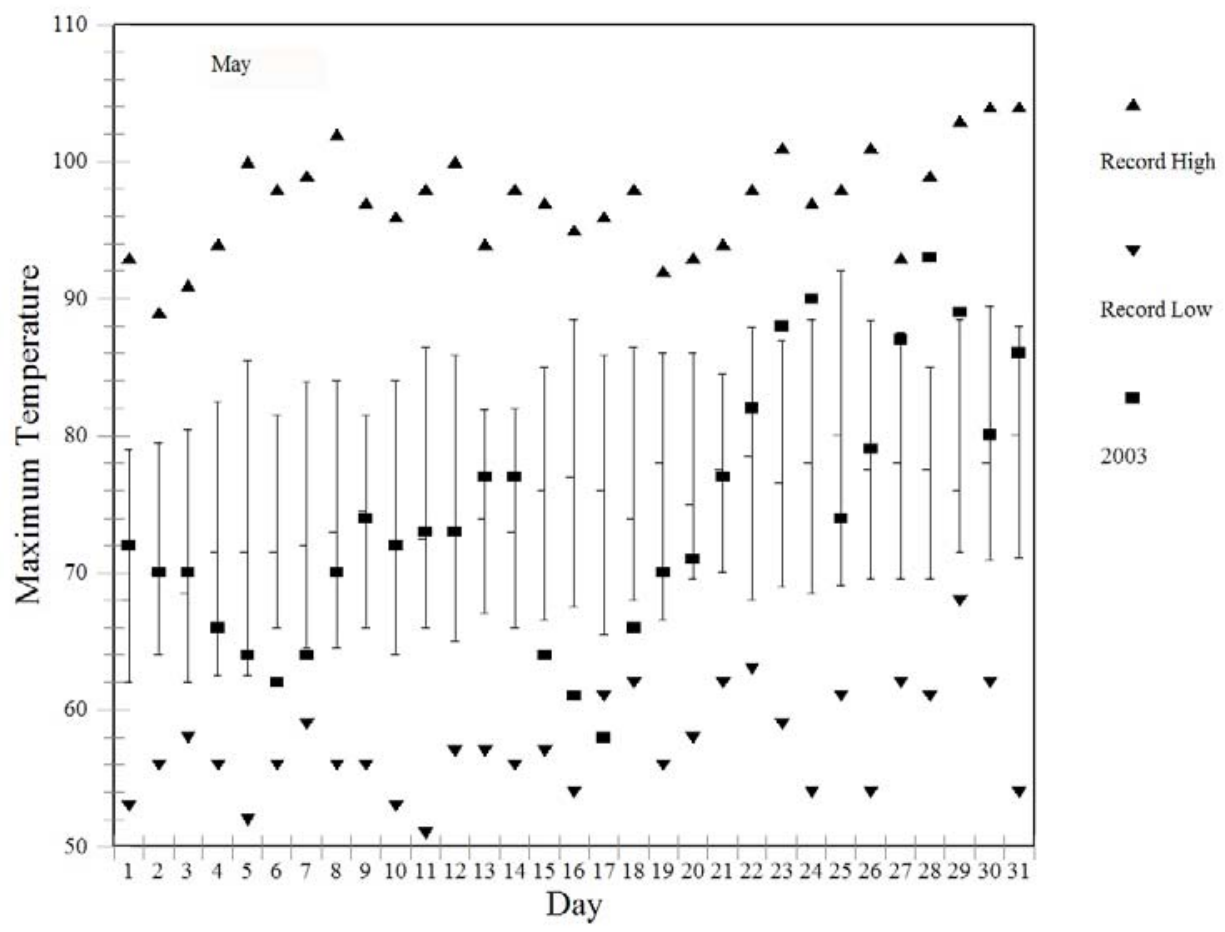

Figure 3.10. Daily Maximum Temperatures $\left({ }^{\circ}\right.$ F), May 2003

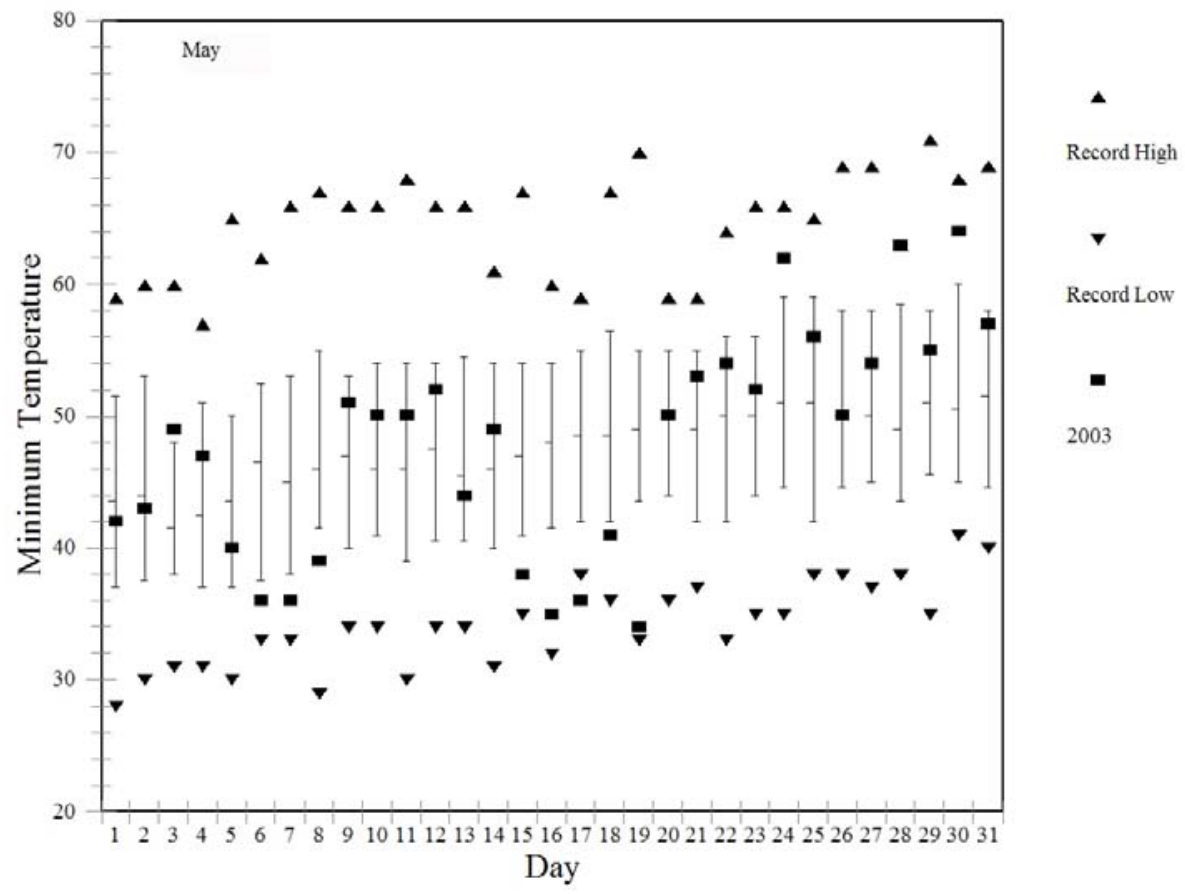

Figure 3.11. Daily Minimum Temperatures $\left({ }^{\circ}\right.$ F), May 2003 


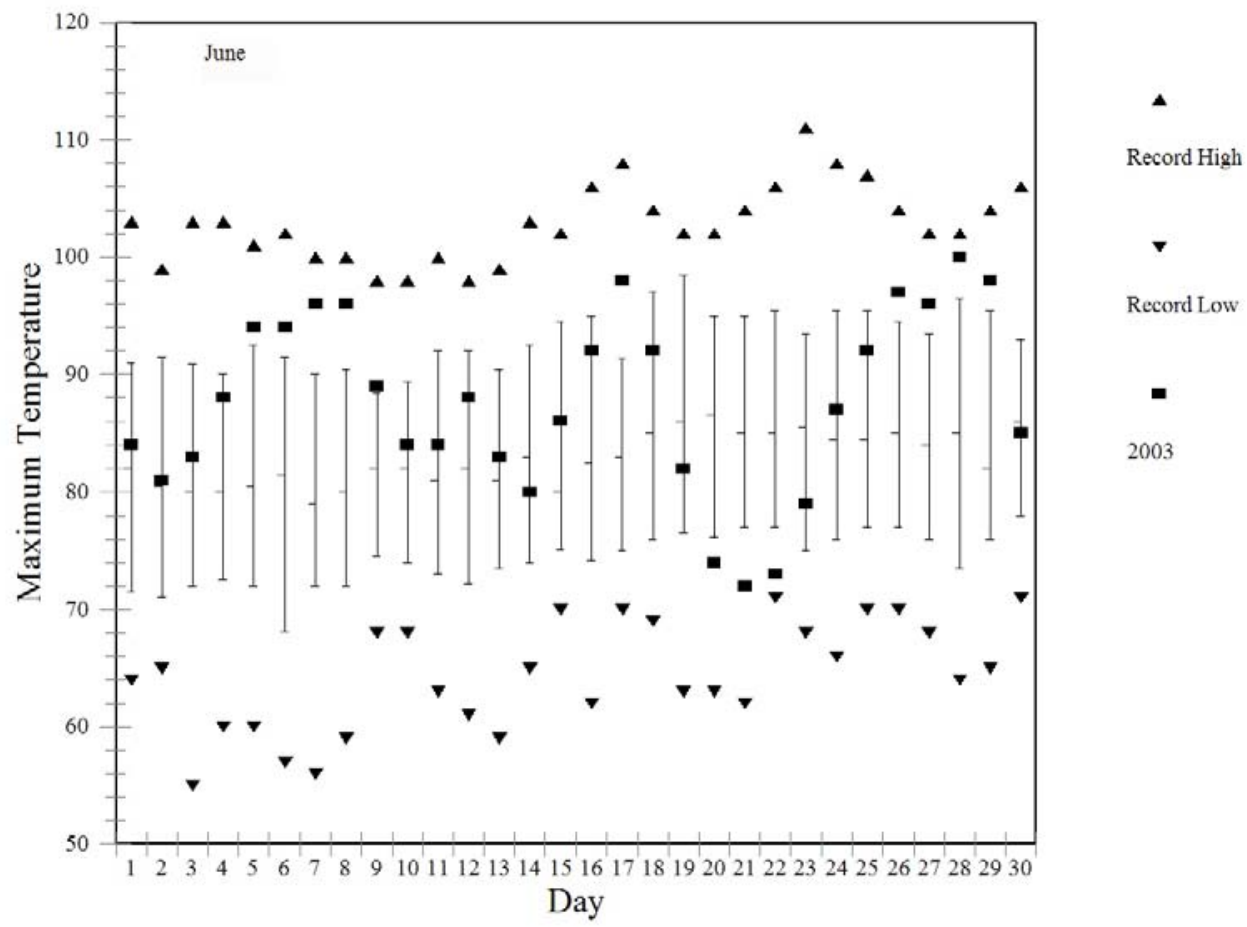

Figure 3.12. Daily Maximum Temperatures $\left({ }^{\circ}\right.$ F), June 2003

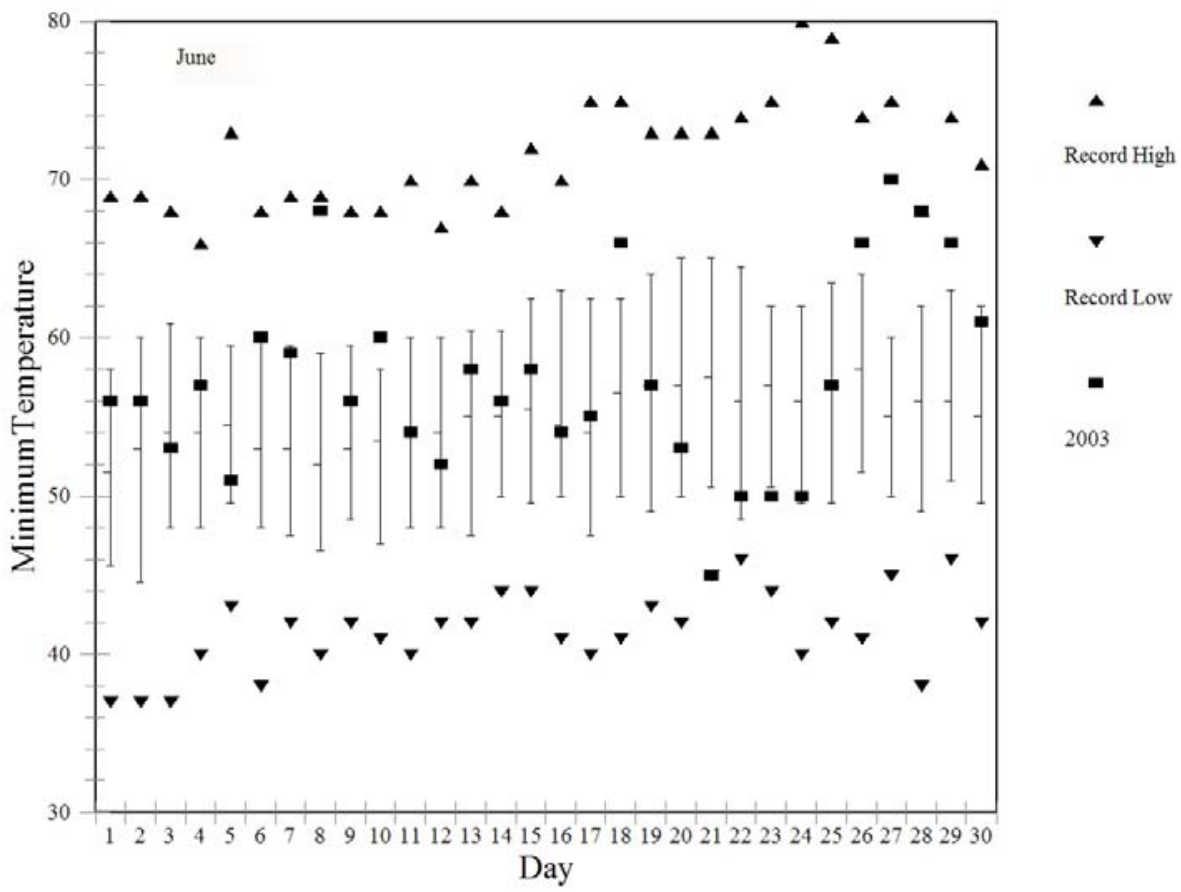

Figure 3.13. Daily Minimum Temperatures $\left({ }^{\circ}\right.$ F), June 2003 


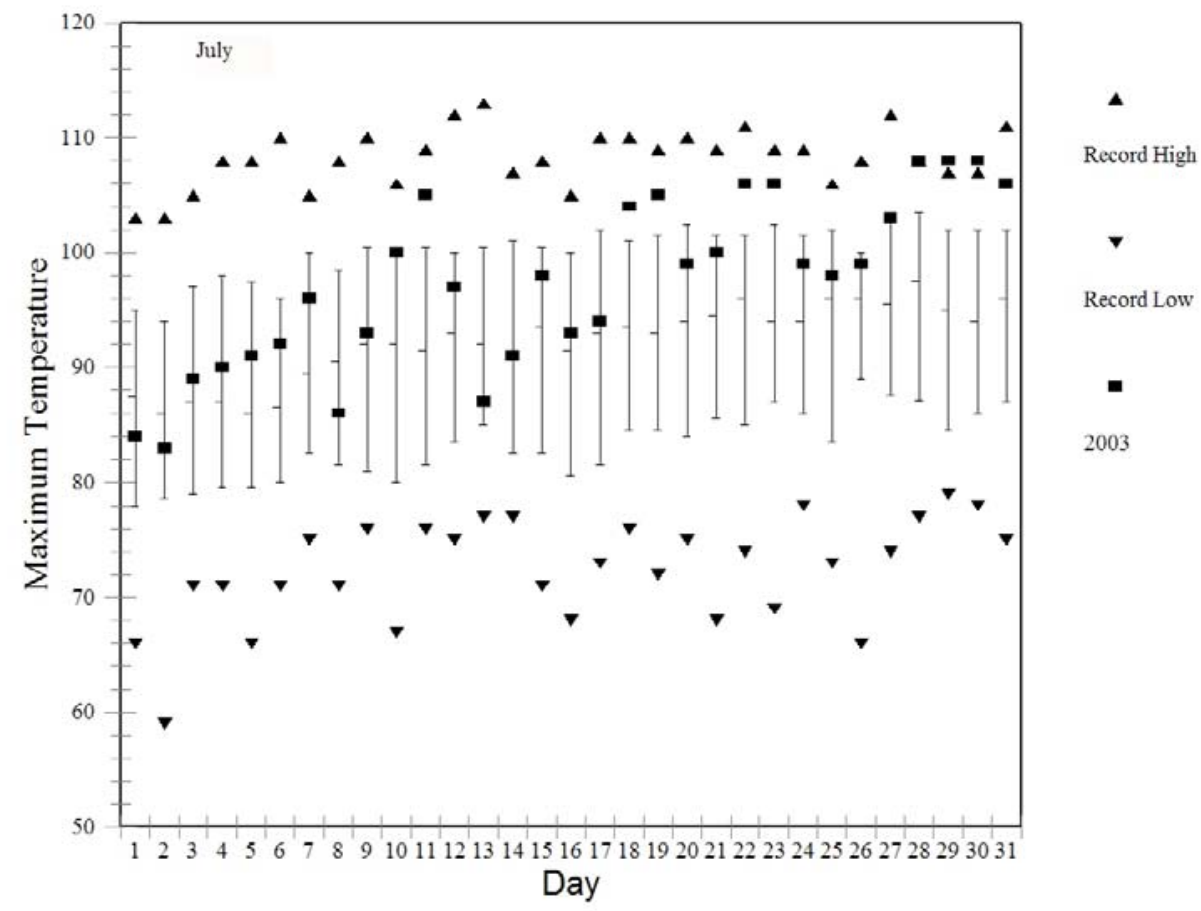

Figure 3.14. Daily Maximum Temperatures $\left({ }^{\circ}\right.$ F), July 2003

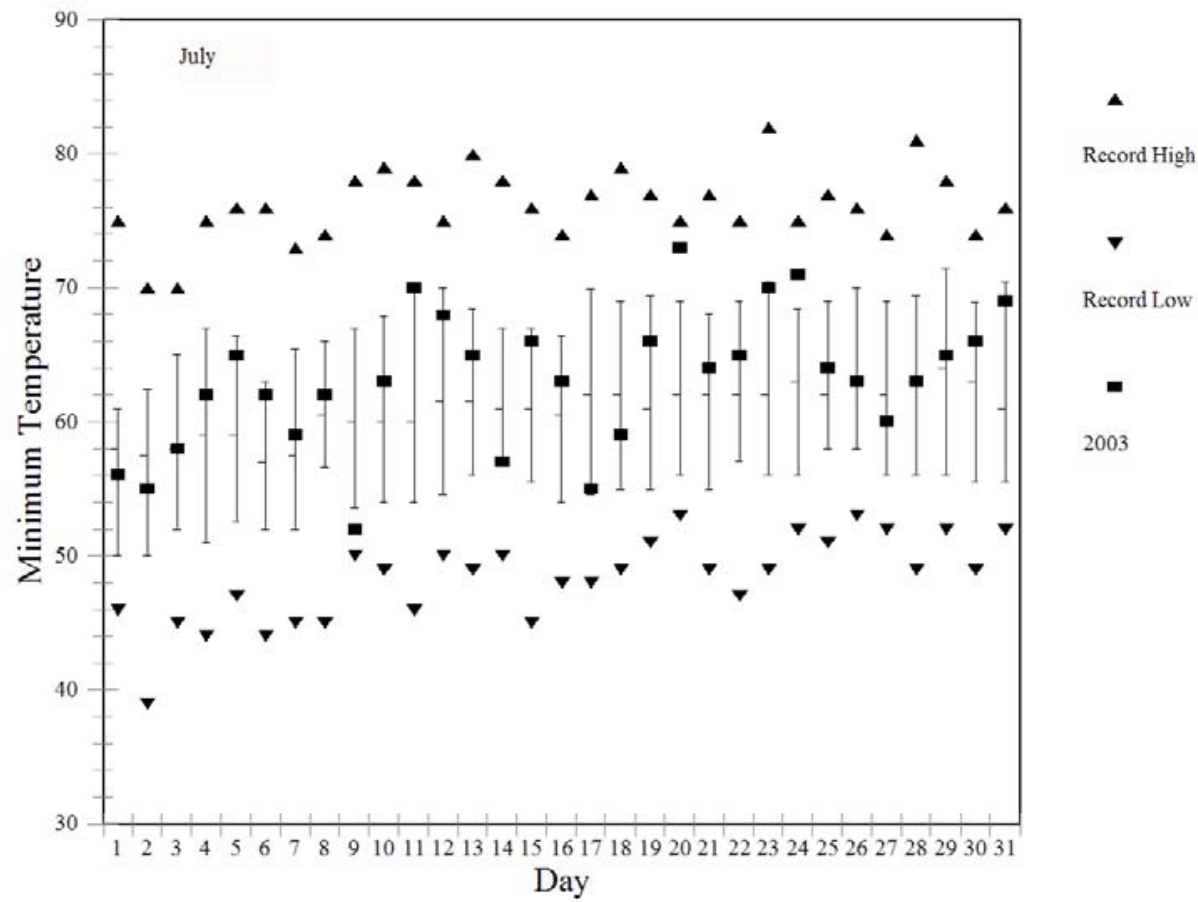

Figure 3.15. Daily Minimum Temperatures $\left({ }^{\circ}\right.$ F), July 2003 


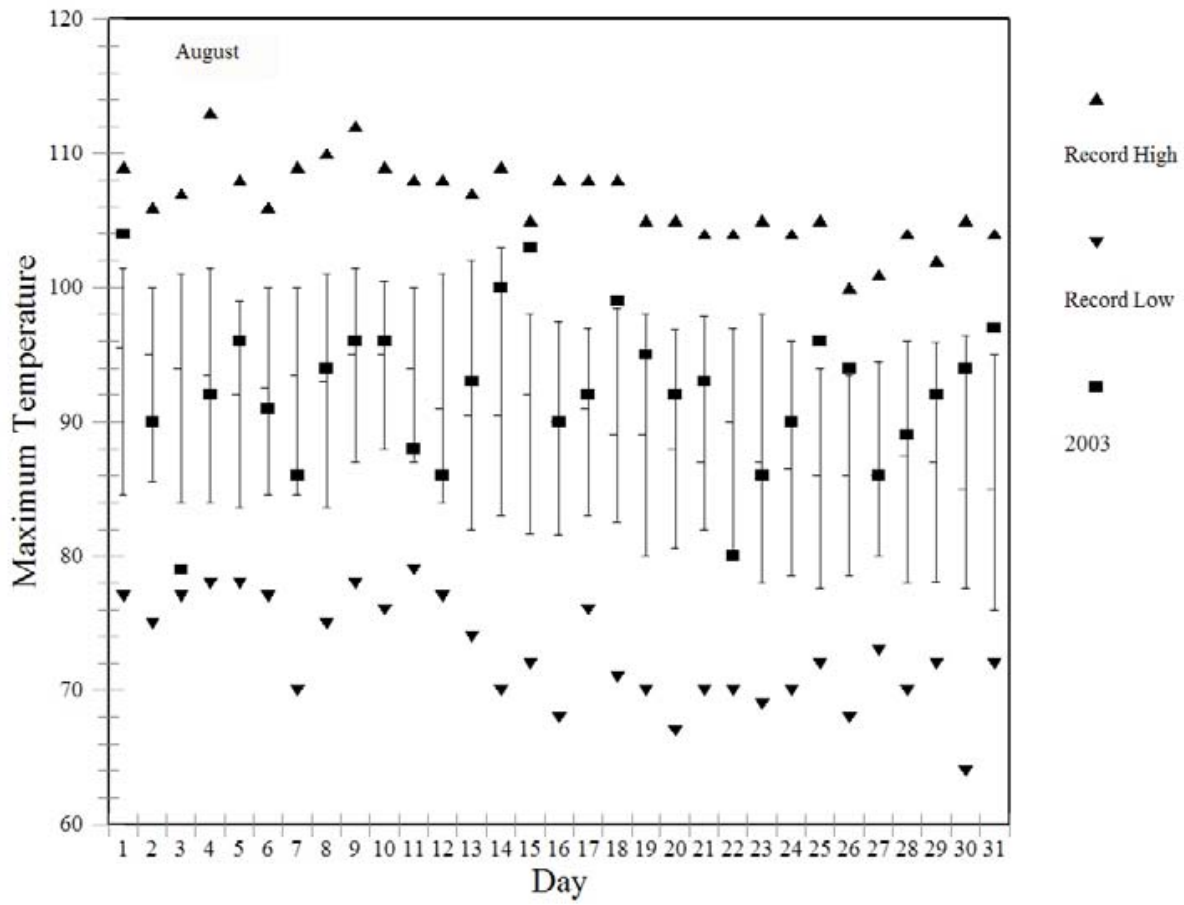

Figure 3.16. Daily Maximum Temperatures $\left({ }^{\circ}\right.$ F), August 2003

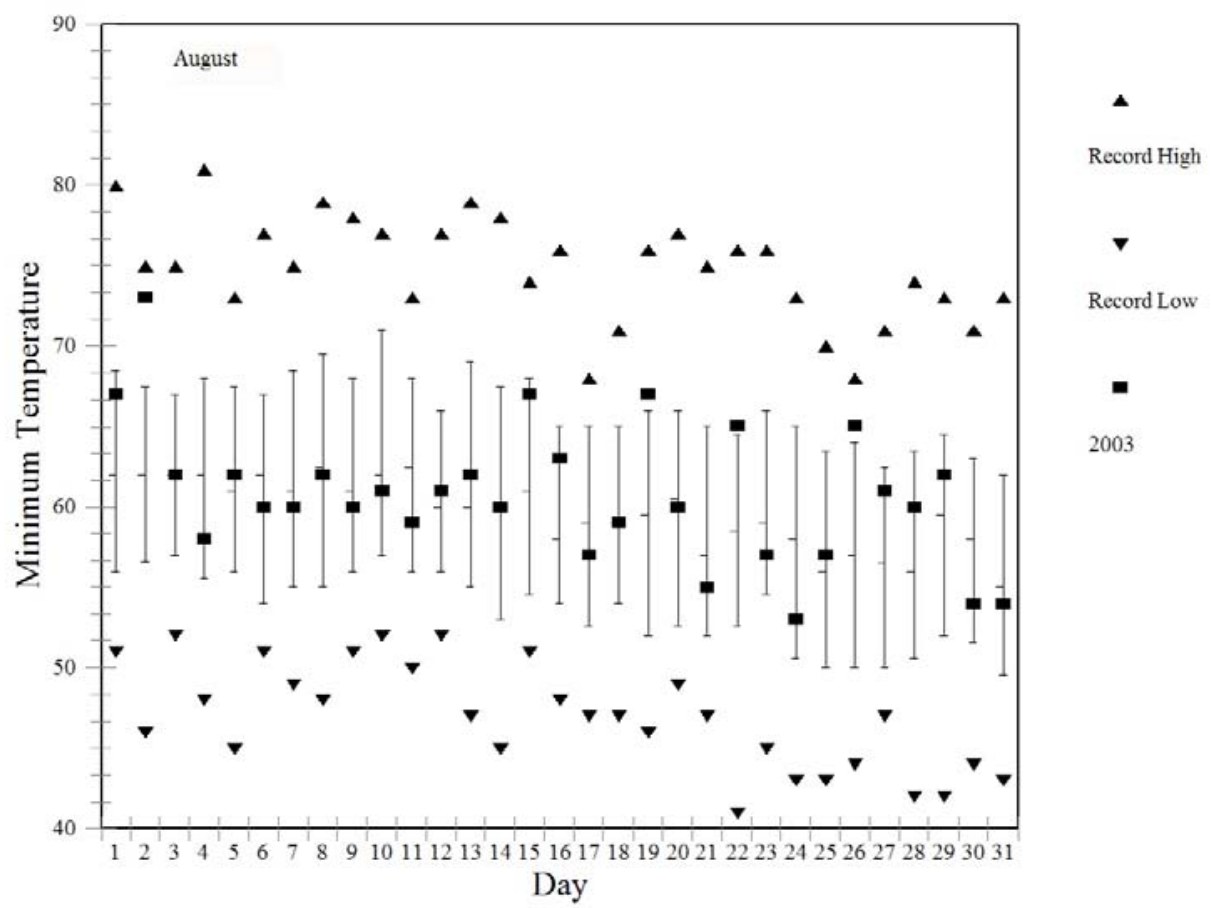

Figure 3.17. Daily Minimum Temperatures $\left({ }^{\circ} \mathbf{F}\right)$, August 2003 


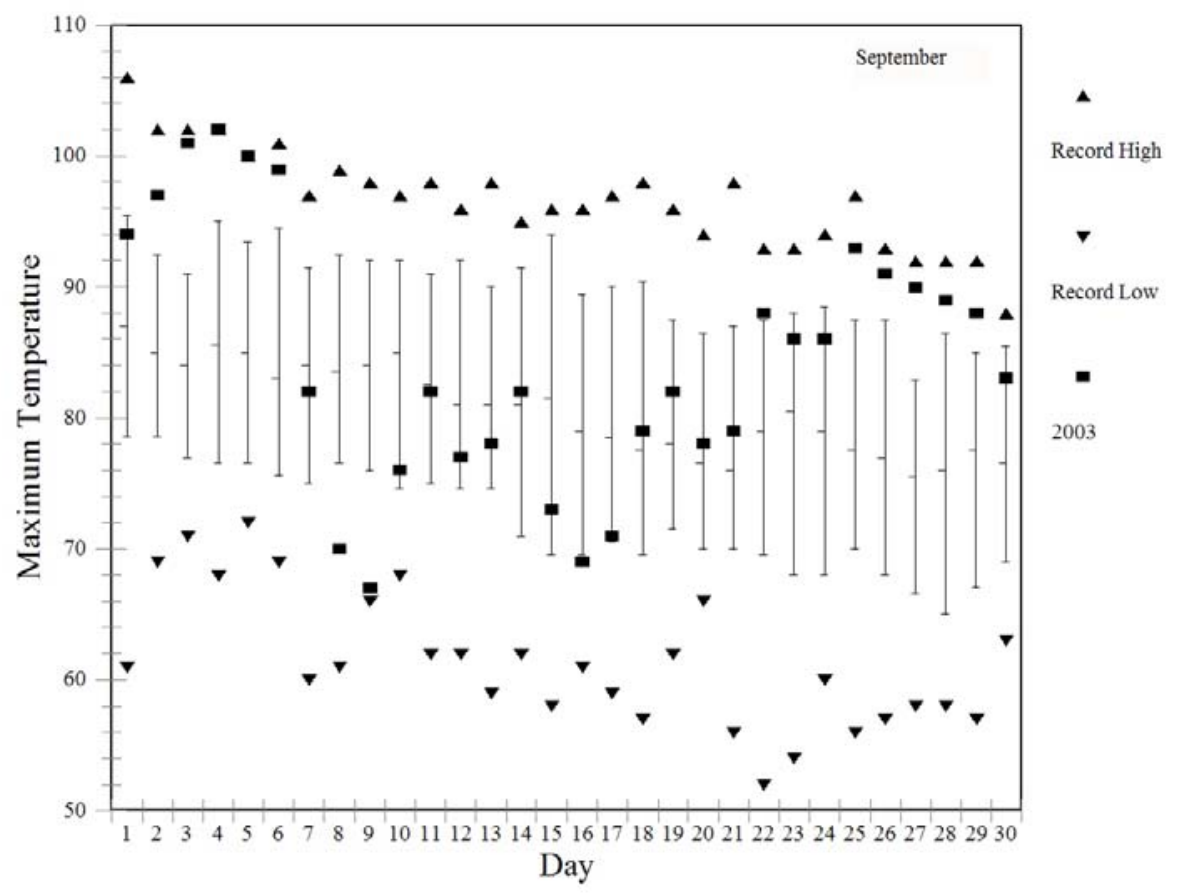

Figure 3.18. Daily Maximum Temperatures $\left({ }^{\circ}\right.$ F), September 2003

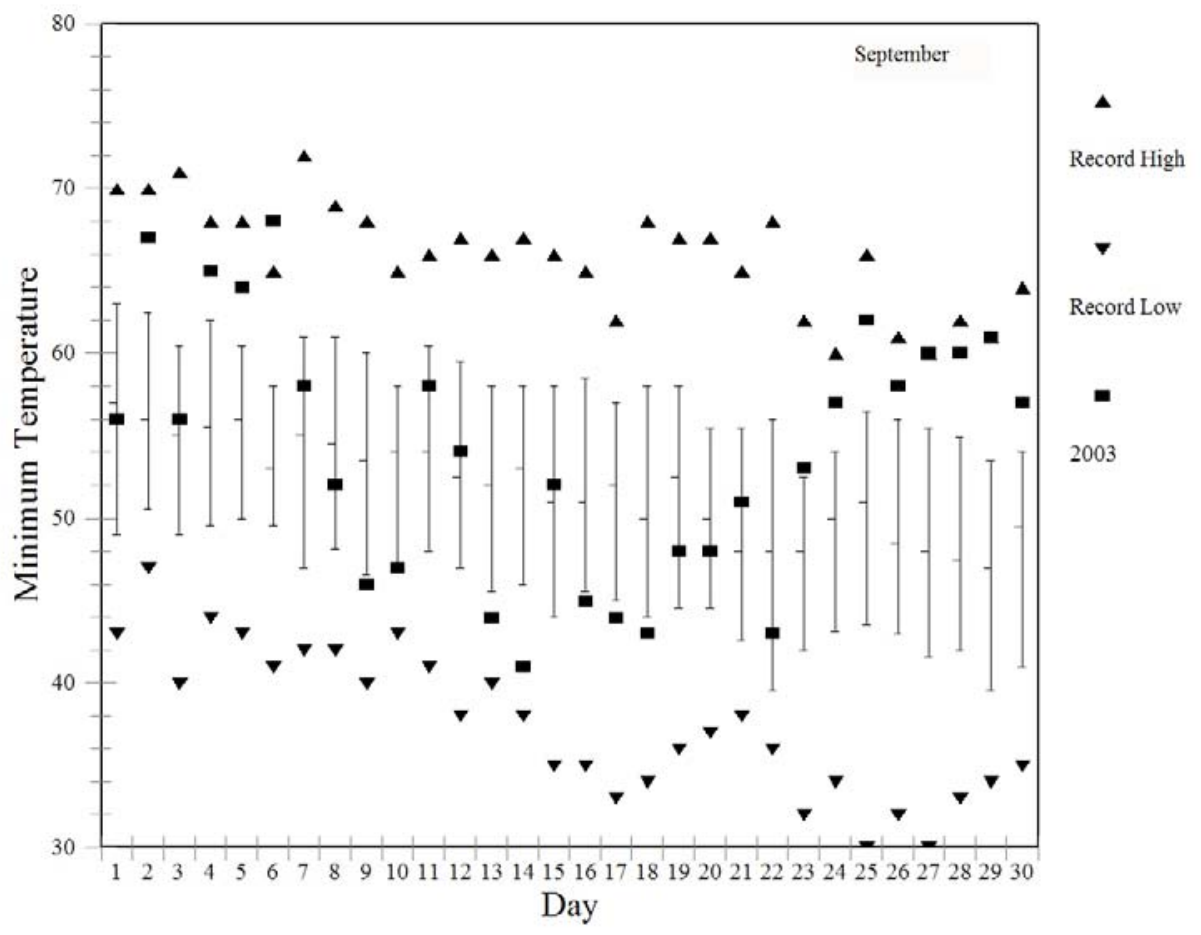

Figure 3.19. Daily Minimum Temperatures $\left({ }^{\circ}\right.$ F), September 2003 


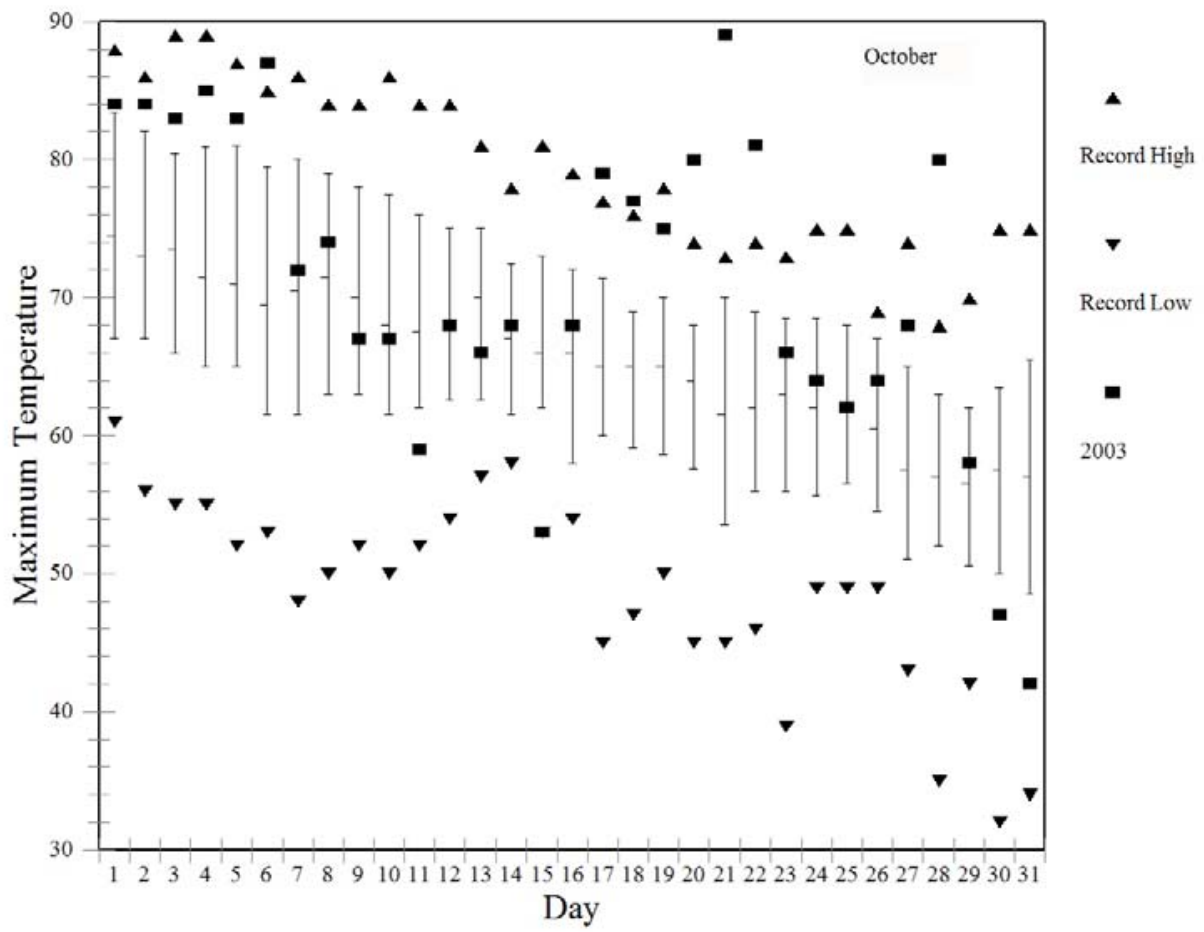

Figure 3.20. Daily Maximum Temperatures $\left({ }^{\circ}\right.$ F), October 2003

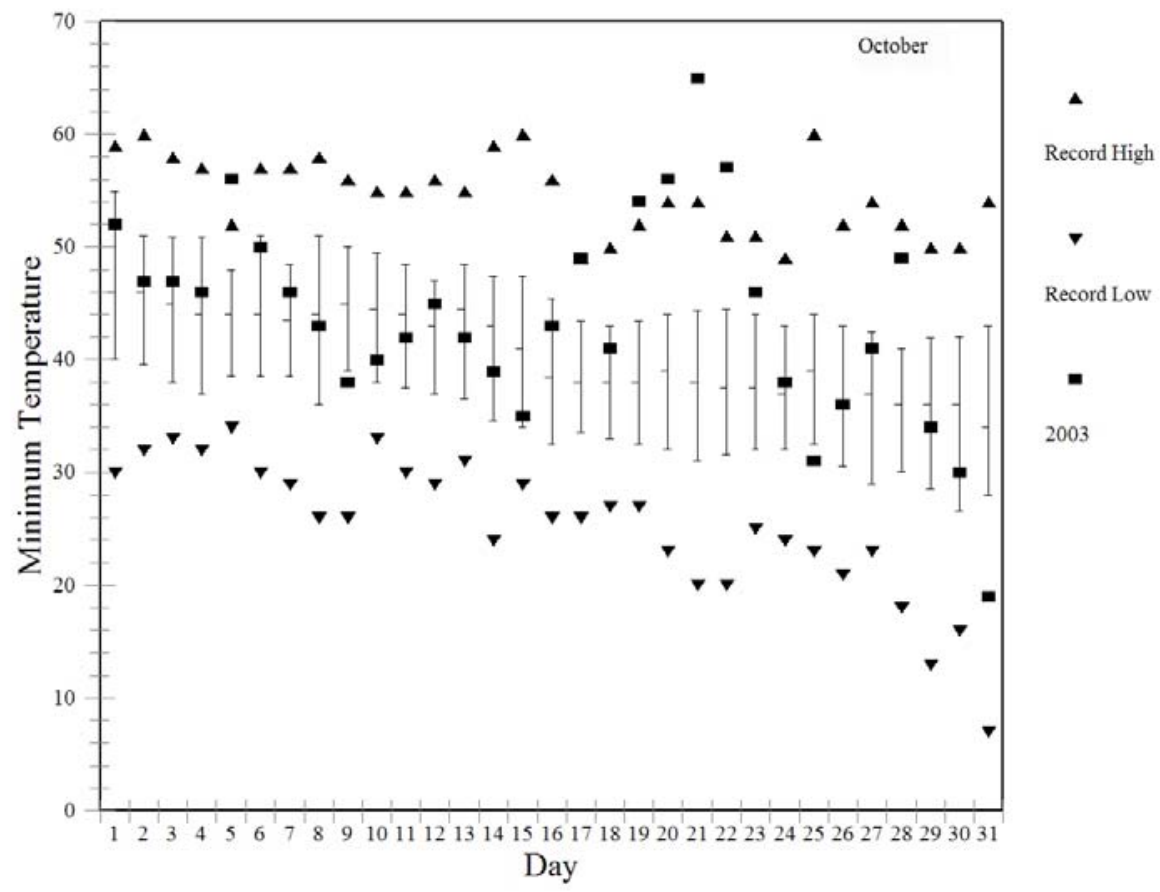

Figure 3.21. Daily Minimum Temperatures $\left({ }^{\circ}\right.$ F), October 2003 


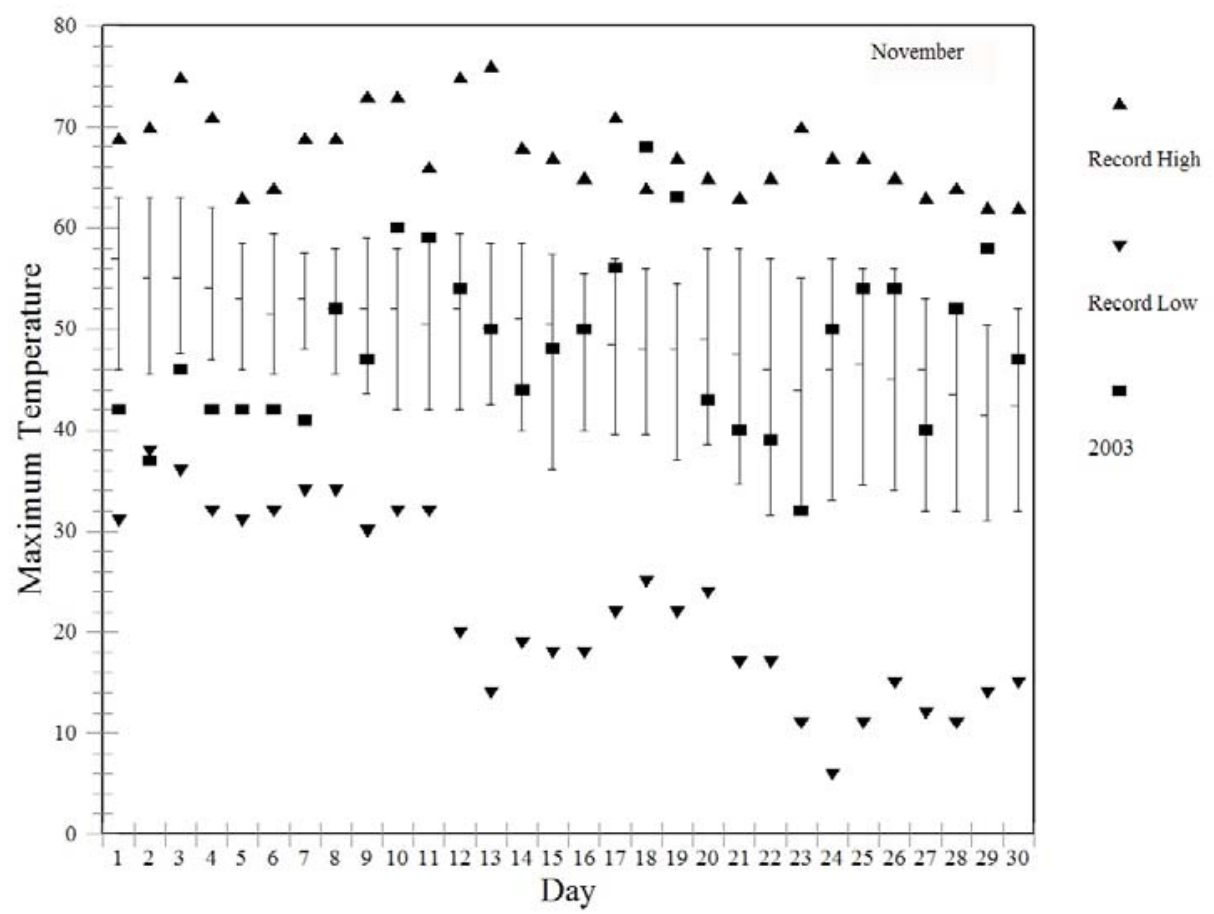

Figure 3.22. Daily Maximum Temperatures $\left({ }^{\circ} \mathbf{F}\right)$, November 2003

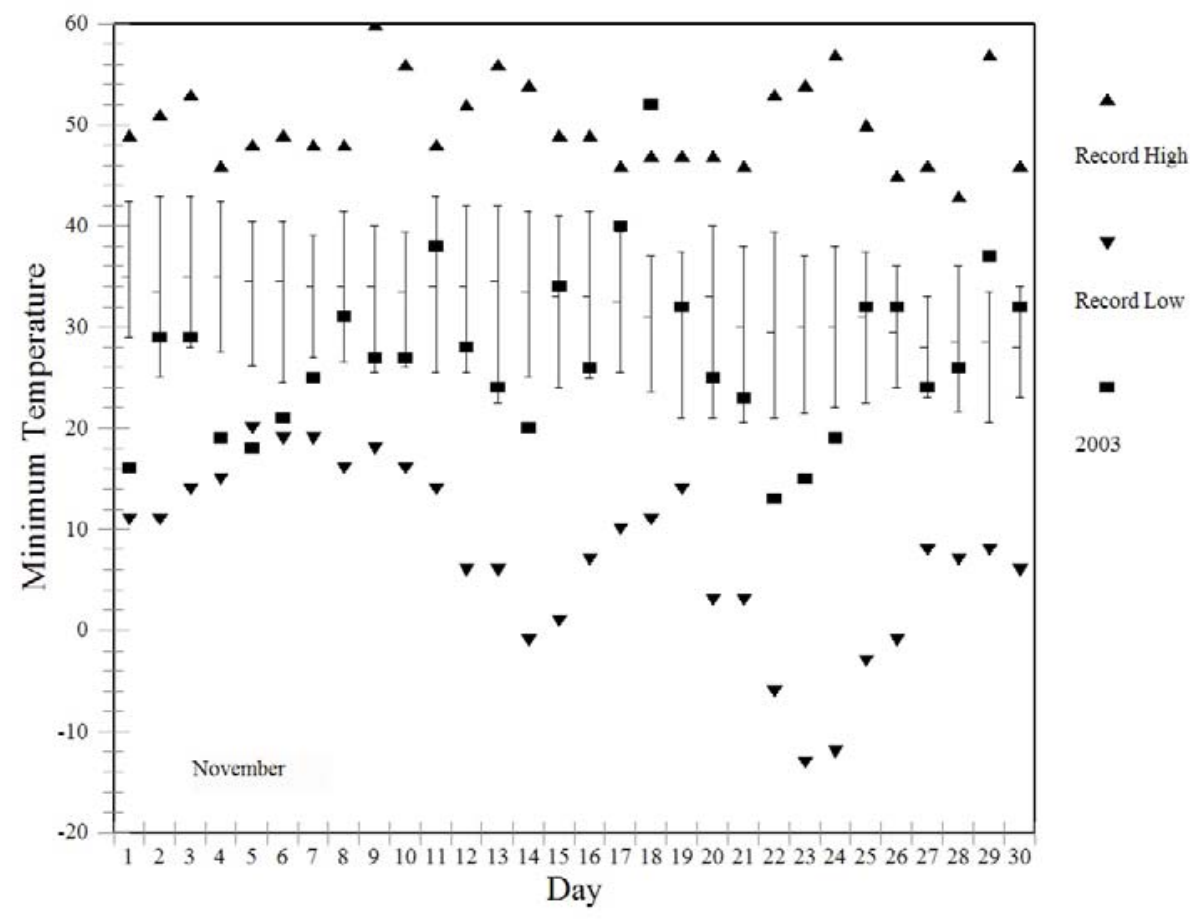

Figure 3.23. Daily Minimum Temperatures $\left({ }^{\circ} \mathbf{F}\right)$, November 2003 


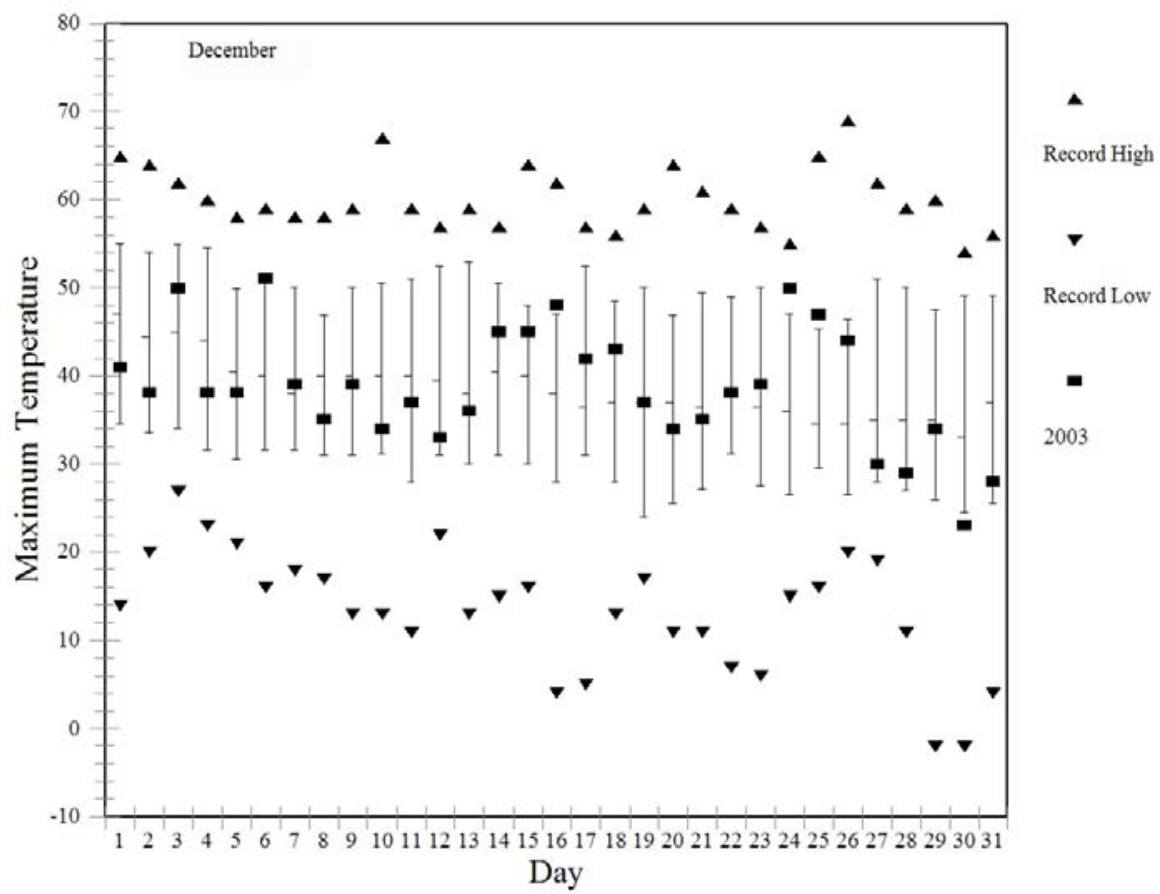

Figure 3.24. Daily Maximum Temperatures $\left({ }^{\circ} \mathrm{F}\right)$, December 2003

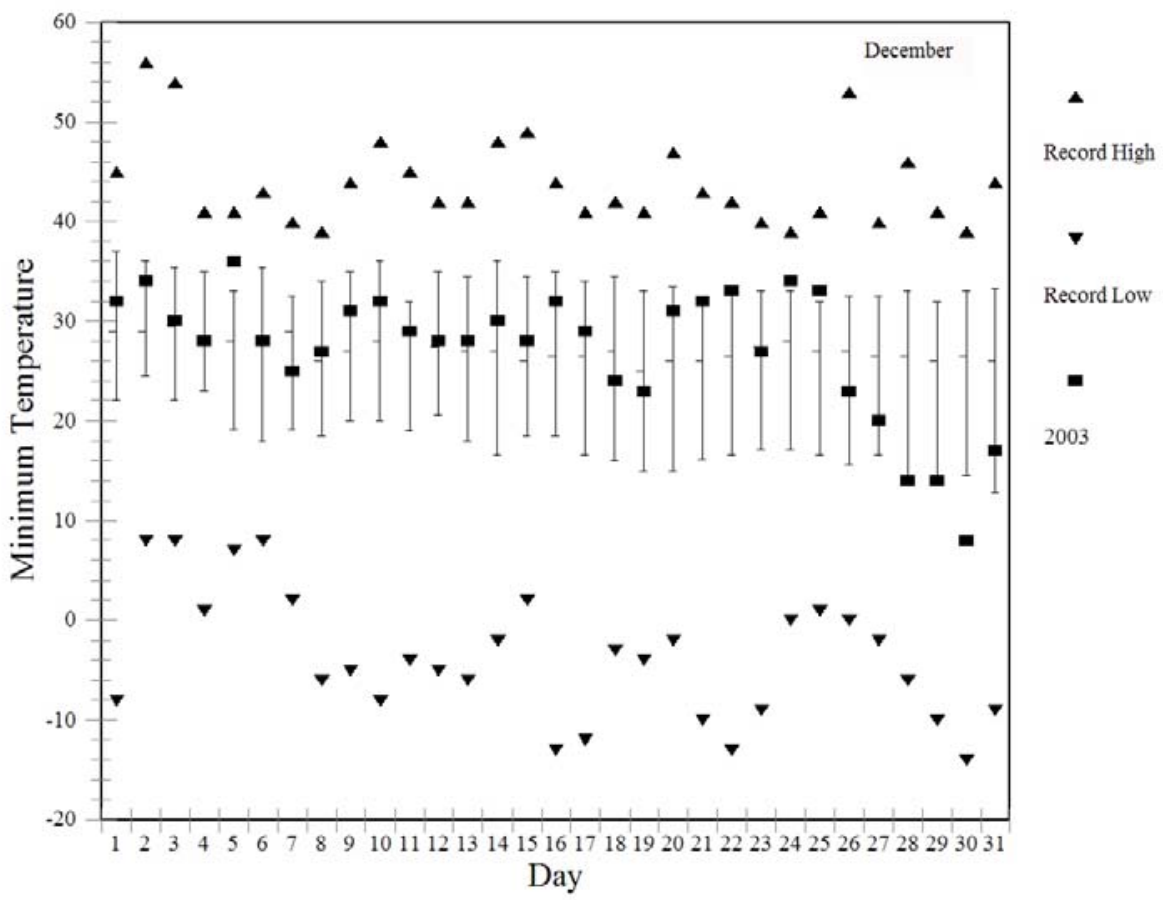

Figure 3.25. Daily Minimum Temperatures $\left({ }^{\circ}\right.$ F), December 2003 
Table 3.11. Average Daily Temperature $\left({ }^{\circ} \mathrm{F}\right)$ Range

\begin{tabular}{|c|c|c|c|c|c|c|c|c|c|c|c|c|c|}
\hline Year & Jan & Feb & Mar & Apr & May & Jun & Jul & Aug & Sep & Oct & Nov & Dec & Annual \\
\hline 1946 & $\overline{17.3}$ & $\overline{21.0}$ & 21.8 & 26.1 & 29.6 & $\overline{25.6}$ & $\overline{30.4}$ & 29.4 & 26.8 & 23.6 & $\overline{17.7}$ & $\overline{15.8}$ & 23.8 \\
\hline 1947 & 18.4 & 22.2 & 25.5 & 27.7 & 29.6 & $25.3^{(\mathrm{a})}$ & 29.3 & 28.8 & 27.3 & $18.1^{(\mathrm{a})}$ & 15.4 & 11.1 & 23.2 \\
\hline 1948 & 15.0 & 17.2 & 23.0 & 23.2 & $22.6^{(\mathrm{a})}$ & 26.4 & 29.1 & 28.0 & 28.7 & 26.5 & 17.8 & 15.9 & 22.8 \\
\hline 1949 & $18.8^{(\mathrm{a})}$ & 19.3 & 20.6 & 30.5 & 28.2 & 30.2 & 30.5 & 30.4 & 27.2 & 26.6 & 16.8 & 16.7 & 24.6 \\
\hline 1950 & 16.0 & 15.6 & 20.0 & 25.3 & 29.6 & 25.7 & 32.3 & 31.6 & 32.4 & 18.2 & 14.7 & 9.7 & 22.6 \\
\hline 1951 & 13.7 & 18.4 & 20.8 & 30.3 & 30.2 & 28.9 & $\mathbf{3 3 . 8}^{(\mathrm{a})}$ & 31.5 & 30.9 & 23.3 & 17.4 & 13.7 & 24.4 \\
\hline 1952 & 12.6 & 17.0 & 22.8 & 30.3 & 28.1 & 27.3 & 32.6 & 32.2 & 32.8 & 29.0 & 16.0 & 9.4 & 24.2 \\
\hline 1953 & 15.6 & 19.8 & 24.4 & 24.0 & 27.9 & 26.4 & 32.8 & 29.1 & 32.5 & 27.8 & 20.3 & $17.7^{\text {(a) }}$ & 24.9 \\
\hline 1954 & 14.3 & 13.8 & 23.7 & 26.3 & 28.1 & 26.8 & 31.4 & 27.6 & 26.2 & 24.5 & 15.8 & 13.2 & 22.6 \\
\hline 1955 & 9.2 & 18.9 & 21.8 & 24.9 & 25.3 & 29.4 & 27.9 & 31.7 & 27.5 & 22.7 & 16.1 & 12.3 & 22.3 \\
\hline 1956 & 12.9 & 15.0 & 20.9 & 28.0 & 26.6 & 26.9 & 30.8 & 28.8 & 30.6 & 22.1 & 13.9 & 13.4 & 22.5 \\
\hline 1957 & 15.2 & 18.3 & $18.4^{(\mathrm{a})}$ & 24.7 & 24.8 & 27.7 & 28.7 & 27.0 & 29.8 & 18.4 & 19.1 & 14.7 & 22.2 \\
\hline 1958 & 13.3 & 15.0 & 22.0 & 23.9 & 29.6 & 27.3 & 30.5 & 33.1 & 27.0 & 27.8 & 17.8 & 10.2 & 23.1 \\
\hline 1959 & 14.1 & 16.2 & 24.3 & 26.9 & 26.5 & 27.1 & 31.1 & 29.9 & 23.8 & 24.4 & 21.9 & 13.4 & 23.3 \\
\hline 1960 & 14.6 & 19.3 & 23.2 & 25.7 & 26.5 & 31.2 & 32.8 & 28.1 & 28.3 & 25.7 & 18.7 & 10.9 & 23.8 \\
\hline 1961 & 12.5 & 17.2 & 20.0 & 25.3 & 25.5 & 31.5 & 30.6 & 30.9 & 26.9 & 25.4 & 20.4 & 15.0 & 23.4 \\
\hline 1962 & 18.1 & 17.4 & 22.0 & 28.9 & 23.2 & 29.9 & 30.2 & 28.3 & 29.9 & 21.4 & 17.3 & 11.4 & 23.2 \\
\hline 1963 & 17.2 & 16.8 & 23.3 & $21.4^{(\mathrm{a})}$ & 28.1 & 26.6 & 27.8 & 30.7 & 29.8 & 24.5 & 16.9 & 9.5 & 22.7 \\
\hline 1964 & 16.0 & $24.1^{(\mathrm{a})}$ & 23.7 & 27.1 & 27.9 & 26.4 & 31.2 & 29.4 & 29.7 & 26.4 & $12.2^{(\mathrm{a})}$ & 14.8 & 24.1 \\
\hline 1965 & 12.3 & 20.3 & 25.6 & 26.4 & 28.3 & 28.1 & 31.1 & 27.7 & 29.8 & 27.4 & 14.2 & 15.7 & 23.9 \\
\hline 1966 & 14.3 & 19.3 & 24.4 & 28.2 & 30.7 & 27.3 & 28.7 & 29.1 & 28.4 & 25.7 & 18.1 & 13.4 & 24.0 \\
\hline 1967 & 17.6 & 24.0 & 24.6 & 24.4 & 27.7 & 28.7 & 32.0 & $34.5^{(\mathrm{a})}$ & 31.7 & 25.5 & 19.9 & 15.7 & $25.5^{(\mathrm{a})}$ \\
\hline 1968 & 17.2 & 20.4 & 23.5 & 27.5 & 27.5 & 27.3 & 31.2 & $26.2^{(\mathrm{a})}$ & 28.2 & 22.8 & 14.2 & 13.9 & 23.3 \\
\hline 1969 & 12.2 & 14.1 & 25.5 & 24.5 & 29.2 & 27.7 & 31.3 & 33.3 & 27.6 & 25.0 & 17.4 & 9.2 & 23.1 \\
\hline 1970 & 12.0 & 16.4 & 23.8 & 25.3 & 29.2 & 29.3 & 31.7 & 33.1 & 27.2 & 26.5 & 17.8 & 15.4 & 24.0 \\
\hline 1971 & 18.4 & 21.1 & 22.8 & 26.8 & 27.7 & 26.9 & 32.0 & 32.3 & 27.8 & 25.8 & 17.8 & 14.8 & 24.5 \\
\hline 1972 & 17.3 & 18.3 & 25.2 & 26.8 & 27.2 & 26.9 & 30.1 & 30.6 & 30.5 & 27.5 & 13.1 & 17.2 & 24.2 \\
\hline 1973 & 15.7 & 16.6 & 24.6 & 29.6 & 31.1 & 29.7 & 32.1 & 32.7 & 27.0 & 22.2 & 12.6 & 12.5 & 23.9 \\
\hline 1974 & 17.8 & 18.8 & 23.2 & 23.4 & 27.3 & $32.7^{(\mathrm{a})}$ & 29.8 & 31.9 & 32.2 & 28.3 & 16.3 & 16.5 & 24.8 \\
\hline 1975 & 15.0 & 17.0 & 21.2 & 24.8 & 29.5 & 28.2 & 30.3 & 28.7 & 32.2 & 22.0 & 20.9 & 14.8 & 23.7 \\
\hline 1976 & 15.2 & 21.0 & 25.3 & 26.0 & 30.6 & 28.8 & 30.5 & 28.0 & 30.5 & 27.5 & 20.3 & 16.6 & 25.0 \\
\hline 1977 & 10.8 & 20.7 & 23.4 & $\mathbf{3 0 . 6}^{(\mathrm{a})}$ & 26.0 & 30.2 & 30.5 & 29.1 & 23.8 & 26.6 & 19.1 & 15.1 & 23.8 \\
\hline 1978 & 11.4 & 15.2 & 23.0 & 23.8 & 27.7 & 31.3 & 31.0 & 29.0 & 25.8 & $\mathbf{3 0 . 3}^{(\mathrm{a})}$ & 18.2 & 16.8 & 23.6 \\
\hline 1979 & 15.5 & 18.7 & 26.0 & 26.5 & 29.4 & 31.1 & 32.9 & 32.0 & 31.1 & 25.6 & 13.0 & 12.9 & 24.6 \\
\hline 1980 & 13.2 & $10.5^{(\mathrm{a})}$ & 22.1 & 27.1 & 25.8 & 25.8 & 31.3 & 29.9 & 27.3 & 24.6 & 15.3 & 11.4 & $22.0^{(a)}$ \\
\hline 1981 & 9.9 & 17.5 & 25.9 & 27.4 & 27.1 & 28.3 & 31.7 & 32.9 & 30.8 & 26.0 & 20.0 & 14.2 & 24.3 \\
\hline 1982 & 16.0 & 21.4 & 24.4 & 28.2 & 29.9 & 28.0 & 30.6 & 29.5 & 27.1 & 24.9 & 16.6 & 13.0 & 24.1 \\
\hline 1983 & 15.5 & 17.3 & 20.7 & 27.9 & 28.4 & 27.9 & 26.3 & 28.4 & 27.5 & 24.7 & 15.5 & 11.0 & 22.6 \\
\hline 1984 & 13.5 & 15.2 & 21.6 & 23.9 & 26.3 & 26.1 & 32.3 & 32.0 & 26.6 & 25.5 & 14.0 & 15.7 & 22.7 \\
\hline 1985 & $6.8^{(\mathbf{a})}$ & 20.4 & 25.4 & 28.7 & 29.2 & 29.4 & 32.0 & 29.9 & 24.3 & 25.4 & 15.8 & $\mathbf{8 . 0}^{(\mathrm{a})}$ & 22.9 \\
\hline 1986 & 12.5 & 17.1 & 22.1 & 26.4 & 26.6 & 29.1 & 28.6 & 31.0 & $23.5^{(\mathrm{a})}$ & 26.8 & 15.1 & 8.4 & 22.3 \\
\hline 1987 & 11.9 & 19.0 & 22.4 & 28.2 & 28.9 & 31.2 & 28.1 & 30.1 & 33.0 & 28.9 & 19.8 & 12.0 & 24.5 \\
\hline 1988 & 13.4 & 23.8 & 25.1 & 25.3 & 27.4 & 26.3 & 30.0 & 32.1 & 31.2 & 26.2 & 16.9 & 11.4 & 24.1 \\
\hline 1989 & 16.5 & 17.3 & 20.2 & 26.2 & 26.0 & 28.5 & 31.1 & 27.3 & 31.1 & 24.0 & 16.0 & 9.4 & 22.8 \\
\hline 1990 & 15.7 & 20.5 & 26.5 & 27.1 & 24.4 & 26.8 & 28.8 & 27.3 & 32.2 & 23.6 & 18.4 & 16.0 & 23.9 \\
\hline 1991 & 16.0 & 21.4 & 22.7 & 26.0 & 25.1 & 25.6 & 30.2 & 29.6 & 31.1 & 26.4 & 13.1 & 13.5 & 23.4 \\
\hline 1992 & 12.5 & 15.2 & 25.9 & 24.6 & $31.9^{(\mathrm{a})}$ & 28.5 & 28.0 & 31.6 & 28.1 & 24.4 & 13.6 & 14.5 & 23.2 \\
\hline 1993 & 12.8 & 15.1 & 18.6 & 23.7 & 29.1 & 27.1 & $25.5^{(\mathrm{a})}$ & 29.5 & $33.5^{(\mathrm{a})}$ & 28.6 & $23.9^{(\mathrm{a})}$ & 10.5 & 23.2 \\
\hline 1994 & 15.7 & 17.2 & $28.4^{(\mathrm{a})}$ & 26.0 & 26.5 & 29.4 & 33.0 & 31.2 & 31.2 & 25.4 & 17.2 & 13.6 & 24.6 \\
\hline 1995 & 13.2 & 20.1 & 23.7 & 25.2 & 28.0 & 26.3 & 29.9 & 30.6 & 30.2 & 24.3 & 19.5 & 11.7 & 23.6 \\
\hline 1996 & 14.0 & 21.2 & 22.9 & 26.0 & 26.6 & 31.0 & 33.7 & 34.1 & 30.8 & 23.9 & 17.0 & 13.1 & 24.5 \\
\hline 1997 & 15.6 & 18.8 & 22.1 & 25.7 & 28.1 & 27.3 & 31.2 & 31.2 & 26.7 & 24.3 & 17.6 & 14.2 & 23.6 \\
\hline 1998 & 15.9 & 19.0 & 23.7 & 28.5 & 27.5 & 29.2 & 30.5 & 33.2 & 31.9 & 26.6 & 16.2 & $17.7^{(\mathrm{a})}$ & 25.0 \\
\hline 1999 & 16.4 & 18.0 & 22.2 & 28.9 & 28.0 & 28.0 & 30.2 & 29.0 & 32.9 & 25.7 & 17.7 & 13.5 & 24.2 \\
\hline 2000 & 14.0 & 16.8 & 23.3 & 28.2 & 26.4 & 29.0 & 31.9 & 32.7 & 27.9 & 23.5 & 14.1 & 9.1 & 23.1 \\
\hline 2001 & 10.5 & 17.7 & 24.3 & 24.2 & 30.6 & 26.7 & 29.5 & 31.4 & 31.0 & 23.8 & 16.2 & 13.4 & 23.3 \\
\hline 2002 & 15.3 & 22.4 & 22.5 & 26.9 & 26.8 & 28.8 & 32.1 & 29.8 & 30.9 & 29.9 & 19.2 & 8.9 & 24.3 \\
\hline 2003 & 10.5 & 20.8 & 23.3 & 24.2 & 26.6 & 30.2 & 34.3 & 31.5 & 30.5 & 27.2 & 21.3 & 11.6 & 24.3 \\
\hline Average $^{(b)}$ & 14.4 & 18.3 & 23.1 & 26.4 & 27.7 & 28.1 & 30.6 & 30.3 & 29.1 & 25.1 & 16.9 & 13.3 & 23.6 \\
\hline Normal & 14.3 & 18.3 & 23.5 & 26.6 & 27.8 & 28.5 & 30.5 & 30.6 & 29.3 & 25.6 & 16.8 & 13.3 & 23.8 \\
\hline
\end{tabular}

(a) Greatest and least values.

(b) Based on entire period of record, 1945 through 2003. 
Table 3.12. Normal and Extreme Daily Maximum and Minimum Temperatures $\left({ }^{\circ}\right.$ F)

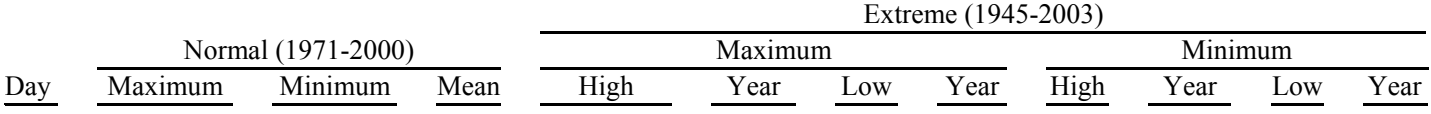

January

\begin{tabular}{|c|c|c|c|c|c|c|c|c|c|c|c|}
\hline 1 & 35 & 21 & 28 & 57 & $98^{(\mathrm{a})}$ & 8 & 69 & 38 & $81^{\text {(a) }}$ & -11 & 79 \\
\hline 2 & 36 & 21 & 28 & 57 & 97 & 15 & 69 & 42 & 63 & -9 & 78 \\
\hline 3 & 36 & 21 & 28 & 63 & 89 & 12 & 50 & 41 & 81 & 0 & 59 \\
\hline 4 & 36 & 21 & 28 & 60 & $94^{(\mathrm{a})}$ & 13 & 59 & 39 & 54 & -6 & 50 \\
\hline 5 & 36 & 21 & 28 & 59 & $90^{(\mathrm{a})}$ & 12 & 50 & 39 & 81 & -7 & 50 \\
\hline 6 & 36 & 22 & 28 & 59 & $90^{\text {(a) }}$ & 10 & 82 & 42 & 98 & -10 & 74 \\
\hline 7 & 36 & 22 & 29 & 63 & $02^{(a)}$ & 9 & 79 & 45 & 90 & -8 & 74 \\
\hline 8 & 36 & 22 & 29 & 59 & 02 & 5 & 74 & 44 & 53 & -9 & 74 \\
\hline 9 & 36 & 22 & 29 & 60 & $90^{\text {(a) }}$ & 5 & 74 & 41 & 90 & -13 & 74 \\
\hline 10 & 36 & 23 & 29 & 61 & 83 & 10 & 74 & 40 & 83 & -5 & 74 \\
\hline 11 & 37 & 23 & 30 & 60 & $83^{(a)}$ & 13 & 63 & 37 & 90 & -6 & 49 \\
\hline 12 & 38 & 24 & 31 & 59 & 53 & 15 & 63 & 47 & 53 & -4 & $63^{\text {(a) }}$ \\
\hline 13 & 39 & 25 & 32 & 61 & 94 & 10 & 50 & 42 & 66 & -4 & $93^{\text {(a) }}$ \\
\hline 14 & 40 & 26 & 33 & 62 & 99 & 7 & 50 & 48 & 61 & -11 & 50 \\
\hline 15 & 40 & 26 & 33 & 60 & $74^{(\mathrm{a})}$ & 5 & 50 & 50 & 74 & -5 & 50 \\
\hline 16 & 41 & 27 & 34 & 61 & 74 & 8 & 50 & 48 & 89 & -8 & 50 \\
\hline 17 & 41 & 27 & 34 & 57 & 98 & 5 & 50 & 40 & 89 & -11 & 50 \\
\hline 18 & 41 & 27 & 34 & 62 & 89 & 10 & 50 & 38 & 89 & -6 & 57 \\
\hline 19 & 41 & 27 & 34 & 63 & 68 & 13 & 50 & 47 & 68 & -2 & 57 \\
\hline 20 & 41 & 27 & 34 & 66 & 68 & 11 & 54 & 47 & 72 & -6 & 54 \\
\hline 21 & 41 & 27 & 34 & 65 & 68 & 14 & 54 & 42 & 72 & 0 & 54 \\
\hline 22 & 41 & 27 & 34 & 56 & 90 & 16 & 69 & 43 & 81 & -2 & 62 \\
\hline 23 & 41 & 27 & 34 & 63 & 53 & 10 & 69 & 43 & 81 & -8 & 69 \\
\hline 24 & 41 & 27 & 34 & 59 & $84^{(\mathrm{a})}$ & 13 & 57 & 45 & 02 & -6 & 49 \\
\hline 25 & 41 & 26 & 34 & 59 & $92^{\text {(a) }}$ & 6 & 50 & 41 & $74^{(\mathrm{a})}$ & -12 & 57 \\
\hline 26 & 41 & 26 & 34 & 67 & 03 & 2 & 57 & 46 & 62 & -22 & 57 \\
\hline 27 & 41 & 26 & 33 & 60 & $84^{(\mathrm{a})}$ & 0 & 57 & 36 & $95^{\text {(a) }}$ & -21 & 57 \\
\hline 28 & 41 & 26 & 33 & 61 & 67 & 6 & 57 & 42 & 99 & -14 & 57 \\
\hline 29 & 40 & 25 & 33 & 62 & 67 & 3 & 50 & 47 & 99 & -18 & 50 \\
\hline 30 & 40 & 25 & 33 & 67 & $89^{(\mathrm{a})}$ & 11 & 57 & 53 & 71 & -13 & 50 \\
\hline 31 & 40 & 25 & 33 & 72 & 71 & -2 & 50 & 45 & 53 & -21 & 50 \\
\hline \multicolumn{12}{|c|}{ February } \\
\hline 1 & 40 & 25 & 32 & 63 & 71 & -3 & 50 & 42 & 92 & -23 & 50 \\
\hline 2 & 40 & 25 & 32 & 61 & $91^{\text {(a) }}$ & 0 & 50 & 42 & 68 & -21 & 50 \\
\hline 3 & 40 & 24 & 32 & 60 & $67^{(\mathrm{a})}$ & 1 & 50 & 46 & 91 & -23 & 50 \\
\hline 4 & 40 & 24 & 32 & 65 & 67 & 11 & 85 & 43 & 68 & -5 & 85 \\
\hline 5 & 40 & 25 & 32 & 61 & 65 & 23 & 85 & 39 & 61 & -5 & 89 \\
\hline 6 & 41 & 25 & 33 & 61 & 67 & 19 & 85 & 44 & 99 & -3 & 89 \\
\hline 7 & 42 & 25 & 34 & 65 & 45 & 20 & 48 & 41 & 55 & 2 & 89 \\
\hline 8 & 43 & 26 & 35 & 63 & 96 & 22 & 56 & 45 & 45 & 5 & $94^{(\mathrm{a})}$ \\
\hline 9 & 45 & 27 & 36 & 65 & 51 & 23 & 56 & 39 & $61^{\text {(a) }}$ & 4 & 85 \\
\hline 10 & 46 & 28 & 37 & 65 & 77 & 30 & $56^{(\mathrm{a})}$ & 50 & 51 & 7 & 85 \\
\hline 11 & 46 & 28 & 37 & 66 & 88 & 27 & 54 & 39 & $93^{\text {(a) }}$ & 9 & 48 \\
\hline 12 & 47 & 29 & 38 & 70 & 77 & 24 & 49 & 40 & 77 & 6 & 48 \\
\hline 13 & 48 & 30 & 39 & 66 & 71 & 25 & 49 & 42 & 47 & 3 & 49 \\
\hline 14 & 49 & 30 & 40 & 62 & $97^{(\mathrm{a})}$ & 22 & 80 & 54 & 82 & 8 & 95 \\
\hline 15 & 49 & 31 & 40 & 67 & 82 & 19 & 56 & 48 & 81 & 5 & 56 \\
\hline 16 & 49 & 31 & 40 & 69 & 77 & 20 & 56 & 48 & 81 & 4 & 56 \\
\hline 17 & 49 & 31 & 40 & 67 & $77^{(\mathrm{a})}$ & 22 & 56 & 48 & 48 & 9 & 56 \\
\hline 18 & 50 & 31 & 40 & 66 & 81 & 24 & 56 & 46 & 81 & 9 & 90 \\
\hline 19 & 50 & 31 & 41 & 68 & 95 & 28 & 56 & 56 & 95 & 14 & $90^{\text {(a) }}$ \\
\hline
\end{tabular}


Table 3.12. (contd)

\begin{tabular}{|c|c|c|c|c|c|c|c|c|c|c|c|}
\hline \multirow[b]{3}{*}{ Day } & \multirow{2}{*}{\multicolumn{3}{|c|}{ Normal (1971-2000) }} & \multicolumn{8}{|c|}{ Extreme (1945-2003) } \\
\hline & & & & \multicolumn{4}{|c|}{ Maximum } & \multicolumn{4}{|c|}{ Minimum } \\
\hline & Maximum & Minimum & Mean & High & Year & Low & Year & High & Year & Low & Year \\
\hline 20 & 51 & 31 & 41 & 68 & 82 & 32 & $57^{(\mathrm{a})}$ & 51 & 61 & 15 & 86 \\
\hline 21 & 51 & 31 & 41 & 71 & 88 & 29 & 57 & 45 & 95 & 13 & 57 \\
\hline 22 & 51 & 31 & 41 & 66 & 02 & 29 & 57 & 44 & 02 & 11 & 93 \\
\hline 23 & 51 & 31 & 41 & 68 & 47 & 34 & $93^{(\mathrm{a})}$ & 43 & $83^{(a)}$ & 19 & 93 \\
\hline 24 & 51 & 31 & 41 & 72 & 86 & 32 & 62 & 60 & 86 & 11 & $93^{(\mathrm{a})}$ \\
\hline 25 & 52 & 31 & 41 & 72 & 86 & 28 & 93 & 49 & 86 & 4 & 93 \\
\hline 26 & 52 & 31 & 41 & 65 & $57^{(\mathrm{a})}$ & 28 & $93^{(\mathrm{a})}$ & 46 & 92 & 10 & 93 \\
\hline 27 & 52 & 31 & 41 & 68 & 72 & 26 & 93 & 44 & 92 & 7 & 62 \\
\hline 28 & 52 & 31 & 41 & 67 & 67 & 25 & 93 & 48 & 72 & 3 & 93 \\
\hline 29 & 52 & 31 & 41 & 63 & $88^{(\mathrm{a})}$ & 40 & 60 & 43 & 92 & 12 & 60 \\
\hline \multicolumn{12}{|c|}{ March } \\
\hline
\end{tabular}

\begin{tabular}{|c|c|c|c|c|c|c|c|c|c|c|c|}
\hline 1 & 52 & 31 & 42 & 67 & 94 & 26 & 93 & 44 & 94 & 15 & $71^{(\mathrm{a})}$ \\
\hline 2 & 52 & 31 & 42 & 66 & 68 & 26 & 60 & 46 & 87 & 14 & 60 \\
\hline 3 & 52 & 31 & 42 & 70 & 94 & 24 & 60 & 50 & 87 & 14 & 89 \\
\hline 4 & 52 & 31 & 42 & 63 & 53 & 26 & 55 & 46 & 87 & 7 & 55 \\
\hline 5 & 53 & 32 & 42 & 68 & 72 & 31 & 45 & 50 & 87 & 6 & 55 \\
\hline 6 & 53 & 33 & 43 & 65 & $67^{(\mathrm{a})}$ & 33 & 57 & 43 & 79 & 18 & 60 \\
\hline 7 & 54 & 33 & 44 & 66 & 53 & 41 & 45 & 42 & $86^{(\mathrm{a})}$ & 21 & $74^{(\mathrm{a})}$ \\
\hline 8 & 55 & 33 & 44 & 67 & 53 & 33 & 51 & 46 & 83 & 20 & $02^{(\mathrm{a})}$ \\
\hline 9 & 56 & 34 & 45 & 69 & 53 & 40 & 51 & 47 & 83 & 22 & $94^{(\mathrm{a})}$ \\
\hline 10 & 57 & 34 & 46 & 69 & 72 & 40 & 48 & 45 & $87^{(\mathrm{a})}$ & 13 & 48 \\
\hline 11 & 57 & 35 & 46 & 67 & $95^{(\mathrm{a})}$ & 32 & 50 & 44 & $03^{(\mathrm{a})}$ & 21 & 50 \\
\hline 12 & 58 & 35 & 46 & 68 & $98^{(\mathrm{a})}$ & 37 & 51 & 48 & 87 & 15 & 56 \\
\hline 13 & 58 & 35 & 46 & 72 & 98 & 38 & 51 & 49 & 03 & 22 & $69^{(\mathrm{a})}$ \\
\hline 14 & 58 & 35 & 47 & 72 & 92 & 40 & 49 & 48 & 03 & 23 & 53 \\
\hline 15 & 59 & 35 & 47 & 73 & 94 & 37 & 49 & 50 & 92 & 23 & 76 \\
\hline 16 & 59 & 36 & 47 & 76 & 72 & 43 & 89 & 47 & 94 & 23 & 55 \\
\hline 17 & 60 & 36 & 48 & 76 & 72 & 38 & 65 & 48 & 69 & 17 & 65 \\
\hline 18 & 60 & 36 & 48 & 76 & 47 & 41 & 65 & 47 & 90 & 14 & 65 \\
\hline 19 & 60 & 36 & 48 & 76 & 47 & 48 & $65^{(\mathrm{a})}$ & 53 & 97 & 16 & 65 \\
\hline 20 & 60 & 36 & 48 & 76 & 47 & 38 & 02 & 49 & 88 & 22 & 74 \\
\hline 21 & 60 & 36 & 48 & 74 & 60 & 41 & 75 & 47 & 03 & 26 & $82^{(\mathrm{a})}$ \\
\hline 22 & 60 & 35 & 48 & 74 & $78^{(\mathrm{a})}$ & 47 & 71 & 47 & 78 & 19 & 94 \\
\hline 23 & 60 & 35 & 48 & 77 & 60 & 39 & 64 & 45 & $98^{(\mathrm{a})}$ & 20 & 48 \\
\hline 24 & 60 & 35 & 48 & 78 & 60 & 38 & 55 & 46 & 01 & 15 & 64 \\
\hline 25 & 60 & 35 & 48 & 83 & 60 & 35 & 55 & 48 & 52 & 21 & 96 \\
\hline 26 & 60 & 35 & 48 & 76 & 46 & 38 & 65 & 49 & $92^{(\mathrm{a})}$ & 21 & 85 \\
\hline 27 & 61 & 35 & 48 & 77 & 94 & 47 & 79 & 46 & 89 & 24 & 75 \\
\hline 28 & 61 & 35 & 48 & 79 & 94 & 42 & 54 & 49 & 78 & 19 & 75 \\
\hline 29 & 61 & 36 & 49 & 78 & $94^{(a)}$ & 49 & 54 & 48 & $94^{(\mathrm{a})}$ & 18 & 54 \\
\hline 30 & 62 & 36 & 49 & 77 & 03 & 52 & 67 & 47 & $92^{(\mathrm{a})}$ & 20 & 54 \\
\hline 31 & 62 & 36 & 49 & 78 & 92 & 45 & 96 & 49 & 03 & 28 & 53 \\
\hline \multicolumn{12}{|c|}{ April } \\
\hline 1 & 63 & 37 & 50 & 80 & 90 & 45 & 76 & 50 & 59 & 24 & 82 \\
\hline 2 & 63 & 37 & 50 & 83 & 92 & 48 & 82 & 50 & 87 & 25 & 76 \\
\hline 3 & 64 & 37 & 50 & 76 & $00^{(\mathrm{a})}$ & 50 & $63^{(a)}$ & 48 & 77 & 23 & 75 \\
\hline 4 & 64 & 38 & 51 & 82 & 60 & 45 & 75 & 56 & 91 & 24 & 03 \\
\hline 5 & 64 & 38 & 51 & 78 & $77^{(\mathrm{a})}$ & 51 & 75 & 54 & 60 & 21 & 75 \\
\hline 6 & 64 & 38 & 51 & 82 & 77 & 50 & 82 & 51 & 62 & 25 & 97 \\
\hline 7 & 64 & 38 & 51 & 85 & 77 & 41 & 45 & 53 & 60 & 26 & 54 \\
\hline 8 & 65 & 38 & 51 & 82 & 96 & 51 & 53 & 50 & 96 & 27 & $92^{\text {(a) }}$ \\
\hline 9 & 65 & 38 & 51 & 80 & 85 & 48 & 92 & 57 & 96 & 28 & 01 \\
\hline 10 & 65 & 38 & 52 & 85 & 68 & 52 & 45 & 50 & 96 & 24 & 81 \\
\hline
\end{tabular}


Table 3.12. (contd)

\begin{tabular}{|c|c|c|c|c|c|c|c|c|c|c|c|}
\hline \multirow[b]{3}{*}{ Day } & & & & \multicolumn{8}{|c|}{ Extreme (1945-2003) } \\
\hline & \multicolumn{3}{|c|}{ Normal (1971-2000) } & \multicolumn{4}{|c|}{ Maximum } & \multicolumn{4}{|c|}{ Minimum } \\
\hline & Maximum & Minimum & Mean & High & Year & Low & Year & High & Year & Low & Year \\
\hline 11 & 65 & 39 & 52 & 79 & 88 & 52 & 83 & 51 & $56^{(\mathrm{a})}$ & 27 & 83 \\
\hline 12 & 66 & 39 & 52 & 83 & 88 & 47 & 95 & 48 & $82^{(a)}$ & 26 & 97 \\
\hline 13 & 66 & 40 & 53 & 88 & 47 & 54 & 55 & 59 & 88 & 23 & 68 \\
\hline 14 & 66 & 40 & 53 & 85 & $62^{(\mathrm{a})}$ & 47 & 75 & 55 & 85 & 28 & 01 \\
\hline 15 & 67 & 40 & 54 & 82 & 88 & 55 & $75^{(a)}$ & 54 & 87 & 27 & 55 \\
\hline 16 & 67 & 41 & 54 & 83 & 54 & 53 & 63 & 54 & 92 & 28 & 82 \\
\hline 17 & 67 & 41 & 54 & 88 & 94 & 48 & 63 & 52 & 90 & 26 & 55 \\
\hline 18 & 68 & 41 & 54 & 88 & 94 & 51 & 67 & 60 & 94 & 29 & 68 \\
\hline 19 & 68 & 41 & 54 & 78 & 56 & 50 & 51 & 56 & 94 & 27 & 66 \\
\hline 20 & 68 & 41 & 55 & 84 & 56 & 45 & 67 & 53 & $90^{(\mathrm{a})}$ & 28 & 82 \\
\hline 21 & 68 & 42 & 55 & 85 & 56 & 52 & 67 & 60 & 56 & 26 & $85^{(a)}$ \\
\hline 22 & 68 & 42 & 55 & 81 & $82^{(a)}$ & 53 & 88 & 55 & 98 & 28 & 72 \\
\hline 23 & 68 & 42 & 55 & 88 & $81^{(\mathrm{a})}$ & 56 & $79^{(a)}$ & 58 & 77 & 30 & 78 \\
\hline 24 & 69 & 42 & 55 & 94 & 77 & 53 & 75 & 56 & 52 & 28 & $86^{(\mathrm{a})}$ \\
\hline 25 & 69 & 42 & 56 & 91 & 46 & 56 & 58 & 59 & 52 & 31 & 55 \\
\hline 26 & 69 & 43 & 56 & 85 & 92 & 53 & 48 & 57 & 78 & 28 & 48 \\
\hline 27 & 70 & 43 & 56 & 90 & 87 & 50 & 90 & 57 & 92 & 27 & 70 \\
\hline 28 & 70 & 43 & 57 & 93 & 87 & 54 & 95 & 64 & 87 & 27 & 67 \\
\hline 29 & 71 & 44 & 57 & 90 & 68 & 47 & 67 & 60 & 87 & 29 & 52 \\
\hline 30 & 71 & 44 & 58 & 92 & 98 & 56 & $67^{(\mathrm{a})}$ & 60 & 98 & 29 & 86 \\
\hline
\end{tabular}

May

\begin{tabular}{|c|c|c|c|c|c|c|c|c|c|c|c|}
\hline 1 & 72 & 44 & 58 & 93 & 98 & 53 & 69 & 59 & $98^{(\mathrm{a})}$ & 28 & 54 \\
\hline 2 & 72 & 44 & 58 & 89 & $98^{(\mathrm{a})}$ & 56 & 88 & 60 & 71 & 30 & 97 \\
\hline 3 & 73 & 45 & 59 & 91 & 66 & 58 & 93 & 60 & 71 & 31 & 49 \\
\hline 4 & 73 & 45 & 59 & 94 & 66 & 56 & 63 & 57 & 46 & 31 & 62 \\
\hline 5 & 73 & 45 & 59 & 100 & 66 & 52 & 61 & 65 & 66 & 30 & 59 \\
\hline 6 & 73 & 45 & 59 & 98 & 92 & 56 & 86 & 62 & 87 & 33 & 00 \\
\hline 7 & 74 & 46 & 60 & 99 & 87 & 59 & 99 & 66 & 92 & 33 & 84 \\
\hline 8 & 74 & 46 & 60 & 102 & 87 & 56 & $99^{\text {(a) }}$ & 67 & $94^{\text {(a) }}$ & 29 & 96 \\
\hline 9 & 74 & 46 & 60 & 97 & 87 & 56 & 48 & 66 & 49 & 34 & $99^{\text {(a) }}$ \\
\hline 10 & 75 & 47 & 61 & 96 & 49 & 53 & 67 & 66 & 49 & 34 & 70 \\
\hline 11 & 75 & 47 & 61 & 98 & 49 & 51 & 67 & 68 & 49 & 30 & 70 \\
\hline 12 & 75 & 47 & 61 & 100 & 93 & 57 & 70 & 66 & 93 & 34 & 85 \\
\hline 13 & 75 & 47 & 61 & 94 & 97 & 57 & 55 & 66 & 97 & 34 & 85 \\
\hline 14 & 76 & 48 & 62 & 98 & 73 & 56 & 55 & 61 & $73^{(\mathrm{a})}$ & 31 & 55 \\
\hline 15 & 76 & 48 & 62 & 97 & 73 & 57 & 59 & 67 & 97 & 35 & 74 \\
\hline 16 & 76 & 48 & 62 & 95 & 73 & 54 & 55 & 60 & 73 & 32 & 74 \\
\hline 17 & 76 & 48 & 62 & 96 & 73 & 58 & 03 & 59 & 85 & 36 & 03 \\
\hline 18 & 76 & 48 & 62 & 98 & 54 & 62 & 74 & 67 & 56 & 36 & 72 \\
\hline 19 & 76 & 48 & 62 & 92 & 93 & 56 & 62 & 70 & 56 & 33 & 75 \\
\hline 20 & 76 & 48 & 62 & 93 & 47 & 58 & 60 & 59 & 56 & 36 & 71 \\
\hline 21 & 77 & 49 & 63 & 94 & 58 & 62 & $60^{(a)}$ & 59 & 58 & 37 & $74^{(\mathrm{a})}$ \\
\hline 22 & 78 & 49 & 63 & 98 & 58 & 63 & 64 & 64 & 58 & 33 & 60 \\
\hline 23 & 78 & 50 & 64 & 101 & 01 & 59 & 62 & 66 & 58 & 35 & 64 \\
\hline 24 & 78 & 50 & 64 & 97 & $01^{(\mathrm{a})}$ & 54 & 62 & 66 & 01 & 35 & 75 \\
\hline 25 & 78 & 50 & 64 & 98 & $92^{(\mathrm{a})}$ & 61 & 98 & 65 & 83 & 38 & $91^{(\mathrm{a})}$ \\
\hline 26 & 78 & 51 & 64 & 101 & $58^{(a)}$ & 54 & 80 & 69 & 47 & 38 & 78 \\
\hline 27 & 78 & 51 & 65 & 93 & 83 & 62 & 89 & 69 & 58 & 37 & 73 \\
\hline 28 & 78 & 51 & 65 & 99 & 83 & 61 & 89 & 63 & $72^{\text {(a) }}$ & 38 & $79^{\text {(a) }}$ \\
\hline 29 & 79 & 51 & 65 & 103 & 83 & 68 & $98^{(\mathrm{a})}$ & 71 & 86 & 35 & 76 \\
\hline 30 & 79 & 51 & 65 & 104 & 86 & 62 & $76^{(\mathrm{a})}$ & 68 & 86 & 41 & $55^{(\mathrm{a})}$ \\
\hline 31 & 79 & 52 & 66 & 104 & 86 & 54 & 71 & 69 & 86 & 40 & $96^{\text {(a) }}$ \\
\hline
\end{tabular}

June

\begin{tabular}{rrrrrrrrrrrr}
\hline 1 & 80 & 52 & 66 & 103 & 86 & 64 & 76 & 69 & 86 & 37 & 84 \\
2 & 80 & 52 & 66 & 99 & 70 & 65 & $99^{(a)}$ & 69 & $89^{(a)}$ & 37 & 76
\end{tabular}


Table 3.12. (contd)

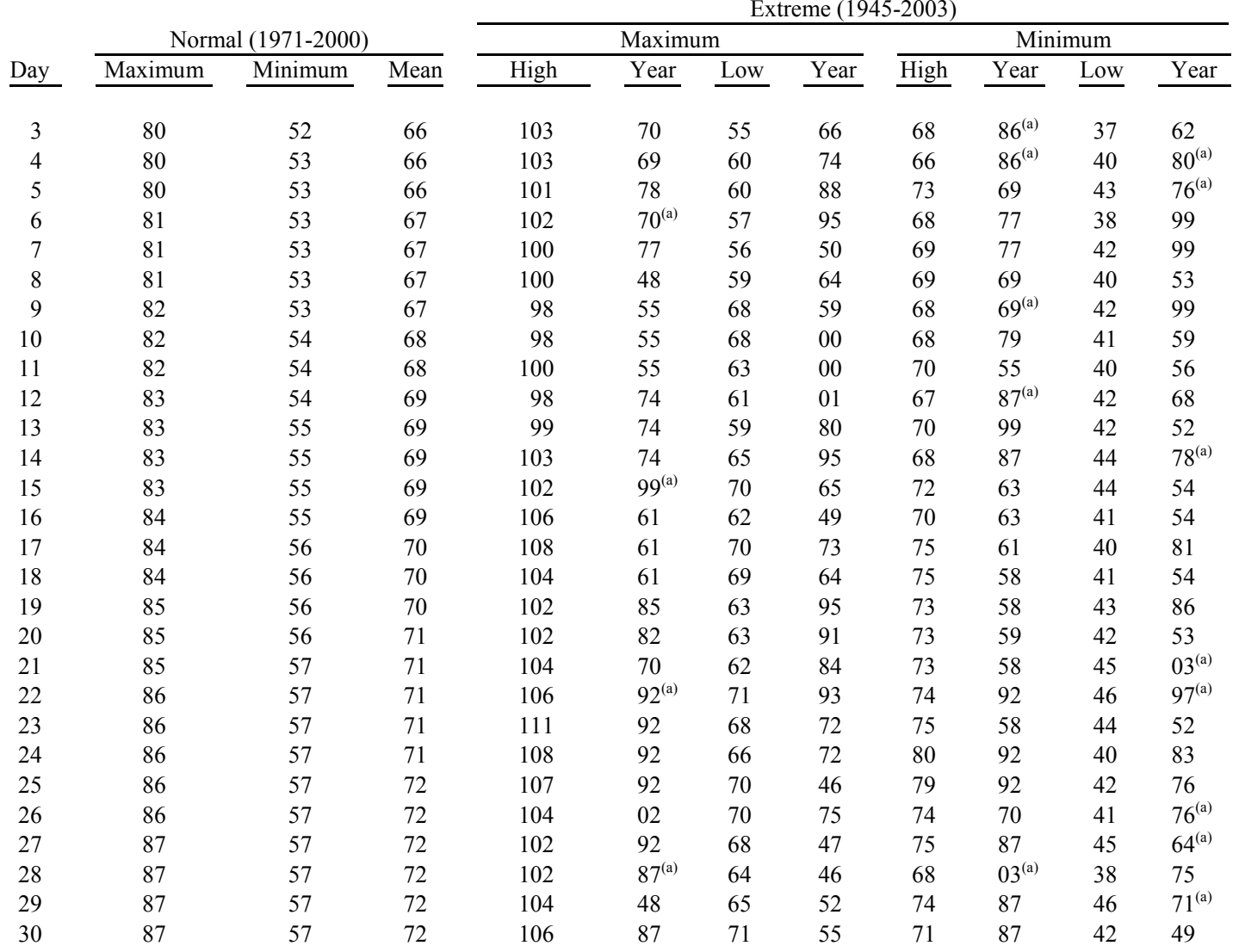

July

\begin{tabular}{rlllllllllll}
\hline & & & & & & & & & & \\
1 & 87 & 57 & 72 & 103 & 87 & 66 & 66 & 75 & 87 & 46 & $73^{(\text {a) }}$ \\
2 & 86 & 57 & 72 & 103 & 96 & 59 & 66 & 70 & 45 & 39 & 79 \\
3 & 86 & 57 & 72 & 105 & $91^{(a)}$ & 71 & 66 & 70 & 67 & 45 & 99 \\
4 & 87 & 57 & 72 & 108 & 68 & 71 & 86 & 75 & 70 & 44 & 00 \\
5 & 87 & 58 & 72 & 108 & 75 & 66 & 51 & 76 & 75 & 47 & $99^{(\text {a) }}$ \\
6 & 88 & 58 & 73 & 110 & 68 & 71 & 55 & 76 & 68 & 44 & 71 \\
7 & 88 & 59 & 73 & 105 & $68^{(a)}$ & 75 & 81 & 73 & 68 & 45 & 71 \\
8 & 88 & 59 & 73 & 108 & 68 & 71 & 72 & 74 & 85 & 45 & 81 \\
9 & 89 & 59 & 74 & 110 & 75 & 76 & 55 & 78 & 75 & 50 & $72^{(a)}$ \\
10 & 89 & 60 & 74 & 106 & 75 & 67 & 74 & 79 & 75 & 49 & $97^{(\text {a) }}$ \\
11 & 90 & 60 & 75 & 109 & $02^{(a)}$ & 76 & 74 & 78 & 75 & 46 & 81 \\
12 & 90 & 60 & 75 & 112 & 02 & 75 & 88 & 75 & 90 & 50 & 74 \\
13 & 90 & 60 & 75 & 113 & 02 & 77 & $93^{(a)}$ & 80 & 02 & 49 & 76 \\
14 & 91 & 61 & 76 & 107 & $87^{(a)}$ & 77 & 83 & 78 & 61 & 50 & 83 \\
15 & 91 & 61 & 76 & 108 & 96 & 71 & 82 & 76 & 55 & 45 & 82 \\
16 & 92 & 61 & 77 & 105 & 70 & 68 & 86 & 74 & 90 & 48 & 74 \\
17 & 92 & 62 & 77 & 110 & 60 & 73 & 93 & 77 & 58 & 48 & 86 \\
18 & 93 & 62 & 77 & 110 & 60 & 76 & 96 & 79 & 60 & 49 & $96^{(\text {a) }}$ \\
19 & 93 & 62 & 78 & 109 & 79 & 72 & 49 & 77 & 79 & 51 & 77 \\
20 & 94 & 63 & 78 & 110 & 79 & 75 & $65^{(a)}$ & 75 & 95 & 53 & $68^{(a)}$ \\
21 & 94 & 63 & 79 & 109 & 94 & 68 & 65 & 77 & 88 & 49 & 49 \\
22 & 94 & 63 & 79 & 111 & 94 & 74 & 92 & 75 & $94^{(a)}$ & 47 & 82 \\
23 & 95 & 63 & 79 & 109 & 94 & 69 & 92 & 82 & 94 & 49 & 63 \\
24 & 95 & 63 & 79 & 109 & 94 & 78 & 63 & 75 & 62 & 52 & $52^{(a)}$ \\
25 & 96 & 64 & 80 & 106 & 84 & 73 & 90 & 77 & 62 & 51 & $49^{(\text {a) }}$ \\
26 & 96 & 64 & 80 & 108 & $98^{(a)}$ & 66 & 55 & 76 & 88 & 53 & 99 \\
27 & 96 & 64 & 80 & 112 & 98 & 74 & 48 & 74 & $98^{(a)}$ & 52 & 86
\end{tabular}


Table 3.12. (contd)

\begin{tabular}{|c|c|c|c|c|c|c|c|c|c|c|c|}
\hline \multirow[b]{3}{*}{ Day } & \multirow{2}{*}{\multicolumn{3}{|c|}{ Normal (1971-2000) }} & \multicolumn{8}{|c|}{ Extreme (1945-2003) } \\
\hline & & & & \multicolumn{4}{|c|}{ Maximum } & \multicolumn{4}{|c|}{ Minimum } \\
\hline & Maximum & Minimum & Mean & High & Year & Low & Year & High & Year & Low & Year \\
\hline 28 & 96 & 64 & 80 & 108 & $03^{(a)}$ & 77 & $50^{(a)}$ & 81 & 98 & 49 & 59 \\
\hline 29 & 96 & 64 & 80 & 108 & 03 & 01 & $93^{(\mathrm{a})}$ & 78 & 82 & 52 & 50 \\
\hline 30 & 96 & 64 & 80 & 108 & 03 & 78 & 75 & 74 & 90 & 49 & 50 \\
\hline 31 & 95 & 64 & 79 & 111 & 71 & 75 & 85 & 76 & 00 & 52 & 95 \\
\hline
\end{tabular}

August

\begin{tabular}{|c|c|c|c|c|c|c|c|c|c|c|c|}
\hline 1 & 95 & 64 & 79 & 109 & 71 & 77 & $76^{(\mathrm{a})}$ & 80 & 49 & 51 & 87 \\
\hline 2 & 95 & 63 & 79 & 106 & 94 & 75 & 56 & 75 & $77^{(\mathrm{a})}$ & 46 & 64 \\
\hline 3 & 95 & 63 & 79 & 107 & 61 & 77 & 62 & 75 & $99^{\text {(a) }}$ & 52 & 59 \\
\hline 4 & 95 & 63 & 79 & 113 & 61 & 78 & $02^{\text {(a) }}$ & 81 & 61 & 48 & 54 \\
\hline 5 & 95 & 63 & 79 & 108 & 90 & 78 & 02 & 72 & 91 & 45 & 69 \\
\hline 6 & 95 & 63 & 79 & 106 & 72 & 77 & 46 & 77 & 90 & 51 & 47 \\
\hline 7 & 95 & 63 & 79 & 109 & 72 & 70 & 62 & 75 & 45 & 49 & 46 \\
\hline 8 & 94 & 63 & 79 & 110 & 72 & 75 & 62 & 79 & $82^{(a)}$ & 48 & 49 \\
\hline 9 & 94 & 63 & 78 & 112 & 71 & 78 & 47 & 78 & 90 & 51 & $95^{(\mathrm{a})}$ \\
\hline 10 & 94 & 62 & 78 & 109 & $96^{(\mathrm{a})}$ & 76 & 85 & 77 & 71 & 52 & 47 \\
\hline 11 & 94 & 62 & 78 & 108 & 71 & 79 & 83 & 73 & 58 & 50 & 85 \\
\hline 12 & 93 & 62 & 77 & 108 & 71 & 77 & 95 & 77 & 92 & 52 & 00 \\
\hline 13 & 92 & 61 & 76 & 107 & 92 & 74 & 68 & 79 & 92 & 47 & 95 \\
\hline 14 & 91 & 60 & 76 & 109 & 92 & 70 & 68 & 78 & $92^{\text {(a) }}$ & 45 & 95 \\
\hline 15 & 90 & 60 & 75 & 105 & 67 & 72 & 60 & 74 & 92 & 51 & 74 \\
\hline 16 & 90 & 60 & 75 & 108 & 67 & 68 & 93 & 76 & 45 & 48 & 76 \\
\hline 17 & 89 & 59 & 74 & 108 & 67 & 76 & 95 & 68 & 91 & 47 & 76 \\
\hline 18 & 89 & 59 & 74 & 108 & 67 & 71 & 80 & 71 & 97 & 47 & 76 \\
\hline 19 & 88 & 59 & 74 & 105 & 67 & 70 & 68 & 76 & 91 & 46 & $80^{(a)}$ \\
\hline 20 & 88 & 59 & 73 & 105 & 67 & 67 & 59 & 77 & 82 & 49 & 52 \\
\hline 21 & 88 & 58 & 73 & 104 & 46 & 70 & 60 & 75 & 46 & 47 & $85^{(\mathrm{a})}$ \\
\hline 22 & 88 & 58 & 73 & 104 & $56^{(\mathrm{a})}$ & 70 & 92 & 76 & $61^{\text {(a) }}$ & 41 & 60 \\
\hline 23 & 88 & 58 & 73 & 105 & 70 & 69 & 92 & 76 & 46 & 45 & 92 \\
\hline 24 & 88 & 57 & 73 & 104 & 58 & 70 & 68 & 73 & 00 & 43 & 92 \\
\hline 25 & 88 & 57 & 72 & 105 & 96 & 72 & 77 & 70 & 46 & 43 & 93 \\
\hline 26 & 88 & 57 & 72 & 100 & 84 & 68 & 56 & 70 & 96 & 44 & $93^{\text {(a) }}$ \\
\hline 27 & 88 & 57 & 72 & 101 & 72 & 73 & 68 & 71 & 67 & 47 & $78^{(\mathrm{a})}$ \\
\hline 28 & 87 & 57 & 72 & 104 & 72 & 70 & 51 & 74 & 86 & 42 & 80 \\
\hline 29 & 87 & 57 & 72 & 102 & 67 & 72 & 51 & 73 & 67 & 42 & 65 \\
\hline 30 & 86 & 57 & 72 & 105 & 67 & 64 & 99 & 71 & 67 & 44 & 64 \\
\hline 31 & 86 & 56 & 71 & 104 & 67 & 72 & $99^{(\mathrm{a})}$ & 73 & 67 & 43 & 99 \\
\hline \multicolumn{12}{|c|}{ September } \\
\hline 1 & 86 & 56 & 71 & 106 & 87 & 61 & 71 & 70 & 87 & 43 & 99 \\
\hline 2 & 86 & 56 & 71 & 102 & $98^{(a)}$ & 69 & 00 & 70 & 49 & 47 & $75^{(\mathrm{a})}$ \\
\hline 3 & 85 & 55 & 70 & 102 & 98 & 71 & 97 & 71 & 95 & 40 & 00 \\
\hline 4 & 85 & 55 & 70 & 102 & $03^{(a)}$ & 68 & 59 & 68 & 55 & 44 & 80 \\
\hline 5 & 85 & 55 & 70 & 100 & $03^{(a)}$ & 72 & 60 & 68 & 63 & 43 & 69 \\
\hline 6 & 85 & 55 & 70 & 101 & 55 & 69 & 46 & 68 & 03 & 41 & 96 \\
\hline 7 & 84 & 54 & 69 & 97 & 58 & 60 & 78 & 72 & 55 & 42 & $92^{(a)}$ \\
\hline 8 & 84 & 54 & 69 & 99 & 81 & 61 & 85 & 69 & 63 & 42 & $76^{(a)}$ \\
\hline 9 & 83 & 53 & 68 & 98 & $81^{\text {(a) }}$ & 66 & 85 & 68 & 69 & 40 & 62 \\
\hline 10 & 83 & 53 & 68 & 97 & 93 & 68 & 85 & 65 & 63 & 43 & $82^{\text {(a) }}$ \\
\hline 11 & 82 & 53 & 68 & 98 & $90^{(\mathrm{a})}$ & 62 & 85 & 66 & 69 & 41 & 88 \\
\hline 12 & 82 & 52 & 67 & 96 & $02^{(a)}$ & 62 & 70 & 67 & 53 & 38 & 49 \\
\hline 13 & 82 & 52 & 67 & 98 & 48 & 59 & 80 & 66 & 01 & 40 & 74 \\
\hline 14 & 81 & 52 & 67 & 95 & 01 & 62 & 92 & 67 & 00 & 38 & 70 \\
\hline 15 & 81 & 52 & 66 & 96 & $79^{(a)}$ & 58 & 59 & 66 & 00 & 35 & 70 \\
\hline 16 & 80 & 52 & 66 & 96 & $81^{(a)}$ & 61 & 46 & 65 & 79 & 35 & 65 \\
\hline 17 & 80 & 51 & 65 & 97 & 81 & 59 & 86 & 62 & 51 & 33 & 65 \\
\hline 18 & 79 & 51 & 65 & 98 & 81 & 57 & 83 & 68 & 00 & 34 & 65 \\
\hline
\end{tabular}


Table 3.12. (contd)

\begin{tabular}{|c|c|c|c|c|c|c|c|c|c|c|c|}
\hline \multirow[b]{3}{*}{ Day } & & & & \multicolumn{8}{|c|}{ Extreme (1945-2003) } \\
\hline & \multicolumn{3}{|c|}{ Normal (1971-2000) } & \multicolumn{4}{|c|}{ Maximum } & \multicolumn{4}{|c|}{ Minimum } \\
\hline & Maximum & Minimum & Mean & High & Year & Low & Year & High & Year & Low & Year \\
\hline 19 & 78 & 50 & 64 & 96 & 67 & 62 & 83 & 67 & 56 & 36 & 57 \\
\hline 20 & 78 & 49 & 64 & 94 & 94 & 66 & $72^{(a)}$ & 67 & 94 & 37 & 83 \\
\hline 21 & 78 & 49 & 63 & 98 & 67 & 56 & 00 & 65 & 62 & 38 & $93^{(a)}$ \\
\hline 22 & 77 & 48 & 62 & 93 & 66 & 52 & 84 & 68 & 66 & 36 & $81^{(\mathrm{a})}$ \\
\hline 23 & 77 & 48 & 62 & 93 & 87 & 54 & 77 & 62 & 92 & 32 & 00 \\
\hline 24 & 77 & 47 & 62 & 94 & $01^{(a)}$ & 60 & 72 & 60 & 50 & 34 & $00^{(\mathrm{a})}$ \\
\hline 25 & 77 & 47 & 62 & 97 & 52 & 56 & 77 & 66 & 49 & 30 & 72 \\
\hline 26 & 77 & 47 & 62 & 93 & 52 & 57 & 48 & 61 & 79 & 32 & 72 \\
\hline 27 & 77 & 47 & 62 & 92 & $63^{(a)}$ & 58 & 77 & 60 & $03^{(a)}$ & 30 & 72 \\
\hline 28 & 77 & 47 & 62 & 92 & 67 & 58 & 77 & 62 & 76 & 33 & 85 \\
\hline 29 & 76 & 47 & 62 & 92 & $96^{(a)}$ & 57 & 77 & 61 & $03^{(a)}$ & 34 & $85^{(a)}$ \\
\hline 30 & 76 & 47 & 61 & 88 & $93^{(a)}$ & 63 & $54^{(\mathrm{a})}$ & 64 & 93 & 35 & 85 \\
\hline
\end{tabular}

October

\begin{tabular}{|c|c|c|c|c|c|c|c|c|c|c|c|}
\hline 1 & 75 & 46 & 61 & 88 & $91^{(\mathrm{a})}$ & 61 & 59 & 59 & 92 & 30 & 54 \\
\hline 2 & 75 & 46 & 60 & 86 & $93^{(\mathrm{a})}$ & 56 & 67 & 60 & 88 & 32 & 54 \\
\hline 3 & 74 & 46 & 60 & 89 & 58 & 55 & 50 & 58 & $88^{(a)}$ & 33 & $99^{\text {(a) }}$ \\
\hline 4 & 73 & 45 & 59 & 89 & 80 & 55 & 50 & 57 & 88 & 32 & 73 \\
\hline 5 & 72 & 44 & 58 & 87 & 58 & 52 & 57 & 56 & 03 & 34 & $82^{\text {(a) }}$ \\
\hline 6 & 72 & 44 & 58 & 87 & 03 & 53 & $57^{(\mathrm{a})}$ & 57 & 60 & 30 & 74 \\
\hline 7 & 72 & 44 & 58 & 86 & 80 & 48 & 57 & 57 & 88 & 29 & 74 \\
\hline 8 & 71 & 44 & 57 & 84 & 65 & 50 & 97 & 58 & 87 & 26 & 85 \\
\hline 9 & 71 & 44 & 57 & 84 & 45 & 52 & 58 & 56 & 96 & 26 & 85 \\
\hline 10 & 70 & 43 & 57 & 86 & 96 & 50 & 62 & 55 & 96 & 33 & 59 \\
\hline 11 & 70 & 43 & 56 & 84 & 52 & 52 & $68^{(a)}$ & 55 & 63 & 30 & 60 \\
\hline 12 & 69 & 43 & 56 & 84 & 45 & 54 & 66 & 56 & 52 & 29 & 02 \\
\hline 13 & 68 & 42 & 55 & 81 & 99 & 57 & 69 & 55 & 88 & 31 & 69 \\
\hline 14 & 68 & 41 & 54 & 78 & 45 & 58 & $90^{\text {(a) }}$ & 59 & 88 & 24 & 69 \\
\hline 15 & 67 & 40 & 54 & 81 & 63 & 53 & 92 & 60 & 88 & 29 & 70 \\
\hline 16 & 66 & 40 & 53 & 79 & 63 & 54 & $92^{\text {(a) }}$ & 56 & 95 & 26 & 46 \\
\hline 17 & 65 & 39 & 52 & 79 & 03 & 45 & 96 & 49 & $03^{(\mathrm{a})}$ & 26 & 96 \\
\hline 18 & 65 & 38 & 51 & 77 & 03 & 47 & 49 & 50 & $00^{(\mathrm{a})}$ & 27 & 49 \\
\hline 19 & 64 & 38 & 51 & 78 & $92^{\text {(a) }}$ & 50 & 45 & 54 & 03 & 27 & $69^{\text {(a) }}$ \\
\hline 20 & 63 & 37 & 50 & 80 & 03 & 45 & 47 & 56 & 03 & 23 & 49 \\
\hline 21 & 63 & 37 & 50 & 89 & 03 & 45 & 96 & 65 & 03 & 20 & 84 \\
\hline 22 & 62 & 37 & 50 & 81 & 03 & 46 & 50 & 57 & 03 & 20 & 84 \\
\hline 23 & 62 & 37 & 49 & 73 & $66^{(\mathrm{a})}$ & 39 & 84 & 51 & 60 & 25 & 84 \\
\hline 24 & 61 & 37 & 49 & 75 & $77^{(\mathrm{a})}$ & 49 & 57 & 49 & 46 & 24 & 02 \\
\hline 25 & 60 & 37 & 48 & 75 & $55^{(\mathrm{a})}$ & 49 & 57 & 60 & 45 & 23 & 02 \\
\hline 26 & 59 & 37 & 48 & 69 & 92 & 49 & $00^{\text {(a) }}$ & 52 & 94 & 21 & 78 \\
\hline 27 & 59 & 36 & 47 & 74 & 85 & 43 & $99^{(\mathrm{a})}$ & 54 & 81 & 23 & 70 \\
\hline 28 & 58 & 36 & 47 & 80 & 03 & 35 & 91 & 52 & 49 & 18 & 71 \\
\hline 29 & 57 & 35 & 46 & 70 & 53 & 42 & $91^{(\mathrm{a})}$ & 50 & 97 & 13 & 71 \\
\hline 30 & 56 & 35 & 45 & 75 & 67 & 32 & 71 & 50 & 97 & 16 & 02 \\
\hline 31 & 56 & 35 & 45 & 75 & 67 & 34 & 84 & 54 & 67 & 7 & 02 \\
\hline \multicolumn{12}{|c|}{ November } \\
\hline 1 & 55 & 35 & 45 & 69 & 88 & 31 & 84 & 49 & 87 & 11 & 02 \\
\hline 2 & 55 & 35 & 45 & 70 & 45 & 37 & 03 & 51 & 85 & 11 & 02 \\
\hline 3 & 54 & 35 & 45 & 75 & 75 & 36 & 73 & 53 & 83 & 14 & 02 \\
\hline 4 & 54 & 35 & 44 & 71 & 75 & 32 & 73 & 46 & $89^{(a)}$ & 15 & 02 \\
\hline 5 & 54 & 35 & 44 & 63 & 89 & 31 & 73 & 48 & 88 & 18 & 03 \\
\hline 6 & 53 & 35 & 44 & 64 & 58 & 32 & 73 & 49 & 89 & 19 & 73 \\
\hline 7 & 52 & 34 & 43 & 69 & 78 & 34 & 45 & 48 & $97^{(a)}$ & 19 & $93^{(a)}$ \\
\hline 8 & 52 & 34 & 43 & 69 & 95 & 34 & 45 & 48 & 89 & 16 & 45 \\
\hline 9 & 51 & 34 & 43 & 73 & 89 & 30 & 45 & 60 & 89 & 18 & 86 \\
\hline
\end{tabular}


Table 3.12. (contd)

\begin{tabular}{|c|c|c|c|c|c|c|c|c|c|c|c|}
\hline \multirow[b]{3}{*}{ Day } & & & & \multicolumn{8}{|c|}{ Extreme (1945-2003) } \\
\hline & \multicolumn{3}{|c|}{ Normal (1971-2000) } & \multicolumn{4}{|c|}{ Maximum } & \multicolumn{4}{|c|}{ Minimum } \\
\hline & Maximum & Minimum & Mean & High & Year & Low & Year & High & Year & Low & Year \\
\hline 10 & 51 & 34 & 43 & 73 & 89 & 32 & 45 & 56 & 89 & 16 & 86 \\
\hline 11 & 51 & 34 & 42 & 66 & 89 & 32 & 85 & 48 & 89 & 14 & 78 \\
\hline 12 & 50 & 33 & 42 & 75 & 99 & 20 & 55 & 52 & 49 & 6 & 55 \\
\hline 13 & 50 & 33 & 41 & 76 & 99 & 14 & 55 & 56 & 98 & 6 & 59 \\
\hline 14 & 50 & 33 & 41 & 68 & 01 & 19 & 55 & 54 & 01 & -1 & 55 \\
\hline 15 & 49 & 33 & 41 & 67 & 98 & 18 & 55 & 49 & 01 & 1 & 55 \\
\hline 16 & 49 & 32 & 40 & 65 & 76 & 18 & 55 & 49 & 54 & 7 & 59 \\
\hline 17 & 48 & 32 & 40 & 71 & 76 & 22 & 55 & 46 & $83^{(a)}$ & 10 & 61 \\
\hline 18 & 48 & 31 & 40 & 68 & 03 & 25 & 55 & 52 & 03 & 11 & 55 \\
\hline 19 & 47 & 31 & 39 & 67 & $02^{\text {(a) }}$ & 22 & 85 & 47 & 02 & 14 & 85 \\
\hline 20 & 46 & 30 & 38 & 65 & 58 & 24 & 85 & 47 & 74 & 3 & 85 \\
\hline 21 & 45 & 29 & 37 & 63 & 58 & 17 & 85 & 46 & 65 & 3 & 85 \\
\hline 22 & 45 & 29 & 37 & 65 & $67^{(a)}$ & 17 & 85 & 53 & 90 & -6 & 85 \\
\hline 23 & 44 & 29 & 37 & 70 & 59 & 11 & 85 & 54 & 90 & -13 & 85 \\
\hline 24 & 44 & 28 & 36 & 67 & $95^{(\mathrm{a})}$ & 6 & 85 & 57 & 90 & -12 & 85 \\
\hline 25 & 43 & 28 & 36 & 67 & 98 & 11 & 85 & 50 & 98 & -3 & 85 \\
\hline 26 & 43 & 28 & 36 & 65 & 49 & 15 & 85 & 45 & 49 & -1 & 85 \\
\hline 27 & 43 & 28 & 36 & 63 & 49 & 12 & 85 & 46 & 49 & 8 & 85 \\
\hline 28 & 43 & 28 & 36 & 64 & 95 & 11 & 85 & 43 & 73 & 7 & 85 \\
\hline 29 & 43 & 28 & 36 & 62 & 95 & 14 & 85 & 57 & 95 & 8 & 85 \\
\hline 30 & 43 & 28 & 36 & 62 & $95^{(a)}$ & 15 & 85 & 46 & 94 & 6 & 85 \\
\hline & & & & & mber & & & & & & \\
\hline
\end{tabular}

\begin{tabular}{|c|c|c|c|c|c|c|c|c|c|c|c|}
\hline 1 & 43 & 28 & 36 & 65 & 72 & 14 & 85 & 45 & 81 & -8 & 85 \\
\hline 2 & 43 & 28 & 35 & 64 & 77 & 20 & 85 & 56 & 75 & 8 & 85 \\
\hline 3 & 42 & 27 & 35 & 62 & $82^{(a)}$ & 27 & $85^{\text {(a) }}$ & 54 & 75 & 8 & 85 \\
\hline 4 & 42 & 27 & 34 & 60 & $75^{(\mathrm{a})}$ & 23 & 72 & 41 & 52 & 1 & 72 \\
\hline 5 & 41 & 26 & 34 & 58 & $91^{\text {(a) }}$ & 21 & 72 & 41 & 87 & 7 & 72 \\
\hline 6 & 40 & 26 & 33 & 59 & 87 & 16 & 56 & 43 & 87 & 8 & 56 \\
\hline 7 & 40 & 26 & 33 & 58 & $73^{(a)}$ & 18 & 56 & 40 & 52 & 2 & 56 \\
\hline 8 & 40 & 26 & 33 & 58 & 89 & 17 & 72 & 48 & 46 & -6 & 72 \\
\hline 9 & 40 & 26 & 33 & 59 & $87^{(\mathrm{a})}$ & 13 & 72 & 44 & 56 & -5 & 72 \\
\hline 10 & 40 & 26 & 33 & 67 & 93 & 13 & 72 & 48 & 46 & -8 & 72 \\
\hline 11 & 40 & 26 & 33 & 59 & $91^{\text {(a) }}$ & 11 & 72 & 45 & 46 & -4 & 72 \\
\hline 12 & 40 & 26 & 33 & 57 & $99^{\text {(a) }}$ & 22 & 72 & 42 & $77^{(\mathrm{a})}$ & -5 & 72 \\
\hline 13 & 40 & 26 & 33 & 59 & 46 & 13 & 72 & 42 & 46 & -6 & 72 \\
\hline 14 & 40 & 26 & 33 & 57 & $79^{\text {(a) }}$ & 15 & 72 & 48 & 79 & -2 & 72 \\
\hline 15 & 39 & 26 & 32 & 64 & 59 & 16 & 72 & 49 & 99 & 2 & 72 \\
\hline 16 & 39 & 25 & 32 & 62 & 99 & 4 & 64 & 44 & 99 & -13 & 64 \\
\hline 17 & 38 & 25 & 31 & 57 & $98^{(\mathrm{a})}$ & 5 & 64 & 41 & 62 & -12 & 64 \\
\hline 18 & 38 & 24 & 31 & 56 & $99^{(a)}$ & 13 & 64 & 42 & 99 & -3 & 84 \\
\hline 19 & 37 & 24 & 30 & 59 & 94 & 17 & 84 & 41 & 94 & -4 & 84 \\
\hline 20 & 37 & 24 & 30 & 64 & 94 & 11 & 84 & 47 & 94 & -2 & $90^{(a)}$ \\
\hline 21 & 36 & 24 & 30 & 61 & 72 & 11 & 90 & 43 & 73 & -10 & 90 \\
\hline 22 & 36 & 24 & 30 & 59 & 80 & 7 & 90 & 42 & 72 & -13 & 83 \\
\hline 23 & 36 & 24 & 30 & 57 & 63 & 6 & 83 & 40 & 72 & -9 & 83 \\
\hline 24 & 36 & 24 & 30 & 55 & $61^{(a)}$ & 15 & $90^{(\mathrm{a})}$ & 39 & $80^{(\mathrm{a})}$ & 0 & 90 \\
\hline 25 & 36 & 24 & 30 & 65 & 80 & 16 & 90 & 41 & 72 & 1 & 90 \\
\hline 26 & 36 & 24 & 30 & 69 & 80 & 20 & $90^{\text {(a) }}$ & 53 & 80 & 0 & 48 \\
\hline 27 & 36 & 24 & 30 & 62 & $80^{(a)}$ & 19 & 48 & 40 & $94^{(\mathrm{a})}$ & -2 & 48 \\
\hline 28 & 35 & 23 & 29 & 59 & 98 & 11 & 96 & 46 & 98 & -6 & 96 \\
\hline 29 & 35 & 23 & 29 & 60 & $98^{(\mathrm{a})}$ & -2 & 68 & 41 & 98 & -10 & $90^{\text {(a) }}$ \\
\hline 30 & 35 & 22 & 29 & 54 & 70 & -2 & 68 & 39 & $88^{(a)}$ & -14 & 68 \\
\hline 31 & 35 & 22 & 28 & 56 & 62 & 4 & 68 & 44 & 80 & -9 & 78 \\
\hline
\end{tabular}

(a) Latest of several occurrences. 
Table 3.13 and Table 3.14 provide monthly heating-degree day and cooling-degree day data, respectively, for the period 1945 through 2003. The heating-degree days are traditionally totaled for the 12-month period July through June of the following year.

The heating degree accumulation begins July 1 of one year and ends June 30 of the following year. Figure 3.26 shows the climatological variation of the accumulation heating-degree days for the period from July 1945 through June 2002. It also shows the accumulation of heating-degree days for the 20022003 heating season. The figure shows a cooler than average fall followed by a warmer than average winter, with cumulative heating-degree days below the range of typical seasons at the end of the season.

Figure 3.27 presents the climatological statistics for cooling-degree days and data for the 2003 cooling season. The figure shows a warm late-spring, followed by a hot summer. Overall, the cooling season was much warmer than typical summers.

The record highs and lows in Figure 3.26 and Figure 3.27 are cumulative values at each date. The record values did not all occur in the same season.

\subsection{Subsurface Soil Temperatures}

Hourly subsurface soil temperature data at depths of $\sim 0.5$ inch, 15 inches, and 36 inches are available for the period from 1955 through 2003. The subsurface soil temperature sensors are installed in the natural soil of the area with the vegetation cover removed. The soil is sandy and mixed with large gravel.

Monthly averages and extremes of monthly averages are presented in Table 3.15. The absolute hourly extremes are also indicated in that table. 
Table 3.13. Monthly and Seasonal Heating-Degree Days

\begin{tabular}{|c|c|c|c|c|c|c|c|c|c|c|c|c|c|}
\hline Year & Jul & Aug & Sep & Oct & Nov & Dec & Jan & Feb & Mar & Apr & May & Jun & Season \\
\hline $1944-45$ & -- & -- & -- & -- & -- & -- & 967 & 738 & 709 & 442 & 141 & 38 & -- \\
\hline $1945-46$ & 0 & 2 & 97 & 277 & 733 & 1,000 & 949 & 710 & 603 & 331 & 79 & 42 & 4,823 \\
\hline $1946-47$ & 0 & 1 & 101 & 479 & 875 & 935 & 1,168 & 702 & 476 & 266 & $36^{(a)}$ & 25 & 5,064 \\
\hline $1947-48$ & 0 & 7 & 70 & 351 & 714 & 989 & 1,024 & 963 & 709 & 471 & 237 & 5 & 5,540 \\
\hline $1948-49$ & 2 & 0 & 109 & 438 & 725 & 1,184 & 1,581 & 928 & 616 & 281 & 85 & 36 & 5,985 \\
\hline $1949-50$ & 1 & 0 & 52 & 456 & 592 & 927 & $1,640^{(a)}$ & 959 & 704 & 452 & 196 & 66 & 6,045 \\
\hline $1950-51$ & 0 & 0 & 64 & 431 & 728 & 895 & 994 & 786 & 773 & 325 & 146 & 45 & 5,187 \\
\hline $1951-52$ & 5 & 19 & 46 & 421 & 763 & 1,164 & 1,235 & 823 & 645 & 311 & 118 & 45 & 5,595 \\
\hline $1952-53$ & 0 & 2 & 34 & $200^{(a)}$ & 929 & 934 & $694^{(a)}$ & 664 & 585 & 419 & 228 & $90^{(\mathrm{a})}$ & 4,779 \\
\hline $1953-54$ & 0 & 3 & 59 & 298 & 649 & 851 & 1,118 & 720 & 722 & 408 & 124 & 77 & 5,029 \\
\hline $1954-55$ & 10 & 4 & 79 & 423 & 567 & 957 & 1,090 & 832 & $794^{(\mathrm{a})}$ & $522^{(\mathrm{a})}$ & 253 & 23 & 5,554 \\
\hline $1955-56$ & $22^{(a)}$ & 0 & 108 & 364 & 1,008 & 1,105 & 1,029 & $1,147^{\text {(a) }}$ & 655 & 273 & 110 & 55 & 5,876 \\
\hline $1956-57$ & 0 & 6 & 32 & 399 & 850 & 940 & 1,499 & 862 & 650 & 308 & 50 & 11 & 5,607 \\
\hline $1957-58$ & 0 & 0 & 37 & 443 & 739 & $822^{(a)}$ & 862 & 576 & 666 & 411 & 72 & 3 & 4,631 \\
\hline $1958-59$ & 0 & 0 & 74 & 339 & 731 & 927 & 1,025 & 827 & 617 & 325 & 248 & 29 & 5,142 \\
\hline $1959-60$ & 4 & 6 & 118 & 359 & 855 & 987 & 1,292 & 799 & 616 & 374 & 227 & 21 & 5,658 \\
\hline $1960-61$ & 0 & $\mathbf{3 2}^{(\mathrm{a})}$ & 35 & 330 & 717 & 1,114 & 930 & 598 & 587 & 380 & 179 & 16 & 4,918 \\
\hline $1961-62$ & 0 & 0 & 91 & 418 & 893 & 974 & 1,090 & 797 & 698 & 287 & 255 & 51 & 5,554 \\
\hline $1962-63$ & 12 & 1 & 60 & 385 & 657 & 874 & 1,228 & 747 & 577 & 456 & 170 & 25 & 5,192 \\
\hline $1963-64$ & 1 & 1 & 25 & 285 & 668 & 1,078 & 913 & 784 & 656 & 445 & 195 & 33 & 5,084 \\
\hline $1964-65$ & 0 & 21 & 94 & 360 & 804 & 1,224 & 1,009 & 686 & 685 & 307 & 171 & 16 & 5,377 \\
\hline $1965-66$ & 5 & 14 & 115 & 247 & 660 & 995 & 963 & 702 & 605 & 311 & 133 & 58 & 4,808 \\
\hline $1966-67$ & 17 & 2 & 26 & 362 & 638 & 829 & 782 & 598 & 639 & 519 & 175 & 12 & 4,599 \\
\hline $1967-68$ & 0 & 0 & 13 & 305 & 704 & 993 & 907 & 670 & 495 & 416 & 117 & 23 & 4,643 \\
\hline $1968-69$ & 0 & 13 & 50 & 458 & 702 & 1,064 & 1,399 & 932 & 591 & 384 & 88 & 6 & 5,687 \\
\hline $1969-70$ & 0 & 5 & 39 & 431 & 745 & 941 & 1,064 & 683 & 625 & 480 & 137 & 23 & 5,173 \\
\hline $1970-71$ & 0 & 0 & 122 & 439 & 758 & 1,063 & 906 & 726 & 752 & 392 & 124 & 50 & 5,332 \\
\hline $1971-72$ & 13 & 3 & 133 & 420 & 728 & 1,064 & 1,065 & 878 & 560 & 463 & 112 & 23 & 5,462 \\
\hline $1972-73$ & 1 & 3 & 179 & 397 & 754 & 1,168 & 1,112 & 742 & 544 & 338 & 144 & 38 & 5,420 \\
\hline $1973-74$ & 2 & 9 & 73 & 389 & 798 & 837 & 1,104 & 675 & 611 & 361 & 236 & 27 & 5,122 \\
\hline $1974-75$ & 8 & 0 & 32 & 388 & 698 & 892 & 996 & 880 & 704 & 504 & 174 & 31 & 5,307 \\
\hline $1975-76$ & 0 & 13 & 25 & 388 & 764 & 949 & 1,024 & 796 & 735 & 422 & 159 & 74 & 5,349 \\
\hline $1976-77$ & 5 & 15 & 23 & 392 & 736 & 1,065 & 1,232 & 684 & 608 & 253 & 258 & 22 & 5,293 \\
\hline $1977-78$ & 5 & 7 & 153 & 401 & 783 & 967 & 1,001 & 761 & 550 & 393 & 203 & 22 & 5,246 \\
\hline $1978-79$ & 1 & 10 & 76 & 390 & 981 & 1,162 & 1,582 & 861 & 571 & 369 & 94 & 21 & $6,118^{(\mathrm{a})}$ \\
\hline $1979-80$ & 13 & 0 & 10 & 266 & 924 & 887 & 1,277 & 888 & 638 & 302 & 138 & 68 & 5,411 \\
\hline $1980-81$ & 5 & 18 & 53 & 394 & 723 & 883 & 838 & 707 & 503 & 345 & 165 & 51 & 4,685 \\
\hline $1981-82$ & 8 & 0 & 108 & 402 & 668 & 998 & 1,092 & 754 & 590 & 469 & 164 & 17 & 5,270 \\
\hline $1982-83$ & 10 & 0 & 75 & 420 & 844 & 1,023 & 855 & 676 & 511 & 419 & 151 & 50 & 5,034 \\
\hline $1983-84$ & 8 & 0 & 125 & 387 & 643 & 1,357 & 1,035 & 763 & 552 & 432 & $292^{\text {(a) }}$ & 70 & 5,664 \\
\hline $1984-85$ & 0 & 3 & 145 & $\mathbf{5 3 2}^{\text {(a) }}$ & 768 & 1,288 & 1,245 & 982 & 651 & 288 & 137 & 21 & 6,060 \\
\hline $1985-86$ & 0 & 10 & $197^{(\mathrm{a})}$ & 475 & $1,206^{(a)}$ & $1,362^{(a)}$ & 959 & 724 & 509 & 426 & 213 & 10 & 6,091 \\
\hline $1986-87$ & 18 & 0 & 153 & 319 & 680 & 1,009 & 1,066 & 696 & 522 & 239 & 85 & 16 & 4,803 \\
\hline $1987-88$ & 1 & 0 & 32 & 304 & 640 & 1,040 & 1,028 & 695 & 591 & 301 & 166 & 65 & 4,863 \\
\hline $1988-89$ & 3 & 0 & 100 & 208 & 625 & 1,033 & 859 & 1,054 & 658 & 254 & 141 & 6 & 4,941 \\
\hline $1989-90$ & 1 & 0 & 12 & 339 & 621 & 985 & 763 & 767 & 530 & 217 & 149 & 28 & 4,412 \\
\hline $1990-91$ & 4 & $0^{\text {(a) }}$ & $\mathbf{0}^{\text {(a) }}$ & 401 & $553^{(\mathrm{a})}$ & 1,269 & 1,124 & $575^{(\mathrm{a})}$ & 649 & 330 & 148 & 55 & 5,108 \\
\hline $1991-92$ & 0 & 0 & 12 & 381 & 710 & 842 & 851 & 648 & $418^{(\mathrm{a})}$ & 278 & 77 & 13 & $4,230^{(a)}$ \\
\hline $1992-93$ & 1 & 18 & 94 & 298 & 716 & 1,084 & 1,247 & 958 & 674 & 374 & 94 & 23 & 5,581 \\
\hline 1993-94 & 1 & 13 & 89 & 303 & 911 & 914 & 819 & 813 & 490 & $217^{(\mathrm{a}, \mathrm{b})}$ & 97 & 22 & 4,689 \\
\hline $1994-95$ & 0 & 0 & 3 & 332 & 759 & 924 & 954 & 614 & 581 & 372 & 89 & 46 & 4,674 \\
\hline $1995-96$ & 0 & 1 & 24 & 398 & 623 & 1,003 & 1,124 & 935 & 623 & 302 & 225 & 14 & 5,272 \\
\hline $1996-97$ & 3 & 0 & 99 & 401 & 797 & 1,090 & 973 & 692 & 544 & 395 & 95 & 2 & 5,091 \\
\hline $1997-98$ & 2 & 0 & 38 & 367 & 658 & 941 & 892 & 642 & 521 & 332 & 131 & $\mathbf{1}^{\text {(a) }}$ & 4,523 \\
\hline 1998-99 & 0 & 0 & 15 & 393 & 582 & 1,000 & 829 & 651 & 581 & 424 & 265 & 62 & 4,802 \\
\hline $1999-00$ & 8 & 17 & 65 & 407 & 576 & 846 & 995 & 760 & 630 & 288 & 143 & 35 & 4,770 \\
\hline $2000-01$ & 8 & 1 & 115 & 400 & 931 & 1,093 & 981 & 820 & 562 & 411 & 138 & 56 & 5,516 \\
\hline 2001-02 & $\mathbf{0}^{(\mathrm{a}, \mathrm{b})}$ & $\mathbf{0}^{(\mathrm{a}, \mathrm{b})}$ & 30 & 363 & 669 & 936 & 854 & 745 & 698 & 359 & 181 & 28 & 4,863 \\
\hline $2002-03$ & 1 & 1 & 54 & 454 & 714 & 865 & 838 & 705 & 500 & 381 & 181 & 12 & 4,706 \\
\hline 2003-04 & 0 & 0 & 47 & 271 & 814 & 993 & -- & -- & -- & -- & -- & -- & -- \\
\hline Average $^{(\mathrm{c})}$ & 4 & 5 & 70 & 374 & 746 & 992 & 1,050 & 771 & 611 & 365 & 155 & 33 & 5,193 \\
\hline Normal & 4 & 5 & 75 & 376 & 747 & 1,032 & 1,028 & 767 & 587 & 350 & 156 & 33 & 5,160 \\
\hline
\end{tabular}

(a) Greatest and least values.

(b) Most recent of numerous occurrences.

(c) Based on entire period of record, 1945 through 2003.

NOTE: Dashes indicate no data are available. 
Table 3.14. Monthly and Annual Cooling-Degree Days

\begin{tabular}{|c|c|c|c|c|c|c|c|c|c|c|c|c|c|}
\hline Year & Jan & $\mathrm{Feb}$ & Mar & Apr & May & Jun & Jul & Aug & Sep & Oct & Nov & Dec & Annual \\
\hline 1945 & 0 & 0 & 0 & 0 & 39 & 113 & 325 & 388 & 86 & 11 & 0 & 0 & 962 \\
\hline 1946 & 0 & 0 & 0 & 9 & 53 & 100 & 345 & 360 & 57 & 0 & 0 & 0 & 924 \\
\hline 1947 & 0 & 0 & 0 & 3 & 153 & 108 & 321 & 217 & 86 & 11 & 0 & 0 & 899 \\
\hline 1948 & 0 & 0 & 0 & 0 & 31 & 230 & 243 & 213 & 90 & 0 & 0 & 0 & 807 \\
\hline 1949 & 0 & 0 & 0 & 4 & 147 & 168 & 311 & 307 & 146 & 0 & 0 & 0 & 1,083 \\
\hline 1950 & 0 & 0 & 0 & 0 & 13 & 112 & 321 & 353 & 139 & 0 & 0 & 0 & 938 \\
\hline 1951 & 0 & 0 & 0 & 1 & 26 & 173 & 370 & 303 & 123 & 0 & 0 & 0 & 996 \\
\hline 1952 & 0 & 0 & 0 & 16 & 46 & 110 & 371 & 281 & 151 & 16 & 0 & 0 & 991 \\
\hline 1953 & 0 & 0 & 0 & 0 & 8 & $\mathbf{2 6}^{\text {(a) }}$ & 336 & 282 & 143 & 1 & 0 & 0 & 796 \\
\hline 1954 & 0 & 0 & 0 & 0 & 55 & 90 & 289 & 204 & 91 & 0 & 0 & 0 & 729 \\
\hline 1955 & 0 & 0 & 0 & 0 & 4 & 174 & 270 & 325 & 13 & 0 & 0 & 0 & 786 \\
\hline 1956 & 0 & 0 & 0 & 10 & 122 & 78 & 430 & 322 & 106 & 0 & 0 & 0 & 1,068 \\
\hline 1957 & 0 & 0 & 0 & 12 & 77 & 185 & 289 & 255 & 160 & 0 & 0 & 0 & 978 \\
\hline 1958 & 0 & 0 & 0 & 0 & $167^{(\mathrm{a})}$ & 282 & 500 & 447 & 93 & 14 & 0 & 0 & $1, \mathbf{5 0 3}^{\text {(a) }}$ \\
\hline 1959 & 0 & 0 & 0 & 0 & 15 & 137 & 397 & 218 & 45 & 0 & 0 & 0 & 812 \\
\hline 1960 & 0 & 0 & 0 & 2 & 26 & 174 & 518 & 233 & 118 & 3 & 0 & 0 & 1,074 \\
\hline 1961 & 0 & 0 & 0 & 0 & 23 & 288 & 447 & 469 & 55 & 0 & 0 & 0 & 1,282 \\
\hline 1962 & 0 & 0 & 0 & 5 & $\mathbf{3}^{\text {(a) }}$ & 148 & 352 & 215 & 125 & 0 & 0 & 0 & 848 \\
\hline 1963 & 0 & 0 & 0 & 0 & 67 & 156 & 232 & 333 & 205 & 5 & 0 & 0 & 998 \\
\hline 1964 & 0 & 0 & 0 & 0 & 30 & 115 & 299 & $171^{(\mathrm{a})}$ & 34 & 0 & 0 & 0 & $649^{(a)}$ \\
\hline 1965 & 0 & 0 & 0 & 0 & 31 & 145 & 362 & 314 & 33 & 2 & 0 & 0 & 887 \\
\hline 1966 & 0 & 0 & 0 & 0 & 80 & 116 & 274 & 332 & 141 & 1 & 0 & 0 & 944 \\
\hline 1967 & 0 & 0 & 0 & 0 & 34 & 237 & 419 & $\mathbf{5 0 8}^{(\mathrm{a})}$ & 216 & 0 & 0 & 0 & 1,414 \\
\hline 1968 & 0 & 0 & 0 & 5 & 35 & 168 & 451 & 213 & 104 & 0 & 0 & 0 & 976 \\
\hline 1969 & 0 & 0 & 0 & 0 & 73 & 310 & 338 & 245 & 110 & 0 & 0 & 0 & 1,076 \\
\hline 1970 & 0 & 0 & 0 & 0 & 29 & 281 & 421 & 351 & 27 & 1 & 0 & 0 & 1,110 \\
\hline 1971 & 0 & 0 & 0 & 0 & 94 & 59 & 437 & 481 & 28 & 10 & 0 & 0 & 1,109 \\
\hline 1972 & 0 & 0 & 0 & 0 & 87 & 164 & 339 & 392 & 67 & 1 & 0 & 0 & 1,050 \\
\hline 1973 & 0 & 0 & 0 & 0 & 87 & 149 & 413 & 285 & 94 & 0 & 0 & 0 & 1,028 \\
\hline 1974 & 0 & 0 & 0 & 0 & 12 & 264 & 303 & 326 & 125 & 0 & 0 & 0 & 1,030 \\
\hline 1975 & 0 & 0 & 0 & 0 & 28 & 102 & 451 & 202 & 117 & 0 & 0 & 0 & 900 \\
\hline 1976 & 0 & 0 & 0 & 0 & 22 & 91 & 319 & 195 & 141 & 3 & 0 & 0 & 771 \\
\hline 1977 & 0 & 0 & 0 & 24 & 5 & 253 & 276 & 447 & 46 & 0 & 0 & 0 & 1,051 \\
\hline 1978 & 0 & 0 & 0 & 0 & 5 & 182 & 332 & 248 & 41 & 0 & 0 & 0 & 808 \\
\hline 1979 & 0 & 0 & 0 & 1 & 65 & 197 & 394 & 299 & 138 & 5 & 0 & 0 & 1,099 \\
\hline 1980 & 0 & 0 & 0 & 7 & 26 & 57 & 305 & 207 & 80 & 9 & 0 & 0 & 691 \\
\hline 1981 & 0 & 0 & 0 & 16 & 25 & 82 & 287 & 438 & 144 & 0 & 0 & 0 & 992 \\
\hline 1982 & 0 & 0 & 0 & 0 & 20 & 261 & 315 & 333 & 88 & 0 & 0 & 0 & 1,017 \\
\hline 1983 & 0 & 0 & 0 & 0 & 115 & 61 & 203 & 291 & 26 & 1 & 0 & 0 & 697 \\
\hline 1984 & 0 & 0 & 0 & 0 & 11 & 88 & 340 & 280 & 60 & 0 & 0 & 0 & 779 \\
\hline 1985 & 0 & 0 & 0 & 3 & 83 & 175 & $\mathbf{5 3 2}^{\text {(a) }}$ & 183 & $11^{(\mathrm{a})}$ & 0 & 0 & 0 & 987 \\
\hline 1986 & 0 & $1^{(\mathrm{a})}$ & 0 & 3 & 125 & 245 & 192 & 442 & 68 & 1 & 0 & 0 & 1,077 \\
\hline 1987 & 0 & 0 & 0 & $26^{(\text {a) }}$ & 125 & 265 & 289 & 359 & 179 & 11 & 0 & 0 & 1,254 \\
\hline 1988 & 0 & 0 & 0 & 6 & 45 & 187 & 385 & 318 & 113 & $44^{(\mathrm{a})}$ & 0 & 0 & 1,098 \\
\hline 1989 & 0 & 0 & 0 & 1 & 34 & 215 & 323 & 260 & 89 & 0 & $\mathbf{1}^{(\mathrm{a})}$ & 0 & 923 \\
\hline 1990 & 0 & 0 & 0 & 3 & 16 & 182 & 491 & 367 & $\mathbf{2 2 2}^{\text {(a) }}$ & 3 & 0 & 0 & 1,284 \\
\hline 1991 & 0 & 0 & 0 & 3 & 6 & 72 & 400 & 427 & 155 & 7 & 0 & 0 & 1,070 \\
\hline 1992 & 0 & 0 & 0 & 11 & 147 & $\mathbf{3 6 5}^{\text {(a) }}$ & 362 & 392 & 81 & 10 & 0 & 0 & 1,368 \\
\hline 1993 & 0 & 0 & 0 & 0 & 139 & 127 & $171^{\text {(a) }}$ & 265 & 135 & 6 & 0 & 0 & 843 \\
\hline 1994 & 0 & 0 & 0 & 15 & 94 & 163 & 501 & 358 & 167 & 3 & 0 & 0 & 1,301 \\
\hline 1995 & 0 & 0 & 0 & 0 & 73 & 142 & 376 & 216 & 174 & 0 & 0 & 0 & 981 \\
\hline 1996 & 0 & 0 & 0 & 4 & 14 & 134 & 450 & 324 & 79 & 9 & 0 & 0 & 1,014 \\
\hline 1997 & 0 & 0 & 0 & 0 & 96 & 118 & 324 & 404 & 92 & 0 & 0 & 0 & 1,034 \\
\hline 1998 & 0 & 0 & 0 & 16 & 55 & 183 & 527 & 398 & 195 & 3 & 0 & 0 & 1,377 \\
\hline 1999 & 0 & 0 & 0 & 0 & 43 & 135 & 281 & 366 & 66 & $\mathbf{0}^{(\mathrm{a}, \mathrm{b})}$ & 0 & 0 & 891 \\
\hline 2000 & 0 & 0 & 0 & 1 & 25 & 185 & 335 & 282 & 74 & 1 & 0 & 0 & 903 \\
\hline 2001 & 0 & 0 & 0 & 7 & 98 & 100 & 343 & 390 & 150 & 4 & 0 & 0 & 1,092 \\
\hline 2002 & 0 & 0 & 0 & 1 & 28 & 224 & 456 & 327 & 94 & 1 & 0 & 0 & 1,131 \\
\hline 2003 & 0 & $0^{(\mathbf{a}, \mathbf{b})}$ & 0 & $\mathbf{0}^{(\mathbf{a}, \mathbf{b})}$ & 58 & 234 & 475 & 358 & 174 & 32 & $0^{(\mathbf{a}, \mathbf{b})}$ & 0 & 1,331 \\
\hline Average $^{(c)}$ & 0 & $<1$ & 0 & 4 & 56 & 164 & 359 & 317 & 105 & 4 & $<1$ & 0 & 1,009 \\
\hline Normal & 0 & $<1$ & 0 & 5 & 57 & 163 & 355 & 326 & 103 & 4 & $<1$ & 0 & 1,014 \\
\hline
\end{tabular}

(a) Greatest and least values.

(b) Most recent of numerous occurrences.

(c) Based on entire period of record, 1945 through 2003. 


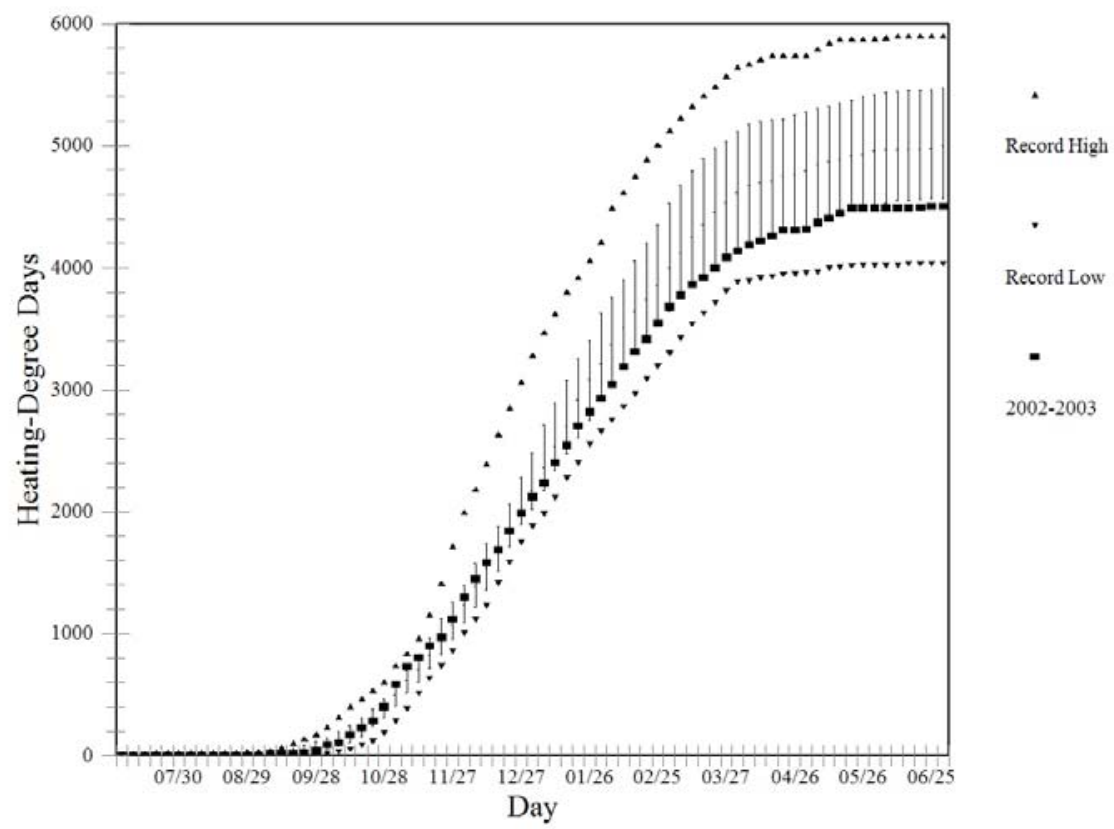

Figure 3.26. Climatological Statistics on Heating-Degree Days with Data for the 2002-2003 Heating Season

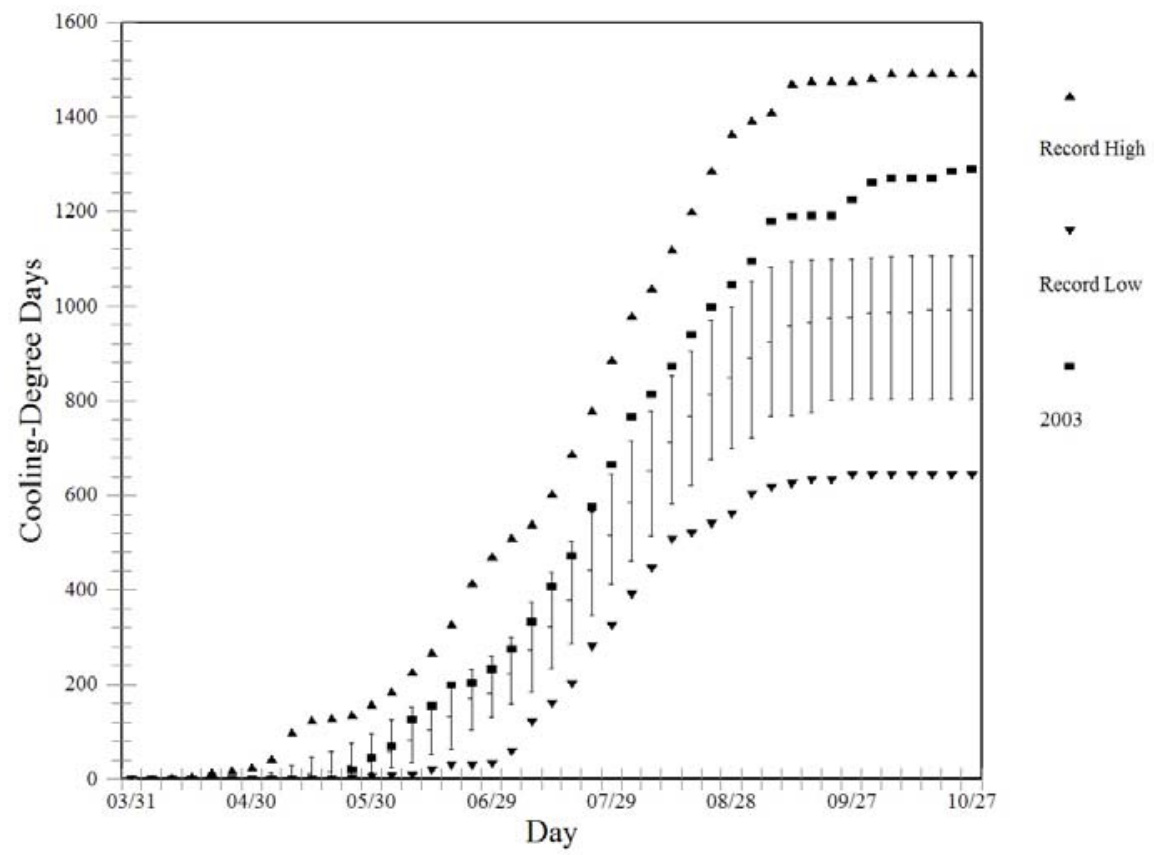

Figure 3.27. Climatological Statistics on Cooling-Degree Days with Data for the 2003 Cooling Season 
Table 3.15. Subsurface Soil Temperatures $\left({ }^{\circ} F\right)$ at Depths of $0.5,15$, and 36 Inches

\begin{tabular}{|c|c|c|c|c|c|c|c|c|c|c|c|c|c|c|c|}
\hline \multirow[b]{4}{*}{ Month } & & & & \multicolumn{4}{|c|}{ 0.5-in. Depth } & \multicolumn{4}{|c|}{ 15-in. Depth } & \multicolumn{4}{|c|}{ 36-in. Depth } \\
\hline & & & & \multirow{3}{*}{$\begin{array}{l}\text { Highest } \\
\text { Monthly } \\
\text { Average } \\
\end{array}$} & \multirow[b]{3}{*}{ Year } & \multirow{3}{*}{$\begin{array}{l}\text { Lowest } \\
\text { Monthly } \\
\text { Average } \\
\end{array}$} & \multirow[b]{3}{*}{ Year } & \multirow{3}{*}{$\begin{array}{l}\text { Highest } \\
\text { Monthly } \\
\text { Average }\end{array}$} & \multirow[b]{3}{*}{ Year } & \multirow{3}{*}{$\begin{array}{l}\text { Lowest } \\
\text { Monthly } \\
\text { Average }\end{array}$} & \multirow[b]{3}{*}{ Year } & \multirow{3}{*}{$\begin{array}{l}\text { Highest } \\
\text { Monthly } \\
\text { Average }\end{array}$} & \multirow[b]{3}{*}{ Year } & \multirow{3}{*}{$\begin{array}{c}\text { Lowest } \\
\text { Monthly } \\
\text { Average } \\
\end{array}$} & \multirow[b]{3}{*}{ Year } \\
\hline & & ly Ave & & & & & & & & & & & & & \\
\hline & $\overline{0.5 \mathrm{in} .}$ & 15 in. & 36 in. & & & & & & & & & & & & \\
\hline Jan & 32.8 & 36.3 & 42.7 & 39.4 & 1967 & 19.2 & 1979 & 42.7 & 1981 & 25.5 & 1979 & 48.7 & 1975 & 36.3 & 1979 \\
\hline Feb & 38.3 & 38.9 & 42.0 & 45.1 & 1958 & 28.6 & 1989 & 44.9 & 1967 & 29.6 & 1957 & 46.9 & 1967 & 33.5 & 1957 \\
\hline Mar & 48.1 & 46.4 & 46.1 & 54.3 & 1992 & 42.4 & 1955 & 52.6 & 1968 & 37.7 & 1956 & 51.7 & 1968 & 38.0 & 1956 \\
\hline Apr & 59.8 & 55.7 & 53.1 & 69.4 & 1977 & 52.4 & 1984 & 62.1 & 1977 & 48.7 & 1955 & 57.4 & 1966 & 47.3 & 1955 \\
\hline May & 72.0 & 65.5 & 60.7 & 81.4 & 1992 & 63.6 & 1984 & 71.4 & 1992 & 58.7 & 1984 & 65.1 & 1987 & 54.8 & 1955 \\
\hline Jun & 82.6 & 75.1 & 68.6 & 90.4 & 1986 & 75.3 & 1956 & 84.5 & 1966 & 70.2 & 1956 & 73.4 & 1969 & 62.8 & 1984 \\
\hline Jul & 90.9 & 81.8 & 75.1 & 96.2 & 1973 & 81.0 & 1993 & 88.2 & 1967 & 75.4 & 1955 & 81.1 & 1967 & 70.8 & 1955 \\
\hline Aug & 87.6 & 82.8 & 78.5 & 94.9 & 1971 & 81.6 & 1960 & 89.2 & 1967 & 77.5 & 1964 & 83.9 & 1967 & 75.3 & $1999^{(a)}$ \\
\hline Sep & 74.0 & 74.7 & 74.9 & 81.0 & 1967 & 65.5 & 1985 & 82.2 & 1967 & 68.8 & 1959 & 81.4 & 1967 & 70.1 & 1978 \\
\hline Oct & 56.5 & 62.5 & 67.3 & 62.6 & 1988 & 52.4 & 1985 & 67.0 & 2003 & 57.9 & 1957 & 72.3 & 1967 & 62.9 & 1959 \\
\hline Nov & 40.7 & 48.1 & 56.8 & 45.7 & 1999 & 31.9 & 1985 & 54.0 & 1974 & 42.5 & 1955 & 62.7 & 1974 & 51.2 & 1955 \\
\hline Dec & 33.5 & 39.1 & 47.8 & 38.7 & 1974 & 26.5 & 1984 & 45.0 & 1974 & 34.1 & 1984 & 54.6 & 1974 & 41.4 & 1955 \\
\hline \multirow[t]{3}{*}{ Annual } & 59.7 & 58.9 & 59.6 & 62.8 & 1967 & 55.9 & 1955 & 63.0 & 1967 & 54.6 & 1955 & 67.3 & 1987 & 55.5 & 1955 \\
\hline & & & & \multicolumn{12}{|c|}{ Absolute Hourly Extremes } \\
\hline & & & & 156.8 & 1996 & -2.0 & 1972 & 93.0 & 1967 & 16.1 & 1979 & 85.3 & 1967 & 32.2 & 1957 \\
\hline
\end{tabular}

(a) Most recent of multiple occurrences. 


\subsection{Precipitation Climatology}

\subsection{Monthly and Annual Totals}

Table 4.1 shows monthly and annual precipitation totals for the period of record, 1946 through 2003. Normal monthly precipitation amounts for the period 1971 through 2000 and averages for the entire period of record are noted on the table, as are monthly and annual extremes. Normal annual precipitation at the Hanford Meteorology Station is 6.98 inches. The wettest year on record was 1995, with 12.31 inches; the driest was 1976, with 2.99 inches.

Historically, the months of November through February provide 3.64 inches $(52 \%)$ of the normal annual precipitation. Although December has the highest average and normal precipitation due to more extreme events (Table 4.1), November has the highest median precipitation (Figure 4.1). July and August are the driest, receiving only 0.27 inch. The wettest month on record was December 1996, with 3.69 inches. September 1999, September 1991, August 1988, and August 1955 received no precipitation.

\subsection{Precipitation Distributions}

The method of presenting climatological data described in Section 3.5 is appropriate for presentation of climatological precipitation data as well, as long as the precipitation data are aggregated for sufficiently long periods of time. Figure 4.1 shows the monthly climatological statistics for the Hanford Meteorological Station for the years from 1947 through 2003. The figure also shows the total precipitation for each month during 2003

Figure 4.2 shows the climatological statistics of seasonal precipitation accumulation and the accumulation for the 2002-2003 season. The precipitation season is defined as beginning July 1 and continuing through June 30 the following year. This definition puts the break between seasons at in the beginning of the driest part of the year, rather than in the middle of the wettest part of the year. The accumulation is shown in Figure 4.2 in 5-day intervals, except for the interval containing February 29, which is a 6-day interval every fourth year.

\subsection{Seasonal Precipitation}

Table 4.2 provides seasonal precipitation information, with normal and average seasonal data noted. The extremes for each season are also noted. The wettest season was the winter of 1996-1997, with 5.45 inches; the driest received only 0.03 inch (summer 1973).

\subsection{Average Number of Days with Specified Amounts of Precipitation}

Table 4.3 presents information on the average number of days per year with precipitation events in six categories. A trace is less than 0.01 inch of precipitation. An average of 122 days per year have a trace or more of precipitation; however, only 24 days receive totals of 0.10 inch or more. During the 58 -year period of record, only 5 days had an inch or more of precipitation. 
Table 4.1. Monthly and Annual Precipitation (inches)

\begin{tabular}{|c|c|c|c|c|c|c|c|c|c|c|c|c|c|}
\hline Year & Jan & $\mathrm{Feb}$ & Mar & Apr & May & Jun & Jul & Aug & Sep & Oct & Nov & Dec & Annual \\
\hline 1946 & -- & -- & -- & -- & -- & -- & 0.15 & 0.35 & 0.52 & 0.65 & 0.66 & 0.11 & -- \\
\hline 1947 & 0.32 & 0.27 & 0.42 & 0.70 & 0.02 & 1.07 & 0.71 & 0.68 & $1.34^{(\mathrm{a})}$ & 2.20 & 0.81 & 0.75 & 9.29 \\
\hline 1948 & 1.36 & 0.69 & 0.07 & 0.95 & 1.71 & 1.47 & 0.40 & 0.39 & 0.16 & 0.45 & 0.95 & 1.11 & 9.71 \\
\hline 1949 & 0.13 & 0.68 & 1.12 & 0.02 & 0.16 & 0.01 & 0.01 & 0.03 & 0.23 & 0.10 & 1.47 & 0.16 & 4.12 \\
\hline 1950 & 1.80 & 1.06 & 0.87 & 0.47 & 0.27 & $2.92^{(\mathrm{a})}$ & 0.07 & $\mathrm{~T}$ & 0.01 & 2.46 & 0.55 & 0.97 & 11.45 \\
\hline 1951 & 0.84 & 0.51 & 0.46 & 0.53 & 0.43 & 1.38 & 0.37 & 0.15 & 0.10 & 0.71 & 0.82 & 0.70 & 7.00 \\
\hline 1952 & 0.65 & 0.50 & 0.06 & 0.13 & 0.58 & 1.07 & $\mathrm{~T}$ & 0.08 & 0.08 & 0.04 & 0.20 & 0.77 & 4.16 \\
\hline 1953 & 2.16 & 0.25 & 0.17 & 0.77 & 0.28 & 0.55 & $\mathrm{~T}$ & 0.96 & 0.13 & 0.20 & 0.96 & 0.49 & 6.92 \\
\hline 1954 & 1.48 & 0.28 & 0.59 & 0.07 & 0.41 & 0.10 & 0.22 & 0.42 & 0.51 & 0.42 & 0.86 & 0.35 & 5.71 \\
\hline 1955 & 0.56 & 0.22 & 0.17 & 0.40 & 0.59 & 0.28 & 0.57 & 0 & 0.77 & 0.40 & 1.54 & 2.03 & 7.53 \\
\hline 1956 & 1.71 & 0.56 & 0.10 & $\mathrm{~T}$ & 0.22 & 0.86 & $\mathrm{~T}$ & 0.38 & 0.01 & 1.03 & 0.15 & 0.58 & 5.60 \\
\hline 1957 & 0.48 & 0.23 & $1.86^{(a)}$ & 0.38 & 0.82 & 0.47 & 0.05 & 0.02 & 0.34 & $2.72^{(\mathrm{a})}$ & 0.39 & 0.53 & 8.29 \\
\hline 1958 & 1.74 & 1.48 & 0.46 & 0.64 & 0.74 & 0.81 & 0.02 & $\mathrm{~T}$ & 0.05 & 0.19 & 0.77 & 1.84 & 8.74 \\
\hline 1959 & 2.05 & 1.17 & 0.40 & 0.20 & 0.50 & 0.23 & $\mathrm{~T}$ & 0.03 & 1.26 & 0.56 & 0.41 & 0.26 & 7.07 \\
\hline 1960 & 0.51 & 0.58 & 0.67 & 0.53 & 0.71 & 0.14 & $\mathrm{~T}$ & 0.26 & 0.23 & 0.23 & 0.92 & 0.64 & 5.42 \\
\hline 1961 & 0.33 & $2.10^{(\mathrm{a})}$ & 1.02 & 0.48 & 0.80 & 0.42 & 0.15 & 0.09 & $\mathrm{~T}$ & 0.07 & 0.49 & 0.89 & 6.84 \\
\hline 1962 & 0.13 & 0.90 & 0.14 & 0.34 & 1.35 & 0.12 & $\mathrm{~T}$ & 0.50 & 0.38 & 0.95 & 0.65 & 0.60 & 6.06 \\
\hline 1963 & 0.95 & 0.69 & 0.53 & 1.17 & 0.43 & 0.28 & 0.31 & 0.01 & 0.02 & 0.04 & 0.74 & 1.14 & 6.31 \\
\hline 1964 & 0.37 & 0.01 & 0.03 & 0.11 & 0.04 & 0.90 & 0.04 & 0.24 & 0.09 & 0.28 & 0.94 & 2.34 & 5.39 \\
\hline 1965 & 0.93 & 0.14 & 0.03 & 0.09 & 0.15 & 0.49 & 0.11 & 0.03 & 0.11 & 0.01 & 1.17 & 0.39 & 3.65 \\
\hline 1966 & 0.68 & 0.03 & 0.39 & 0.03 & 0.05 & 0.43 & 0.81 & $\mathrm{~T}$ & 0.27 & 0.39 & 2.25 & 0.60 & 5.93 \\
\hline 1967 & 0.32 & $\mathrm{~T}$ & 0.14 & 0.90 & 0.56 & 0.57 & $\mathrm{~T}$ & $\mathrm{~T}$ & 0.05 & 0.13 & 0.16 & 0.43 & 3.26 \\
\hline 1968 & 0.88 & 0.58 & $\boldsymbol{0 . 0 2}^{(\mathrm{a})}$ & 0.01 & 0.06 & 0.19 & 0.04 & 0.51 & 0.25 & 0.93 & 1.23 & 1.25 & 5.95 \\
\hline 1969 & 1.24 & 0.54 & 0.10 & 1.22 & 0.51 & 0.75 & $\mathrm{~T}$ & $\mathrm{~T}$ & 0.48 & 0.10 & 0.13 & 1.29 & 6.36 \\
\hline 1970 & $2.47^{\text {(a) }}$ & 0.75 & 0.27 & 0.45 & 0.54 & 0.25 & 0.01 & $\mathrm{~T}$ & 0.03 & 0.24 & 0.71 & 0.61 & 6.33 \\
\hline 1971 & 0.78 & 0.10 & 1.02 & 0.07 & 0.56 & 0.71 & 0.13 & 0.09 & 1.13 & 0.18 & 0.46 & 1.07 & 6.30 \\
\hline 1972 & 0.19 & 0.27 & 0.58 & 0.10 & $2.03^{(a)}$ & 0.66 & 0.16 & 0.56 & 0.02 & $\mathrm{~T}$ & 0.55 & 1.27 & 6.39 \\
\hline 1973 & 0.90 & 0.21 & 0.08 & $\mathrm{~T}$ & 0.24 & 0.01 & $\mathrm{~T}$ & 0.02 & 0.43 & 1.72 & 2.64 & 2.02 & 8.27 \\
\hline 1974 & 0.90 & 0.41 & 0.52 & 0.46 & 0.28 & 0.12 & 0.71 & $\mathrm{~T}$ & 0.01 & 0.21 & 0.71 & 0.97 & 5.30 \\
\hline 1975 & 1.43 & 0.98 & 0.33 & 0.42 & 0.38 & 0.24 & 0.32 & 1.16 & 0.03 & 0.87 & 0.60 & 0.70 & 7.46 \\
\hline 1976 & 0.56 & 0.36 & 0.23 & 0.41 & 0.08 & 0.11 & 0.13 & 0.96 & $\mathrm{~T}$ & 0.04 & $\mathbf{T}^{(\mathrm{a})}$ & $\mathbf{0 . 1 1}{ }^{(\mathrm{a}, \mathrm{b})}$ & $2.99^{(a)}$ \\
\hline 1977 & $\mathbf{0 . 0 8}^{(\mathrm{a})}$ & 0.57 & 0.41 & $\mathrm{~T}$ & 0.65 & 0.37 & 0.06 & $1.36^{(a)}$ & 0.66 & 0.15 & 0.63 & 1.47 & 6.41 \\
\hline 1978 & 1.72 & 0.92 & 0.30 & 0.46 & 0.41 & 0.09 & 0.52 & 0.57 & 0.11 & $\mathrm{~T}$ & 1.21 & 0.26 & 6.57 \\
\hline 1979 & 0.54 & 0.17 & 0.54 & 0.52 & 0.10 & $\mathrm{~T}$ & 0.09 & 0.38 & 0.20 & 0.67 & 1.36 & 0.99 & 5.56 \\
\hline 1980 & 1.32 & 1.30 & 0.30 & 0.86 & 1.41 & 0.96 & $\mathrm{~T}$ & 0.02 & 0.85 & 0.33 & 0.44 & 1.89 & 9.68 \\
\hline 1981 & 0.56 & 0.60 & 0.70 & 0.02 & 0.99 & 0.43 & 0.19 & 0.03 & 0.60 & 0.39 & 1.08 & 1.45 & 7.04 \\
\hline 1982 & 0.33 & 0.57 & 0.30 & 0.75 & 0.28 & 0.75 & 0.22 & 0.20 & 0.55 & 1.33 & 0.91 & 1.79 & 7.98 \\
\hline 1983 & 1.44 & 1.36 & 1.00 & 0.42 & 0.52 & 0.68 & 0.31 & 0.12 & 0.46 & 0.52 & 2.12 & 2.12 & 11.07 \\
\hline 1984 & 0.23 & 0.94 & 1.01 & 0.60 & 0.55 & 0.99 & 0.06 & $\mathrm{~T}$ & 0.42 & 0.07 & 1.83 & 0.57 & 7.27 \\
\hline 1985 & 0.34 & 0.82 & 0.36 & 0.01 & 0.12 & 0.15 & 0.12 & 0.01 & 0.63 & 0.46 & 1.24 & 0.84 & 5.10 \\
\hline 1986 & 1.76 & 1.37 & 0.76 & $\mathrm{~T}$ & 0.30 & $\mathrm{~T}$ & 0.21 & 0.02 & 0.96 & 0.29 & 0.65 & 0.77 & 7.09 \\
\hline 1987 & 0.80 & 0.19 & 1.05 & 0.14 & 0.17 & 0.11 & 0.50 & 0.07 & 0.01 & $\mathbf{T}^{(\mathrm{a}, \mathrm{b})}$ & 0.40 & 1.63 & 5.07 \\
\hline 1988 & 0.48 & $\mathbf{T}^{(\mathrm{a}, \mathrm{b})}$ & 0.39 & 1.12 & 0.33 & 0.11 & 0.13 & $\mathbf{0}^{(\mathrm{a}, \mathrm{b})}$ & 0.39 & 0.01 & 0.82 & 0.40 & 4.18 \\
\hline 1989 & 0.21 & 1.67 & 1.56 & 0.84 & 0.59 & 0.01 & 0.01 & 0.26 & 0.02 & 0.42 & 1.04 & 0.29 & 6.92 \\
\hline 1990 & 0.77 & 0.09 & 0.10 & 0.40 & 0.86 & 0.36 & 0.14 & 0.83 & $\mathrm{~T}$ & 0.78 & 0.02 & 0.72 & 5.07 \\
\hline 1991 & 0.33 & 0.19 & 1.12 & 0.45 & 0.49 & 1.44 & 0.29 & 0.07 & 0 & 0.53 & 1.44 & 0.40 & 6.75 \\
\hline 1992 & 0.44 & 0.94 & 0.09 & 0.94 & $\mathbf{T}^{\text {(a) }}$ & 1.14 & 0.38 & 0.20 & 0.27 & 0.61 & 1.07 & 1.82 & 7.90 \\
\hline 1993 & 1.30 & 1.17 & 0.67 & 0.71 & 0.60 & 0.12 & $1.76^{\text {(a) }}$ & 0.24 & 0.04 & 0.09 & 0.19 & 0.94 & 7.83 \\
\hline 1994 & 0.44 & 0.11 & 0.03 & 0.61 & 1.27 & 0.38 & 0.15 & 0.08 & 0.08 & 0.93 & 0.68 & 1.36 & 6.12 \\
\hline 1995 & 2.14 & 0.69 & 0.95 & 1.54 & 0.79 & 0.77 & 0.34 & 0.07 & 0.79 & 0.87 & 1.04 & 2.32 & $12.31^{(\mathrm{a})}$ \\
\hline 1996 & 1.42 & 1.22 & 0.83 & 0.43 & 0.62 & 0.05 & 0.14 & 0.02 & 0.22 & 0.88 & $2.67^{(a)}$ & $3.69^{(a)}$ & 12.19 \\
\hline 1997 & 1.51 & 0.25 & 0.70 & 0.33 & 0.33 & 0.46 & 0.19 & 0.06 & 0.32 & 0.92 & 1.01 & 0.31 & 6.39 \\
\hline 1998 & 1.24 & 1.15 & 0.50 & 0.07 & 0.52 & 0.48 & 0.34 & 0.04 & 0.10 & 0.28 & 1.29 & 0.44 & 6.45 \\
\hline 1999 & 0.89 & 0.70 & 0.06 & $\mathbf{T}^{(\mathrm{a}, \mathrm{b})}$ & 0.34 & 0.31 & 0.07 & 0.57 & $\mathbf{0}^{(\mathrm{a}, \mathrm{b})}$ & 0.48 & 0.26 & 0.07 & 3.75 \\
\hline 2000 & 1.09 & 1.12 & 0.94 & 0.57 & 0.77 & 0.25 & 0.46 & $\mathrm{~T}$ & 0.56 & 0.57 & 1.08 & 0.67 & 8.08 \\
\hline 2001 & 0.29 & 0.42 & 0.67 & 0.83 & 0.08 & 1.27 & 0.05 & 0.08 & 0.13 & 0.37 & 1.67 & 0.80 & 6.66 \\
\hline 2002 & 0.42 & 0.67 & 0.19 & 0.29 & 0.16 & 0.65 & 0.16 & 0.01 & $\mathrm{~T}$ & 0.12 & 0.38 & 2.26 & 5.41 \\
\hline 2003 & 1.87 & 0.82 & 0.26 & $2.23^{(a)}$ & 0.08 & $T^{(\mathbf{a}, \mathrm{b})}$ & $0^{\text {(a) }}$ & 0.46 & 0.24 & 0.07 & 0.15 & 1.96 & 8.14 \\
\hline Average $^{(c)}$ & 0.93 & 0.64 & 0.50 & 0.48 & 0.51 & 0.53 & 0.21 & 0.23 & 0.30 & 0.53 & 0.89 & 1.04 & 6.79 \\
\hline Normal & 0.87 & 0.68 & 0.58 & 0.44 & 0.55 & 0.41 & 0.27 & 0.27 & 0.33 & 0.49 & 0.98 & 1.11 & 6.98 \\
\hline
\end{tabular}

(a) Greatest and least values.

(b) Most recent of multiple occurrences.

(c) Based on the entire period of record, 1946 through 2003.

NOTE: Dashes indicate no data are available. 


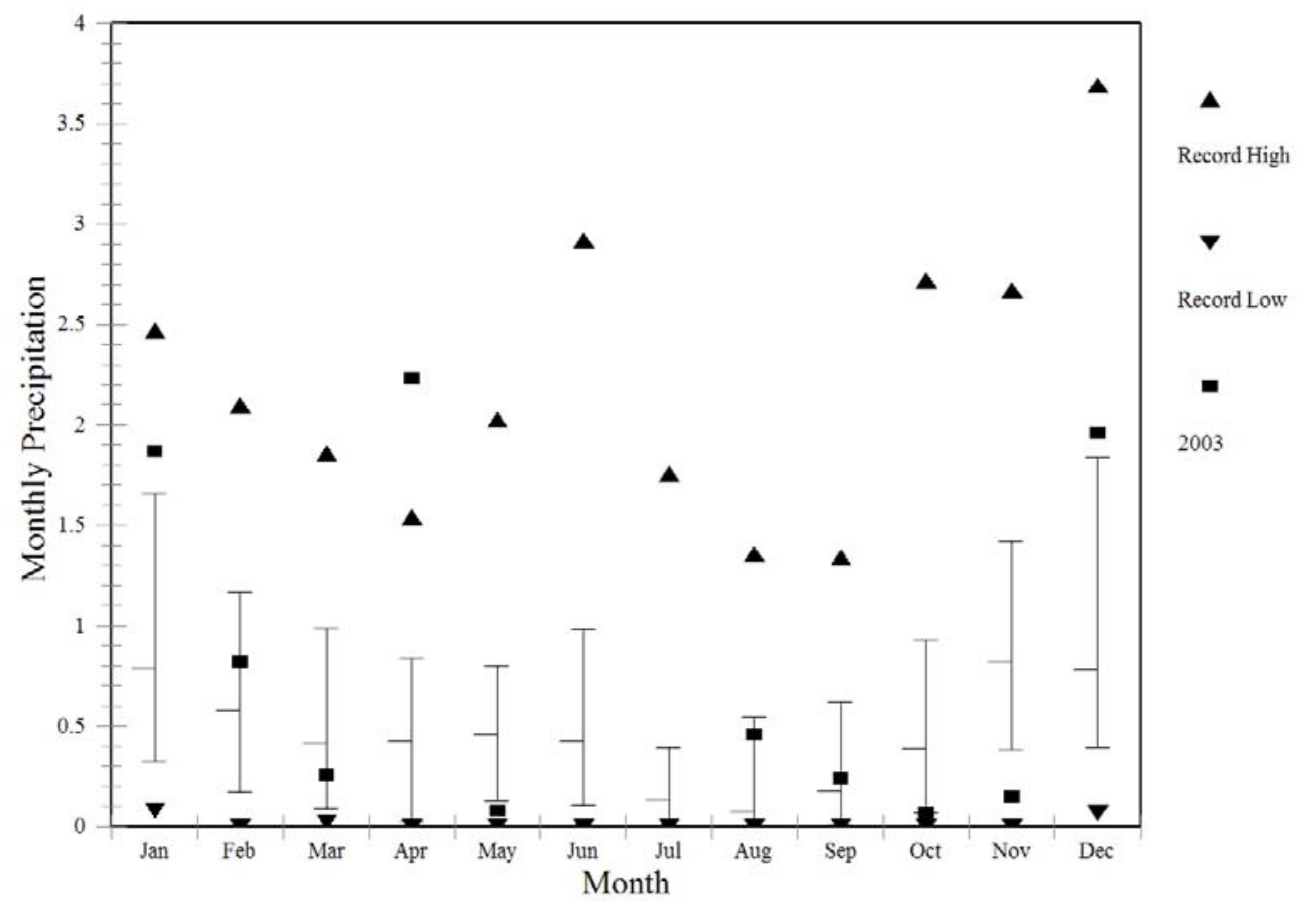

Figure 4.1. Monthly Precipitation Totals (inches, water equivalent)

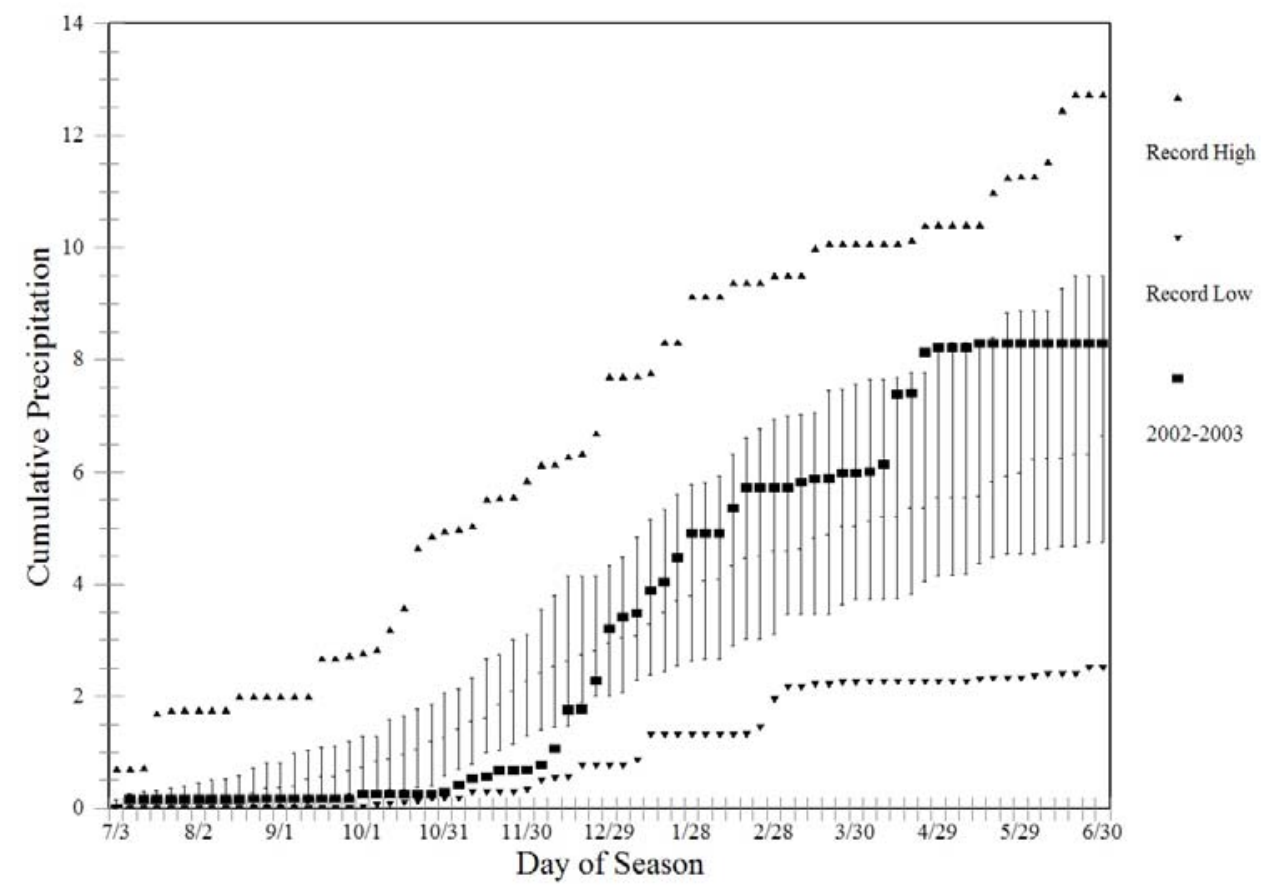

Figure 4.2. Seasonal Precipitation Accumulation (inches, water equivalent) 
Table 4.2. Seasonal Precipitation (inches)

\begin{tabular}{|c|c|c|c|c|}
\hline Year & $\begin{array}{l}\text { Winter }^{(a)} \\
\text { Dec-Feb } \\
\end{array}$ & $\begin{array}{c}\text { Spring } \\
\text { Mar-May } \\
\end{array}$ & $\begin{array}{l}\text { Summer } \\
\text { Jun-Aug } \\
\end{array}$ & $\begin{array}{l}\text { Autumn } \\
\text { Sep-Nov } \\
\end{array}$ \\
\hline 1946 & -- & -- & -- & 1.83 \\
\hline 1947 & $0.70^{(\mathrm{b})}$ & 1.14 & 2.46 & 4.35 \\
\hline 1948 & 2.80 & 2.73 & 2.26 & 1.56 \\
\hline 1949 & 1.92 & 1.30 & 0.05 & 1.80 \\
\hline 1950 & 3.02 & 1.61 & $2.99^{(\mathrm{b})}$ & 3.02 \\
\hline 1951 & 2.32 & 1.42 & 1.90 & 1.63 \\
\hline 1952 & 1.85 & 0.77 & 1.15 & 0.32 \\
\hline 1953 & 3.18 & 1.22 & 1.51 & 1.29 \\
\hline 1954 & 2.25 & 1.07 & 0.74 & 1.79 \\
\hline 1955 & 1.13 & 1.16 & 0.85 & 2.71 \\
\hline 1956 & 4.30 & 0.32 & 1.24 & 1.19 \\
\hline 1957 & 1.29 & 3.06 & 0.54 & 3.45 \\
\hline 1958 & 3.75 & 1.84 & 0.83 & 1.01 \\
\hline 1959 & 5.06 & 1.10 & 0.26 & 2.23 \\
\hline 1960 & 1.35 & 1.91 & 0.40 & 1.38 \\
\hline 1961 & 3.07 & 2.30 & 0.66 & 0.56 \\
\hline 1962 & 1.92 & 1.83 & 0.62 & 1.98 \\
\hline 1963 & 2.24 & 2.13 & 0.60 & 0.80 \\
\hline 1964 & 1.52 & 0.18 & 1.18 & 1.31 \\
\hline 1965 & 3.41 & 0.27 & 0.63 & 1.29 \\
\hline 1966 & 1.10 & 0.47 & 1.24 & 2.91 \\
\hline 1967 & 0.92 & 1.60 & 0.57 & 0.34 \\
\hline 1968 & 1.89 & $0.09^{(\mathrm{b})}$ & 0.74 & 2.41 \\
\hline 1969 & 3.03 & 1.83 & 0.75 & 0.71 \\
\hline 1970 & 4.51 & 1.26 & 0.26 & 0.98 \\
\hline 1971 & 1.49 & 1.65 & 0.93 & 1.77 \\
\hline 1972 & 1.53 & 2.71 & 1.38 & 0.57 \\
\hline 1973 & 2.38 & 0.32 & $\mathbf{0 . 0 3}^{(\mathrm{b})}$ & $4.79^{(\mathrm{b})}$ \\
\hline 1974 & 3.33 & 1.26 & 0.83 & 0.93 \\
\hline 1975 & 3.65 & 1.13 & 1.72 & 1.50 \\
\hline 1976 & 1.62 & 0.72 & 1.20 & $0.04^{(\mathrm{b})}$ \\
\hline 1977 & 0.76 & 1.06 & 1.79 & 1.44 \\
\hline 1978 & 3.91 & 1.17 & 1.18 & 1.32 \\
\hline 1979 & 0.97 & 1.16 & 0.47 & 2.23 \\
\hline 1980 & 3.61 & 2.57 & 0.98 & 1.62 \\
\hline 1981 & 3.05 & 1.71 & 0.65 & 2.07 \\
\hline 1982 & 2.35 & 1.33 & 1.17 & 2.79 \\
\hline 1983 & 4.59 & 1.94 & 1.11 & 3.10 \\
\hline 1984 & 3.29 & 2.16 & 1.05 & 2.32 \\
\hline 1985 & 1.73 & 0.49 & 0.28 & 2.33 \\
\hline 1986 & 3.97 & 1.06 & 0.23 & 1.90 \\
\hline 1987 & 1.76 & 1.36 & 0.68 & 0.41 \\
\hline 1988 & 2.11 & 1.84 & 0.24 & 1.22 \\
\hline 1989 & 2.28 & 2.99 & 0.28 & 1.48 \\
\hline 1990 & 1.15 & 1.36 & 1.33 & 0.80 \\
\hline 1991 & 1.24 & 2.06 & 1.80 & 1.97 \\
\hline 1992 & 1.78 & 1.03 & 1.72 & 1.95 \\
\hline 1993 & 4.29 & 1.98 & 2.12 & 0.32 \\
\hline 1994 & 1.49 & 1.91 & 0.61 & 1.69 \\
\hline 1995 & 4.19 & $3.28^{(\text {b) }}$ & 1.18 & 2.70 \\
\hline 1996 & 4.96 & 1.88 & 0.21 & 3.77 \\
\hline 1997 & $5.45^{(\mathrm{b})}$ & 1.36 & 0.71 & 2.25 \\
\hline 1998 & 2.70 & 1.09 & 0.86 & 1.67 \\
\hline 1999 & 2.03 & 0.40 & 0.95 & 0.74 \\
\hline 2000 & 2.28 & 2.28 & 0.71 & 2.21 \\
\hline 2001 & 1.38 & 1.58 & 1.40 & 2.17 \\
\hline 2002 & 1.89 & 0.64 & 0.82 & 0.50 \\
\hline 2003 & 5.05 & 2.57 & 0.46 & 0.46 \\
\hline Average $^{(\mathrm{c})}$ & 2.61 & 1.49 & 0.97 & 1.72 \\
\hline Normal & 2.66 & 1.58 & 0.95 & 1.80 \\
\hline
\end{tabular}

(a) For the winter season, December is included in the previous year.

(b) Greatest and least values.

(c) Based on entire period of record, 1945 through 2003

NOTE: Dashes indicate no data are available. 
Table 4.3. Average Number of Days with Precipitation of Specified Amount

\begin{tabular}{|c|c|c|c|c|c|c|}
\hline Month & $\begin{array}{c}\text { Trace } \\
\text { or more }\end{array}$ & $\begin{array}{l}0.01 \text { in. } \\
\text { or more }\end{array}$ & $\begin{array}{l}0.10 \text { in. } \\
\text { or more }\end{array}$ & $\begin{array}{l}0.25 \text { in. } \\
\text { or more }\end{array}$ & $\begin{array}{l}0.50 \text { in. } \\
\text { or more }\end{array}$ & $\begin{array}{l}1.00 \mathrm{in} . \\
\text { or more }\end{array}$ \\
\hline Jan & 16 & 9 & 3 & 1 & (a) & 0 \\
\hline Feb & 12 & 7 & 2 & 1 & (a) & 0 \\
\hline Mar & 11 & 6 & 2 & (a) & (a) & (a) \\
\hline Apr & 10 & 5 & 2 & 1 & (a) & 0 \\
\hline May & 10 & 5 & 2 & 1 & (a) & 0 \\
\hline Jun & 9 & 4 & 2 & 1 & (a) & (a) \\
\hline Jul & 5 & 2 & 1 & (a) & (a) & (a) \\
\hline Aug & 5 & 2 & 1 & (a) & (a) & 0 \\
\hline Sep & 6 & 3 & 1 & (a) & (a) & 0 \\
\hline Oct & 9 & 5 & 2 & 1 & (a) & (a) \\
\hline Nov & 14 & 9 & 3 & 1 & (a) & (a) \\
\hline Dec & 16 & 10 & 4 & 1 & (a) & 0 \\
\hline Annual $^{(b)}$ & 122 & 68 & 23 & 7 & $1^{(\mathrm{c})}$ & (a) \\
\hline
\end{tabular}

\footnotetext{
(a) Used to denote an average of less than $1 / 2$ day.

(b) Annual totals may differ from summation of monthly events because of rounding.

(c) Although the number of days with 0.50 inch or more averages less than $1 / 2$ day for any one month, 80 such days were recorded during 58 years of record.
}

\subsection{Total Time with Precipitation Observed}

The total time during which precipitation was observed at the Hanford Meteorology Station includes all types of precipitation. Observations of precipitation are recorded in hours and minutes, with the weather observer recording the starting and ending time of each precipitation event. These data are presented in Table 4.4. No record was kept for the hours 1600 through 2400 from July 1971 through June 1974; therefore, a 3-year gap exists in the record for those hours. Also, beginning in late April 1995, operations at the Hanford Meteorology Station were decreased to 8 hours (0600 to 1400) on weekends and holidays. However, a combination of precipitation sensors and computer programs was initiated to help ascertain the beginning and ending times of precipitation events during periods when the Hanford Meteorology Station is not staffed. Table 4.5 lists total hours of precipitation by month for the period 1946 through 2003. As previously noted, complete precipitation duration data for the period July 1971 through June 1974 are not available, and incomplete data are not included.

The months of November through February, which contribute more than half of the annual precipitation, received precipitation $10.1 \%$ of the time, three times more than the other 8 months of the year $(3.3 \%)$. 
Table 4.4. Monthly and Annual Averages and Extremes in Total Time with Precipitation Observed: July 1946 through June 1971, July 1974 through December 2003

\begin{tabular}{|c|c|c|c|c|c|c|c|c|}
\hline \multirow[b]{2}{*}{ Month } & \multicolumn{2}{|c|}{ Averages } & \multicolumn{3}{|c|}{ Greatest } & \multicolumn{3}{|c|}{ Least } \\
\hline & $\begin{array}{l}\text { No. of } \\
\text { Hours }\end{array}$ & $\begin{array}{l}\% \text { of } \\
\text { Time }\end{array}$ & $\begin{array}{l}\text { No. of } \\
\text { Hours }\end{array}$ & $\begin{array}{l}\% \text { of } \\
\text { Time }\end{array}$ & Year & $\begin{array}{l}\text { No. of } \\
\text { Hours }\end{array}$ & $\begin{array}{l}\% \text { of } \\
\text { Time }\end{array}$ & Year \\
\hline Jan & 87.8 & 11.8 & 212.0 & 28.5 & 1969 & 29.1 & 3.9 & 2002 \\
\hline Feb & 55.5 & 8.2 & 151.6 & 22.6 & 1980 & 2.5 & 0.4 & 1988 \\
\hline Mar & 38.7 & 5.2 & 135.2 & 18.2 & 1957 & 6.4 & 0.9 & 1994 \\
\hline Apr & 29.4 & 4.1 & 69.2 & 9.6 & 1953 & 1.6 & 0.2 & 1985 \\
\hline May & 29.8 & 4.0 & 89.9 & 12.1 & 1948 & 1.2 & 0.2 & 1992 \\
\hline Jun & 26.3 & 3.7 & 80.8 & 11.2 & 1950 & 0.3 & $<0.1$ & 2003 \\
\hline Jul & 10.0 & 1.3 & 38.2 & 5.1 & 1966 & 0.0 & 0.0 & 2003 \\
\hline Aug & 11.6 & 1.6 & 61.7 & 8.3 & 1968 & 0.0 & 0.0 & $1988^{(a)}$ \\
\hline Sep & 15.2 & 2.1 & 66.4 & 9.2 & 1977 & 0.0 & 0.0 & $1999^{(a)}$ \\
\hline Oct & 30.9 & 4.2 & 119.9 & 16.1 & 1947 & 0.4 & 0.1 & 1978 \\
\hline Nov & 60.0 & 8.3 & 146.5 & 20.3 & 1985 & 4.8 & 0.7 & 1976 \\
\hline Dec & 88.9 & 11.9 & 230.5 & 31.0 & 1985 & 15.8 & 2.1 & 1976 \\
\hline Annual & 484.0 & 5.5 & 738.0 & 8.4 & 1950 & 286.7 & 3.3 & 1990 \\
\hline
\end{tabular}

(a) Most recent of several occurrences.

\subsection{Notable Wet Periods}

Eleven periods are listed when precipitation was particularly high:

\begin{tabular}{|c|c|c|c|c|c|}
\hline \multirow[b]{2}{*}{ Period } & \multicolumn{2}{|c|}{$\begin{array}{c}\text { Number of Days with } \\
\text { Trace or More }\end{array}$} & \multicolumn{3}{|c|}{ Total Amount (inches) } \\
\hline & Altogether & $\begin{array}{c}\text { Greatest } \\
\text { Consecutive }\end{array}$ & $\begin{array}{l}\text { Measurable } \\
\text { Precipitation }\end{array}$ & $\begin{array}{c}\text { Water } \\
\text { Equivalent }\end{array}$ & Snowfall \\
\hline Oct 7 - Nov 4, 1947 & 23 out of 29 & 10 & 17 & 2.21 & 0.0 \\
\hline Jan $3-28,1950$ & 21 out of 26 & 10 & 15 & 1.80 & 23.4 \\
\hline Nov 11 - Dec 19, 1950 & 33 out of 39 & 12 & 20 & 1.37 & 3.7 \\
\hline Nov 16 - Dec 22, 1955 & 31 out of 37 & 15 & 24 & 3.19 & 22.7 \\
\hline Oct $31-$ Dec 7, 1973 & 32 out of 38 & 14 & 20 & 3.45 & 8.1 \\
\hline Nov 15 - Dec 7, 1985 & 17 out of 23 & 8 & 14 & 1.96 & 25.2 \\
\hline Dec 27, 1992 - Jan 23, 1993 & 26 out of 29 & 12 & 19 & 2.02 & 26.8 \\
\hline Nov 13 - 27, 1996 & 12 out of 15 & 7 & 10 & 2.66 & 11.9 \\
\hline Dec 20 - 31, 1996 & 11 out of 12 & 9 & 9 & 3.00 & 20.1 \\
\hline Dec 2, 2002 - Jan 4, 2003 & 28 out of 34 & 12 & 24 & 2.74 & 0.6 \\
\hline Nov 28, 2003 - Jan 7, 2004 & 32 out of 41 & 17 & 27 & 3.82 & 26.5 \\
\hline
\end{tabular}


Table 4.5. Total Duration (hours) of Precipitation by Month and Year

\begin{tabular}{|c|c|c|c|c|c|c|c|c|c|c|c|c|c|}
\hline Year & Jan & Feb & Mar & Apr & May & Jun & Jul & Aug & Sep & Oct & Nov & Dec & Annual \\
\hline 1946 & -- & -- & -- & -- & -- & -- & 6.0 & 16.3 & 12.2 & 38.2 & 66.0 & 31.5 & -- \\
\hline 1947 & 34.6 & 29.8 & 30.2 & 32.7 & 4.8 & 38.4 & 17.4 & 12.6 & 36.4 & $119.9^{(\mathrm{a})}$ & 48.1 & 71.1 & 476.0 \\
\hline 1948 & 70.7 & 70.4 & 16.6 & 43.8 & $89.9^{(a)}$ & 36.4 & 15.2 & 16.6 & 15.8 & 19.7 & 44.4 & 81.9 & 521.4 \\
\hline 1949 & $29.2^{(\mathrm{a})}$ & 69.9 & 63.5 & 5.6 & 13.2 & 3.2 & 2.2 & 1.6 & 21.2 & 15.0 & 98.7 & 32.1 & 355.4 \\
\hline 1950 & 147.4 & 78.0 & 72.0 & 23.3 & 13.9 & $\mathbf{8 0 . 8}^{\text {(a) }}$ & 5.0 & 2.3 & 1.3 & 112.3 & 92.9 & 108.8 & $738.0^{(a)}$ \\
\hline 1951 & 66.3 & 55.9 & 34.8 & 21.5 & 23.7 & 60.8 & 12.8 & 17.7 & 26.6 & 66.5 & 66.0 & 86.7 & 539.3 \\
\hline 1952 & 151.0 & 57.8 & 19.3 & 22.1 & 25.4 & 77.7 & 4.3 & 5.4 & 7.1 & 3.6 & 71.2 & 137.0 & 581.9 \\
\hline 1953 & 89.3 & 29.7 & 32.3 & $69.2^{(\mathrm{a})}$ & 20.6 & 37.1 & 2.0 & 25.2 & 7.1 & 23.6 & 59.4 & 32.2 & 427.7 \\
\hline 1954 & 92.6 & 90.0 & 35.8 & 18.4 & 33.7 & 32.4 & 17.8 & 22.2 & 15.1 & 17.9 & 74.3 & 39.9 & 490.1 \\
\hline 1955 & 116.4 & 33.4 & 20.0 & 57.2 & 47.8 & 10.2 & 36.9 & 0.0 & 40.1 & 42.3 & 132.2 & 141.0 & 677.5 \\
\hline 1956 & 126.9 & 74.4 & 15.9 & 1.8 & 35.8 & 30.8 & 4.2 & 17.4 & 6.6 & 65.7 & 71.0 & 98.8 & 549.3 \\
\hline 1957 & 140.6 & 46.4 & $135.2^{(a)}$ & 19.5 & 43.4 & 20.8 & 2.6 & 5.7 & 23.1 & 72.0 & 21.4 & 49.3 & 580.0 \\
\hline 1958 & 82.8 & 106.8 & 37.5 & 54.5 & 24.2 & 24.2 & 1.2 & 2.0 & 13.4 & 13.6 & 58.5 & 107.7 & 526.4 \\
\hline 1959 & 129.5 & 98.2 & 32.6 & 17.5 & 33.0 & 29.8 & 4.2 & 15.9 & 52.2 & 27.2 & 44.8 & 51.8 & 536.7 \\
\hline 1960 & 86.8 & 48.0 & 49.9 & 32.8 & 47.2 & 6.3 & 3.5 & 27.3 & 15.8 & 34.8 & 64.1 & 120.8 & 537.3 \\
\hline 1961 & 91.8 & 94.4 & 60.7 & 39.2 & 48.7 & 23.9 & 4.2 & 17.2 & 2.0 & 15.9 & 57.2 & 99.0 & 554.2 \\
\hline 1962 & 43.9 & 58.8 & 55.1 & 24.8 & 80.2 & 13.9 & 4.3 & 24.9 & 21.6 & 71.5 & 44.4 & 139.6 & 583.0 \\
\hline 1963 & 56.3 & 88.4 & 31.2 & 66.5 & 51.3 & 37.1 & 20.9 & 4.4 & 11.2 & 26.4 & 61.0 & 179.6 & 634.3 \\
\hline 1964 & 49.1 & 5.2 & 8.3 & 15.7 & 6.1 & 46.8 & 14.5 & 14.1 & 7.2 & 19.2 & 109.0 & 149.0 & 444.2 \\
\hline 1965 & 153.3 & 18.8 & 14.2 & 30.9 & 15.2 & 28.6 & 6.8 & 18.4 & 11.2 & 11.2 & 89.1 & 57.8 & 455.5 \\
\hline 1966 & 51.7 & 12.4 & 42.9 & 9.1 & 7.2 & 30.4 & $38.2^{(\mathrm{a})}$ & 3.7 & 15.9 & 26.3 & 103.5 & 75.6 & 416.9 \\
\hline 1967 & 34.1 & 4.7 & 30.6 & 60.9 & 52.9 & 23.3 & 2.2 & 1.7 & 12.1 & 29.4 & 27.0 & 88.2 & 367.1 \\
\hline 1968 & 99.1 & 42.0 & 7.3 & 18.6 & 29.9 & 38.3 & 5.6 & $61.7^{(\mathrm{a})}$ & 17.2 & 45.3 & 68.9 & 134.2 & 568.1 \\
\hline 1969 & $212.0^{(a)}$ & 75.4 & 9.7 & 52.2 & 51.9 & 38.7 & 1.3 & 0.3 & 26.8 & 20.4 & 44.1 & 148.3 & 681.1 \\
\hline 1970 & 157.2 & 72.9 & 34.0 & 19.2 & 27.2 & 31.1 & 6.9 & 2.3 & 5.3 & 32.2 & 85.8 & 83.9 & 558.0 \\
\hline 1971 & 49.5 & 14.8 & 68.0 & 25.0 & 43.7 & 52.7 & (b) & (b) & (b) & (b) & (b) & (b) & (b) \\
\hline 1972 & (b) & (b) & (b) & (b) & (b) & (b) & (b) & (b) & (b) & (b) & (b) & (b) & (b) \\
\hline 1973 & (b) & (b) & (b) & (b) & (b) & (b) & (b) & (b) & (b) & (b) & (b) & (b) & (b) \\
\hline 1974 & (b) & (b) & (b) & (b) & (b) & (b) & 21.8 & 0.8 & 1.3 & 26.2 & 58.9 & 64.9 & (b) \\
\hline 1975 & 105.7 & 103.8 & 42.3 & 29.5 & 29.2 & 20.8 & 13.5 & 26.8 & 1.0 & 65.6 & 56.1 & 76.6 & 570.9 \\
\hline 1976 & 79.8 & 28.6 & 35.2 & 36.8 & 16.7 & 10.1 & 15.1 & 38.8 & 2.2 & 10.7 & $4.8^{(\mathrm{a})}$ & 15.8 & 294.6 \\
\hline 1977 & 138.7 & 37.1 & 37.8 & 4.6 & 45.9 & 24.8 & 14.2 & 28.9 & $66.4^{(\mathrm{a})}$ & 15.8 & 77.1 & 98.1 & 589.4 \\
\hline 1978 & 147.9 & 98.6 & 46.2 & 53.4 & 45.2 & 8.1 & 27.6 & 37.7 & 27.9 & $0.4^{(\mathrm{a})}$ & 71.6 & 43.7 & 608.3 \\
\hline 1979 & 111.5 & 73.7 & 45.0 & 29.6 & 14.0 & 4.5 & 8.6 & 27.4 & 10.0 & 47.3 & 86.9 & 104.2 & 562.7 \\
\hline 1980 & 118.5 & $151.6^{(\mathrm{a})}$ & 35.4 & 33.8 & 60.2 & 45.0 & 1.5 & 8.8 & 24.5 & 22.0 & 44.2 & 121.2 & 666.7 \\
\hline 1981 & 72.0 & 31.7 & 32.0 & 3.4 & 28.4 & 21.1 & 7.5 & 1.0 & 17.4 & 24.8 & 34.1 & 91.8 & 365.2 \\
\hline 1982 & 47.5 & 29.2 & 27.8 & 33.6 & 9.4 & 21.8 & 5.8 & 5.3 & 23.2 & 40.1 & 42.8 & 81.7 & 368.2 \\
\hline 1983 & 72.5 & 76.1 & 59.4 & 15.2 & 13.2 & 22.4 & 17.9 & 13.7 & 12.5 & 19.8 & 79.3 & 133.2 & 535.2 \\
\hline 1984 & 32.6 & 58.1 & 40.8 & 35.2 & 34.2 & 37.9 & 0.5 & 0.5 & 20.5 & 7.1 & 97.6 & 75.5 & 440.5 \\
\hline 1985 & 151.2 & 54.9 & 26.0 & $1.6^{(\mathrm{a})}$ & 7.0 & 17.4 & 3.2 & 1.2 & 30.8 & 17.2 & $146.5^{(\mathrm{a})}$ & $230.5^{(\mathrm{a})}$ & 687.5 \\
\hline 1986 & 107.6 & 68.9 & 47.4 & 14.4 & 22.3 & 2.9 & 13.3 & 1.1 & 28.2 & 10.3 & 31.3 & 143.8 & 491.5 \\
\hline 1987 & 64.6 & 20.8 & 74.0 & 10.8 & 14.6 & 11.8 & 16.1 & 4.5 & 0.5 & 1.8 & 21.1 & 125.6 & 366.2 \\
\hline 1988 & 92.4 & $2.5^{(\mathrm{a})}$ & 24.8 & 36.2 & 18.3 & 17.4 & 6.2 & $\mathbf{0 . 0 ^ { ( \mathrm { a } , \mathrm { c } ) }}$ & 13.8 & 2.2 & 55.8 & 62.7 & 332.3 \\
\hline 1989 & 35.2 & 114.4 & 102.2 & 36.8 & 25.8 & 5.4 & 3.8 & 14.4 & 0.7 & 23.1 & 33.2 & 57.9 & 452.9 \\
\hline 1990 & 34.6 & 20.5 & 18.2 & 29.9 & 31.0 & 8.6 & 7.3 & 15.4 & 0.1 & 49.1 & 7.1 & 64.9 & 286.7 \\
\hline 1991 & 57.5 & 28.0 & 43.8 & 15.8 & 39.0 & 41.8 & 9.8 & 4.2 & 0.0 & 42.9 & 70.2 & 48.2 & 401.2 \\
\hline 1992 & 36.2 & 56.2 & 7.9 & 42.0 & $1.2^{(\mathrm{a})}$ & 35.9 & 22.5 & 6.6 & 23.6 & 36.6 & 53.0 & 92.6 & 414.3 \\
\hline 1993 & 171.0 & 64.4 & 65.2 & 57.0 & 38.7 & 13.0 & 35.2 & 12.1 & 3.0 & 6.8 & 30.3 & 58.2 & 554.9 \\
\hline 1994 & 40.5 & 55.8 & 6.4 & 43.2 & 40.7 & 21.2 & 4.7 & 3.8 & 8.9 & 37.6 & 52.9 & 72.2 & 387.9 \\
\hline 1995 & 113.8 & 39.2 & 47.3 & 56.2 & 27.3 & 52.6 & 8.3 & 7.9 & 14.5 & 33.0 & 47.1 & 62.2 & 509.4 \\
\hline 1996 & 102.1 & 73.0 & 55.4 & 23.2 & 30.8 & 3.6 & 8.2 & 3.3 & 13.7 & 46.8 & 81.5 & 124.4 & 566.0 \\
\hline 1997 & 69.5 & 17.7 & 36.1 & 13.7 & 19.2 & 14.7 & 12.7 & 4.1 & 19.7 & 29.4 & 43.6 & $15.5^{(\mathrm{a})}$ & 295.9 \\
\hline 1998 & 60.0 & 72.3 & 34.2 & 8.6 & 45.7 & 13.7 & 12.7 & 1.8 & 6.8 & 14.8 & 45.1 & 54.1 & 369.8 \\
\hline 1999 & 52.9 & 56.6 & $4.8^{(a)}$ & 3.1 & 15.2 & 9.4 & 2.1 & 9.4 & $0.0^{(a, c)}$ & 20.9 & 44.8 & 35.2 & $254.4^{(\mathrm{a})}$ \\
\hline 2000 & 85.8 & 78.8 & 39.6 & 17.3 & 25.7 & 19.8 & 7.1 & 0.8 & 27.5 & 23.0 & 56.4 & 49.9 & 431.7 \\
\hline 2001 & 43.8 & 55.0 & 47.4 & 44.6 & 8.4 & 34.6 & 2.9 & 10.1 & 4.9 & 34.7 & 66.5 & 49.3 & 402.2 \\
\hline 2002 & 29.1 & 24.2 & 28.3 & 17.9 & 12.6 & 27.2 & 1.5 & 0.9 & 1.9 & 12.1 & 31.4 & 154.4 & 341.5 \\
\hline 2003 & 106.1 & 27.9 & 20.8 & 59.7 & 9.1 & $0.3^{(\text {a) }}$ & $0.0^{(a)}$ & 16.2 & 7.0 & 10.5 & 29.6 & 133.7 & 420.9 \\
\hline Average $^{(d)}$ & 89.5 & 56.6 & 39.1 & 28.8 & 30.9 & 26.7 & 10.5 & 11.8 & 15.8 & 31.6 & 61.0 & 87.5 & 489.6 \\
\hline Normal & 83.4 & 56.6 & 40.9 & 26.3 & 27.5 & 20.7 & 11.4 & 10.4 & 14.8 & 25.0 & 54.6 & 81.7 & 453.0 \\
\hline
\end{tabular}

(a) Greatest and least values.

(b) Incomplete data not included. See Section 4.5.

(c) Most recent of numerous occurrences.

(d) Based on entire period of record, 1945 through 2003

NOTE: Dashes indicate no data are available. 
The period from November 28 through December 14, 2003 established a new record for number of consecutive days with precipitation with 17 days. The previous record had been 15 days on four separate occasions, the most recent being December 19, 1985 through January 2, 1986.

From a precipitation standpoint, 1973 was an unusual year. Total precipitation for 1973 was 8.27 inches, $132 \%$ of normal (6.26 inches). The period March 30 through September 18, 1973, was extremely dry, receiving only 0.29 inch of precipitation during that 173-day period; however, the period October 31 through December 7, 1973 was a notable wet period. During the months of October, November, and December 1973, 6.38 inches of precipitation were recorded, 289\% of normal (2.21 inches) for those months. November and December 1996 received 6.36 inches of precipitation, 328\% of normal (1.94 inches) for those months, which is greater than the normal precipitation amount for an entire year (6.26 inches).

\subsection{Notable Dry Periods}

The Hanford Meteorology Station is in a semiarid region; thus, it experiences many dry periods. January, March, and December are the only months that have always received measurable precipitation (1946 through 2003). In 2002, a 130-day dry period occurred between June 30 and November 6, when total precipitation measured 0.29 inch.

During 1946 through 2003, there were 42 months without measurable precipitation, with the months of July and August accounting for 22 of those months. The record number of consecutive days with no precipitation (not even a trace) occurred in 1988, when the period July 14 through September 17 (66 days) was totally dry. The following list indicates some long periods with small amounts of precipitation.

\begin{tabular}{|c|c|c|c|c|}
\hline \multicolumn{5}{|c|}{ Notable Dry Periods } \\
\hline Year & From & To & $\begin{array}{l}\text { Number } \\
\text { of Days }\end{array}$ & $\begin{array}{c}\text { Total Precipitation } \\
\text { (inch) }\end{array}$ \\
\hline 1952 & Jun 30 & Nov 10 & 134 & 0.20 \\
\hline 1967 & Jun 22 & Nov 7 & 139 & 0.18 \\
\hline 1968 & Feb 24 & Aug 13 & 172 & 0.32 \\
\hline 1973 & Mar 30 & Sep 18 & 173 & 0.29 \\
\hline 1976 & Aug 26 & Dec 31 & 128 & 0.15 \\
\hline 1985 & Mar 31 & Sep 7 & 161 & 0.43 \\
\hline 1986 & May 6 & Sep 12 & 129 & 0.30 \\
\hline 1987 & Jul 19 & Oct 31 & 105 & 0.08 \\
\hline 1988 & Jun 6 & Sep 17 & 105 & 0.13 \\
\hline 2002 & Jun 30 & Nov 6 & 130 & 0.29 \\
\hline
\end{tabular}

The driest year on record was 1976, which had 2.99 inches recorded (less than 50\% of normal). During the period September through December 1976, total precipitation was 0.15 inch, which was $6 \%$ of normal (2.52 inches) for those months.

\subsection{Snowfall}

Snowfall, which includes all frozen precipitation, varied from a seasonal total of 0.3 to 56.1 inches in 1957-1958 and 1992-1993, respectively. Table 4.6 provides information on monthly and seasonal snowfall amounts, as well as the dates and amounts of earliest and latest snowfall each season. The earliest measurable snowfall ( $0.3 \mathrm{inch})$ was recorded on October 26,1957 ; the latest measurable snowfall (1 inch) 
Table 4.6. Monthly and Seasonal Snowfall (inches), Including First and Last Dates of Both Trace and Measurable Snowfalls

\begin{tabular}{|c|c|c|c|c|c|c|c|c|c|c|c|c|c|c|c|c|}
\hline \multirow[b]{2}{*}{ Season } & \multirow[b]{2}{*}{ Oct } & \multirow[b]{2}{*}{ Nov } & \multirow[b]{2}{*}{ Dec } & \multirow[b]{2}{*}{ Jan } & \multirow[b]{2}{*}{ Feb } & \multirow[b]{2}{*}{ Mar } & \multirow[b]{2}{*}{ Apr } & \multirow[b]{2}{*}{ Total } & \multicolumn{2}{|c|}{ First } & \multicolumn{2}{|c|}{ First Measurable } & \multicolumn{2}{|c|}{ Last Measurable } & \multicolumn{2}{|c|}{ Last } \\
\hline & & & & & & & & & Date & Amount & Date & Amount & Date & Amount & Date & Amount \\
\hline 1945-1946 & -- & -- & -- & -- & -- & -- & -- & -- & -- & -- & -- & -- & & & & \\
\hline 1946-1947 & $\mathrm{T}$ & 7.2 & 0.5 & 3.3 & $\mathrm{~T}$ & $\mathrm{~T}$ & 0 & 11.0 & & & $11 / 17$ & 0.2 & $01 / 31$ & 2.2 & & \\
\hline 1948-1949 & 0 & 1.7 & 8.1 & 1.8 & 6.9 & $\mathrm{~T}$ & 0 & 18.5 & $11 / 07$ & $\mathrm{~T}$ & $11 / 18$ & 0.2 & $02 / 21$ & 0.2 & $03 / 15$ & $\mathrm{~T}$ \\
\hline $1949-1950$ & $\mathrm{~T}$ & 0 & 0.7 & $23.4^{(\mathrm{a})}$ & 3.1 & 1.5 & $\mathrm{~T}$ & 28.7 & $10 / 18$ & $\mathrm{~T}$ & $12 / 16$ & 0.1 & $03 / 13$ & 0.3 & $04 / 02$ & $\mathrm{~T}$ \\
\hline 1950-1951 & 0 & 0.8 & 2.9 & 5.3 & 5.3 & $4.2^{(a)}$ & 0 & 18.5 & $11 / 19$ & $\mathrm{~T}$ & $11 / 30$ & 0.8 & $03 / 12$ & 1.1 & & \\
\hline 1951-1952 & 0 & 0.5 & 4.4 & 7.5 & 3.1 & $\mathrm{~T}$ & 0 & 15.5 & & & $11 / 25$ & 0.5 & $02 / 24$ & 0.1 & $03 / 20$ & $\mathrm{~T}$ \\
\hline 1953-1954 & 0 & 0 & 1.0 & 14.3 & 1.6 & $\mathrm{~T}$ & 0 & 16.9 & & & $12 / 08$ & 1.0 & $02 / 11$ & 1.6 & $03 / 10$ & $\mathrm{~T}$ \\
\hline 1954-1955 & 0 & 0 & 1.8 & 6.0 & 2.4 & 0.7 & $\mathrm{~T}$ & 10.9 & $12 / 03$ & $\mathrm{~T}$ & $12 / 04$ & 1.8 & $03 / 25$ & 0.7 & $04 / 02$ & $\mathrm{~T}$ \\
\hline $1955-1956$ & 0 & 12.7 & 13.4 & 10.2 & 2.2 & $\mathrm{~T}$ & 0 & 38.5 & & & $11 / 02$ & 0.2 & $02 / 23$ & 0.1 & $03 / 26$ & $\mathrm{~T}$ \\
\hline 1956-1957 & $\mathrm{T}$ & 0.1 & 2.5 & 7.9 & 1.4 & 4.0 & $\mathrm{~T}$ & 15.9 & $10 / 26$ & $\mathrm{~T}$ & $11 / 26$ & 0.1 & $03 / 06$ & 1.7 & $03 / 12$ & $\mathrm{~T}$ \\
\hline $1957-1958$ & 0.3 & 0 & $\mathrm{~T}$ & $\mathrm{~T}$ & 0 & $\mathrm{~T}$ & 0 & $0.3^{(a)}$ & & & $10 / 26$ & 0.3 & $10 / 26$ & 0.3 & $03 / 16$ & $\mathrm{~T}$ \\
\hline 1958-1959 & 0 & $\mathrm{~T}$ & 0.9 & 4.5 & 12.7 & 0 & 0 & 18.1 & $11 / 14$ & $\mathrm{~T}$ & $12 / 06$ & 0.4 & $02 / 19$ & 1.2 & & \\
\hline 1959-1960 & 0 & 0.3 & 1.0 & 5.9 & $\mathrm{~T}$ & 1.5 & 0 & 8.7 & $11 / 04$ & $\mathrm{~T}$ & $11 / 15$ & 0.1 & $03 / 05$ & 1.4 & & \\
\hline $1960-1961$ & 0 & 0 & 3.3 & 1.9 & 0 & 1.6 & 0 & 6.8 & $12 / 09$ & $\mathrm{~T}$ & $12 / 10$ & 0.1 & $03 / 05$ & 1.6 & & \\
\hline 1961-1962 & 0 & 0.5 & 6.1 & 0.4 & 2.4 & 0.9 & 0 & 10.3 & $11 / 18$ & $\mathrm{~T}$ & $11 / 23$ & 0.1 & 03/09 & 0.1 & $03 / 11$ & $\mathrm{~T}$ \\
\hline 1963-1964 & 0 & $\mathrm{~T}$ & 6.4 & 2.9 & $\mathrm{~T}$ & $\mathrm{~T}$ & $\mathrm{~T}$ & 9.3 & $11 / 19$ & $\mathrm{~T}$ & $12 / 08$ & 4.3 & $01 / 24$ & 1.5 & $03 / 22$ & $\mathrm{~T}$ \\
\hline $1964-1965$ & 0 & 0.1 & 19.1 & 6.6 & $\mathrm{~T}$ & $\mathrm{~T}$ & 0 & 25.8 & $11 / 21$ & $\mathrm{~T}$ & $11 / 29$ & 0.1 & $01 / 23$ & 3.1 & $03 / 27$ & $\mathrm{~T}$ \\
\hline $1965-1966$ & 0 & $\mathrm{~T}$ & 6.9 & 2.6 & $\mathrm{~T}$ & $\mathrm{~T}$ & 0 & 9.5 & $11 / 23$ & $\mathrm{~T}$ & $12 / 23$ & 0.6 & $01 / 22$ & 0.2 & $03 / 21$ & $\mathrm{~T}$ \\
\hline 1966-1967 & 0 & 0.4 & 2.8 & 0.1 & 0 & 0 & 0 & 3.3 & & & $11 / 11$ & 0.2 & $01 / 26$ & 0.1 & & \\
\hline $1967-1968$ & 0 & 0 & 5.7 & 4.5 & 0.3 & 0 & $\mathrm{~T}$ & 10.5 & $12 / 06$ & $\mathrm{~T}$ & $12 / 09$ & 0.6 & $02 / 17$ & 0.3 & $04 / 16$ & $\mathrm{~T}$ \\
\hline 1968-1969 & 0 & $\mathrm{~T}$ & 9.7 & 15.9 & 2.1 & 0 & 0 & 27.7 & $11 / 16$ & $\mathrm{~T}$ & $12 / 19$ & 0.1 & $02 / 23$ & 2.0 & $02 / 28$ & $\mathrm{~T}$ \\
\hline $1969-1970$ & 0 & $\mathrm{~T}$ & 2.7 & 6.6 & $\mathrm{~T}$ & 0.2 & 0 & 9.5 & $11 / 29$ & $\mathrm{~T}$ & $12 / 08$ & 1.3 & 03/01 & 0.2 & & \\
\hline $1970-1971$ & 0 & 0.5 & 4.4 & 2.0 & $\mathrm{~T}$ & 0.6 & 0 & 7.5 & $11 / 22$ & $\mathrm{~T}$ & $11 / 30$ & 0.5 & $03 / 14$ & 0.1 & $03 / 22$ & $\mathrm{~T}$ \\
\hline 1971-1972 & 0.6 & $\mathrm{~T}$ & 8.1 & 4.9 & 1.4 & 0.1 & $\mathrm{~T}$ & 15.1 & $11 / 27$ & $\mathrm{~T}$ & $11 / 29$ & 0.1 & $02 / 05$ & 0.1 & $04 / 12$ & $\mathrm{~T}$ \\
\hline $1972-1973$ & 0 & $\mathrm{~T}$ & 7.2 & 4.3 & 1.7 & 0 & 0 & 13.2 & $12 / 02$ & $\mathrm{~T}$ & $12 / 03$ & 1.7 & $02 / 10$ & 1.7 & $02 / 13$ & $\mathrm{~T}$ \\
\hline 1973-1974 & $1.5^{(\mathrm{a})}$ & 6.6 & 7.5 & 3.9 & 0 & $\mathrm{~T}$ & 0 & 19.5 & & & $10 / 31$ & 1.5 & $01 / 12$ & 2.3 & $03 / 06$ & $\mathrm{~T}$ \\
\hline $1974-1975$ & 0 & 0 & 0.7 & 2.5 & 12.1 & $\mathrm{~T}$ & $\mathrm{~T}$ & 15.3 & $12 / 02$ & $\mathrm{~T}$ & $12 / 12$ & 0.3 & $02 / 09$ & 1.7 & $04 / 04$ & $\mathrm{~T}$ \\
\hline $1975-1976$ & 0 & 1.7 & 3.8 & 6.0 & 0.2 & $\mathrm{~T}$ & $\mathrm{~T}$ & 11.7 & & & $11 / 10$ & 0.6 & $02 / 03$ & 0.2 & $04 / 01$ & $\mathrm{~T}$ \\
\hline 1976-1977 & 0 & 0 & 0.2 & 2.9 & $\mathrm{~T}$ & $\mathrm{~T}$ & 0 & 3.1 & $12 / 04$ & $\mathrm{~T}$ & $12 / 23$ & 0.2 & $01 / 31$ & 0.2 & $03 / 27$ & $\mathrm{~T}$ \\
\hline $1977-1978$ & 0 & 2.1 & 3.4 & 2.9 & 0.9 & $\mathrm{~T}$ & 0 & 9.3 & $11 / 15$ & $\mathrm{~T}$ & $11 / 18$ & 0.1 & $02 / 26$ & 0.1 & $03 / 05$ & $\mathrm{~T}$ \\
\hline $1978-1979$ & 0 & 10.1 & 1.4 & 10.3 & 0.5 & 0.1 & 0 & 22.6 & $11 / 15$ & $\mathrm{~T}$ & $11 / 18$ & 5.3 & $03 / 03$ & 0.1 & & \\
\hline $1979-1980$ & 0 & 5.6 & 7.3 & 8.7 & 4.5 & 0.3 & 0 & 26.2 & & & $11 / 22$ & 1.4 & 03/05 & 0.3 & & \\
\hline
\end{tabular}


Table 4.6. (contd)

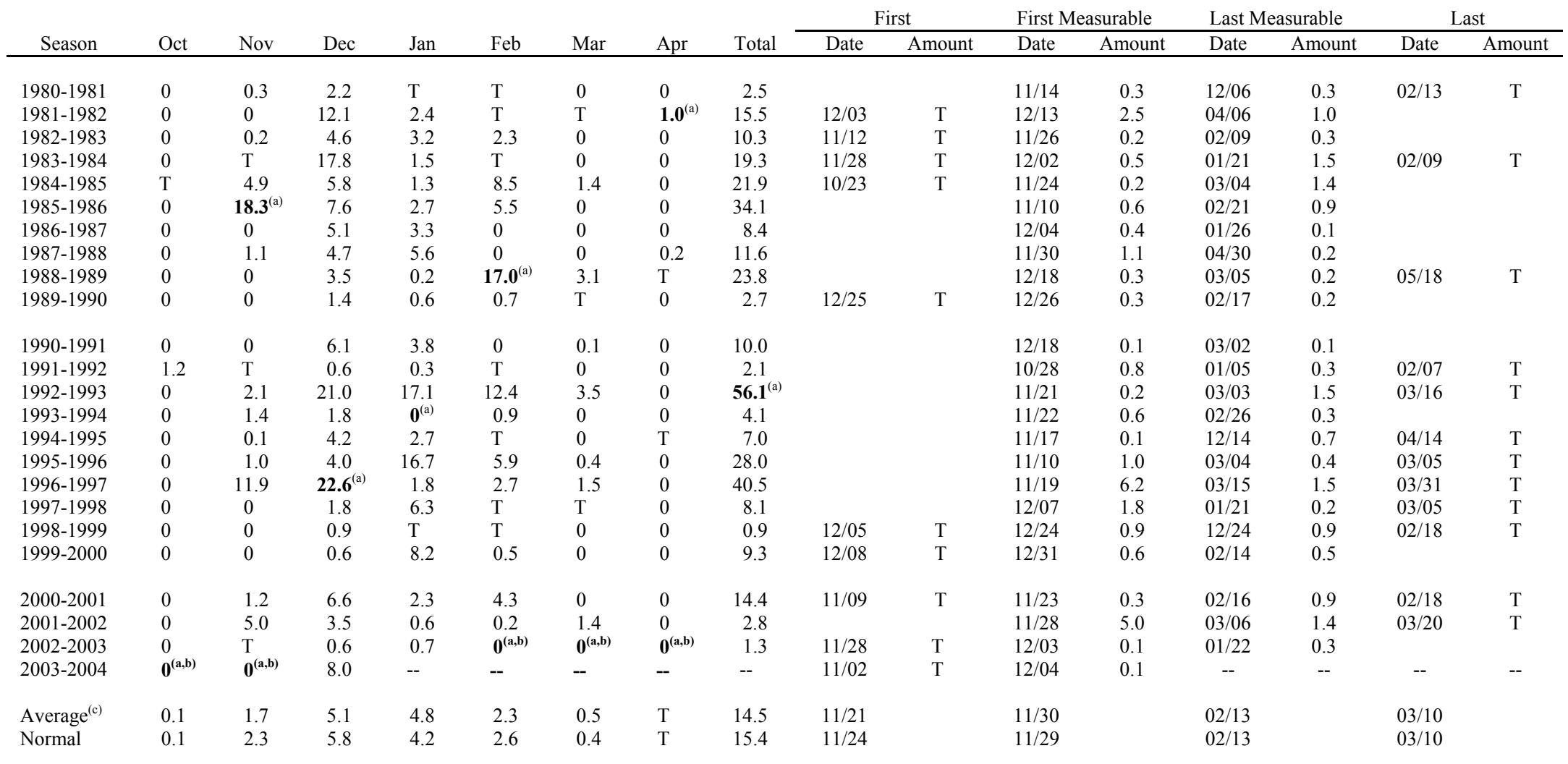

(a) Greatest and least values.

(b) Most recent of multiple occurrences.

(c) Based on entire period of record, 1946 through 2003

$\mathrm{T}=$ Trace.

NOTE: Dashes indicate no data are available. 
was recorded on April 6, 1982. The average date of the first measurable snow is November 30; the average last measurable snow date is February 13. Normal snowfall for the period 1971 through 2000 and averages for the entire period of record are noted on the table, as are monthly and seasonal extremes.

Table 4.7 lists the greatest single storm snowfall amounts by month for the period 1946 through 2002 . The greatest single snowstorm, on February 18-20, 1993, produced 12.4 inches of snow. During the winter of 1957-58 (the only snowfall was recorded in October), the greatest single snowstorm produced only 0.3 inch.

Table 4.8 lists some miscellaneous snowfall statistics for the Hanford Meteorology Station for the period 1946 through 2003. Included in this table are average number of days per month with snow depth above certain threshold values, greatest number of days per month with snow depth above certain threshold values, record number of consecutive days with snow depth above certain threshold values, record monthly snow depth, and 24-hour snowfall amounts. The record snow depth at the Hanford Meteorology Station is 15.6 inches, recorded in December 1985. The record number of days with snow depth $\geq 6$ inches was 43 days in the winter of 1992-1993.

\subsection{Normal and Maximum Daily Precipitation}

Table 4.9 contains annual maximum precipitation statistics for the time periods 1, 2, 3, 6, 12, and 24 hours, including the dates of occurrence for each time period, 1947 through 2003. Table 4.10 contains normal and maximum values of precipitation (minimum values are not needed because every day of the year has a minimum value of 0 ). The normal precipitation values are based on the period 1971 through 2000; the daily maximum values are for the entire period of record (1945 through 2003). The maximum daily value for each month is noted on the table.

October 1, 1957, recorded the greatest precipitation in one day, 1.60 inches. There have been only 5 days during the period of record that have never received measurable precipitation. However, all have received a trace. 
Table 4.7. Snowfall (inches) - Greatest Amount from a Single Storm

\begin{tabular}{|c|c|c|c|c|c|c|c|c|c|c|c|c|c|}
\hline Year & Jul & Aug & Sep & Oct & Nov & Dec & Jan & Feb & Mar & Apr & May & Jun & $\underline{\text { Season }}$ \\
\hline 1946-47 & 0 & 0 & 0 & $\mathrm{~T}$ & 4.5 & 0.3 & 2.2 & $\mathrm{~T}$ & $\mathrm{~T}$ & 0 & 0 & 0 & 4.5 \\
\hline $1947-48$ & 0 & 0 & 0 & 0 & $\mathrm{~T}$ & 1.8 & 2.6 & 5.2 & 0.1 & $\mathrm{~T}$ & 0 & 0 & 5.2 \\
\hline $1948-49$ & 0 & 0 & 0 & 0 & 1.7 & 1.9 & 0.8 & 4.4 & $\mathrm{~T}$ & 0 & 0 & 0 & 4.4 \\
\hline $1949-50$ & 0 & 0 & 0 & $\mathrm{~T}$ & 0 & 0.4 & 5.1 & 2.2 & 1.2 & $\mathrm{~T}$ & 0 & 0 & 5.1 \\
\hline $1950-51$ & 0 & 0 & 0 & 0 & 0.8 & 2.1 & 2.3 & 3.5 & 2.2 & 0 & 0 & 0 & 3.5 \\
\hline $1951-52$ & 0 & 0 & 0 & 0 & 0.5 & 2.1 & 3.0 & 2.5 & $\mathrm{~T}$ & 0 & 0 & 0 & 3.0 \\
\hline $1952-53$ & 0 & 0 & 0 & 0 & $\mathrm{~T}$ & 1.1 & 2.7 & 0 & $\mathrm{~T}$ & 0 & 0 & 0 & 2.7 \\
\hline $1953-54$ & 0 & 0 & 0 & 0 & 0 & 1.0 & $9.6^{(\mathrm{a})}$ & 1.6 & $\mathrm{~T}$ & 0 & 0 & 0 & 9.6 \\
\hline 1954-55 & 0 & 0 & 0 & 0 & 0 & 1.8 & 1.7 & 2.2 & 0.7 & $\mathrm{~T}$ & $\mathrm{~T}$ & 0 & 2.2 \\
\hline $1955-56$ & 0 & 0 & 0 & 0 & 4.8 & 3.4 & 4.4 & 0.6 & $\mathrm{~T}$ & 0 & 0 & 0 & 4.8 \\
\hline $1956-57$ & 0 & 0 & 0 & $\mathrm{~T}$ & 0.1 & 2.4 & 3.5 & 1.2 & 2.2 & 0 & 0 & 0 & 3.5 \\
\hline $1957-58$ & 0 & 0 & 0 & 0.3 & 0 & $\mathrm{~T}$ & $\mathrm{~T}$ & 0 & $\mathrm{~T}$ & 0 & 0 & 0 & $\mathbf{0 . 3 ^ { ( b ) }}$ \\
\hline $1958-59$ & 0 & 0 & 0 & 0 & $\mathrm{~T}$ & 0.4 & 2.7 & 5.9 & 0 & 0 & 0 & 0 & 5.9 \\
\hline $1959-60$ & 0 & 0 & 0 & 0 & 0.2 & 0.6 & 3.6 & $\mathrm{~T}$ & 1.5 & 0 & $\mathrm{~T}$ & 0 & 3.6 \\
\hline 1960-61 & 0 & 0 & 0 & 0 & 0 & 2.0 & 1.9 & 0 & 1.6 & 0 & 0 & 0 & 2.0 \\
\hline $1961-62$ & 0 & 0 & 0 & 0 & 0.4 & 3.0 & 0.3 & 2.0 & 0.8 & 0 & 0 & 0 & 3.0 \\
\hline $1962-63$ & 0 & 0 & 0 & 0 & 0 & $\mathrm{~T}$ & 7.1 & 0.6 & 0 & 0 & 0 & 0 & 7.1 \\
\hline 1963-64 & 0 & 0 & 0 & 0 & $\mathrm{~T}$ & 4.3 & 1.5 & $\mathrm{~T}$ & $\mathrm{~T}$ & $\mathrm{~T}$ & $\mathrm{~T}$ & 0 & 4.3 \\
\hline 1964-65 & 0 & 0 & 0 & 0 & 0.1 & 5.3 & 3.2 & $\mathrm{~T}$ & $\mathrm{~T}$ & 0 & 0 & 0 & 5.3 \\
\hline $1965-66$ & 0 & 0 & 0 & 0 & $\mathrm{~T}$ & 5.4 & 1.9 & $\mathrm{~T}$ & $\mathrm{~T}$ & 0 & 0 & 0 & 5.4 \\
\hline $1966-67$ & 0 & 0 & 0 & 0 & 0.4 & 0.3 & 0.1 & 0 & $\mathrm{~T}$ & 0 & 0 & 0 & 0.4 \\
\hline $1967-68$ & 0 & 0 & 0 & 0 & 0 & 3.3 & 2.9 & 0.3 & 0 & $\mathrm{~T}$ & 0 & 0 & 3.3 \\
\hline $1968-69$ & 0 & 0 & 0 & 0 & $\mathrm{~T}$ & 3.6 & 6.4 & 2.0 & 0 & 0 & 0 & 0 & 6.4 \\
\hline 1969-70 & 0 & 0 & 0 & 0 & $\mathrm{~T}$ & 1.3 & 3.0 & $\mathrm{~T}$ & 0.2 & 0 & 0 & 0 & 3.0 \\
\hline 1970-71 & 0 & 0 & 0 & 0 & 0.5 & 3.1 & 1.8 & $\mathrm{~T}$ & 0.5 & 0 & 0 & 0 & 3.1 \\
\hline 1971-72 & 0 & 0 & 0 & 0.6 & $\mathrm{~T}$ & 3.4 & 3.9 & 1.3 & 0.1 & $\mathrm{~T}$ & 0 & 0 & 3.9 \\
\hline $1972-73$ & 0 & 0 & 0 & 0 & $\mathrm{~T}$ & 4.0 & 2.8 & 1.7 & 0 & 0 & 0 & 0 & 4.0 \\
\hline $1973-74$ & 0 & 0 & 0 & $1.5^{(\mathrm{a})}$ & 3.9 & 5.8 & 2.3 & 0 & 0 & 0 & 0 & 0 & 5.8 \\
\hline 1974-75 & 0 & 0 & 0 & 0 & 0 & 0.4 & 0.9 & 5.6 & $\mathrm{~T}$ & $\mathrm{~T}$ & 0 & 0 & 5.6 \\
\hline $1975-76$ & 0 & 0 & 0 & 0 & 1.7 & 3.1 & 2.4 & 0.2 & $\mathrm{~T}$ & 0 & 0 & 0 & 3.1 \\
\hline 1976-77 & 0 & 0 & 0 & 0 & 0 & 0.2 & 1.8 & $\mathrm{~T}$ & $\mathrm{~T}$ & 0 & 0 & 0 & 1.8 \\
\hline $1977-78$ & 0 & 0 & 0 & 0 & 1.8 & 2.5 & 2.2 & 0.8 & $\mathrm{~T}$ & 0 & 0 & 0 & 2.5 \\
\hline $1978-79$ & 0 & 0 & 0 & 0 & $9.1^{(\mathrm{a})}$ & 1.0 & 5.0 & 0.3 & 0 & 0 & 0 & 0 & 9.1 \\
\hline 1979-80 & 0 & 0 & 0 & 0 & 3.4 & 3.6 & 6.4 & 4.5 & 0.3 & 0 & 0 & 0 & 6.4 \\
\hline $1980-81$ & 0 & 0 & 0 & 0 & 0.3 & 1.9 & $\mathrm{~T}$ & $\mathrm{~T}$ & 0 & 0 & 0 & 0 & 1.9 \\
\hline 1981-82 & 0 & 0 & 0 & 0 & 0 & 3.8 & 1.0 & $\mathrm{~T}$ & $\mathrm{~T}$ & $1.0^{\text {(a) }}$ & 0 & 0 & 3.8 \\
\hline $1982-83$ & 0 & 0 & 0 & 0 & 0.2 & 2.6 & 2.0 & 2.0 & 0 & 0 & $\mathrm{~T}$ & 0 & 2.6 \\
\hline $1983-84$ & 0 & 0 & 0 & 0 & $\mathrm{~T}$ & 5.1 & 1.5 & $\mathrm{~T}$ & 0 & 0 & 0 & 0 & 5.1 \\
\hline $1984-85$ & 0 & 0 & 0 & $\mathrm{~T}$ & 4.7 & 2.4 & 1.3 & 2.9 & 0 & 0 & 0 & 0 & 4.7 \\
\hline $1985-86$ & 0 & 0 & 0 & 0 & 8.8 & $\mathbf{6 . 6}^{(\mathrm{a})}$ & 1.1 & 2.7 & 0 & 0 & 0 & 0 & 8.8 \\
\hline $1986-87$ & 0 & 0 & 0 & 0 & 0 & 2.1 & 0.8 & 0 & 0 & 0 & 0 & 0 & 2.1 \\
\hline $1987-88$ & 0 & 0 & 0 & 0 & 1.1 & 4.4 & 2.3 & 0 & 0 & 0 & 0 & 0 & 4.4 \\
\hline 1988-89 & 0 & 0 & 0 & 0 & 0 & 1.7 & 0.2 & 10.0 & $2.7^{(\mathrm{a})}$ & $\mathrm{T}$ & $\mathbf{T}^{(\mathrm{a}, \mathrm{c})}$ & 0 & 10.0 \\
\hline 1989-90 & 0 & 0 & 0 & 0 & 0 & 1.1 & 0.6 & 0.7 & $\mathrm{~T}$ & 0 & 0 & 0 & 1.1 \\
\hline $1990-91$ & 0 & 0 & 0 & 0 & 0 & 2.8 & 2.1 & 0 & 0.1 & 0 & 0 & 0 & 2.8 \\
\hline 1991-92 & 0 & 0 & 0 & 0.9 & $\mathrm{~T}$ & 0.6 & 0.3 & $\mathrm{~T}$ & 0 & 0 & 0 & 0 & 0.9 \\
\hline $1992-93$ & 0 & 0 & 0 & 0 & 1.6 & 3.8 & 7.3 & $12.4^{(\mathrm{a})}$ & 2.0 & 0 & 0 & 0 & $12.4^{(\mathrm{a})}$ \\
\hline $1993-94$ & 0 & 0 & 0 & 0 & 0.6 & 1.0 & 0 & 0.3 & 0 & 0 & 0 & 0 & 1.0 \\
\hline 1994-95 & 0 & 0 & 0 & 0 & 0.1 & 1.7 & 1.9 & $\mathrm{~T}$ & 0 & $\mathrm{~T}$ & 0 & 0 & 1.9 \\
\hline $1995-96$ & 0 & 0 & 0 & 0 & 1.0 & 2.7 & 3.5 & 4.0 & 0.4 & 0 & 0 & 0 & 4.0 \\
\hline $1996-97$ & 0 & 0 & 0 & 0 & 7.1 & 6.0 & 0.9 & 2.7 & 1.5 & 0 & 0 & 0 & 7.1 \\
\hline $1997-98$ & 0 & 0 & 0 & 0 & 0 & 1.8 & 3.3 & $\mathrm{~T}$ & $\mathrm{~T}$ & 0 & 0 & 0 & 3.3 \\
\hline $1998-99$ & 0 & 0 & 0 & 0 & 0 & 0.9 & $\mathrm{~T}$ & 0 & 0 & 0 & 0 & 0 & 0.9 \\
\hline $1999-2000$ & 0 & 0 & 0 & 0 & 0 & 0.6 & 2.2 & 0.5 & 0 & 0 & 0 & 0 & 2.2 \\
\hline $2000-2001$ & 0 & 0 & 0 & 0 & 0.7 & 1.4 & 0.9 & 2.2 & 0 & 0 & 0 & 0 & 2.2 \\
\hline $2001-2002$ & 0 & 0 & 0 & 0 & 5.0 & 1.9 & 0.6 & 0.2 & 0 & 0 & 0 & 0 & 5.0 \\
\hline $2002-2003$ & 0 & 0 & 0 & 0 & $\mathrm{~T}$ & 0.3 & 0.7 & 0 & 0 & 0 & 0 & 0 & 0.7 \\
\hline 2003-2004 & 0 & 0 & 0 & 0 & $\mathrm{~T}$ & 3.6 & -- & -- & -- & -- & -- & -- & -- \\
\hline
\end{tabular}

(a) Greatest value.

(b) Seasonal low.

(c) Most recent of multiple occurrences.

$\mathrm{T}=$ Trace

NOTE: Dashes indicate no data are available. 
Table 4.8. Miscellaneous Snowfall Statistics, 1946 through 2003

\begin{tabular}{|c|c|c|c|c|c|c|c|}
\hline & Oct & Nov & Dec & Jan & Feb & Mar & Season \\
\hline \multicolumn{8}{|c|}{$\begin{array}{l}\text { Average Number } \\
\text { of Days of Given } \\
\text { Depth at } 0400 \text { PST }\end{array}$} \\
\hline$\geq 1$ inch & (a) & 1 & 6 & 8 & 4 & (a) & 20 \\
\hline$\geq 3$ inches & 0 & 1 & 2 & 5 & 2 & (a) & 11 \\
\hline$\geq 6$ inches & 0 & (a) & 1 & 2 & 1 & (a) & 4 \\
\hline$\geq 12$ inches & 0 & 0 & (a) & (a) & 0 & 0 & (a) \\
\hline \multicolumn{8}{|l|}{$\begin{array}{l}\text { Record Greatest } \\
\text { Number of Days } \\
\text { of Given Depth } \\
\text { at } 0400 \text { PST }\end{array}$} \\
\hline$\geq 1$ inch & 0 & $12(1996)^{(b)}$ & $31(1985)$ & 31 (1969) & 20 (1989) & 7 (1993) & 72 (1992-93) \\
\hline$\geq 3$ inches & 0 & $12(1996)$ & $31(1985)$ & 27 (1993) & $16(1950)$ & $6(1993)$ & $58(1985-86)$ \\
\hline$\geq 6$ inches & 0 & $9(1985)$ & $23(1985)$ & 25 (1993) & 9 (1993) & 5 (1993) & 43 (1992-93) \\
\hline$\geq 12$ inches & 0 & 0 & 4 (1964) & $10(1993)$ & 0 & 0 & $10(1992-93)$ \\
\hline $\begin{array}{l}\text { Record Greatest } \\
\text { Depth }\end{array}$ & $1.5(1973)$ & $10.0(1985)$ & $15.6(1985)$ & $15.0(1993)$ & $10.0(1969)$ & 9.1 (1993) & 15.6 (Dec 1985) \\
\hline $\begin{array}{l}\text { Greatest in } \\
24 \text { hours }\end{array}$ & $1.5(1973)$ & $8.8(1985)$ & $6.6(1985)$ & $7.1(1954)$ & $10.2(1993)$ & 2.7 (1989) & $10.2($ Feb 1993) \\
\hline
\end{tabular}

Record Consecutive

Number of Days of

Given Depth at

0400 PST

\section{Number}

$\geq 1$ inch

$\geq 3$ inches

$\geq 6$ inches

$\geq 12$ inches

\begin{tabular}{c} 
of Days \\
\hline 60 \\
57 \\
32 \\
6
\end{tabular}

6

$\frac{\text { From }}{\text { November 20, } 1985}$
November 22, 1985
December 20, 1964
January 15, 1993

\begin{tabular}{c} 
To \\
\hline January 18,1986 \\
January 17,1986 \\
January 20, 1965 \\
January 20, 1993
\end{tabular}

(a) Denotes less than $1 / 2$ day.

(b) Year of occurrence in parentheses.

PST $=$ Pacific Standard Time. 
Table 4.9. Maximum Precipitation (inches)

\begin{tabular}{|c|c|c|c|c|c|c|c|c|c|c|c|c|}
\hline Year & $1 \mathrm{~h}$ & Date & $2 \mathrm{~h}$ & Date & $3 \mathrm{~h}$ & Date & $6 \mathrm{~h}$ & Date & $12 \mathrm{~h}$ & Date & $24 \mathrm{~h}$ & Date \\
\hline 1947 & 0.48 & $08-29$ & 0.51 & 06-07 & 0.54 & $06-07$ & 0.67 & $09-15$ & 0.75 & $09 / 16-17$ & 0.88 & $10 / 19-20$ \\
\hline 1948 & 0.24 & $05-19$ & 0.30 & $06-11$ & 0.31 & $06 / 10-11$ & 0.50 & $01 / 6-7$ & 0.65 & $01-06$ & 1.08 & 01/06-07 \\
\hline 1949 & 0.18 & $11-23$ & 0.28 & $11-23$ & 0.41 & $11-23$ & 0.60 & $11-23$ & 0.63 & $11-23$ & 0.65 & $11 / 23-24$ \\
\hline 1950 & 0.30 & $06-17$ & 0.52 & $06-17$ & 0.58 & $06-17$ & 0.87 & $06-17$ & 1.05 & $06-17$ & 1.24 & $06 / 16-17$ \\
\hline 1951 & 0.28 & $04-28$ & 0.41 & $04-28$ & 0.44 & $04-28$ & 0.45 & $06-06$ & 0.47 & $06-06$ & 0.70 & 06/05-06 \\
\hline 1952 & 0.27 & $05-10$ & 0.27 & $05-10$ & 0.27 & $05-10$ & 0.29 & $06-29$ & 0.39 & $06-29$ & 0.48 & $06-29$ \\
\hline 1953 & 0.35 & $08-26$ & 0.35 & $08-26$ & 0.35 & $08-26$ & 0.44 & 01/08-09 & 0.77 & 01/08-09 & 0.83 & 01/08-09 \\
\hline 1954 & 0.16 & 03-19 & 0.19 & $05-26$ & 0.27 & $01-16$ & 0.52 & $01-16$ & 0.72 & $01 / 15-16$ & 0.77 & $01 / 15-16$ \\
\hline 1955 & 0.13 & $12-31$ & 0.21 & $12-21$ & 0.31 & $12-21$ & 0.49 & $12-21$ & 0.61 & $12-21$ & 0.64 & $11 / 26-27$ \\
\hline 1956 & 0.16 & $06-14$ & 0.22 & $06-14$ & 0.27 & $06-14$ & $0.28^{(a)}$ & $01-15$ & 0.44 & $01 / 14-15$ & 0.73 & $01 / 14-15$ \\
\hline 1957 & 0.47 & $10-01$ & $0.88^{(a)}$ & $10-01$ & $1.08^{(a)}$ & $10-01$ & $1.68^{(a)}$ & $10 / 01-02$ & $1.88^{(a)}$ & $10 / 01-02$ & $1.91^{(\mathrm{a})}$ & $10 / 01-02$ \\
\hline 1958 & 0.43 & $06-12$ & 0.43 & 06-12 & 0.43 & 06-12 & 0.65 & $12 / 10-11$ & 0.88 & $12 / 10-11$ & 1.00 & $12 / 10-11$ \\
\hline 1959 & 0.18 & $05-17$ & 0.18 & $05-17^{(\mathrm{b})}$ & 0.23 & $09-14^{(\mathrm{b})}$ & 0.40 & $01-11$ & 0.54 & $01 / 11-12$ & 0.82 & $01 / 11-12$ \\
\hline 1960 & 0.22 & $03-27$ & 0.23 & $03-27$ & 0.33 & 05/06-07 & 0.43 & 05/06-07 & 0.44 & 05/06-07 & 0.44 & 05/06-07 \\
\hline 1961 & 0.21 & $02-01$ & 0.39 & $02-01$ & 0.42 & $02-01$ & 0.46 & $05 / 09-10$ & 0.72 & $02-01$ & 0.72 & $02-01$ \\
\hline 1962 & 0.19 & $11-30$ & 0.27 & $11-30$ & 0.34 & $02-09$ & 0.40 & $10-12$ & 0.52 & $10-12$ & 0.52 & $10-12$ \\
\hline 1963 & 0.22 & $01-31$ & 0.37 & $01-31$ & 0.44 & $01-31$ & 0.54 & $01-31$ & 0.94 & $01-31 / 02-01$ & 0.98 & $01-31 / 02-01$ \\
\hline 1964 & 0.16 & $12-22$ & 0.20 & 06-08 & 0.32 & $12-21$ & 0.42 & $12-21$ & 0.54 & $12-21$ & 0.60 & $12 / 21-22$ \\
\hline 1965 & $0.10^{(a)}$ & $05-19$ & $\mathbf{0 . 1 4} 4^{(\mathrm{a})}$ & $11-24^{(b)}$ & $0.18^{(a)}$ & $06-17$ & 0.29 & $06-17$ & 0.39 & $06-17$ & 0.48 & $06-17$ \\
\hline 1966 & 0.14 & $07-02$ & 0.17 & 07-02 & 0.22 & $11-19$ & 0.37 & $11-19$ & 0.74 & $11 / 19-20$ & 0.78 & $11 / 19-20$ \\
\hline 1967 & 0.15 & $04-18$ & 0.26 & $06-21$ & 0.31 & $06-21$ & 0.31 & $06-21$ & $0.32^{(a)}$ & $06-21$ & $0.37^{(a)}$ & $04 / 17-18$ \\
\hline 1968 & 0.12 & $12-24$ & 0.21 & $12-24$ & 0.28 & $12-24$ & 0.36 & $12-24$ & 0.43 & $10-11$ & 0.54 & $10 / 07-08$ \\
\hline 1969 & $0.55^{(\mathrm{a})}$ & $06-12$ & 0.59 & $06-12$ & 0.59 & $06-12$ & 0.60 & $06-12$ & 0.60 & $06 / 12-13$ & 0.60 & $06-12$ \\
\hline 1970 & 0.15 & $05-12$ & 0.29 & $05-12$ & 0.37 & $05-12$ & 0.47 & $05-12$ & 0.50 & $05-12$ & 0.61 & $01 / 22-23$ \\
\hline 1971 & 0.15 & $03-15$ & 0.26 & $03-25$ & 0.35 & $01-16$ & 0.48 & $03 / 25-26$ & 0.53 & $01-16$ & 0.53 & $01-16$ \\
\hline 1972 & 0.18 & $05-20$ & 0.32 & $05-30$ & 0.45 & $05-20$ & 0.80 & $05 / 20-21$ & 1.24 & $5 / 20-21$ & 1.39 & $05 / 20-21$ \\
\hline 1973 & 0.15 & $10-31$ & 0.21 & $10-31^{(\mathrm{b})}$ & 0.30 & $11-12$ & 0.53 & $10-31$ & 0.64 & $10-31$ & 0.64 & $11 / 11-12$ \\
\hline 1974 & 0.45 & $07-19$ & 0.45 & 07-19 & 0.45 & $07-19$ & 0.45 & $07-19$ & 0.45 & $07-19$ & 0.45 & $07-19$ \\
\hline 1975 & 0.30 & 08-18 & 0.47 & $08-18$ & 0.55 & $08-18$ & 0.69 & $08-18$ & 0.69 & $08-18$ & 0.69 & $08-18$ \\
\hline 1976 & 0.32 & 08-07 & 0.33 & $08-07$ & 0.33 & $08-07$ & 0.33 & $08-07$ & 0.33 & $08-07$ & 0.40 & $08 / 24-25$ \\
\hline 1977 & 0.16 & $12-13$ & 0.28 & $12-13$ & 0.36 & $12-13$ & 0.61 & $12-13$ & 0.75 & $12-13$ & 0.89 & $08 / 29-30$ \\
\hline 1978 & 0.15 & $04-27$ & 0.22 & $04-27$ & 0.23 & $04-27$ & 0.31 & $11-18^{(b)}$ & 0.58 & $11 / 18-19$ & 0.67 & $11 / 18-19$ \\
\hline 1979 & 0.11 & $04-17$ & 0.18 & $03-27$ & 0.22 & $03-27$ & 0.29 & $03-27$ & 0.40 & $03-27$ & 0.42 & $11-16+$ \\
\hline 1980 & 0.14 & $12-25$ & 0.24 & $04-20$ & 0.29 & $04-20$ & 0.47 & $05 / 25-26$ & 0.74 & $09-13$ & 0.90 & $05 / 25-26$ \\
\hline 1981 & 0.22 & $05-25$ & 0.34 & $05-25$ & 0.38 & $05-25$ & 0.73 & $05-25$ & 0.74 & $05-25$ & 0.74 & $05-25$ \\
\hline 1982 & 0.22 & $07-07$ & 0.33 & $11-18$ & 0.40 & $11-18$ & 0.64 & $10-28$ & 0.95 & $10 / 28-29$ & 0.97 & $10 / 28-29$ \\
\hline 1983 & 0.24 & 09-01 & 0.31 & $11-10$ & 0.39 & $11-10$ & 0.45 & $11 / 23-24$ & 0.60 & $11 / 23-24$ & 0.66 & $11-10$ \\
\hline 1984 & 0.20 & $06-28$ & 0.38 & $03-20$ & 0.39 & $03-20$ & 0.48 & $03 / 20-21$ & 0.51 & $03 / 20-21$ & 0.53 & $03 / 20-21$ \\
\hline 1985 & 0.14 & $12-07$ & 0.22 & $11-21$ & 0.29 & $11-21$ & 0.46 & $11-21$ & 0.52 & $11 / 21-22$ & 0.52 & $11 / 21-22$ \\
\hline 1986 & 0.24 & $09-15$ & 0.43 & $09-15$ & 0.45 & $09-15$ & 0.47 & $09-15$ & 0.47 & $09-15$ & 0.54 & $09-15$ \\
\hline 1987 & 0.21 & 07-09 & 0.24 & 07-09 & 0.27 & 07-09 & 0.31 & $12-09$ & 0.34 & $12-09$ & 0.55 & $12-09$ \\
\hline 1988 & 0.31 & $04-28$ & 0.42 & $04-28$ & 0.42 & $04-28$ & 0.42 & $04-28$ & 0.48 & $04-28$ & 0.49 & $04 / 27-28$ \\
\hline 1989 & 0.16 & $04-25$ & 0.25 & $04-25$ & 0.26 & $04-25$ & 0.31 & $05-23$ & 0.38 & $02 / 16-17$ & 0.56 & $02 / 16-17$ \\
\hline 1990 & 0.25 & $06-06$ & 0.33 & $08-21^{(b)}$ & 0.43 & $08-21$ & 0.66 & $08-21$ & 0.77 & $08 / 20-21$ & 0.77 & $08 / 20-21$ \\
\hline 1991 & 0.49 & $06-29$ & 0.50 & $06-29$ & 0.51 & $06-29$ & 0.51 & $06-29$ & 0.53 & $06-29$ & 0.59 & $06 / 05-06$ \\
\hline 1992 & 0.17 & $06-12$ & 0.25 & $06-12$ & 0.31 & $06-12$ & 0.44 & $06-12$ & 0.70 & $06-12$ & 0.79 & $06-12$ \\
\hline 1993 & 0.32 & $07-17$ & 0.45 & $07-17$ & 0.55 & $07-17$ & 0.82 & $07-17$ & 1.01 & $07 / 16-17$ & 1.39 & $07 / 16-17$ \\
\hline 1994 & 0.27 & $05-15$ & 0.32 & $10-14$ & 0.37 & $05-15$ & 0.49 & $05-15$ & 0.58 & $05-15$ & 0.59 & $05 / 14-15$ \\
\hline 1995 & 0.48 & 05-09 & 0.53 & 05-09 & 0.53 & 05-09 & 0.55 & $12-12$ & 0.65 & $12-12$ & 1.04 & $12 / 11-12$ \\
\hline 1996 & 0.16 & $12-31^{(b)}$ & 0.29 & $12-29$ & 0.40 & $12-29$ & 0.65 & $12-29$ & 0.90 & $11-19$ & 1.70 & $11 / 18-19$ \\
\hline 1997 & 0.27 & $10-08$ & 0.36 & $10-08$ & 0.40 & $10-08$ & 0.48 & $01-31$ & 0.57 & $11-07$ & 0.70 & $01-17$ \\
\hline 1998 & 0.19 & $11-05$ & 0.29 & $11-05$ & 0.36 & $11-05$ & 0.49 & $11-05$ & 0.62 & $11-05$ & 0.62 & $11-05$ \\
\hline 1999 & 0.40 & $08-05$ & 0.40 & $08-05$ & 0.47 & $08-05$ & 0.48 & $08-05$ & 0.48 & $08-05$ & 0.51 & $01 / 22-23$ \\
\hline 2000 & 0.18 & $07-16$ & 0.23 & $05-31$ & 0.29 & $05-31$ & 0.40 & $05-31$ & 0.50 & $04-13$ & 0.54 & $04-13$ \\
\hline 2001 & 0.15 & 06-05 & 0.25 & $06-26$ & 0.34 & $06-26$ & 0.47 & $06 / 26-27$ & 0.48 & $06 / 26-27$ & 0.72 & $06 / 26-27$ \\
\hline 2002 & 0.22 & $02-07$ & 0.32 & $02-07$ & 0.39 & $02-07$ & 0.51 & $02-07$ & 0.53 & $02-07$ & 0.64 & $12 / 13-14$ \\
\hline 2003 & 0.23 & $04-14$ & 0.39 & $04-14$ & 0.54 & $04-14$ & 0.92 & $04-14$ & 1.19 & 04-14 & 1.24 & $04 / 14$ \\
\hline
\end{tabular}

(a) Greatest and least values.

(b) Last of multiple occurrences. 
Table 4.10. Normal and Maximum Daily Precipitation (inches)

\begin{tabular}{|c|c|c|c|c|c|c|c|}
\hline \multirow[b]{3}{*}{ Day } & \multicolumn{5}{|c|}{ Normal Period (1971-2000) } & \multicolumn{2}{|c|}{$\begin{array}{c}\text { Historical Period } \\
(1945-2003)\end{array}$} \\
\hline & \multirow[b]{2}{*}{ Normal } & \multicolumn{2}{|c|}{ Number of Years } & \multirow[b]{2}{*}{ Maximum } & \multirow{3}{*}{ Year } & \multirow{3}{*}{ Maximum } & \multirow{3}{*}{ Year } \\
\hline & & W/Meas. & W/Trace & & & & \\
\hline \multicolumn{5}{|c|}{ January } & & & \\
\hline 1 & 0.02 & 8 & 3 & 0.20 & 1987 & 0.20 & 1987 \\
\hline 2 & 0.02 & 10 & 6 & 0.17 & 1983 & 0.27 & 1953 \\
\hline 3 & 0.02 & 10 & 6 & 0.20 & 1975 & 0.28 & 1966 \\
\hline 4 & 0.02 & 8 & 8 & 0.17 & 1976 & 0.25 & 1956 \\
\hline 5 & 0.03 & 7 & 6 & 0.19 & 1986 & 0.23 & 1966 \\
\hline 6 & 0.03 & 4 & 8 & 0.50 & 1983 & 0.87 & 1948 \\
\hline 7 & 0.03 & 13 & 3 & 0.31 & 1990 & 0.31 & 1990 \\
\hline 8 & 0.03 & 9 & 9 & 0.34 & 1993 & 0.59 & 1953 \\
\hline 9 & 0.03 & 9 & 9 & 0.41 & 1995 & 0.41 & $1995^{\text {(a) }}$ \\
\hline 10 & 0.04 & 14 & 5 & 0.22 & 1995 & 0.22 & 1995 \\
\hline 11 & 0.04 & 11 & 7 & 0.19 & 1995 & 0.48 & 1959 \\
\hline 12 & 0.04 & 7 & 11 & 0.32 & 1973 & 0.58 & 1958 \\
\hline 13 & 0.04 & 9 & 7 & 0.33 & 1980 & 0.37 & 1950 \\
\hline 14 & 0.04 & 16 & 2 & 0.27 & 1993 & 0.40 & $1968^{(\mathrm{a})}$ \\
\hline 15 & 0.04 & 7 & 6 & 0.43 & 1978 & 0.43 & 1978 \\
\hline 16 & 0.04 & 14 & 5 & 0.53 & 1971 & 0.70 & 1954 \\
\hline 17 & 0.03 & 8 & 6 & 0.31 & 1997 & 0.31 & 1997 \\
\hline 18 & 0.03 & 8 & 8 & 0.28 & 1996 & 0.28 & 1996 \\
\hline 19 & 0.03 & 8 & 3 & 0.12 & 1983 & 0.39 & 1950 \\
\hline 20 & 0.02 & 8 & 2 & 0.26 & 1985 & 0.32 & 1953 \\
\hline 21 & 0.02 & 7 & 6 & 0.16 & 1997 & 0.16 & $1997^{(\mathrm{a})}$ \\
\hline 22 & 0.02 & 7 & 7 & 0.47 & 1999 & 0.54 & 1970 \\
\hline 23 & 0.03 & 11 & 5 & 0.13 & 1998 & 0.27 & 1965 \\
\hline 24 & 0.03 & 8 & 4 & 0.26 & 1996 & 0.26 & 1996 \\
\hline 25 & 0.03 & 7 & 4 & 0.72 & 1975 & 0.72 & 1975 \\
\hline 26 & 0.02 & 7 & 4 & 0.20 & 1983 & 0.36 & 1970 \\
\hline 27 & 0.02 & 9 & 5 & 0.21 & 1996 & 0.32 & 1954 \\
\hline 28 & 0.02 & 8 & 5 & 0.19 & $1995^{(\mathrm{a})}$ & 0.19 & $1995^{\text {(a) }}$ \\
\hline 29 & 0.02 & 6 & 4 & 0.21 & 1986 & 0.33 & 1958 \\
\hline 30 & 0.02 & 7 & 6 & 0.24 & 1995 & 0.27 & 2003 \\
\hline 31 & 0.02 & 9 & 9 & 0.69 & 1997 & $0.94^{(\mathrm{b})}$ & $1963^{(\mathrm{b})}$ \\
\hline \multicolumn{8}{|c|}{ February } \\
\hline 1 & 0.02 & 8 & 6 & 0.26 & 1985 & 0.72 & 1961 \\
\hline 2 & 0.02 & 4 & 9 & 0.12 & 1980 & 0.26 & 1963 \\
\hline 3 & 0.02 & 6 & 6 & 0.31 & 1998 & 0.31 & 1998 \\
\hline 4 & 0.02 & 6 & 3 & 0.28 & 1975 & 0.28 & 1975 \\
\hline 5 & 0.02 & 7 & 7 & 0.10 & 1996 & 0.15 & 1953 \\
\hline 6 & 0.02 & 9 & 4 & 0.16 & $1983^{(\mathrm{a})}$ & 0.18 & 1961 \\
\hline 7 & 0.02 & 8 & 6 & 0.34 & 1995 & 0.53 & 2002 \\
\hline 8 & 0.02 & 5 & 5 & 0.12 & 1985 & 0.12 & 1985 \\
\hline 9 & 0.02 & 7 & 4 & 0.21 & 1992 & 0.43 & 1959 \\
\hline 10 & 0.02 & 2 & 5 & 0.15 & 1973 & 0.64 & 1961 \\
\hline 11 & 0.02 & 10 & 5 & 0.25 & 1997 & 0.30 & 1969 \\
\hline 12 & 0.02 & 9 & 5 & 0.37 & 1998 & 0.42 & 1958 \\
\hline 13 & 0.02 & 9 & 7 & 0.21 & 1981 & 0.21 & 1981 \\
\hline 14 & 0.03 & 7 & 8 & 0.39 & 1986 & 0.39 & 1986 \\
\hline 15 & 0.03 & 10 & 4 & 0.20 & 1982 & 0.30 & 1970 \\
\hline 16 & 0.04 & 5 & 8 & 0.42 & 1989 & 0.42 & 1989 \\
\hline 17 & 0.04 & 10 & 5 & 0.42 & 1989 & 0.42 & 1989 \\
\hline 18 & 0.03 & 11 & 5 & 0.34 & 1983 & 0.34 & $1983^{(\mathrm{a})}$ \\
\hline 19 & 0.03 & 10 & 5 & 0.78 & 1993 & $0.78^{(b)}$ & $1993^{(b)}$ \\
\hline 20 & 0.03 & 10 & 4 & 0.18 & 1984 & 0.18 & 1984 \\
\hline 21 & 0.03 & 10 & 5 & 0.20 & 1986 & 0.36 & 1956 \\
\hline 22 & 0.02 & 3 & 2 & 0.15 & 1989 & 0.21 & 1949 \\
\hline
\end{tabular}


Table 4.10. (contd)

\begin{tabular}{|c|c|c|c|c|c|c|c|}
\hline \multirow[b]{3}{*}{ Day } & \multicolumn{5}{|c|}{ Normal Period (1971-2000) } & \multicolumn{2}{|c|}{$\begin{array}{c}\text { Historical Period } \\
(1945-2003)\end{array}$} \\
\hline & \multirow[b]{2}{*}{ Normal } & \multicolumn{2}{|c|}{ Number of Years } & \multirow[b]{2}{*}{ Maximum } & \multirow[b]{2}{*}{ Year } & \multirow[b]{2}{*}{ Maximum } & \multirow[b]{2}{*}{ Year } \\
\hline & & W/Meas. & $\mathrm{W} /$ Trace & & & & \\
\hline 23 & 0.02 & 7 & 4 & 0.16 & 1986 & 0.22 & 1968 \\
\hline 24 & 0.02 & 7 & 7 & 0.13 & 1996 & 0.33 & 1950 \\
\hline 25 & 0.02 & 9 & 7 & 0.22 & $1996^{(\mathrm{a})}$ & 0.25 & 1948 \\
\hline 26 & 0.02 & 7 & 4 & 0.30 & $2000^{(\mathrm{a})}$ & 0.30 & $2000^{(a)}$ \\
\hline 27 & 0.02 & 9 & 5 & 0.32 & 1999 & 0.32 & 1999 \\
\hline 28 & 0.02 & 8 & 2 & 0.33 & 1977 & 0.33 & 1977 \\
\hline 29 & 0.02 & 2 & 2 & 0.04 & 1984 & 0.04 & 1984 \\
\hline \multicolumn{8}{|c|}{ March } \\
\hline 1 & 0.02 & 11 & 3 & 0.15 & 1972 & 0.15 & 1972 \\
\hline 2 & 0.02 & 8 & 7 & 0.20 & 1991 & 0.20 & $1991^{(\mathrm{a})}$ \\
\hline 3 & 0.02 & 8 & 6 & 0.15 & $1991^{(\mathrm{a})}$ & 0.15 & $1991^{(\mathrm{a})}$ \\
\hline 4 & 0.02 & 12 & 5 & 0.48 & 2000 & 0.48 & 2000 \\
\hline 5 & 0.02 & 5 & 6 & 0.23 & 1989 & 0.23 & 1989 \\
\hline 6 & 0.02 & 5 & 2 & 0.07 & $1971^{\text {(a) }}$ & 0.24 & 1957 \\
\hline 7 & 0.02 & 6 & 4 & 0.21 & 1986 & 0.21 & 1986 \\
\hline 8 & 0.02 & 7 & 4 & 0.19 & 1988 & 0.23 & 1951 \\
\hline 9 & 0.02 & 4 & 8 & 0.42 & 1995 & 0.42 & 1995 \\
\hline 10 & 0.02 & 14 & 3 & 0.21 & 1995 & 0.21 & 1995 \\
\hline 11 & 0.02 & 6 & 7 & 0.24 & 1989 & 0.24 & 1989 \\
\hline 12 & 0.02 & 7 & 3 & 0.42 & 1987 & 0.42 & 1987 \\
\hline 13 & 0.02 & 8 & 8 & 0.35 & 1983 & 0.35 & 1983 \\
\hline 14 & 0.02 & 6 & 5 & 0.16 & 1995 & 0.16 & 1995 \\
\hline 15 & 0.02 & 7 & 3 & 0.18 & 1987 & 0.25 & 1949 \\
\hline 16 & 0.02 & 9 & 5 & 0.34 & 1997 & 0.34 & $1997^{(\mathrm{a})}$ \\
\hline 17 & 0.02 & 3 & 6 & 0.03 & 1975 & 0.16 & 1949 \\
\hline 18 & 0.02 & 7 & 6 & 0.08 & 1997 & 0.25 & 1949 \\
\hline 19 & 0.02 & 3 & 3 & 0.12 & 1987 & 0.12 & 1987 \\
\hline 20 & 0.01 & 7 & 2 & 0.43 & 1984 & 0.43 & 1984 \\
\hline 21 & 0.02 & 4 & 2 & 0.10 & $1984^{(\mathrm{a})}$ & 0.18 & 1958 \\
\hline 22 & 0.02 & 6 & 6 & 0.21 & 1971 & 0.22 & 1961 \\
\hline 23 & 0.02 & 6 & 6 & 0.26 & 1986 & 0.26 & 1986 \\
\hline 24 & 0.02 & 4 & 0 & 0.52 & 1991 & $0.52^{(\mathfrak{b})}$ & $1991^{(\mathrm{b})}$ \\
\hline 25 & 0.02 & 7 & 2 & 0.43 & 1971 & 0.43 & 1971 \\
\hline 26 & 0.02 & 4 & 5 & 0.50 & 1981 & 0.50 & 1981 \\
\hline 27 & 0.02 & 4 & 3 & 0.42 & 1979 & 0.42 & 1979 \\
\hline 28 & 0.02 & 4 & 2 & 0.13 & 1982 & 0.13 & 1982 \\
\hline 29 & 0.01 & 6 & 1 & 0.15 & 1983 & 0.15 & 1983 \\
\hline 30 & 0.01 & 3 & 6 & 0.23 & 1974 & 0.23 & 1974 \\
\hline 31 & 0.01 & 5 & 5 & 0.26 & 1996 & 0.26 & 1996 \\
\hline \multicolumn{8}{|c|}{ April } \\
\hline 1 & 0.01 & 6 & 7 & 0.18 & 1983 & 0.22 & 1958 \\
\hline 2 & 0.01 & 5 & 4 & 0.11 & 1993 & 0.18 & 1948 \\
\hline 3 & 0.01 & 3 & 4 & 0.10 & 1993 & 0.18 & 1947 \\
\hline 4 & 0.01 & 4 & 5 & 0.13 & 1984 & 0.18 & 1948 \\
\hline 5 & 0.01 & 5 & 4 & 0.07 & 1972 & 0.44 & 1969 \\
\hline 6 & 0.01 & 6 & 3 & 0.36 & 1982 & 0.36 & 1982 \\
\hline 7 & 0.01 & 3 & 3 & 0.22 & 1984 & 0.30 & 1953 \\
\hline 8 & 0.01 & 7 & 3 & 0.18 & 1991 & 0.18 & 1991 \\
\hline 9 & 0.01 & 4 & 9 & 0.32 & 1992 & 0.32 & 1992 \\
\hline 10 & 0.02 & 3 & 6 & 0.06 & 1995 & 0.26 & 2001 \\
\hline 11 & 0.02 & 4 & 9 & 0.23 & 1982 & 0.25 & 2001 \\
\hline 12 & 0.01 & 6 & 2 & 0.36 & 1995 & 0.36 & 1995 \\
\hline 13 & 0.01 & 5 & 2 & 0.54 & 2000 & 0.54 & 2000 \\
\hline
\end{tabular}


Table 4.10. (contd)

\begin{tabular}{|c|c|c|c|c|c|c|c|}
\hline \multirow[b]{3}{*}{ Day } & \multicolumn{5}{|c|}{ Normal Period (1971-2000) } & \multicolumn{2}{|c|}{$\begin{array}{c}\text { Historical Period } \\
(1945-2003)\end{array}$} \\
\hline & \multirow[b]{2}{*}{$\underline{\text { Normal }}$} & \multicolumn{2}{|c|}{ Number of Years } & \multirow[b]{2}{*}{$\underline{\text { Maximum }}$} & \multirow[b]{2}{*}{ Year } & \multirow[b]{2}{*}{ Maximum } & \multirow[b]{2}{*}{ Year } \\
\hline & & W/Meas. & W/Trace & & & & \\
\hline 14 & 0.01 & 5 & 5 & 0.17 & $1975^{(\mathrm{a})}$ & 1.04 & $2003^{(b)}$ \\
\hline 15 & 0.01 & 2 & 6 & 0.17 & 1991 & 0.17 & 1991 \\
\hline 16 & 0.01 & 4 & 6 & 0.08 & $1979^{(\mathrm{a})}$ & 0.11 & 1948 \\
\hline 17 & 0.01 & 3 & 7 & 0.36 & 1988 & 0.36 & 1988 \\
\hline 18 & 0.01 & 1 & 4 & 0.12 & 1984 & 0.31 & 1967 \\
\hline 19 & 0.02 & 5 & 8 & 0.22 & 1994 & 0.41 & 1970 \\
\hline 20 & 0.02 & 5 & 3 & 0.56 & 1980 & 0.56 & 1980 \\
\hline 21 & 0.02 & 1 & 4 & 0.07 & 1989 & 0.07 & 1989 \\
\hline 22 & 0.02 & 7 & 4 & 0.28 & 1996 & 0.28 & 1996 \\
\hline 23 & 0.02 & 7 & 4 & 0.22 & 1974 & 0.22 & 1974 \\
\hline 24 & 0.02 & 4 & 7 & 0.22 & 1975 & 0.22 & 1975 \\
\hline 25 & 0.02 & 5 & 6 & 0.35 & 1989 & 0.35 & 1989 \\
\hline 26 & 0.02 & 2 & 3 & 0.04 & 1989 & 0.67 & 2003 \\
\hline 27 & 0.02 & 6 & 5 & 0.34 & 1995 & 0.34 & 1995 \\
\hline 28 & 0.02 & 7 & 4 & 0.48 & 1988 & 0.51 & 1951 \\
\hline 29 & 0.02 & 2 & 1 & 0.10 & 1992 & 0.30 & 1961 \\
\hline 30 & 0.02 & 8 & 0 & 0.12 & 1984 & 0.12 & 1984 \\
\hline \multicolumn{8}{|c|}{ May } \\
\hline 1 & 0.01 & 4 & 5 & 0.19 & 1984 & 0.19 & 1984 \\
\hline 2 & 0.01 & 6 & 2 & 0.17 & 1975 & 0.17 & 1975 \\
\hline 3 & 0.01 & 6 & 5 & 0.29 & 1977 & 0.29 & 1977 \\
\hline 4 & 0.01 & 6 & 3 & 0.06 & 1973 & 0.10 & 1967 \\
\hline 5 & 0.02 & 8 & 2 & 0.07 & 2000 & 0.28 & 1963 \\
\hline 6 & 0.02 & 4 & 6 & 0.20 & 1986 & 0.20 & 1986 \\
\hline 7 & 0.02 & 4 & 2 & 0.39 & 1983 & 0.39 & 1983 \\
\hline 8 & 0.02 & 5 & 7 & 0.55 & 1972 & 0.55 & 1972 \\
\hline 9 & 0.02 & 3 & 5 & 0.53 & 1995 & 0.53 & 1995 \\
\hline 10 & 0.02 & 5 & 5 & 0.15 & 1980 & 0.39 & 1961 \\
\hline 11 & 0.02 & 6 & 3 & 0.11 & 1975 & 0.39 & 1951 \\
\hline 12 & 0.01 & 4 & 5 & 0.14 & 1996 & 0.50 & 1970 \\
\hline 13 & 0.01 & 4 & 3 & 0.11 & 1985 & 0.15 & 1952 \\
\hline 14 & 0.01 & 6 & 6 & 0.25 & 1978 & 0.25 & 1978 \\
\hline 15 & 0.01 & 5 & 5 & 0.58 & 1994 & 0.58 & 1994 \\
\hline 16 & 0.02 & 1 & 5 & 0.14 & 1991 & 0.14 & 1991 \\
\hline 17 & 0.02 & 8 & 2 & 0.23 & 1998 & 0.25 & 1959 \\
\hline 18 & 0.02 & 6 & 3 & 0.13 & 1981 & 0.13 & 1981 \\
\hline 19 & 0.02 & 5 & 3 & 0.20 & 1994 & 0.55 & 1948 \\
\hline 20 & 0.02 & 5 & 1 & 0.70 & 1972 & 0.70 & 1972 \\
\hline 21 & 0.02 & 4 & 1 & 0.69 & 1972 & 0.69 & 1972 \\
\hline 22 & 0.02 & 5 & 4 & 0.12 & 1984 & 0.12 & 1984 \\
\hline 23 & 0.03 & 5 & 5 & 0.33 & 1990 & 0.33 & 1990 \\
\hline 24 & 0.02 & 5 & 3 & 0.14 & 1998 & 0.51 & 1962 \\
\hline 25 & 0.02 & 8 & 1 & 0.74 & 1981 & 0.74 & 1981 \\
\hline 26 & 0.02 & 4 & 7 & 0.79 & 1980 & $0.79^{(\mathfrak{b})}$ & $1980^{(\mathrm{b})}$ \\
\hline 27 & 0.02 & 7 & 3 & 0.11 & 1990 & 0.16 & 2002 \\
\hline 28 & 0.02 & 8 & 4 & 0.28 & 1988 & 0.28 & 1988 \\
\hline 29 & 0.02 & 4 & 3 & 0.09 & 1996 & 0.11 & 1961 \\
\hline 30 & 0.02 & 7 & 1 & 0.14 & 1987 & 0.14 & 1987 \\
\hline 31 & 0.02 & 6 & 3 & 0.45 & 2000 & 0.45 & 2000 \\
\hline \multicolumn{8}{|c|}{ June } \\
\hline 1 & 0.02 & 2 & 3 & 0.29 & 1977 & 0.29 & 1977 \\
\hline 2 & 0.02 & 4 & 2 & 0.10 & 1975 & 0.12 & 1966 \\
\hline 3 & 0.02 & 4 & 11 & 0.30 & 1971 & 0.30 & 1971 \\
\hline
\end{tabular}


Table 4.10. (contd)

\begin{tabular}{|c|c|c|c|c|c|c|c|}
\hline \multirow[b]{3}{*}{ Day } & \multicolumn{5}{|c|}{ Normal Period (1971-2000) } & \multicolumn{2}{|c|}{$\begin{array}{c}\text { Historical Period } \\
(1945-2003)\end{array}$} \\
\hline & \multicolumn{3}{|c|}{ Number of Years } & \multirow[b]{2}{*}{ Maximum } & \multirow[b]{2}{*}{ Year } & \multirow[b]{2}{*}{ Maximum } & \multirow[b]{2}{*}{ Year } \\
\hline & $\underline{\text { Normal }}$ & W/Meas. & W/Trace & & & & \\
\hline 4 & 0.02 & 7 & 2 & 0.25 & 1984 & 0.45 & 1951 \\
\hline 5 & 0.02 & 5 & 4 & 0.49 & 1991 & 0.49 & 1991 \\
\hline 6 & 0.02 & 5 & 4 & 0.36 & 1990 & 0.54 & 1951 \\
\hline 7 & 0.02 & 7 & 4 & 0.15 & 1972 & 0.71 & 1947 \\
\hline 8 & 0.02 & 6 & 3 & 0.21 & 1981 & 0.49 & 1964 \\
\hline 9 & 0.02 & 2 & 3 & 0.04 & 1972 & 0.22 & 1948 \\
\hline 10 & 0.01 & 1 & 4 & 0.08 & 1983 & 0.14 & 1956 \\
\hline 11 & 0.01 & 4 & 4 & 0.06 & 1997 & 0.39 & 1950 \\
\hline 12 & 0.01 & 5 & 7 & 0.79 & 1992 & 0.79 & 1992 \\
\hline 13 & 0.01 & 6 & 3 & 0.35 & 1980 & 0.49 & 1948 \\
\hline 14 & 0.01 & 4 & 1 & 0.10 & 1995 & 0.37 & 1956 \\
\hline 15 & 0.01 & 6 & 2 & 0.03 & $1998^{(a)}$ & 0.15 & 1964 \\
\hline 16 & 0.01 & 3 & 5 & 0.14 & 1980 & 0.18 & 1948 \\
\hline 17 & 0.01 & 2 & 5 & 0.06 & 1975 & $1.09^{(b)}$ & $1950^{(b)}$ \\
\hline 18 & 0.01 & 3 & 3 & 0.09 & 1994 & 0.09 & 1994 \\
\hline 19 & 0.01 & 4 & 2 & 0.29 & 1998 & 0.29 & 1998 \\
\hline 20 & 0.01 & 6 & 2 & 0.24 & 1984 & 0.24 & 1984 \\
\hline 21 & 0.01 & 4 & 2 & 0.03 & $1991^{(\mathrm{a})}$ & 0.32 & 1967 \\
\hline 22 & 0.01 & 4 & 6 & 0.14 & 1971 & 0.14 & 1971 \\
\hline 23 & 0.01 & 6 & 1 & 0.05 & $1996^{(\mathrm{a})}$ & 0.17 & 1963 \\
\hline 24 & 0.01 & 7 & 2 & 0.21 & 1972 & 0.21 & 1972 \\
\hline 25 & 0.01 & 3 & 5 & 0.02 & 1980 & 0.03 & 1954 \\
\hline 26 & 0.02 & 3 & 4 & 0.27 & 1982 & 0.39 & 2001 \\
\hline 27 & 0.02 & 3 & 1 & 0.37 & 1983 & 0.37 & 1983 \\
\hline 28 & 0.01 & 5 & 6 & 0.24 & 1992 & 0.24 & 1992 \\
\hline 29 & 0.01 & 5 & 2 & 0.53 & 1991 & 0.53 & 1991 \\
\hline 30 & 0.01 & 1 & 2 & 0.06 & 1976 & 0.06 & 1976 \\
\hline \multicolumn{8}{|c|}{ July } \\
\hline 1 & 0.01 & 2 & 2 & 0.16 & 1978 & 0.31 & 1966 \\
\hline 2 & 0.01 & 3 & 6 & 0.07 & 1986 & 0.34 & 1966 \\
\hline 3 & 0.01 & 3 & 3 & 0.31 & 1978 & 0.31 & 1978 \\
\hline 4 & 0.01 & 5 & 2 & 0.10 & 1986 & 0.10 & 1986 \\
\hline 5 & 0.01 & 3 & 2 & 0.19 & 1981 & 0.36 & 1951 \\
\hline 6 & 0.01 & 4 & 2 & 0.25 & 2000 & 0.25 & 2000 \\
\hline 7 & 0.01 & 3 & 1 & 0.22 & 1982 & 0.30 & 1963 \\
\hline 8 & 0.01 & 7 & 2 & 0.20 & 1995 & 0.20 & 1995 \\
\hline 9 & 0.01 & 6 & 1 & 0.27 & 1987 & 0.27 & 1987 \\
\hline 10 & 0.01 & 4 & 5 & 0.12 & 1997 & 0.16 & 1954 \\
\hline 11 & 0.01 & 2 & 3 & 0.04 & 1979 & 0.04 & 1979 \\
\hline 12 & 0.01 & 0 & 2 & $\mathrm{~T}$ & $1982^{(\mathrm{a})}$ & $\mathrm{T}$ & $1982^{\text {(a) }}$ \\
\hline 13 & 0.01 & 4 & 4 & 0.28 & 1975 & 0.28 & 1975 \\
\hline 14 & 0.01 & 0 & 2 & $\mathrm{~T}$ & $1993^{(\mathrm{a})}$ & 0.05 & 1957 \\
\hline 15 & 0.01 & 3 & 1 & 0.08 & 1991 & 0.08 & 1991 \\
\hline 16 & 0.02 & 6 & 2 & 0.50 & 1993 & 0.50 & 1993 \\
\hline 17 & 0.01 & 6 & 2 & 0.89 & 1993 & $0.89^{(\mathrm{b})}$ & $1993^{(\mathrm{b})}$ \\
\hline 18 & 0.01 & 2 & 2 & 0.12 & 1987 & 0.12 & 1987 \\
\hline 19 & 0.01 & 2 & 2 & 0.45 & 1974 & 0.45 & 1974 \\
\hline 20 & 0.01 & 2 & 3 & 0.01 & $1992^{(\mathrm{a})}$ & 0.09 & 1965 \\
\hline 21 & $\mathrm{~T}$ & 1 & 2 & 0.01 & 1997 & 0.02 & 1965 \\
\hline 22 & $\mathrm{~T}$ & 0 & 3 & $\mathrm{~T}$ & $1993^{(\mathrm{a})}$ & $\mathrm{T}$ & $1993^{\text {(a) }}$ \\
\hline 23 & $\mathrm{~T}$ & 2 & 0 & 0.28 & 1992 & 0.28 & 1992 \\
\hline 24 & 0.01 & 2 & 2 & 0.06 & 1990 & 0.07 & 1955 \\
\hline 25 & 0.01 & 3 & 2 & 0.23 & 1983 & 0.23 & 1983 \\
\hline 26 & 0.01 & 1 & 2 & 0.04 & 1995 & 0.22 & 1955 \\
\hline
\end{tabular}


Table 4.10. (contd)

\begin{tabular}{|c|c|c|c|c|c|c|c|}
\hline \multirow[b]{3}{*}{ Day } & \multicolumn{5}{|c|}{ Normal Period (1971-2000) } & \multicolumn{2}{|c|}{$\begin{array}{c}\text { Historical Period } \\
(1945-2003)\end{array}$} \\
\hline & \multirow[b]{2}{*}{$\underline{\text { Normal }}$} & \multicolumn{2}{|c|}{ Number of Years } & \multirow[b]{2}{*}{ Maximum } & \multirow[b]{2}{*}{ Year } & \multirow[b]{2}{*}{ Maximum } & \multirow[b]{2}{*}{ Year } \\
\hline & & W/Meas. & W/Trace & & & & \\
\hline 27 & 0.01 & 1 & 0 & 0.02 & 1983 & 0.31 & 1947 \\
\hline 28 & 0.01 & 1 & 3 & 0.06 & 1984 & 0.28 & 1947 \\
\hline 29 & 0.01 & 3 & 2 & 0.05 & 1997 & 0.05 & 1997 \\
\hline 30 & $\mathrm{~T}$ & 0 & 4 & $\mathrm{~T}$ & $1997^{(\mathrm{a})}$ & $\mathrm{T}$ & $2001^{(a)}$ \\
\hline 31 & $\mathrm{~T}$ & 3 & 2 & 0.16 & 1998 & 0.16 & 1998 \\
\hline \multicolumn{8}{|c|}{ August } \\
\hline 1 & $\mathrm{~T}$ & 1 & 0 & 0.08 & $1976^{(\mathrm{a})}$ & 0.08 & $1976^{(\mathrm{a})}$ \\
\hline 2 & $\mathrm{~T}$ & 2 & 0 & 0.01 & 1996 & 0.01 & $1996^{(a)}$ \\
\hline 3 & $\mathrm{~T}$ & 0 & 1 & $\mathrm{~T}$ & 1971 & 0.29 & 1962 \\
\hline 4 & $\mathrm{~T}$ & 1 & 0 & 0.01 & 1985 & 0.04 & 1948 \\
\hline 5 & $\mathrm{~T}$ & 1 & 3 & 0.48 & 1999 & 0.48 & 1999 \\
\hline 6 & 0.01 & 2 & 1 & 0.11 & 1976 & 0.11 & 1976 \\
\hline 7 & 0.01 & 2 & 1 & 0.33 & 1976 & 0.36 & 2003 \\
\hline 8 & 0.01 & 1 & 3 & 0.01 & 1994 & 0.08 & 1952 \\
\hline 9 & 0.01 & 2 & 1 & 0.10 & 1982 & 0.10 & 1982 \\
\hline 10 & $\mathrm{~T}$ & 1 & 3 & 0.06 & 1995 & 0.06 & 1947 \\
\hline 11 & $\mathrm{~T}$ & 1 & 1 & 0.01 & 1983 & 0.11 & 1947 \\
\hline 12 & $\mathrm{~T}$ & 1 & 2 & 0.01 & 1972 & 0.18 & 1962 \\
\hline 13 & 0.01 & 4 & 3 & 0.04 & $1987^{(\mathrm{a})}$ & 0.04 & $1987^{(\mathrm{a})}$ \\
\hline 14 & 0.01 & 4 & 3 & 0.09 & 1979 & 0.09 & 1979 \\
\hline 15 & 0.01 & 3 & 1 & 0.42 & 1972 & 0.42 & 1972 \\
\hline 16 & 0.01 & 1 & 1 & 0.24 & 1993 & 0.24 & 1993 \\
\hline 17 & 0.01 & 0 & 3 & $\mathrm{~T}$ & $1995^{(\mathrm{a})}$ & $\mathrm{T}$ & $1995^{\text {(a) }}$ \\
\hline 18 & 0.01 & 3 & 1 & 0.69 & 1975 & 0.69 & 1975 \\
\hline 19 & 0.01 & 3 & 4 & 0.05 & 1979 & 0.18 & 1954 \\
\hline 20 & 0.01 & 6 & 3 & 0.03 & 1978 & 0.22 & 1953 \\
\hline 21 & 0.02 & 3 & 4 & 0.76 & 1990 & $0.76^{(\mathrm{b})}$ & $1990^{(\mathrm{b})}$ \\
\hline 22 & 0.02 & 6 & 0 & 0.18 & 1978 & 0.18 & 1978 \\
\hline 23 & 0.02 & 3 & 1 & 0.14 & 1975 & 0.14 & 1975 \\
\hline 24 & 0.02 & 6 & 2 & 0.38 & 1977 & 0.38 & 1977 \\
\hline 25 & 0.01 & 1 & 2 & 0.29 & 1976 & 0.29 & 1976 \\
\hline 26 & 0.01 & 3 & 2 & 0.02 & $1994^{(\mathrm{a})}$ & 0.38 & 1953 \\
\hline 27 & 0.01 & 3 & 2 & 0.14 & 1989 & 0.14 & 1989 \\
\hline 28 & 0.01 & 2 & 5 & 0.13 & 1975 & 0.13 & 1975 \\
\hline 29 & 0.01 & 6 & 2 & 0.28 & 1977 & 0.51 & 1947 \\
\hline 30 & 0.01 & 3 & 3 & 0.61 & 1977 & 0.61 & 1977 \\
\hline 31 & 0.01 & 1 & 4 & 0.01 & 1973 & 0.02 & $1961^{(\mathrm{a})}$ \\
\hline \multicolumn{8}{|c|}{ September } \\
\hline 1 & 0.01 & 6 & 0 & 0.43 & 1971 & 0.43 & 1971 \\
\hline 2 & 0.01 & 3 & 3 & 0.17 & 1971 & 0.17 & 1971 \\
\hline 3 & 0.01 & 5 & 0 & 0.15 & 1997 & 0.15 & 1997 \\
\hline 4 & 0.01 & 1 & 2 & 0.02 & 1977 & 0.19 & 1960 \\
\hline 5 & 0.01 & 3 & 1 & 0.19 & 1971 & 0.19 & 1971 \\
\hline 6 & 0.01 & 4 & 0 & 0.48 & 1995 & 0.48 & 1995 \\
\hline 7 & 0.01 & 3 & 2 & 0.19 & 1995 & 0.23 & 1947 \\
\hline 8 & 0.01 & 2 & 4 & 0.10 & 1985 & 0.21 & 2003 \\
\hline 9 & 0.01 & 3 & 4 & 0.07 & 1985 & 0.07 & 1985 \\
\hline 10 & 0.01 & 2 & 3 & 0.27 & 2000 & 0.27 & 2000 \\
\hline 11 & 0.01 & 2 & 1 & 0.05 & 1982 & 0.10 & 1966 \\
\hline 12 & 0.01 & 0 & 1 & $\mathrm{~T}$ & $1980^{(a)}$ & 0.03 & 1958 \\
\hline 13 & 0.01 & 3 & 1 & 0.79 & 1980 & $0.79^{(\mathrm{b})}$ & $1980^{(b)}$ \\
\hline 14 & 0.01 & 5 & 3 & 0.14 & 1996 & 0.41 & 1959 \\
\hline 15 & 0.01 & 3 & 5 & 0.54 & 1986 & 0.54 & 1986 \\
\hline
\end{tabular}


Table 4.10. (contd)

\begin{tabular}{|c|c|c|c|c|c|c|c|}
\hline \multirow[b]{3}{*}{ Day } & \multicolumn{5}{|c|}{ Normal Period (1971-2000) } & \multicolumn{2}{|c|}{$\begin{array}{c}\text { Historical Period } \\
(1945-2003)\end{array}$} \\
\hline & & \multicolumn{2}{|c|}{ Number of Years } & \multirow[b]{2}{*}{ Maximum } & \multirow[b]{2}{*}{ Year } & \multirow[b]{2}{*}{ Maximum } & \multirow[b]{2}{*}{ Year } \\
\hline & Normal & W/Meas. & W/Trace & & & & \\
\hline 16 & 0.01 & 2 & 5 & 0.03 & 1985 & 0.66 & 1947 \\
\hline 17 & 0.02 & 3 & 4 & 0.16 & 1985 & 0.26 & 1969 \\
\hline 18 & 0.02 & 4 & 5 & 0.22 & 1983 & 0.41 & 1959 \\
\hline 19 & 0.02 & 6 & 4 & 0.26 & 1973 & 0.26 & 1973 \\
\hline 20 & 0.01 & 5 & 6 & 0.13 & 1988 & 0.13 & 1988 \\
\hline 21 & 0.01 & 4 & 2 & 0.05 & 2000 & 0.05 & 2000 \\
\hline 22 & 0.01 & 3 & 1 & 0.20 & 1984 & 0.20 & 1984 \\
\hline 23 & 0.01 & 5 & 0 & 0.21 & 1986 & 0.21 & 1986 \\
\hline 24 & 0.01 & 4 & 1 & 0.10 & 1977 & 0.10 & 1977 \\
\hline 25 & 0.01 & 4 & 3 & 0.25 & 1982 & 0.25 & 1982 \\
\hline 26 & 0.01 & 4 & 2 & 0.22 & 1981 & 0.22 & 1981 \\
\hline 27 & 0.01 & 4 & 0 & 0.38 & 1981 & 0.43 & 1955 \\
\hline 28 & 0.01 & 4 & 2 & 0.11 & 1977 & 0.34 & 1962 \\
\hline 29 & 0.01 & 3 & 1 & 0.07 & 1986 & 0.07 & 1986 \\
\hline 30 & 0.01 & 1 & 3 & 0.02 & 1995 & 0.03 & $1953^{(a)}$ \\
\hline \multicolumn{8}{|c|}{ October } \\
\hline 1 & 0.01 & 2 & 4 & 0.14 & 2000 & $1.60^{(b)}$ & $1957^{(b)}$ \\
\hline 2 & 0.01 & 3 & 5 & 0.09 & 1995 & 0.31 & 1957 \\
\hline 3 & 0.01 & 4 & 1 & 0.38 & 1995 & 0.38 & 1995 \\
\hline 4 & 0.01 & 1 & 1 & 0.01 & 1996 & 0.15 & 1950 \\
\hline 5 & $\mathrm{~T}$ & 0 & 4 & $\mathrm{~T}$ & $1981^{(\mathrm{a})}$ & 0.25 & 1950 \\
\hline 6 & 0.01 & 5 & 2 & 0.22 & 1973 & 0.22 & 1973 \\
\hline 7 & 0.01 & 1 & 1 & 0.25 & 1985 & 0.25 & 1985 \\
\hline 8 & 0.01 & 2 & 3 & 0.46 & 1997 & 0.49 & 1950 \\
\hline 9 & 0.01 & 1 & 3 & 0.04 & 1975 & 0.32 & 1947 \\
\hline 10 & 0.01 & 5 & 4 & 0.22 & 2000 & 0.32 & 1959 \\
\hline 11 & 0.01 & 2 & 3 & 0.18 & 1995 & 0.43 & 1968 \\
\hline 12 & 0.01 & 3 & 4 & 0.09 & 1996 & 0.52 & 1962 \\
\hline 13 & 0.01 & 4 & 2 & 0.16 & 1994 & 0.16 & 1994 \\
\hline 14 & 0.01 & 4 & 3 & 0.22 & 1994 & 0.43 & 1950 \\
\hline 15 & 0.01 & 1 & 1 & 0.01 & 1980 & 0.15 & 1947 \\
\hline 16 & 0.01 & 1 & 2 & 0.02 & 1993 & 0.24 & 1947 \\
\hline 17 & 0.01 & 3 & 1 & 0.09 & 1995 & 0.23 & 1950 \\
\hline 18 & 0.01 & 4 & 1 & 0.28 & 1979 & 0.28 & 1979 \\
\hline 19 & 0.01 & 4 & 3 & 0.12 & 1979 & 0.64 & 1947 \\
\hline 20 & 0.02 & 5 & 4 & 0.18 & 2000 & 0.37 & 1947 \\
\hline 21 & 0.02 & 6 & 3 & 0.45 & 1975 & 0.45 & 1975 \\
\hline 22 & 0.02 & 6 & 4 & 0.20 & 1983 & 0.23 & 1957 \\
\hline 23 & 0.02 & 4 & 2 & 0.39 & 1973 & 0.39 & 1973 \\
\hline 24 & 0.02 & 6 & 3 & 0.12 & 1991 & 0.12 & 1991 \\
\hline 25 & 0.03 & 8 & 3 & 0.22 & 1975 & 0.22 & 1975 \\
\hline 26 & 0.03 & 8 & 2 & 0.12 & 1989 & 0.18 & 1956 \\
\hline 27 & 0.03 & 7 & 4 & 0.36 & 1999 & 0.36 & 1999 \\
\hline 28 & 0.03 & 8 & 6 & 0.93 & 1982 & 0.93 & 1982 \\
\hline 29 & 0.03 & 11 & 4 & 0.18 & 1986 & 0.38 & 1950 \\
\hline 30 & 0.03 & 5 & 4 & 0.52 & 1990 & 0.52 & 1990 \\
\hline 31 & 0.03 & 7 & 6 & 0.64 & 1973 & 0.64 & 1973 \\
\hline \multicolumn{8}{|c|}{ November } \\
\hline 1 & 0.03 & 8 & 3 & 0.18 & 1987 & 0.26 & 1948 \\
\hline 2 & 0.03 & 4 & 2 & 0.25 & 1984 & 0.25 & 1984 \\
\hline 3 & 0.03 & 6 & 3 & 0.15 & 1972 & 0.28 & 1965 \\
\hline 4 & 0.03 & 10 & 3 & 0.24 & 1991 & 0.24 & 1991 \\
\hline
\end{tabular}


Table 4.10. (contd)

\begin{tabular}{|c|c|c|c|c|c|c|c|}
\hline \multirow[b]{3}{*}{ Day } & \multicolumn{5}{|c|}{ Normal Period (1971-2000) } & \multicolumn{2}{|c|}{$\begin{array}{c}\text { Historical Period } \\
(1945-2003)\end{array}$} \\
\hline & \multicolumn{3}{|c|}{ Number of Years } & \multirow[b]{2}{*}{$\underline{\text { Maximum }}$} & \multirow[b]{2}{*}{ Year } & \multirow[b]{2}{*}{ Maximum } & \multirow[b]{2}{*}{ Year } \\
\hline & Normal & W/Meas. & W/Trace & & & & \\
\hline 5 & 0.03 & 10 & 2 & 0.62 & 1998 & 0.62 & 1998 \\
\hline 6 & 0.03 & 10 & 3 & 0.30 & 1980 & 0.30 & $1980^{(a)}$ \\
\hline 7 & 0.03 & 8 & 4 & 0.57 & 1997 & 0.57 & 1997 \\
\hline 8 & 0.03 & 9 & 5 & 0.53 & 2000 & 0.53 & 2000 \\
\hline 9 & 0.03 & 8 & 6 & 0.16 & 1973 & 0.27 & 1949 \\
\hline 10 & 0.03 & 8 & 5 & 0.66 & 1983 & 0.66 & 1983 \\
\hline 11 & 0.03 & 10 & 3 & 0.19 & 1973 & 0.26 & 1970 \\
\hline 12 & 0.03 & 7 & 5 & 0.57 & 1973 & 0.57 & 1973 \\
\hline 13 & 0.03 & 8 & 5 & 0.47 & 1981 & 0.47 & 1981 \\
\hline 14 & 0.03 & 4 & 5 & 0.10 & 1981 & 0.35 & 1966 \\
\hline 15 & 0.03 & 11 & 5 & 0.19 & 1994 & 0.19 & 1994 \\
\hline 16 & 0.04 & 13 & 2 & 0.42 & 1979 & 0.45 & 2001 \\
\hline 17 & 0.04 & 11 & 6 & 0.12 & 1974 & 0.18 & 1955 \\
\hline 18 & 0.04 & 6 & 2 & 0.51 & 1996 & 0.51 & 1996 \\
\hline 19 & 0.04 & 12 & 5 & 1.39 & 1996 & $1.39^{(\mathrm{b})}$ & $1996^{(b)}$ \\
\hline 20 & 0.04 & 3 & 5 & 0.26 & 1984 & 0.42 & 1966 \\
\hline 21 & 0.04 & 10 & 5 & 0.50 & 1985 & 0.50 & 1985 \\
\hline 22 & 0.04 & 9 & 8 & 0.30 & 1979 & 0.30 & 1979 \\
\hline 23 & 0.04 & 15 & 4 & 0.42 & 1983 & 0.63 & 1949 \\
\hline 24 & 0.03 & 9 & 6 & 0.33 & 1996 & 0.37 & 1965 \\
\hline 25 & 0.03 & 8 & 7 & 0.30 & 2000 & 0.30 & 2000 \\
\hline 26 & 0.03 & 7 & 6 & 0.37 & 1991 & 0.54 & 1955 \\
\hline 27 & 0.03 & 12 & 5 & 0.49 & 1984 & 0.49 & 1984 \\
\hline 28 & 0.03 & 9 & 4 & 0.19 & 1986 & 0.46 & 2001 \\
\hline 29 & 0.03 & 11 & 4 & 0.18 & 1978 & 0.18 & 1978 \\
\hline 30 & 0.03 & 10 & 2 & 0.34 & 1998 & 0.34 & 1998 \\
\hline \multicolumn{8}{|c|}{ December } \\
\hline 1 & 0.03 & 8 & 2 & 0.11 & 1987 & 0.32 & 2003 \\
\hline 2 & 0.03 & 12 & 4 & 0.34 & $1985^{(\mathrm{a})}$ & 0.34 & $1985^{(a)}$ \\
\hline 3 & 0.03 & 7 & 5 & 0.56 & 1980 & 0.56 & 1980 \\
\hline 4 & 0.04 & 10 & 2 & 0.28 & 1974 & 0.28 & 1974 \\
\hline 5 & 0.04 & 10 & 5 & 0.32 & 1983 & 0.43 & 1963 \\
\hline 6 & 0.04 & 12 & 8 & 0.18 & 1985 & 0.18 & 1985 \\
\hline 7 & 0.04 & 10 & 3 & 0.32 & 1983 & 0.36 & 1948 \\
\hline 8 & 0.04 & 6 & 6 & 0.33 & 1993 & 0.36 & 1963 \\
\hline 9 & 0.04 & 7 & 10 & 0.55 & 1987 & 0.55 & 1987 \\
\hline 10 & 0.04 & 8 & 8 & 0.46 & 1992 & 0.54 & 1958 \\
\hline 11 & 0.04 & 8 & 4 & 0.40 & 1995 & 0.53 & 1958 \\
\hline 12 & 0.04 & 11 & 2 & 0.65 & 1995 & 0.65 & 1995 \\
\hline 13 & 0.04 & 8 & 5 & 0.76 & 1977 & 0.76 & 1977 \\
\hline 14 & 0.04 & 9 & 5 & 0.23 & 1981 & 0.50 & 2002 \\
\hline 15 & 0.03 & 10 & 1 & 0.22 & 1981 & 0.22 & 1981 \\
\hline 16 & 0.03 & 8 & 8 & 0.37 & 1994 & 0.37 & 1994 \\
\hline 17 & 0.03 & 8 & 5 & 0.22 & 1973 & 0.22 & 1973 \\
\hline 18 & 0.03 & 9 & 6 & 0.22 & 1981 & 0.27 & 1960 \\
\hline 19 & 0.03 & 10 & 7 & 0.16 & 1981 & 0.20 & 1953 \\
\hline 20 & 0.03 & 9 & 6 & 0.33 & 1982 & 0.33 & 1982 \\
\hline 21 & 0.03 & 10 & 6 & 0.30 & 1980 & 0.61 & 1955 \\
\hline 22 & 0.03 & 8 & 4 & 0.21 & 1972 & 0.59 & 1964 \\
\hline 23 & 0.04 & 6 & 8 & 0.31 & 1975 & 0.31 & 1975 \\
\hline 24 & 0.04 & 12 & 4 & 0.15 & 1980 & 0.36 & 1968 \\
\hline 25 & 0.04 & 12 & 7 & 0.32 & 1996 & 0.32 & 1996 \\
\hline 26 & 0.04 & 8 & 4 & 0.58 & 1996 & 0.58 & 1996 \\
\hline 27 & 0.04 & 8 & 10 & 0.36 & 1973 & 0.36 & 1973 \\
\hline
\end{tabular}


Table 4.10. (contd)

\begin{tabular}{|c|c|c|c|c|c|c|c|}
\hline \multirow[b]{3}{*}{ Day } & \multicolumn{5}{|c|}{ Normal Period (1971-2000) } & \multicolumn{2}{|c|}{$\begin{array}{l}\text { Historical Period } \\
(1945-2003)\end{array}$} \\
\hline & \multirow[b]{2}{*}{ Normal } & \multicolumn{2}{|c|}{ Number of Years } & \multirow[b]{2}{*}{ Maximum } & \multirow[b]{2}{*}{ Year } & & \\
\hline & & W/Meas. & $\overline{\mathrm{W} / \text { Trace }}$ & & & Maximum & Year \\
\hline 28 & 0.04 & 10 & 9 & 0.09 & $1990^{\text {(a) }}$ & 0.25 & 2002 \\
\hline 29 & 0.04 & 12 & 5 & 0.80 & 1996 & $0.80^{(\mathrm{b})}$ & $1996^{(b)}$ \\
\hline 30 & 0.03 & 8 & 3 & 0.29 & 1995 & 0.41 & 2002 \\
\hline 31 & 0.03 & 8 & 7 & 0.72 & 1996 & 0.72 & 1996 \\
\hline
\end{tabular}

(a) Most recent of several occurrences.

(b) Greatest monthly value and year of occurrence.

$\mathrm{T}=$ Trace . 


\subsection{Wind Climatology}

\subsection{Monthly and Annual Prevailing Wind Directions, Average Speeds, and Peak Gusts}

At the Hanford Meteorology Station, the prevailing wind direction for every month of the year is either WNW or NW (Table 5.1), and the peak gusts for every month are from the SSW, SW, or WSW. Hourly observations of wind direction and speed are taken at the 50-foot level of a 408-foot instrumented tower. The highest monthly average wind speeds occur in June, the lowest in December. The variability in monthly average wind speeds is much greater in the winter months than during the remainder of the year. The highest January average of $10.3 \mathrm{mph}$ is more than 3.5 times greater than the lowest $(2.9 \mathrm{mph})$; however, in June, the highest average $(10.7 \mathrm{mph})$ is only 1.4 times greater than the lowest $(7.7 \mathrm{mph})$.

Table 5.1. Monthly and Annual Prevailing Wind Directions, Average Speeds, and Peak Gusts at 50-Foot Level, 1945 through 2003

\begin{tabular}{|c|c|c|c|c|c|c|c|c|c|}
\hline \multirow[b]{2}{*}{ Month } & \multirow{2}{*}{$\begin{array}{l}\text { Prevailing } \\
\text { Direction }\end{array}$} & \multirow{2}{*}{$\begin{array}{c}\text { Average } \\
\text { Speed, mph }\end{array}$} & \multicolumn{2}{|c|}{ Highest Average } & \multicolumn{2}{|c|}{ Lowest Average } & \multicolumn{3}{|c|}{ Peak Gusts } \\
\hline & & & $\mathrm{mph}$ & Year & $\mathrm{mph}$ & Year & Speed, mph & Direction & Year \\
\hline Jan & NW & 6.3 & 10.3 & 1972 & 2.9 & 1985 & 80 & SW & 1972 \\
\hline Feb & NW & 7.1 & 11.1 & 1999 & 4.6 & 1963 & 65 & SW & 1971 \\
\hline Mar & WNW & 8.2 & 10.7 & $1977^{(a)}$ & 5.9 & 1958 & 70 & SW & 1956 \\
\hline Apr & WNW & 8.8 & 11.1 & $1972^{(a)}$ & 7.4 & $1989^{(a)}$ & 73 & SSW & 1972 \\
\hline May & WNW & 8.8 & 10.7 & 1983 & 5.8 & 1957 & 71 & SSW & 1948 \\
\hline Jun & NW & 9.1 & 10.7 & $1983^{(a)}$ & 7.7 & $1950^{(a)}$ & 72 & SW & 1957 \\
\hline Jul & NW & 8.6 & 10.7 & 1983 & 6.8 & 1955 & 69 & WSW & 1979 \\
\hline Aug & WNW & 8.0 & 9.5 & 1996 & 6.0 & 1956 & 66 & SW & 1961 \\
\hline Sep & WNW & 7.5 & 9.2 & 1961 & 5.4 & 1957 & 65 & SSW & 1953 \\
\hline Oct & NW & 6.6 & 9.1 & 1946 & 4.4 & 1952 & 72 & SW & 1997 \\
\hline Nov & NW & 6.4 & 10.0 & 1990 & 2.9 & 1956 & 67 & WSW & 1993 \\
\hline Dec & NW & 6.0 & 8.3 & 1968 & 3.3 & 1985 & 71 & SW & 1955 \\
\hline Annual & NW & 7.6 & 8.8 & 1999 & 6.2 & 1989 & 80 & SW & Jan 1972 \\
\hline
\end{tabular}

(a) Also in earlier years.

\subsection{Days with Peak Gusts Above or Below Specific Thresholds}

Table 5.2 lists the number of days by month and year with peak wind gusts (at 50-foot level) above or below specific threshold wind speeds. June and July have the highest average number of days with gusts $\geq 25 \mathrm{mph}$ (nearly 20 each); however, January, March, April, and December have the highest average number of days with gusts $\geq 40 \mathrm{mph}$ (nearly 3 days), and January and December have the highest average number of days with gusts $\geq 50 \mathrm{mph}$ ( 0.9 and 0.8 day, respectively). January also has the record highest number of gusts $\geq 40$ and $\geq 50 \mathrm{mph}$ at 11 and 7 days, respectively, in 1990. Calendar year 1990 recorded the most days with gusts $\geq 40$ and $\geq 50 \mathrm{mph}$ at 57 and 18 days, respectively. Of particular interest is that previous records for these categories were 41 days $\geq 40 \mathrm{mph}$ in 1961 and 10 days $\geq 50 \mathrm{mph}$ in 1972 . 


\subsection{Frequency of Monthly and Annual Wind Direction and Speed at 50-Foot Level}

Table 5.3 presents Hanford Meteorology Station data on the percent frequency of monthly and annual wind direction and wind speed at the 50-foot level. This table shows that for every month of the year the prevailing wind direction is either from the WNW or NW. Winds are relatively evenly distributed from the NNE through the SSW at between $2 \%$ and $4 \%$ on an annual average for each direction.

The wind speed class with the highest frequency of occurrence is 4 to $7 \mathrm{mph}$, with winds in that category $37 \%$ of the time. The speed class with the second highest frequency is 8 to $12 \mathrm{mph}$, at $25 \%$. Winds averaging greater than $25 \mathrm{mph}$ occur only $1 \%$ of the time on an annual basis, with the highest frequency occurring in March (1.6\%).

\subsection{Composite Wind Roses and Joint Frequency Distributions for the Hanford Meteorological Monitoring Network}

Figure 5.1 and Table 5.4 contain composite wind roses and joint frequency distributions at the 30 -foot level for the entire Hanford Meteorological Monitoring Network (see Table 1.1 and Figure 1.1) for the entire period of operation 1982 through 2003.

Figure 5.2 and Table 5.5 contain composite wind roses and joint frequency distributions at the 60-meter level for stations 9, 11, 13, and 21 for the entire period of operation 1986 through 2003. 
Table 5.2. Number of Days with Peak Gusts Above or Below Specific Thresholds at 50-Foot Level, 1945 through 2003

\begin{tabular}{|c|c|c|c|c|c|c|c|c|c|c|c|c|c|c|c|c|c|c|c|c|}
\hline \multirow[b]{2}{*}{ Month } & \multicolumn{5}{|c|}{ Days with Peak Gusts $\leq 12 \mathrm{mph}$} & \multicolumn{5}{|c|}{ Days with Peak Gusts $\geq 25 \mathrm{mph}$} & \multicolumn{5}{|c|}{ Days with Peak Gusts $\geq 40 \mathrm{mph}$} & \multicolumn{5}{|c|}{ Days with Peak Gusts $\geq 50 \mathrm{mph}$} \\
\hline & Avg & Max & Year & Min & Year & Avg & Max & Year & Min & Year & Avg & Max & Year & Min & Year & Avg & Max & Year & Min & Year \\
\hline Jan & 9.6 & 29 & 1985 & 3 & 1968 & 7.6 & 21 & 1953 & 0 & $1985^{(\mathrm{a})}$ & 2.8 & 11 & $1990^{(a)}$ & 0 & $2003^{(a)}$ & 0.9 & 7 & 1990 & 0 & $2003^{(\mathrm{a})}$ \\
\hline Feb & 6.2 & 16 & 1963 & 0 & 1990 & 8.6 & 17 & $1976^{(\mathrm{a})}$ & 2 & $1952^{\text {(a) }}$ & 2.4 & 10 & $1999^{(a)}$ & 0 & $2001^{\text {(a) }}$ & 0.6 & 4 & 1972 & 0 & $2002^{(\mathrm{a})}$ \\
\hline Mar & 2.6 & 8 & 1992 & 0 & $2003^{(a)}$ & 13.2 & 21 & 1977 & 4 & 1992 & 2.8 & 9 & 1956 & 0 & $1998^{(a)}$ & 0.6 & 4 & 1956 & 0 & $2000^{(\mathrm{a})}$ \\
\hline Apr & 0.6 & 6 & 1951 & 0 & $2003^{(a)}$ & 17.0 & 26 & 1954 & 8 & 1946 & 2.8 & 8 & 1991 & 0 & $1998^{(\mathrm{a})}$ & 0.4 & 2 & $1997^{(\mathrm{a})}$ & 0 & $2003^{(\mathrm{a})}$ \\
\hline May & 0.3 & 3 & 1955 & 0 & $2003^{(a)}$ & 18.8 & 26 & 1978 & 9 & 1945 & 2.4 & 7 & 2002 & 0 & $1997^{(\mathrm{a})}$ & 0.2 & 2 & $1993^{(\mathrm{a})}$ & 0 & $2003^{(a)}$ \\
\hline Jun & 0.1 & 1 & $1980^{(a)}$ & 0 & $2003^{(a)}$ & 19.7 & 26 & 1963 & 11 & $1950^{(a)}$ & 2.4 & 7 & 1985 & 0 & $1982^{(\mathrm{a})}$ & 0.2 & 2 & $1992^{(a)}$ & 0 & $2003^{(a)}$ \\
\hline Jul & 0.1 & 1 & $1957^{(\mathrm{a})}$ & 0 & $2003^{(a)}$ & 19.5 & 26 & 1995 & 11 & 1955 & 1.8 & 5 & $1995^{\text {(a) }}$ & 0 & $1981^{(\mathrm{a})}$ & 0.2 & 1 & $2002^{(a)}$ & 0 & $2003^{(a)}$ \\
\hline Aug & 0.2 & 2 & 1972 & 0 & $2003^{(a)}$ & 15.9 & 24 & 2000 & 7 & 1945 & 1.3 & 5 & 1951 & 0 & $2000^{(a)}$ & 0.1 & 1 & $1998^{(\mathrm{a})}$ & 0 & $2003^{(\mathrm{a})}$ \\
\hline Sep & 2.4 & 9 & 1987 & 0 & $2003^{(a)}$ & 11.3 & 17 & $2002^{(a)}$ & 7 & $1975^{\text {(a) }}$ & 1.4 & 4 & 1946 & 0 & $2002^{(a)}$ & 0.2 & 2 & 1953 & 0 & $2003^{(a)}$ \\
\hline Oct & 6.7 & 15 & 1974 & 2 & $2003^{(a)}$ & 9.0 & 19 & 2003 & 3 & $1987^{(\mathrm{a})}$ & 1.9 & 8 & $2003^{(a)}$ & 0 & $1993^{(a)}$ & 0.2 & 2 & $2003^{(a)}$ & 0 & $2002^{(\mathrm{a})}$ \\
\hline Nov & 9.2 & 20 & $1956^{(\mathrm{a})}$ & 2 & $1977^{(\mathrm{a})}$ & 8.3 & 16 & 1990 & 0 & 1979 & 2.3 & 8 & 1990 & 0 & $2002^{(\mathrm{a})}$ & 0.6 & 4 & $1998^{(a)}$ & 0 & $2002^{(\mathrm{a})}$ \\
\hline Dec & 11.1 & 23 & 1985 & 3 & 1968 & 7.4 & 15 & 1968 & 0 & 1985 & 2.6 & 8 & $1957^{(\mathrm{a})}$ & 0 & $2003^{(a)}$ & 0.8 & 5 & 2001 & 0 & $2003^{(\mathrm{a})}$ \\
\hline Annual & 49.2 & 87 & 1952 & 28 & 1973 & 156.6 & 192 & 1999 & 123 & 1952 & 27.0 & 57 & 1990 & 10 & 1978 & 5.0 & 18 & 1990 & 0 & 1985 \\
\hline
\end{tabular}

(a) Most recent of multiple occurrences. 
Table 5.3. Frequency (\%) of Monthly and Annual Wind Direction and Speed at 50-Foot Level, 1955 through 2003

\begin{tabular}{|c|c|c|c|c|c|c|c|c|c|c|c|c|c|}
\hline Direction & Jan & Feb & Mar & Apr & May & Jun & Jul & Aug & Sep & Oct & Nov & Dec & Total \\
\hline $\mathrm{N}$ & 4.2 & 4.8 & 4.6 & 4.1 & 3.7 & 3.6 & 4.6 & 4.7 & 5.5 & 5.0 & 4.4 & 4.2 & 4.4 \\
\hline NNE & 3.7 & 4.5 & 4.4 & 3.7 & 3.4 & 3.2 & 4.1 & 3.9 & 5.4 & 4.1 & 3.5 & 3.3 & 3.9 \\
\hline NE & 3.0 & 3.7 & 3.4 & 3.4 & 3.3 & 3.1 & 3.5 & 3.5 & 4.2 & 3.6 & 3.0 & 2.9 & 3.4 \\
\hline ENE & 2.4 & 2.1 & 2.1 & 2.4 & 2.4 & 2.2 & 2.5 & 2.5 & 2.4 & 2.6 & 2.5 & 2.5 & 2.4 \\
\hline E & 2.6 & 2.2 & 2.3 & 2.5 & 2.5 & 2.5 & 2.9 & 3.1 & 3.1 & 3.0 & 2.7 & 2.8 & 2.7 \\
\hline ESE & 2.9 & 2.6 & 2.7 & 2.6 & 2.7 & 2.7 & 2.9 & 3.3 & 3.2 & 3.8 & 3.3 & 3.2 & 3.0 \\
\hline SE & 4.0 & 3.6 & 3.8 & 3.0 & 3.1 & 2.9 & 3.0 & 3.5 & 3.9 & 4.9 & 4.4 & 4.5 & 3.7 \\
\hline SSE & 3.4 & 3.3 & 3.4 & 3.0 & 3.0 & 2.8 & 2.5 & 2.8 & 3.3 & 4.0 & 4.0 & 3.8 & 3.3 \\
\hline S & 3.3 & 3.2 & 3.5 & 3.2 & 2.7 & 2.7 & 2.5 & 2.6 & 2.7 & 3.6 & 4.1 & 3.6 & 3.1 \\
\hline SSW & 4.8 & 4.4 & 5.0 & 4.4 & 3.6 & 3.5 & 2.8 & 3.0 & 3.3 & 4.2 & 5.2 & 4.7 & 4.1 \\
\hline SW & 6.6 & 7.7 & 9.2 & 8.9 & 7.0 & 6.5 & 5.6 & 5.9 & 5.8 & 6.9 & 7.7 & 6.7 & 7.0 \\
\hline WSW & 6.7 & 7.4 & 10.6 & 12.0 & 10.5 & 9.6 & 8.2 & 8.8 & 9.2 & 8.7 & 8.1 & 7.3 & 8.9 \\
\hline $\mathrm{W}$ & 6.7 & 8.3 & 9.7 & 11.5 & 11.5 & 10.9 & 9.7 & 10.8 & 11.2 & 10.4 & 8.4 & 7.2 & 9.7 \\
\hline WNW & 15.3 & 15.1 & 14.5 & 16.2 & 18.4 & 19.1 & 19.6 & 18.1 & 15.3 & 13.3 & 12.8 & 13.8 & 15.9 \\
\hline NW & 19.2 & 18.0 & 14.4 & 14.2 & 17.5 & 19.5 & 20.0 & 17.6 & 14.6 & 13.5 & 15.6 & 18.1 & 16.9 \\
\hline NNW & 7.5 & 6.9 & 5.8 & 4.5 & 4.3 & 4.8 & 5.4 & 5.4 & 5.9 & 6.4 & 7.0 & 7.1 & 5.9 \\
\hline Calm & 3.8 & 2.2 & 0.7 & 0.4 & 0.4 & 0.4 & 0.3 & 0.4 & 0.9 & 2.0 & 3.4 & 4.3 & 1.6 \\
\hline Total & 100.0 & 100.0 & 100.0 & 100.0 & 100.0 & 100.0 & 100.0 & 100.0 & 100.0 & 100.0 & 100.0 & 100.0 & 100.0 \\
\hline Speed, mph & Jan & Feb & Mar & Apr & May & Jun & Jul & Aug & Sep & Oct & Nov & Dec & Total \\
\hline Calm & 3.8 & 2.2 & 0.7 & 0.4 & 0.5 & 0.4 & 0.3 & 0.4 & 0.9 & 2.0 & 3.5 & 4.4 & 1.6 \\
\hline $1-3$ & 30.4 & 24.2 & 16.8 & 13.0 & 11.6 & 9.6 & 10.8 & 13.4 & 18.0 & 26.0 & 29.4 & 33.3 & 19.7 \\
\hline $4-7$ & 35.1 & 36.0 & 36.5 & 35.2 & 35.2 & 35.8 & 39.2 & 42.2 & 41.6 & 39.2 & 36.1 & 34.1 & 37.2 \\
\hline $8-12$ & 20.2 & 24.4 & 27.7 & 28.9 & 30.6 & 30.3 & 29.3 & 27.7 & 26.0 & 21.7 & 20.2 & 18.3 & 25.4 \\
\hline $13-18$ & 6.7 & 8.5 & 12.2 & 15.4 & 15.7 & 16.6 & 14.3 & 11.7 & 9.8 & 7.8 & 7.0 & 6.2 & 11.0 \\
\hline $19-24$ & 2.5 & 3.2 & 4.5 & 5.5 & 5.4 & 6.2 & 5.3 & 4.0 & 3.2 & 2.6 & 2.7 & 2.6 & 4.0 \\
\hline $25-31$ & 1.0 & 1.2 & 1.4 & 1.4 & 1.0 & 1.1 & 0.9 & 0.6 & 0.5 & 0.6 & 1.0 & 0.9 & 1.0 \\
\hline $32-38$ & 0.3 & 0.3 & 0.2 & 0.1 & 0.0 & 0.0 & 0.0 & 0.0 & 0.0 & 0.1 & 0.2 & 0.2 & 0.1 \\
\hline $39-46$ & 0.0 & 0.0 & 0.0 & 0.0 & 0.0 & 0.0 & 0.0 & 0.0 & 0.0 & 0.0 & 0.0 & 0.0 & 0.0 \\
\hline$>46$ & 0.0 & 0.0 & 0.0 & 0.0 & 0.0 & 0.0 & 0.0 & 0.0 & 0.0 & 0.0 & 0.0 & 0.0 & 0.0 \\
\hline Total & 100.0 & 100.0 & 100.0 & 100.0 & 100.0 & 100.0 & 100.0 & 100.0 & 100.0 & 100.0 & 100.0 & 100.0 & 100.0 \\
\hline
\end{tabular}




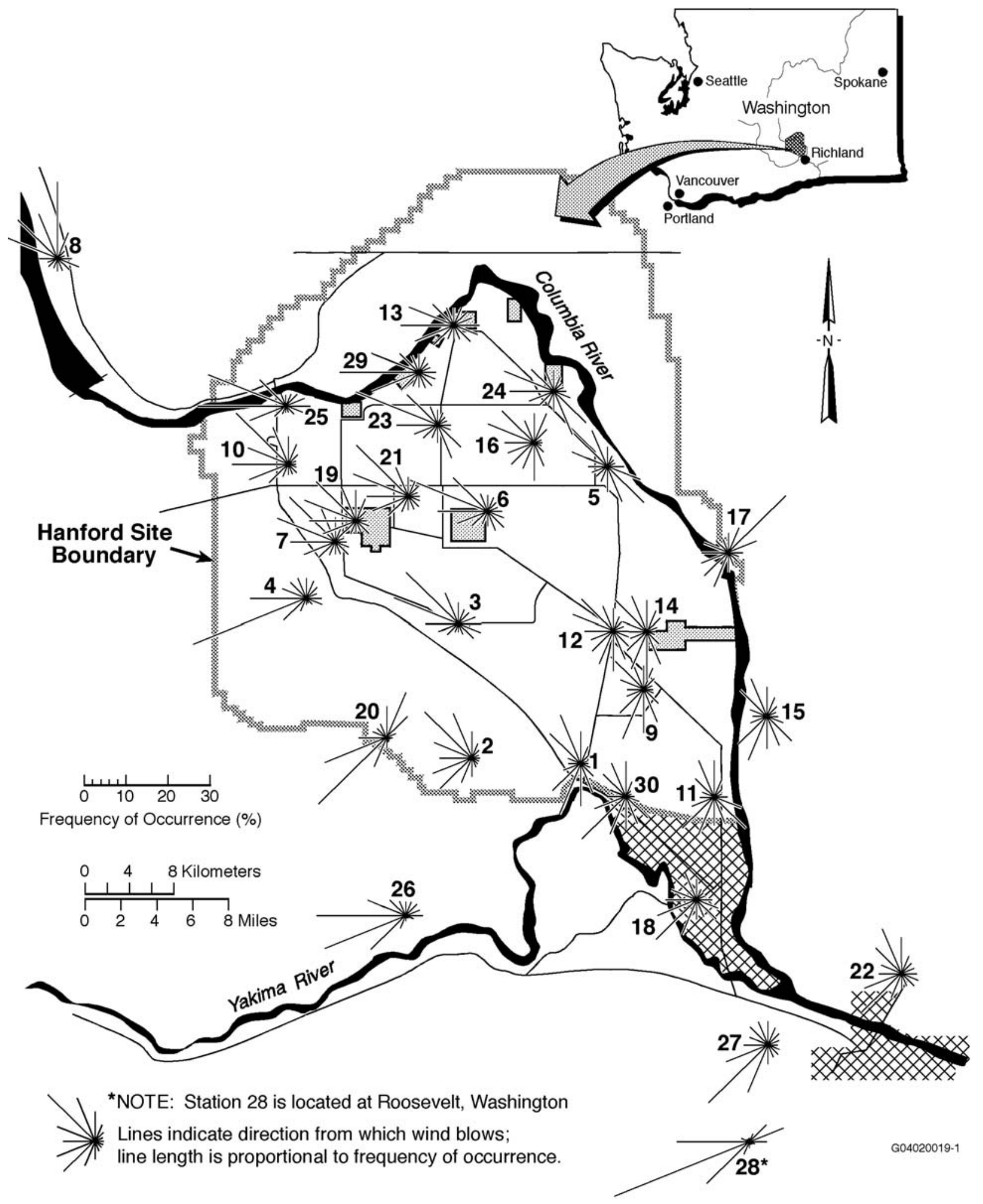

Figure 5.1. Hanford Meteorological Monitoring Network Wind Roses at 30 Feet, 1982 through 2003 (Refer to Table 1.1 for the names of the numbered locations on this map.) 
Table 5.4. Joint Frequency Distributions (\%) for Hanford Meteorological Monitoring Network Wind Stations at 30 Feet, 1982 through 2003

\begin{tabular}{|c|c|c|c|c|c|c|c|c|c|c|c|c|c|c|c|c|c|c|}
\hline tat & & & & & & & 170 & & & & & & & & & & & \\
\hline & & & & & & & & REC & $T \mathrm{~T}$ & & & & & & & & & \\
\hline PEED & $\mathrm{N}$ & NNE & $\mathrm{NE}$ & ENE & $\mathrm{E}$ & $\mathrm{ESE}$ & SE & SSE & $\mathrm{S}$ & SSW & SW & WSW & W & WNW & NW & NNW & CALM & I TOTA \\
\hline CALM & 0.0 & 0.0 & 0.0 & 0.0 & 0.0 & 0.0 & 0.0 & 0.0 & 0.0 & 0.0 & 0.0 & 0.0 & 0.0 & 0.0 & 0.0 & 0.0 & 1.1 & \\
\hline & 2.1 & & 1.2 & 0.9 & 1. & 1.2 & & 2.2 & 2.4 & 2. & & & .1 & & 2.1 & 2. & & 26 \\
\hline & 3.2 & 1.8 & 1.0 & 0.7 & 0.8 & 1.1 & 2.2 & 4.2 & 4.6 & 3. & 2.0 & 0 . & 0.7 & 1. & 3.1 & 4. & & \\
\hline & 1.9 & 0 & & & & .2 & & & 2 . & & & & & & & & & \\
\hline & 0 & .2 & & & & .0 & & & 0.5 & 2. & & & & . & 1. & & & \\
\hline & 0.1 & 0.1 & 0.0 & 0.0 & 0. & 0.0 & 0. & 0 . & 0.0 & 0. & 0.7 & & 0.2 & 0 . & 0.5 & 0 . & & \\
\hline & 0.0 & 0.0 & 0.0 & 0.0 & 0.0 & 0.0 & 0.0 & 0.0 & 0.0 & 0.1 & 0.3 & 0 . & 0.0 & 0.0 & 0.1 & 0.0 & & \\
\hline $32-38$ & 0.0 & 0.0 & 0.0 & 0.0 & 0.0 & 0.0 & 0.0 & 0.0 & 0.0 & 0.0 & 0.1 & 0. & 0.0 & 0.0 & 0.0 & 0.0 & 0. & \\
\hline & 0.0 & 0.0 & 0.0 & 0.0 & 0.0 & 0.0 & 0.0 & & 0.0 & & 0.0 & & & 0.0 & & 0.0 & & \\
\hline & & 0.0 & & & & & 0. & 0.0 & 0.0 & & & & & 0.0 & 0.0 & & & \\
\hline OTAL & 7.8 & 4.4 & 2.7 & 1.7 & 1.9 & 2.5 & 4.4 & 7.5 & 10.1 & 13.2 & 9.2 & 4.3 & 3.0 & 3.2 & 10.2 & 12.7 & 1.1 & 100 \\
\hline
\end{tabular}

Station: ( 2) EOC

Begin: 1/1982 End: 12/2003 Total Hours: 188694 DIRECTION

\begin{tabular}{|c|c|c|c|c|c|c|c|c|c|c|c|c|c|c|c|c|c|c|}
\hline EED & $\mathrm{N}$ & NNE & $\mathrm{NE}$ & ENE & $E$ & ESE & SE & SSE & S & SSW & SW & WSW & W & WNW & NW & NNW & CALM & TOTAL \\
\hline CALM & 0.0 & 0.0 & 0.0 & 0.0 & 0.0 & 0.0 & 0.0 & 0.0 & 0.0 & 0.0 & 0.0 & 0.0 & 0.0 & 0.0 & 0.0 & 0.0 & 1.0 & \\
\hline $1-3$ & 1.2 & 1.1 & 1.1 & 1.0 & 0.9 & 0.8 & 0.8 & 0.9 & 1.2 & 1.2 & 1.3 & 1.2 & 1.6 & 1.8 & 1.8 & 1.4 & 0.0 & \\
\hline $4-7$ & 2.7 & 1.9 & 1.3 & 0.9 & 1.0 & & 1.1 & & 1.9 & . & & .2 & 2. & .6 & & 3.2 & & \\
\hline $8-12$ & 1.8 & 0.6 & 0.2 & & 0.0 & & 3 & & 1.1 & & & & 1 & & & & & \\
\hline $3-18$ & 0.6 & 0.2 & 0.1 & 0.0 & 0.0 & 0. & 0.0 & . & 0.2 & D. & 2.2 & 2.3 & 1.4 & 0.7 & 3 & 2.4 & .0 & \\
\hline $9-24$ & 0.1 & 0.0 & 0.0 & 0.0 & 0.0 & 0. & 0.0 & . & 0.0 & 0.2 & 1.5 & 2.0 & 0.9 & 0.2 & 0. & 0.4 & 0.0 & \\
\hline $5-31$ & 0.0 & 0.0 & 0.0 & 0. & 0.0 & 0 . & 0.0 & 0. & 0.0 & 0. & 1.0 & 1.0 & 0.3 & 0.1 & 0. & 0.0 & & \\
\hline-38 & 0.0 & 0.0 & 0.0 & 0.0 & 0.0 & 0.0 & 0.0 & 0. & 0.0 & 0 . & 0.3 & 0.2 & 0 . & 0.0 & 0 & 0.0 & & \\
\hline $39-46$ & 0.0 & 0.0 & 0.0 & 0 . & 0.0 & 0 & . & & 0.0 & & & 0.0 & & & & 0.0 & & \\
\hline & 0.0 & 0.0 & .0 & & 0.0 & & & & 0 . & & & & & & & 0.0 & & \\
\hline OTAL & 6.4 & 3.8 & 2.6 & 1.9 & 2.0 & 2.0 & 2.2 & 2.5 & 4.4 & 6.4 & 10.5 & 9.9 & 7.7 & 9.5 & 15.7 & 11.4 & 1.0 & \\
\hline
\end{tabular}

Station: ( 3) ARMY

\begin{tabular}{|c|c|c|c|c|c|c|c|c|c|c|c|c|c|c|c|c|c|c|}
\hline \\
\hline SPEED & $\mathrm{N}$ & NNE & $\mathrm{NE}$ & ENE & $E$ & ESE & $\mathrm{SE}$ & SSE & S & SSW & SW & WSW & W & WNW & NW & NNW & CALM & TOTAI \\
\hline CALM & 0.0 & 0.0 & 0.0 & 0.0 & 0.0 & 0.0 & 0.0 & 0.0 & 0.0 & 0.0 & 0.0 & 0.0 & 0.0 & 0.0 & 0.0 & 0.0 & 1.1 & \\
\hline $1-3$ & 1.9 & 1.7 & 2.0 & 2.2 & & .3 & 1.8 & & & & & & 2.2 & & & & & 30.3 \\
\hline $4-7$ & 2.0 & 1. & & . & & ? & 2.1 & & & & & & 2.4 & & & & & \\
\hline $8-12$ & 0.9 & 0.5 & 0.3 & 0.2 & 0.2 & 0. & 0.9 & 0 . & 0.4 & . & 0. & & 1.9 & 4. & 4 & & & \\
\hline $3-18$ & 0.2 & 0.2 & 0.1 & 0.0 & 0. & 0. & 0.2 & 0 . & 0.2 & 0. & 0. & & 1.0 & 1. & & 4 & & \\
\hline $9-24$ & 0.0 & 0.1 & 0.0 & 0.0 & 0.0 & 0.0 & 0.0 & 0.0 & 0.0 & 0.2 & 0.5 & 0 . & 0.3 & 0 . & 0.7 & & & \\
\hline & 0.0 & 0.0 & 0.0 & 0.0 & 0.0 & 0.0 & 0.0 & 0.0 & 0.0 & & & & 0.1 & & & & & \\
\hline & 0.0 & & & & & & & & & & & & & & & & & \\
\hline & 0.0 & & & & & & & & & & & & & & & & & \\
\hline$>46$ & 0.0 & 0.0 & 0.0 & 0.0 & 0 . & 0 . & 0.0 & 0 . & 0.0 & ). & 0 & & 0.0 & 0.0 & 0.0 & 0 & 0 & \\
\hline OTAL & 5.1 & 3.8 & 3.8 & 4.3 & 5.5 & 5.9 & 5.1 & 2.8 & 2.0 & 2.2 & 3.7 & 5.2 & 7.9 & 16.5 & 17.3 & 7.9 & 1.1 & \\
\hline
\end{tabular}

Station: ( 4) RSPG

\begin{tabular}{|c|c|c|c|c|c|c|c|c|c|c|c|c|c|c|c|c|c|c|}
\hline \\
\hline PEED & $\mathrm{N}$ & NNE & $\mathrm{NE}$ & ENE & $E$ & ESE & $\mathrm{SE}$ & SSE & S & SSW & SW & WSW & W & WNW & NW & NNW & CALM & OTE \\
\hline CALM & 0.0 & 0.0 & 0.0 & 0.0 & 0.0 & 0.0 & 0.0 & 0.0 & 0.0 & 0.0 & 0.0 & 0.0 & 0.0 & 0.0 & 0.0 & 0.0 & 1.0 & \\
\hline $1-3$ & 1.6 & 1.8 & 1.9 & 1.6 & 1.8 & 1.7 & 1.1 & 0.8 & 0.8 & 1. & 1.8 & 2. & 1.9 & 1.2 & 1.1 & 1.2 & 0.0 & 23. \\
\hline $4-7$ & 2.6 & 2.1 & 1.5 & 1.1 & 1.8 & 2.1 & 0.8 & & 4 & & & 9. & & & & & & \\
\hline $8-1$ & & 0 . & .2 & & & & 1 & & & & & 14. & & & & & & . \\
\hline & 0. & 0. & 0.1 & & 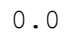 & & & & & & & & & & & & & \\
\hline & 0.0 & 0.0 & 0. & 0. & 0 . & 0 . & 0.0 & . & .0 & & 0. & & 0.2 & & & & & \\
\hline-31 & 0.0 & 0.0 & 0.0 & 0.0 & 0.0 & 0.0 & 0.0 & 0.0 & 0.0 & 0 . & 0.2 & 0. & 0.0 & 0 . & 0 & 0.0 & & \\
\hline-38 & 0.0 & 0.0 & 0.0 & 0.0 & 0.0 & 0.0 & 0.0 & 0 . & 0.0 & . & 0.0 & 0. & 0.0 & 0 . & & .0 & & \\
\hline $39-46$ & 0.0 & 0.0 & 0.0 & 0.0 & 0.0 & 0.0 & 0.0 & & 0. & & & & & & & .0 & & \\
\hline$>46$ & 0.0 & 0.0 & 0. & 0. & . & & 0. & & 0. & & 0. & & & & & & & \\
\hline & 4. & 4.5 & 3.7 & 1.8 & 3.7 & t & t. & & 10 & 3.3 & 1.6 & 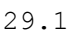 & 5 & 5.2 & 5.4 & 5.2 & 1.0 & \\
\hline
\end{tabular}


Table 5.4. (contd)

Station: ( 5) EDNA

\begin{tabular}{|c|c|c|c|c|c|c|c|c|c|c|c|c|c|c|c|c|c|c|}
\hline & & & & & & & 98 & & & $d$ & & & & & & & & 39200 \\
\hline & & & & & & & & {$[R E C]$} & & & & & & & & & & \\
\hline SPEED & $\mathrm{N}$ & NNE & $\mathrm{NE}$ & ENE & E & ESE & $\mathrm{SE}$ & SSE & S & SSW & SW & WSW & W & WNW & NW & NNW & CALM & TOTAL \\
\hline CALM & 0.0 & 0.0 & 0.0 & 0.0 & 0.0 & 0.0 & 0.0 & 0.0 & 0.0 & 0.0 & 0.0 & 0.0 & 0.0 & 0.0 & 0.0 & 0.0 & 1.2 & 1.2 \\
\hline $1-3$ & 1.2 & 0.8 & 0.7 & 0.8 & 1.1 & 2.0 & 3.5 & 3.9 & 3.0 & 1.9 & 1.4 & 1.2 & 1.7 & 2.8 & 3.2 & 2.1 & 0.0 & 31.6 \\
\hline $4-7$ & 2.2 & 1.1 & 0.9 & 1.0 & 2.0 & 4.6 & 7.2 & 3.8 & 1.7 & 0.8 & 0.7 & 0.7 & 1.0 & 2.5 & 6.0 & 4.8 & 0.0 & 41.0 \\
\hline $8-12$ & 1.2 & 0.6 & 0.4 & 0.2 & 0.8 & 1.8 & 1.4 & 1.1 & 0.9 & 0.6 & 0.7 & 0.9 & 1.1 & 1.7 & 2.3 & 2.0 & 0.0 & 17.4 \\
\hline $13-18$ & 0.2 & 0.2 & 0.2 & 0.1 & 0.0 & 0.1 & 0.2 & 0.3 & 0.5 & 0.4 & 0.5 & 0.7 & 0.6 & 1.4 & 1.0 & 0.2 & 0.0 & 6.5 \\
\hline $19-24$ & 0.0 & 0.1 & 0.1 & 0.0 & 0.0 & 0.0 & 0.0 & 0.0 & 0.1 & 0.2 & 0.2 & 0.3 & 0.2 & 0.5 & 0.3 & 0.0 & 0.0 & 1.9 \\
\hline & 0.0 & 0.0 & 0.0 & 0.0 & 0. & 0.0 & 0.0 & 0. & 0.0 & 0.0 & 0.1 & 0 . & 0.0 & 0. & 0.0 & 0. & 0.0 & 0.4 \\
\hline $32-38$ & 0.0 & 0.0 & 0.0 & 0.0 & 0.0 & 0.0 & 0.0 & 0.0 & 0.0 & 0.0 & 0.0 & 0.0 & 0.0 & 0.0 & 0.0 & 0.0 & 0.0 & 0.0 \\
\hline $39-46$ & 0.0 & 0.0 & 0.0 & 0.0 & 0.0 & 0.0 & 0.0 & 0.0 & 0.0 & 0.0 & 0.0 & 0.0 & 0.0 & 0.0 & 0.0 & 0.0 & 0.0 & 0.0 \\
\hline$>46$ & 0.0 & 0.0 & 0.0 & 0.0 & 0.0 & 0.0 & 0.0 & 0.0 & 0.0 & 0.0 & 0.0 & 0.0 & 0.0 & 0.0 & 0.0 & 0.0 & 0.0 & 0.0 \\
\hline TOTAL & 4.8 & 2.8 & 2.2 & 2.1 & 3.9 & 8.5 & 12.3 & 9.1 & 6.2 & 3.9 & 3.5 & 3.9 & 4.7 & 9.0 & 12.9 & 9.1 & 1.2 & 100.0 \\
\hline
\end{tabular}

Station: (6) 200E

Begin: 1/1982 End: 12/2003

Total Hours: 188774 DIRECTION

\begin{tabular}{|c|c|c|c|c|c|c|c|c|c|c|c|c|c|c|c|c|c|c|}
\hline SPEED & $\mathrm{N}$ & NNE & $\mathrm{NE}$ & ENE & $\mathrm{E}$ & ESE & SE & SSE & S & SSW & SW & WSW & W & WNW & NW & NNW & CALM & TOTAI \\
\hline CALM & 0.0 & 0.0 & 0.0 & 0.0 & 0.0 & 0.0 & 0.0 & 0.0 & 0.0 & 0.0 & 0.0 & 0.0 & 0.0 & 0.0 & 0.0 & 0.0 & 1.1 & \\
\hline $1-3$ & 1.5 & 1.3 & 1.4 & .5 & .7 & .7 & & & 1 & & & & .4 & .8 & & .6 & 0.0 & \\
\hline $4-7$ & 1.6 & 1.3 & 1.0 & 1.0 & 1.5 & 2.1 & 3. & 2. & 1.5 & & 1. & & 4. & 0. & & .1 & & \\
\hline $8-12$ & 0.7 & 0.7 & 0.3 & 0.1 & 0.3 & 0 . & 0 . & 1. & 0.7 & . & 1. & 2 . & 4.5 & 7. & 2 & .7 & & \\
\hline $3-18$ & 0.2 & 0.2 & 0.1 & 0.0 & 0.0 & 0 . & 0.1 & 0 . & 0.3 & & & 3 & 1.4 & 3. & & & & \\
\hline & 0.0 & 1 & 0.0 & & 0. & & 0. & & 0.1 & & & & & & & & & \\
\hline & & & 0 . & & & & & & & & & & & & & & & \\
\hline-38 & 0.0 & 0. & 0.0 & 0. & 0. & 0. & . & & 0.0 & & 0. & & 0.0 & 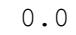 & & .0 & & \\
\hline $39-46$ & 0.0 & 0. & 0.0 & 0. & 0. & 0 . & 0 . & D. & 0.0 & & 0. & & 0. & 0. & & .0 & & \\
\hline$>46$ & 0.0 & 0.0 & 0.0 & 0.0 & 0.0 & 0.0 & 0.0 & 0.0 & 0.0 & 0 . & 0.0 & 0.0 & 0.0 & 0.0 & 0.0 & 0.0 & 0.0 & \\
\hline OTAL & 3.9 & 3.6 & 2.9 & 2.6 & 3.5 & 4.3 & 5.6 & 5.0 & 3.5 & 3.2 & 5.1 & 7.8 & 11.9 & 20.1 & 11.3 & 4.5 & 1.1 & \\
\hline
\end{tabular}

Station: ( 7) 200w

\begin{tabular}{|c|c|c|c|c|c|c|c|c|c|c|c|c|c|c|c|c|c|c|}
\hline \multirow[b]{3}{*}{ SPEED } & \multirow[b]{3}{*}{$\mathrm{N}$} & \multirow[b]{3}{*}{ NNE } & \multirow{3}{*}{$\mathrm{NE}$} & & \multirow{2}{*}{\multicolumn{2}{|c|}{ Begin: }} & \multicolumn{2}{|c|}{ /1982 } & \multicolumn{4}{|c|}{ End: $12 / 2003$} & \multirow[b]{3}{*}{ W } & \multirow[b]{3}{*}{ WNW } & \multirow{2}{*}{\multicolumn{3}{|c|}{ Total Hours: }} & \multirow[t]{2}{*}{174638} \\
\hline & & & & & & & & s & & & & & & & & & & \\
\hline & & & & & $\mathrm{E}$ & ESE & $S E$ & SSE & $\mathrm{S}$ & SSW & SW & WSW & & & NW & NNW & CALM & TOTAL \\
\hline CALM & 0.0 & 0.0 & 0.0 & 0.0 & 0.0 & 0.0 & 0.0 & 0.0 & 0.0 & 0.0 & 0.0 & 0.0 & 0.0 & 0.0 & 0.0 & 0.0 & 1.6 & 1.6 \\
\hline $1-3$ & 2.2 & 1.8 & 1.5 & 1.4 & 1.6 & 1.8 & 2.1 & 2.0 & 1.8 & 1.6 & 1.9 & 2.4 & 3.4 & 4.2 & 3.6 & 2.6 & 0.0 & 35.9 \\
\hline $4-7$ & 2.9 & 1.6 & 1.0 & 0.8 & 1.0 & 1.5 & 1.8 & 1.0 & 0.7 & 0.8 & 1.2 & 1.8 & 3.8 & 6.7 & 5.2 & 3.4 & 0.0 & 35.2 \\
\hline $8-12$ & 0.7 & 0.5 & 0.2 & 0.1 & 0.1 & 0.3 & 0.4 & 0.2 & 0.2 & 0.6 & 1.1 & 1.7 & 2.5 & 3.1 & 3.4 & 1.9 & 0.0 & 16.9 \\
\hline $13-18$ & 0.1 & 0.2 & 0.1 & 0.0 & 0.0 & 0.0 & 0.0 & 0.0 & 0.1 & 0.4 & 0.9 & 1.4 & 0.8 & 0.8 & 2.0 & 0.5 & 0.0 & 7.4 \\
\hline $19-24$ & 0.0 & 0.0 & 0.0 & 0.0 & 0.0 & 0.0 & 0.0 & 0.0 & 0.0 & 0.1 & 0.5 & 0.5 & 0.2 & 0.1 & 0.8 & 0.1 & 0.0 & 2.4 \\
\hline $25-31$ & 0.0 & 0.0 & 0.0 & 0.0 & 0.0 & 0.0 & 0.0 & 0 . & 0.0 & 0.0 & 0.1 & 0. & 0.0 & 0 . & 0.1 & 0 & 0.0 & 0.5 \\
\hline $32-38$ & 0.0 & 0.0 & 0.0 & 0.0 & 0.0 & 0.0 & 0.0 & 0.0 & 0.0 & 0.0 & 0.0 & 0.0 & 0.0 & 0.0 & 0.0 & 0.0 & 0.0 & 0.1 \\
\hline $39-46$ & 0.0 & 0.0 & 0.0 & 0.0 & 0.0 & 0.0 & 0.0 & 0.0 & 0.0 & 0.0 & 0.0 & 0.0 & 0.0 & 0.0 & 0.0 & 0.0 & 0.0 & 0.0 \\
\hline$>46$ & 0.0 & 0.0 & 0.0 & 0.0 & 0.0 & 0.0 & 0.0 & 0.0 & 0.0 & 0.0 & 0.0 & 0.0 & 0.0 & 0.0 & 0.0 & 0.0 & 0.0 & 0.0 \\
\hline TOTAL & 6.0 & 4.1 & 2.9 & 2.3 & 2.7 & 3.6 & 4.3 & 3.2 & 2.9 & 3.5 & 5.7 & 8.0 & 10.7 & 14.9 & 15.2 & 8.4 & 1.6 & 100.0 \\
\hline
\end{tabular}

Station: ( 8) BVLY

\begin{tabular}{rrrrrrrrrrrrrrrrrrr} 
SPEED & N & NNE & NE & ENE & E & ESE & SE & SSE & S & SSW & SW & WSW & W & WNW & NW & NNW & CALM TOTAL \\
\hline CALM & 0.0 & 0.0 & 0.0 & 0.0 & 0.0 & 0.0 & 0.0 & 0.0 & 0.0 & 0.0 & 0.0 & 0.0 & 0.0 & 0.0 & 0.0 & 0.0 & 1.0 & 1.0 \\
$1-3$ & 2.9 & 2.2 & 1.7 & 1.4 & 1.7 & 1.8 & 1.9 & 1.7 & 1.6 & 1.2 & 1.1 & 1.1 & 1.3 & 1.6 & 2.1 & 2.5 & 0.0 & 27.9 \\
$4-7$ & 8.5 & 2.8 & 0.6 & 0.4 & 1.4 & 3.1 & 1.7 & 1.1 & 1.0 & 0.8 & 0.7 & 0.8 & 1.5 & 2.7 & 4.9 & 6.9 & 0.0 & 38.9 \\
$8-12$ & 6.5 & 1.6 & 0.1 & 0.0 & 0.3 & 0.7 & 0.3 & 0.3 & 0.2 & 0.3 & 0.4 & 0.4 & 1.2 & 3.7 & 3.3 & 1.9 & 0.0 & 21.3 \\
$13-18$ & 0.4 & 0.2 & 0.1 & 0.0 & 0.0 & 0.0 & 0.0 & 0.1 & 0.1 & 0.2 & 0.5 & 0.3 & 0.5 & 3.3 & 2.0 & 0.1 & 0.0 & 7.9 \\
$19-24$ & 0.0 & 0.0 & 0.0 & 0.0 & 0.0 & 0.0 & 0.0 & 0.0 & 0.0 & 0.1 & 0.2 & 0.1 & 0.1 & 1.2 & 0.6 & 0.0 & 0.0 & 2.3 \\
$25-31$ & 0.0 & 0.0 & 0.0 & 0.0 & 0.0 & 0.0 & 0.0 & 0.0 & 0.0 & 0.0 & 0.1 & 0.0 & 0.0 & 0.3 & 0.1 & 0.0 & 0.0 & 0.0 \\
$32-38$ & 0.0 & 0.0 & 0.0 & 0.0 & 0.0 & 0.0 & 0.0 & 0.0 & 0.0 & 0.0 & 0.0 & 0.0 & 0.0 & 0.0 & 0.0 & 0.0 & 0.0 & 0.1 \\
$39-46$ & 0.0 & 0.0 & 0.0 & 0.0 & 0.0 & 0.0 & 0.0 & 0.0 & 0.0 & 0.0 & 0.0 & 0.0 & 0.0 & 0.0 & 0.0 & 0.0 & 0.0 & 0.0 \\
> 46 & 0.0 & 0.0 & 0.0 & 0.0 & 0.0 & 0.0 & 0.0 & 0.0 & 0.0 & 0.0 & 0.0 & 0.0 & 0.0 & 0.0 & 0.0 & 0.0 & 0.0 & 0.0 \\
TOTAL & 18.3 & 6.8 & 2.5 & 1.9 & 3.4 & 5.7 & 4.0 & 3.2 & 3.1 & 2.6 & 3.0 & 2.6 & 4.5 & 12.9 & 13.1 & 11.5 & 1.0 & 100.0
\end{tabular}


Table 5.4. (contd)

\begin{tabular}{|c|c|c|c|c|c|c|c|c|c|c|c|c|c|c|c|c|c|c|}
\hline & & & & & & & & & & $d:$ & & & & & & & rs: & 188278 \\
\hline & & & & & & & & BF & ION & & & & & & & & & \\
\hline SPEED & $\mathrm{N}$ & NNE & $\mathrm{NE}$ & ENE & E & ESE & $\mathrm{SE}$ & SSE & $\mathrm{S}$ & SSW & SW & WSW & W & WNW & NW & NNW & CALM & I TOTAL \\
\hline CALM & 0.0 & 0.0 & 0.0 & 0.0 & 0.0 & 0.0 & 0.0 & 0.0 & 0.0 & 0.0 & 0.0 & 0.0 & 0.0 & 0.0 & 0.0 & 0.0 & 0.5 & 0.5 \\
\hline $1-$ & 1.2 & 1.1 & 0.9 & 0. & 0. & & 1. & & 1.2 & 1. & & & & & & & & 16.9 \\
\hline $4-7$ & 2.8 & 2.7 & 1. & 1. & 1. & 1. & 2. & & 4.2 & 3. & 2 & & & 2. & & 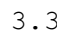 & & 8.8 \\
\hline $8-12$ & 1.4 & 1.3 & 0.7 & 0.2 & 0.2 & 0. & 1.2 & 3. & 3.9 & 4. & 1. & 0. & 0. & 1.7 & 3 & 2.7 & & 28.6 \\
\hline $3-18$ & 0.3 & 0.3 & 0.2 & 0.0 & 0.0 & 0.0 & 0.1 & 0.3 & 0.9 & 3. & 1.8 & 0.7 & 0.5 & 0.8 & 1.7 & 0.5 & & 11.2 \\
\hline $9-24$ & 0.0 & 0.1 & 0.1 & 0.0 & 0.0 & 0.0 & 0.0 & 0. & 0.1 & 0 . & 0.8 & 0. & 0.2 & 0.2 & 0.5 & 0.1 & 0. & 3.0 \\
\hline-31 & 0.0 & 0.0 & 0.0 & 0.0 & 0.0 & 0.0 & 0.0 & 0.0 & 0.0 & 0 . & 0.3 & 0.1 & & 0.0 & 0.1 & 0.0 & & 0.8 \\
\hline & . & 0 & c & & 0. & 0. & & & & & & & & & & & & 0.2 \\
\hline-46 & 0.0 & 0.0 & 0.0 & 0. & 0. & 0. & 0 & 0 . & 0.0 & 0 & 0. & 0 & 0. & & 0.0 & 0.0 & 0.0 & 0.0 \\
\hline$>46$ & 0.0 & 0.0 & 0.0 & 0.0 & 0. & 0.0 & 0. & 0.0 & 0.0 & 0. & 0. & 0. & 0. & 0.0 & 0 & 0.0 & 0.0 & 0.0 \\
\hline OTAL & 5.7 & 5.5 & 3.7 & 2.2 & 2.2 & 2.6 & 5.0 & 8.4 & 0.3 & 2.9 & 8.0 & 4.3 & 4.0 & 5.9 & 0.9 & 7.8 & 0.5 & 0.0 \\
\hline
\end{tabular}

Station: (10) YAKB

\begin{tabular}{|c|c|c|c|c|c|c|c|c|c|c|c|c|c|c|c|c|c|c|}
\hline PEED & $\mathrm{N}$ & $\mathrm{NE}$ & $\mathrm{NE}$ & ENE & $\mathrm{E}$ & ESE & $\mathrm{SE}$ & SSE & S & SSW & SW & WSW & W & WNW & NW & NNW & CALM & $01 \mathrm{~F}$ \\
\hline CALM & 0.0 & 0.0 & 0.0 & 0.0 & 0.0 & 0.0 & 0.0 & 0.0 & 0.0 & 0.0 & 0.0 & 0.0 & 0.0 & 0.0 & 0.0 & 0.0 & 0.6 & \\
\hline $1-3$ & 1.7 & 1.6 & 1.4 & 1.1 & 0.9 & 1.0 & 1.1 & 1.2 & 1.2 & 1.1 & 1.4 & 1.9 & 2.4 & 2.0 & 1.7 & 1.6 & 0.0 & 23. \\
\hline $4-7$ & 3.7 & 2.9 & 1.6 & 0.9 & 0.8 & 1.0 & 1.5 & 1.2 & 0.9 & & 1.6 & & & & & & & \\
\hline $8-12$ & 1.3 & 0.5 & 0.2 & 0.1 & 0 . & 0.2 & 0 . & 0. & 0.3 & & 1.5 & & 3 & & & & & 2 . \\
\hline-18 & 0.1 & 0.2 & 0.1 & 0.0 & 0 & 0.0 & 0. & 0 & 0 . & & 1.2 & & 0 & & & & & 10 \\
\hline $19-24$ & 0.0 & 0.0 & 0.0 & 0.0 & 0.0 & 0.0 & 0.0 & 0.0 & 0.0 & 0.2 & 0.5 & 0.4 & 0.1 & 0.3 & 1.9 & 0.1 & 0.0 & \\
\hline $25-31$ & 0.0 & 0.0 & 0.0 & 0.0 & 0.0 & 0.0 & 0.0 & 0.0 & 0.0 & 0 . & 0.2 & 0 . & 0.0 & 0.1 & 0. & 0.0 & 0 & \\
\hline & 0.0 & 0.0 & 0.0 & 0.0 & 0.0 & 0.0 & 0.0 & 0.0 & 0.0 & 0 . & 0.0 & 0 . & 0.0 & 0 . & & 0 & & \\
\hline & 0.0 & 0.0 & 0.0 & 0.0 & . & 0 . & 0. & 0. & 0 . & & & & & & & & & \\
\hline & 0.0 & 0.0 & 0.0 & 0.0 & 0.0 & 0.0 & 0.0 & 0 . & 0.0 & & 0.0 & U. & 0.0 & 0.0 & 0.0 & 0.0 & 0.0 & \\
\hline OTAL & 6.8 & 5.2 & 3.3 & 2.1 & 1.8 & 2.2 & 2.9 & & & & & & & & & & & \\
\hline
\end{tabular}

Station: (11) 300A

\begin{tabular}{|c|c|c|c|c|c|c|c|c|c|c|c|c|c|c|c|c|c|c|}
\hline PEED & $\mathrm{N}$ & NNE & $\mathrm{NE}$ & ENE & E & ESE & $\mathrm{SE}$ & SSE & 5 & SSW & SW & WSW & W & WNW & NW & NNW & CALM & TOTAL \\
\hline CALM & 0.0 & 0.0 & 0.0 & 0.0 & 0.0 & 0.0 & 0.0 & 0.0 & 0.0 & 0.0 & 0.0 & 0.0 & 0.0 & 0.0 & 0.0 & 0.0 & 0.5 & \\
\hline $1-3$ & 1.2 & 0.7 & 0.6 & 0.6 & 0.8 & 1.2 & 1.7 & 1.8 & 1.7 & 1.4 & 1.3 & 1.2 & 1.2 & 1.3 & 1.8 & 1.7 & 0.0 & 0. \\
\hline $4-7$ & 3.5 & 1.6 & 1.0 & 1.2 & 1.9 & 4.4 & 6.8 & 3.7 & 3.0 & 2.5 & 1.9 & L. 2 & 0.9 & 1.0 & 2.1 & .0 & .0 & \\
\hline $8-12$ & 3.4 & 1.9 & 0.8 & 0.3 & 0.4 & 1.3 & 1.7 & 0.9 & 1.6 & 3.2 & 3.3 & 1.6 & 0.6 & 0.4 & 1.1 & 2.6 & 0.0 & \\
\hline $13-18$ & 0.6 & 0.5 & 0.1 & 0.1 & 0.0 & 0.0 & 0.1 & 0.1 & 0.4 & 1.5 & 2.5 & 1.3 & 0.4 & 0.2 & 0.9 & 0.9 & 0. & \\
\hline $19-24$ & 0.1 & 0.1 & 0.0 & 0.0 & 0.0 & 0.0 & 0.0 & 0.0 & 0.1 & 0.4 & 0.9 & 0.5 & 0.2 & 0.0 & 0.3 & .2 & & \\
\hline & 0.0 & 0.0 & 0.0 & 0.0 & 0.0 & 0.0 & 0.0 & 0.0 & 0.0 & 0.1 & 0.4 & 0 . & 0.1 & 0.0 & 0.1 & .0 & & \\
\hline & 0.0 & 0.0 & 0. & 0. & 0.0 & 0. & 0. & 0. & 0.0 & & & & & 0. & & & & \\
\hline $39-46$ & 0.0 & 0.0 & 0.0 & 0.0 & 0.0 & 0.0 & 0.0 & 0.0 & 0.0 & 0.0 & 0 & 0.0 & 0.0 & 0.0 & 0 & 0.0 & .0 & \\
\hline$>46$ & 0.0 & 0.0 & 0.0 & 0.0 & 0.0 & 0.0 & 0.0 & 0.0 & 0.0 & 0.0 & 0.0 & 0.0 & 0.0 & 0.0 & 0.0 & 0.0 & 0.0 & \\
\hline COTAL & 8.7 & 4.8 & 2.7 & 2.1 & 3.1 & 6.9 & 10.4 & 6.5 & 6.8 & 9.2 & 10.4 & 5.9 & 3.4 & 3.0 & 6.3 & 9.4 & 0.6 & \\
\hline
\end{tabular}

Station: (12) WYEB

DIRECTION

\begin{tabular}{|c|c|c|c|c|c|c|c|c|c|c|c|c|c|c|c|c|c|c|}
\hline SPEED & $\mathrm{N}$ & NNE & $\mathrm{NE}$ & ENE & $\mathrm{E}$ & ESE & $\mathrm{SE}$ & SSE & S & SSW & SW & WSW & W & WNW & NW & NNW & CALM & TOTAI \\
\hline CALM & 0.0 & 0.0 & 0.0 & 0.0 & 0.0 & 0.0 & 0.0 & 0.0 & 0.0 & 0.0 & 0.0 & 0.0 & 0.0 & 0.0 & 0.0 & 0.0 & 0.4 & \\
\hline $1-3$ & & & 2 & .2 & & & & & & & & & 2 & & & & & 20 \\
\hline $4-7$ & 2.6 & .6 & 1.3 & 1.4 & 2 & . & & - & 3. & & 2. & & .5 & & & & & \\
\hline $8-12$ & 1.2 & 0.6 & 0.3 & 0.2 & 0.3 & 0.4 & U. & 2.0 & 2.9 & 2. & 1.5 & 1. & 2.1 & 4. & 3. & 5 & & \\
\hline $3-18$ & 0.3 & 0.2 & 0.1 & 0.0 & 0.0 & 0 . & 0 . & 0. & 1.0 & & 1. & & 0.9 & 5 & & & & \\
\hline & 0 & 0.1 & & & & & & & 0 . & & & & & & & & & \\
\hline & & & & & & & & & & & & & & & & & & \\
\hline-38 & 0.0 & 0. & 0.0 & 0 & . & c & & & 0.0 & & 0. & & 0.0 & & & & & \\
\hline $39-46$ & 0.0 & 0. & 0.0 & 0.0 & 0 . & 0 . & & 0. & 0. & & 0. & & 0.0 & & & & & \\
\hline$>46$ & 0.0 & 0.0 & 0.0 & 0. & 0 . & 0.0 & & 0. & 0.0 & & 0. & & 0.0 & 0. & & 0. & & \\
\hline AI & 5.4 & 3.7 & 3.0 & 2.8 & 3.8 & 4.0 & 5.3 & 7.3 & 9.3 & .5 & 6.9 & 5.7 & 6.9 & 0.2 & 0.5 & 6.3 & 0.4 & 100 \\
\hline
\end{tabular}


Table 5.4. (contd)

Station: (13) $100 \mathrm{~N}$

\begin{tabular}{|c|c|c|c|c|c|c|c|c|c|c|c|c|c|c|c|c|c|c|}
\hline \multirow[b]{3}{*}{ SPEED } & \multirow[b]{3}{*}{$\mathrm{N}$} & \multirow[b]{3}{*}{ NNE } & & \multicolumn{2}{|c|}{ Begin: } & \multicolumn{3}{|c|}{$1 / 1982$} & \multicolumn{3}{|c|}{ End: $12 / 2003$} & \multirow[b]{3}{*}{ W } & \multirow[b]{3}{*}{ WNW } & \multirow{2}{*}{\multicolumn{3}{|c|}{ Total Hours: }} & \multirow[t]{2}{*}{188576} \\
\hline & & & & & & & & IRECI & ION & & & & & & & & & \\
\hline & & & $\mathrm{NE}$ & ENE & $E$ & ESE & $\mathrm{SE}$ & SSE & S & SSW & SW & WSW & & & NW & NNW & CALM & TOTAL \\
\hline CALM & 0.0 & 0.0 & 0.0 & 0.0 & 0.0 & 0.0 & 0.0 & 0.0 & 0.0 & 0.0 & 0.0 & 0.0 & 0.0 & 0.0 & 0.0 & 0.0 & 0.9 & 0.9 \\
\hline $1-3$ & 2.1 & 1.8 & 2.1 & 2.3 & 3.1 & 3.1 & 2.5 & 1.8 & 1.7 & 1.6 & 2.1 & 2.6 & 3.2 & 3.3 & 3.1 & 2.5 & 0.0 & 38.9 \\
\hline $4-7$ & 1.5 & 1.6 & 1.6 & 2.0 & 2.7 & 2.7 & 2.2 & 1.3 & 0.9 & 1.0 & 2.2 & 4.4 & 4.9 & 3.2 & 2.1 & 1.5 & 0.0 & 35.7 \\
\hline $8-12$ & 0.4 & 0.8 & 0.6 & 0.2 & 0.2 & 0.4 & 0.8 & 0.5 & 0.3 & 0.5 & 1.2 & 2.1 & 3.2 & 2.3 & 0.7 & 0.4 & 0.0 & 14.5 \\
\hline $13-18$ & 0.2 & 0.4 & 0.2 & 0.1 & 0.0 & 0.0 & 0.1 & 0.1 & 0.1 & 0.3 & 0.8 & 0.6 & 1.2 & 2.0 & 0.7 & 0.1 & 0.0 & 7.0 \\
\hline $19-24$ & 0.0 & 0.1 & 0.1 & 0.0 & 0.0 & 0.0 & 0.0 & 0.0 & 0.0 & 0.1 & 0.3 & 0.2 & 0.2 & 0. & 0.4 & 0. & 0.0 & 2.3 \\
\hline $25-31$ & 0.0 & 0.0 & 0.0 & 0.0 & 0.0 & 0.0 & 0.0 & 0.0 & 0.0 & 0.1 & 0.1 & 0.0 & 0.0 & 0.2 & 0.1 & 0.0 & 0.0 & 0.6 \\
\hline $32-38$ & 0.0 & 0.0 & 0.0 & 0.0 & 0.0 & 0.0 & 0.0 & 0.0 & 0.0 & 0.0 & 0.0 & 0.0 & 0.0 & 0.0 & 0.0 & 0.0 & 0.0 & 0.0 \\
\hline $39-46$ & 0.0 & 0.0 & 0.0 & 0.0 & 0.0 & 0.0 & 0.0 & 0.0 & 0.0 & 0.0 & 0.0 & 0.0 & 0.0 & 0.0 & 0.0 & 0.0 & 0.0 & 0.0 \\
\hline$>46$ & 0.0 & 0.0 & 0.0 & 0.0 & 0.0 & 0.0 & 0.0 & 0.0 & 0.0 & 0.0 & 0.0 & 0.0 & 0.0 & 0.0 & 0.0 & 0.0 & 0.0 & 0.0 \\
\hline TOTAL & 4.2 & 4.7 & 4.6 & 4.7 & 6.1 & 6.2 & 5.7 & 3.7 & 3.1 & 3.6 & 6.7 & 9.9 & 12.8 & 11.6 & 7.1 & 4.5 & 0.9 & 100.0 \\
\hline
\end{tabular}

Station: (14) WPPS

Begin: 1/1982 End: 12/2003

Total Hours: 189104

DIRECTION

\begin{tabular}{|c|c|c|c|c|c|c|c|c|c|c|c|c|c|c|c|c|c|c|}
\hline PEED & $\mathrm{N}$ & NNE & $\mathrm{NE}$ & ENE & $E$ & ESE & $\mathrm{SE}$ & SSE & S & SSW & SW & WSW & W & WNW & NW & NNW & CALM & TOTAL \\
\hline CALM & 0.0 & 0.0 & 0.0 & 0.0 & 0.0 & 0.0 & 0.0 & 0.0 & 0.0 & 0.0 & 0.0 & 0.0 & 0.0 & 0.0 & 0.0 & 0.0 & 0.9 & \\
\hline $1-3$ & 2.5 & 2.1 & 1.8 & 1.3 & 1.1 & & 1.4 & & 2.1 & & & & 1.6 & & & 2.7 & & \\
\hline $4-7$ & 3. & 2. & 2 . & 1. & & & & & & & & & & & & & & \\
\hline $8-12$ & 1.2 & 0.7 & 0.5 & 0.2 & 0. & . & 0 . & 2. & 3.4 & & 1. & & 0. & & 2 & & & \\
\hline-18 & 0.3 & 0.2 & 0.1 & 0.0 & 0. & 0. & 0. & 0. & 0.7 & & 1.2 & & 0. & B & & 3 & & \\
\hline & 0.0 & 0.1 & 0.0 & 0.0 & 0.0 & 0.0 & 0.0 & 0.0 & 0.1 & & 0.6 & 0. & 0.1 & 0 . & 0 . & & & \\
\hline & 0.0 & 0.0 & 0.0 & 0.0 & 0.0 & 0.0 & 0.0 & 0 . & 0.0 & 0 . & 0.2 & 0. & & 0 . & & & & \\
\hline & & & 0.0 & & & & & & & & & & & & & & & \\
\hline $9-46$ & 0 . & & & & & & & & & & & & & & & & & \\
\hline$>46$ & 0.0 & 0. & 0. & 0 . & 0 . & 0 . & 0. & 0 . & 0.0 & & & & & & & 0 & & \\
\hline TOTAL & 7.5 & 5.5 & 4.6 & 2.6 & 1.9 & 2.1 & 4.1 & 8.3 & 11.6 & 9.6 & 6.7 & 4.2 & 4.4 & 6.2 & 10.7 & 9.0 & 1.0 & \\
\hline
\end{tabular}

Station: (15) FRNK

\begin{tabular}{|c|c|c|c|c|c|c|c|c|c|c|c|c|c|c|c|c|c|c|}
\hline PEED & $\mathrm{N}$ & $\mathrm{NE}$ & $\mathrm{NE}$ & ENE & $\mathrm{E}$ & ESE & SE & SSE & S & SSW & SW & WSW & $W$ & WNW & NW & NNW & CALM & $101 \mathrm{~T}$ \\
\hline CALM & 0.0 & 0.0 & 0.0 & 0.0 & 0.0 & 0.0 & 0.0 & 0.0 & 0.0 & 0.0 & 0.0 & 0.0 & 0.0 & 0.0 & 0.0 & 0.0 & 0.7 & \\
\hline $1-3$ & 1.2 & 1.0 & 1.0 & 0.8 & 0.9 & 1.1 & 1.4 & 1.4 & 1.2 & 1.1 & 1.1 & 1.1 & 1.3 & 1.4 & 1.6 & 1.4 & 0.0 & 19. \\
\hline $4-7$ & 4.1 & 2.7 & 1.7 & 1.2 & 1.5 & & 5.0 & & 3.5 & & & & & . & & .4 & & \\
\hline $8-12$ & 1.6 & 0.9 & 0.5 & 0 & 0.3 & & 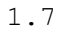 & & 2.5 & & & & & & & & & \\
\hline $3-18$ & 0.1 & 0.2 & 0.2 & 0 . & 0.0 & 0 . & 0.1 & 0.2 & 0.4 & 1. & 1.8 & . & 0.2 & 0.2 & 0. & .2 & & \\
\hline $9-24$ & 0.0 & 0.0 & 0.0 & 0.0 & 0.0 & 0 . & 0.0 & 0.0 & 0.0 & 0.3 & 0.5 & 0.2 & 0.1 & 0.0 & 0 . & 0.0 & 0. & \\
\hline $5-31$ & 0.0 & 0.0 & 0.0 & 0.0 & 0.0 & 0 . & 0.0 & 0.0 & 0.0 & 0. & 0.2 & 0 . & 0.0 & 0.0 & 0 & 0.0 & & \\
\hline-38 & 0.0 & 0.0 & 0.0 & 0.0 & 0.0 & 0 . & 0.0 & 0. & 0.0 & 0 . & & 0. & 0. & 0 . & & .0 & & \\
\hline-46 & 0.0 & 0.0 & 0 . & 0 . & . & 0 . & & . & . & & & & & 0. & & & & \\
\hline$>46$ & 0.0 & 0.0 & 0.0 & 0 . & 0. & 0. & 0. & 0 . & 0.0 & 0. & & & 0. & 0. & & .0 & & \\
\hline OTAL & 7.0 & 4.9 & 3.4 & 2.4 & 2.7 & 4.3 & 8.3 & 7.5 & 7.8 & 11.2 & 9.5 & 4.2 & 3.5 & 4.2 & 8.9 & 9.5 & 0.7 & \\
\hline
\end{tabular}

Station: (16) GABL

\begin{tabular}{|c|c|c|c|c|c|c|c|c|c|c|c|c|c|c|c|c|c|c|}
\hline EED & $\mathrm{N}$ & $\mathrm{NE}$ & $\mathrm{NE}$ & ENE & $E$ & ESE & SE & SSE & S & SSW & SW & DSW & W & WNW & NW & NNW & CALM & \\
\hline CALM & 0.0 & 0.0 & 0.0 & 0.0 & 0.0 & 0.0 & 0.0 & 0.0 & 0.0 & 0.0 & 0.0 & 0.0 & 0.0 & 0.0 & 0.0 & 0.0 & 0.8 & \\
\hline $1-$ & 1.0 & 0.9 & 0.8 & 0.6 & 0.6 & 0.6 & 0.7 & 0. & 1.0 & 1. & 0.9 & 0 . & 0.7 & 0.7 & 0. & 0.9 & b. & 12. \\
\hline $4-7$ & 2.3 & 2.3 & 1.6 & 0.9 & 0.9 & 0.9 & 1.3 & 2.1 & 3.2 &. & 1.8 & 1. & 1.5 & 1.7 & 2 & . & & 28. \\
\hline $8-$ & 2.0 & 2.2 & & & & & & & & & & & & & & & & \\
\hline & 1 & 1.4 & 0 . & & & & & & & & & & & & & & & 17. \\
\hline & 0.4 & 0.5 & 0.3 & 0.0 & 0 . & 0. & 0. & 0. & .6 & & 0. & & 0.9 & 2. & & & & \\
\hline $5-3$ & 0.1 & 0.1 & 0.2 & 0.0 & 0.0 & 0. & 0. & 0 . & 0.2 & . & 0.6 & & 0.2 & 1.3 & 0. & .0 & . & \\
\hline $32-38$ & 0.0 & 0.0 & 0.1 & 0.0 & 0.0 & 0. & 0.0 & 0. & 0.1 & 0.2 & 0.3 & 0. & 0.0 & 0.2 & 0 & .0 & & \\
\hline $39-46$ & 0.0 & 0.0 & 0.0 & 0.0 & 0. & 0 . & 0 . & 0 . & 0.0 & D. & 0.1 & 0. & 0.0 & 0 . & & & & \\
\hline & 0.0 & 0.0 & 0. & 0.0 & & & 0. & & 0 . & & & & & 0.0 & & & & \\
\hline TOTAL & 7.0 & 7.5 & 4.5 & 2.0 & 2.0 & 2.1 & 3.2 & 6.1 & 9.0 & 6.8 & 7.3 & 6.7 & 7.0 & 11.7 & 10.6 & 5.7 & 0.8 & \\
\hline
\end{tabular}


Table 5.4. (contd)

Station: (17) RING

Begin: 1/1982

End: $12 / 2003$

Total Hours: 188267

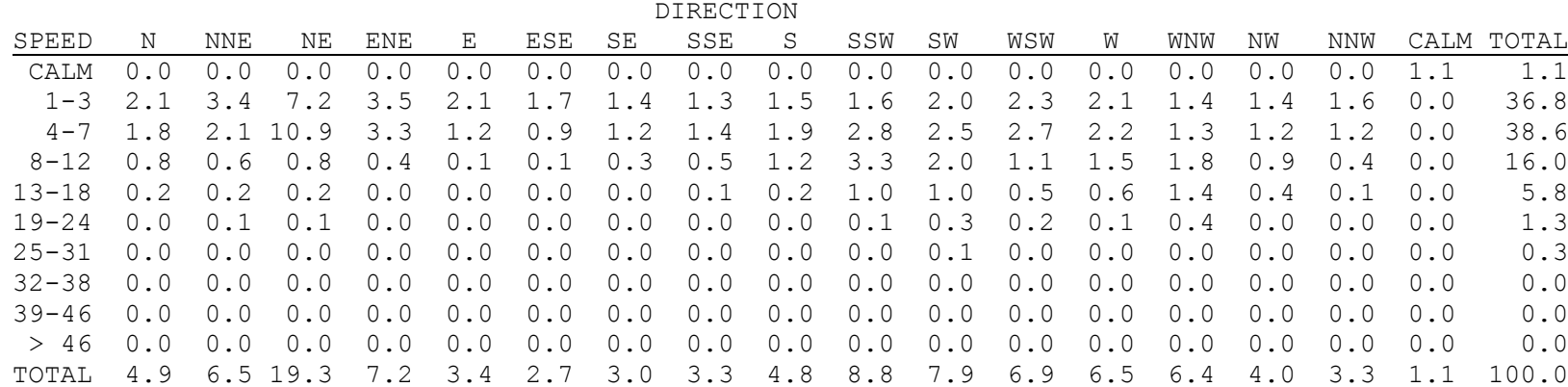

Station: (18) RICH

Begin: 1/1982 End: 12/2003

Total Hours: 189348

DIRECTION

\begin{tabular}{|c|c|c|c|c|c|c|c|c|c|c|c|c|c|c|c|c|c|c|}
\hline EED & $\mathrm{N}$ & NNE & $\mathrm{NE}$ & ENE & $E$ & ESE & SE & SSE & S & SSW & SW & WSW & W & WNW & NW & NNh & CATN & TTA \\
\hline CALM & 0.0 & 0.0 & 0.0 & 0.0 & 0.0 & 0.0 & 0.0 & 0.0 & 0.0 & 0.0 & 0.0 & & 0.0 & 0.0 & 0.0 & 0.0 & 1.1 & 1.1 \\
\hline $1-3$ & 1.3 & 0.9 & 0.9 & 1.0 & 1.6 & 2.6 & 3.1 & 2.6 & 2.2 & 2.1 & 2.3 & 2.4 & 2 & 2.7 & 5 & 1.9 & 0.0 & 32.5 \\
\hline $4-7$ & 2.0 & 0.9 & 0.9 & 1.0 & 1.8 & 2.8 & 3.2 & 1. & 1.7 & 3.0 & 4.1 & 3.2 & 2.3 & 2.7 & 3. & 2.6 & 0.0 & 36.9 \\
\hline $8-12$ & 1.3 & 0.7 & 0.4 & 0.3 & 0.2 & 0.2 & & 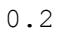 & 0.6 & 2.6 & & 2.7 & & & & 1.5 & & 18.6 \\
\hline & 0 & 0 & 0. & & 0. & & & & & & & & & & & & & .4 \\
\hline & c & 0 & 0. & & 0. & & & & & & & & & & & & & 2.1 \\
\hline-31 & 0. & 0.0 & 0.0 & 0. & 0.0 & & 0 & & 0. & 0. & 0. & 0.1 & 0 & 0.0 & & 0.0 & 0 & 0.4 \\
\hline $2-38$ & 0. & 0.0 & 0. & 0.0 & 0.0 & 0. & 0 . & 0. & 0.0 & 0.0 & 0.0 & 0.0 & 0. & 0.0 & 0 & 0.0 & 0.0 & 0.0 \\
\hline $39-46$ & 0.0 & 0.0 & 0.0 & 0.0 & 0.0 & 0. & 0. & 0. & 0.0 & 0 . & 0. & 0. & 0 & 0.0 & & .0 & 0 & 0.0 \\
\hline$>46$ & 0.0 & 0.0 & 0.0 & 0.0 & 0.0 & 0. & & & 0 . & 0. & 0.0 & 0.0 & & & & .0 & 0.0 & 0.0 \\
\hline OTAL & .0 & 2.7 & 2.3 & 2.3 & 3.7 & 5.6 & 6.6 & 4.5 & 4.7 & 9.0 & 13.5 & 10.0 & 7.5 & 6.7 & 7.9 & 6.9 & 1.1 & 100. \\
\hline
\end{tabular}

Station: (19) PFP

Begin: 2/1994 End: 12/2003

Total Hours: 86475 DIRECTION

\begin{tabular}{|c|c|c|c|c|c|c|c|c|c|c|c|c|c|c|c|c|c|c|}
\hline SEEED & $\mathrm{N}$ & NNE & $\mathrm{NE}$ & ENE & $E^{\prime}$ & ESE & SE & SSE & S & SSW & SW & WSW & W & WNW & NW & NNW & CALM & TOTAL \\
\hline CALM & 0.0 & 0.0 & 0.0 & 0.0 & 0.0 & 0.0 & 0.0 & 0.0 & 0.0 & 0.0 & 0.0 & 0.0 & 0.0 & 0.0 & 0.0 & 0.0 & 2.1 & 2.1 \\
\hline $1-3$ & 3.4 & 3.2 & 2.0 & 1.4 & 1.4 & 1.8 & 2.2 & 1.8 & 1.8 & 1.8 & 2.3 & 3.2 & 5.0 & 5.1 & 3.8 & 3.2 & 0.0 & 43.3 \\
\hline $4-7$ & 3.4 & 2.1 & 0.9 & 0.6 & 0.8 & 1.5 & 1.9 & 0.8 & 0.6 & 0.7 & 1.2 & .9 & 4.2 & 5.5 & 5. & .7 & .0 & \\
\hline $8-12$ & 0.4 & 0.5 & 0.2 & 0.1 & 0.1 & 0.2 & 0.3 & 0.2 & 0.3 & 0.6 & 1.3 & 1.9 & 1.5 & 1.6 & 3.2 & 1.2 & 0.0 & \\
\hline $13-18$ & 0.0 & 0.1 & 0.1 & 0.0 & 0.0 & 0.0 & 0.0 & 0.0 & 0.1 & 0.3 & 0.8 & 1.0 & 0.4 & 0.2 & 1.2 & 0.2 & 0.0 & \\
\hline $19-24$ & 0.0 & 0.0 & 0.0 & 0.0 & 0.0 & 0.0 & 0.0 & 0.0 & 0.0 & 0.1 & 0.2 & 0.2 & 0.0 & 0.0 & 0. & .0 & 0. & \\
\hline & 0.0 & 0.0 & 0.0 & 0.0 & 0.0 & 0.0 & 0.0 & 0.0 & 0.0 & 0. & 0.0 & 0 & 0.0 & 0.0 & 0. & .0 & & \\
\hline & 0.0 & 0.0 & 0. & 0.0 & 0.0 & & 0 & 0. & 0.0 & & & & & 0. & & & & \\
\hline $39-46$ & 0.0 & 0.0 & 0.0 & 0.0 & 0.0 & 0. & 0.0 & 0.0 & 0.0 & 0 . & .0 & 0 & 0.0 & 0.0 & 0. & .0 & .0 & \\
\hline$>46$ & 0.0 & 0.0 & 0.0 & 0.0 & 0.0 & 0.0 & 0.0 & 0.0 & 0.0 & 0.0 & .0 & 0 & 0.0 & 0.0 & 0.0 & .0 & .0 & \\
\hline TOTAL & 7.3 & 5.8 & 3.3 & 2.1 & 2.4 & 3.4 & 4.4 & 2.9 & 2.8 & 3.4 & 5.9 & 8.3 & 1.1 & 3.2 & 3.3 & 8.3 & 2.1 & \\
\hline
\end{tabular}

Station: (20) RMTN

Begin: 1/1982 End: 12/2003

Total Hours: 186776

DIRECTION

\begin{tabular}{|c|c|c|c|c|c|c|c|c|c|c|c|c|c|c|c|c|c|c|}
\hline SPEED & $\mathrm{N}$ & NNE & $\mathrm{NE}$ & ENE & E & ESE & $\mathrm{SE}$ & SSE & $\mathrm{S}$ & SSW & SW & WSW & W & WNW & NW & NNW & CALM & $\mathrm{OTH}$ \\
\hline CALM & 0.0 & 0.0 & 0.0 & 0.0 & 0.0 & 0.0 & 0.0 & 0.0 & 0.0 & 0.0 & 0.0 & 0.0 & 0.0 & 0.0 & 0.0 & 0.0 & 0.6 & \\
\hline $1-3$ & 0.5 & 0.5 & 0.5 & 0.4 & & & 0.4 & & & & & & .4 & & & & & \\
\hline $4-7$ & 1.4 & 1. & 1. & .9 & & & . & & & & & & & & & & & \\
\hline $8-12$ & 2.2 & 2.5 & 1.8 & 0.8 & 0.4 & 0. & 0.2 & 0. & 0.7 & 1. & 3.5 & 2. & 1.4 & U. & 0 & 1 & & \\
\hline $3-18$ & 2.1 & 2.8 & 1.3 & 0.3 & 0. & 0 . & 0.1 & 0 . & 0.4 & 1. & 4. & 3. & 1.6 & 0 . & & & & \\
\hline & 1.0 & 2.0 & 0.8 & 0.1 & 0. & 0 . & 0.0 & 0 . & 0.1 & . & & 2. & 1.2 & 0. & & & & \\
\hline & & & & & & & & & & & & & & & & & & \\
\hline & 0.7 & & & & & & & & & & & & & & & & & \\
\hline $9-46$ & 0.0 & 0 . & 0.2 & 0. & 0 . & & 1. & D. & 0.0 & & & & 0.1 & & & & & \\
\hline$>46$ & 0.0 & 0.2 & 0.2 & 0.0 & 0.0 & . & 0.0 & 0 . & 0.0 & & 1.1 & 0 & 0.0 & 0 . & & & & \\
\hline OTAL & 7.6 & 11.7 & 7.1 & 2.6 & 1.5 & 1.1 & 1.1 & 1.3 & 2.6 & 7.5 & 22.8 & 15.8 & 6.7 & 3.6 & 3.0 & 3.5 & 0.6 & \\
\hline
\end{tabular}


Table 5.4. (contd)

Station: (21) HMS

\begin{tabular}{|c|c|c|c|c|c|c|c|c|c|c|c|c|c|c|c|c|c|c|}
\hline \multirow[b]{3}{*}{ SPFFD } & \multirow[b]{3}{*}{$\mathrm{N}$} & \multirow[b]{3}{*}{ NNE } & & \multicolumn{2}{|c|}{ Begin: } & \multicolumn{3}{|c|}{$1 / 1982$} & \multicolumn{3}{|c|}{ End: $12 / 2003$} & \multirow[b]{3}{*}{ W } & \multicolumn{4}{|c|}{ Total Hours: } & \multirow[t]{2}{*}{191465} \\
\hline & & & & & & & & IREC & ION & & & & & & & & & \\
\hline & & & $\mathrm{NE}$ & ENE & $E$ & ESE & $\mathrm{SE}$ & SSE & S & SSW & SW & WSW & & WNW & NW & NNW & CALM & TOTAL \\
\hline CALM & 0.0 & 0.0 & 0.0 & 0.0 & 0.0 & 0.0 & 0.0 & 0.0 & 0.0 & 0.0 & 0.0 & 0.0 & 0.0 & 0.0 & 0.0 & 0.0 & 1.1 & 1.1 \\
\hline $1-3$ & 2.0 & 1.7 & 1.7 & 1.4 & 1.4 & 1.5 & 1.7 & 1.3 & 1.2 & 1.2 & 1.3 & 1.4 & 1.6 & 1.9 & 2.1 & 2.1 & 0.0 & 25.5 \\
\hline $4-7$ & 2.2 & 1.4 & 1.1 & 1.0 & 1.2 & 1.4 & 1.7 & 1.6 & 1.4 & 1.5 & 2.3 & 3.7 & 5.0 & 6.2 & 6.7 & 3.8 & 0.0 & 42.3 \\
\hline $8-12$ & 0.5 & 0.5 & 0.3 & 0.1 & 0.2 & 0.1 & 0.2 & 0.4 & 0.4 & 0.6 & 1.3 & 2.4 & 2.5 & 4.5 & 5.3 & 1.1 & 0.0 & 20.4 \\
\hline $13-18$ & 0.1 & 0.2 & 0.1 & 0.0 & 0.0 & 0.0 & 0.0 & 0.1 & 0.2 & 0.4 & 1.1 & 1.2 & 0.6 & 1.4 & 2.5 & 0.3 & 0.0 & 8.2 \\
\hline $19-24$ & 0.0 & 0.0 & 0.0 & 0.0 & 0.0 & 0.0 & 0.0 & 0.0 & 0.0 & 0.2 & 0.5 & 0.3 & 0.1 & 0.2 & 0.7 & 0 & 0.0 & 2.2 \\
\hline $25-31$ & 0.0 & 0.0 & 0.0 & 0.0 & 0.0 & 0.0 & 0.0 & 0.0 & 0.0 & 0.1 & 0.1 & 0.1 & 0.0 & 0.0 & 0.1 & 0.0 & 0.0 & 0.3 \\
\hline $32-38$ & 0.0 & 0.0 & 0.0 & 0.0 & 0.0 & 0.0 & 0.0 & 0.0 & 0.0 & 0.0 & 0.0 & 0.0 & 0.0 & 0.0 & 0.0 & 0.0 & 0.0 & 0.0 \\
\hline $39-46$ & 0.0 & 0.0 & 0.0 & 0.0 & 0.0 & 0.0 & 0.0 & 0.0 & 0.0 & 0.0 & 0.0 & 0.0 & 0.0 & 0.0 & 0.0 & 0.0 & 0.0 & 0.0 \\
\hline$>46$ & 0.0 & 0.0 & 0.0 & 0.0 & 0.0 & 0.0 & 0.0 & 0.0 & 0.0 & 0.0 & 0.0 & 0.0 & 0.0 & 0.0 & 0.0 & 0.0 & 0.0 & 0.0 \\
\hline TOTAL & 4.8 & 3.9 & 3.3 & 2.5 & 2.8 & 3.0 & 3.6 & 3.4 & 3.2 & 4.0 & 6.6 & 9.1 & 9.9 & 14.2 & 17.4 & 7.3 & 1.1 & 100.0 \\
\hline
\end{tabular}

Station: (22) PASC

Begin: 10/1987 End: 12/2003

Total Hours: 137184

DIRECTION

\begin{tabular}{|c|c|c|c|c|c|c|c|c|c|c|c|c|c|c|c|c|c|c|}
\hline PEED & $\mathrm{N}$ & NNE & $\mathrm{NE}$ & ENE & $E$ & ESE & $\mathrm{SE}$ & SSE & S & SSW & SW & WSW & W & WNW & NW & NNW & CALM & TOTA \\
\hline CALM & 0.0 & 0.0 & 0.0 & 0.0 & 0.0 & 0.0 & 0.0 & 0.0 & 0.0 & 0.0 & 0.0 & 0.0 & 0.0 & 0.0 & 0.0 & 0.0 & 1.0 & \\
\hline $1-3$ & 4.6 & 2.8 & 2.4 & 2.3 & 2.5 & 2.3 & 1.9 & & 1.3 & & & & & & & & & \\
\hline $4-7$ & 2.8 & 1.2 & 0 . & & & & & & & & & & & & & & & \\
\hline $8-12$ & 1.0 & 0.4 & 0.2 & 0 & 0. & 0.2 & 0.3 & . & .5 & & 4 & & 0.9 & & & & & \\
\hline-18 & 0.2 & 0.2 & 0.1 & 0.0 & 0. & 0. & 0. & 0. & 0.1 & & 3. & & 0. & & & 3 & & \\
\hline & 0.0 & 0.0 & 0.0 & 0.0 & 0.0 & 0.0 & 0.0 & 0.0 & 0.0 & 0 . & 1.0 & 1 . & 0.2 & 0. & & & & \\
\hline & 0.0 & 0.0 & 0.0 & 0.0 & 0.0 & 0.0 & 0.0 & 0 . & 0.0 & 0 . & 0.3 & 0. & 0.1 & & & & & \\
\hline & 0.0 & & 0.0 & & & & & & & & & & & & & & & \\
\hline $39-46$ & 0 . & & & & & & & & & & & & & & & & & \\
\hline$>46$ & 0.0 & 0 . & 0. & 0 . & 0 . & 0 . & 0. & 0. & 0.0 & & & & & & & 0. & & \\
\hline TOTAL & 8.6 & 4.6 & 3.4 & 3.3 & 3.8 & 4.4 & 4.2 & 3.2 & 3.5 & 6.4 & 13.4 & 9.3 & 5.5 & 5.3 & 8.6 & 11.5 & 1.1 & \\
\hline
\end{tabular}

Station: (23) GABW

Begin: 3/1986 End: 12/2003

Total Hours: 153360

DIRECTION

\begin{tabular}{|c|c|c|c|c|c|c|c|c|c|c|c|c|c|c|c|c|c|c|}
\hline EED & $\mathrm{N}$ & $\mathrm{NE}$ & $\mathrm{NE}$ & ENE & $E$ & ESE & $\mathrm{SE}$ & SSE & S & SSW & SW & WSW & W & WNW & NW & NNW & CALM & TOTE \\
\hline CALM & 0.0 & 0.0 & 0.0 & 0.0 & 0.0 & 0.0 & 0.0 & 0.0 & 0.0 & 0.0 & 0.0 & 0.0 & 0.0 & 0.0 & 0.0 & 0.0 & 1.3 & \\
\hline $1-3$ & 1.5 & 1.3 & 1.2 & 1.3 & 1.5 & 1.7 & 2.3 & 2.5 & 2.1 & 1.6 & 1.6 & 1.7 & 2.6 & 3.6 & 3.1 & 2.0 & 0.0 & \\
\hline $4-7$ & 1.5 & 1.1 & 0.9 & 1.0 & 1.3 & & 4.0 & 4. & 1.6 & & 1.0 & & 3.1 & & & .9 & & \\
\hline $8-12$ & 0.5 & 0.5 & 0.3 & 0 & 0.2 & &. & & 0.3 & & & & 2. & & & & & \\
\hline $3-18$ & 0.1 & 0.2 & 0.1 & 0.0 & 0.0 & 0. & 0.1 & 0. & 0.2 & ). & .8 & . & 1.0 & 3.5 & 1. & .1 & & \\
\hline $9-24$ & 0.0 & 0.1 & 0.0 & 0.0 & 0.0 & 0. & 0.0 & 0. & 0.0 & 0 . & 0.4 & 0 . & 0.2 & 1.1 & 0. & 0.0 & 0 . & \\
\hline $5-31$ & 0.0 & 0.0 & 0.0 & 0.0 & 0.0 & 0 . & 0.0 & 0. & 0.0 & 0 . & 0.1 & 0 . & 0.0 & 0.1 & 0 & 0.0 & & \\
\hline-38 & 0.0 & 0.0 & 0.0 & 0.0 & 0.0 & 0. & 0.0 & 0 . & 0.0 & o. & 0 . & 0. & 0.0 & 0 . & & .0 & & \\
\hline & 0. & 0.0 & 0 . & 0. & . & 0. & & J. & 0 . & & & & & & & & & \\
\hline$>46$ & 0.0 & 0.0 & 0.0 & 0 . & 0.0 & 0. & 0. & 0 . & 0.0 & & & & 0.0 & 0.0 & 0. & .0 & & \\
\hline OTAL & 3.7 & 3.1 & 2.6 & 2.5 & 3.1 & 3.3 & 7.7 & 8.5 & 4.2 & 3.4 & 4.8 & 6.1 & 9.8 & 21.0 & 10.1 & 4.6 & 1.3 & \\
\hline
\end{tabular}

Station: (24) 100F

Begin: 3/1986 End: 12/2003

Total Hours: 153493 DIRECTION

\begin{tabular}{|c|c|c|c|c|c|c|c|c|c|c|c|c|c|c|c|c|c|c|}
\hline SPEED & $\mathrm{N}$ & NNE & $\mathrm{NE}$ & ENE & $E$ & ESE & SE & SSE & $\mathrm{S}$ & SSW & SW & WSW & $\mathrm{W}$ & WNW & NW & NNW & CALM & TOTAI \\
\hline CALM & 0.0 & 0.0 & 0.0 & 0.0 & 0.0 & 0.0 & 0.0 & 0.0 & 0.0 & 0.0 & 0.0 & 0.0 & 0.0 & 0.0 & 0.0 & 0.0 & 1.6 & \\
\hline $1-3$ & 1.8 & 1.4 & 1.2 & 1.1 & & .8 & 2.7 & & .2 & & & & 3.6 & & & & & \\
\hline $4-7$ & 2.0 & 1.3 & 1.1 & 1.1 & .2 & .7 & 5. & 5. & .6 & & & & 3.3 & & & & & \\
\hline $8-12$ & 1.0 & 0.7 & 0.3 & 0.3 & 0. & 0 . & 2.5 & 2. & 0.6 & & 0.8 & 3 & 2.5 & 2. & & .5 & & \\
\hline & 0.2 & 0.2 & 0.1 & 0.0 & 0.0 & 0.0 & 0.3 & 0 . & 0.3 & . & 0.6 & 0 . & 1.0 & 1. & & & & \\
\hline & 0.0 & 0.1 & 0.1 & 0.0 & 0. & 0.0 & 0.0 & 0 . & 0.1 & & & . & 0.2 & & & & & \\
\hline & & & & & & & & & & & & & & & & & & \\
\hline & & & & & 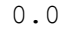 & & & & & & & & & & & & & \\
\hline $9-46$ & 0.0 & 0. & 0.0 & 0.0 & 0 . & 0. & 0. & 0. & 0.0 & & 0. & & 0.0 & 0 . & & .0 & & \\
\hline$>46$ & 0.0 & 0.0 & 0.0 & 0.0 & 0.0 & 0.0 & 0.0 & 0.0 & 0.0 & & 0.0 & 0 & 0.0 & 0.0 & 0 & 0.0 & 0.0 & \\
\hline OTAL & 5.0 & 3.7 & 2.8 & 2.6 & 2.9 & 4.2 & 10.8 & 11.2 & 4.8 & 3.6 & 4.6 & 6.4 & 10.6 & 12.8 & 7.4 & 5.0 & 1.6 & 10 \\
\hline
\end{tabular}


Table 5.4. (contd)

Station: (25) VERN

Begin: 2/1988

Total Hours: 137607

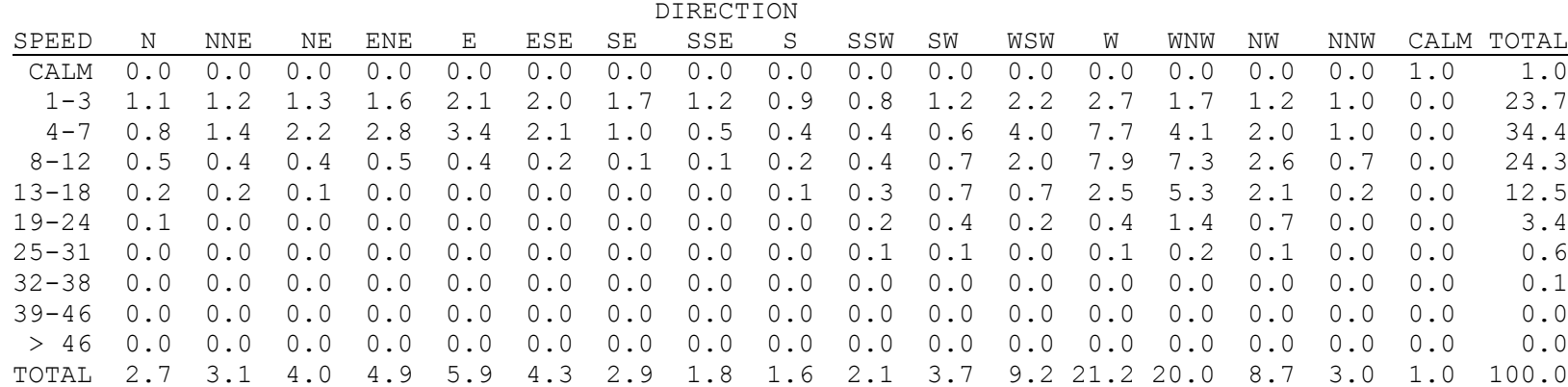

Station: (26) BENT

DIRECTION

\begin{tabular}{|c|c|c|c|c|c|c|c|c|c|c|c|c|c|c|c|c|c|c|}
\hline SPEED & $\mathrm{N}$ & $\mathrm{NNE}$ & $\mathrm{NE}$ & ENE & $\mathrm{E}$ & ESE & $\mathrm{SE}$ & SSE & $\mathrm{S}$ & SSW & SW & WSW & W & WNW & NW & NN & CALM & M TOTAL \\
\hline CALM & 0.0 & 0.0 & 0.0 & 0.0 & 0.0 & 0.0 & 0.0 & 0.0 & 0.0 & 0.0 & 0.0 & 0.0 & 0.0 & 0.0 & 0.0 & 0.0 & 0.3 & \\
\hline $1-3$ & 1.1 & 1.0 & 0.9 & 0.8 & 0.8 & 0.7 & 0.5 & 0.4 & 0.5 & 0.7 & 1.2 & 1.8 & 2.5 & 2.0 & 1.5 & 1. & 0.0 & 17.7 \\
\hline $4-7$ & 1.4 & 1.1 & 1.5 & 2.0 & 2.3 & 1.2 & 0.3 & 0.3 & 0.5 & 1.3 & 5.3 & 12.0 & 13.6 & 5.6 & 2.8 & 2 . & 0.0 & 3 \\
\hline $8-12$ & 0.5 & 0.7 & 1.0 & 0.9 & 1.0 & 0.3 & 0.0 & 0.0 & 0.2 & 0.8 & 3.2 & 5.9 & 4.7 & 1.9 & 1.1 & 0 . & 0.0 & 2 \\
\hline $13-18$ & 0.3 & 0.6 & 0.4 & 0.0 & 0.0 & 0.0 & 0.0 & 0.0 & 0.1 & 0.4 & 1.4 & 0.9 & 0.5 & 0.2 & 0.1 & 0 . & 0.0 & \\
\hline $19-24$ & 0.1 & 0.2 & 0.1 & 0.0 & 0.0 & 0.0 & 0.0 & 0.0 & 0.0 & 0.1 & 0.4 & 0.2 & 0.1 & 0.0 & 0.0 & 0 . & 0.0 & \\
\hline $25-31$ & 0.0 & 0.0 & 0.0 & 0.0 & 0.0 & 0.0 & 0.0 & 0.0 & 0.0 & 0.0 & 0.1 & 0.0 & 0.0 & 0.0 & 0.0 & 0 . & 0.0 & \\
\hline $32-38$ & 0.0 & 0.0 & 0.0 & 0.0 & 0.0 & 0.0 & 0.0 & 0.0 & 0.0 & 0.0 & 0.0 & 0.0 & 0.0 & 0.0 & 0.0 & 0 . & 0.0 & \\
\hline $39-46$ & 0.0 & 0.0 & 0.0 & 0.0 & 0.0 & 0.0 & 0.0 & 0.0 & 0.0 & 0.0 & 0.0 & 0.0 & 0.0 & 0.0 & 0.0 & U. & 0.0 & \\
\hline$>46$ & 0.0 & 0.0 & 0.0 & 0.0 & 0.0 & 0.0 & 0.0 & 0.0 & 0.0 & 0.0 & 0.0 & 0.0 & 0.0 & 0.0 & 0.0 & 0 . & 0.0 & \\
\hline & 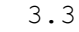 & & & 3 & & & & & & & & & & & & & & \\
\hline
\end{tabular}

Station: (27) VSTA

\begin{tabular}{|c|c|c|c|c|c|c|c|c|c|c|c|c|c|c|c|c|c|c|}
\hline PEED & $\mathrm{N}$ & NNE & $\mathrm{NE}$ & ENE & $\mathrm{E}$ & ESE & SE & SSE & S & SSW & SW & WSW & W & WNW & NW & NNW & CALM & TOTAL \\
\hline CALM & 0.0 & 0.0 & 0.0 & 0.0 & 0.0 & 0.0 & 0.0 & 0.0 & 0.0 & 0.0 & 0.0 & 0.0 & 0.0 & 0.0 & 0.0 & 0.0 & 0.7 & 0.7 \\
\hline $1-3$ & 2.2 & 2.2 & 2.0 & 1.6 & 1.6 & 1.8 & 2.0 & 1.8 & 2.1 & 2.4 & 2.7 & 2.5 & 2.2 & 1.8 & 1.8 & 1.7 & 0.0 & 32.5 \\
\hline $4-7$ & 3.0 & 2.0 & 1.6 & 1.2 & 0.8 & 1.1 & 1.1 & 1.2 & 2.0 & 4. & 5.9 & 4. & 2.4 & 2.5 & 3.1 & 3.0 & J. & 39. \\
\hline $8-12$ & 0.5 & 0.2 & 0.1 & 0.0 & 0.0 & 0.1 & 0.1 & 0.2 & 0.6 & 3. & 6.0 & 2.7 & 0.8 & 0.4 & 0 & 1.2 & 0.0 & \\
\hline $13-18$ & 0.0 & 0.0 & 0.0 & 0.0 & 0.0 & 0.0 & 0.0 & 0.0 & 0.2 & 1.6 & 4.0 & 1.1 & 0.4 & 0.2 & 0.1 & 0.1 & 0.0 & \\
\hline $19-24$ & 0.0 & 0.0 & 0.0 & 0.0 & 0.0 & 0.0 & 0.0 & 0.0 & 0.0 & 0.2 & 1.1 & 0.3 & 0.1 & 0.0 & 0. & 0.0 & 0.0 & \\
\hline $25-31$ & 0.0 & 0.0 & 0.0 & 0.0 & 0.0 & 0.0 & 0.0 & 0.0 & 0.0 & 0.0 & 0.1 & 0.0 & 0.0 & 0.0 & 0. & 0.0 & & \\
\hline $32-38$ & 0.0 & 0.0 & 0.0 & 0.0 & 0.0 & 0.0 & 0.0 & 0.0 & 0.0 & 0 . & 0.0 & 0 . & 0.0 & 0.0 & 0 & 0.0 & & \\
\hline $39-46$ & 0.0 & 0.0 & 0.0 & 0.0 & 0 . & 0 . & . & 0 . & 0.0 & & & & 0 & 0 . & & 0.0 & & \\
\hline$>46$ & 0.0 & 0.0 & 0.0 & 0.0 & 0.0 & 0.0 & 0.0 & 0.0 & 0.0 & 0.0 & 0.0 & 0.0 & 0.0 & 0.0 & 0.0 & 0.0 & 0.0 & \\
\hline TOTAL & 5.7 & 4.5 & 3.7 & 2.9 & 2.4 & 3.0 & 3.3 & 3.2 & 4.8 & 2.3 & 9.9 & 0.6 & 5.9 & 4.9 & 6.1 & 6.0 & 0.7 & \\
\hline
\end{tabular}

Station: (28) SURF

DIRECTION

\begin{tabular}{|c|c|c|c|c|c|c|c|c|c|c|c|c|c|c|c|c|c|c|}
\hline SPEED & $\mathrm{N}$ & NNE & $\mathrm{NE}$ & ENE & $\mathrm{E}$ & ESE & $\mathrm{SE}$ & SSE & S & SSW & SW & WSW & W & WNW & NW & NNW & CALM & TOTAI \\
\hline CALM & 0.0 & 0.0 & 0.0 & 0.0 & 0.0 & 0.0 & 0.0 & 0.0 & 0.0 & 0.0 & 0.0 & 0.0 & 0.0 & 0.0 & 0.0 & 0.0 & 0.7 & \\
\hline $1-3$ & & & & .2 & & & & & & & & & & & & & & \\
\hline $4-7$ & 0.2 & 0 & 2.4 & 3.9 & . & & & & & & & 6. & 1. & & & & & \\
\hline $8-12$ & 0.2 & 0.6 & 1.9 & 3.0 & 0.7 & 0.0 & U. & 0 . & 0.0 & U. & 4.4 & 10. & 3.6 & & & 0 & & \\
\hline & 0.2 & 0.3 & 0.2 & 0.5 & 0.2 & 0 . & . & 0 & 0.0 & & 2.1 & 9 & 5. & & & 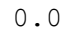 & & \\
\hline & & 0.1 & & & & & & & & & & & & & & & & \\
\hline & & & & & & & & & & & & & & & & & & \\
\hline-38 & 0.0 & 0. & 0.0 & 0 & . & & & & 0.0 & & & 0 & 0. & & & & & \\
\hline $39-46$ & 0.0 & 0. & 0.0 & 0 . & 0 . & 0 . & & 0 & 0.0 & & 0.0 & & 0.0 & & & & & \\
\hline$>46$ & 0.0 & 0.0 & 0.0 & 0.0 & 0. & 0.0 & 0 & 0 & 0.0 & & 0.0 & 0. & 0.0 & & & 0. & & \\
\hline AI & 0.9 & 2.1 & 5.7 & 8.7 & 4.1 & 1.5 & 1.0 & 0.9 & 1.6 & 3.3 & 4.3 & 34.8 & 17.3 & 2.2 & 0.6 & 0.5 & 0.7 & 100 \\
\hline
\end{tabular}


Table 5.4. (contd)

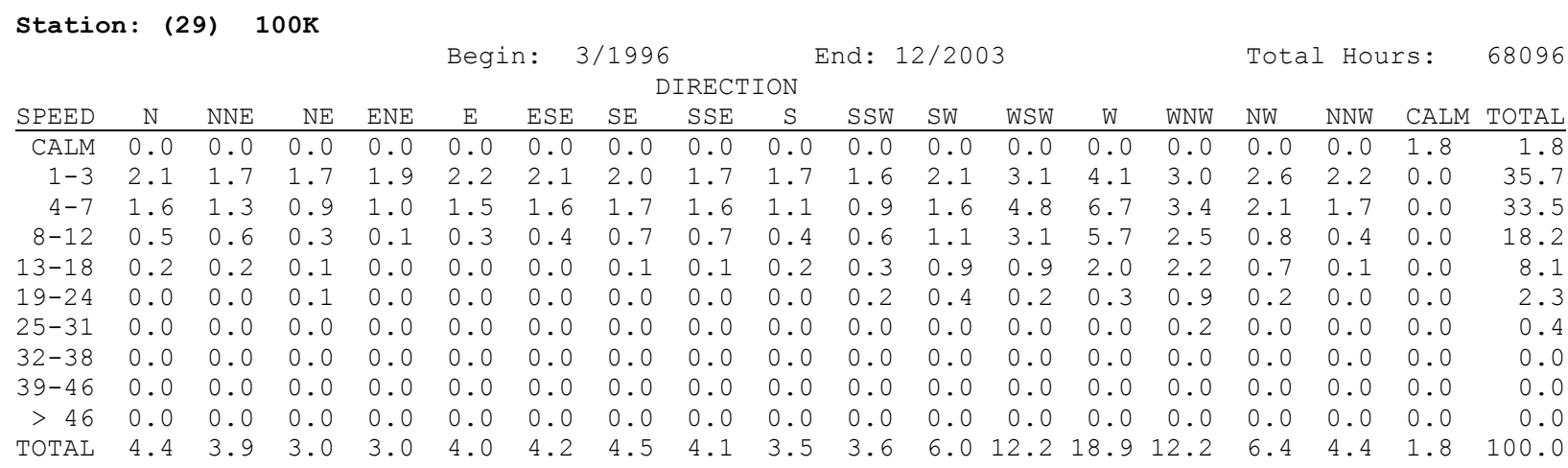

Station: (30) HAMR

Begin: 1/1998 End: 12/2003 Total Hours: 52451

\begin{tabular}{|c|c|c|c|c|c|c|c|c|c|c|c|c|c|c|c|c|c|c|}
\hline \multicolumn{19}{|c|}{ DIRECTION } \\
\hline SPEED & $\mathrm{N}$ & NNE & $\mathrm{NE}$ & ENE & $\mathrm{E}$ & ESE & $\mathrm{SE}$ & SSE & $\mathrm{S}$ & SSW & SW & WSW & W & WNW & NW & NNW & CALM & TOTAL \\
\hline CALM & 0.0 & 0.0 & 0.0 & 0.0 & 0.0 & 0.0 & 0.0 & 0.0 & 0.0 & 0.0 & 0.0 & 0.0 & 0.0 & 0.0 & 0.0 & 0.0 & 0.5 & 0.5 \\
\hline $1-3$ & 1.2 & 0.8 & 0.8 & 0.6 & 0.8 & 1.1 & 1.9 & 2.2 & 2.1 & 1.6 & 1.4 & 1.2 & 1.2 & 1.3 & 1.4 & 1.2 & 0.0 & 20.9 \\
\hline $4-7$ & 4.0 & 1.8 & 0.8 & 0.7 & 1.0 & 2.1 & 4.8 & 4.4 & 4.1 & 3.8 & 4.0 & 2.4 & 1.6 & 1.8 & 2.8 & 3.7 & 0.0 & 44.0 \\
\hline $8-12$ & 2.5 & 1.0 & 0.3 & 0.1 & 0.3 & 0.3 & 0.4 & 0.4 & 1.2 & 3.0 & 6.0 & 2.6 & 0.7 & 0.6 & 1.6 & 2.4 & 0.0 & 23.5 \\
\hline $13-18$ & 0.6 & 0.2 & 0.0 & 0.0 & 0.0 & 0.0 & 0.0 & 0.1 & 0.2 & 0.9 & 2.4 & 1.3 & 0.5 & 0.2 & 1.3 & 1.0 & 0.0 & 8.7 \\
\hline $19-24$ & 0.1 & 0.1 & 0.0 & 0.0 & 0.0 & 0.0 & 0.0 & 0.0 & 0.1 & 0.2 & 0.6 & 0.3 & 0.2 & 0.0 & 0.3 & 0.1 & 0.0 & 2.0 \\
\hline $25-31$ & 0.0 & 0.0 & 0.0 & 0.0 & 0.0 & 0.0 & 0.0 & 0.0 & 0.0 & 0.1 & 0.2 & 0.1 & 0.1 & 0.0 & 0.0 & 0.0 & 0.0 & 0.5 \\
\hline-38 & 0.0 & 0.0 & 0.0 & 0.0 & 0.0 & 0 . & 0.0 & 0.0 & 0.0 & 0 . & 0. & 0 . & 0.0 & 0. & 0. & 0 & & 0.0 \\
\hline $39-46$ & 0.0 & 0.0 & 0.0 & 0.0 & 0.0 & 0.0 & 0.0 & 0.0 & 0.0 & 0.0 & 0.0 & 0.0 & 0.0 & 0.0 & 0.0 & 0.0 & 0.0 & 0.0 \\
\hline$>46$ & 0.0 & 0.0 & 0.0 & 0.0 & 0.0 & 0.0 & 0.0 & 0.0 & 0.0 & 0.0 & 0.0 & 0.0 & 0.0 & 0.0 & 0.0 & 0.0 & 0.0 & 0.0 \\
\hline TOTAL & 8.3 & 3.8 & 1.9 & 1.5 & 2.1 & 3.5 & 7.2 & 7.1 & 7.8 & 9.6 & 14.6 & 7.9 & 4.3 & 3.9 & 7.4 & 8.4 & 0.5 & 100.0 \\
\hline
\end{tabular}




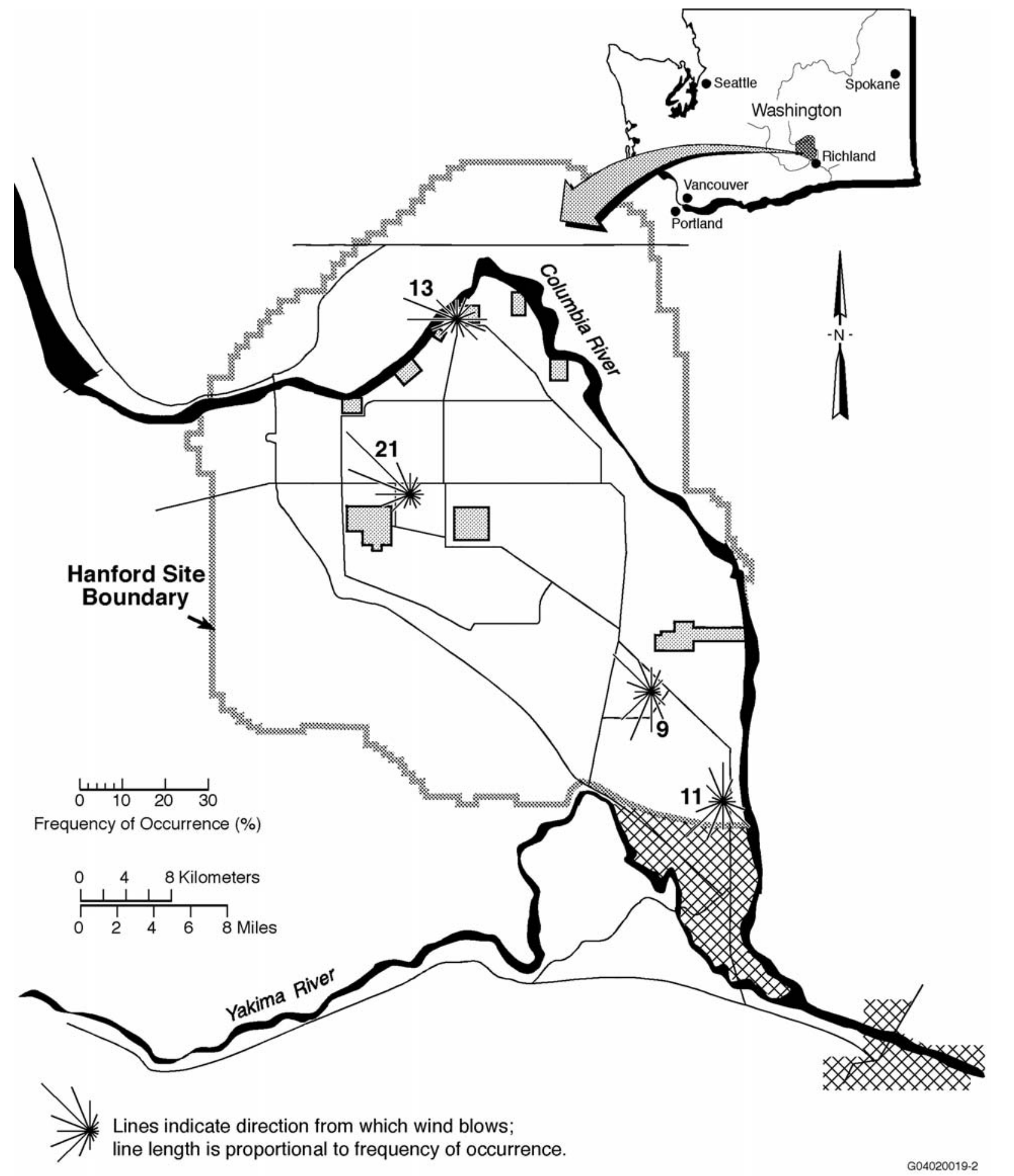

Figure 5.2. Hanford Meteorological Monitoring Network Wind Roses at 60-Meter Level, 1986 through 2003 (Refer to Table 1.1 for the names of the numbered locations on this map.) 
Table 5.5. Joint Frequency Distributions (\%) for Hanford Meteorological Monitoring Network Wind Stations at 60-Meter Level, 1986 through 2003

\begin{tabular}{|c|c|c|c|c|c|c|c|c|c|c|c|c|c|c|c|c|c|c|}
\hline \multirow{4}{*}{$\begin{array}{l}\text { SPEED } \\
\text { CATM }\end{array}$} & \multirow{3}{*}{$\mathrm{N}$} & \multirow{3}{*}{ NNE } & \multirow[b]{3}{*}{$\mathrm{NE}$} & \multirow{2}{*}{\multicolumn{3}{|c|}{ Begin: }} & \multicolumn{3}{|c|}{$1 / 1986$} & \multirow[t]{2}{*}{ End: } & \multicolumn{2}{|c|}{$12 / 2003$} & \multirow[b]{3}{*}{ W } & \multirow[b]{3}{*}{ WNW } & Total & $1 \mathrm{H}$ & Es: & 151963 \\
\hline & & & & & & & & & & & & & & & & & & \\
\hline & & & & ENE & $\mathrm{E}$ & ESE & $\mathrm{SE}$ & SSE & $\mathrm{S}$ & SSW & SW & WSW & & & NW & NNW & CALM & I TOTAL \\
\hline & 0.0 & 0.0 & 0.0 & 0.0 & 0.0 & 0.0 & 0.0 & 0.0 & 0.0 & 0.0 & 0.0 & 0.0 & 0.0 & 0.0 & 0.0 & 0.0 & 0.7 & 0.7 \\
\hline $1-3$ & 1.7 & 1.6 & 1.8 & 2.2 & .2 & 3.0 & 2.4 & 1.7 & 1.4 & 1.2 & 1.3 & & 1.9 & 2.0 & 1.9 & 1.7 & 0.0 & 30.4 \\
\hline $4-7$ & 1.7 & 1.9 & 1.6 & 1.8 & .1 & 3.2 & 2.6 & 1.5 & 1.0 & 0.8 & 1.3 & 2.0 & 3.0 & 3.1 & 2.2 & 1.4 & 0.0 & 32.1 \\
\hline $8-12$ & 0.7 & 1.0 & 0.8 & 0.4 & .5 & 0.7 & 1.2 & 0.6 & 0.5 & 0.5 & 1.0 & 1.3 & 2.6 & 2.6 & 0.9 & 0.5 & 0.0 & 16.0 \\
\hline $13-18$ & 0.4 & 0.6 & 0.4 & 0.2 & 0.1 & 0.2 & 0.5 & 0.4 & 0.3 & 0.4 & 0.8 & 0.9 & 2.6 & 3.0 & 0.7 & 0.2 & 0.0 & 11.6 \\
\hline $19-24$ & 0.2 & 0.3 & 0.2 & .1 & .1 & 0.1 & 0.1 & 0.1 & 0.1 & 0.2 & 0.5 & 0.4 & 1.0 & 1.9 & 0.6 & 0.1 & 0. & 6.0 \\
\hline $25-31$ & 0.1 & 0.1 & 0.1 & 0.0 & & 0.0 & 0.0 & 0.0 & 0.1 & 0.2 & 0.3 & 0 . & 0.3 & 0.7 & & 0.0 & 0.0 & .5 \\
\hline $32-38$ & 0.0 & .0 & 0.0 & .0 & 0 & 0.0 & 0.0 & 0.0 & & 0.1 & 0.1 & & 0.1 & 0.1 & 0.1 & 0.0 & 0.0 & .6 \\
\hline $39-46$ & 0.0 & 0.0 & 0.0 & 0.0 & .0 & 0.0 & 0.0 & 0.0 & 0.0 & 0.0 & 0.0 & 0.0 & 0.0 & 0.0 & 0.0 & 0.0 & 0.0 & \\
\hline$>46$ & 0.0 & 0.0 & 0.0 & 0.0 & .0 & 0.0 & 0.0 & 0.0 & 0.0 & .0 & 0.0 & 0.0 & 0.0 & 0.0 & 0.0 & 0.0 & 0.0 & 0.0 \\
\hline COTAL & 4.8 & 5.5 & 4.9 & 4.8 & 7.0 & 7.2 & 6.8 & 4.4 & 3.3 & 3.4 & 5.3 & 6.4 & 11.4 & 13.4 & 6.6 & 4.1 & 0.7 & \\
\hline & 200 & & & & & & & & & & & & & & & & & \\
\hline & & & & & eg & & 1198 & & & End: & $12 /$ & 03 & & & Total & $1 \mathrm{HOl}$ & IS: & 57604 \\
\hline EED & N & $\mathrm{NF}$ & (זע口 & (2) & $\Gamma$ & תחת & & $\mathrm{SSF}$ & & & & & & & & & & \\
\hline CALM & 0.0 & 10 & ND & $N E$ & $\frac{1}{0}$ & ESE & SE & DL & S & SWW & SW & WS & W & WNW & & NNW & CALM & \\
\hline & & & & & & 0.0 & 0.0 & & & .0 & 0.0 & 0.0 & 0.0 & 0.0 & 0.0 & 0.0 & & \\
\hline & & & & & & .0 & & & & & & & & & & & & \\
\hline & 2 . & & 1.5 & 1.3 & .6 & 1.3 & 1.8 & 1. & 1.0 & 1.0 & 1.2 & 1. & 2.0 & 2.9 & 4.2 & 3.7 & & 31. \\
\hline $8-12$ & 1.0 & 0.7 & 0.5 & 0.3 & 0.4 & 0.3 & 0.4 & 0.7 & 0.4 & 0.6 & 1.2 & 1.9 & 2.7 & 4.8 & 6.2 & 2.2 & 0.0 & 24.4 \\
\hline $3-18$ & 0.2 & 0.3 & 0.2 & 0.1 & 0.1 & 0.0 & 0.1 & 0.3 & 0.3 & 0.5 & 1.1 & 1.9 & 2.0 & 4.6 & 5.6 & 0.6 & 0.0 & 17.9 \\
\hline & 0.0 & 1 & 0.1 & .0 & & 0.0 & 0.0 & 0 . & & & & & & & & .1 & & 7.4 \\
\hline & 0. & & 0. & & & 0.0 & 0. & & & & & & & & & .0 & & .2 \\
\hline $32-38$ & 0.0 & 0 & 0. & c & & 0.0 & 0.0 & 0 . & 0.0 & 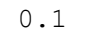 & 0.2 & & 0.0 & 0. & 0.1 & 0.0 & 0 & . \\
\hline $39-46$ & 0.0 & 0.0 & 0.0 & 0.0 & .0 & 0.0 & 0.0 & 0.0 & 0.0 & 0.0 & 0.1 & 0. & 0.0 & 0.0 & 0.0 & 0.0 & 0.0 & 0 . \\
\hline$>46$ & 0.0 & 0.0 & 0.0 & .0 & . & 0.0 & 0.0 & 0 . & 0.0 & 0 . & 0.0 & 0 . & 0.0 & 0.0 & 0.0 & 0.0 & 0.0 & \\
\hline TOTAL & 5.0 & 2 & 3.4 & 2.7 & 3 & 2.7 & 3.5 & 3.6 & 2.6 & 3.5 & 5.9 & 7.2 & 8.1 & 15.5 & 20.8 & 7.9 & 0.3 & \\
\hline & 300 & & & & & & & & & & & & & & & & & \\
\hline & & & & & eg & & 198 & & & End: & $12 / 2$ & & & & Total & $1 \mathrm{HOr}$ & Is: & 1076 \\
\hline & & & & & & & & RFG & & & & & & & & & & \\
\hline SPEED & $\mathrm{N}$ & $\mathrm{NF}$ & $\mathrm{NE}$ & ENE & $E$ & ESE & $\mathrm{SE}$ & & $\mathrm{S}$ & SSW & N & WSW & & WNW & NW & NNW & CALM & I TOTAI \\
\hline$\overline{\mathrm{CA}}$ & 0.0 & & 0.0 & .0 & 0.0 & 0.0 & 0.0 & & 0.0 & & & & 0.0 & & & 0.0 & & 0.6 \\
\hline $1-$ & 1.0 & 0 & 0.7 & 0.6 & & 1.1 & 1.2 & 1.2 & 1.1 & .9 & 0.8 & 7 & 0.7 & 0.8 & 0.9 & 1.0 & 0. & 14.2 \\
\hline $4-7$ & 2.8 & 1.8 & 1.2 & 1.2 & & 2.8 & 4.2 & 3.1 & 2.8 & 2.5 & 2.0 & 1.2 & 0.9 & 0.8 & 1.3 & 2.4 & 0.0 & 33.1 \\
\hline $8-12$ & 3.2 & 2.0 & 0.9 & .4 & 0.4 & 1.2 & 2.7 & 1.5 & 1.9 & 3.5 & 3.3 & 1.7 & .7 & 0.6 & 1.2 & 2.6 & & 28.0 \\
\hline & 1.7 & & 0.2 & & & 0.2 & 0.4 & 0. & 0. & & 3.3 & 1. & .6 & & 11 & 1.9 & & 15.6 \\
\hline & 0.2 & & & & & 0.0 & 0 . & & & & & & 3 & & & 0.4 & & 5.5 \\
\hline & 0. & & & & & 0.0 & & & & & & & & & & 0.1 & & .1 \\
\hline $32-38$ & 0.0 & 0 . & 0.0 & 0 & & 0.0 & 0.0 & 0 . & 0.0 & 0.1 & 0.3 & 0.1 & 0.0 & 0.0 & 0.0 & 0.0 & 0.0 & .6 \\
\hline $39-46$ & 0.0 & 0.0 & 0.0 & 0 & 0.0 & 0.0 & 0.0 & 0 . & 0.0 & 0.0 & 0.1 & 0. & 0.0 & 0.0 & 0.0 & 0.0 & 0.0 & .2 \\
\hline$>46$ & 0.0 & 0 & 0 . & 0 & & 0.0 & 0.0 & 0 . & 0. & & 0.0 & & & & & 0.0 & 0.0 & 0 \\
\hline TOTAL & 9.0 & 5.7 & 3.1 & 2.3 & 3.0 & 5.3 & 8.6 & 6.1 & 6.6 & 9.8 & 12.0 & 6.7 & 3.4 & 2.8 & 5.5 & 8.3 & 0.6 & 100.0 \\
\hline & & & & & & & & & & & & & & & & & & \\
\hline & & & & & Beg & & 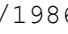 & & & End: & $12 / 2$ & 03 & & & Total & $1 \mathrm{HOU}$ & rs: & 152899 \\
\hline & & & & & & & & & & & & & & & & & & \\
\hline SPEED & $\mathrm{N}$ & & $\mathrm{NE}$ & $\mathrm{NE}$ & $\mathrm{E}$ & ESE & SE & & $\mathrm{S}$ & SSW & SW & & & & & NNW & CALM & I TOTAI \\
\hline$\overline{\text { CALM }}$ & 0.0 & & 0.0 & & & & 0.0 & & & & & & & & & 0.0 & & \\
\hline & 0. & & 0. & & & 0.8 & 0. & & & & 0.8 & & & & 0.7 & 0.8 & 0.0 & 12. \\
\hline & 2.2 & 2.0 & 1.6 & 1 & & 1.3 & 2.0 & 2. & 3.0 & 2.3 & 1.6 & 1. & 1.2 & 1. & 2.1 & 2.2 & & 28.9 \\
\hline $8-12$ & 1.9 & 1.7 & 1.0 & 0.4 & 0.3 & 0.3 & 1.3 & 2.3 & 3.5 & 3.7 & 2.1 & 0.9 & 0.9 & 1.4 & 2.9 & 2.7 & 0.0 & 27.4 \\
\hline $13-18$ & 0.7 & 0.6 & 0.3 & .1 & 0.0 & 0.0 & 0.5 & 0. & 1.6 & 3.7 & 2.4 & 0.8 & 0.6 & 1.5 & 3.7 & 1.8 & 0.0 & 19.0 \\
\hline & 0.1 & & 0. & & & 0.0 & 0.1 & 0 & & & & & & & 2.2 & 0.3 & & \\
\hline & 0.0 & & 0 . & 0 & & 0.0 & 0 . & 0 . & 0 . & 0 . & 0.7 & 0 & 0.1 & 0 . & 0.6 & 0.1 & 0.0 & 2 . \\
\hline & 0.0 & & 0. & & & 0.0 & 0 . & & 0. & & 0.3 & & & & 0.1 & 0.0 & 0.0 & 0 . \\
\hline $39-46$ & 0.0 & 0 & 0. & 0 & 0. & 0.0 & 0.0 & 0 . & 0 . & 0.1 & 0.1 & 0 . & 0.0 & 0.0 & 0.0 & 0.0 & 0.0 & \\
\hline$>46$ & 0.0 & 0.0 & 0. & 0.0 & 0.0 & 0.0 & 0.0 & 0.0 & 0.0 & 0.0 & 0.0 & 0.0 & 0.0 & 0.0 & 0.0 & 0.0 & 0.0 & 0.1 \\
\hline TOTAL & 5.8 & 5.3 & 3.8 & 2.2 & 2.1 & 2.5 & 4.8 & 7.0 & 9.4 & 12.5 & 9.5 & 4.5 & 3.9 & 6.1 & 12.4 & 7.9 & 0.3 & 100.0 \\
\hline
\end{tabular}




\subsection{Miscellaneous Climatological Statistics}

\subsection{Sky Cover}

The term sky cover is used to express the portion of the celestial dome that is (1) covered, but not necessarily hidden, by clouds or obscuring phenomena aloft; (2) hidden by an obscuring phenomenon on the ground (such as fog or smoke); or (3) a combination of both 1 and 2. The sky cover is determined hourly by scanning the sky and estimating the number of tenths that are covered ( 0 denotes clear and 10 denotes overcast). Average monthly sunrise-to-sunset sky covers for the period 1946 through 2003 are shown in Table 6.1. Also shown in Table 6.1 is the number of clear, partly cloudy, and cloudy days for the period 1954 through 2003. The number of clear, partly cloudy, and cloudy days is the result of assigning each day to one of the following categories based on its average sky cover for that day:

\begin{tabular}{|c|c|}
\hline Category & $\begin{array}{c}\text { Average } \\
\text { Sky Cover }\end{array}$ \\
\hline Clear & $0-3$ tenths \\
\hline Partly cloudy & $4-7$ tenths \\
\hline Cloudy & $8-10$ tenths \\
\hline
\end{tabular}

During the period of record (1954 through 2003), an average of 203 sunny days (the sum of the clear and partly cloudy days) was recorded per year at the Hanford Meteorology Station. The longest consecutive number of cloudy days was 28, from January 30 through February 26, 1958.

\subsection{Fog and Dense Fog}

Table 6.2 shows the average monthly and annual number of days with fog and dense fog. Fog is reported any time horizontal visibility is reduced to 6 miles or less because of the suspension of water droplets in the surface layer of the atmosphere. Dense fog is reported when horizontal visibility is reduced to 0.25 mile or less. Most fog at the Hanford Meteorology Station is radiation fog, a common type of fog that forms during nights characterized by light wind, clear sky, and moist air in the lower levels of the atmosphere. Nearly $90 \%$ of both fog and dense fog at the Hanford Meteorology Station occurs during the late autumn and winter months, though fog is observed every month of the year.

\subsection{Psychrometric Data}

Psychrometric data include observations of dry bulb, wet bulb, dew point temperatures, and relative humidity. The dry bulb temperature is the temperature of the ambient air; the wet bulb temperature is the lowest temperature to which a parcel of air, under constant pressure, can be cooled by evaporating water into it. The dew point temperature is the temperature to which a given parcel of air, under constant watervapor content, must be cooled to attain saturation. Relative humidity is the ratio of the actual water-vapor content of the air to the one where saturation would occur if the pressure and temperature remained unchanged. Relative humidity has a diurnal cycle, with the highest values generally between 4 a.m. and 6 a.m., and the lowest values between 2 p.m. and 4 p.m. 
Table 6.1. Average Sky Cover (sunrise to sunset), 1946 through 2003, and Number of Days Clear, Partly Cloudy, and Cloudy, 1954 through 2003

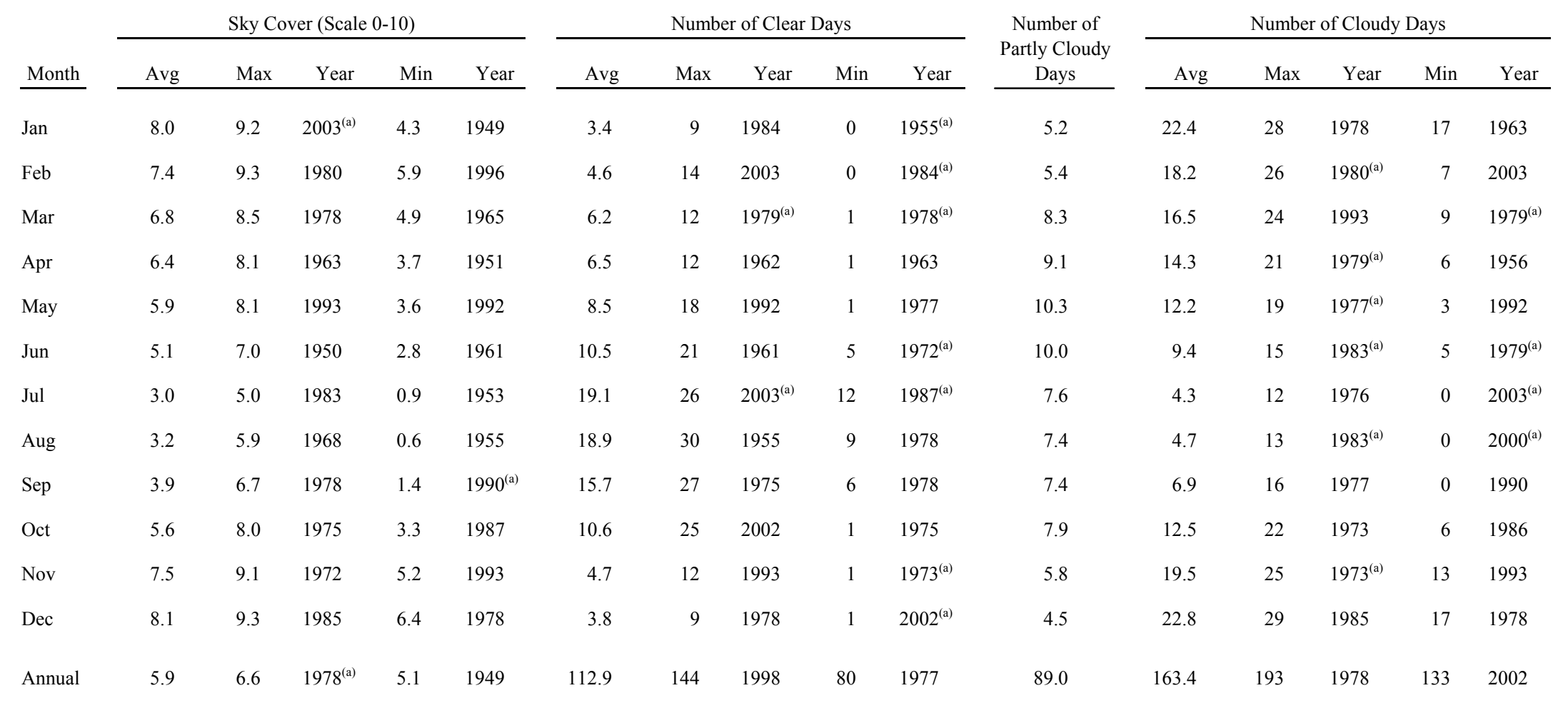

(a) Most recent of multiple occurrences. 
Table 6.2. Monthly and Annual Number of Days with Fog and Dense Fog, 1945 through 2003

\begin{tabular}{|c|c|c|c|c|c|c|c|c|c|c|}
\hline \multirow[b]{2}{*}{ Month } & \multicolumn{5}{|c|}{ Days with Fog (Visibility $\leq 6$ miles) } & \multicolumn{5}{|c|}{ Days with Dense Fog (Visibility $\leq 0.25$ mile) } \\
\hline & Avg & $\operatorname{Max}$ & Year & Min & Year & Avg & $\operatorname{Max}$ & Year & Min & Year \\
\hline Jan & 11.7 & 25 & 1979 & 0 & 1949 & 6.2 & 15 & $1994^{(\mathrm{a})}$ & 0 & 1949 \\
\hline Feb & 6.6 & 20 & 1963 & 0 & $1988^{(\mathrm{a})}$ & 3.2 & 11 & 1963 & 0 & $1999^{(\mathrm{a})}$ \\
\hline Mar & 2.0 & 10 & 1993 & 0 & $2003^{(\mathrm{a})}$ & 0.8 & 5 & $1993^{(\mathrm{a})}$ & 0 & $2003^{(\mathrm{a})}$ \\
\hline Apr & 0.5 & 3 & 1992 & 0 & $2002^{(\mathrm{a})}$ & 0.1 & 1 & $2003^{(a)}$ & 0 & $2002^{(\mathrm{a})}$ \\
\hline May & 0.2 & 3 & 1948 & 0 & $2003^{(\mathrm{a})}$ & $<0.1$ & 1 & 1958 & 0 & $2002^{\text {(a) }}$ \\
\hline Jun & 0.1 & 2 & 1971 & 0 & $2003^{(\mathrm{a})}$ & $<0.1$ & 1 & 1971 & 0 & $2003^{(\mathrm{a})}$ \\
\hline Jul & $<0.1$ & 1 & 1966 & 0 & $2003^{(a)}$ & 0 & 0 & 0 & & \\
\hline Aug & 0.1 & 1 & $1985^{(\mathrm{a})}$ & 0 & $2003^{(a)}$ & $<0.1$ & 1 & $1985^{(\mathrm{a})}$ & 0 & $2003^{(a)}$ \\
\hline Sep & 0.3 & 2 & $1985^{(\mathrm{a})}$ & 0 & $2003^{(\mathrm{a})}$ & 0.1 & 1 & $1995^{(\mathrm{a})}$ & 0 & $2003^{(\mathrm{a})}$ \\
\hline Oct & 2.0 & 9 & 1962 & 0 & $1989^{(\mathrm{a})}$ & 1.0 & 7 & 1980 & 0 & $1998^{(a)}$ \\
\hline Nov & 9.89 & 19 & $1985^{(\mathrm{a})}$ & 0 & 1990 & 5.6 & 14 & 2001 & 0 & $1990^{(a)}$ \\
\hline Dec & 14.3 & 25 & $1989^{(a)}$ & 2 & 1968 & 7.5 & 17 & 1950 & 2 & $1996^{(\mathrm{a})}$ \\
\hline Annual & 47.5 & 84 & $1985-86$ & 22 & $1948-49$ & 24.8 & 42 & $1950-51$ & 9 & $1948-49$ \\
\hline
\end{tabular}

(a) Most recent of multiple occurrences.

Longest duration of fog: 113.7 hours, December 16-20, 1985.

Longest duration of dense fog: 47.0 hours, December 1957.

Table 6.3 presents monthly averages and extremes of dry bulb, wet bulb, dew point temperatures, and relative humidity from the Hanford Meteorology Station for the period 1950 through 2003. These variables are collected hourly and are averaged on a monthly (as opposed to a daily) basis. Prior to 1975, wet bulb temperatures $\geq 75^{\circ} \mathrm{F}$ had never been observed at the Hanford Meteorology Station. On July 8, 9, and $10,1975,7$ hourly observations were made of wet bulb temperatures $\geq 75^{\circ} \mathrm{F}$.

\subsection{Solar Radiation}

Table 6.4 presents average and extreme daily solar radiation values by month for the period 1953 through 2003. These data are reported in langleys (a langley is a unit defined as 1 gram calorie per square centimeter) and are integrated over an hour period and totaled for a daily value.

The highest daily values occur with a clear sky and clean air; the lowest commonly occur on days overcast with low stratus clouds. The lowest midday values of hourly solar radiation occurred on May 18, 1980, as the dense ash cloud from the morning eruption of Mount St. Helens passed over eastern Washington. Hourly solar radiation values dropped to 0 at 1100 hours and remained at 0 for the rest of that day.

\subsection{Thunderstorms, Dust, and Glaze}

A thunderstorm day is one in which thunder is heard at the observing station one or more times during a calendar day. If a thunderstorm were to begin before midnight and continue until after midnight, it is possible to have two thunderstorm days from a single storm. 
Table 6.3. Monthly Averages and Extremes of Psychrometric Data, 1950 through 2003

\begin{tabular}{|c|c|c|c|c|c|c|c|c|c|c|c|c|c|}
\hline \multirow[b]{2}{*}{ Category $^{(\mathrm{a})}$} & \multicolumn{13}{|c|}{ Monthly Averages } \\
\hline & Jan & Feb & Mar & Apr & May & Jun & Jul & Aug & Sep & Oct & Nov & Dec & Annual \\
\hline Dry bulb & 31.4 & 37.6 & 45.2 & 53.2 & 62.1 & 69.9 & 77.3 & 75.7 & 66.5 & 53.0 & 40.1 & 32.6 & 53.7 \\
\hline Wet bulb & 29 & 34 & 39 & 44 & 50 & 55 & 58 & 58 & 53 & 45 & 37 & 31 & 44 \\
\hline Rel. hum. & 77.5 & 70.4 & 56.6 & 47.5 & 42.9 & 39.5 & 33.3 & 35.6 & 42.1 & 56.1 & 73.6 & 80.4 & 54.6 \\
\hline \multirow[t]{2}{*}{ Dew point } & 24.8 & 27.8 & 29.0 & 31.8 & 37.0 & 41.6 & 43.8 & 44.1 & 40.4 & 36.0 & 31.5 & 26.7 & 34.5 \\
\hline & \multicolumn{13}{|c|}{ Extremes of Monthly Averages } \\
\hline \multicolumn{14}{|l|}{ Dry Bulb } \\
\hline Highest & 43.0 & 44.6 & 51.6 & 58.6 & 68.7 & 77.3 & 83.3 & 82.5 & 72.7 & 59.5 & 46.4 & 38.8 & 56.6 \\
\hline Year & 1953 & 1991 & 1992 & 1987 & 1958 & 1992 & 1985 & 1967 & 1990 & 1988 & 1999 & 1953 & 1992 \\
\hline Lowest & 12.9 & 25.8 & 39.6 & 48.3 & 57.0 & 64.2 & 71.3 & 70.6 & 58.9 & 48.1 & 25.7 & 21.9 & 50.2 \\
\hline Year & 1950 & 1956 & 1955 & 1955 & 1984 & 1953 & 1986 & 1964 & 1985 & 1984 & 1985 & 1985 & 1985 \\
\hline \multicolumn{14}{|l|}{ Wet Bulb } \\
\hline$\overline{\text { Highest }}$ & 39 & 41 & 44 & 47 & 55 & 59 & 63 & 61 & 56 & 50 & 42 & 36 & 47 \\
\hline Year & 1953 & 1956 & 1992 & 1992 & 1958 & $1992^{(\mathrm{b})}$ & 1998 & $1999^{(\mathrm{b})}$ & $1995^{(\mathrm{b})}$ & 1988 & $1999^{(\mathrm{b})}$ & $1991^{(\mathrm{b})}$ & 1992 \\
\hline Lowest & 12 & 23 & 33 & 39 & 45 & 51 & 56 & 55 & 48 & 40 & 24 & 21 & 41 \\
\hline Year & 1950 & 1956 & 1955 & 1955 & 1959 & $1983^{(\mathrm{b})}$ & $1986^{(\mathrm{b})}$ & $1980^{(\mathrm{b})}$ & 1970 & 1984 & 1978 & $1985^{(\mathrm{b})}$ & 1985 \\
\hline \multicolumn{14}{|c|}{ Relative Humidity } \\
\hline Highest & 88.8 & 86.9 & 69.1 & 64.5 & 61.9 & 53.5 & 45.6 & 47.8 & 55.5 & 74.2 & 88.7 & 90.5 & 58.9 \\
\hline Year & 1960 & 1963 & 1993 & 1963 & 1948 & 1950 & 1993 & 1976 & 1977 & 1962 & 1979 & 1950 & 1978 \\
\hline Lowest & 60.0 & 54.0 & 44.0 & 36.9 & 31.2 & 30.0 & 21.9 & 24.5 & 33.2 & 42.5 & 62.8 & 69.0 & 49.4 \\
\hline Year & 1963 & 1967 & 1965 & 1966 & 1966 & 1949 & 1959 & 1967 & 1974 & 1952 & 1976 & 1968 & 1967 \\
\hline \multicolumn{14}{|l|}{ Dew Point } \\
\hline Highest & 34.4 & 36.7 & 37.2 & 37.1 & 43.9 & 47.5 & 50.1 & 48.4 & 45.4 & 43.5 & 38.3 & 34.3 & 37.7 \\
\hline Year & 1953 & $1992^{(b)}$ & 1986 & $1992^{(\mathrm{b})}$ & 1998 & 1958 & 1975 & 1976 & 1963 & 1962 & 1954 & 1950 & 1958 \\
\hline Lowest & 6.5 & 17.3 & 20.8 & 26.0 & 30.4 & 37.5 & 35.4 & 38.4 & 33.8 & 30.2 & 19.4 & 15.1 & 31.5 \\
\hline Year & 1950 & 1956 & $1965^{(\mathrm{b})}$ & 1982 & 1964 & 1954 & 1959 & 1955 & 1970 & 1984 & 1985 & 1983 & 1955 \\
\hline
\end{tabular}

(a) Dry bulb, wet bulb, and dew point temperatures in ${ }^{\circ} \mathrm{F}$, relative humidity in $\%$.

(b) Most recent of multiple occurrences.

Table 6.4. Average and Extreme Solar Radiation Daily Values (langley), 1953 through 2003

\begin{tabular}{|c|c|c|c|c|c|c|c|c|c|c|c|c|c|}
\hline & Jan & Feb & Mar & Apr & May & Jun & Jul & Aug & Sep & Oct & Nov & Dec & Annual \\
\hline Average & 105 & 185 & 317 & 446 & 546 & 604 & 628 & 535 & 402 & 252 & 123 & 82 & 352 \\
\hline Highest & 277 & 422 & 542 & 704 & 838 & 821 & 808 & 721 & 591 & 434 & 295 & 196 & 838 \\
\hline Year & 1969 & 1958 & 1968 & 1972 & 1977 & 1971 & 1974 & 1957 & 1970 & 1973 & 1971 & 1972 & May 1977 \\
\hline Lowest & 16 & 11 & 44 & 75 & 67 & 92 & 118 & 82 & 61 & 33 & 13 & 6 & 6 \\
\hline Year & $1976^{(a)}$ & 1995 & 1979 & 1974 & 1962 & 1992 & 1972 & 2003 & 1957 & 1974 & 2001 & 2002 & Dec 2002 \\
\hline
\end{tabular}

(a) Most recent of multiple occurrences.

Table 6.5 shows that thunderstorms occurred in every month of the year, except January and November. The thunderstorm season is essentially from April through September. The average number of thunderstorm days per year is 10; however, the total varies from a low of 3 in 1949 to a high of 23 in 1948. The largest number of thunderstorms in any single month was 8 in July 1998, July 1983, June 1972, and August 1953. 
Table 6.5. Average Number of Days of Various Meteorological Phenomena, 1945 through 2003

\begin{tabular}{|c|c|c|c|c|c|c|c|c|c|c|c|c|c|}
\hline Phenomenon & Jan & Feb & Mar & Apr & May & Jun & Jul & Aug & Sep & Oct & Nov & Dec & Annual \\
\hline Thunderstorm & 0 & $\leq 0.1$ & 0.2 & 0.8 & 1.5 & 2.2 & 2.2 & 2.0 & 0.7 & 0.2 & 0 & $\leq 0.1$ & 9.8 \\
\hline Dust or blowing dust & 0.4 & 0.4 & 0.5 & 0.6 & 0.6 & 0.4 & 0.4 & 0.2 & 0.5 & 0.3 & 0.2 & 0.2 & 4.6 \\
\hline Glaze & 2.0 & 0.6 & $\leq 0.1$ & 0 & 0 & 0 & 0 & 0 & 0 & 0 & 0.8 & 2.4 & 6.0 \\
\hline
\end{tabular}

The criterion for both dust and blowing dust is that horizontal visibility be reduced to 6 miles or less. Dust is carried into the area from a distant source and may occur without strong winds. Blowing dust occurs when dust is picked up locally and occurs with stronger winds. Both dust and blowing dust occur at the Hanford Meteorology Station; however, in most cases, it is blowing dust. Table 6.5 presents the average number of days per month and year of dust and blowing dust during the period 1945 through 2003. The average number of days per year with dust or blowing dust is 5 . The greatest number of such days in any year was 20 in 1980; the fewest was 0 in 1987 and earlier years. The greatest number of days with dust or blowing dust in any month was 9 in May 1980. This peak in the number of days with dust or blowing dust (for either a month or a year) resulted from the eruption of Mount St. Helens on May 18, 1980, and subsequent dates.

Glaze is a coating of ice formed when rain or drizzle freezes on contact with any surface having a temperature that is below freezing. Table 6.5 provides data on the number of days per month and year with glaze for the period 1945 through 2003. The average number of days with freezing rain or freezing drizzle is 6 . The highest number of days with glaze in any winter season was 18 during the winter of 1969-1970; the least, 1 day during the winter of 1987-1988 and earlier winters. The greatest number of such days in any single month was 9 in January 1970.

\subsection{Atmospheric Pressure}

Table 6.6 contains atmospheric pressure data for the period 1955 through 2003. This table lists both station and sea-level pressure, including extremes and years of occurrence. Atmospheric pressure may be indicated in several different units, including inches of mercury, millimeters of mercury, millibars, or Pascals; however, in this table, pressure is stated in inches of mercury. Station pressure is the barometric pressure measured at the Hanford Meteorology Station (at an elevation of 733 feet); sea-level pressure is the station pressure adjusted to sea level. Most are familiar with barometric pressure adjusted to sea level, which allows atmospheric pressures for all locations to be compared, regardless of the elevation of the station where the data are measured.

The highest sea-level pressure ever recorded at the Hanford Meteorology Station was 31.12 inches in January 1979; the lowest was 28.91 inches in December 2002.

Some rapid pressure changes occurred on November 3, 1958, falling 0.492 inch over a 6-hour period (0.082 inch per hour), including a 1-hour fall of $0.160 \mathrm{inch}$. On the same day, the pressure rose 0.554 inch during a 6-hour period ( 0.090 inch per hour), including a 1-hour rise of 0.145 inch. The greatest sea-level pressure change during a 1-day period was 1.02 inches (December 8, 1971). 
Table 6.6. Average and Extreme Station and Sea-Level Pressure Data, 1955 through 2003 (inches of mercury)

\begin{tabular}{|c|c|c|c|c|c|c|c|c|c|c|}
\hline \multirow[b]{2}{*}{ Month } & \multicolumn{6}{|c|}{ Station Pressure } & \multicolumn{4}{|c|}{ Sea-Level Pressure } \\
\hline & Average & High & Year & Low & Year & $\begin{array}{c}\text { Greatest Daily } \\
\text { Range } \\
\end{array}$ & High & Year & Low & Year \\
\hline Jan & 29.32 & 30.23 & $1979^{(\mathrm{a})}$ & 28.18 & 1980 & 0.77 & 31.12 & 1979 & 28.94 & 1964 \\
\hline Feb & 29.27 & 30.08 & 1956 & 28.23 & $1958^{(\mathrm{a})}$ & 0.86 & 30.97 & $1956^{(\mathrm{a})}$ & 28.98 & $1958^{(\mathrm{a})}$ \\
\hline Mar & 29.20 & 29.92 & 1955 & 28.34 & 1995 & 0.85 & 30.79 & 1955 & 29.11 & 1995 \\
\hline Apr & 29.19 & 29.91 & 1999 & 28.49 & $1962^{(\mathrm{a})}$ & 0.81 & 30.73 & 1999 & 29.26 & 1962 \\
\hline May & 29.16 & 29.72 & 2003 & 28.61 & 1999 & 0.47 & 30.53 & 2003 & 29.38 & $1999^{(\mathrm{a})}$ \\
\hline Jun & 29.14 & 29.60 & $1987^{(\mathrm{a})}$ & 28.67 & $1992^{(\mathrm{a})}$ & 0.54 & 30.40 & 1987 & 29.42 & 1992 \\
\hline Jul & 29.14 & 29.56 & $1993^{(\mathrm{a})}$ & 28.80 & $2002^{(a)}$ & 0.48 & 30.34 & $1993^{(\mathrm{a})}$ & 29.55 & $2002^{(\mathrm{a})}$ \\
\hline Aug & 29.14 & 29.55 & 1968 & 28.75 & 1980 & 0.39 & 30.32 & 1968 & 29.52 & 1980 \\
\hline Sep & 29.18 & 29.79 & $1983^{(\mathrm{a})}$ & 28.48 & $1986^{(\mathrm{a})}$ & 0.56 & 30.60 & 1983 & 29.25 & 1986 \\
\hline Oct & 29.25 & 29.86 & 1999 & 28.39 & 1962 & 0.74 & 30.68 & $1999^{(a)}$ & 29.15 & 1962 \\
\hline Nov & 29.28 & 30.06 & $1979^{(\mathrm{a})}$ & 28.36 & $2002^{(a)}$ & 0.78 & 30.90 & $1979^{(a)}$ & 29.13 & $2002^{(\mathrm{a})}$ \\
\hline Dec & 29.32 & 30.20 & 1978 & 28.15 & 2002 & 1.02 & 31.07 & $1978^{(a)}$ & 28.91 & 2002 \\
\hline Annual & 29.22 & 30.23 & Jan $1979^{(a)}$ & 28.15 & Dec 2002 & 1.02 & 31.12 & Jan 1979 & 28.91 & Dec 2002 \\
\hline
\end{tabular}

(a) Most recent of several occurrences. 


\subsection{Sunrise and Sunset Times for the Hanford Meteorological Station}

Table 6.7 lists the sunrise and sunset times for the Hanford Meteorological Station in Pacific Standard Time (PST). The longest days of the year (period between sunrise and sunset) are June 22 and 23 at 15 hours and 50 minutes each. The shortest days of the year are December 19 through 23 at 8 hours and 34 minutes. Sunrise varies from 4:04 am PST (earliest) on June 11 through 14 to 7:42 am PST (latest) from December 28 through January 7. Sunset varies from 4:11 pm PST (earliest) from December 5 through 16 to 7:55 pm PST from June 22 through July 1. 
Table 6.7. Sunrise and Sunset Times (PST) at the Hanford Meteorological Station

\begin{tabular}{|c|c|c|c|c|c|c|c|c|c|c|c|c|c|c|c|c|c|c|c|c|c|c|c|c|}
\hline \multirow[b]{2}{*}{ Day } & \multicolumn{2}{|c|}{ Jan } & \multicolumn{2}{|c|}{ Feb } & \multicolumn{2}{|c|}{ Mar } & \multicolumn{2}{|c|}{ Apr } & \multicolumn{2}{|c|}{ May } & \multicolumn{2}{|c|}{ Jun } & \multicolumn{2}{|c|}{ Jul } & \multicolumn{2}{|c|}{ Aug } & \multicolumn{2}{|c|}{ Sep } & \multicolumn{2}{|c|}{ Oct } & \multicolumn{2}{|c|}{ Nov } & \multicolumn{2}{|c|}{ Dec } \\
\hline & $\begin{array}{l}\text { Rise } \\
\text { a.m. }\end{array}$ & $\begin{array}{l}\text { Set } \\
\text { p.m. }\end{array}$ & $\begin{array}{l}\text { Rise } \\
\text { a.m. }\end{array}$ & $\begin{array}{l}\text { Set } \\
\text { p.m. }\end{array}$ & $\begin{array}{l}\text { Rise } \\
\text { a.m. }\end{array}$ & $\begin{array}{l}\text { Set } \\
\text { p.m. }\end{array}$ & $\begin{array}{l}\text { Rise } \\
\text { a.m. }\end{array}$ & \begin{tabular}{|l} 
Set \\
p.m.
\end{tabular} & $\begin{array}{l}\text { Rise } \\
\text { a.m. }\end{array}$ & \begin{tabular}{|r} 
Set \\
p.m.
\end{tabular} & $\begin{array}{l}\text { Rise } \\
\text { a.m. }\end{array}$ & \begin{tabular}{|l} 
Set \\
p.m.
\end{tabular} & $\begin{array}{l}\text { Rise } \\
\text { a.m. }\end{array}$ & \begin{tabular}{|l} 
Set \\
p.m.
\end{tabular} & $\begin{array}{l}\text { Rise } \\
\text { a.m. }\end{array}$ & \begin{tabular}{|l} 
Set \\
p.m.
\end{tabular} & $\begin{array}{l}\text { Rise } \\
\text { a.m. }\end{array}$ & \begin{tabular}{|r} 
Set \\
p.m.
\end{tabular} & $\begin{array}{l}\text { Rise } \\
\text { a.m. }\end{array}$ & \begin{tabular}{|l} 
Set \\
p.m.
\end{tabular} & $\begin{array}{l}\text { Rise } \\
\text { a.m. }\end{array}$ & $\begin{array}{l}\text { Set } \\
\text { p.m. }\end{array}$ & $\begin{array}{l}\text { Rise } \\
\text { a.m. }\end{array}$ & \begin{tabular}{|r} 
Set \\
p.m.
\end{tabular} \\
\hline 1 & 0742 & 1621 & 0722 & 1702 & 0639 & 1743 & 0538 & 1827 & 0444 & 1907 & 0409 & 1943 & 0408 & 1955 & 0438 & 1930 & 0517 & 1838 & 0557 & 1738 & 0640 & 1643 & 0721 & 1613 \\
\hline 2 & 0742 & 1622 & 0722 & 1703 & 0637 & 1745 & 0537 & 1828 & 0443 & 1908 & 0408 & 1944 & 0409 & 1954 & 0439 & 1929 & 0519 & 1836 & 0558 & 1736 & 0641 & 1642 & 0722 & 1612 \\
\hline 3 & 0742 & 1623 & 0719 & 1705 & 0635 & 1746 & 0534 & 1830 & 0440 & 1910 & 0408 & 1945 & 0410 & 1954 & 0440 & 1927 & 0520 & 1834 & 0600 & 1734 & 0643 & 1640 & 0723 & 1612 \\
\hline 4 & 0742 & 1624 & 0719 & 1706 & 0632 & 1748 & 0532 & 1831 & 0439 & 1911 & 0407 & 1946 & 0411 & 1954 & 0442 & 1926 & 0521 & 1832 & 0601 & 1732 & 0643 & 1639 & 0725 & 1612 \\
\hline 5 & 0742 & 1625 & 0717 & 1708 & 0631 & 1749 & 0530 & 1832 & 0439 & 1912 & 0407 & 1946 & 0411 & 1953 & 0443 & 1924 & 0522 & 1830 & 0602 & 1730 & 0646 & 1637 & 0726 & 1611 \\
\hline 6 & 0742 & 1626 & 0716 & 1709 & 0529 & 1750 & 0528 & 1834 & 0436 & 1914 & 0406 & 1947 & 0412 & 1953 & 0444 & 1923 & 0523 & 1829 & 0602 & 1729 & 0647 & 1636 & 0727 & 1611 \\
\hline 7 & 0742 & 1627 & 0714 & 1711 & 0628 & 1752 & 0526 & 1835 & 0435 & 1915 & 0406 & 1948 & 0413 & 1953 & 0446 & 1921 & 0525 & 1827 & 0604 & 1727 & 0648 & 1635 & 0728 & 1611 \\
\hline 8 & 0741 & 1629 & 0714 & 1712 & 0626 & 1753 & 0524 & 1836 & 0434 & 1916 & 0406 & 1949 & 0414 & 1952 & 0446 & 1920 & 0526 & 1825 & 0605 & 1725 & 0650 & 1633 & 0729 & 1611 \\
\hline 9 & 0741 & 1630 & 0711 & 1714 & 0623 & 1755 & 0522 & 1838 & 0432 & 1918 & 0405 & 1949 & 0414 & 1952 & 0449 & 1918 & 0527 & 1823 & 0607 & 1723 & 0652 & 1632 & 0730 & 1611 \\
\hline 10 & 0741 & 1631 & 0710 & 1715 & 0622 & 1756 & 0521 & 1839 & 0431 & 1919 & 0405 & 1950 & 0415 & 1951 & 0449 & 1917 & 0528 & 1821 & 0609 & 1721 & 0652 & 1631 & 0731 & 1611 \\
\hline 11 & 0740 & 1632 & 0708 & 1717 & 0619 & 1758 & 0519 & 1840 & 0429 & 1920 & 0404 & 1951 & 0416 & 1950 & 0451 & 1915 & 0530 & 1819 & 0610 & 1719 & 0653 & 1630 & 0732 & 1611 \\
\hline 12 & 0740 & 1633 & 0707 & 1718 & 0617 & 1759 & 0517 & 1842 & 0428 & 1922 & 0404 & 1951 & 0417 & 1950 & 0452 & 1913 & 0531 & 1817 & 0611 & 1717 & 0656 & 1628 & 0733 & 1611 \\
\hline 13 & 0739 & 1635 & 0705 & 1720 & 0616 & 1800 & 0515 & 1843 & 0427 & 1923 & 0404 & 1952 & 0418 & \begin{tabular}{|l|l|}
1949 \\
\end{tabular} & 0453 & 1912 & 0532 & 1815 & 0613 & 1715 & 0657 & 1627 & 0733 & 1611 \\
\hline 14 & 0739 & 1636 & 0704 & 1721 & 0613 & 1802 & 0513 & 1844 & 0425 & 1924 & 0404 & 1952 & 0419 & 1948 & 0455 & 1910 & 0533 & 1813 & 0614 & 1713 & 0659 & 1626 & 0734 & 1611 \\
\hline 15 & 0738 & 1637 & 0702 & 1723 & 0611 & 1803 & 0511 & 1846 & 0424 & 1925 & 0405 & 1953 & 0420 & 1948 & 0456 & 1908 & 0535 & 1811 & 0615 & 1712 & 0700 & 1625 & 0735 & 1611 \\
\hline 16 & 0738 & 1638 & 0701 & 1724 & 0610 & 1805 & 0510 & 1847 & 0423 & 1926 & 0405 & 1953 & 0420 & 1947 & 0457 & 1907 & 0536 & 1809 & 0617 & 1710 & 0702 & 1624 & 0735 & 1611 \\
\hline 17 & 0737 & 1640 & 0659 & 1726 & 0608 & 1806 & 0508 & 1848 & 0422 & 1927 & 0405 & 1953 & 0421 & 1946 & 0458 & 1905 & 0538 & 1806 & 0618 & 1708 & 0703 & 1623 & 0736 & 1612 \\
\hline 18 & 0736 & 1641 & 0657 & 1727 & 0605 & 1808 & 0506 & 1850 & 0421 & 1929 & 0405 & 1954 & 0422 & 1945 & 0500 & 1903 & 0538 & 1805 & 0619 & 1705 & 0704 & 1622 & 0737 & 1612 \\
\hline 19 & 0736 & 1642 & 0656 & 1729 & 0604 & 1809 & \begin{tabular}{|l}
0504 \\
\end{tabular} & 1851 & 0420 & 1930 & 0405 & 1954 & 0423 & 1944 & 0501 & 1901 & 0541 & 1802 & 0621 & 1705 & 0706 & 1621 & 0738 & 1612 \\
\hline 20 & 0735 & 1644 & 0654 & 1730 & 0602 & 1810 & 0503 & 1852 & 0419 & 1931 & 0405 & 1954 & 0424 & 1944 & 0502 & 1900 & 0542 & 1800 & 0622 & 1703 & 0707 & 1620 & 0739 & 1613 \\
\hline 21 & 0734 & 1645 & 0652 & 1732 & 0600 & 1812 & 0501 & 1854 & 0418 & 1932 & 0405 & 1954 & 0426 & 1943 & 0503 & 1858 & 0543 & 1758 & 0624 & 1701 & 0708 & 1619 & 0739 & 1613 \\
\hline 22 & 0733 & 1647 & 0651 & 1733 & 0558 & 1813 & 0459 & 1855 & 0417 & 1933 & 0405 & 1955 & 0427 & 1942 & 0505 & 1856 & 0544 & 1756 & 0625 & 1659 & 0710 & 1618 & 0739 & 1613 \\
\hline 23 & 0732 & 1648 & 0649 & 1735 & 0555 & 1815 & 0457 & 1856 & 0416 & 1934 & 0405 & 1955 & 0428 & 1941 & 0506 & 1854 & 0546 & 1754 & 0626 & 1658 & 0711 & 1617 & 0740 & 1614 \\
\hline 24 & 0731 & 1649 & 0647 & 1736 & 0554 & 1816 & 0455 & 1858 & 0415 & 1935 & 0406 & 1955 & 0429 & 1939 & 0507 & 1853 & 0547 & 1752 & 0628 & 1656 & 0712 & 1617 & 0740 & 1615 \\
\hline 25 & 0730 & 1651 & 0646 & 1737 & 0552 & 1817 & 0454 & 1859 & 0414 & 1936 & 0406 & 1955 & 0430 & 1938 & 0508 & 1851 & 0548 & 1750 & 0629 & 1654 & 0714 & 1616 & 0741 & 1616 \\
\hline 26 & 0730 & 1652 & 0544 & 1739 & 0549 & 1819 & 0452 & 1900 & 0413 & 1937 & 0406 & 1955 & 0431 & 1937 & 0510 & 1849 & 0551 & 1748 & 0631 & 1653 & 0715 & 1615 & 0741 & 1616 \\
\hline 27 & 0728 & 1654 & 0642 & 1740 & 0548 & 1820 & 0450 & 1902 & 0412 & 1938 & 0407 & 1955 & 0432 & 1936 & 0511 & 1847 & 0551 & 1746 & 0632 & 1651 & 0716 & 1615 & 0741 & 1617 \\
\hline 28 & 0727 & 1655 & 0640 & 1742 & 0546 & 1821 & 0449 & 1903 & 0412 & 1939 & 0407 & 1955 & 0433 & 1935 & 0512 & 1845 & 0553 & 1744 & 0633 & 1650 & 0718 & 1614 & 0742 & 1618 \\
\hline 29 & 0726 & 1657 & 0639 & 1743 & 0543 & 1823 & 0448 & 1904 & 0411 & 1940 & 0408 & 1955 & 0434 & 1934 & 0513 & 1844 & 0554 & 1742 & 0635 & 1648 & 0719 & 1614 & 0742 & 1618 \\
\hline 30 & 0725 & 1658 & & & 0542 & 1824 & 0445 & 1906 & 0410 & 1941 & 0408 & 1955 & 0436 & 1932 & 0515 & 1842 & 0556 & 1740 & 0635 & 1647 & 0720 & 1613 & 0742 & 1619 \\
\hline 31 & 0724 & 1700 & & & 0540 & 1826 & & & 0410 & 1942 & & & 0437 & 1931 & 0516 & 1840 & & & 0638 & 1645 & & & 0742 & 1620 \\
\hline
\end{tabular}




\subsection{Extreme Values}

Extreme values are generally described in terms of probability of occurrence or in terms of return period. For low probability events, the return period is simply the reciprocal of the probability when the probability is expressed as the likelihood of the event occurring in a given year. As with all estimated extreme values, the uncertainty in the estimates increases as the return period increases. In addition, the extreme value estimates assume that the climate in the future will be the same as it has been since the Hanford Meteorology Station was established. This section was not updated to include 2003 data.

\subsection{Annual Temperature Extremes}

Annual maximum and minimum temperatures with return periods from 2 to 1,000 years are listed in Table 7.1. The probabilities of exceeding various maximum and minimum temperatures are shown in Figure 7.1 and Figure 7.2 along with the maximum and minimum temperatures observed at the Hanford Meteorology Station from 1945 through 2002. The curves were estimated by assuming that the annual extreme temperatures may be fit using a normal distribution and calculating distribution parameters from the observed data.

\subsection{Precipitation Rates}

Maximum precipitation rates for return periods of 2 to 1,000 years are listed in Table 7.2. The corresponding precipitation amounts are listed in Table 7.3. The precipitation rate estimates are based on precipitation measurements made at the Hanford Meteorology Station from 1947 through 2002. The precipitation rates were estimated for each return period assuming a lognormal distribution and distribution parameters calculated from the data. Figure 7.3 shows the predicted rates for 1, 3, 6, and 12 hours duration along with the observed data.

\subsection{Snow}

Snow extremes for return periods from 2 to 1,000 years are listed in Table 7.4. The estimates are based on data from the Hanford Meteorology Station for the 1946-1947 through 2001-2002 snow seasons. The values in the tables were estimated assuming a Type 1 (Gumbel) extreme value distribution (Johnson et al. 1995) using maximum-likelihood estimates (Kinnison 1985) of the distribution parameter values calculated from the Hanford Meteorology Station data. Figure 7.4, Figure 7.5, and Figure 7.6 show the probabilities of seasonal maximum snowfall, maximum single storm snowfall, and maximum snow depth, respectively with the corresponding Hanford Meteorology Station data.

\subsection{Peak Wind Gusts}

Peak wind gusts for return periods of 2 to 10,000 years are listed in Table 7.5 for heights of 30, 50, 200, and 400 feet above ground. The peak wind gust estimates are based on wind measurements made at the 50-, 200-, 400-foot levels of the tower at the Hanford Meteorology Station. The peak wind gusts for each return period for these levels were estimated assuming a Type 1 extreme value distribution and maximum likelihood distribution parameters calculated from the Hanford Meteorology Station data. The 
peak wind gusts for the 30 -foot level were made by first adjusting the peak gusts observed at 50 feet to 30 feet using the technique described by Peterka and Shahid (1998) and then calculating the distribution parameters using maximum likelihood techniques. Figure 7.7 shows the probabilities of peak wind gusts at all four levels along with the Hanford Meteorology Station peak wind gust data for 50, 200, and 400 feet.

\section{Table 7.1. $\quad$ Return Periods ${ }^{(a)}$ for Annual Maximum and Minimum Temperatures}

\begin{tabular}{|c|c|c|}
\hline $\begin{array}{l}\text { Return Period } \\
\text { (years) }\end{array}$ & $\begin{array}{c}\text { Maximum Temperature } \\
\left({ }^{\circ} \mathrm{F}\right)\end{array}$ & $\begin{array}{c}\text { Minimum Temperature } \\
\left({ }^{\circ} \mathrm{F}\right)\end{array}$ \\
\hline 2 & 106.2 & 0.1 \\
\hline 5 & 108.8 & -8.5 \\
\hline 10 & 110.2 & -13.1 \\
\hline $\begin{array}{l}20 \\
25\end{array}$ & $\begin{array}{l}111.4 \\
111.7\end{array}$ & $\begin{array}{l}-16.8 \\
-17.9\end{array}$ \\
\hline 50 & 112.7 & -21.0 \\
\hline 100 & 113.6 & -23.8 \\
\hline 200 & 114.8 & -26.3 \\
\hline 500 & 115.5 & -29.4 \\
\hline 1,000 & 116.2 & -31.6 \\
\hline
\end{tabular}

(a) Return periods are the frequency we may expect these temperatures to occur.

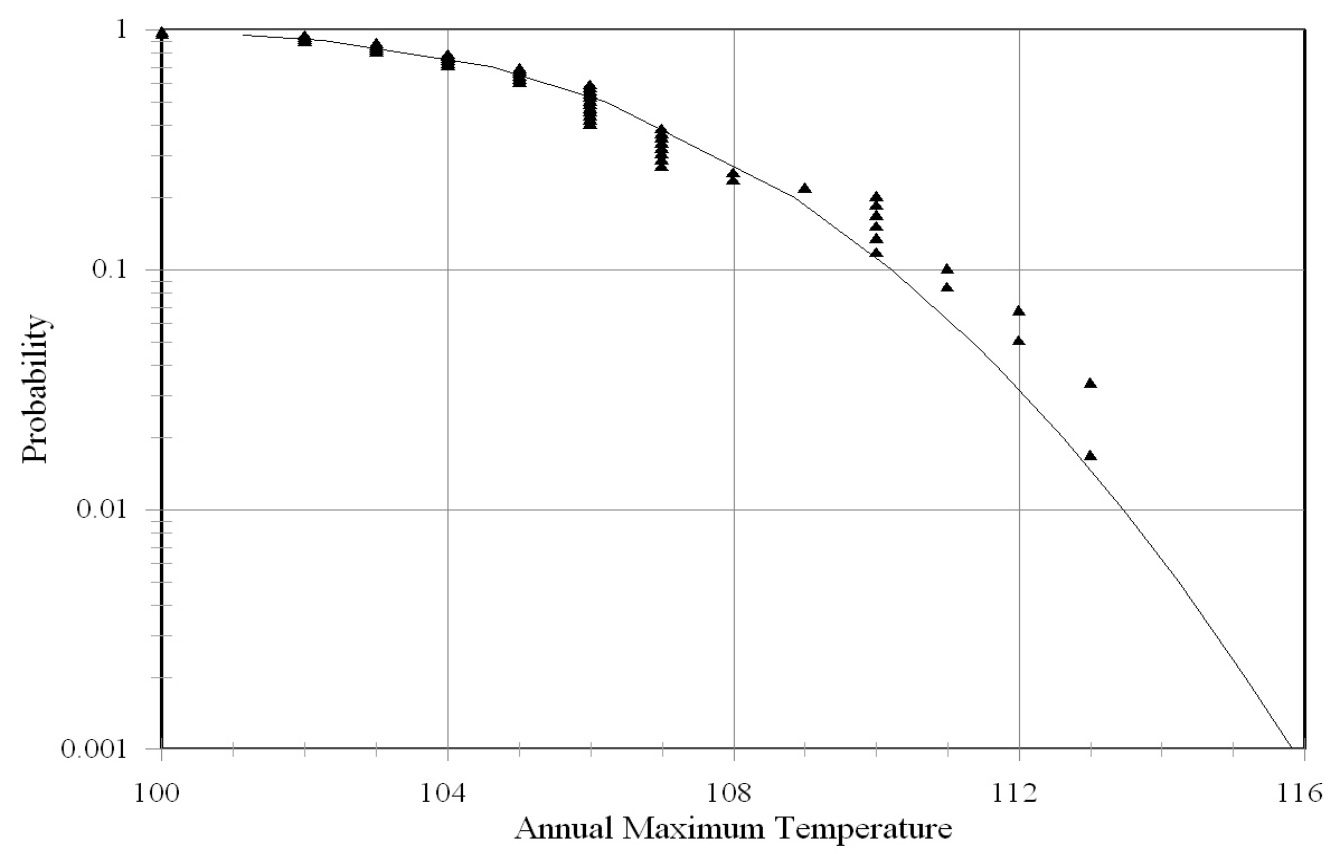

Figure 7.1. Probability (1/yr) of an Annual Maximum Temperature $\left({ }^{\circ} \mathrm{F}\right)$ Exceeding a Given Value 


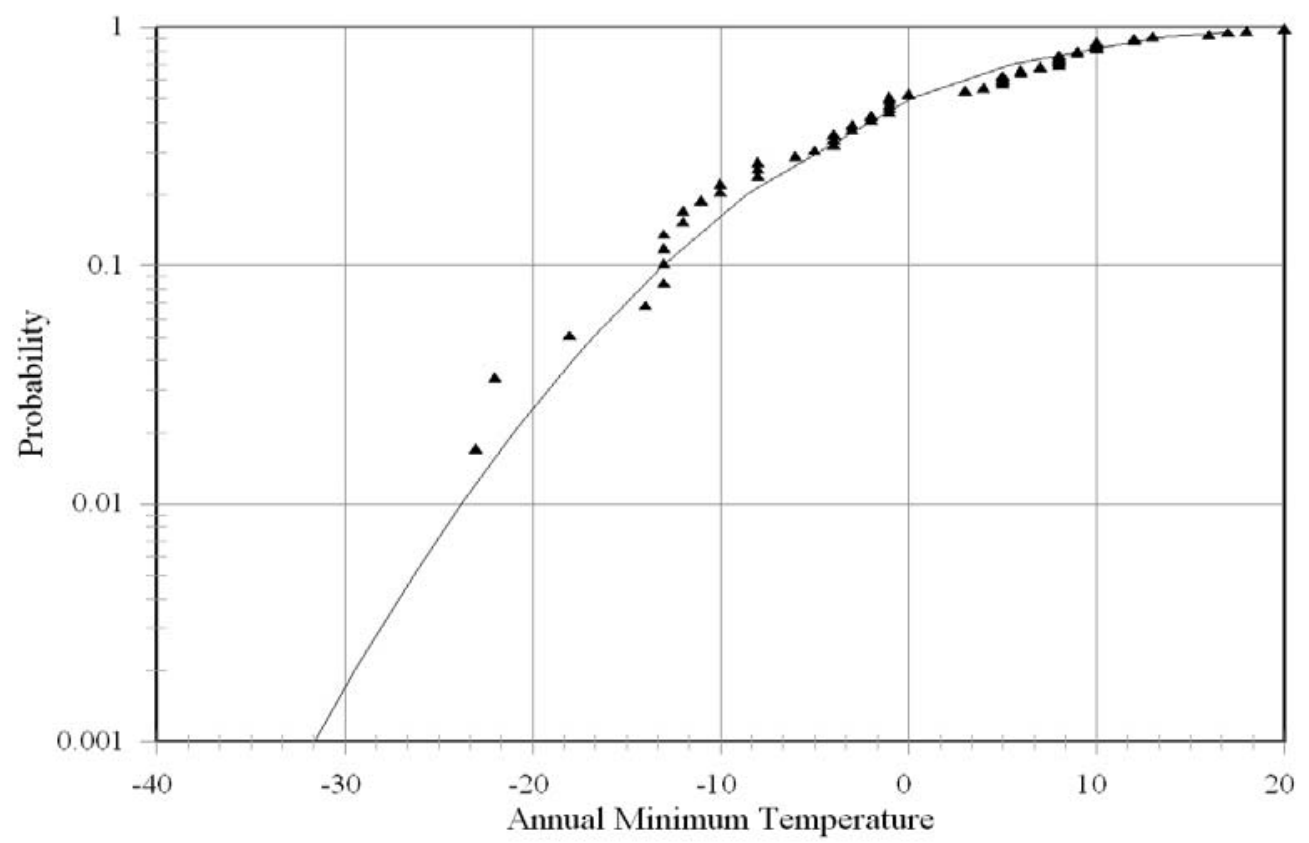

Figure 7.2. Probability (1/yr) of an Annual Minimum Temperature $\left({ }^{\circ} \mathrm{F}\right)$ Being Less Than a Given Value

Table 7.2. Precipitation Rates (inches per hour) for 1 to 24 Hours Duration and Return Periods from 2 to 1,000 Years

\begin{tabular}{|c|c|c|c|c|c|c|}
\hline \multirow{2}{*}{$\begin{array}{l}\text { Return Period } \\
\text { (years) }\end{array}$} & \multicolumn{6}{|c|}{ Duration } \\
\hline & 1 hour & 2 hours & 3 hours & 6 hours & 12 hours & 24 hours \\
\hline 2 & 0.22 & 0.15 & 0.12 & 0.08 & 0.05 & 0.03 \\
\hline 5 & 0.31 & 0.21 & 0.16 & 0.11 & 0.07 & 0.04 \\
\hline 10 & 0.37 & 0.24 & 0.18 & 0.12 & 0.08 & 0.04 \\
\hline $\begin{array}{l}20 \\
25\end{array}$ & $\begin{array}{l}0.43 \\
0.45\end{array}$ & $\begin{array}{l}0.27 \\
0.28\end{array}$ & $\begin{array}{l}0.20 \\
0.21\end{array}$ & $\begin{array}{l}0.14 \\
0.14\end{array}$ & $\begin{array}{l}0.09 \\
0.09\end{array}$ & $\begin{array}{l}0.05 \\
0.05\end{array}$ \\
\hline 50 & 0.52 & 0.32 & 0.23 & 0.16 & 0.10 & 0.06 \\
\hline 100 & 0.58 & 0.35 & 0.25 & 0.17 & 0.11 & 0.06 \\
\hline 200 & 0.64 & 0.38 & 0.26 & 0.18 & 0.12 & 0.07 \\
\hline 500 & 0.73 & 0.42 & 0.29 & 0.20 & 0.13 & 0.08 \\
\hline 1,000 & 0.79 & 0.46 & 0.31 & 0.22 & 0.14 & 0.08 \\
\hline
\end{tabular}


Table 7.3. Precipitation Amounts (inches) for 1 to 24 Hours in Periods and Return Periods from 2 to 1,000 Years

\begin{tabular}{|c|c|c|c|c|c|c|}
\hline \multirow{2}{*}{$\begin{array}{l}\text { Return Period } \\
\text { (years) }\end{array}$} & \multicolumn{6}{|c|}{ Duration } \\
\hline & 1 hour & 2 hours & 3 hours & 6 hours & 12 hours & 24 hours \\
\hline 2 & 0.22 & 0.31 & 0.36 & 0.48 & 0.60 & 0.69 \\
\hline 5 & 0.31 & 0.41 & 0.47 & 0.63 & 0.79 & 0.93 \\
\hline 10 & 0.37 & 0.48 & 0.54 & 0.73 & 0.92 & 1.08 \\
\hline 20 & 0.43 & 0.55 & 0.60 & 0.82 & 1.04 & 1.23 \\
\hline 25 & 0.45 & 0.57 & 0.62 & 0.85 & 1.08 & 1.27 \\
\hline 50 & 0.52 & 0.63 & 0.68 & 0.93 & 1.19 & 1.42 \\
\hline 100 & 0.58 & 0.70 & 0.74 & 1.02 & 1.30 & 1.56 \\
\hline 200 & 0.64 & 0.76 & 0.79 & 1.10 & 1.42 & 1.70 \\
\hline 500 & 0.73 & 0.85 & 0.87 & 1.22 & 1.57 & 1.88 \\
\hline 1,000 & 0.79 & 0.91 & 0.93 & 1.30 & 1.69 & 2.03 \\
\hline
\end{tabular}

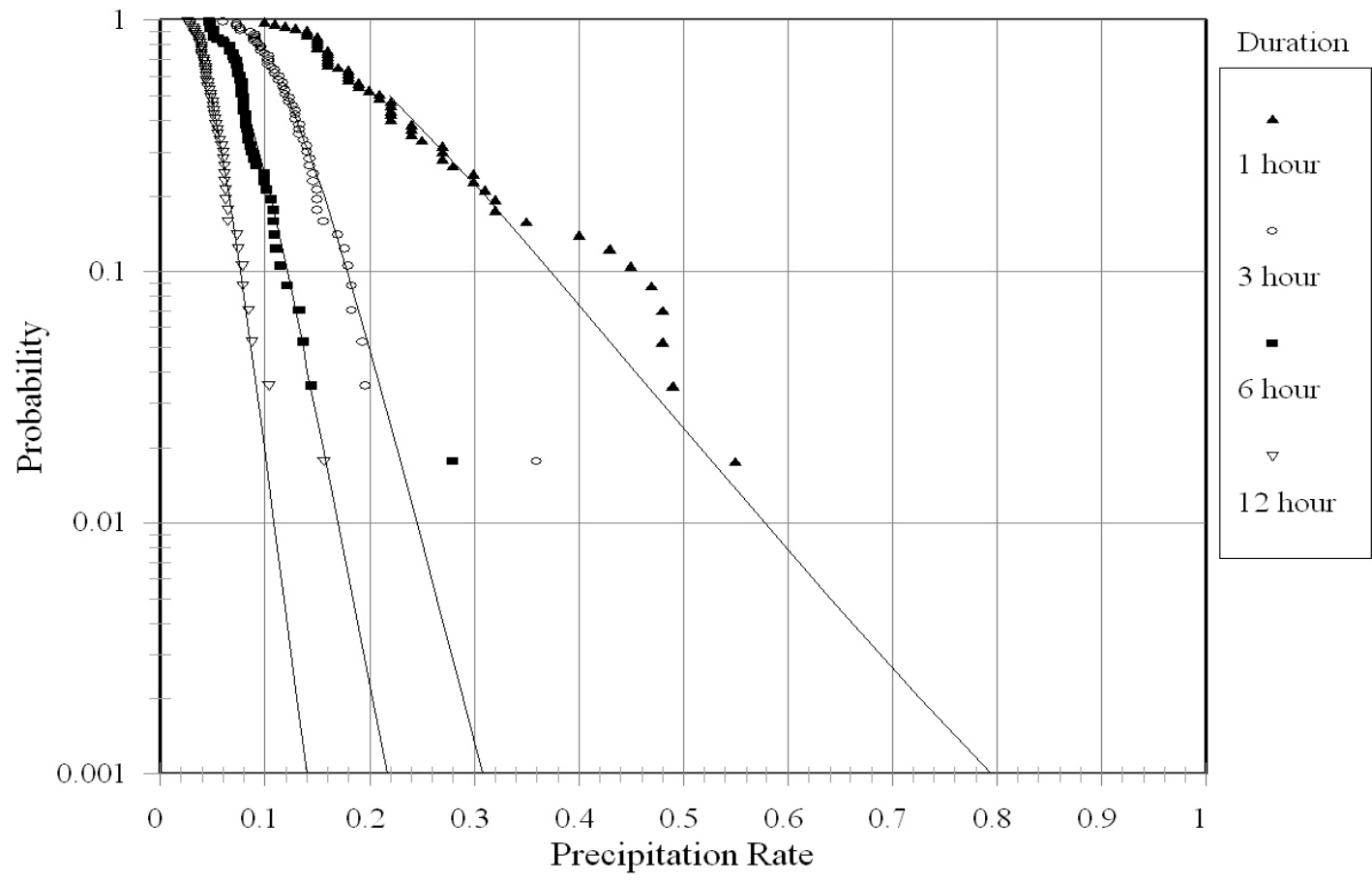

Figure 7.3. Probability (1/yr) of Precipitation Rate (inches per hour) Exceeding Given Values by Duration 
Table 7.4. Snowfall Extremes for Return Periods from 2 to 1,000 Years

\begin{tabular}{|c|c|c|c|}
\hline $\begin{array}{l}\text { Return Period } \\
\text { (years) }\end{array}$ & $\begin{array}{l}\text { Seasonal Total } \\
\text { (inches) }\end{array}$ & $\begin{array}{l}\text { Single Storm } \\
\text { (inches) }\end{array}$ & $\begin{array}{l}\text { Maximum on } \\
\text { Ground (inches) }\end{array}$ \\
\hline 2 & 12.9 & 3.7 & 4.9 \\
\hline 5 & 21.3 & 5.9 & 7.9 \\
\hline 10 & 26.9 & 7.3 & 10.0 \\
\hline $\begin{array}{l}20 \\
25\end{array}$ & $\begin{array}{l}32.3 \\
34.0\end{array}$ & $\begin{array}{l}8.7 \\
9.1\end{array}$ & $\begin{array}{l}11.9 \\
12.6\end{array}$ \\
\hline 50 & 39.2 & 10.5 & 14.5 \\
\hline 100 & 44.4 & 11.8 & 16.4 \\
\hline 200 & 49.6 & 13.1 & 18.3 \\
\hline 500 & 56.4 & 14.9 & 20.8 \\
\hline 1,000 & 61.6 & 16.2 & 22.6 \\
\hline
\end{tabular}

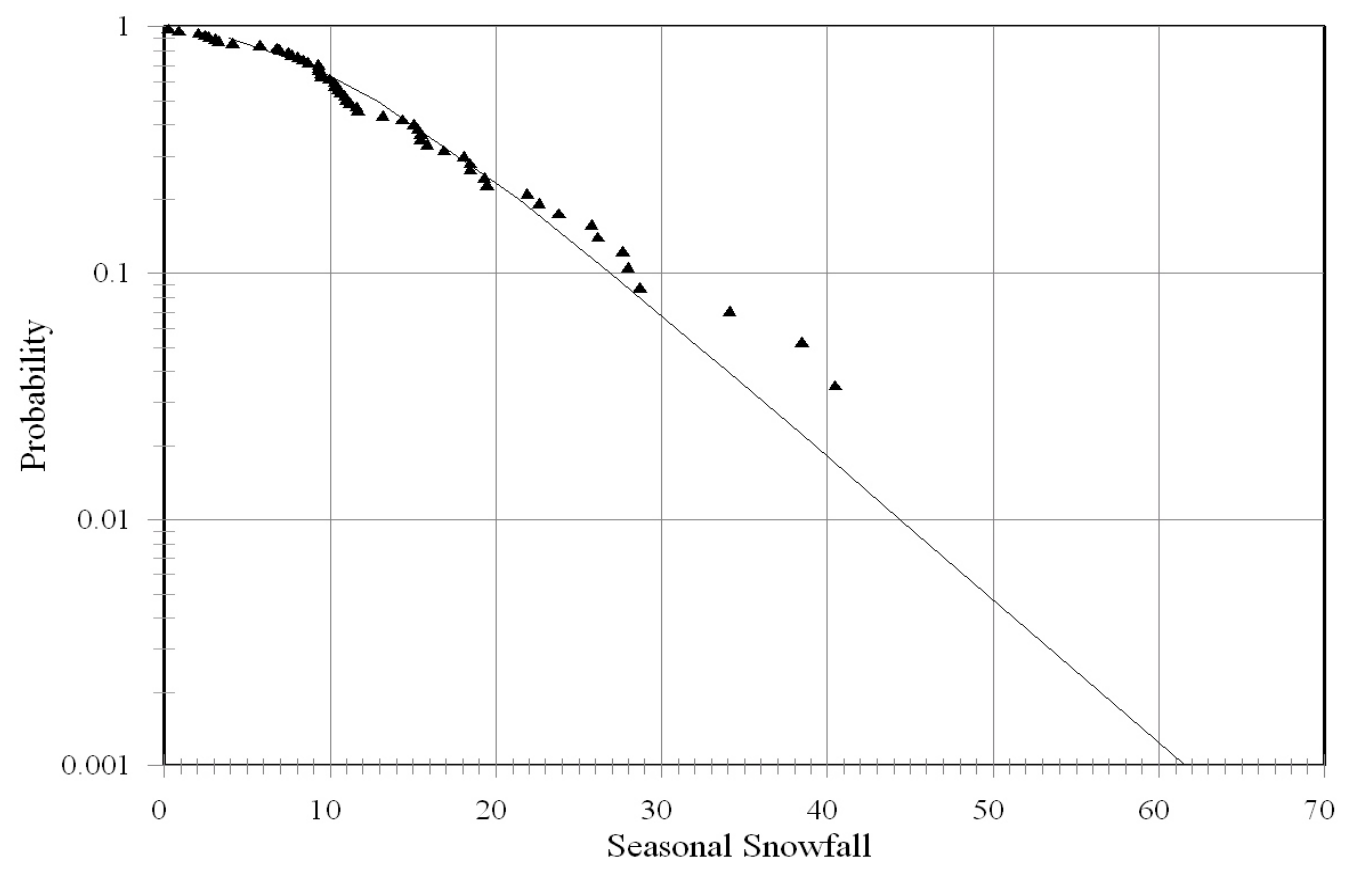

Figure 7.4. Probability (1/yr) of Exceeding a Given Seasonal Snowfall (inches) 


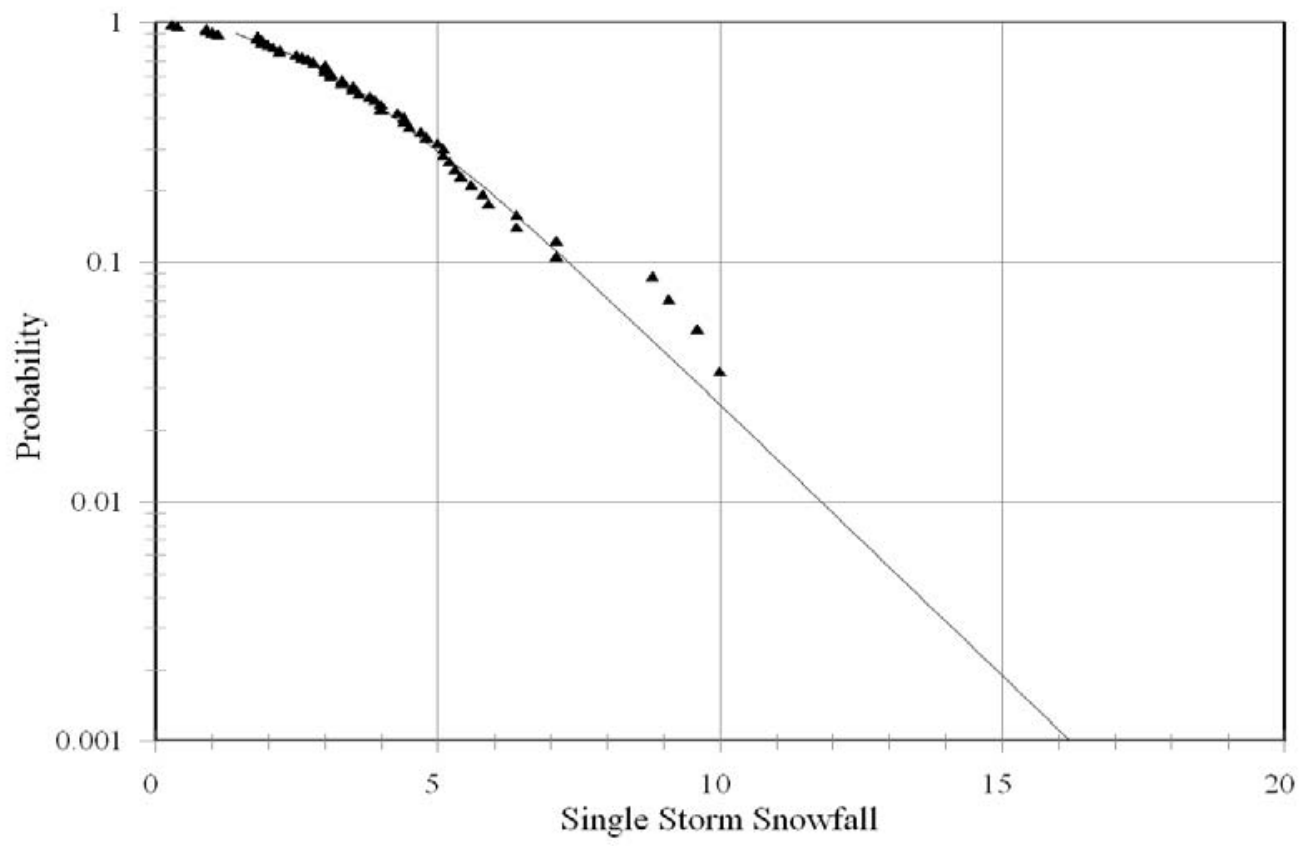

Figure 7.5. Probability (1/yr) of Exceeding a Given Snowfall (inches) in a Single Storm

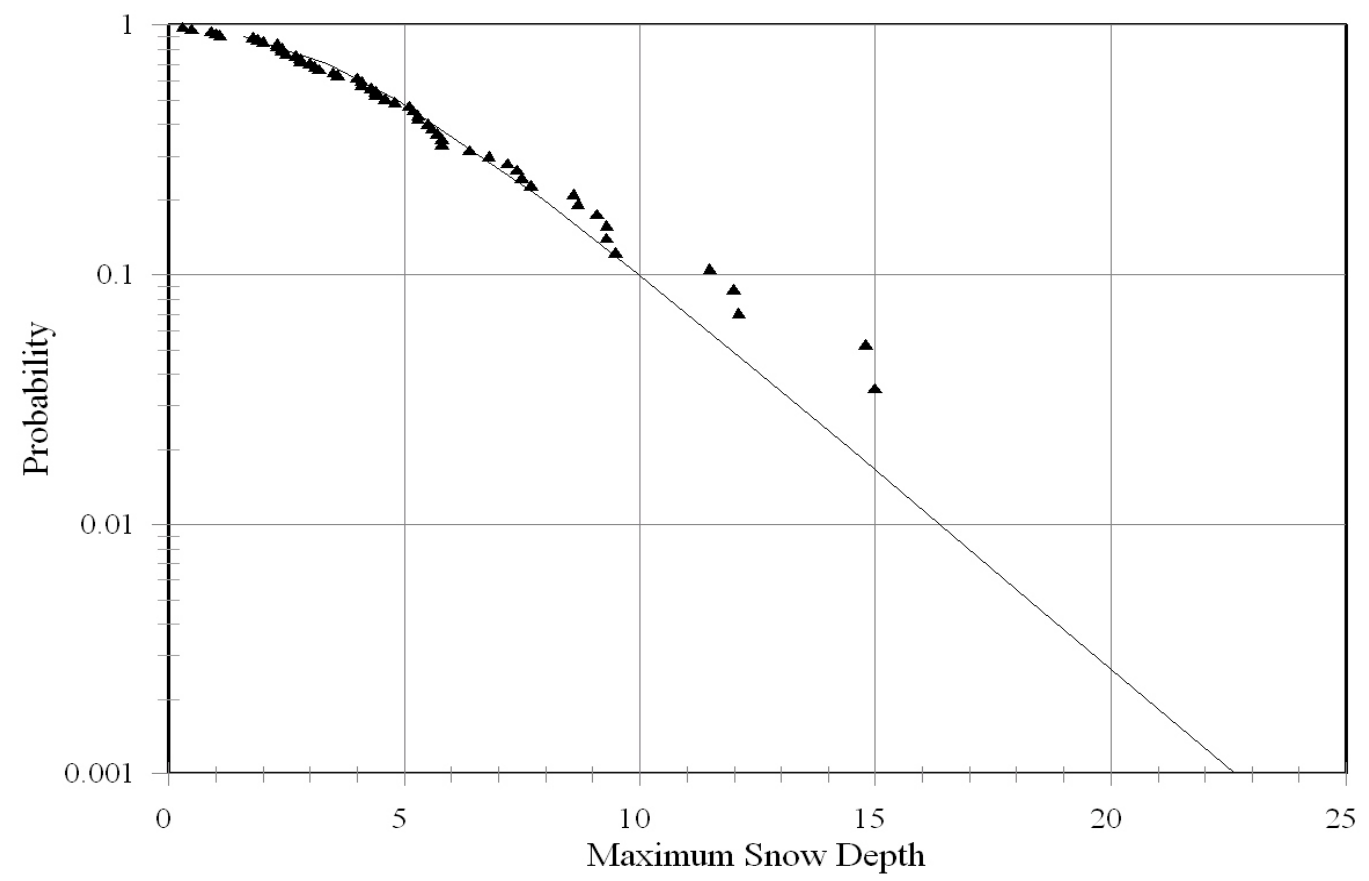

Figure 7.6. Probability (1/yr) of Exceeding a Given Snow Depth (inches) 
Table 7.5. Peak Wind Gusts (mph) for Return Periods from 2 to 10,000 Years

\begin{tabular}{|c|c|c|c|c|}
\hline \multirow{2}{*}{$\begin{array}{l}\text { Return Period } \\
\text { (years) }\end{array}$} & \multicolumn{4}{|c|}{ Height Above Ground } \\
\hline & 30 feet & 50 feet & 200 feet & 400 feet \\
\hline 2 & 57.6 & 60.3 & 67.4 & 71.6 \\
\hline 5 & 63.9 & 66.8 & 75.0 & 80.3 \\
\hline 10 & 68.1 & 71.2 & 80.0 & 86.1 \\
\hline 20 & 72.0 & 75.3 & 84.8 & 91.7 \\
\hline 25 & 73.3 & 76.6 & 86.4 & 93.4 \\
\hline 50 & 77.2 & 80.7 & 91.1 & 98.9 \\
\hline 100 & 81.1 & 84.7 & 95.7 & 104.2 \\
\hline 200 & 85.0 & 88.7 & 100.4 & 109.6 \\
\hline 500 & 90.1 & 94.0 & 106.5 & 116.7 \\
\hline 1,000 & 93.9 & 98.0 & 111.2 & 122.0 \\
\hline 2,000 & 97.8 & 102.0 & 115.8 & 127.4 \\
\hline 5,000 & 102.8 & 107.2 & 122.0 & 134.4 \\
\hline 10,000 & 106.7 & 111.2 & 126.6 & 139.8 \\
\hline
\end{tabular}

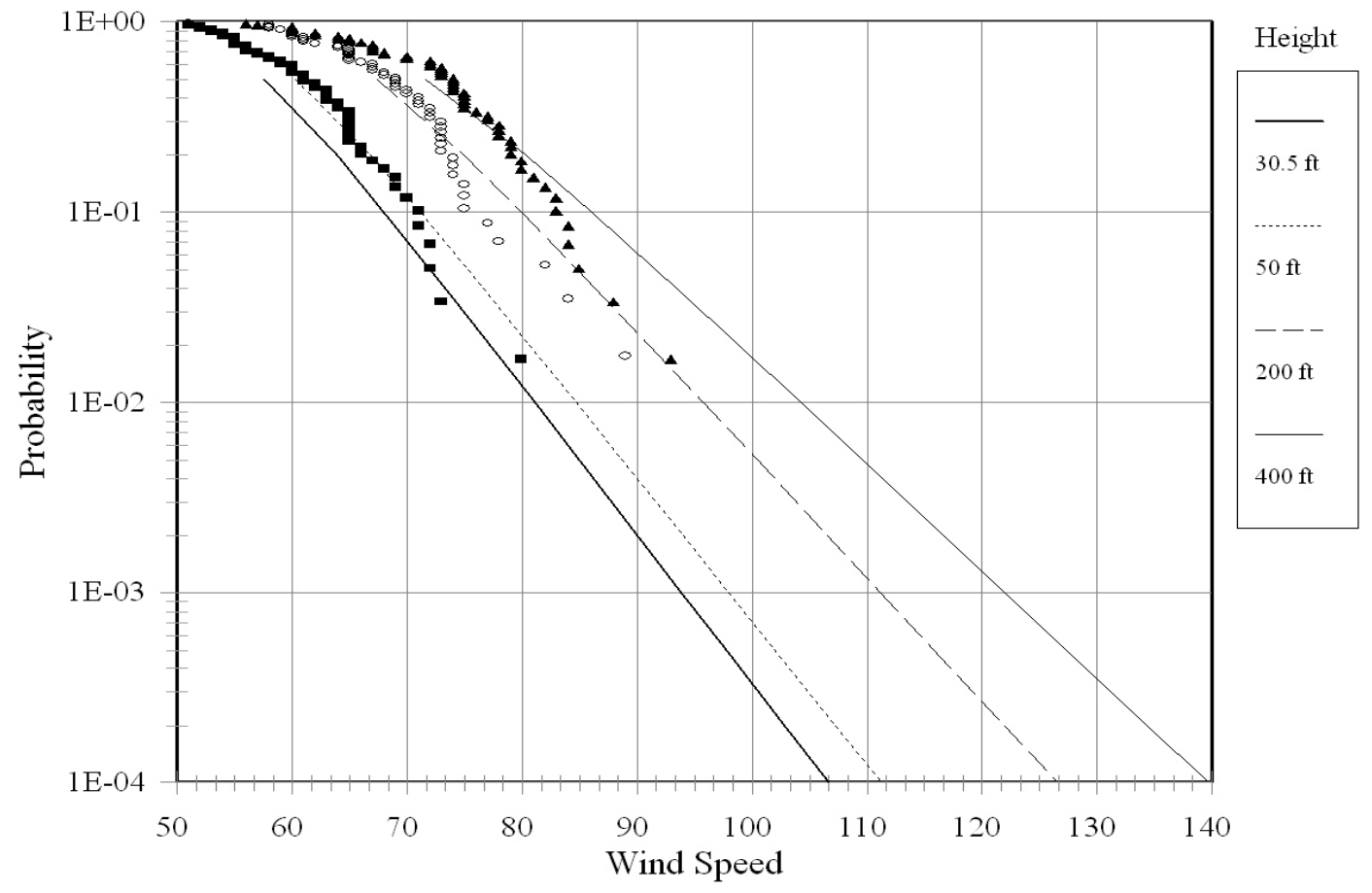

Figure 7.7. Probabilities (1/yr) of Peak Wind Gusts (miles per hour) Exceeding Given Values 


\subsection{References}

DOE. 1999. Final Hanford Comprehensive Land-Use Plan Environmental Impact Statement.

DOE/EIS-0222. Online report. http://nepa.eh.doe.gov/eis/eis0222/eis0222.html

Hoitink, D. J. and K. W. Burk. 1994. Climatological Data Summary 1993, with Historical Data. PNL-9809, Pacific Northwest Laboratory, Richland, Washington.

Hoitink, D. J. and K. W. Burk. 1995. Climatological Data Summary 1994, with Historical Data. PNL-10553, Pacific Northwest Laboratory, Richland, Washington.

Hoitink, D. J. and K. W. Burk. 1996. Climatological Data Summary 1995, with Historical Data. PNNL-11107, Pacific Northwest National Laboratory, Richland, Washington.

Hoitink, D. J. and K. W. Burk. 1997. Climatological Data Summary 1996, with Historical Data. PNNL-11471, Pacific Northwest National Laboratory, Richland, Washington.

Hoitink, D. J. and K. W. Burk. 1998. Climatological Data Summary 1997, with Historical Data. PNNL-11794, Pacific Northwest National Laboratory, Richland, Washington.

Hoitink, D. J., K. W. Burk, and J. V. Ramsdell, Jr. 1999. Climatological Data Summary 1998, with Historical Data. PNNL-12087, Pacific Northwest National Laboratory, Richland, Washington.

Hoitink, D. J., K. W. Burk, and J. V. Ramsdell, Jr. 2000. Climatological Data Summary 1999, with Historical Data. PNNL-13117, Pacific Northwest National Laboratory, Richland, Washington.

Hoitink, D. J., K.W. Burk, and J.V. Ramsdell. 2001. Hanford Site Climatological Data Summary 2000 with Historical Data. PNNL-13469. Pacific Northwest National Laboratory, Richland, Washington.

Hoitink, D. J., K. W. Burk, J. V. Ramsdell Jr., and W. J. Shaw. 2002. Hanford Site Climatological Data Summary 2001 with Historical Data. PNNL-13859. Pacific Northwest National Laboratory, Richland, Washington.

Hoitink, D. J., K. W. Burk, J. V. Ramsdell Jr., and W. J. Shaw. 2003. Hanford Site Climatological Data Summary 2002 with Historical Data. PNNL-14242. Pacific Northwest National Laboratory, Richland, Washington.

Jenne, D. E. and R. E. Kerns. 1959. A Climatological Study of the Hanford Area. HW-57722, General Electric, Hanford Atomic Products Operation, Richland, Washington.

Johnson, N. L., S. Kotz, and N. Balakrishnan. 1995. Continuous Univariate Distributions, Vol. 2, John Wiley \& Sons, New York.

Kinnison, R. R. 1985. Applied Extreme Value Statistics. Macmillan, New York. 
Peterka, J. A. and S. Shahid. 1998. "Design Gust Wind Speeds in the United States." Journal of Structural Engineering 124:207-214.

Stone, W. A., D. E. Jenne, and J. M. Thorp. 1972. Climatography of the Hanford Area. BNWL-1605, Pacific Northwest Laboratory, Richland, Washington.

Stone, W. A., J. M. Thorp, O. P. Gifford, and D. J. Hoitink. 1983. Climatological Summary for the Hanford Area. PNL-4622, Pacific Northwest Laboratory, Richland, Washington. 


\section{Appendix A}

\section{Climate Classification of the Mid-Columbia Region}




\section{Appendix A}

\section{Climate Classification of the Mid-Columbia Region}

What is the proper description of the climate of the Mid-Columbia Region? The local Tri-City Herald has a Sunday newspaper section called "Desert Living." Numerous place names in eastern Washington contain the word "desert," since people commonly regard any region that is too dry to support trees, except perhaps in river bottoms, as desert. On the other hand, popular and other publications of both the Nature Conservancy and the National Audubon Society carefully describe this region as a shrub-steppe.

The classification of the climate of the Mid-Columbia Region has not been consistent among the numerous meteorological and ecological publications that have described the area, either. In these publications, one finds the Mid-Columbia alternately described as shrub-steppe (or semi-arid) and desert (or arid)-sometimes within the same publication (e.g., Rogers and Rickard 1988; p. 1ff and p. 8). To further confuse the issue, the terms "arid" or "desert" and "semiarid" or "steppe" are used as a subdivision of the general term "arid," which represents a climatic condition in which the potential evaporation exceeds precipitation on average (American Meteorological Society 2000). In fairness to Rogers and Rickard, their use of "arid" cited above was most likely intended in the general sense rather than as a contrast to "semiarid."

Climate, an abstract concept of the weather conditions at a particular location over a long period of time, is popularly understood among laypeople and scientists alike in terms of its effects. In their introductory text on climate, Trewartha and Horn (1980) note that climate fundamentally controls the distribution of natural things such as plants, animals, and soils. They further comment (p. 218) that if "one disregards the distribution of non-climatic phenomena [e.g., plants, animals, and soils], it is difficult to provide meaningful temperature-rainfall limits of climatic types." In other words, assessing the climate of a particular area, especially near boundaries of climate types, requires consideration of not only meteorological variables but also those natural things that respond to them.

The non-climatic phenomenon that is most frequently associated with climate is the distribution of vegetation. Because the vegetation distribution is heavily determined by temperature and available moisture, most of the classification systems for climate have been empirically developed by correlating vegetation and some measure of temperature and humidity. In fact, the general correspondence between patterns of climate variables and patterns of vegetation is so strong that categories of some climate types are named for the dominant vegetation type (e.g., tropical rainforest or steppe) that they generate. Climate classification systems have generally sought to create indices that are indicative of specific climate types for specified ranges of the index variable. 


\section{A.1 Climate Classification Systems}

Numerous classification schemes have been developed for climate. Gedzelman (1985) in his introductory chapter to the Handbook of Applied Meteorology provides a helpful and detailed overview of the more prominent of these systems. Much of the discussion that follows is based on his writing.

One of the earliest and still most widely used climate classification schemes was developed by Köppen in the early twentieth century. This system sought to describe regions of similar vegetation in terms of temperature and humidity, whose cumulative effect Köppen expressed in terms of combinations of three letters. The first letter (A-E) denotes temperature, except for "B," which indicates a lack of moisture. The second letter indicates quantity of precipitation, and the third, if present, relates to mean monthly temperatures. As the scheme applies to the Columbia Basin, the classification is either BS (steppe) or BW (desert), and is determined as follows:

(a) If 70\% or more of the mean annual precipitation $\bar{R}$ occurs in the six cooler months (October through March in the Northern Hemisphere), then the classification is given in terms of $\bar{R}(\mathrm{~cm})$ and the mean annual temperature $\bar{T}\left({ }^{\circ} \mathrm{C}\right)$ as

$$
\begin{array}{ll}
\bar{T} \leq \bar{R}<2 \bar{T} & \text { station climate is steppe (BS) } \\
\bar{R}<\bar{T} & \text { station climate is desert (BW) }
\end{array}
$$

(b) If 70\% or more of $\bar{R}$ occurs in the six warmer months, then the classification is

$$
\begin{array}{ll}
\bar{T}+14 \leq \bar{R}<2(\bar{T}+14) & \text { station climate is BS } \\
\bar{R}<\bar{T}+14 & \text { station climate is BW }
\end{array}
$$

(c) If precipitation is evenly distributed throughout the year [i.e., neither (a) nor (b) applies], then the classification is

$$
\begin{array}{ll}
\bar{T}+7 \leq \bar{R}<2(\bar{T}+7) & \text { station climate is BS } \\
\bar{R}<\bar{T}+7 & \text { station climate is BW }
\end{array}
$$

Note that the boundary between desert and steppe in each of these sets of inequalities occurs at half the value of precipitation required for the boundary between steppe and humid climates.

From the historical averages through 2000 at the Hanford Meteorological Station, the mean annual temperature is $53.4^{\circ} \mathrm{F}\left(11.9^{\circ} \mathrm{C}\right)$, and the mean annual precipitation is 6.79 inches ( 17.2 centimeters) of which $66.6 \%$ falls from October through March. Since this percentage falls just short of Köppen's criterion of $70 \%$ for a winter maximum of precipitation, one might infer that (c) applies and the climate classification is BW, or desert. These formulas exhibit an inconsistent behavior, however. If the precipitation were the same in the winter but less in the summer months by 0.33 inch, the appropriate formulas would be (a) in which case the climate classification would be BS, or steppe. Thus by reducing 
annual rainfall in this manner, the Hanford Site would move to a more moist Köppen classification. This suggests that it is not useful to be too inflexible in the application of classification formulas, especially near zone boundaries.

Patton (1962) offered a simplification of Köppen's system that expressed the boundary between humid and semiarid climates as a single, easily memorized equation:

$$
R^{\prime}=\frac{1}{2} T^{\prime}-\frac{1}{4} P^{\prime} w
$$

where $\quad R^{\prime}=$ annual precipitation in inches

$T^{\prime}=$ temperature in degrees Fahrenheit

$P^{\prime} w=$ the percentage of precipitation that falls in the winter months.

Mean precipitation greater than $R^{\prime}$ results in a humid climate. Precipitation less than $R^{\prime}$ but greater than $1 / 2 R^{\prime}$ generates a steppe climate, and less than $1 / 2 R^{\prime}$ generates desert conditions. This formula was shown to give results that are not significantly different from Köppen's set of three relations. Patton's relation, incidentally, also solves the problem of the inconsistency noted above in Köppen's formulas near the precipitation regime boundaries. Applying Patton's result to the Hanford Meteorological Station data

$$
\begin{aligned}
\frac{1}{2} R^{\prime} & =\frac{1}{4} T^{\prime}-\frac{1}{8} P^{\prime} w \\
& =\frac{1}{4}(53.4)-\frac{1}{8}(66.6) \\
& =5.03 \mathrm{in}
\end{aligned}
$$

This precipitation value for the boundary between steppe and desert is significantly lower than the Hanford Meteorological Station mean annual precipitation of 6.79 inches, placing the Hanford Site in a steppe climate by this representation of the Köppen system.

Another classification for climate that has been widely used is that given by Thornthwaite (1931). Thornthwaite was the first to attempt to develop a numerical index by which climate zones could be defined. In fact, he developed two climate indices: a "temperature efficiency" index $T E$ and a "precipitation effectiveness" index $P E$. Like Köppen, he based his indices on temperature and humidity for a region in such a way as to try to make them representative of patterns of plant communities. These indices are defined as follows:

$$
T E=\frac{1}{4} \sum_{k=1}^{12}\left(\overline{T_{k}}-32\right)
$$

where $\overline{T_{k}}$ is the mean temperature in ${ }^{o} F$ for month $k$, and 


$$
P E=115 \sum_{k=1}^{12}\left(\frac{\overline{r_{k}}}{\overline{T_{k}}-10}\right)^{10 / 9}
$$

where $\overline{r_{k}}$ is the mean precipitation for month $k$ in inches.

Using the same Hanford Meteorological Station data as those for calculating the Köppen classification above, the Thornthwaite indices for the Hanford Site are $T E=64.5$ and $P E=16.1$. The $T E$ value is near the boundary of 63.5 between Thornthwaite's "microthermal" (cool) and "mesothermal" (warm, but not hot) climates. The $P E$ value for the Hanford Site, of primary interest here, places the Hanford Meteorological Station in a semiarid climate but is very near Thornthwaite's boundary value between arid $(P E<16)$ and semiarid $(16 \leq P E<31)$.

Thornthwaite (1948) updated his classification in an effort to tie it more closely to the physical water balance. The result was more philosophically satisfying but also considerably more complicated, since not only temperature and precipitation but also soil characteristics must be considered. Daubenmire (1988), in his definitive study of eastern Washington vegetation, found that the resulting values of Thornthwaite's Moisture Index overlapped distinct steppe zones and that the index was not as practical as simpler formulations. Knapp (1985) suggested that Thornthwaite's index may not be applicable to dry regions because its correlations were developed from the humid zones of the central and eastern United States.

\section{A.2 Reliability of Precipitation}

Another important distinction between desert and steppe climates is the reliability of precipitation. True desert regions are generally characterized by sporadic albeit sometimes heavy precipitation events. Because of this, the routine availability of moisture is often much less than would be suggested by the mean annual precipitation value. Thus, one region, with long periods between significant precipitation events, may only be able to support desert vegetation while another, with a similar mean value of temperature and precipitation, may support the grasses and shrubs of the steppe. Daubenmire (1988) criticized the Thornwaite index for this reason because it uses mean annual rainfall rather than the probably more appropriate median value.

Under the definition of "semiarid zone," the Glossary of Meteorology (American Meteorological Society 2000) notes that the coefficient of variation for arid zones exceeds $50 \%$, while the value for semiarid zones is $30-50 \%$. This quantity is defined as

$$
C_{r}=100 \frac{\sigma_{r}}{\bar{r}}
$$

where $\quad \bar{r}=$ the mean of the annual precipitation.

$\sigma_{r}=$ its standard deviation. 
Using historical data from the Hanford Meteorological Station through 2000, this value is $C_{r}=30.5 \%$, far from the variability that characterizes desert regions.

\section{A.3 Vegetation of the Mid-Columbia Region}

Vegetation may be viewed as an integrator of weather that reflects the climate. The Mid-Columbia region, where the surface is undisturbed, is characterized by a substantial cover of xerophytic shrubs (i.e., those adapted to dry regions, such as sagebrush) and perennial grasses. From Daubenmire's (1988) perspective

"reasonable limits would be to consider desert as regions too dry to support a noticeable cover of perennial grasses on zonal soils, and steppe as regions with moisture relations adequate to support an appreciable cover of perennial grasses on zonal soil, yet not enough for arborescent vegetation. Since even the driest part of eastern Washington can support a heavy cover of perennial grasses wherever there is a zonal soil, a continuous film of cryptogams covers the soil surface, and at least 0.7 metric tons/ha/yr of dry matter is produced, its classification as steppe rather than desert seems preferable."

Some writers have attempted to disregard vegetation in classifying the climate of the intermountain West. In a recent textbook, Bailey (1996) relied on an apparently rigid application of the KöppenTrewartha system to conclude that, despite the widespread support of semi-desert shrubs and other vegetation, the Great Basin and Columbia Basin were true deserts. This is a curious result, since the climate mapping produced by Trewartha himself (Trewartha and Horn 1980) classifies the northern Great Basin and the Columbia Basin as steppe.

\section{A.4 Summary}

From virtually all perspectives but the popular criterion of lack of naturally growing trees, the climate of the Hanford Site as well as the rest of the Mid-Columbia region is best classified as steppe (or, equivalently, semiarid), although it is on the dry side of that classification. With respect to classification schemes, only the most rigid application of the Köppen formulation places the area in the desert class. The precipitation falls with a reliability that is characteristic of steppes rather than deserts, and the vegetation that is present is considered typical of a shrub-steppe ecosystem. For these reasons, we believe that the most appropriate term for the local climate is "steppe."

Table A.1 organizes the terminology discussed above. 
Table A.1. Description of the Uses of the Terms "arid" and "semiarid" with Respect to Climate.

\begin{tabular}{|c|c|c|c|}
\hline \multicolumn{4}{|c|}{ Arid Climate } \\
\hline $\begin{array}{l}\text { Conventional } \\
\text { Boundary of } \\
\text { Classification: }\end{array}$ & \multicolumn{3}{|c|}{$\begin{array}{l}\text { On an annual average, more moisture can leave the vegetation and soil surfaces than falls } \\
\text { as precipitation }\end{array}$} \\
\hline Effect: & \multicolumn{3}{|c|}{ No arborescent vegetation (i.e., trees), except perhaps in river bottoms } \\
\hline $\begin{array}{l}\text { Equivalent } \\
\text { Terms: }\end{array}$ & \multicolumn{3}{|c|}{ None in this general sense, except perhaps "dry climate" } \\
\hline & Semiarid Climate & & Arid Climate \\
\hline $\begin{array}{l}\text { Conventional } \\
\text { Boundary of } \\
\text { Classification: }\end{array}$ & $\begin{array}{l}\text { Within the general arid designa- } \\
\text { tion, the annual precipitation is half } \\
\text { or more of the moisture that could } \\
\text { escape to the atmosphere from the } \\
\text { soil and plant surfaces }\end{array}$ & $\begin{array}{l}\text { Conventional } \\
\text { Boundary of } \\
\text { Classification: }\end{array}$ & $\begin{array}{l}\text { Within the general arid designa- } \\
\text { tion, the annual precipitation is less } \\
\text { than half of the moisture that could } \\
\text { escape to the atmosphere from the } \\
\text { soil and plant surfaces. }\end{array}$ \\
\hline Effect: & $\begin{array}{l}\text { Widespread coverage of undis- } \\
\text { turbed soil surface by annual and } \\
\text { perennial grasses and, in some } \\
\text { areas, by shrubs adapted to dry } \\
\text { climates }\end{array}$ & Effect: & $\begin{array}{l}\text { Sparse coverage of undisturbed } \\
\text { soil surface by shrubs and perhaps } \\
\text { grasses; a significant fraction of } \\
\text { the soil surface is free of } \\
\text { vegetation }\end{array}$ \\
\hline $\begin{array}{l}\text { Equivalent } \\
\text { Terms: }\end{array}$ & $\begin{array}{l}\text { steppe, shrub-steppe (if shrubs are } \\
\text { present), semi-desert }\end{array}$ & $\begin{array}{l}\text { Equivalent } \\
\text { Term: }\end{array}$ & desert \\
\hline
\end{tabular}

NOTE that "arid" can mean either "dry" or "desert" depending on context. Because of the tight link between climate and vegetation, terms that fundamentally describe ecosystems, such as "steppe," are widely used interchangeably with terms such as "semiarid" that describe the physical water balance of a region.

\section{A.5 References}

American Meteorological Society. 2000. Glossary of Meteorology, $2^{\text {nd }}$ Edition. Boston.

Bailey, R. G. 1996. Ecosystem Geography. Springer-Verlag, New York.

Daubenmire, R. F. 1988. Steppe Vegetation of Washington. Cooperative Extension Publication EB1446, Washington State University, Pullman, Washington.

Gedzelman, S. D. 1985. “Atmospheric Circulation Systems.” in Handbook of Applied Meteorology, D. D. Houghton (ed.), John Wiley \& Sons, New York. 
Knapp, S. D. 1985. “Evaporation and Transpiration” In Handbook of Applied Meteorology. D. D. Houghton (ed.), John Wiley \& Sons, New York.

Patton, C. P. 1962. "A Note on the Classification of Dry Climates in the Köppen System.” California Geographer, 2:105-112.

Rogers, L. E. and W. H. Rickard. 1988. "Introduction: Shrub-steppe lands." Shrub-steppe: Balance and Change in a Semi-Arid Terrestrial Ecosystem. W. H. Rickard, L. E. Rogers, B. E. Vaughan, and S. F. Liebetrau (eds.), Elsevier.

Thornthwaite, C. W. 1931. "The Climates of North America According to a New Classification." Geographical Review., 21:633-635.

Thornthwaite, C. W. 1948. “An Approach Toward a Rational Classification of Climate." Geographical Review, 38:55-94.

Trewartha, G. T. and L. H. Horn. 1980. An Introduction to Climate, $5^{\text {th }}$ edition. McGraw-Hill, New York. 


\section{Appendix B}

\section{Wind Climatology}




\section{Appendix B}

\section{Wind Climatology}

This appendix provides the 2003 station-specific wind roses (Figures B.1[a] and B.2[a]) from the Hanford Meteorological Monitoring Network. Each petal of the wind rose represents the proportional amount of time that the wind blew from that direction. The width of the petal corresponds to each wind speed category. Starting from the center of the rose, the narrowest petal represents winds in the 1- to 3-miles per hour ( $\mathrm{mph}$ ) class, the next widest represents the 4- to 7-mph class, and so forth. The length of each of these petals is proportional to the frequency of occurrence for each speed class.

The wind speed histograms (Figures B.1[b] and A.2[b]) represent the proportional amount of time in each speed class.

Table B.1 lists joint frequency distributions (at 30 feet) of wind direction versus wind speed class for the individual stations (see Figure 2.1 in text for locations). Table B.2 lists joint frequency distributions (at 60 meters) for stations 9, 11, 13, and 21 (see Figure 2.2 in text for locations). 


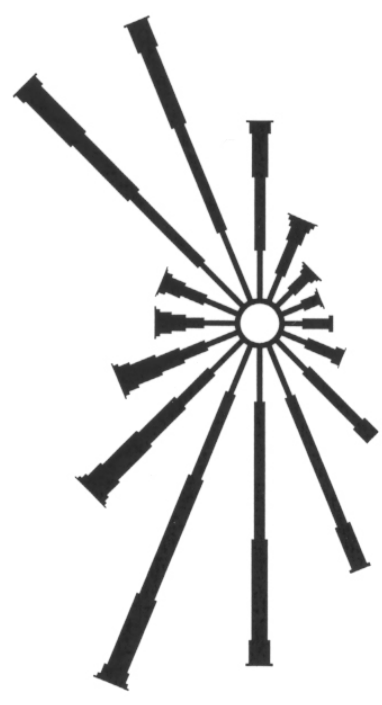

(a) Wind Rose

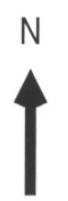

Station \# 1 - PROS

Period: $1 / 2003-12 / 2003$

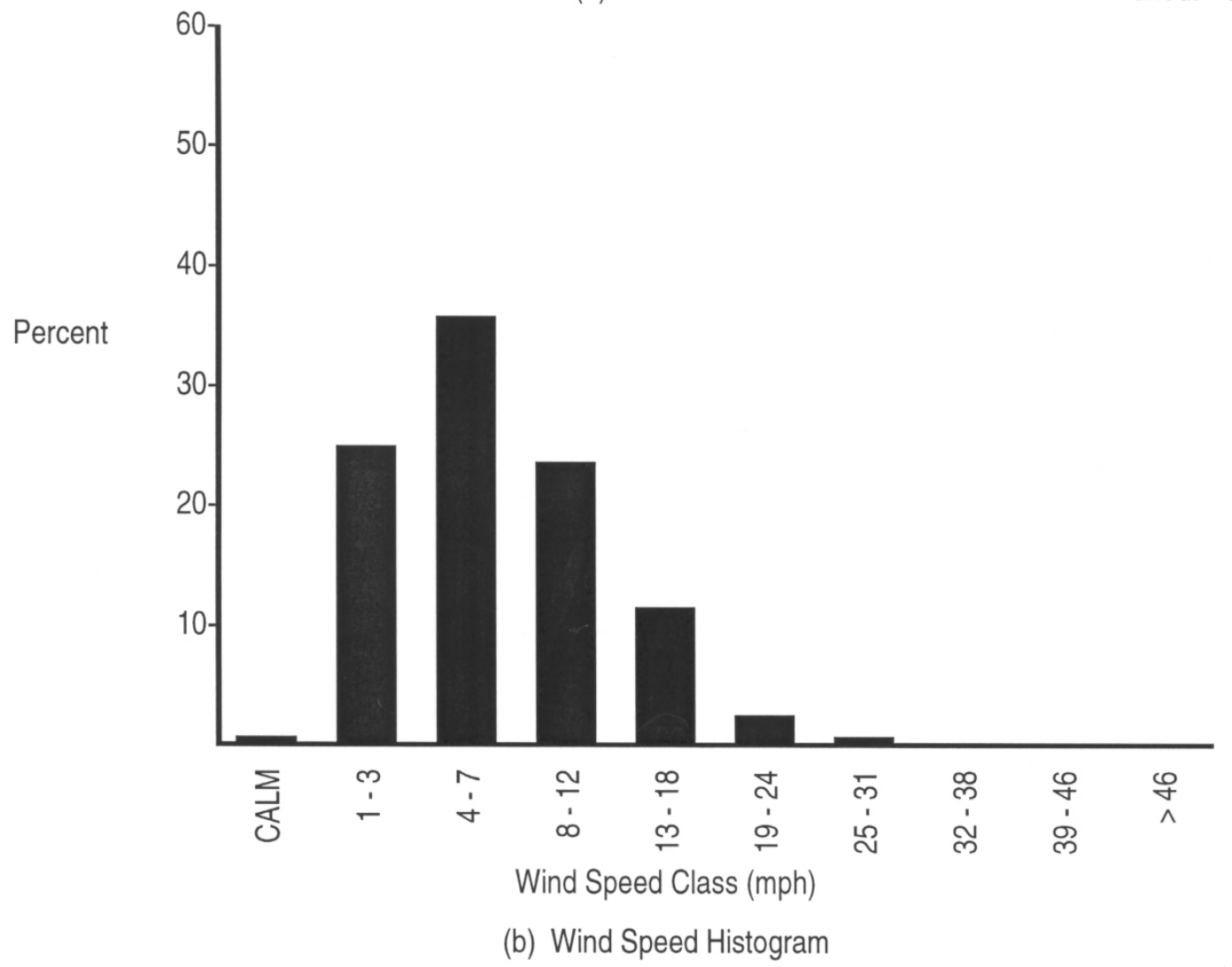

Figure B.1. Wind Rose and Wind Speed Histogram, 30 Feet 

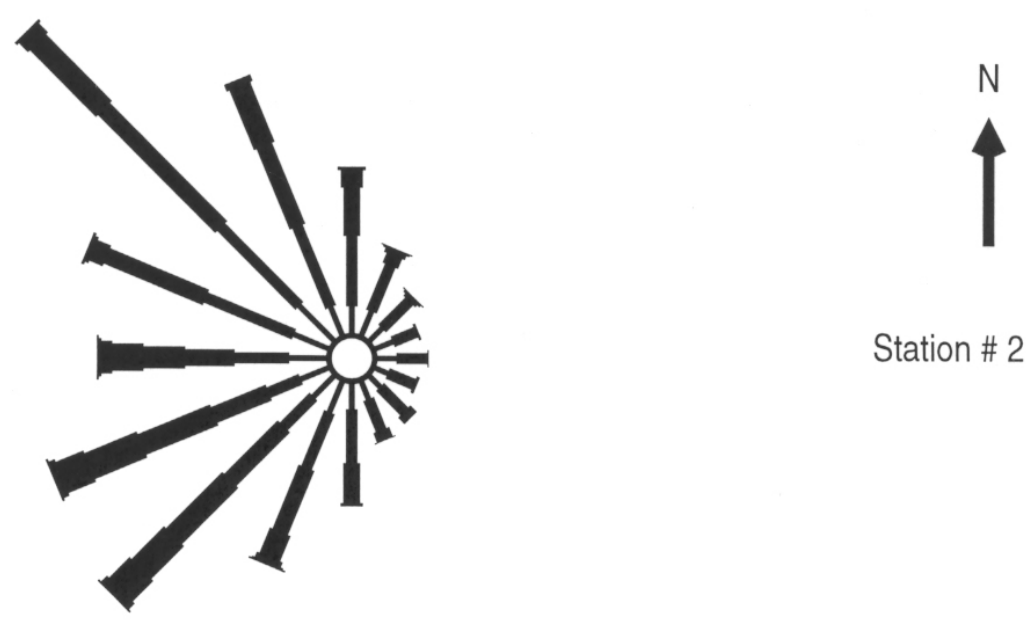

Station \# 2 - EOC

(a) Wind Rose

Period: $1 / 2003-12 / 2003$

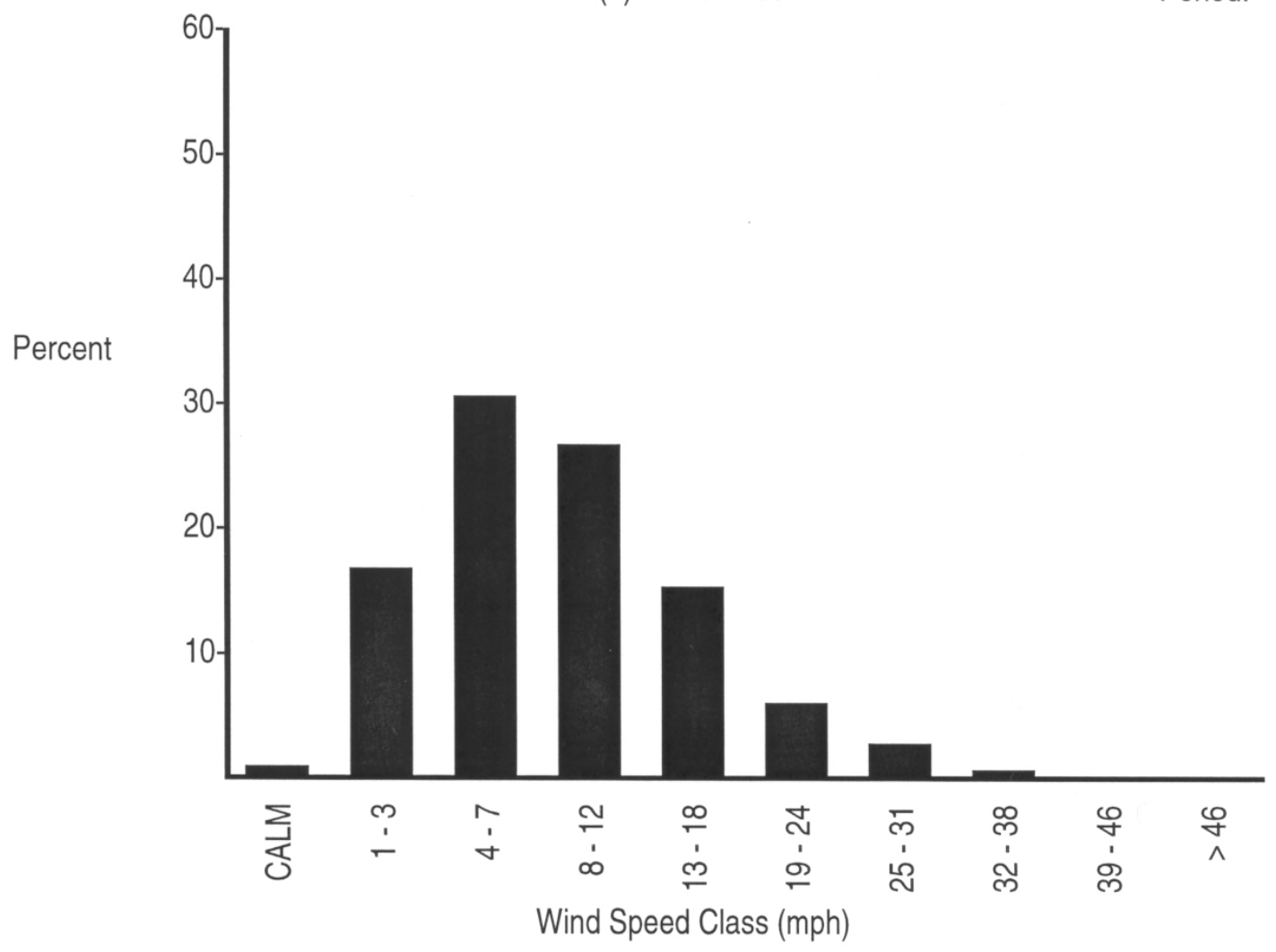

(b) Wind Speed Histogram

Figure B.1. (contd) 

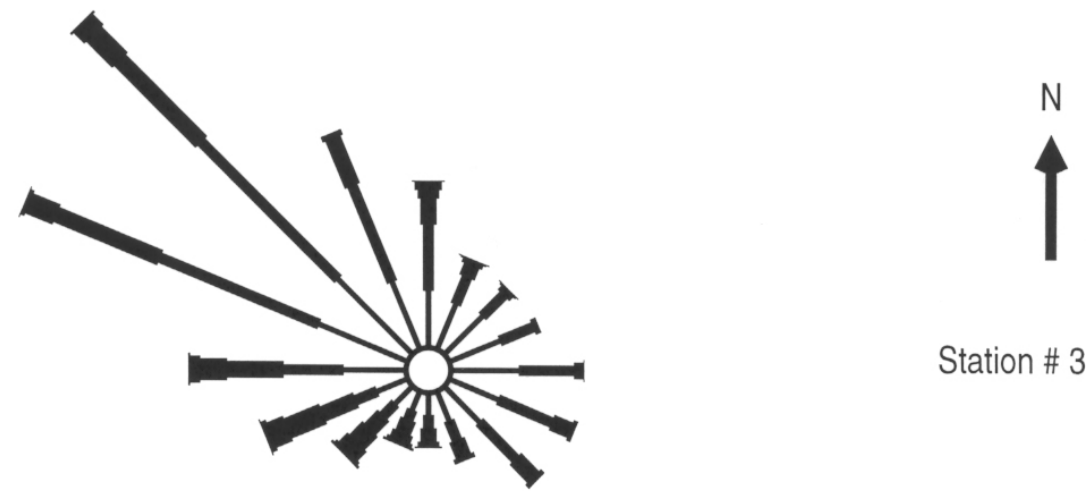

Station \# 3 - ARMY

(a) Wind Rose

Period: $1 / 2003-12 / 2003$

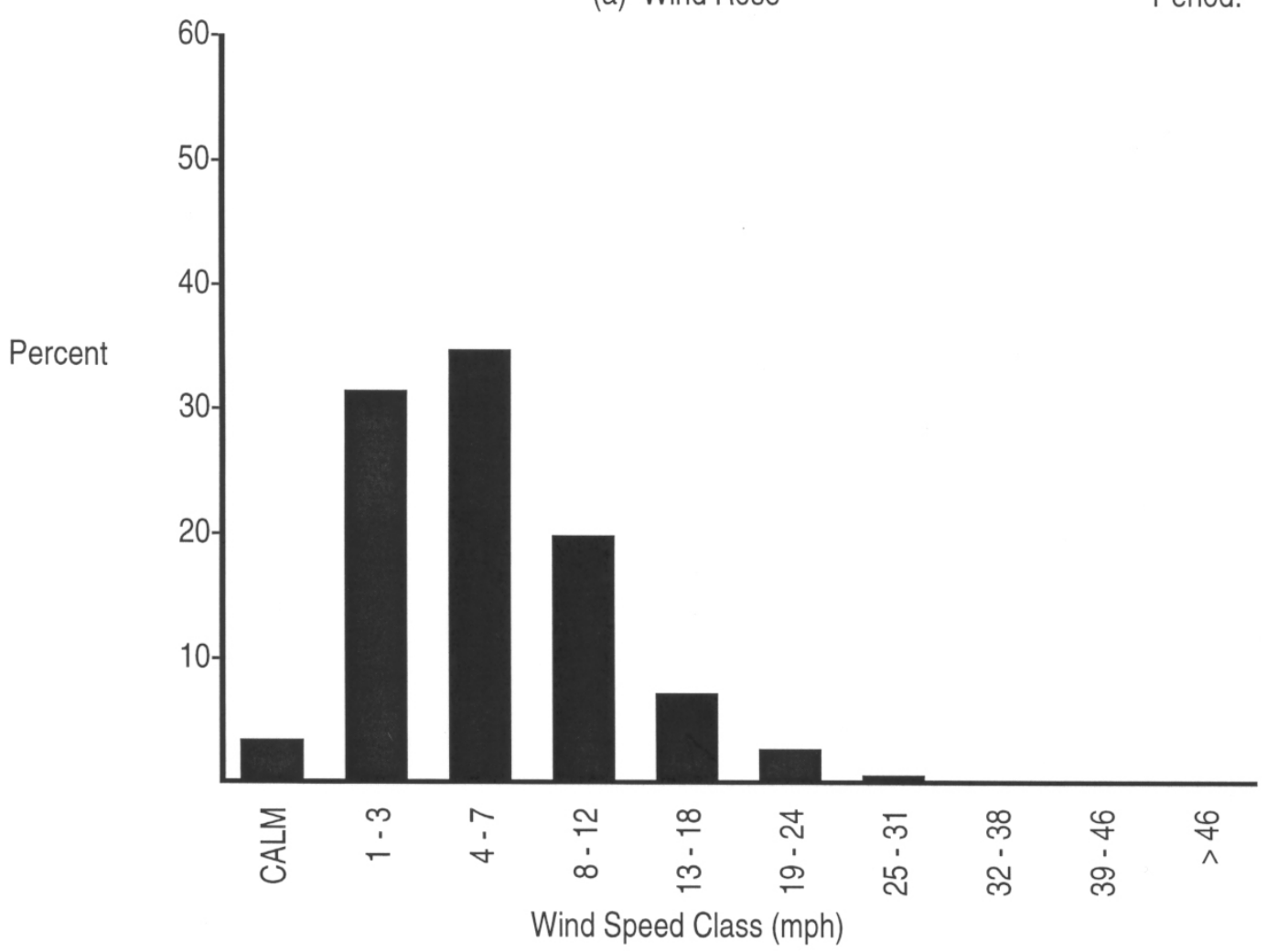

(b) Wind Speed Histogram

Figure B.1. (contd) 


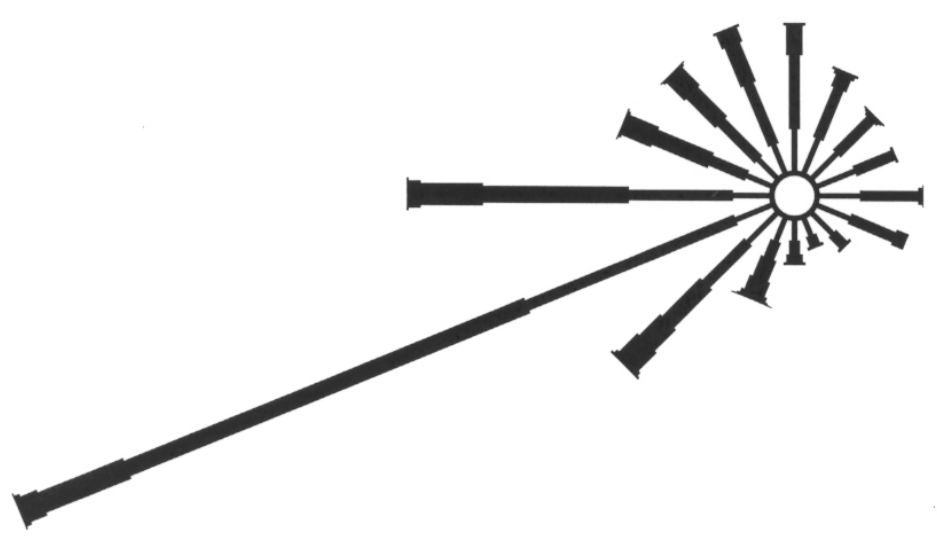

(a) Wind Rose

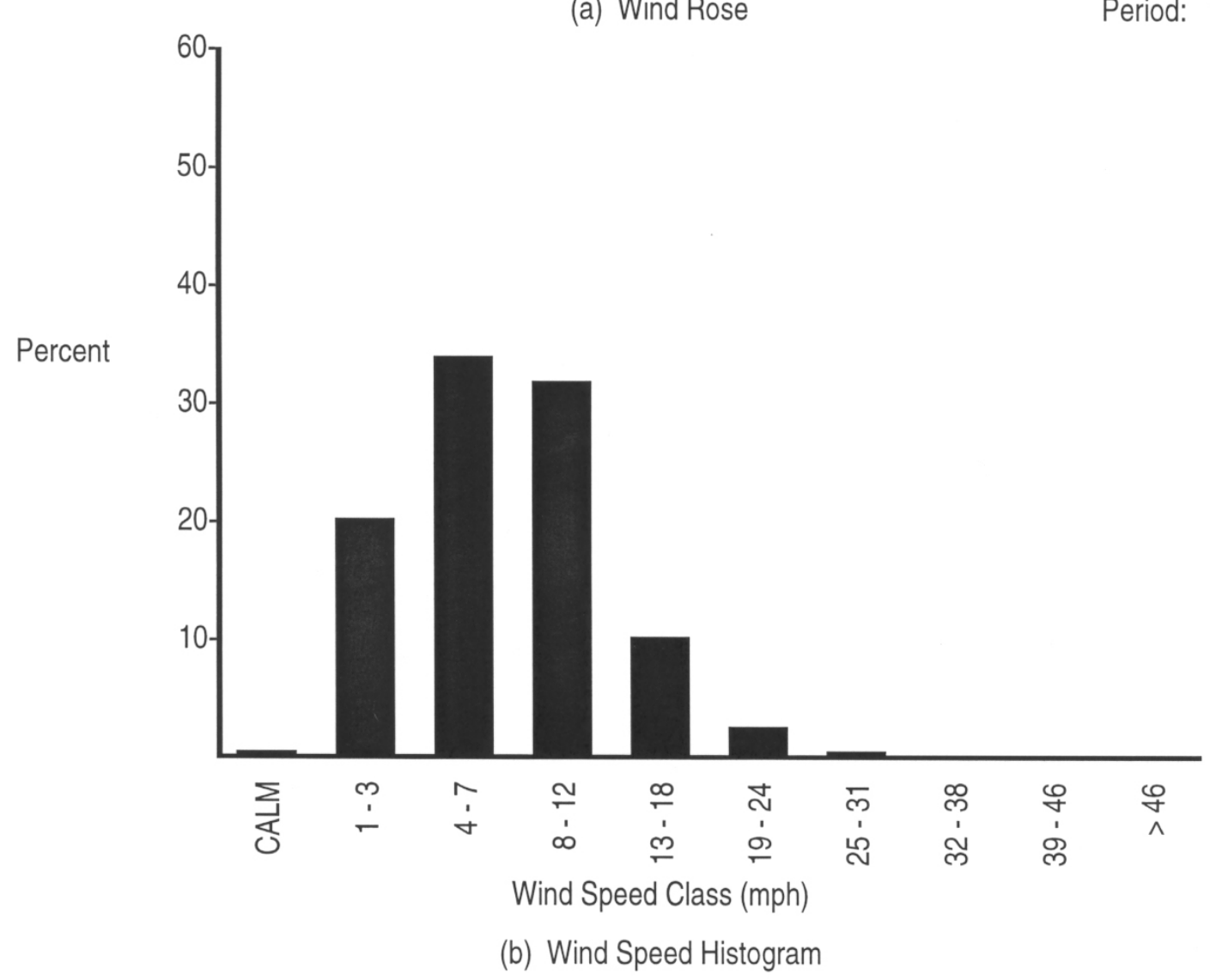

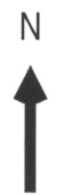

Station \# 4 - RSPG

Period: 1/2003 - 12/2003

Figure B.1. (contd) 


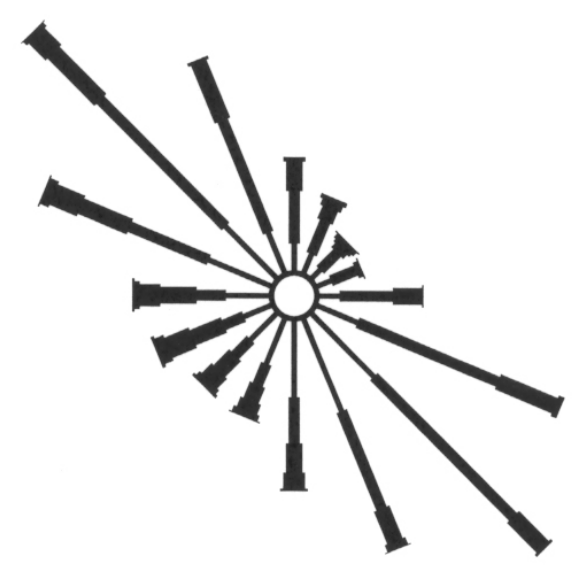

(a) Wind Rose

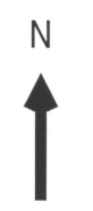

Station \# 5 - EDNA

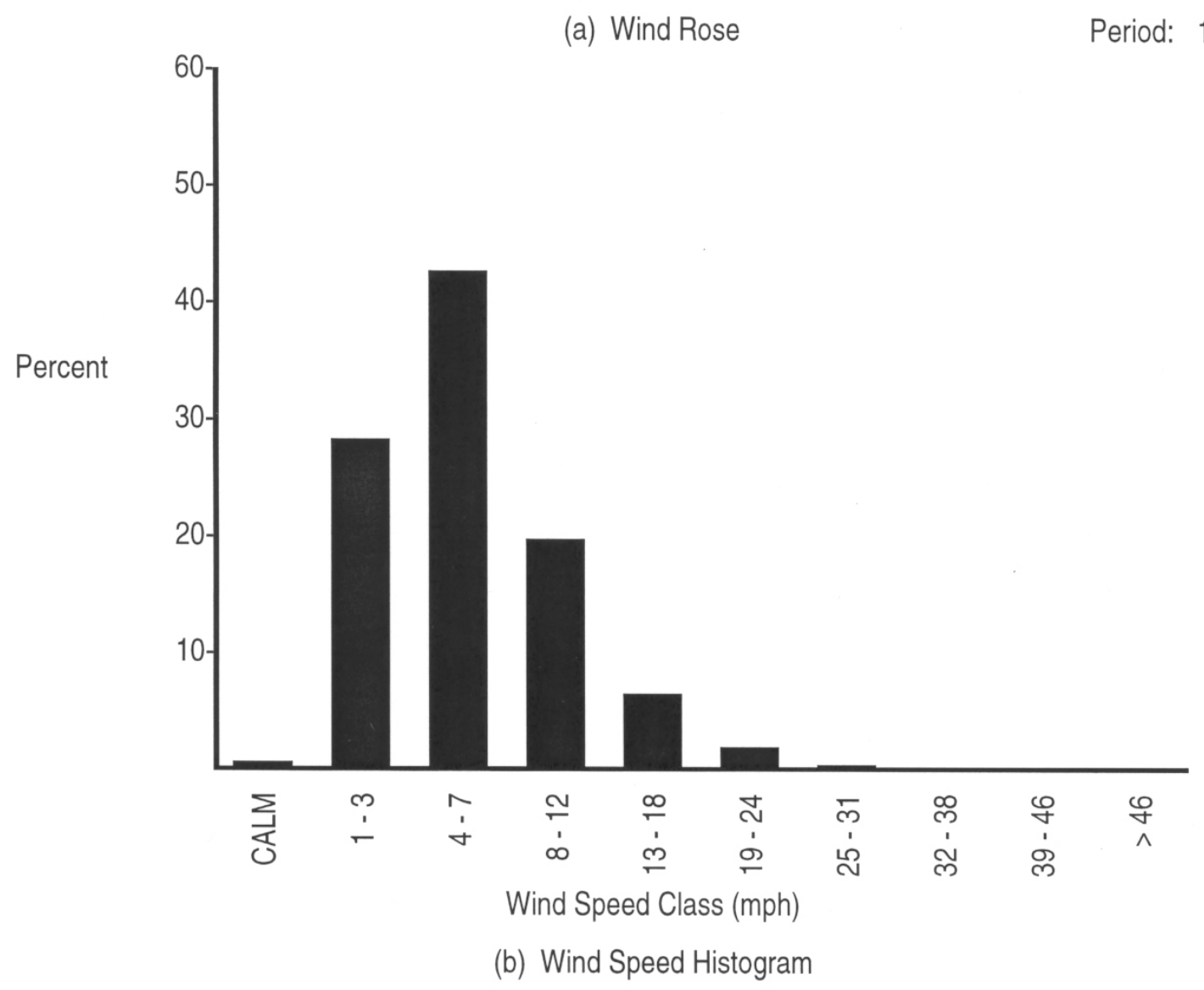

Figure B.1. (contd) 


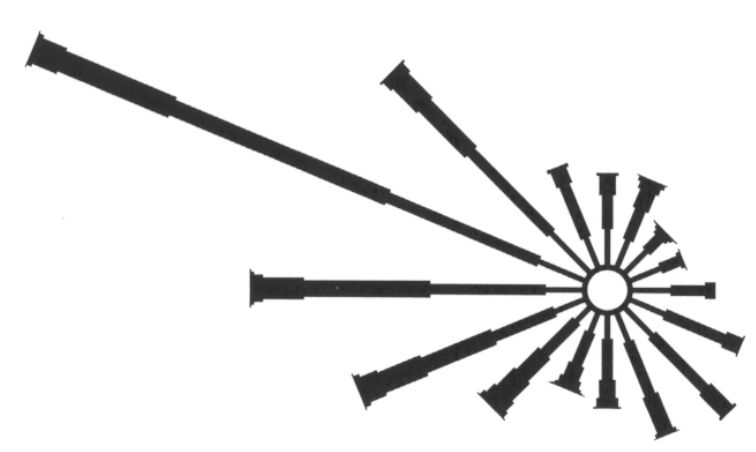

(a) Wind Rose

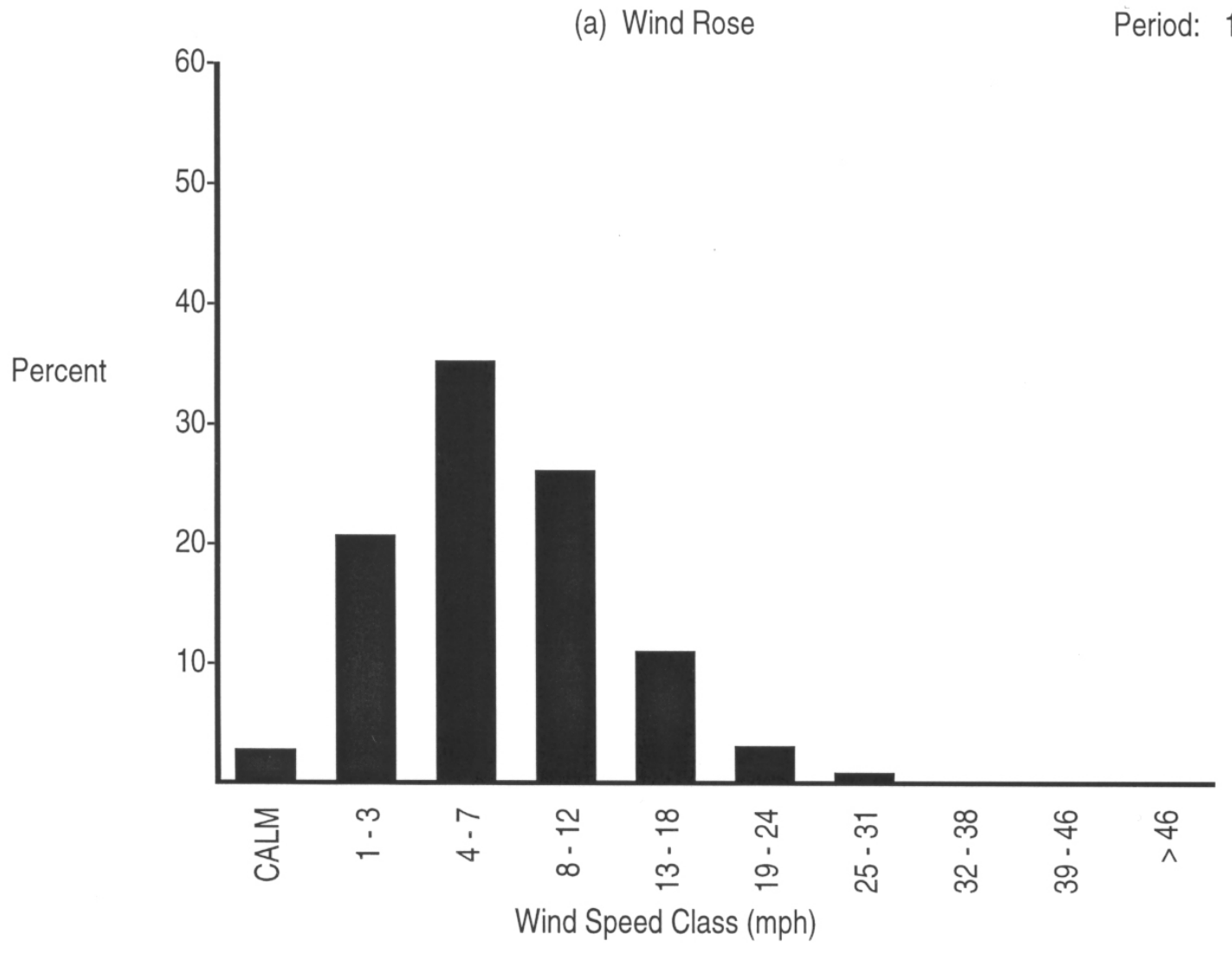

(b) Wind Speed Histogram

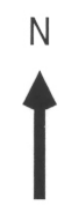

Station \# 6 - 200E

Period: 1/2003 - 12/2003 

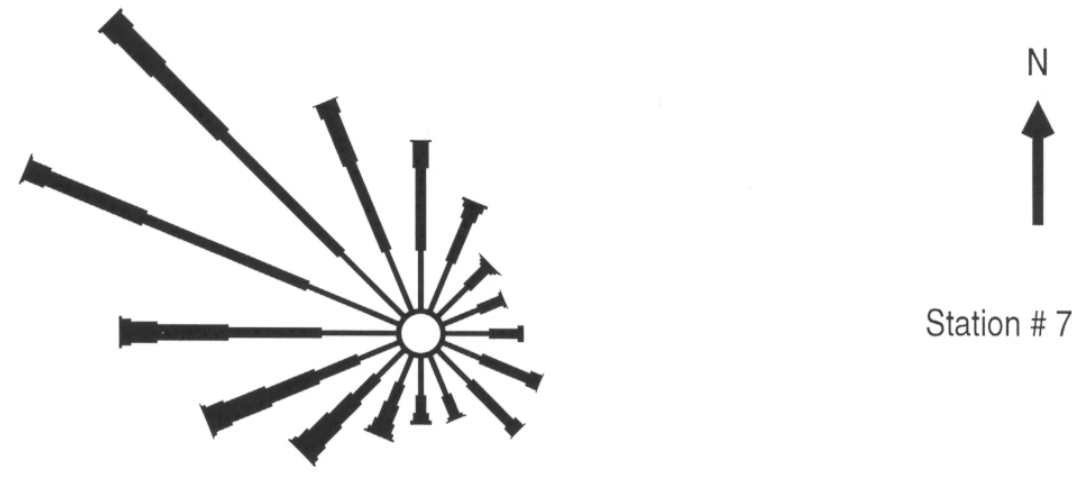

Station \# 7 - 200W

(a) Wind Rose

Period: 1/2003-12/2003

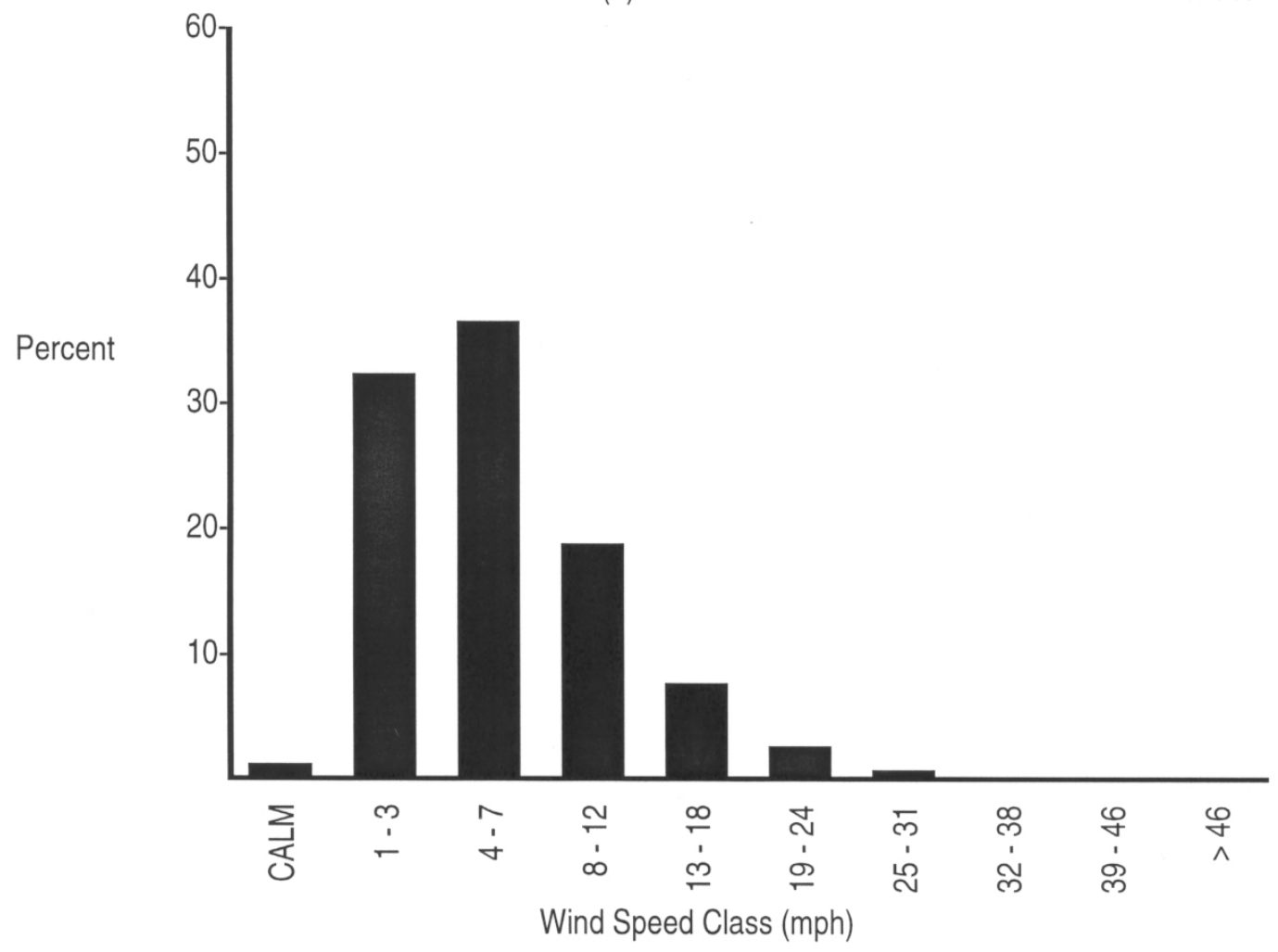

(b) Wind Speed Histogram

Figure B.1. (contd) 


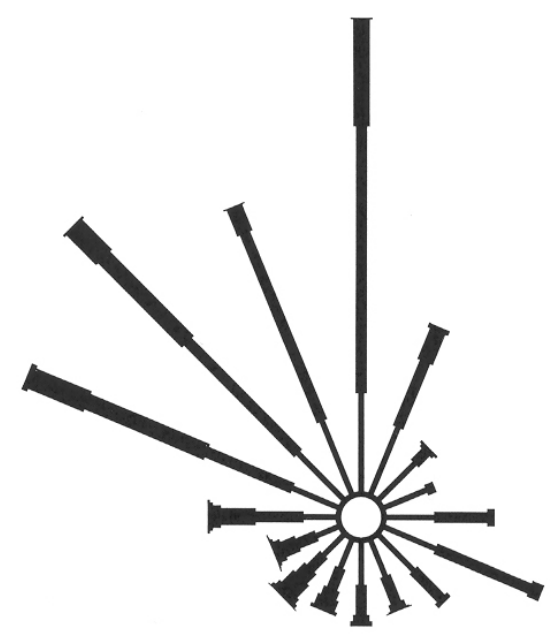

(a) Wind Rose

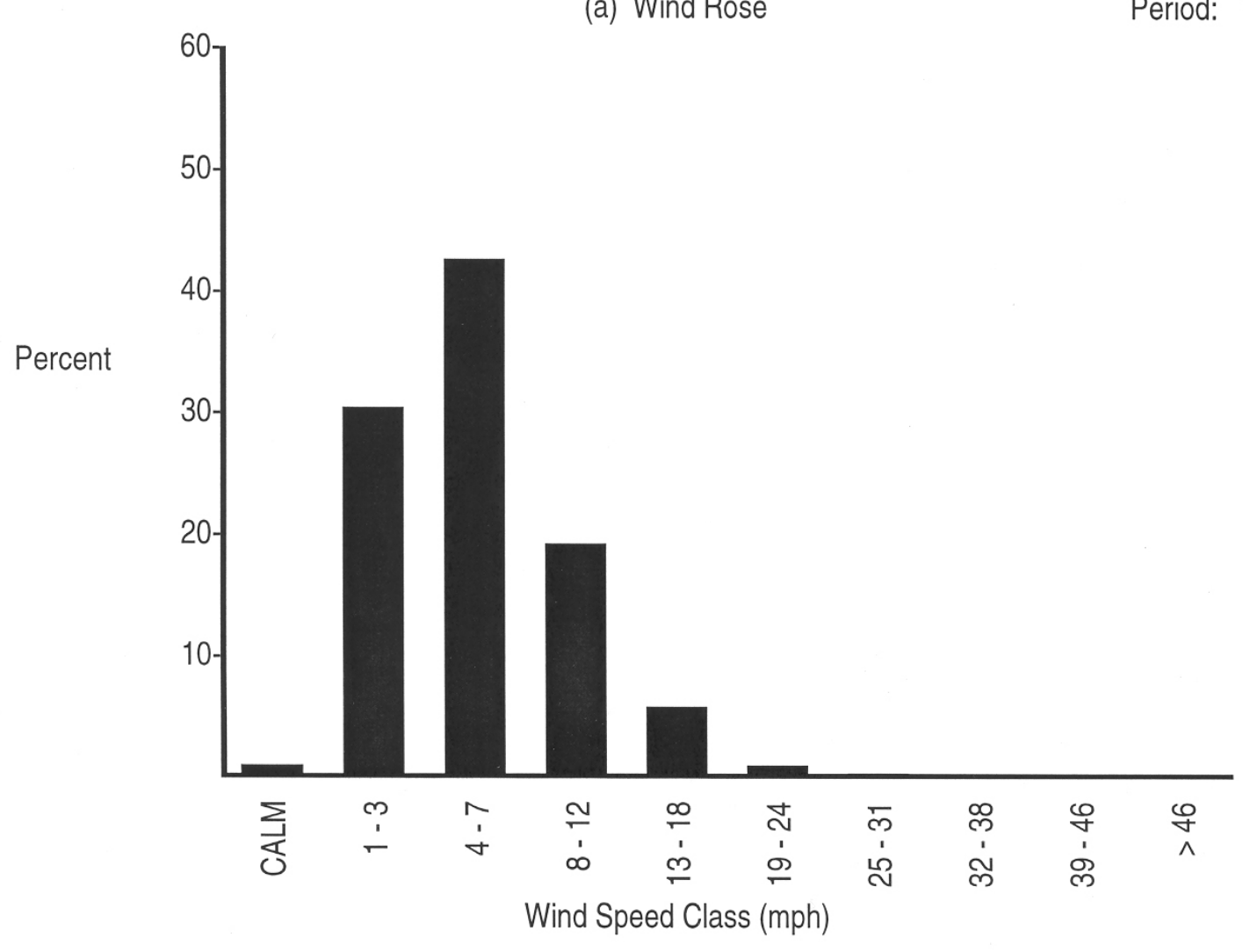

(b) Wind Speed Histogram

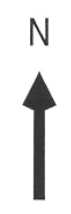

Station \# 8 - BVLY

Period: $1 / 2003-12 / 2003$

Figure B.1. (contd) 


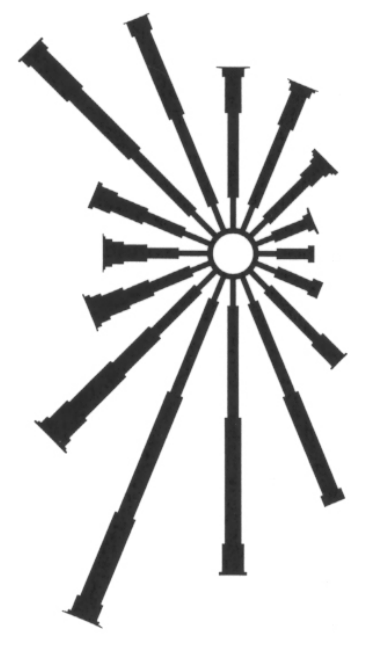

(a) Wind Rose

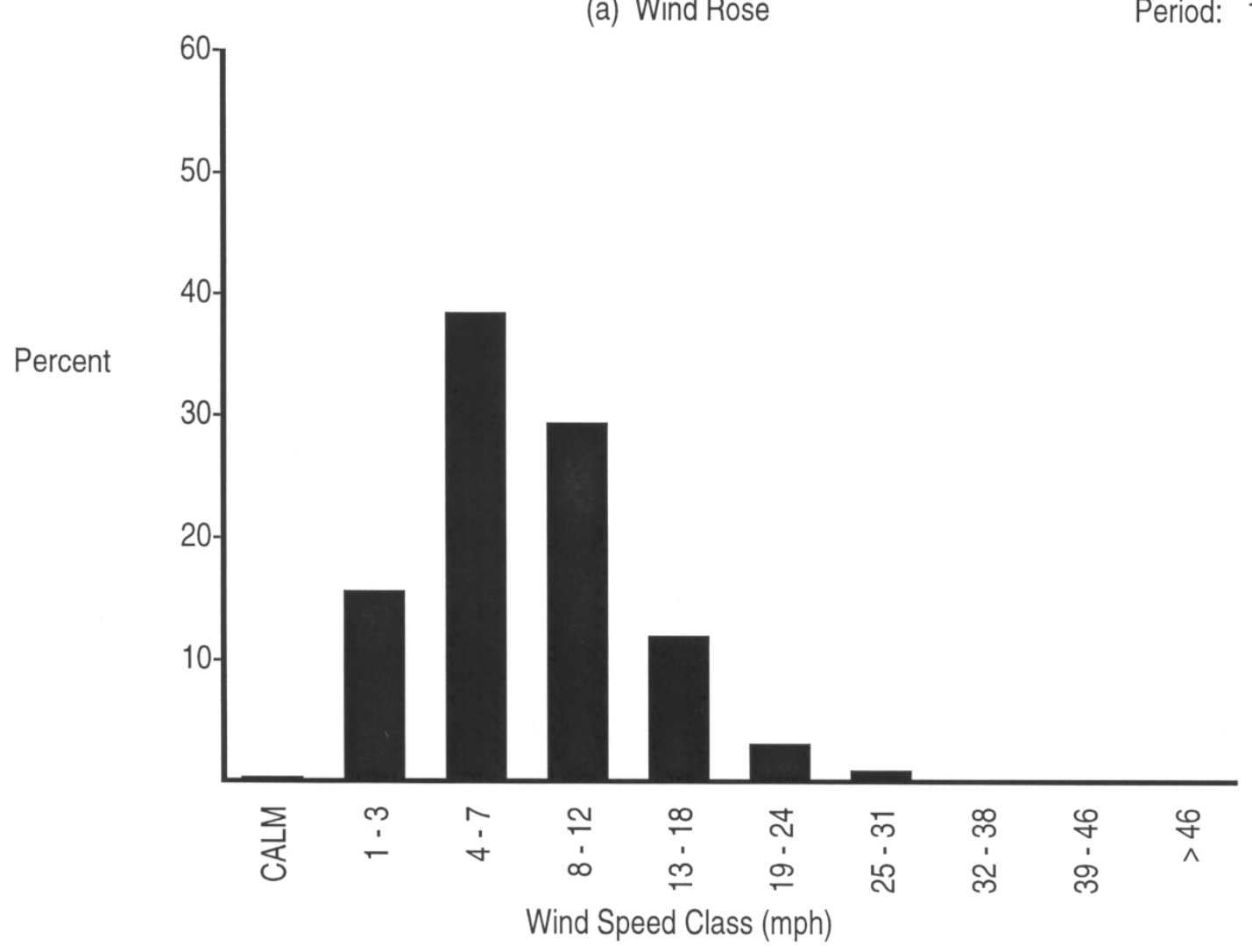

(b) Wind Speed Histogram

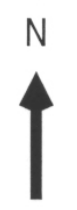

Station \# 9 - FFTF

Period: 1/2003-12/2003

Figure B.1. (contd) 

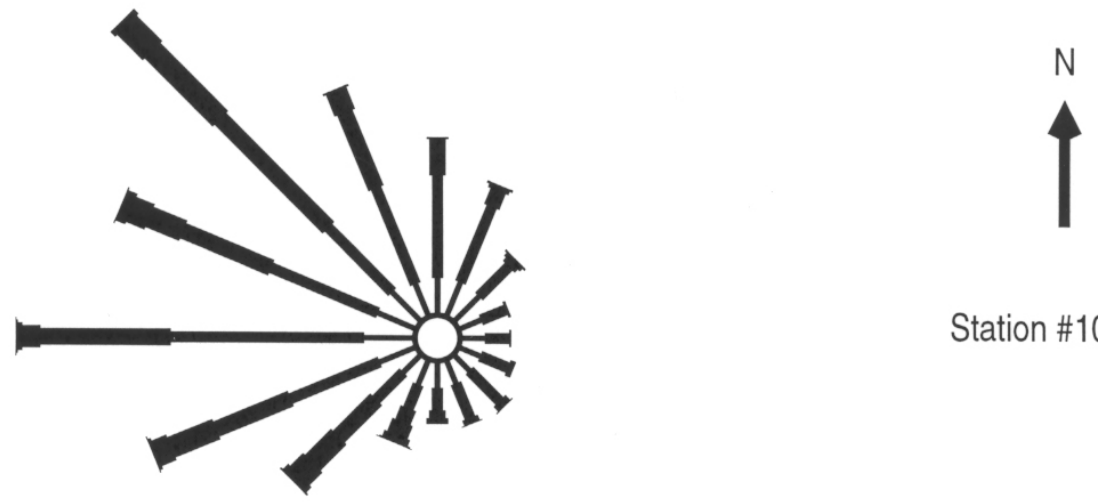

Station \#10 - YAKB

(a) Wind Rose

Period: $1 / 2003-12 / 2003$

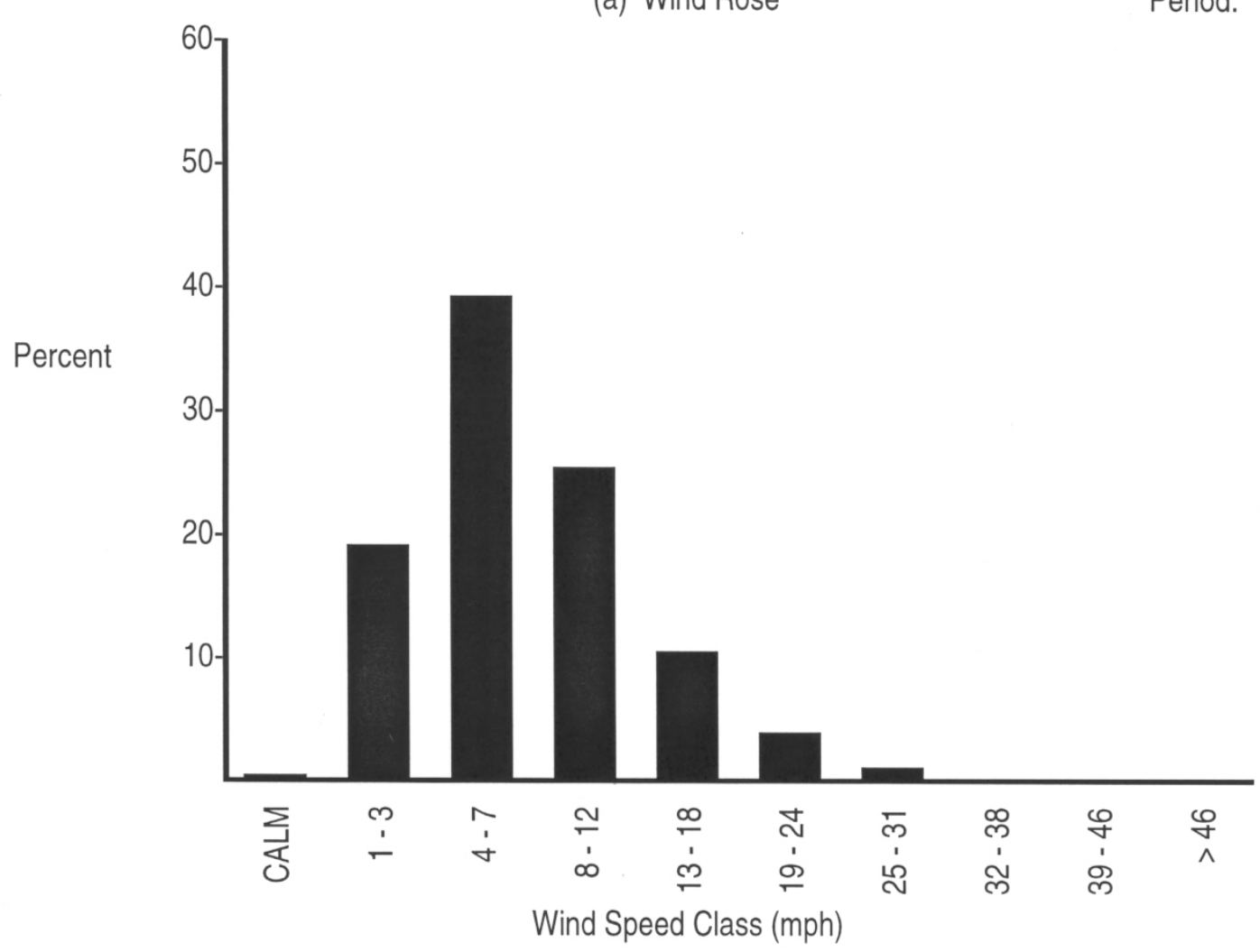

(b) Wind Speed Histogram

Figure B.1. (contd) 


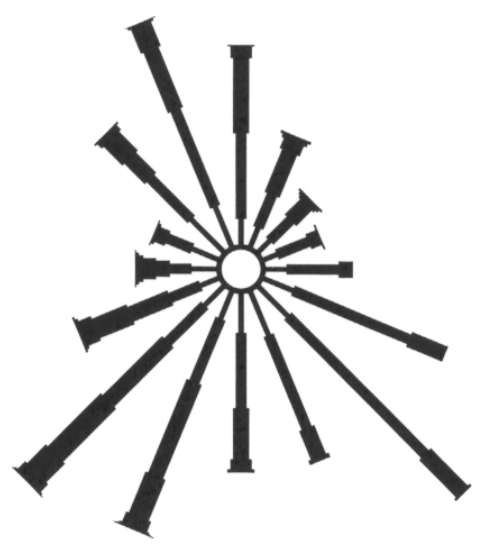

(a) Wind Rose

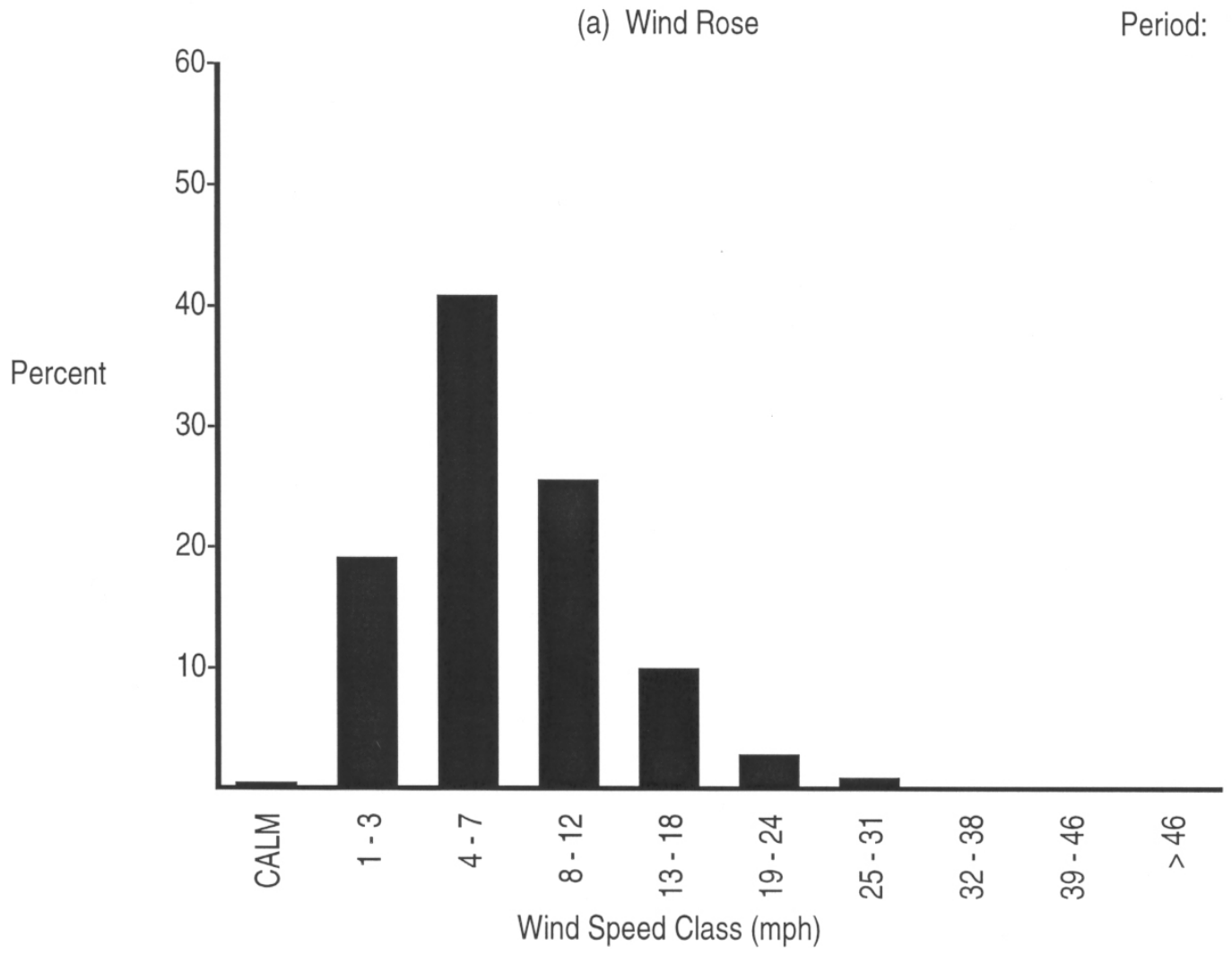

(b) Wind Speed Histogram

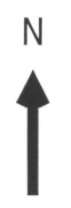

Station \#11 - 300A

Period: 1/2003 - 12/2003

Figure B.1. (contd) 


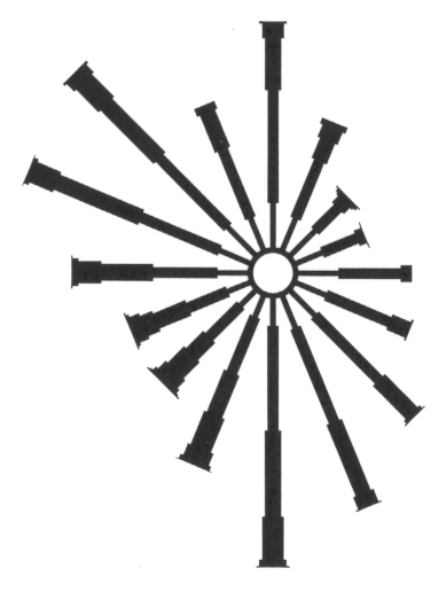

(a) Wind Rose

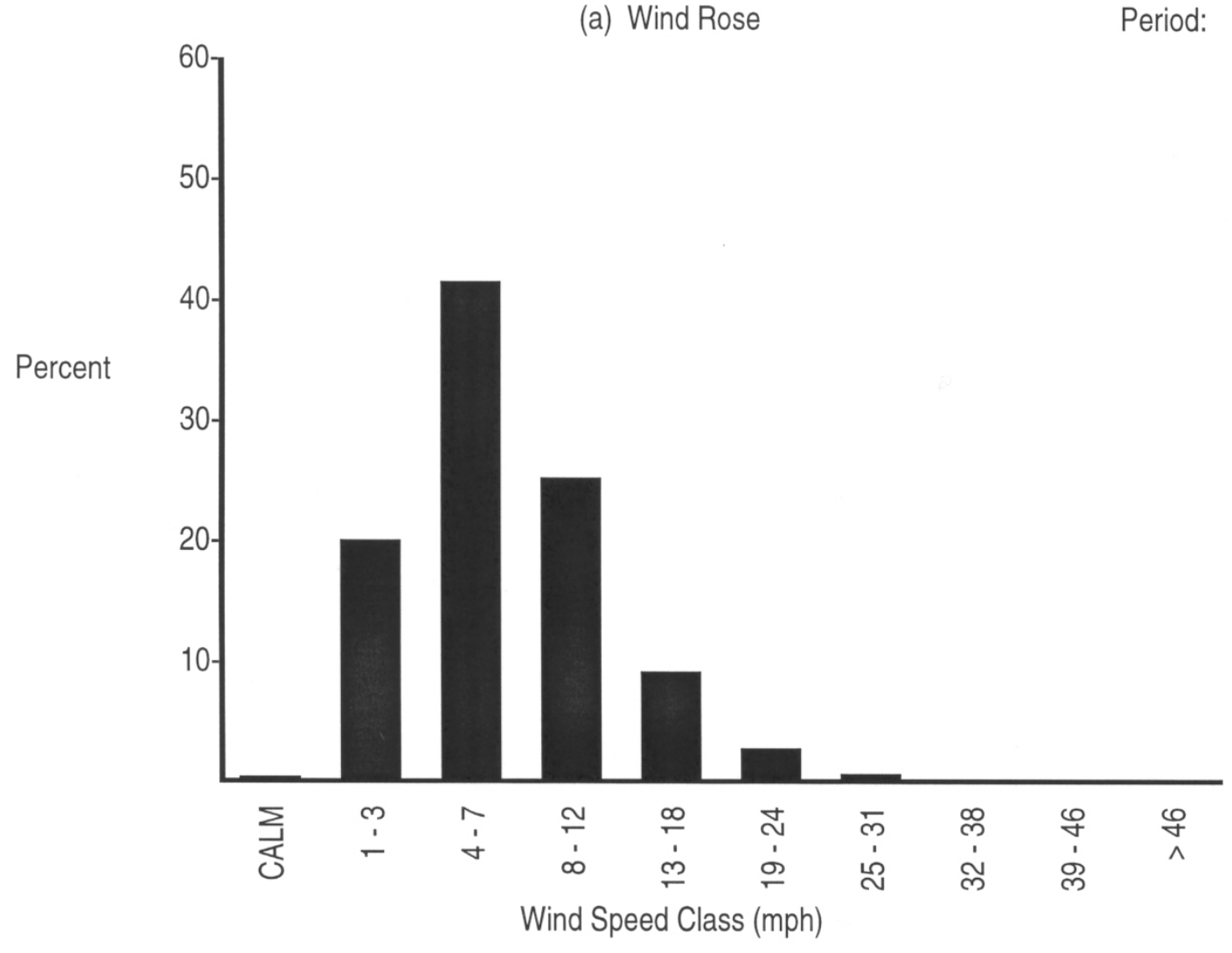

(b) Wind Speed Histogram

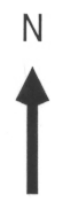

Station \#12 - WYEB

Period: 1/2003 - 12/2003

Figure B.1. (contd) 


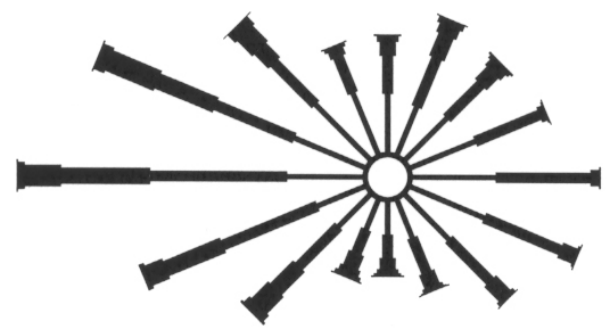

(a) Wind Rose

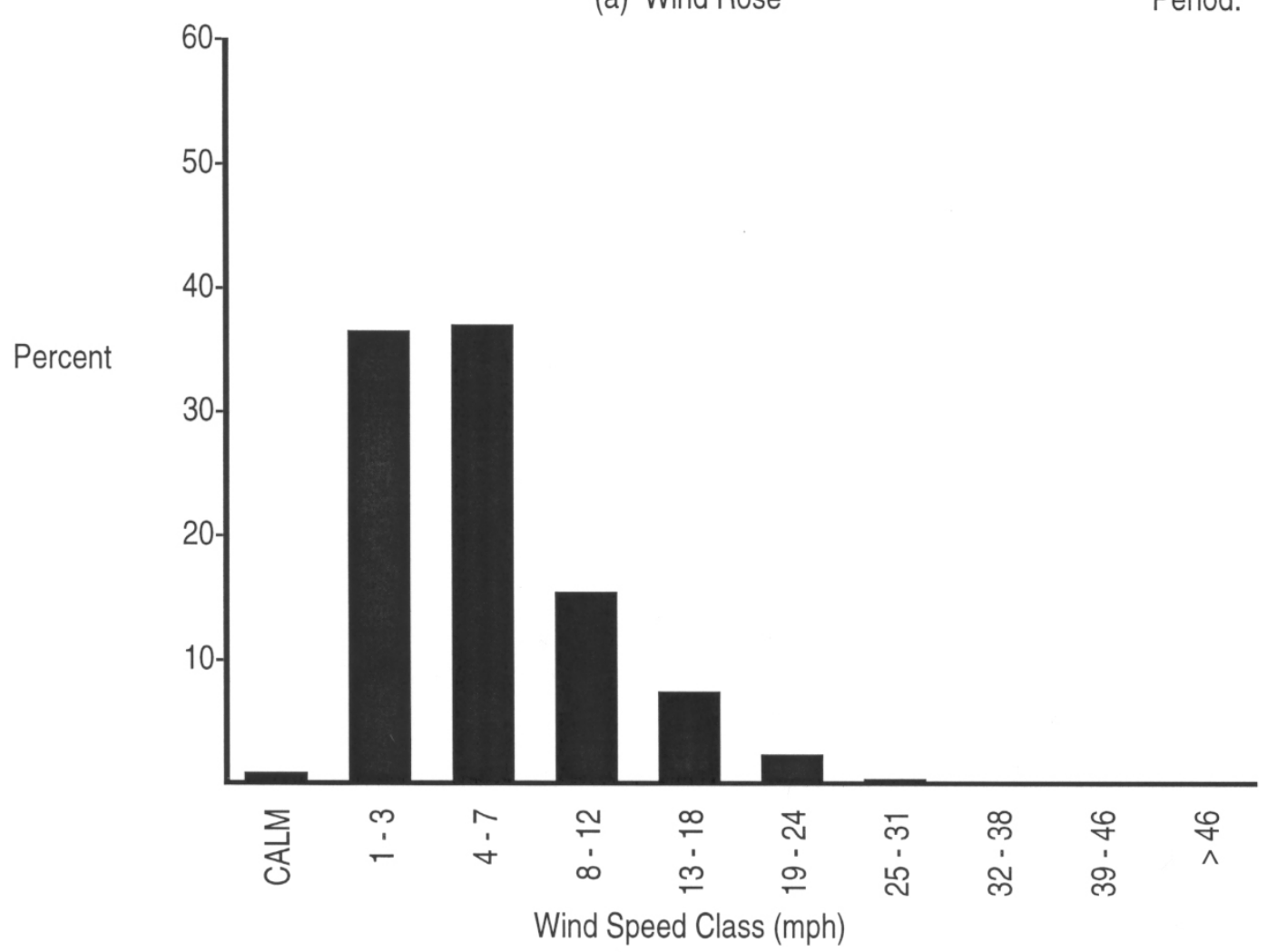

(b) Wind Speed Histogram

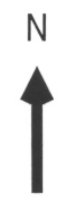

Station \#13-100N

Period: 1/2003 - 12/2003

Figure B.1. (contd) 


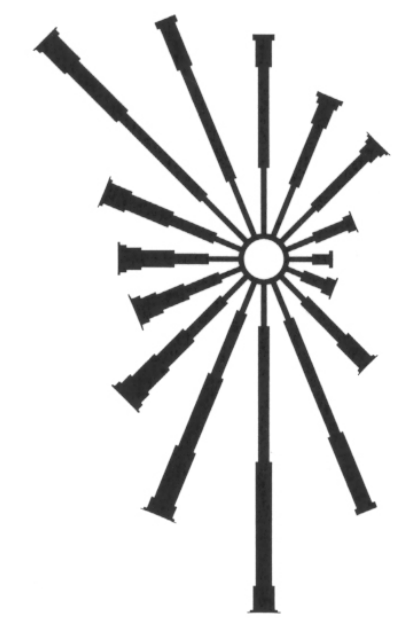

(a) Wind Rose

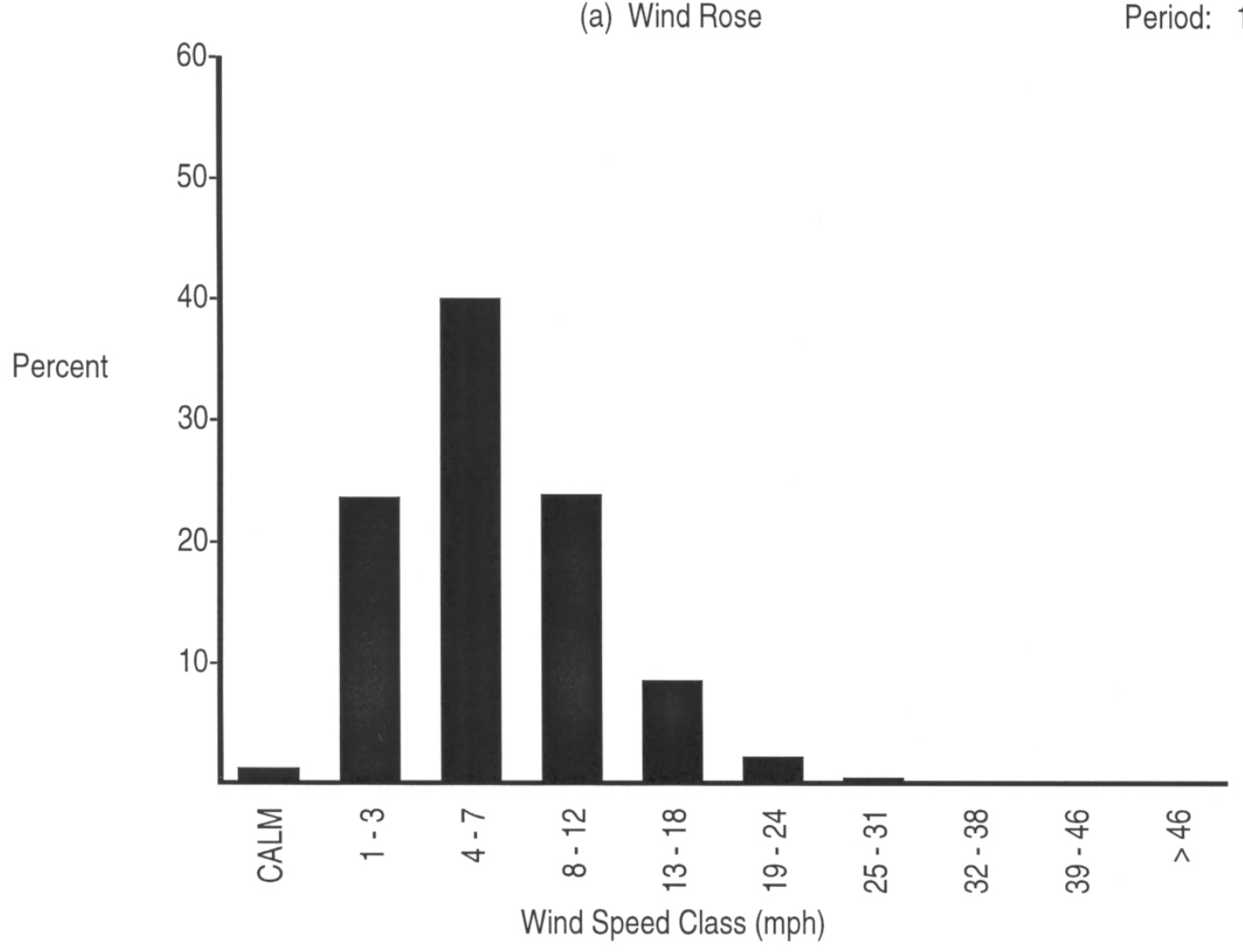

(b) Wind Speed Histogram

Figure B.1. (contd)

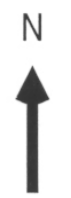

Station \#14 - WPPS

Period: 1/2003 - 12/2003 


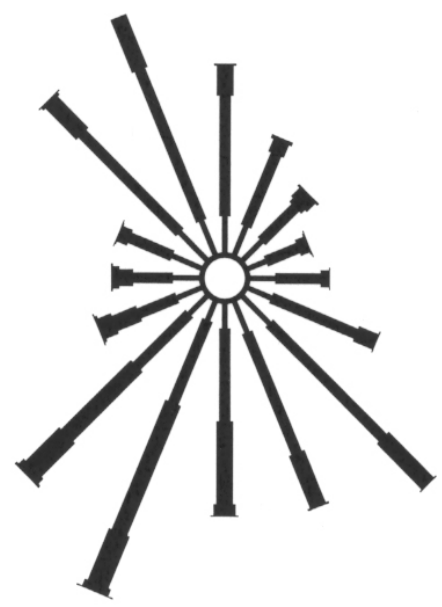

(a) Wind Rose

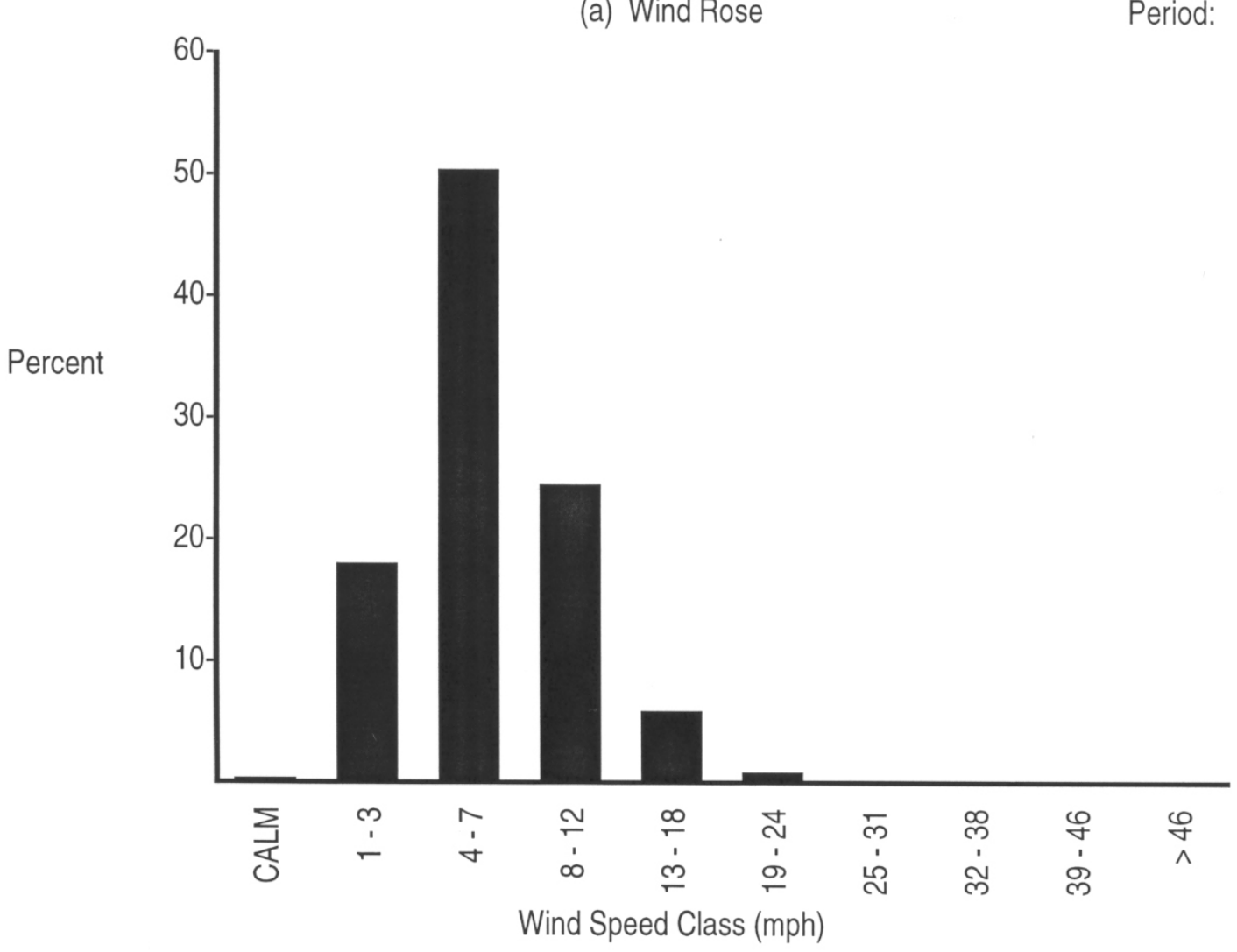

(b) Wind Speed Histogram

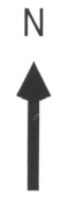

Station \#15 - FRNK

Period: 1/2003 - 12/2003

Figure B.1. (contd) 

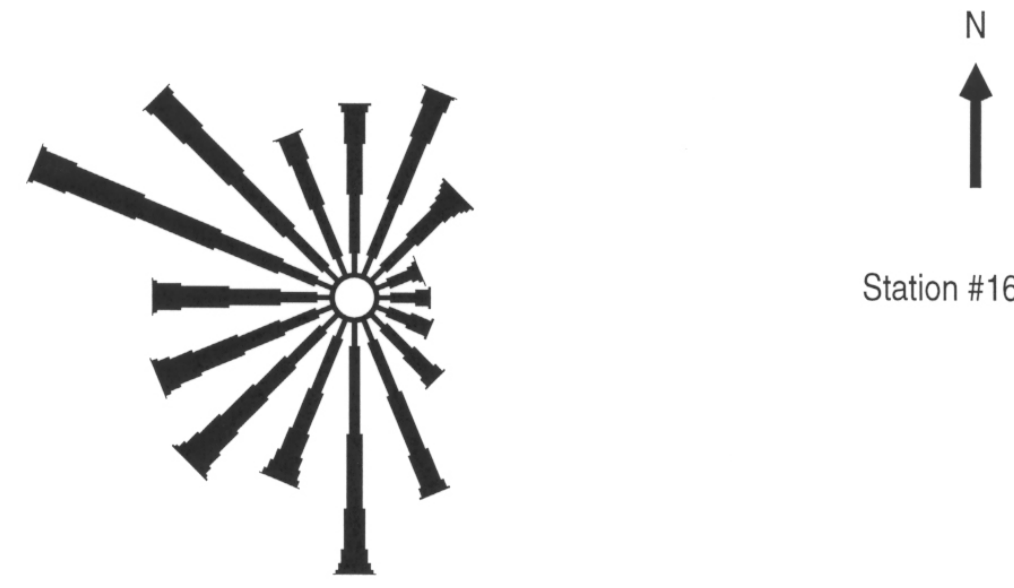

Station \#16 - GABL

(a) Wind Rose

Period: $1 / 2003-12 / 2003$

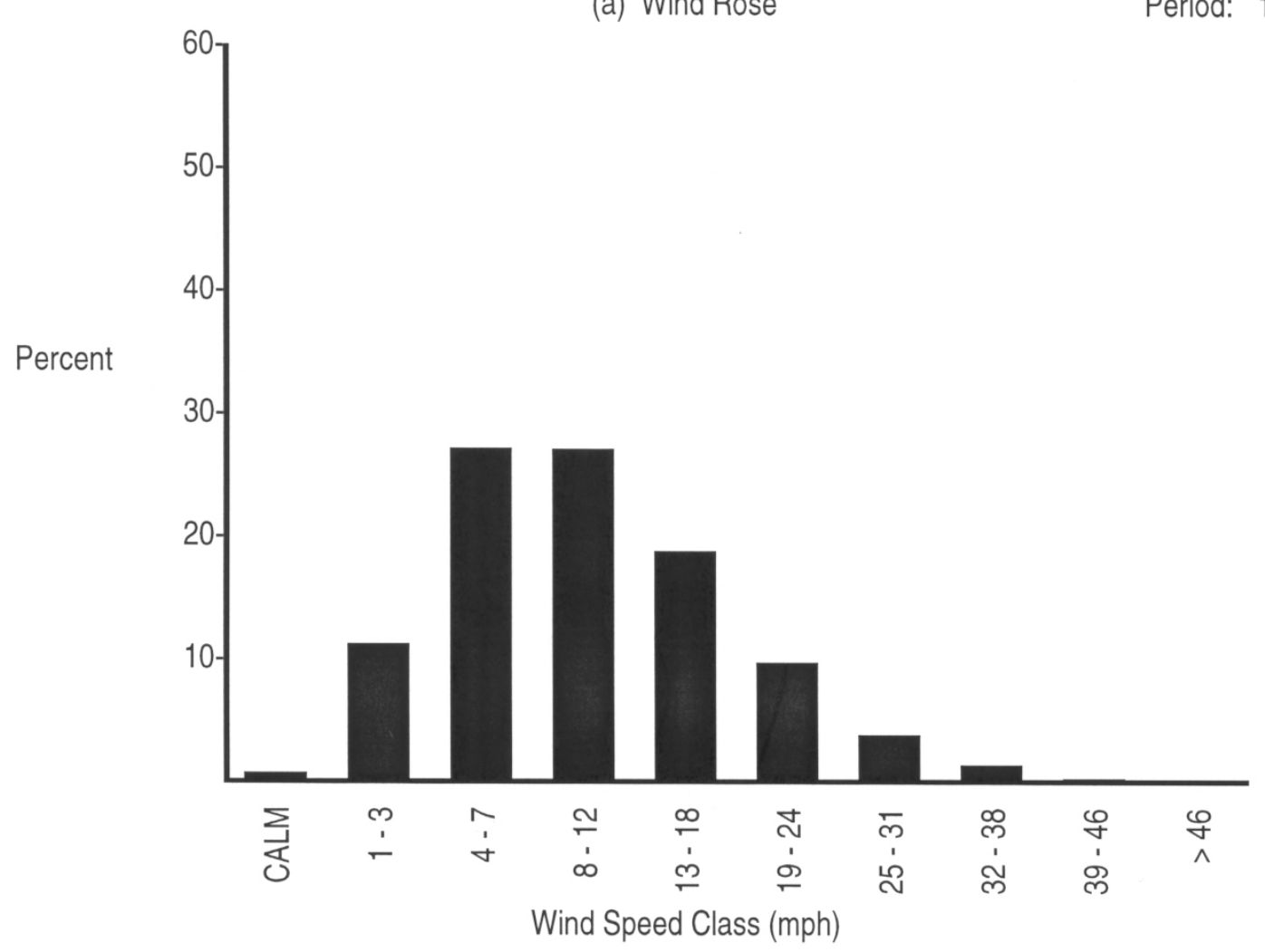

(b) Wind Speed Histogram

Figure B.1. (contd) 

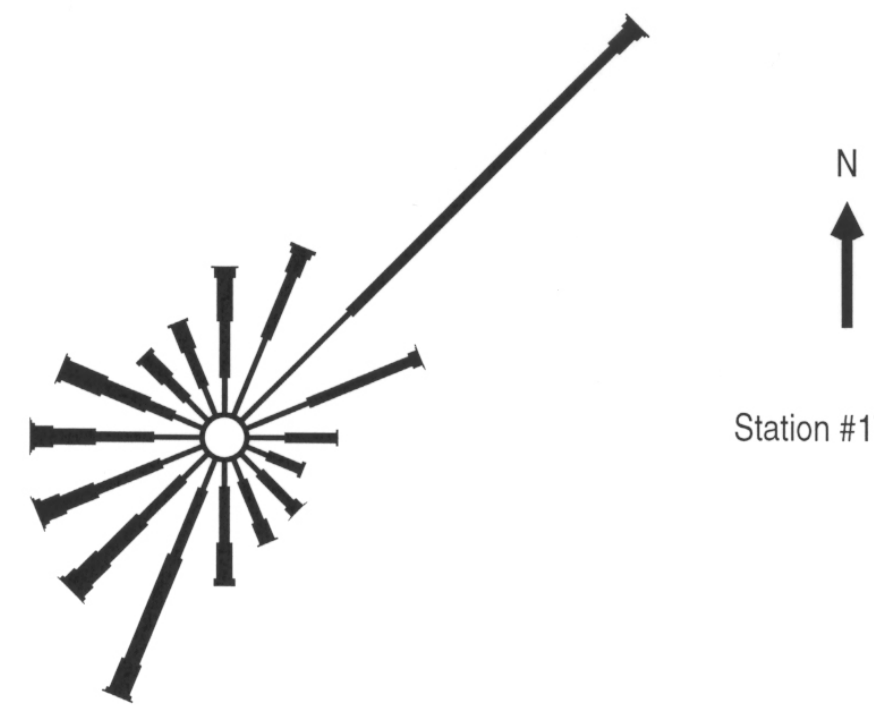

Station \#17 - RING

(a) Wind Rose

Period: 1/2003 - 12/2003

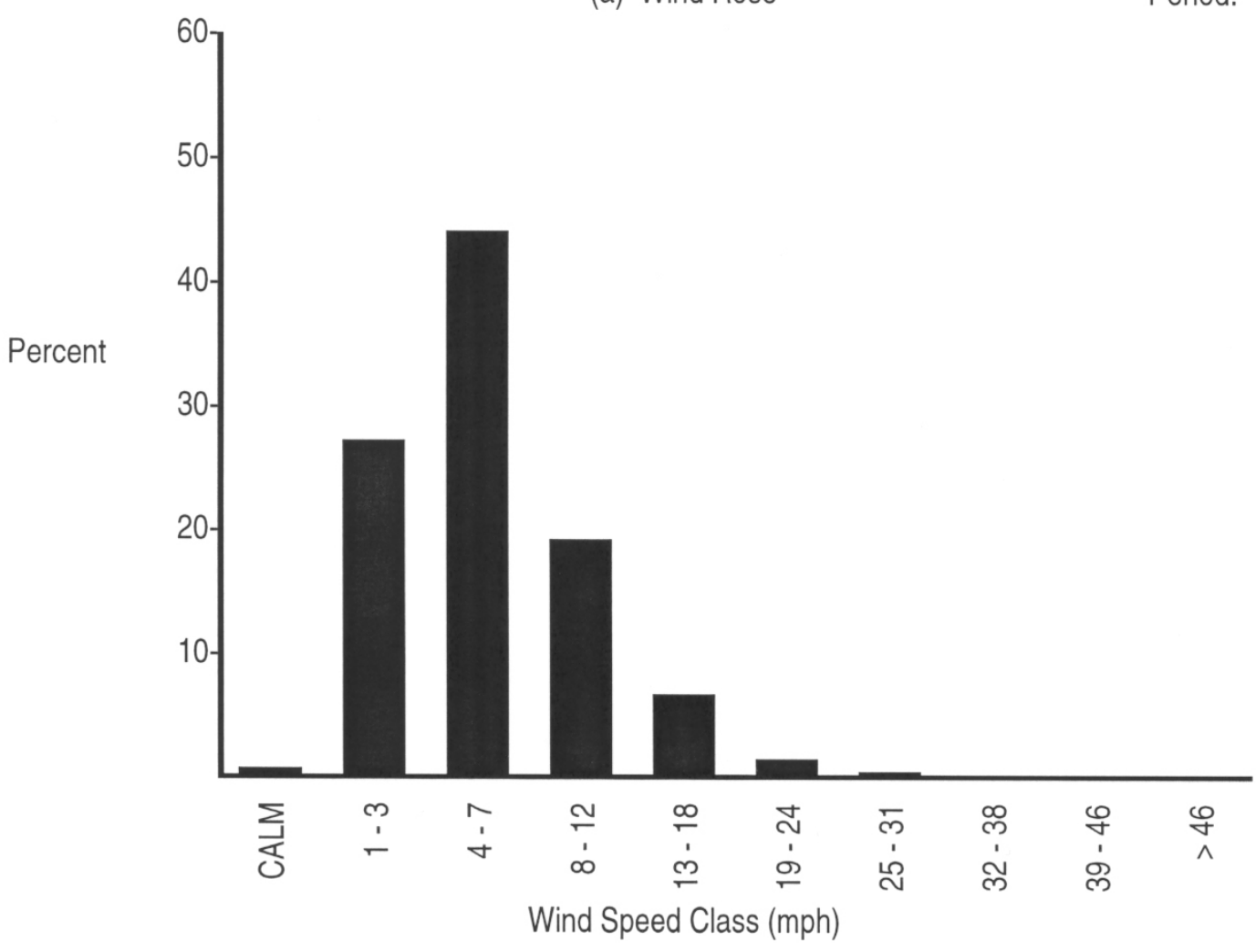

(b) Wind Speed Histogram

Figure B.1. (contd)

B. 18 


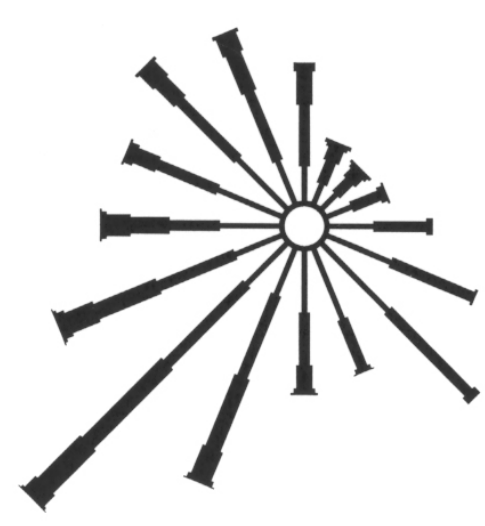

(a) Wind Rose

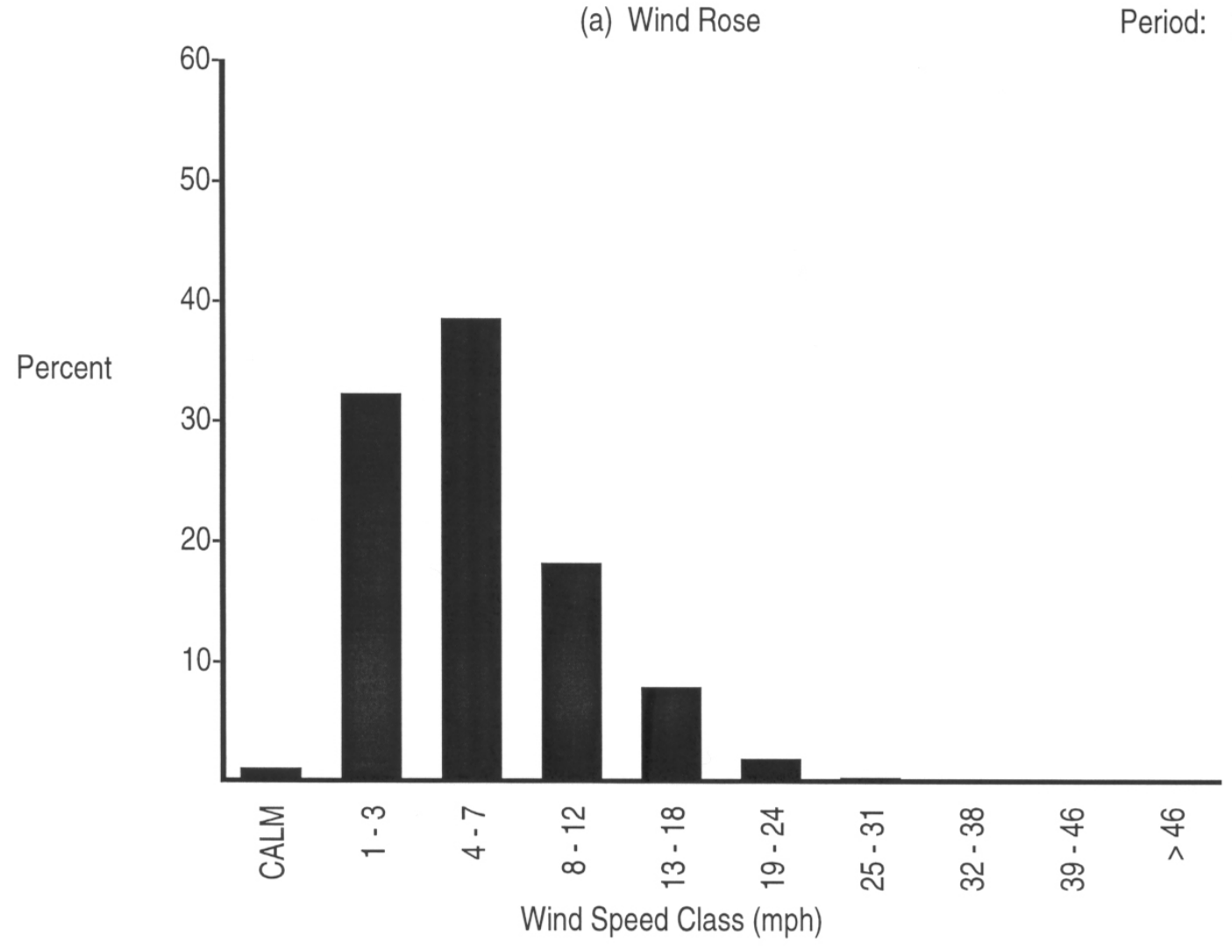

(b) Wind Speed Histogram

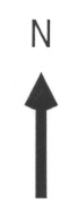

Station \#18 - RICH

Period: 1/2003 - 12/2003

Figure B.1. (contd) 


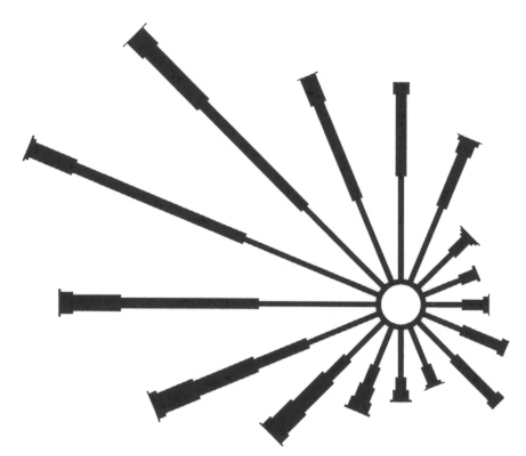

(a) Wind Rose

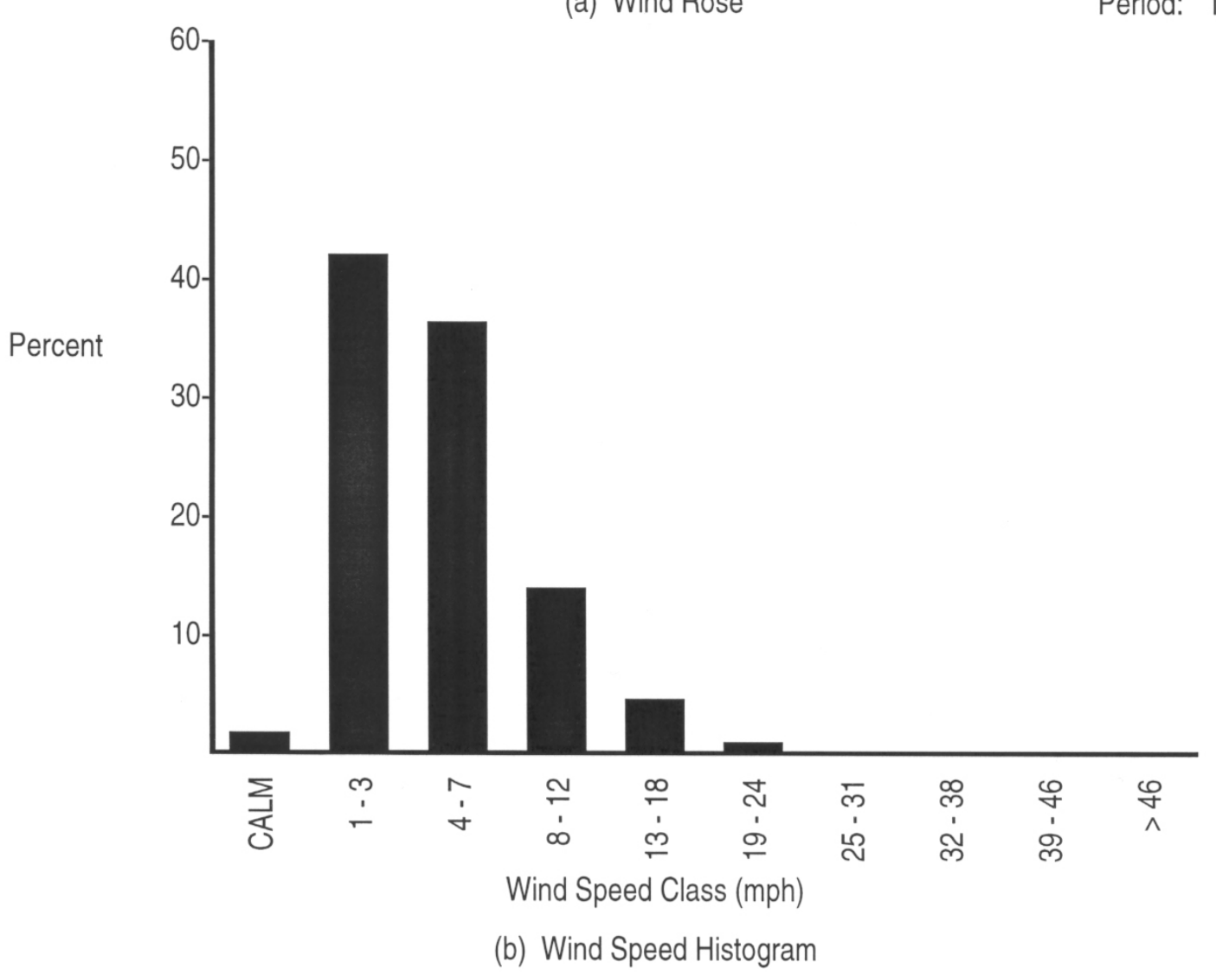

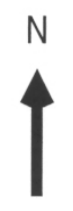

Station \#19 - PFP

Period: 1/2003 - 12/2003

Figure B.1. (contd) 


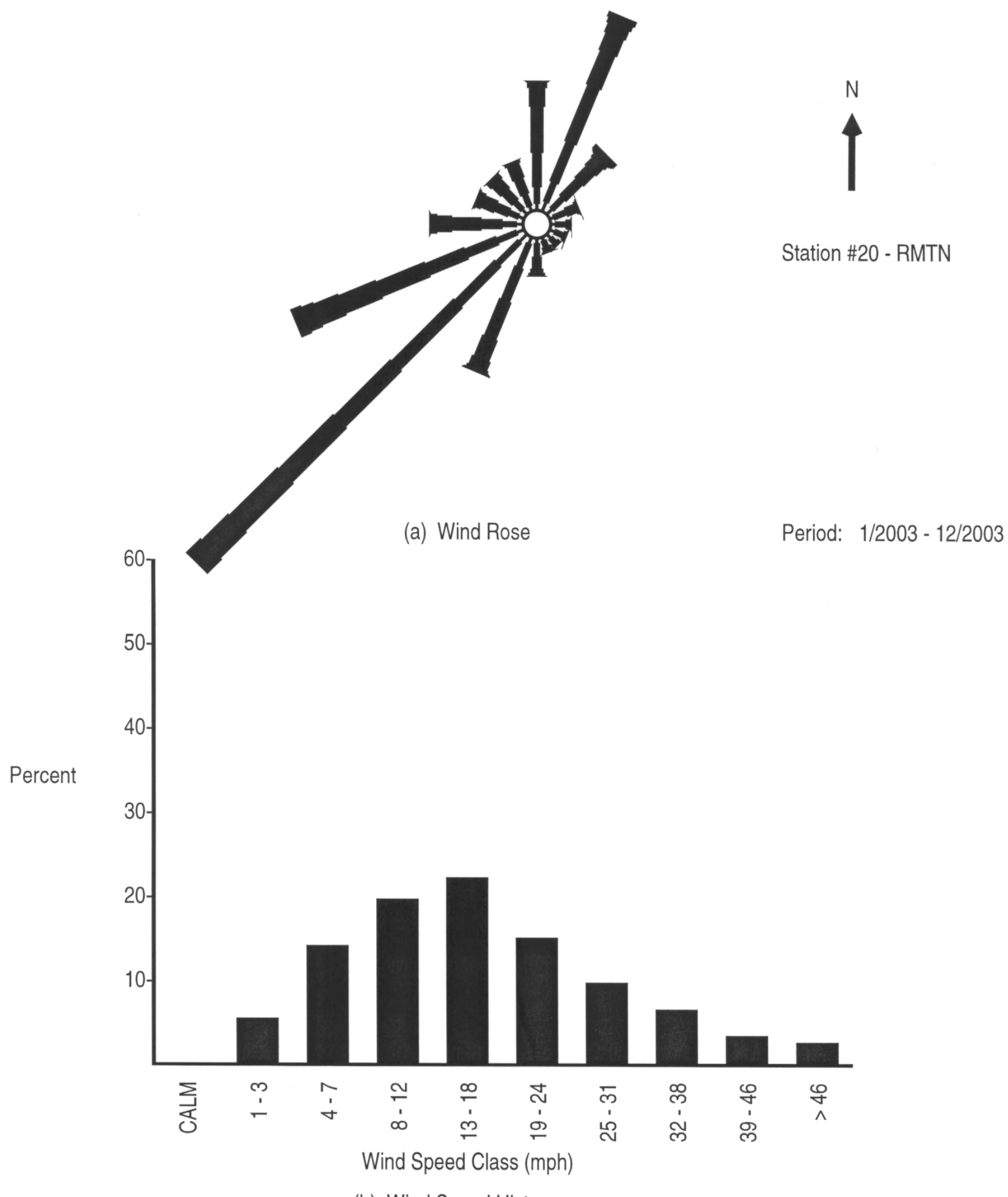

(b) Wind Speed Histogram

Figure B.1. (contd) 


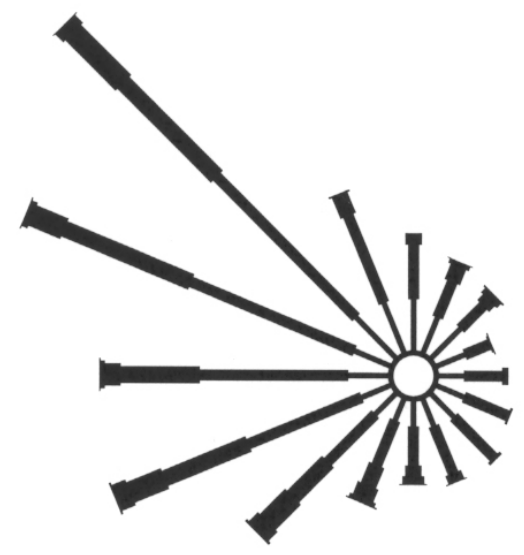

(a) Wind Rose

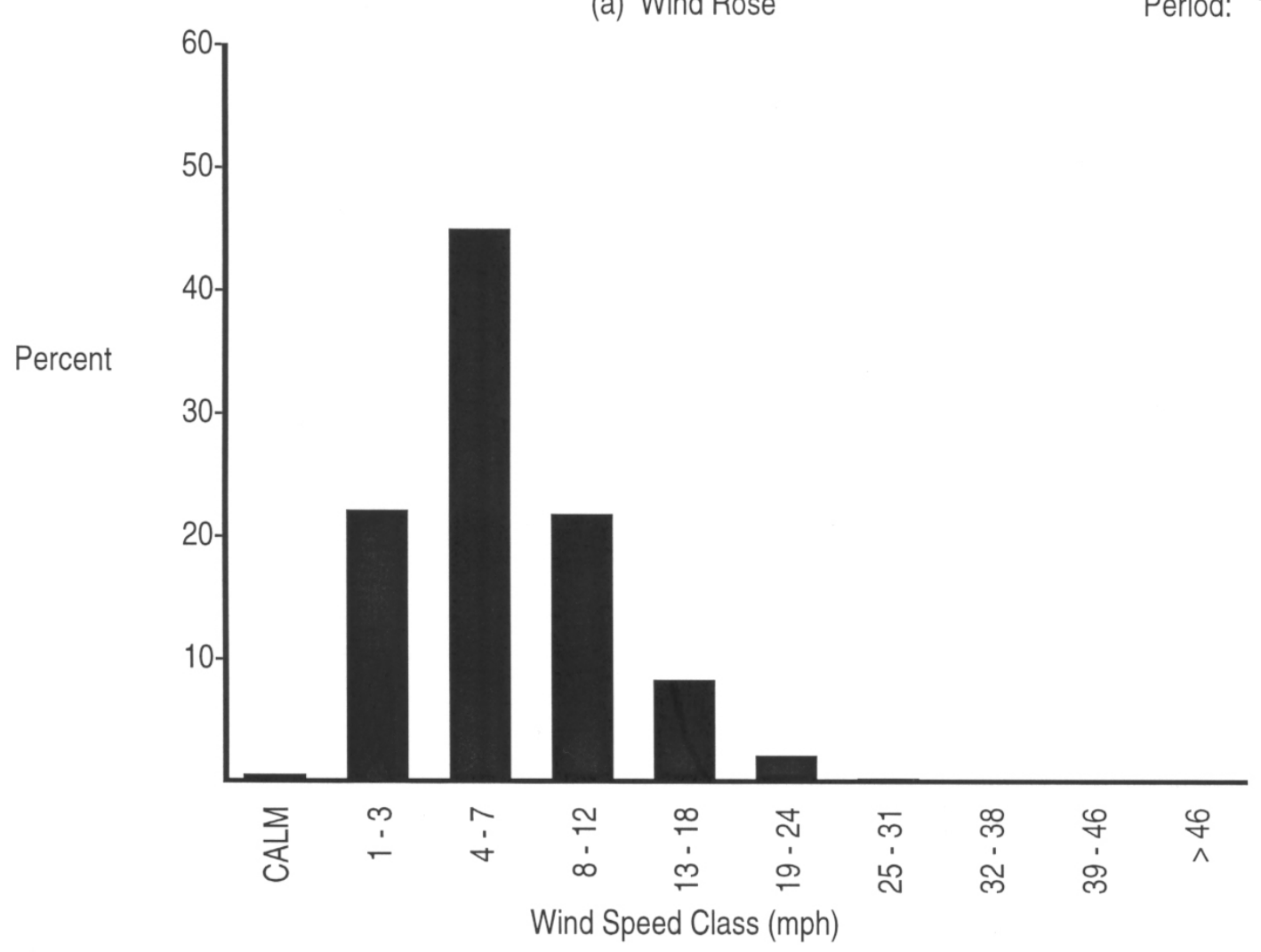

(b) Wind Speed Histogram

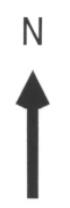

Station \#21 - HMS

Period: 1/2003 - 12/2003

Figure B.1. (contd) 


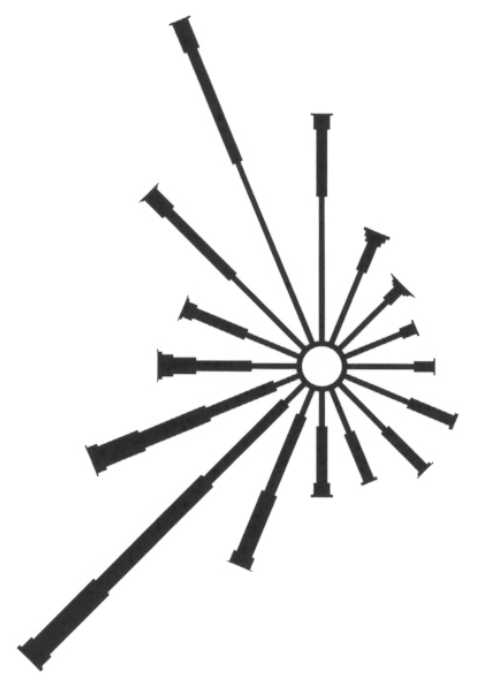

(a) Wind Rose

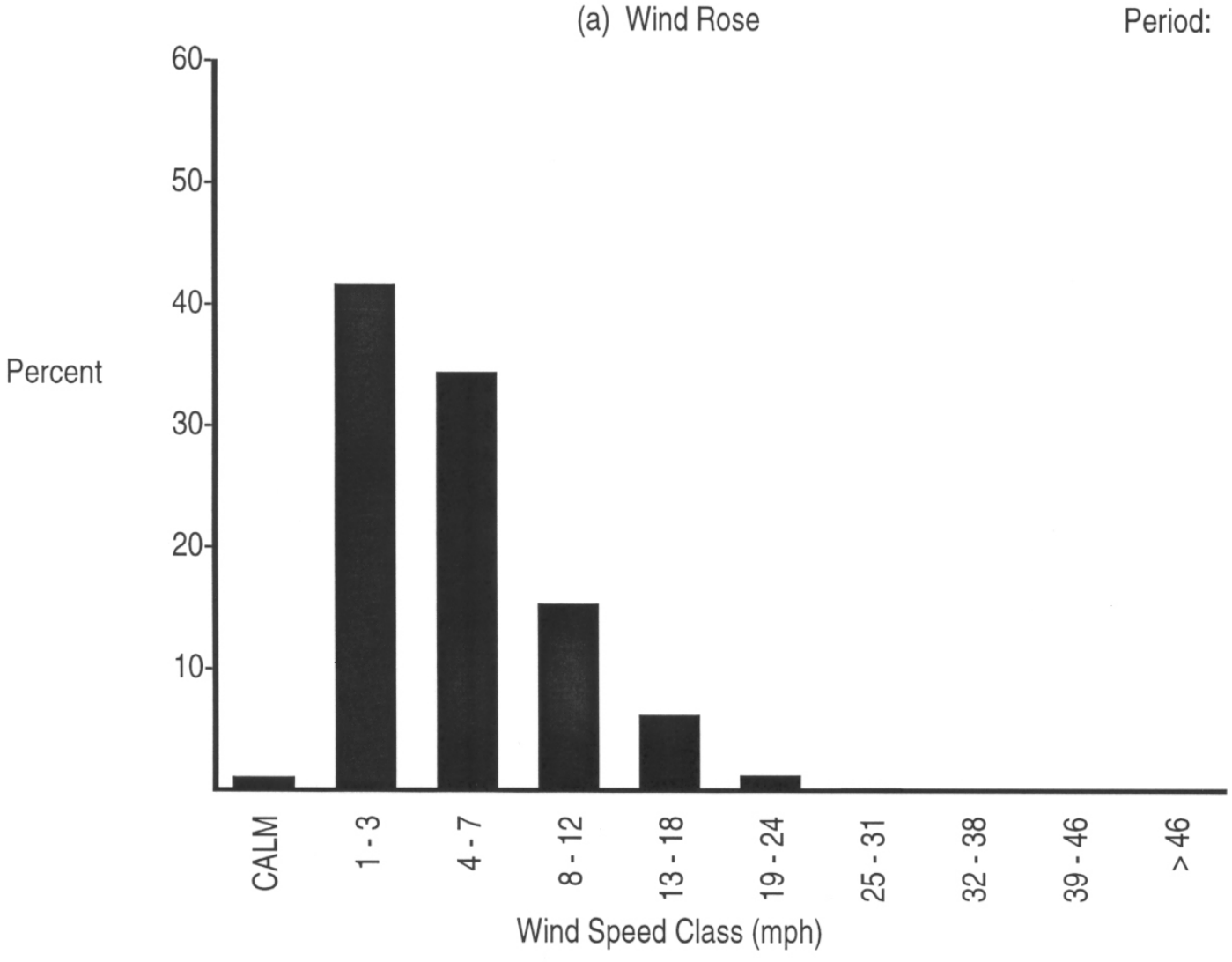

(b) Wind Speed Histogram

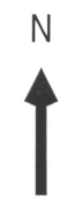

Station \#22 - PASC

Period: 1/2003 - 12/2003

Figure B.1. (contd) 


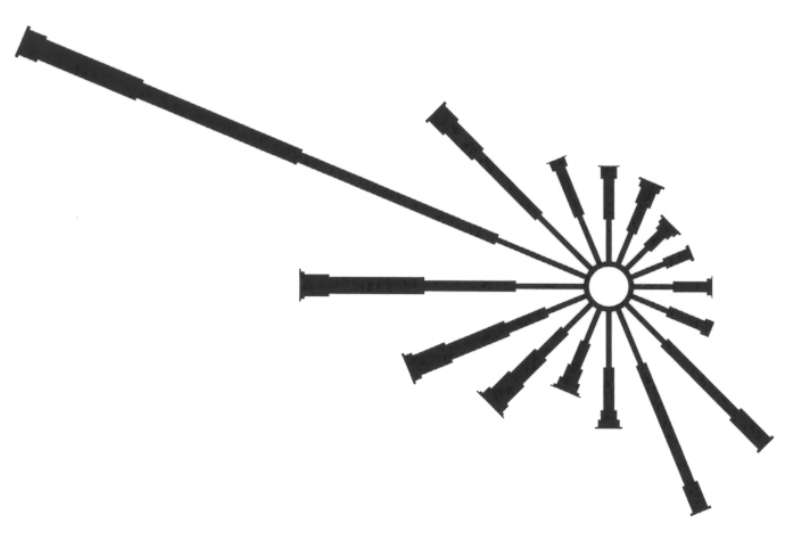

(a) Wind Rose

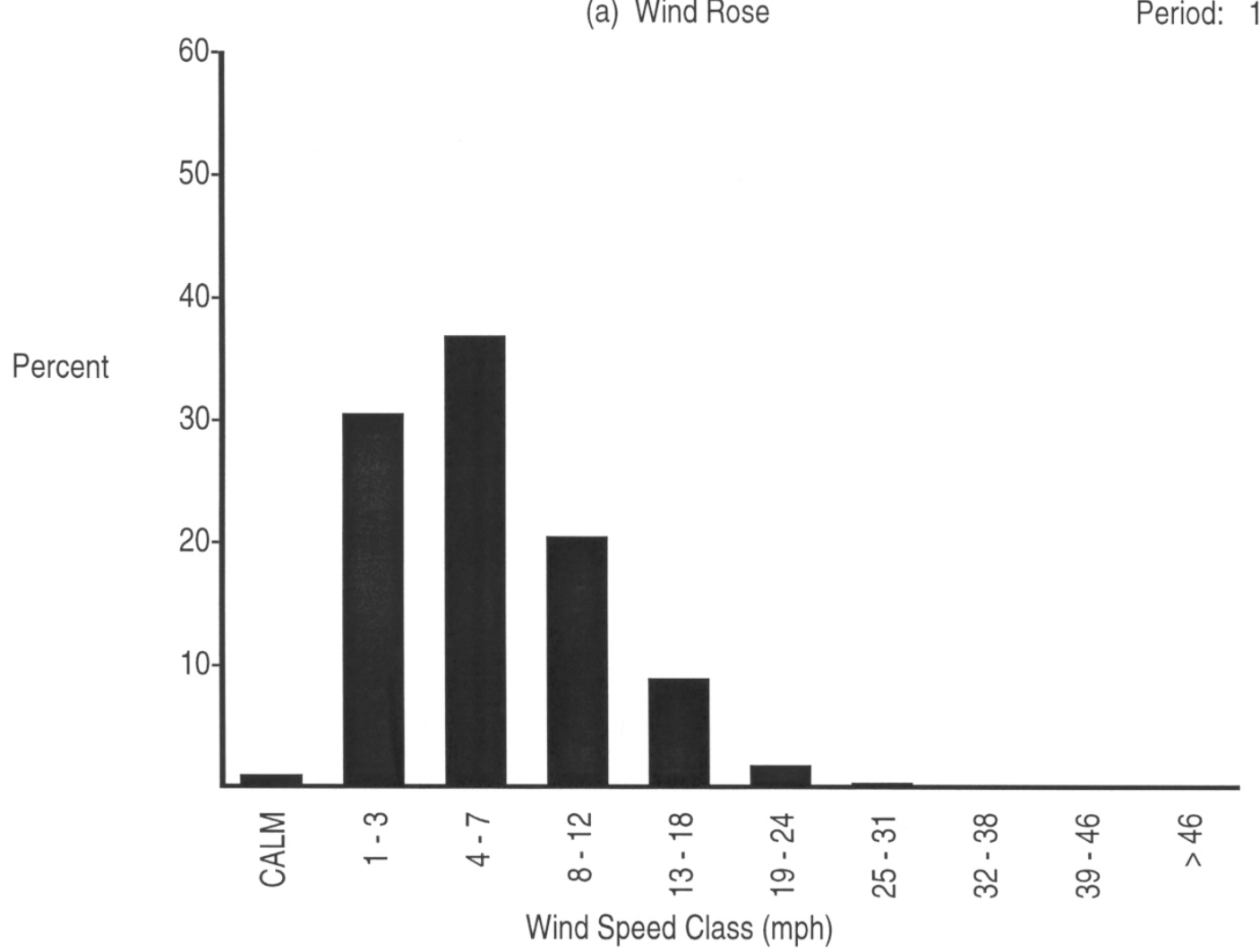

(b) Wind Speed Histogram

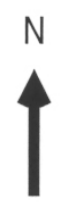

Station \#23 - GABW

Period: 1/2003 - 12/2003

Figure B.1. (contd) 


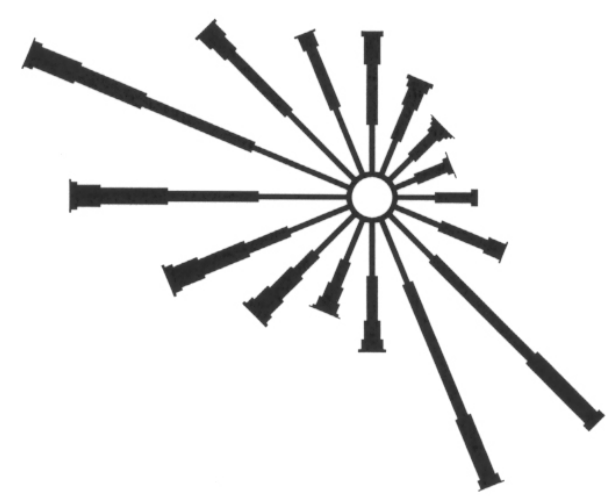

(a) Wind Rose

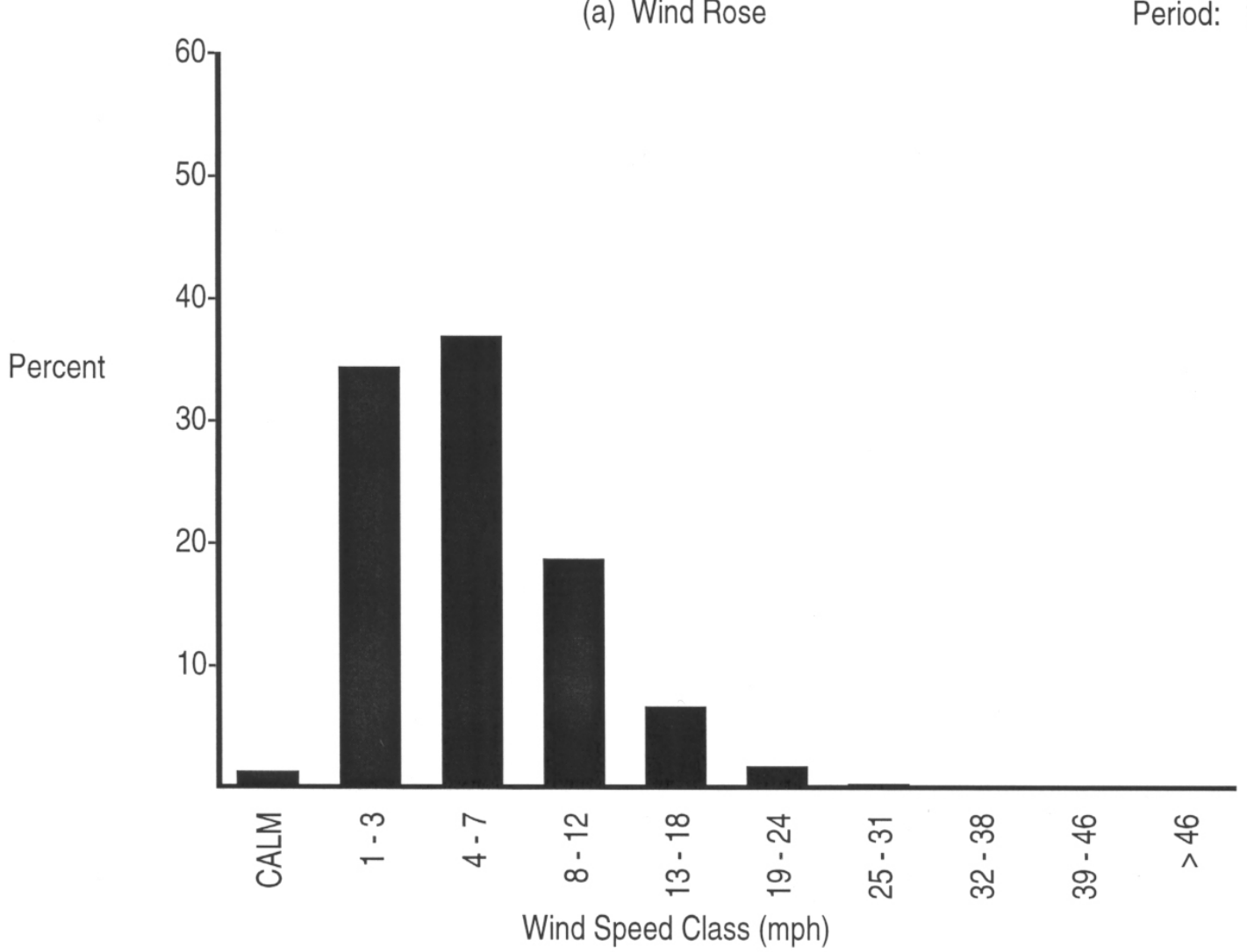

(b) Wind Speed Histogram

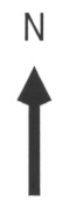

Station \#24 - 100F

Period: 1/2003-12/2003

Figure B.1. (contd) 


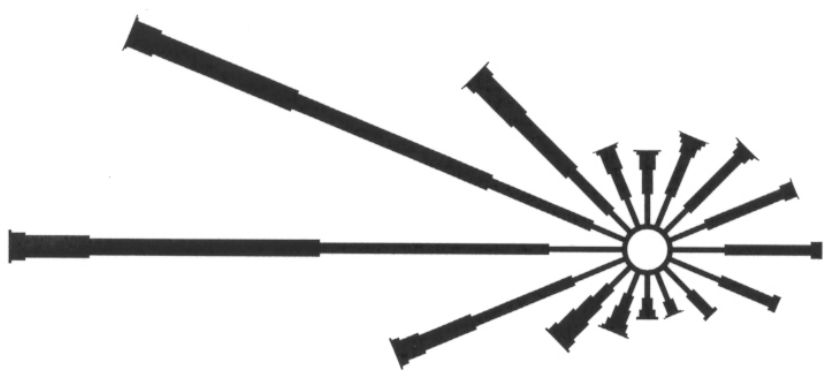

(a) Wind Rose

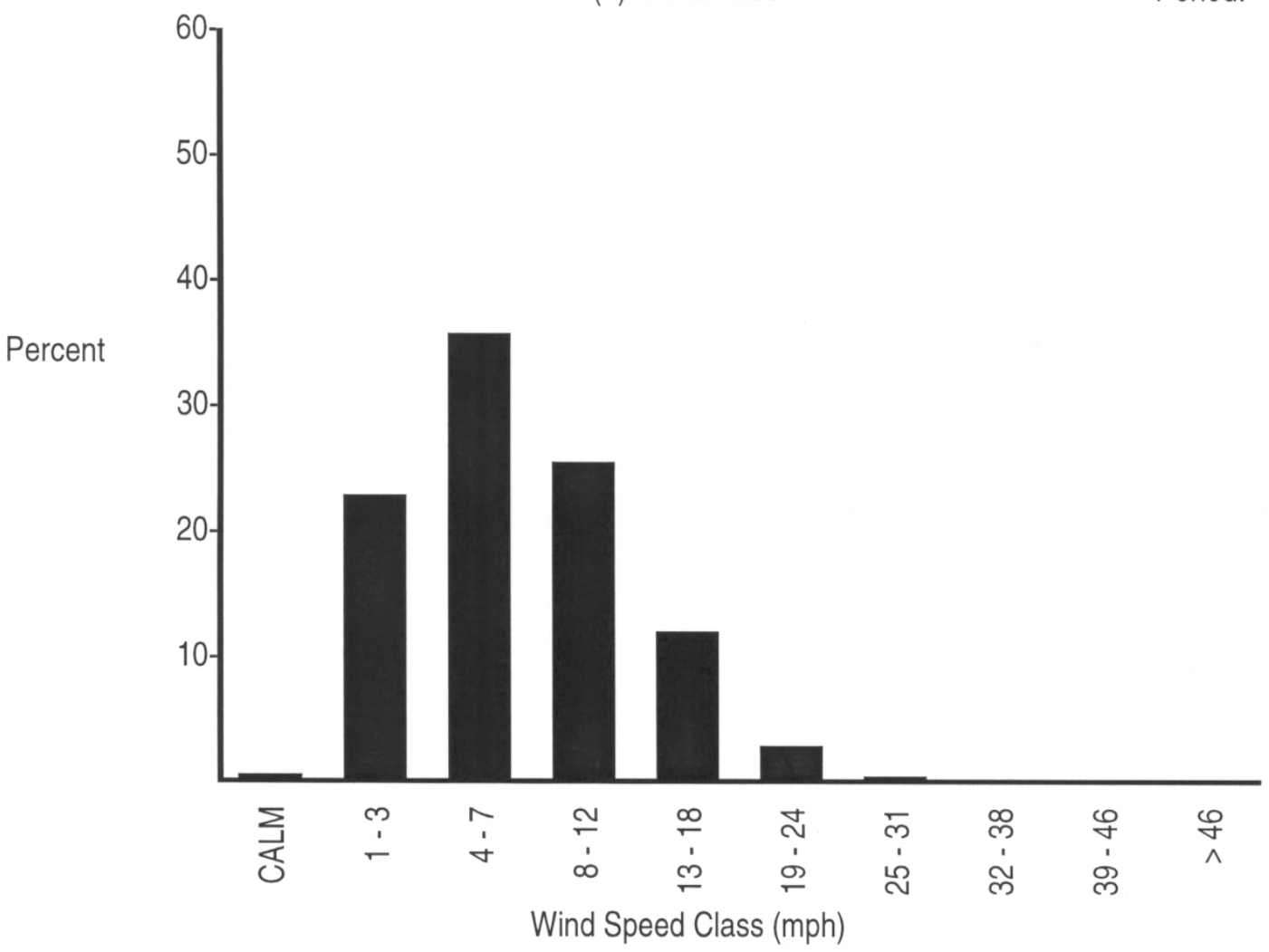

(b) Wind Speed Histogram

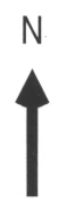

Station \#25 - VERN

Period: $1 / 2003-12 / 2003$

Figure B.1. (contd) 


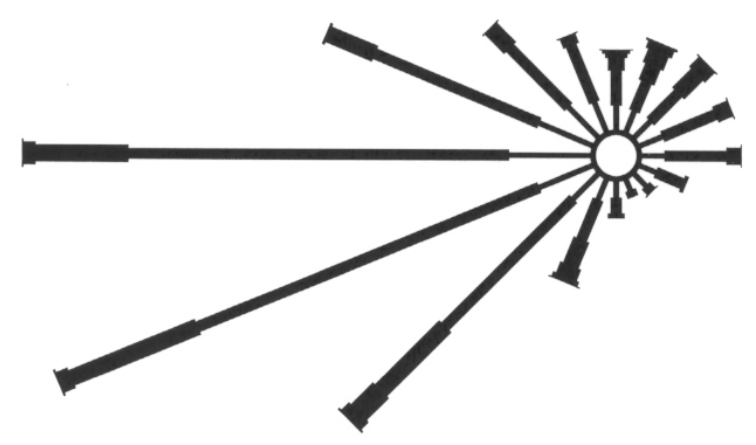

(a) Wind Rose

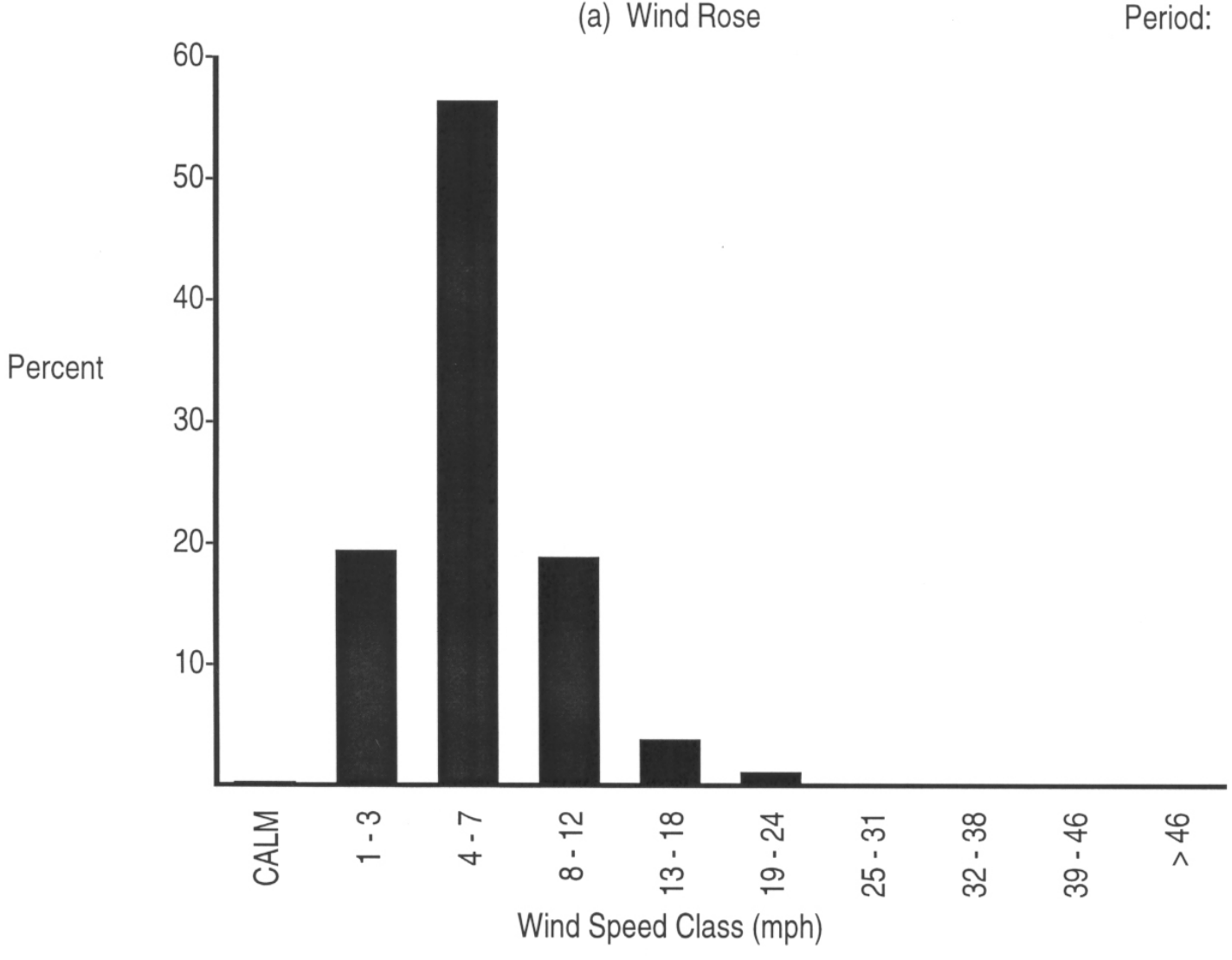

(b) Wind Speed Histogram

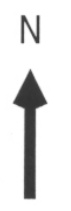

Station \#26 - BENT

Period: 1/2003 - 12/2003

Figure B.1. (contd) 


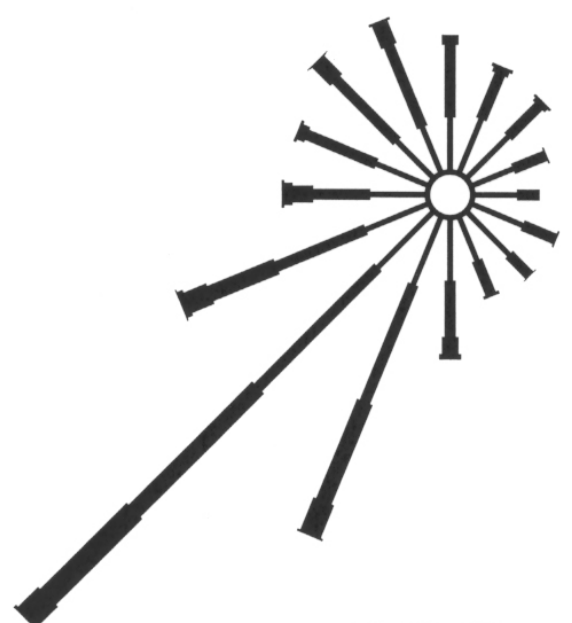

(a) Wind Rose

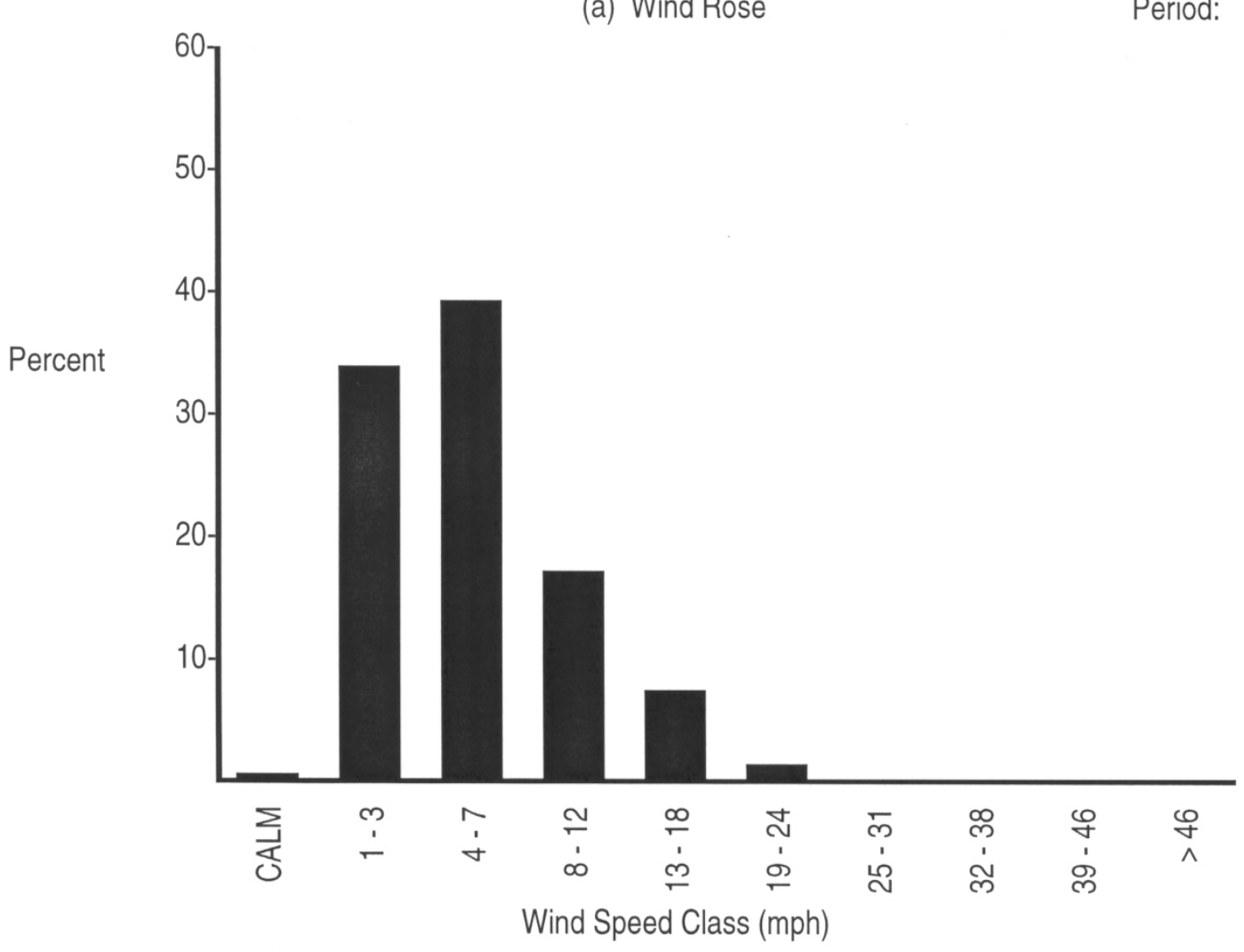

(b) Wind Speed Histogram

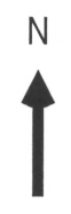

Station \#27 - VSTA

Period: 1/2003 - 12/2003

Figure B.1. (contd) 

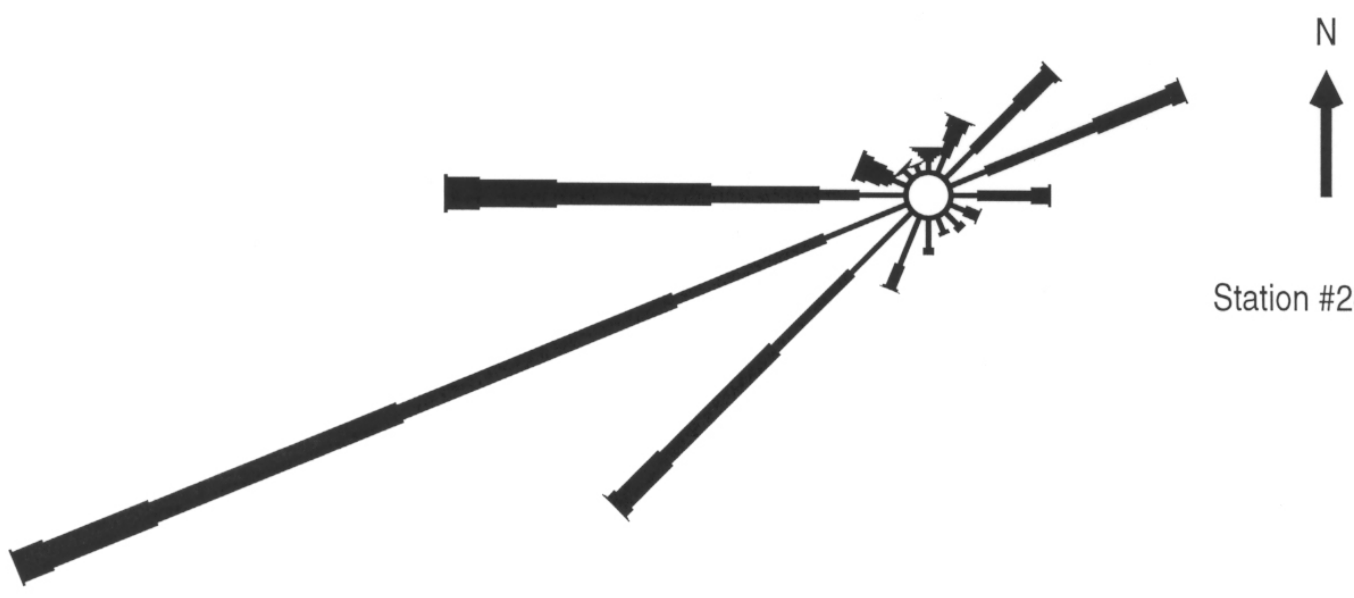

Station \#28 - SURF

(a) Wind Rose

Period: 1/2003-12/2003

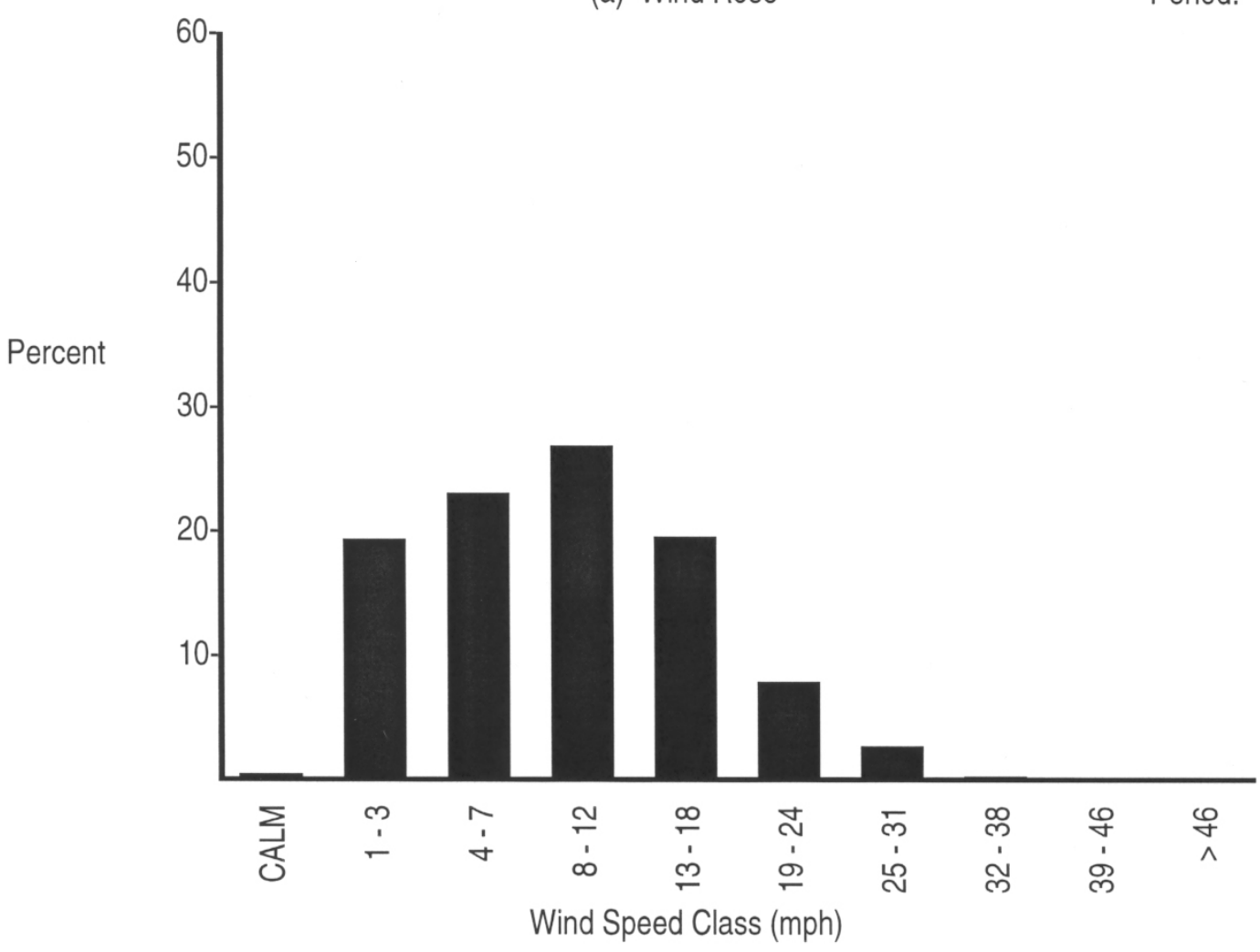

(b) Wind Speed Histogram

Figure B.1. (contd) 


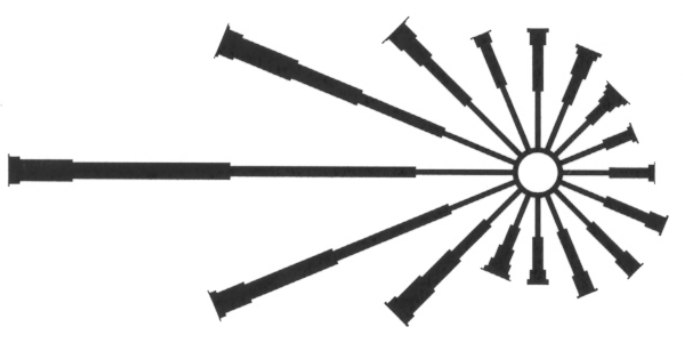

(a) Wind Rose

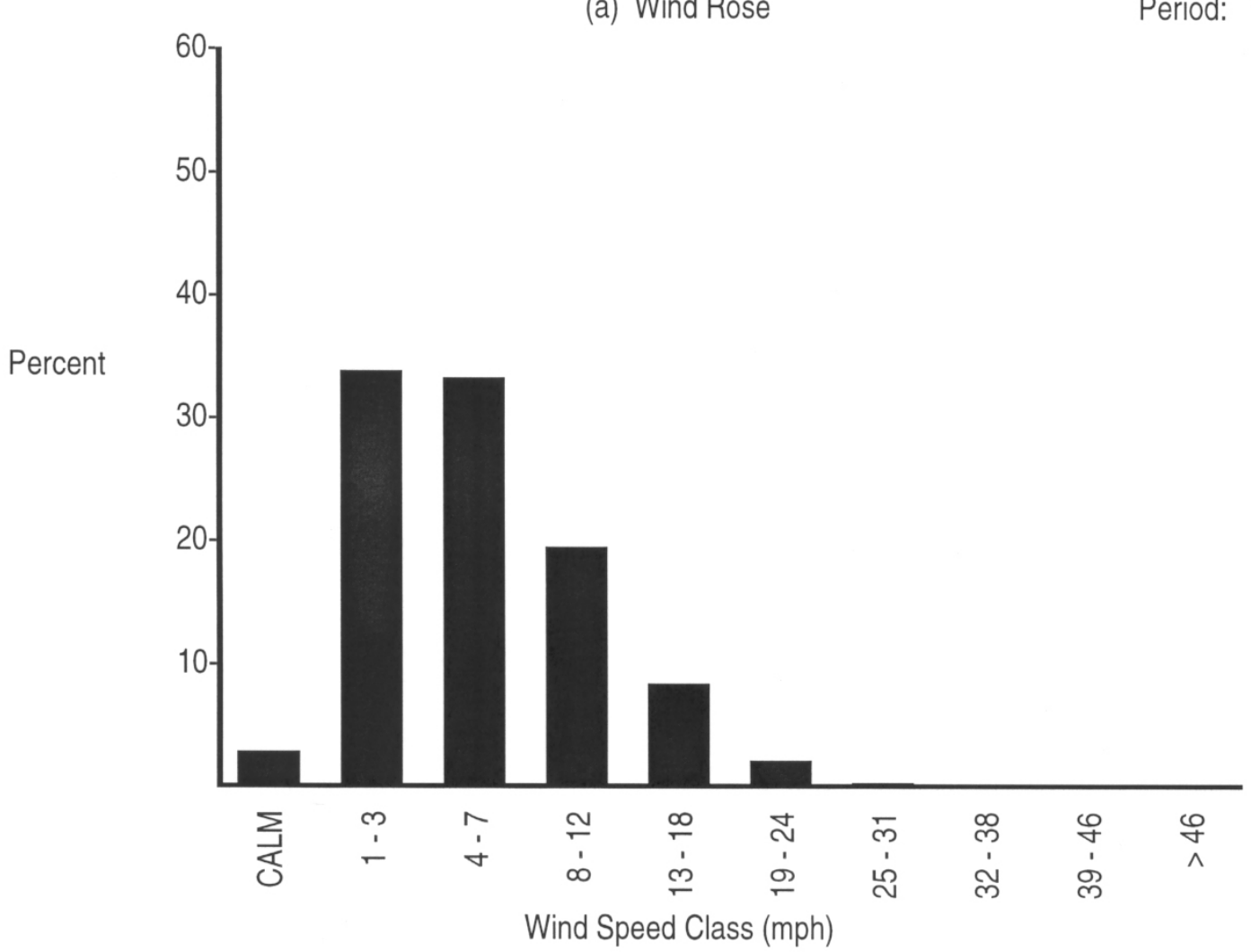

(b) Wind Speed Histogram

Figure B.1. (contd)

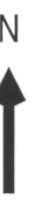

Station \#29 - 100K

Period: 1/2003 - 12/2003 


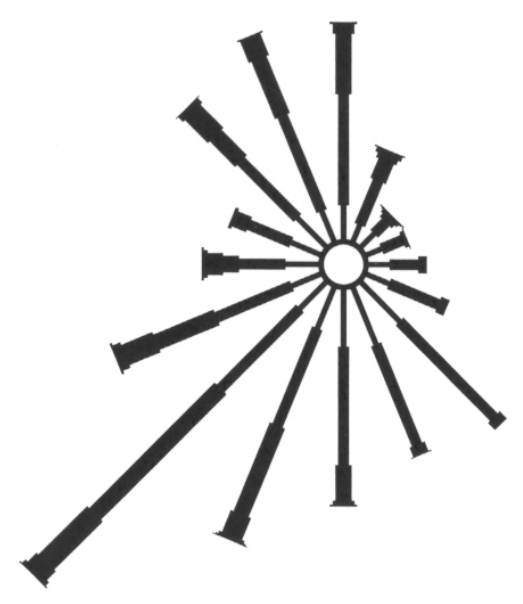

(a) Wind Rose

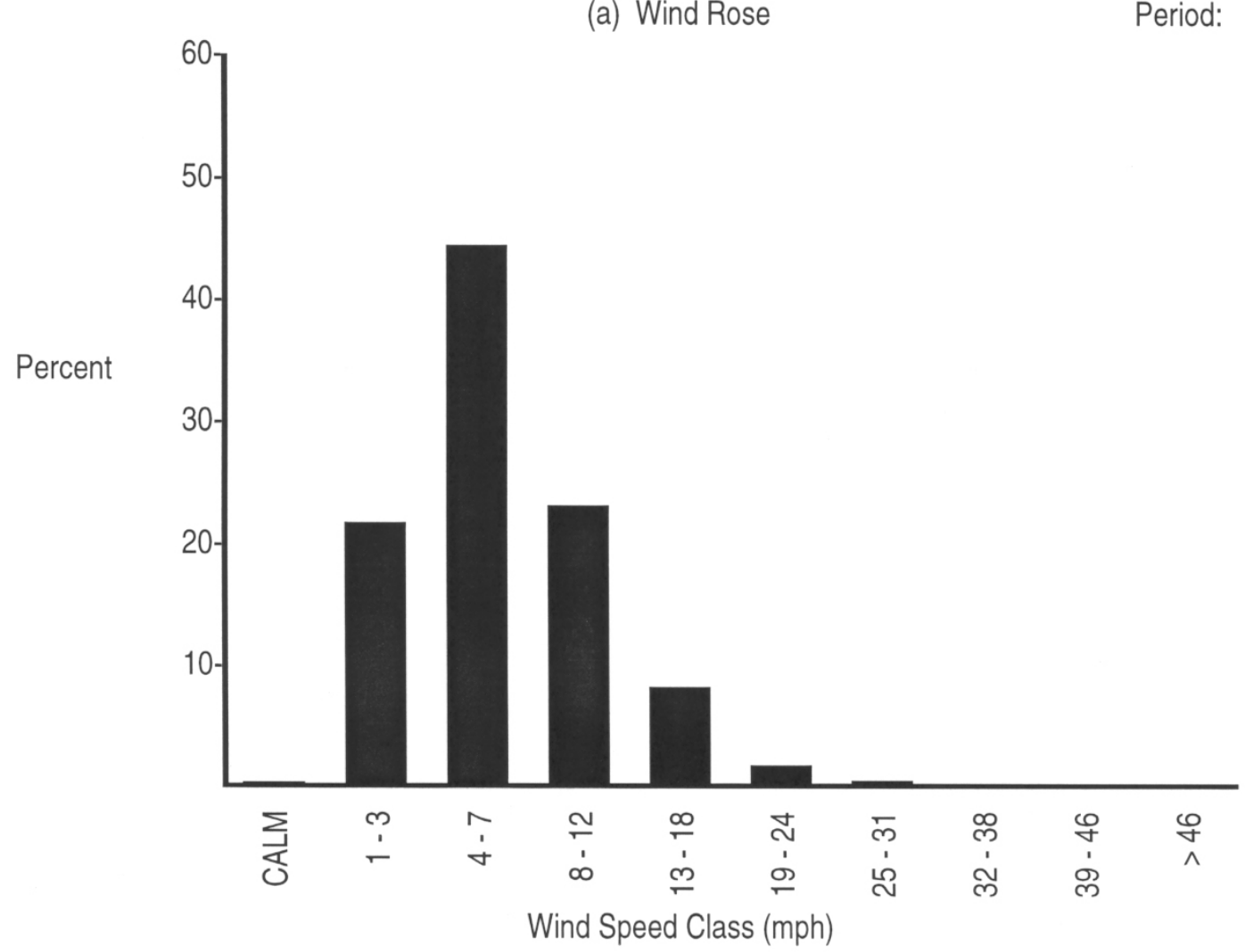

(b) Wind Speed Histogram

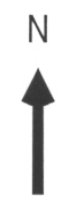

Station \#30 - HAMR

Period: 1/2003 - 12/2003

Figure B.1. (contd) 


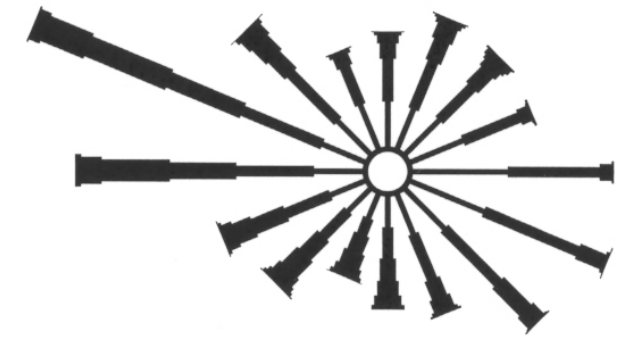

(a) Wind Rose

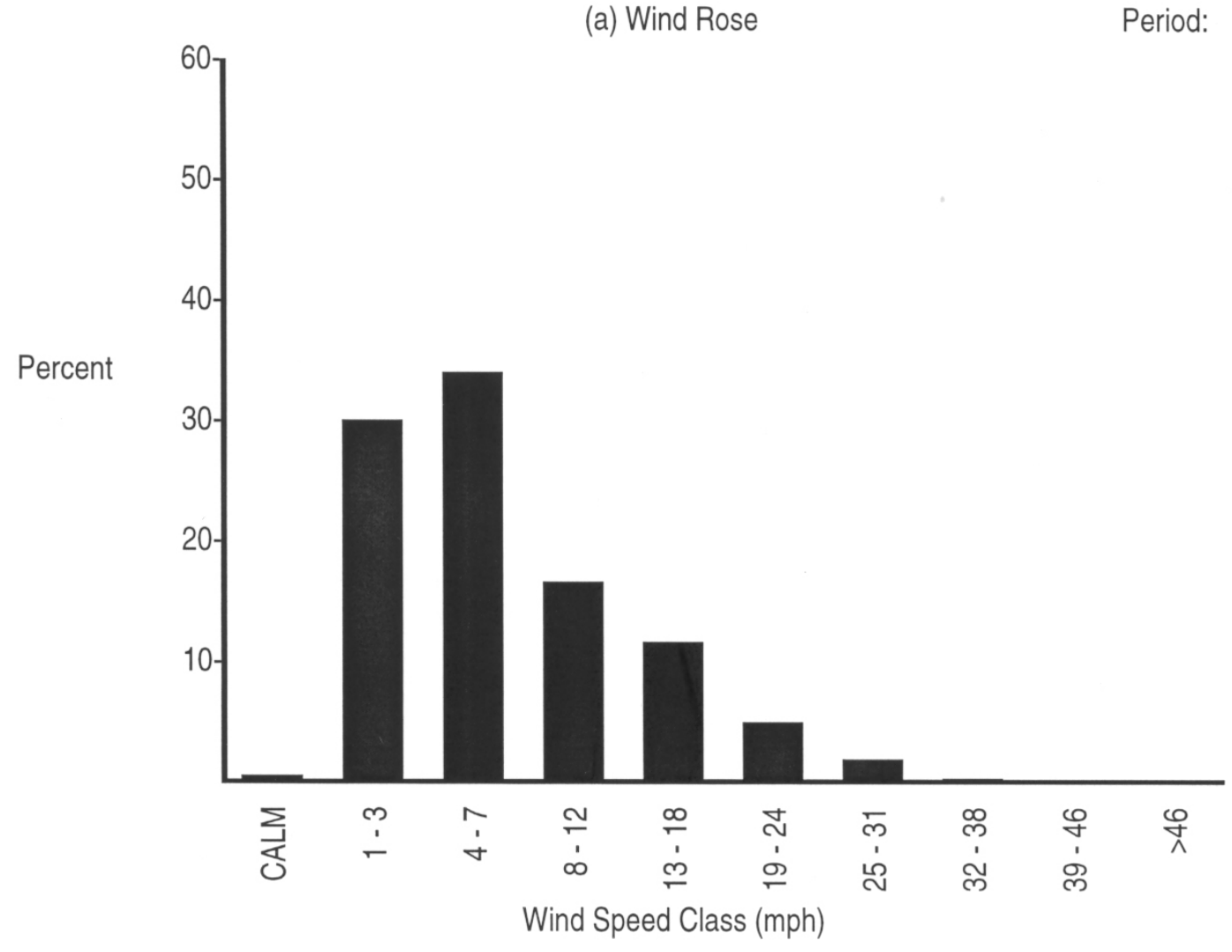

(b) Wind Speed Histogram

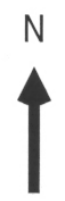

100 Area Tower

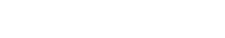

Period: $1 / 2003-12 / 2003$

Figure B.2. Wind Rose and Wind Speed Histogram, 60 meters 

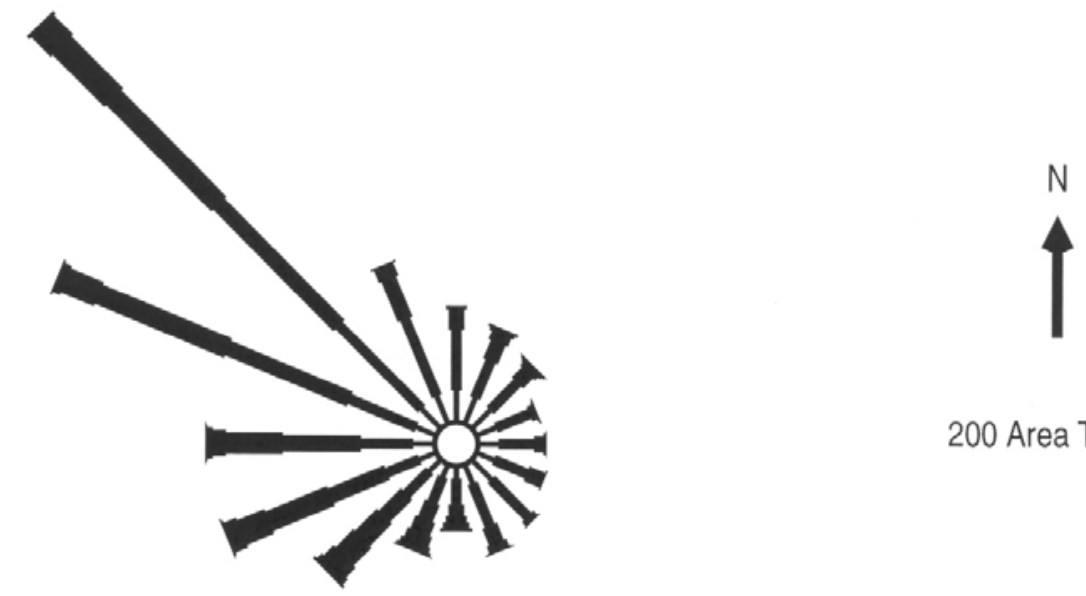

200 Area Tower

(a) Wind Rose

Period: 1/2003-12/2003

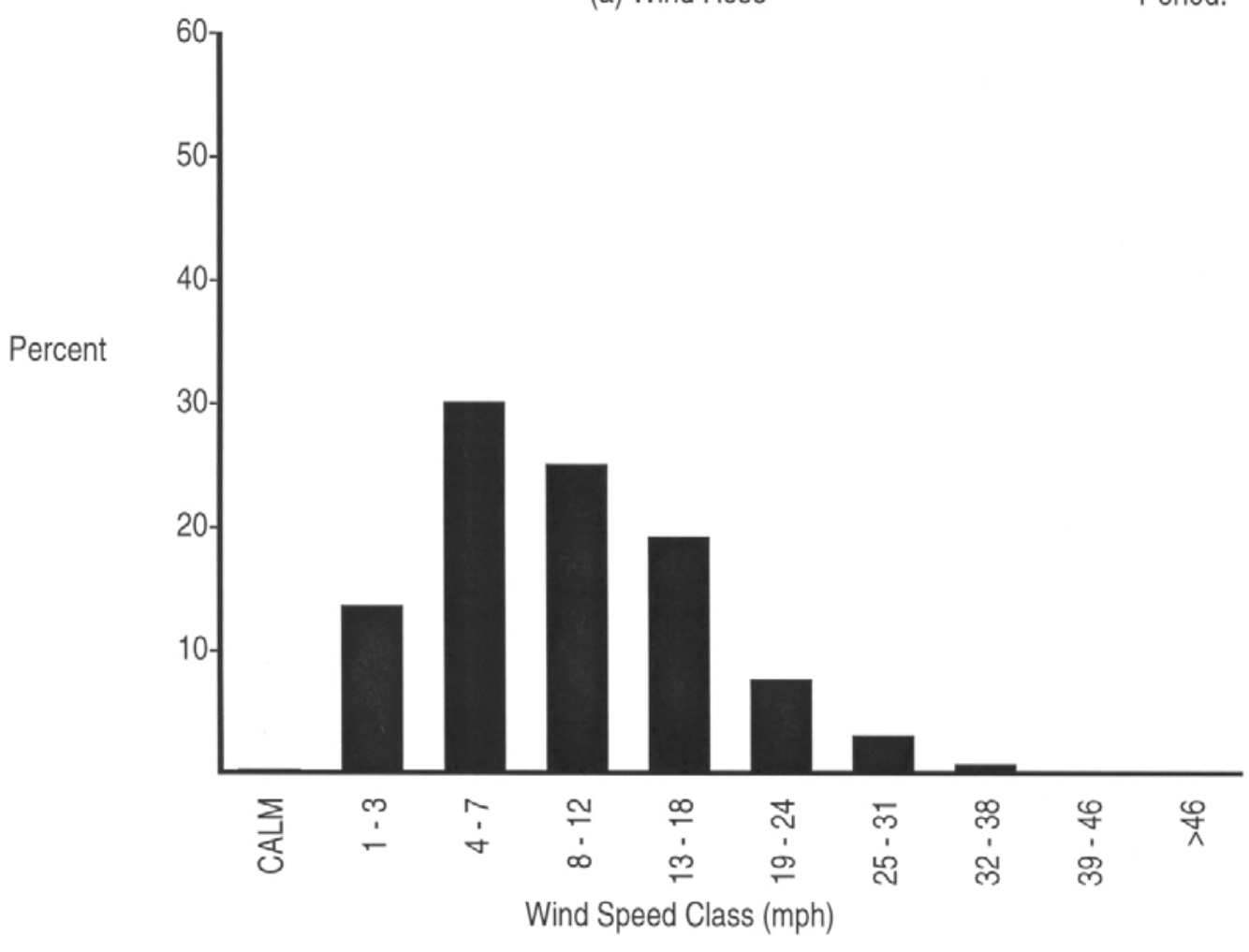

(b) Wind Speed Histogram

Figure B.2. (contd) 


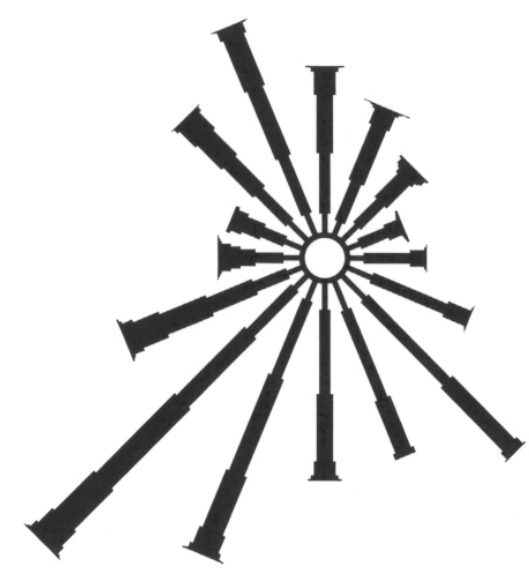

(a) Wind Rose

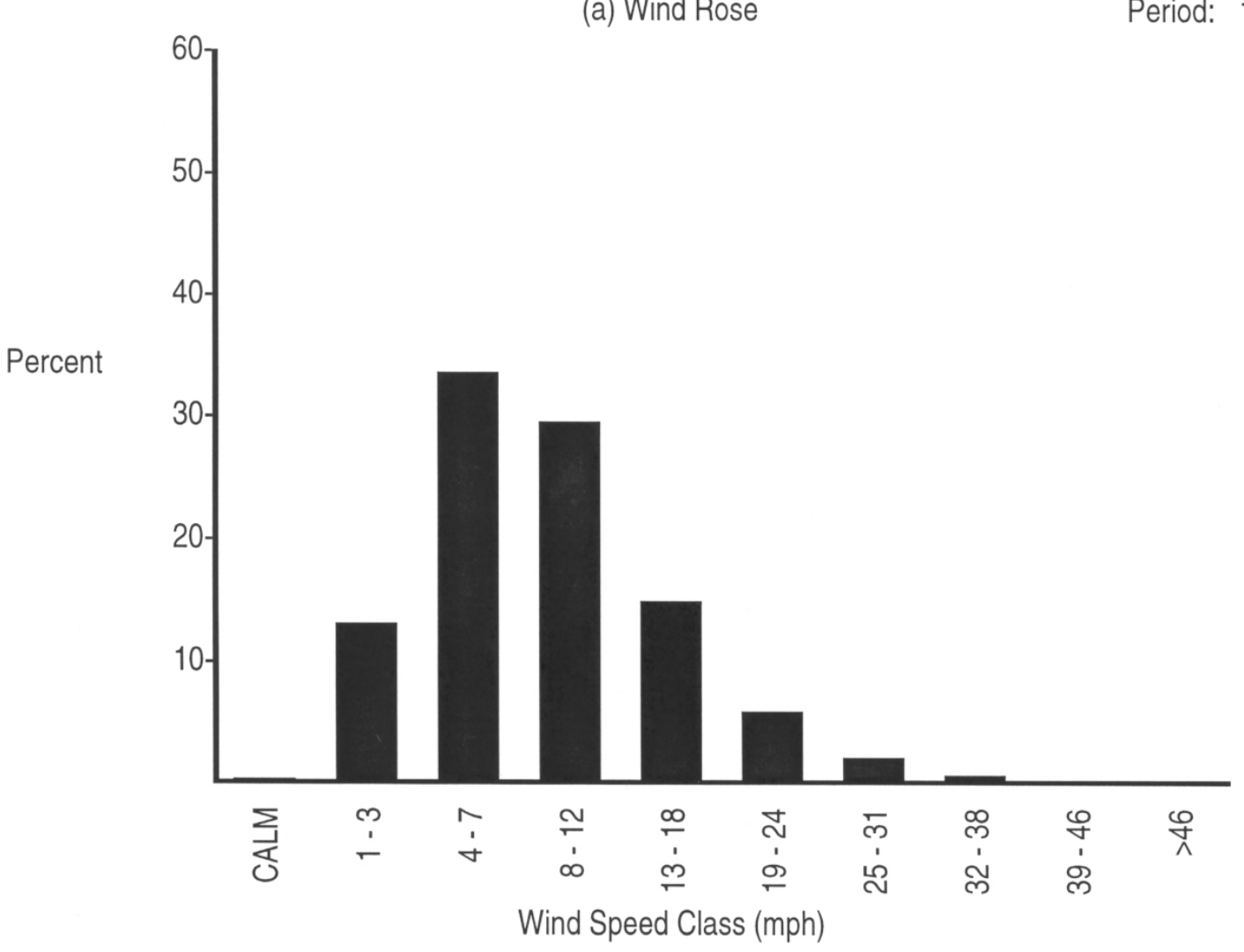

(b) Wind Speed Histogram

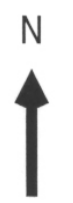

300 Area Tower

Period: 1/2003 - 12/2003

Figure B.2. (contd) 


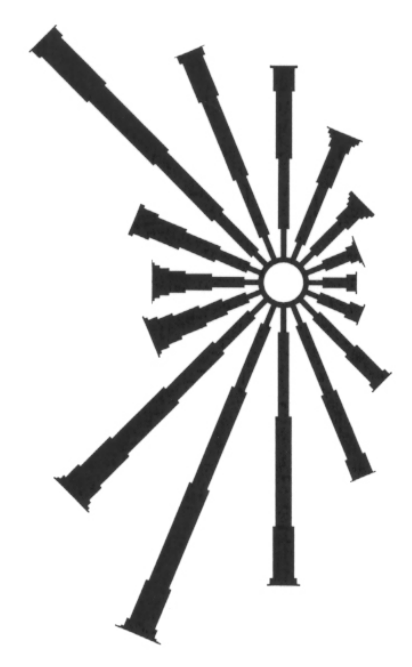

(a) Wind Rose

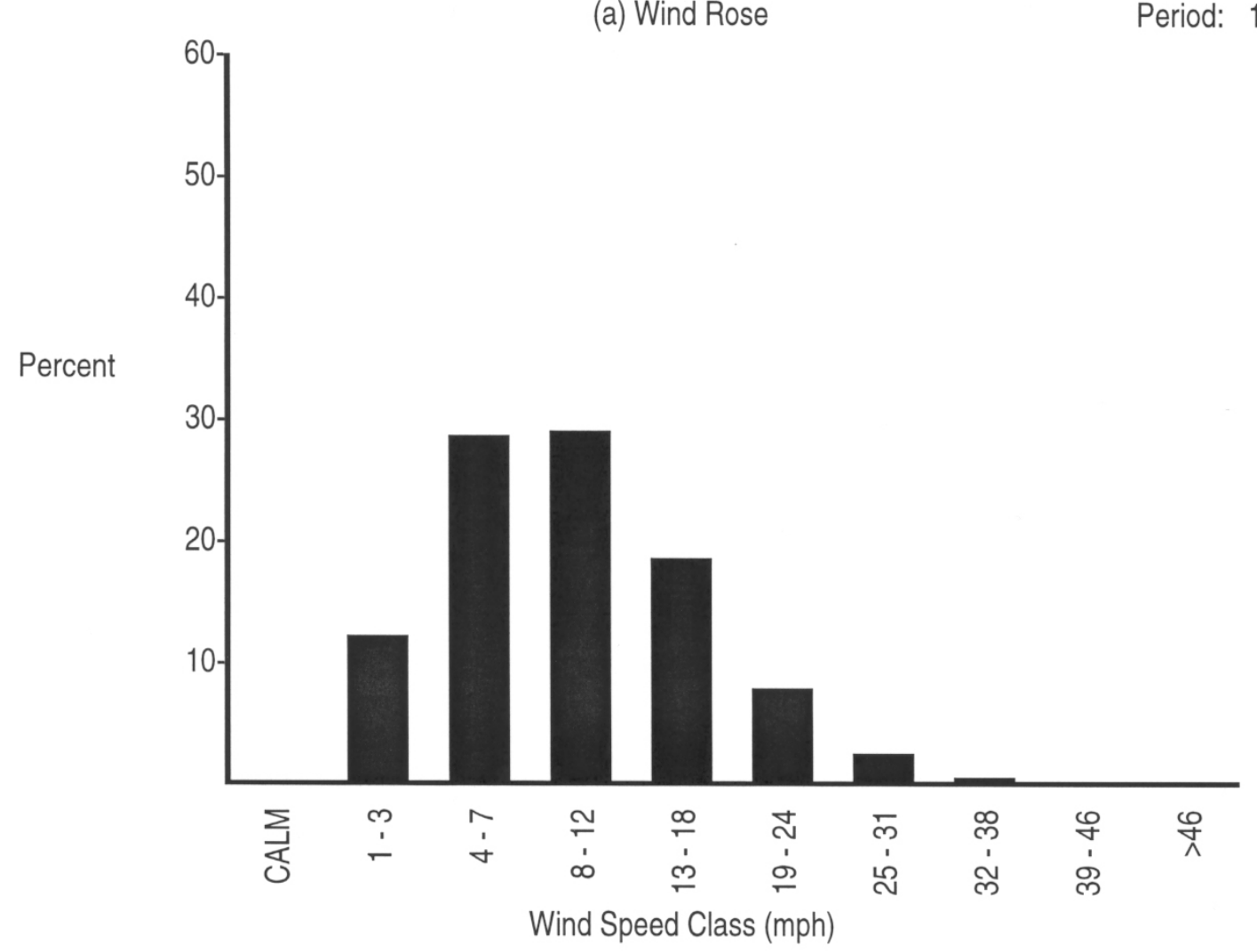

(b) Wind Speed Histogram

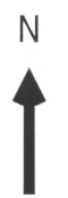

400 Area Tower

Period: 1/2003 - 12/2003

Figure B.2. (contd) 


\section{Table B.1. Joint Frequency Distributions (\%) for Hanford Meteorological Monitoring Network Wind Stations at 30 Feet, 2003}

\begin{tabular}{|c|c|c|c|c|c|c|c|c|c|c|c|c|c|c|c|c|c|c|}
\hline cat & & & & & & & & & & & & & & & & & & \\
\hline & & & & & & & & IRECI & ION & & & & & & & & & \\
\hline SPEED & $\mathrm{N}$ & NNE & $\mathrm{NE}$ & ENE & $E$ & ESE & $\mathrm{SE}$ & SSE & S & SSW & SW & WSW & W & WNW & NW & NNW & CALM & TOTAL \\
\hline CALM & 0.0 & 0.0 & 0.0 & 0.0 & 0.0 & 0.0 & 0.0 & 0.0 & 0.0 & 0.0 & 0.0 & 0.0 & 0.0 & 0.0 & 0.0 & 0.0 & .7 & \\
\hline & 1.9 & 1.1 & .8 & .9 & & & 1.6 & & 2.1 & 2. & 1. & & 1. & 1.4 & & .8 & 0.0 & 25.0 \\
\hline $4-7$ & 2.7 & 1.3 & .7 & .6 & 1.0 & 1. & 2.8 & 5. & 5.1 & 3. & 1.6 & 1.0 & .7 & .9 & & 4.2 & 0. & 5.8 \\
\hline $8-12$ & 1.6 & .5 & .2 & .1 & .2 & .3 & .7 & 1.7 & 3.7 & 4. & 1.9 & .9 & .5 & .6 & 2. & 3.0 & 0. & 23.6 \\
\hline $13-18$ & .4 & .2 & .1 & 0.0 & 0.0 & 0.0 & 0.0 & .1 & .9 & 2.9 & 1.8 & 1.0 & .4 & .2 & 2.4 & 1.1 & 0. & 11.5 \\
\hline & .1 & .2 & 0.0 & & 0.0 & 0.0 & 0.0 & 0.0 & .1 & .4 & .7 & .4 & .2 & .1 & .4 & & & 2.5 \\
\hline & 0.0 & 0.0 & c & & 0. & & 0. & ) & 0.0 & . & . & . & 0 & 0.0 & & 0.0 & & .7 \\
\hline & 0.0 & 0.0 & 0.0 & 0.0 & 0 . & 0. & 0. & 0 . & 0.0 & . & .1 & 0.0 & & 0.0 & 0. & 0.0 & 0. & .2 \\
\hline $39-46$ & 0.0 & 0.0 & 0.0 & 0.0 & 0.0 & 0.0 & 0.0 & 0. & 0.0 & 0 . & 0.0 & 0.0 & 0.0 & 0.0 & 0.0 & 0.0 & 0.0 & 0.0 \\
\hline$>46$ & 0.0 & 0.0 & 0.0 & 0.0 & 0.0 & 0.0 & 0.0 & 0.0 & 0.0 & 0. & 0.0 & 0.0 & 0.0 & 0.0 & 0.0 & 0.0 & 0.0 & 0.0 \\
\hline OTAL & 6.7 & 3.3 & 1.9 & 1.6 & 1.9 & 2.5 & 5.1 & 9.0 & 11.8 & 13.6 & 8.1 & 4.8 & 3.1 & 3.1 & 11.5 & 11.2 & .7 & 100.0 \\
\hline
\end{tabular}

\begin{tabular}{|c|c|c|c|c|c|c|c|c|c|c|c|c|c|c|c|c|c|c|}
\hline cat & & & & & & & & & & & & & & & & & & \\
\hline & & & & & & & & $T R E$ & & & & & & & & & & \\
\hline SPEED & $\mathrm{N}$ & NNE & $\mathrm{NE}$ & ENE & $\mathrm{E}$ & ESE & $\mathrm{SE}$ & SSE & S & SSW & SW & WSW & W & WNW & NW & NNW & CALM & TOTAL \\
\hline CALM & 0.0 & 0.0 & 0.0 & 0.0 & 0.0 & 0.0 & 0.0 & 0.0 & 0.0 & 0.0 & 0.0 & 0.0 & 0.0 & 0.0 & 0.0 & 0.0 & .9 & .9 \\
\hline & 1.0 & 1.0 & .7 & .8 & & .6 & .6 & . & & 1. & & & & & & .2 & 0.0 & 16.8 \\
\hline & 2. & 1.8 & 1.4 & . & & & 1.2 & & 1.9 & & & & & & & & & 0.6 \\
\hline $8-12$ & 1.7 & .4 & .1 & .1 & & . & .3 &. & 1.5 & & 2. & & 1. & 3.9 & & 3.1 & & 26.7 \\
\hline $13-18$ & .6 & .2 & .1 & 0.0 & 0.0 & 0.0 & 0.0 & 0.0 & .1 & 1.0 & 2.9 & 2.8 & 1.5 & .5 & 3.3 & 2.3 & 0.0 & 15.3 \\
\hline $9-24$ & .1 & .1 & 0.0 & 0.0 & 0.0 & 0.0 & 0.0 & 0.0 & 0.0 & .2 & 1.7 & 2.0 & 1.1 & .1 & .5 & .2 & 0.0 & 6.0 \\
\hline & .0 & 0.0 & & & & & & & & .1 & & 1. & .4 & .1 & & 0.0 & 0. & 2.8 \\
\hline & c & & & & & & & & & . & & & & 0.0 & & 0.0 & 0. & 7 \\
\hline $99-4$ & 0. & 0.0 & 0.0 & & & & & 0 . & 0.0 & 0. & 0. & . & & & & 0. & & .2 \\
\hline$>46$ & 0.0 & 0.0 & 0.0 & 0.0 & 0.0 & 0.0 & 0.0 & 0.0 & 0.0 & 0.0 & 0.0 & 0.0 & 0.0 & 0.0 & 0.0 & 0.0 & 0.0 & 0.0 \\
\hline OTAL & 6.1 & 3.4 & 2.3 & 1.8 & 1.9 & 1.7 & 2.2 & 2.3 & 4.5 & 7.2 & 11.3 & 10.8 & 8.4 & 9.5 & 15.7 & 10.0 & .9 & 100.0 \\
\hline
\end{tabular}

Station: ( 3) ARMY

\begin{tabular}{|c|c|c|c|c|c|c|c|c|c|c|c|c|c|c|c|c|c|c|}
\hline PEED & $\mathrm{N}$ & NNE & $\mathrm{NE}$ & ENE & $\mathrm{E}$ & ESE & SE & SSE & $\mathrm{S}$ & SSW & SW & WSW & $W$ & WNW & NW & NNW & CALM & TOTAL \\
\hline CALM & 0.0 & 0.0 & 0.0 & 0.0 & 0.0 & 0.0 & 0.0 & 0.0 & 0.0 & 0.0 & 0.0 & 0.0 & 0.0 & 0.0 & 0.0 & 0.0 & 3.4 & 3.4 \\
\hline $1-3$ & 2.1 & 1.8 & 2.0 & 2.0 & 2.5 & 2.0 & 1.8 & 1.2 & .8 & .7 & .9 & 1.2 & 2.3 & 3.5 & 3.9 & 2.7 & 0.0 & 31.4 \\
\hline $4-7$ & 2.5 & 1.1 & 1.1 & 1.3 & 2.0 & 2.1 & 1.9 & .8 & .5 & .4 & .6 & .9 & 2.2 & 6.3 & 7.1 & 3.8 & 0.0 & 34.7 \\
\hline $8-12$ & 1.0 & .5 & .2 & .2 & .4 & .7 & .9 & .6 & .5 & .5 & .7 & 1.3 & 2.1 & & .2 & 1 & & 19.8 \\
\hline $3-1 \varepsilon$ & .3 & .1 & .1 & 0.0 & 0.0 & .2 & .2 & .2 & .2 & .3 & .7 & 1.4 & 1.0 & & 1.4 & & .0 & 7.2 \\
\hline-24 & .3 & .1 & 0.0 & 0.0 & 0.0 & 0.0 & 0.0 & 0.0 & 0.0 & .1 & .5 & .6 & .4 & .2 & .5 & & 0.0 & 2.7 \\
\hline $5-31$ & 0.0 & 0.0 & 0.0 & 0.0 & 0.0 & 0.0 & 0.0 & 0.0 & 0.0 & .1 & .1 & .2 & 0.0 & 0.0 & .1 & 0.0 & 0.0 & .6 \\
\hline-38 & 0.0 & 0.0 & 0.0 & 0. & 0.0 & 0.0 & 0.0 & 0.0 & 0.0 & 0.0 & .1 & 0.0 & 0.0 & 0 & 0.0 & 0 & 0.0 & .1 \\
\hline-46 & 0.0 & 0.0 & 0.0 & 0.0 & 0.0 & 0.0 & 0.0 & 0.0 & 0.0 & 0.0 & 0.0 & 0.0 & 0.0 & 0 & 0.0 & & .0 & 0.0 \\
\hline$>46$ & 0.0 & 0 . & 0.0 & 0 . & 0.0 & 0 . & 0 . & 0.0 & 0. & 0.0 & 0. & & 0.0 & & 0.0 & & 0.0 & 0.0 \\
\hline DTA & 6.2 & 3.6 & 3.4 & 3.6 & 4.9 & 5.0 & 4.8 & 2.7 & 2.0 & 2.1 & 3.6 & 5.7 & 8.0 & 15.2 & 17.2 & 8.6 & 3.4 & 100.0 \\
\hline
\end{tabular}

Station: ( 4) RSPG

\begin{tabular}{|c|c|c|c|c|c|c|c|c|c|c|c|c|c|c|c|c|c|c|}
\hline SPEED & $\mathrm{N}$ & NNE & $\mathrm{NE}$ & ENE & $\mathrm{E}$ & ESE & $\mathrm{SE}$ & SSE & S & SSW & SW & WSW & W & WNW & NW & NNW & CALM & TOTAL \\
\hline CALM & 0.0 & 0.0 & 0.0 & 0.0 & 0.0 & 0.0 & 0.0 & 0.0 & 0.0 & 0.0 & 0.0 & 0.0 & 0.0 & 0.0 & 0.0 & 0.0 & .5 & \\
\hline $1-3$ & .6 & 1.4 & 1.4 & 1.5 & 1.6 & $\cdot$ & 1.1 & .7 & .8 & .0 & 6 & 1.5 & 1. & .1 & & .2 & 0 & 0.2 \\
\hline $4-7$ & 2.7 & 2.0 & 1.7 & 1.5 & 2.1 & 1.8 & .6 & .4 & .5 & .7 & 2. & 8.3 & 3. & 1.4 & & 2.4 & .0 & 3.9 \\
\hline $8-12$ & 1.1 & .5 & .1 & .1 & .2 & . & .1 & .1 & .3 & .8 & 2.0 & 15.7 & 5.3 & 2.2 & 1.5 & 1.5 & 0.0 & 31.9 \\
\hline $13-1$ & .1 & .1 & .1 & 0.0 & 0.0 & 0.0 & 0.0 & 0.0 & .1 & .6 & 1.1 & 3.3 & 2.3 & 1.1 & .9 & .6 & 0.0 & 10.2 \\
\hline & 0.0 & .1 & 0.0 & 0.0 & 0.0 & 0. & 0.0 & .0 & 0.0 & .2 & .9 & .6 & .4 & & & .1 & 0.0 & 2.6 \\
\hline & 0.0 & 0.0 & 0.0 & & & & & & & .1 & .1 & .2 & & & & & . & \\
\hline-38 & 0.0 & 0.0 & 0.0 & & 0.0 & & 0. & & 0.0 & 0.0 & .1 & 0.0 & 0.0 & .0 & & .0 & 0.0 & \\
\hline-46 & 0.0 & 0.0 & 0.0 & 0. & 0.0 & 1 & 0.0 & ). & 0.0 & 0.0 & 0.0 & 0.0 & 0.0 & .0 & & 0.0 & 0.0 & 0.0 \\
\hline$>46$ & 0.0 & 0.0 & 0.0 & 0.0 & 0.0 & 0. & 0.0 & 0 . & 0.0 & 0.0 & 0.0 & 0.0 & 0.0 & 0.0 & & 0.0 & 0.0 & 0.0 \\
\hline OTAL & 5.5 & 4.1 & 3.3 & 3.2 & 3.9 & 3.6 & 1.8 & 1.2 & 1.7 & 3.3 & 8.0 & 29.6 & 13.3 & 6.0 & 5.5 & 5.7 & 5 & 0 \\
\hline
\end{tabular}




\section{Table B.1. (contd)}

\section{Station: ( 5) EDNA}

\begin{tabular}{|c|c|c|c|c|c|c|c|c|c|c|c|c|c|c|c|c|c|c|}
\hline \multirow[b]{2}{*}{ SPEED } & \multirow[b]{2}{*}{$\mathrm{N}$} & \multirow[b]{2}{*}{ NNE } & \multirow[b]{2}{*}{$\mathrm{NE}$} & \multirow[b]{2}{*}{ ENE } & \multicolumn{2}{|c|}{ Begin: } & \multicolumn{3}{|c|}{$1 / 2003$} & \multicolumn{3}{|c|}{ nd: $12 / 2003$} & \multirow[b]{2}{*}{ W } & \multirow[b]{2}{*}{ WNW } & \multicolumn{3}{|c|}{ Total Hours: } & \\
\hline & & & & & $E$ & ESE & $\mathrm{SE}$ & SSE & $\mathrm{S}$ & SSW & SW & WSW & & & NW & NNW & CALM & TOTAL \\
\hline CALM & 0.0 & 0.0 & 0.0 & 0.0 & 0.0 & 0.0 & 0.0 & 0.0 & 0.0 & 0.0 & 0.0 & 0.0 & 0.0 & 0.0 & 0.0 & 0.0 & .6 & \\
\hline $1-3$ & 1.1 & .8 & .5 & .6 & .8 & 1.7 & 3.3 & 3.7 & 2.8 & 1.8 & 1.4 & 1.2 & 1.7 & 2.5 & 2.7 & 1.6 & 0.0 & 28.3 \\
\hline $4-7$ & 1.9 & 1.3 & .9 & 1.0 & 1.9 & 5.5 & 7.2 & 3.8 & 1.7 & .8 & .8 & .8 & 1.1 & 3.1 & 6.4 & 4.4 & 0.0 & 42.6 \\
\hline $8-12$ & 1.1 & .5 & .2 & .2 & 1.1 & 2.2 & 1.5 & 1.3 & 1.1 & .6 & .8 & 1.2 & 1.3 & 1.9 & 2.4 & 2.3 & 0.0 & 19.7 \\
\hline $13-18$ & .2 & .2 & .1 & 0.0 & .1 & .2 & .1 & .3 & .6 & .4 & .5 & 1.0 & .8 & 1.1 & .9 & .1 & 0.0 & 6.4 \\
\hline $9-24$ & 0.0 & .2 & .2 & 0.0 & 0.0 & 0.0 & 0.0 & 0.0 & .1 & .2 & .2 & .3 & .2 & .3 & .2 & 0.0 & 0.0 & 1.9 \\
\hline $25-31$ & 0.0 & 0.0 & 0.0 & 0.0 & 0.0 & 0.0 & 0.0 & 0.0 & 0.0 & 0.0 & .1 & .1 & .1 & 0.0 & 0.0 & 0.0 & 0.0 & .4 \\
\hline $32-38$ & 0.0 & 0.0 & 0.0 & 0.0 & 0.0 & 0.0 & 0.0 & 0.0 & 0.0 & 0.0 & 0.0 & 0.0 & 0.0 & 0.0 & 0.0 & 0.0 & 0.0 & 0.0 \\
\hline $39-46$ & 0.0 & 0.0 & 0.0 & 0.0 & 0.0 & 0.0 & 0.0 & 0.0 & 0.0 & 0.0 & 0.0 & 0.0 & 0.0 & 0.0 & 0.0 & 0.0 & 0.0 & 0.0 \\
\hline$>46$ & 0.0 & 0.0 & 0.0 & 0.0 & 0.0 & 0.0 & 0.0 & 0.0 & 0.0 & 0.0 & 0.0 & 0.0 & 0.0 & 0.0 & 0.0 & 0.0 & 0.0 & 0.0 \\
\hline TOTAL & 4.2 & 3.0 & 1.9 & 1.8 & 3.9 & 9.7 & 12.1 & 9.1 & 6.2 & 3.9 & 3.8 & 4.6 & 5.1 & 9.0 & 12.6 & 8.4 & .6 & 100.0 \\
\hline
\end{tabular}

Station: ( 6) 200E

8734

\begin{tabular}{|c|c|c|c|c|c|c|c|c|c|c|c|c|c|c|c|c|c|c|}
\hline PEED & $\mathrm{N}$ & JNE & $\mathrm{NE}$ & ENE & $E$ & ESE & $\mathrm{SE}$ & SSE & S & SSW & SW & WSW & W & WNW & NW & NNW & CALM & TO \\
\hline CALM & 0.0 & 0.0 & 0.0 & 0.0 & 0.0 & 0.0 & 0.0 & 0.0 & 0.0 & 0.0 & 0.0 & 0.0 & 0.0 & 0.0 & 0.0 & 0.0 & 2.8 & 2.8 \\
\hline $1-3$ & 1.4 & 1.1 & 1.2 & 1.3 & 1.5 & 1.4 & 1.5 & 1.2 & .9 & .8 & .8 & 1.2 & 1.4 & 1.9 & 2.1 & 1.2 & 0.0 & 20.7 \\
\hline $4-7$ & 1.2 & 1.2 & .7 & .7 & 1.3 & 2.3 & 2.7 & 2.1 & 1.5 & 1.3 & 1.6 & 2.4 & 4.2 & 5 & 4.1 & 2.0 & & 35.3 \\
\hline $8-12$ & .7 & .7 & .2 & .1 & .4 & .6 & 1. & 1.4 & .6 & .5 & 1. & 2.7 & 4. & 8 & , & .6 & .0 & 26.2 \\
\hline $3-18$ & .1 & .3 & .1 & 0.0 & 0.0 & 0.0 &. & .2 &. & .3 & .9 & 1.7 & 1.5 & 4 & 1.2 & .1 & 0.0 & 11.0 \\
\hline $9-24$ & 0.0 & .2 & 0.0 & 0.0 & 0.0 & 0.0 & 0.0 & 0.0 & .1 & .1 & .4 & .6 & .3 & .9 & .4 & 0.0 & 0.0 & 3.1 \\
\hline $5-31$ & 0.0 & 0.0 & 0.0 & 0. & 0.0 & 0. & 0. & 0.0 & 0.0 & .1 & .2 & .2 & .1 & .2 & .1 & 0 & 0.0 & .9 \\
\hline-38 & 0.0 & 0.0 & 0.0 & 0.0 & 0.0 & 0. & 0. & 0.0 & 0.0 & 0.0 & 0.0 & 0.0 & 0.0 & 0.0 & 0.0 & & .0 & .1 \\
\hline $39-46$ & 0.0 & 0. & 0.0 & 0. & 0. & 0. & & 0 & J. & 0.0 & & & 0 . & & 0.0 & & & 0.0 \\
\hline$>46$ & 0.0 & 0. & 0.0 & 0. & 0. & 0. & & 0 & & 0 & & 0 & 0.0 & 0. & 0.0 & & 0 & 0.0 \\
\hline OTAL & 3.4 & 3.5 & 2.2 & 2.1 & 3.1 & 4.4 & 5.4 & 4.8 & 3.4 & 3.0 & 5.1 & 8.9 & 12.1 & 21.6 & 10.2 & 4.0 & 2.8 & 100.0 \\
\hline
\end{tabular}

Station: ( 7) 200W

\begin{tabular}{|c|c|c|c|c|c|c|c|c|c|c|c|c|c|c|c|c|c|c|}
\hline PEED & $\mathrm{N}$ & NNE & $\mathrm{NE}$ & ENE & $E$ & ESE & SE & SSE & S & SSW & SW & WSW & W & WNW & NW & NNW & CALM & 'ОT \\
\hline CALM & 0.0 & 0.0 & 0.0 & 0.0 & 0.0 & 0.0 & 0.0 & 0.0 & 0.0 & 0.0 & 0.0 & 0.0 & 0.0 & 0.0 & 0.0 & 0.0 & 1.2 & 1.2 \\
\hline $1-3$ & 2.3 & 2.0 & 1.6 & 1.5 & 1.7 & 1.6 & 1.7 & 1.6 & 1.5 & 1.2 & 1.5 & 1.7 & 2.9 & .7 & 4 & 2.5 & .0 & 32.3 \\
\hline $4-7$ & 3.1 & 1.7 & 1.0 & .9 & 1.1 & 1.9 & 2.1 & .8 & .7 & .8 & 1. & 1.7 & 3. & 6.6 & & 3.5 & & 36.6 \\
\hline $8-12$ & 1.0 & .4 & .1 & .1 & .2 & .5 & .4 & .1 & .3 & .7 & 1.1 & 2.0 & 2. & 3.9 & 3. & 1.9 & ). & 18.8 \\
\hline & 0 & .2 & .1 & 0.0 & 0.0 & & 0.0 & 0.0 & .2 & .4 & & 1.6 & 1. & .8 & & .5 & & \\
\hline 24 & c & .1 & .0 & & 0.0 & & & & 0.0 & .2 & .5 & .7 & .3 & .1 & t & 0 & & 2.6 \\
\hline-31 & 0. & 0.0 & 0.0 & 0.0 & 0.0 & 0. & 0 . & ). & 0.0 & .1 & .2 & .2 & .1 & 0.0 & .1 & 0.0 & .0 & \\
\hline $2-38$ & 0.0 & 0.0 & 0.0 & 0.0 & 0.0 & 0.0 & 0.0 & 0.0 & 0.0 & 0.0 & .1 & 0.0 & 0.0 & 0.0 & 0.0 & 0.0 & .0 & \\
\hline $39-46$ & 0.0 & 0.0 & 0.0 & 0.0 & 0.0 & 0. & 0.0 & 0. & 0.0 & 0.0 & 0.0 & 0.0 & 0. & 0.0 & & .0 & & \\
\hline$>46$ & . & 0.0 & 0.0 & 0.0 & 0.0 & & & & 0. & & & 0.0 & 0.0 & 0.0 & 0.0 & .0 & & \\
\hline OTAL & 6.4 & 4.5 & 2.8 & 2.5 & 3.0 & 4.0 & 4.3 & 2.6 & 2.6 & 3.3 & 5.5 & 7.8 & 10.4 & 15.1 & 15.6 & 8.5 & 1.2 & 100. \\
\hline
\end{tabular}

Station: ( 8) BVLY

\begin{tabular}{|c|c|c|c|c|c|c|c|c|c|c|c|c|c|c|c|c|c|c|}
\hline PEED & $\mathrm{N}$ & NNE & $\mathrm{NE}$ & ENE & $E$ & ESE & $\mathrm{SE}$ & SSE & S & SSW & SW & WSW & W & WNW & NW & NNW & CALM & TOTAL \\
\hline CALM & 0.0 & 0.0 & 0.0 & 0.0 & 0.0 & 0.0 & 0.0 & 0.0 & 0.0 & 0.0 & 0.0 & 0.0 & 0.0 & 0.0 & 0.0 & 0.0 & .9 & \\
\hline $1-3$ & 3.6 & 2.7 & 1.9 & 1.7 & 1.8 & .1 & 1.9 & .4 & 1.6 & 1.1 & 1. & .9 & 3 & .9 & 4 & 9 & 0.0 & 30.4 \\
\hline $4-7$ & 9.6 & 2.6 & .6 & .3 & 1.9 & 3.7 & 1.4 & 1.0 & 1.1 & .8 & .7 & .7 & 1. & 3.4 & 5.7 & 7.3 & 0.0 & 42.6 \\
\hline $8-12$ & 3.9 & 1.2 & .1 & 0.0 & .3 & .5 & .2 & .2 & .2 & .3 & . & .6 & 1. & 4.7 & 4. & & 0.0 & 19.2 \\
\hline $13-18$ & 0.0 & .2 & .1 & 0.0 & 0.0 & 0.0 & 0.0 & 0.0 & .2 & .4 & . & .2 & .3 & 2.2 & 1. & & .0 & \\
\hline & 0.0 & 0.0 & 0.0 & & 0.0 & & & & 0.0 & .1 & .2 & .1 & .1 & .2 & 1 & & .0 & .8 \\
\hline & 0.0 & & 0.0 & & 0.0 & & & & & .0 & & 0.0 & & & & & .0 & \\
\hline-38 & 0.0 & 0. & 0.0 & 0.0 & 0.0 & D. & 0.0 & ). & 0.0 & 0.0 & 0.0 & 0.0 & 0.0 & 0.0 & 0.0 & 0 & 0.0 & 0.0 \\
\hline $9-46$ & 0.0 & 0.0 & 0.0 & 0.0 & 0.0 & 0 & 0.0 & & 0.0 & 0.0 & D. & 0.0 & 0 . & 0.0 & 0.0 & 0 & 0.0 & 0.0 \\
\hline$>46$ & 0.0 & 0.0 & 0.0 & 0.0 & 0.0 & 0 . & 0.0 & 0 . & 0.0 & 0.0 & 0 . & 0.0 & 0.0 & 0.0 & 0.0 & 0.0 & 0.0 & 0.0 \\
\hline OTAL & 17.2 & 6.6 & 2.7 & 2.1 & 4.0 & 6.3 & 3.5 & 2.8 & 3.1 & 2.8 & 3.2 & 2.6 & 4.7 & 12.3 & 14.0 & 11.3 & .9 & 100.0 \\
\hline
\end{tabular}




\section{Table B.1. (contd)}

Station: ( 9) FFTF

Begin: 1/2003 End: 12/2003

Total Hours:

8728

DIRECTION

\begin{tabular}{|c|c|c|c|c|c|c|c|c|c|c|c|c|c|c|c|c|c|c|}
\hline PEED & $\mathrm{N}$ & NNE & $\mathrm{NE}$ & ENE & $\mathrm{E}$ & ESE & $\mathrm{SE}$ & SSE & $\mathrm{S}$ & SSW & SW & WSW & W & WNW & NW & NNW & CALM & TOTAI \\
\hline CALM & 0.0 & 0.0 & 0.0 & 0.0 & 0.0 & 0.0 & 0.0 & 0.0 & 0.0 & 0.0 & 0.0 & 0.0 & 0.0 & 0.0 & 0.0 & 0.0 & .3 & \\
\hline $1-3$ & 1.2 & 1.0 & .9 & .7 & .8 & .9 & .9 & 1.0 & 1.0 & 1.0 & 1.0 & .8 & 1.1 & 1.1 & 1.2 & 1.0 & 0.0 & 15.6 \\
\hline $4-7$ & 3.1 & 3.1 & 2.0 & 1.3 & 1.1 & 1.3 & 2.5 & 3.6 & 4.0 & 3.7 & 2.2 & 1.6 & 1.1 & 1.5 & 3.0 & 3.4 & 0.0 & 38.4 \\
\hline $8-12$ & 1.3 & 1.3 & .6 & .2 & .2 & .4 & 1.1 & 3.8 & 4.5 & 4.7 & 1.9 & 1.0 & .8 & 1.2 & 3.0 & 3.3 & 0.0 & 29.4 \\
\hline $3-18$ & .3 & .2 & .2 & 0.0 & 0.0 & 0.0 & .1 & .4 & 1.0 & 3.3 & 2.1 & .8 & .4 & .6 & 1.9 & .5 & 0.0 & 12.0 \\
\hline-24 & 0.0 & .1 & .1 & .1 & 0.0 & 0.0 & 0.0 & 0.0 & .1 & .6 & .9 & .4 & .3 & .2 & .4 & 0.0 & 0.0 & 3.1 \\
\hline & 0.0 & 0.0 & .1 & 0.0 & 0.0 & 0.0 & & 0.0 & 0.0 & .1 & .3 & .2 & .1 & 0.0 & .1 & 0 & .0 & .9 \\
\hline & 0. & 0.0 & 0.0 & 0.0 & 0. & 0. & & & 0.0 & 0. & .1 & .0 & 0.0 & & & & & 1 \\
\hline $9-46$ & 0.0 & 0.0 & 0.0 & 0.0 & 0.0 & 0.0 & 0. & 0. & 0.0 & 0. & 0.0 & 0.0 & 0.0 & & 0.0 & 0.0 & 0.0 & \\
\hline$>46$ & 0.0 & 0.0 & 0.0 & 0.0 & 0.0 & 0.0 & 0.0 & 0.0 & 0.0 & 0.0 & 0.0 & 0.0 & 0.0 & 0 . & 0.0 & 0.0 & 0.0 & 0.0 \\
\hline TOTAL & 5.9 & 5.8 & 3.9 & 2.3 & 2.1 & 2.6 & 4.6 & 8.8 & 10.7 & 13.4 & 8.5 & 4.8 & 3.9 & 4.6 & 9.5 & 8.3 & .3 & 100.0 \\
\hline
\end{tabular}

Station: (10) YAKB

DIRECTION

\begin{tabular}{|c|c|c|c|c|c|c|c|c|c|c|c|c|c|c|c|c|c|c|}
\hline SPEED & $\mathrm{N}$ & NNE & $\mathrm{NE}$ & ENE & $\mathrm{E}$ & ESE & SE & SSE & S & SSW & SW & WSW & W & WNW & NW & NNW & CALM & TOTAI \\
\hline CALM & 0.0 & 0.0 & 0.0 & 0.0 & 0.0 & 0.0 & 0.0 & 0.0 & 0.0 & 0.0 & 0.0 & 0.0 & 0.0 & 0.0 & 0.0 & 0.0 & .5 & .5 \\
\hline $1-3$ & 1.4 & 1.4 & 1.3 & .9 & .9 & .9 & .8 & 1.0 & 1.0 & 1.0 & .9 & 1.5 & 1.9 & 1.5 & 1.5 & 1.3 & 0.0 & 19.2 \\
\hline $4-7$ & 3.7 & 3.0 & 1.6 & 1.0 & .9 & 1.1 & 1.6 & 1.4 & .8 & .9 & 1.4 & 3.5 & 7.1 & 4.2 & 3.3 & 3.8 & 0.0 & 39.3 \\
\hline $8-12$ & 1.3 & .6 & .2 & .1 & .1 & .2 & .2 & .2 & .3 & .5 & 1.9 & 2.9 & 4.7 & 3.5 & 5.6 & 3.0 & 0.0 & 25.4 \\
\hline $13-18$ & 0.0 & .1 & .1 & 0.0 & 0.0 & 0.0 & 0.0 & 0.0 & .2 & .5 & 1.3 & 1.8 & .6 & 1.3 & 3.5 & .8 & 0.0 & 10.5 \\
\hline $19-24$ & 0.0 & .1 & .1 & 0.0 & 0.0 & 0.0 & 0.0 & 0.0 & 0.0 & .2 & .7 & .6 & .2 & .7 & 1.3 & .1 & 0.0 & 3.9 \\
\hline $25-31$ & 0.0 & 0.0 & 0.0 & 0.0 & 0.0 & 0.0 & 0.0 & 0.0 & 0.0 & .1 & .3 & .1 & 0.0 & .4 & .2 & 0.0 & 0.0 & 1.1 \\
\hline $2-38$ & 0.0 & 0.0 & 0.0 & 0.0 & 0.0 & 0.0 & 0.0 & 0.0 & 0.0 & 0.0 & 0.0 & 0.0 & 0.0 & 0.0 & 0.0 & 0.0 & 0.0 & \\
\hline $39-46$ & 0.0 & 0.0 & 0.0 & 0.0 & 0.0 & 0.0 & 0.0 & 0.0 & 0.0 & 0.0 & 0.0 & 0.0 & 0.0 & 0.0 & 0.0 & 0.0 & 0.0 & \\
\hline$>46$ & 0.0 & 0.0 & 0.0 & 0.0 & 0.0 & 0.0 & 0.0 & 0.0 & 0.0 & 0.0 & 0.0 & 0.0 & 0.0 & 0.0 & 0.0 & 0.0 & 0.0 & D. \\
\hline דגד ד & 6.5 & 5.2 & 3.2 & 2.0 & 1.9 & 2.2 & 2.7 & 2.5 & 2.3 & 3.3 & 6.6 & 0.4 & 4.6 & 1.7 & 5.5 & 9.0 & 5 & \\
\hline
\end{tabular}

Station: (11) 300A

\begin{tabular}{|c|c|c|c|c|c|c|c|c|c|c|c|c|c|c|c|c|c|c|}
\hline DEED & $\mathrm{N}$ & NNE & $\mathrm{NE}$ & ENE & $E$ & ESE & $\mathrm{SE}$ & SSE & S & SSW & SW & WSW & W & WNW & NW & NNW & CALM & TOTAI \\
\hline CALM & 0.0 & 0.0 & 0.0 & 0.0 & 0.0 & 0.0 & 0.0 & 0.0 & 0.0 & 0.0 & 0.0 & 0.0 & 0.0 & 0.0 & 0.0 & 0.0 & .5 & \\
\hline $1-3$ & 1.1 & .8 & .7 & .6 & .9 & 1.3 & 1.6 & 1.8 & 1.6 & 1.2 & 1.1 & .8 & 1.0 & 1.1 & 1.8 & 1.7 & 0.0 & 19 \\
\hline $4-7$ & 3.2 & 1.3 & 1.1 & 1.3 & 1.9 & 4.7 & 7.2 & 3.7 & 2.8 & 2.6 & 1.9 & 1.2 & .8 & 1.0 & 2.1 & 3.9 & 0.0 & 0. \\
\hline $8-12$ & 2.8 & 1.7 & .8 & .4 & .5 & 1.4 & 2.0 & 1.1 & 1.9 & 3.8 & 3.1 & 1.6 & .5 & .4 & 1.1 & & . & \\
\hline $13-18$ & .5 & .4 & .1 & 0.0 & 0.0 & 0.0 & .1 & .2 & .4 & 1.6 & 2 & 1.4 & .5 & .1 & 1.0 & & .0 & D \\
\hline-24 & 0.0 & .1 & .1 & 0.0 & 0.0 & 0.0 & 0. & 0.0 & .1 & .4 & 1. & .5 & .2 & 0.0 & .2 & 1 & 0.0 & \\
\hline $5-31$ & 0.0 & .1 & .1 & 0.0 & 0.0 & 0.0 & 0.0 & 0.0 & 0.0 & .1 & .4 & .1 & .1 & 0.0 & .1 & 0.0 & 0.0 & \\
\hline $2-38$ & 0.0 & 0.0 & 0.0 & 0.0 & 0.0 & 0.0 & 0.0 & 0.0 & 0.0 & 0.0 & .1 & .1 & 0.0 & 0.0 & 0.0 & 0 & 0.0 & \\
\hline $39-46$ & 0.0 & 0.0 & 0.0 & 0.0 & 0.0 & 0.0 & 0.0 & 0.0 & 0.0 & 0.0 & 0.0 & 0.0 & 0.0 & 0.0 & 0.0 & 0 & 0.0 & \\
\hline$>46$ & 0.0 & 0.0 & 0.0 & 0.0 & 0.0 & 0.0 & 0.0 & 0.0 & 0 . & 0.0 & 0.0 & 0.0 & 0.0 & & 0.0 & & 0.0 & \\
\hline OTAL & 7.5 & 4.6 & 2.9 & 2.4 & 3.3 & 7.4 & 10.9 & 6.9 & 6.7 & 9.7 & 10.4 & 5.7 & 3.1 & 2.7 & 6.3 & 9.1 & .5 & \\
\hline
\end{tabular}

Station: (12) WYEB

\begin{tabular}{rrrrrrrrrrrrrrrrrrr} 
SPEED & N & NNE & NE & ENE & \multicolumn{1}{c}{ E } & ESE & SE & SSE & \multicolumn{1}{c}{ S } & SSW & SW & WSW & W & WNW & NW & NNW & CALM TOTAL \\
\hline CALM & 0.0 & 0.0 & 0.0 & 0.0 & 0.0 & 0.0 & 0.0 & 0.0 & 0.0 & 0.0 & 0.0 & 0.0 & 0.0 & 0.0 & 0.0 & 0.0 & .4 & .4 \\
$1-3$ & 1.7 & 1.3 & 1.3 & 1.1 & 1.6 & 1.3 & 1.3 & 1.3 & 1.1 & .9 & .8 & 1.0 & 1.2 & 1.3 & 1.5 & 1.3 & 0.0 & 20.1 \\
$4-7$ & 4.1 & 2.2 & 1.2 & 1.4 & 2.3 & 2.5 & 3.3 & 3.7 & 3.8 & 2.2 & 1.6 & 1.6 & 2.3 & 3.1 & 3.5 & 2.7 & 0.0 & 41.6 \\
$8-12$ & 1.9 & .8 & .3 & .3 & .4 & .7 & 1.7 & 2.8 & 3.1 & 1.7 & .9 & 1.3 & 1.9 & 3.3 & 2.8 & 1.5 & 0.0 & 25.3 \\
$13-18$ & .5 & .5 & .1 & 0.0 & 0.0 & 0.0 & .1 & .5 & 1.6 & 1.3 & .8 & .5 & .8 & .9 & 1.1 & .3 & 0.0 & 9.2 \\
$19-24$ & .1 & .2 & .1 & 0.0 & 0.0 & 0.0 & 0.0 & 0.0 & .2 & .5 & .4 & .4 & .2 & .2 & .4 & 0.0 & 0.0 & 2.0 \\
$25-31$ & 0.0 & 0.0 & 0.0 & 0.0 & 0.0 & 0.0 & 0.0 & 0.0 & 0.0 & .2 & .2 & .1 & 0.0 & 0.0 & .1 & 0.0 & 0.0 & .0 \\
$32-38$ & 0.0 & 0.0 & 0.0 & 0.0 & 0.0 & 0.0 & 0.0 & 0.0 & 0.0 & 0.0 & .1 & 0.0 & 0.0 & 0.0 & 0.0 & 0.0 & 0.0 & .1 \\
$39-46$ & 0.0 & 0.0 & 0.0 & 0.0 & 0.0 & 0.0 & 0.0 & 0.0 & 0.0 & 0.0 & 0.0 & 0.0 & 0.0 & 0.0 & 0.0 & 0.0 & 0.0 & 0.0 \\
>6 & 0.0 & 0.0 & 0.0 & 0.0 & 0.0 & 0.0 & 0.0 & 0.0 & 0.0 & 0.0 & 0.0 & 0.0 & 0.0 & 0.0 & 0.0 & 0.0 & 0.0 & 0.0 \\
TOTAL & 8.3 & 5.1 & 3.1 & 2.8 & 4.2 & 4.6 & 6.5 & 8.3 & 9.8 & 6.7 & 4.9 & 4.8 & 6.5 & 8.8 & 9.4 & 5.8 & .4 & 100.0
\end{tabular}




\section{Table B.1. (contd)}

\begin{tabular}{|c|c|c|c|c|c|c|c|c|c|c|c|c|c|c|c|c|c|c|}
\hline & - & & & & & & & & & & & & & & & & & \\
\hline & & & & & & & 12003 & & & nd: & $2 / 20$ & & & & Tot & $1 \mathrm{Hol}$ & rs: & 8732 \\
\hline & & & & & & & & IREC' & ION & & & & & & & & & \\
\hline PEED & $\mathrm{N}$ & NNE & $\mathrm{NE}$ & ENE & $\mathrm{E}$ & ESE & $\mathrm{SE}$ & SSE & $\mathrm{S}$ & SSW & SW & WSW & W & WNW & NW & NNW & CALM & TOTAL \\
\hline CALM & 0.0 & 0.0 & 0.0 & 0.0 & 0.0 & 0 . & 0.0 & 0.0 & 0.0 & 0.0 & 0.0 & 0.0 & 0.0 & 0.0 & 0.0 & .0 & .8 & \\
\hline $1-3$ & 2.2 & 2.0 & 2.3 & 2.7 & 3.1 & 3. & 2.4 & 1.5 & 1.3 & 1. & 1. & 2.0 & 2 & 2.9 & 2. & 2.5 & 0.0 & 36.5 \\
\hline $4-7$ & 1.4 & 1.9 & 1.8 & 2.6 & 3.5 & 3.0 & 2.2 & 1.4 & .8 & .9 & 2.1 & 3.8 & 5. & 3. & 2.1 & 1.4 & 0 & 37.0 \\
\hline $8-12$ & .5 & .9 & .6 & .2 & .3 & .6 & .8 & .5 & .4 & .6 & 1.2 & 2.3 & 3. & 2. & .7 & .3 & 0. & 15.4 \\
\hline & .2 & .4 & .3 & .1 & .1 & .7 & .2 & & .2 & .2 &. & .7 & & & & 1 & & 7.4 \\
\hline & .1 & .2 & .1 & 0.0 & 0.0 & 0.0 & 0.0 & 20. &. & . & . & .2 & & & & 0.0 & D. & 2.4 \\
\hline & 0.0 & 0.0 & .1 & 0.0 & 0.0 & 0.0 & 0.0 & 0 . & 0.0 & 0.0 & .1 & 0.0 & 0.0 & 0.0 & 0.0 & 0.0 & 0.0 & .4 \\
\hline $2-38$ & 0.0 & 0.0 & 0.0 & 0.0 & 0.0 & 0.0 & 0.0 & 0.0 & 0.0 & 0.0 & 0.0 & 0.0 & 0 . & 0.0 & 0.0 & 0.0 & 0. & 0.0 \\
\hline $39-46$ & 0.0 & 0.0 & 0.0 & 0.0 & 0.0 & 0.0 & 0.0 & 0 . & 0.0 & 0 . & 0.0 & 0.0 & 0 & 0 . & & 0.0 & 0 . & 0.0 \\
\hline$>46$ & 0.0 & 0.0 & 0.0 & 0.0 & 0.0 & 0.0 & 0.0 & 0.0 & 0.0 & 0.0 & 0.0 & 0.0 & 0.0 & 0.0 & 0.0 & 0.0 & 0.0 & 0.0 \\
\hline TOTAL & 4.4 & 5.4 & 5.1 & 5.5 & 7.0 & 6.7 & 5.4 & 3.6 & 2.8 & 3.1 & 6.2 & 8.9 & 12.7 & 10.7 & 7.0 & 4.4 & .8 & 100.0 \\
\hline
\end{tabular}

Station: (14) WPPS

Begin: 1/2003 End: 12/2003 Total Hours: 8734 DIRECTION

\begin{tabular}{|c|c|c|c|c|c|c|c|c|c|c|c|c|c|c|c|c|c|c|}
\hline SPEED & $\mathrm{N}$ & NNE & $\mathrm{NE}$ & ENE & $\mathrm{E}$ & ESE & SE & SSE & $\mathrm{S}$ & SSW & SW & WSW & W & WNW & NW & NNW & CALM & TOTAL \\
\hline CALM & 0.0 & 0.0 & 0.0 & 0.0 & 0.0 & 0.0 & 0.0 & 0.0 & 0.0 & 0.0 & 0.0 & 0.0 & 0.0 & 0.0 & 0.0 & 0.0 & 1.2 & 1.2 \\
\hline $1-3$ & 2.5 & 2.0 & 1.8 & 1.2 & .9 & .7 & 1. & & 1 & & 2 & .9 & & .1 & & 3 & .0 & 3.6 \\
\hline $4-7$ & 3.7 & 2.3 & 2.6 & 1.2 & .6 & .9 & 2.0 & $\theta$ & 4.9 & 2.5 & 1. & 1.3 & 1 . & 1.7 & - & 4.4 & .0 & .0 \\
\hline $8-12$ & 1.0 & .8 & .4 & .3 & .1 & .4 & 1.2 & 2.8 & 4.5 & 2.9 & 1. & 1.1 & 1.2 & 1.7 & 2. & 1.7 & 0 & 23.9 \\
\hline $13-18$ & .2 & .2 & .2 & 0.0 & 0.0 & 0.0 & .1 & .2 & .9 & 2.1 & 1.1 & .0 & .5 & .8 & 1.2 & .3 & 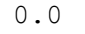 & 8.5 \\
\hline & 0.0 & .2 & .1 & 0.0 & 0.0 & 0.0 & 0.0 & 0.0 & .1 & .5 & .5 & .3 & .2 & .1 & & 0.0 & D & 2.2 \\
\hline & 0.0 & 0.0 & 0.0 & & 0.0 & & & & 0.0 & .1 & & 0.0 & 1 & 0.0 & & & & \\
\hline & 0.0 & & .0 & & & & & & & & & & & & & & & \\
\hline-46 & 0.0 & 0.0 & 0.0 & & 0.0 & & 0. & & 0.0 & .0 & & 0.0 & 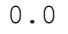 & 0.0 & & .0 & .0 & 0.0 \\
\hline 46 & 0.0 & 0.0 & 0.0 & 0 . & 0.0 & 0.0 & 0.0 & 0 . & 0.0 & 0 . & 0 . & 0.0 & 0 . & 0.0 & 0. & 0.0 & 0.0 & 0.0 \\
\hline OTAL & 7.3 & 5.6 & 5.1 & 2.7 & 1.7 & 1.9 & 4.6 & 9.0 & 12.0 & 9.2 & 6.3 & 4.2 & 4.5 & 5.5 & 10.5 & 8.6 & 1.2 & 00 . \\
\hline
\end{tabular}

Station: (15) FRNK

Begin: 1/2003 End: 12/2003

Total Hours:

8734

\begin{tabular}{|c|c|c|c|c|c|c|c|c|c|c|c|c|c|c|c|c|c|c|}
\hline \multicolumn{19}{|c|}{ DIRECTION } \\
\hline SPEED & $\mathrm{N}$ & NNE & $\mathrm{NE}$ & ENE & $\mathrm{E}$ & ESE & $\mathrm{SE}$ & $\mathrm{SSE}$ & $\mathrm{S}$ & SSW & SW & WSW & W & WNW & NW & NNW & CALM & TOTAL \\
\hline CALM & 0.0 & 0.0 & 0.0 & 0.0 & 0.0 & 0.0 & 0.0 & 0.0 & 0.0 & 0.0 & 0.0 & 0.0 & 0.0 & 0.0 & 0.0 & 0.0 & .4 & \\
\hline $1-3$ & 1.4 & 1.2 & 1.1 & .8 & 1.0 & 1.0 & 1.4 & 1.3 & 1.0 & .8 & 1.0 & 1.0 & 1.0 & 1.2 & 1.5 & 1.4 & 0.0 & 18.0 \\
\hline $4-7$ & 4.4 & 2.8 & 1.7 & 1.3 & 1.6 & 3.3 & 5.8 & 4.7 & 3.4 & 3.2 & 2.7 & 1.5 & 1.4 & 1.7 & 5.0 & 5.9 & 0.0 & 0.3 \\
\hline $8-12$ & 1.1 & .5 & .2 & .3 & .3 & .8 & 2.3 & 2.0 & 3.0 & 5.0 & 3.6 & .9 & .5 & .4 & 1.6 & 2.0 & 0.0 & 24.5 \\
\hline $13-18$ & .1 & .1 & .4 & .1 & 0.0 & 0.0 & 0.0 & .1 & .4 & 2.0 & 1.7 & .5 & .2 & 0.0 & .1 & 0.0 & 0.0 & 5.9 \\
\hline $19-24$ & 0.0 & 0.0 & .1 & 0.0 & 0.0 & 0.0 & 0.0 & 0.0 & 0.0 & .3 & .3 & .1 & .1 & 0.0 & 0.0 & 0.0 & 0.0 & .8 \\
\hline $25-31$ & 0.0 & 0.0 & 0.0 & 0.0 & 0.0 & 0.0 & 0.0 & 0.0 & 0.0 & 0.0 & .1 & 0.0 & 0.0 & 0.0 & 0.0 & 0.0 & 0.0 & .1 \\
\hline $32-38$ & 0.0 & 0.0 & 0.0 & 0.0 & 0.0 & 0.0 & 0.0 & 0.0 & 0.0 & 0.0 & 0.0 & 0.0 & 0.0 & 0.0 & 0.0 & 0.0 & 0.0 & 0.0 \\
\hline $39-46$ & 0.0 & 0.0 & 0.0 & 0.0 & 0.0 & 0.0 & 0.0 & 0.0 & 0.0 & 0.0 & 0.0 & 0.0 & 0.0 & 0.0 & 0.0 & 0.0 & 0.0 & 0.0 \\
\hline$>46$ & 0.0 & 0.0 & 0.0 & 0.0 & 0.0 & 0.0 & 0.0 & 0.0 & 0.0 & 0.0 & 0.0 & 0.0 & 0.0 & 0.0 & 0.0 & 0 & 0.0 & 0.0 \\
\hline TOTAL & 6.9 & 4.6 & 3.5 & 2.4 & 3.0 & 5.1 & 9.6 & 8.0 & 7.7 & 11.4 & 9.3 & 4.1 & 3.3 & 3.3 & 8.2 & 9.3 & .4 & 100.0 \\
\hline
\end{tabular}

Station: (16) GABL

Begin: 1/2003 End: 12/2003

Total Hours: 8678

DIRECTION

\begin{tabular}{|c|c|c|c|c|c|c|c|c|c|c|c|c|c|c|c|c|c|c|}
\hline \\
\hline EED & $\mathrm{N}$ & NNE & $\mathrm{NE}$ & ENE & E & ESE & SE & SSE & $\mathrm{S}$ & SSW & SW & WSW & W & WNW & NW & NNW & CALM & TOTAI \\
\hline CALM & 0.0 & 0.0 & 0.0 & 0.0 & 0.0 & 0.0 & 0.0 & 0.0 & 0.0 & 0.0 & 0.0 & 0.0 & 0.0 & 0.0 & 0.0 & 0.0 & .7 & \\
\hline $1-3$ & .8 & .8 & .7 & .5 & .5 & .5 & .7 & .9 & 1.0 & .8 & .8 & .7 & .5 & .7 & .7 & .8 & 0.0 & 11.2 \\
\hline $4-7$ & 2.2 & 2.2 & 1.5 & .9 & .9 & .8 & 1.3 & 2.1 & 3.2 & 2.1 & 1.7 & 1.4 & 1.4 & 1.4 & 1.9 & 2.0 & 0.0 & 27.1 \\
\hline-12 & 1.9 & 2.5 & 1.3 & .2 & .5 & .8 & 1.0 & 2.3 & 2.7 & 1.6 & 1.6 & 1.7 & 1.9 & 2. & 2.8 & 1.8 & & 27.1 \\
\hline$-1 \varepsilon$ & 1.2 & 1.3 & .4 & .2 & .1 & .1 & .4 & 1.3 & 1.5 & .9 & 1.7 & 1.8 & 1.8 & 3 & 2.3 & 1.0 & 0.0 & 18.7 \\
\hline & .3 & .5 & .3 & 0.0 & 0.0 & 0.0 & .1 & .3 & .5 & .5 & 1.0 & .9 & .9 & 2.7 & 1.6 & .1 & 0.0 & 9.7 \\
\hline-31 & .1 & .1 & .2 & 0.0 & 0.0 & 0.0 & 0.0 & .1 & .2 & .4 & .6 & .4 & .1 & 1.2 & .3 & 0.0 & 0.0 & 3.8 \\
\hline-38 & 0.0 & 0.0 & .2 & 0.0 & 0.0 & 0.0 & 0.0 & 0.0 & .1 & .2 & .3 & .2 & 0.0 & .2 & 0.0 & 0.0 & 0.0 & 1.3 \\
\hline-46 & 0.0 & 0.0 & .1 & 0.0 & 0.0 & 0.0 & 0.0 & 0.0 & 0.0 & 0.0 & .1 & 0.0 & 0.0 & 0.0 & 0.0 & 0.0 & 0.0 & .3 \\
\hline 46 & 0.0 & 0.0 & 0.0 & 0.0 & 0.0 & 0.0 & 0.0 & 0.0 & 0.0 & 0.0 & .1 & 0.0 & 0.0 & 0.0 & 0.0 & 0.0 & 0.0 & \\
\hline DTAL & 6.4 & 7.4 & 4.6 & 1.8 & 2.0 & 2.2 & 3.4 & 7.0 & 9.3 & 6.5 & 7.8 & 7.0 & 6.7 & 12.0 & 9.6 & 5.6 & .7 & 100.0 \\
\hline
\end{tabular}




\section{Table B.1. (contd)}

\begin{tabular}{|c|c|c|c|c|c|c|c|c|c|c|c|c|c|c|c|c|c|c|}
\hline tati & & & & & & & & & & & & & & & & & & \\
\hline & & & & & & & & $R E C$ & & & & & & & & & & \\
\hline SPEED & $\mathrm{N}$ & NNE & $\mathrm{NE}$ & ENE & $\mathrm{E}$ & ESE & $\mathrm{SE}$ & SSE & S & SSW & SW & WSW & W & WNW & NW & NNW & CALM & TOTAL \\
\hline CALM & 0.0 & 0.0 & 0.0 & 0.0 & 0.0 & 0.0 & 0.0 & 0.0 & 0.0 & 0.0 & 0.0 & 0.0 & 0.0 & 0.0 & 0.0 & 0.0 & .7 & .7 \\
\hline & 1.4 & 3.1 & 5.7 & 2.5 & 1.4 & .9 & 1.0 & .9 & & 1. & 1.3 & 1. & 1.8 & 1. & & 1.2 & 0.0 & 27.2 \\
\hline $4-7$ & 2.1 & 2.6 & 13.8 & 4.1 & 1.9 & 1.3 & 1.6 & 1.6 & 2.1 & 2. & 2. & 2.6 & 2 . & 1.0 & & .5 & .0 & 44.1 \\
\hline $8-12$ & 1.6 & .7 & .8 & .3 & .1 &. & . & .8 & 1.3 & 4.2 & 2. & 1.3 & 1.6 & 1.7 & 1.1 & 1.0 & 0.0 & 19.2 \\
\hline $13-18$ & .4 & .2 & .3 & 0.0 & 0.0 & 0.0 & 0.0 & .1 & .3 & 1.2 & 1.0 & .7 & .7 & 1.6 & .2 & .1 & 0.0 & 6.7 \\
\hline $19-24$ & .1 & .1 & .1 & 0.0 & 0.0 & 0.0 & 0.0 & 0.0 & 0.0 & .2 & .5 & .2 & .1 & .2 & 0.0 & 0.0 & 0.0 & 1.5 \\
\hline $5-31$ & 0.0 & 0.0 & .1 & 0.0 & 0.0 & 0.0 & 0.0 & 0.0 & 0.0 & .1 & .2 & .1 & .1 & 0.0 & & 0.0 & 0. & .4 \\
\hline & 0.0 & 0.0 & 0.0 & 0. & & 0. & 0. & 0.0 & 0.0 & 0.0 & 0.0 & 0.0 & 0.0 & & & 0 & 0. & .1 \\
\hline $39-46$ & 0.0 & 0.0 & 0.0 & 0. & & . & & .0 & 0. & 0 . & 0. & 0.0 & D. & & & 0.0 & 0. & 0.0 \\
\hline$>46$ & 0.0 & 0.0 & 0.0 & 0.0 & 0 . & 0. & 0 . & 0.0 & 0.0 & 0. & 0 . & 0.0 & 0 . & 0.0 & 0 & 0.0 & 0.0 & 0.0 \\
\hline TOTAL & 5.5 & 6.8 & 20.7 & 7.0 & 3.4 & 2.3 & 3.0 & 3.4 & 4.6 & 9.5 & 7.2 & 6.6 & 6.4 & 5.7 & 3.4 & 3.8 & .7 & 100.0 \\
\hline
\end{tabular}

Station: (18) RICH

Begin: 1/2003 End: 12/2003 Total Hours: 8734

DIRECTION

\begin{tabular}{|c|c|c|c|c|c|c|c|c|c|c|c|c|c|c|c|c|c|c|}
\hline PEED & $\mathrm{N}$ & NNE & $\mathrm{NE}$ & ENE & $\mathrm{E}$ & ESE & $\mathrm{SE}$ & SSE & $\mathrm{S}$ & SSW & SW & WSW & W & WNW & NW & NNW & CALM & TOTAI \\
\hline CALM & 0.0 & 0.0 & 0.0 & 0.0 & 0.0 & 0.0 & 0.0 & 0.0 & 0.0 & 0.0 & 0.0 & 0.0 & 0.0 & 0.0 & 0.0 & 0.0 & 1.0 & 1.0 \\
\hline $1-3$ & 1.4 & .8 & .8 & 1.0 & 1.7 & 2.6 & 2 & 2.8 & 2.3 & 1.9 & 2.2 & 隹 & 2.3 & 2. & 2.6 & 1.7 & 0.0 & 32.2 \\
\hline $4-7$ & 2.3 & .9 & .7 & 1.1 & 1.9 & 3.3 & 4.2 & 1.9 & 1.9 & 3.2 & 4.1 & 3.0 & 1.8 & 2.4 & 3.0 & 2.7 & 0.0 & 38.5 \\
\hline $8-12$ & 1.2 & .5 & .4 & .2 & .2 & .1 & .3 & .2 & .9 & 2.8 & 4.1 & 2.5 & 1.4 & .9 & 1.0 & 1.5 & 0.0 & 18.2 \\
\hline $13-18$ & .3 & .1 & .1 & .1 & 0.0 & 0.0 & 0.0 & 0.0 & .2 & 1.1 & 2.2 & 1.1 & .9 & .3 & .7 & .7 & .0 & 7.8 \\
\hline $1-24$ & 0.0 & .1 & .1 & 0.0 & 0.0 & 0.0 & 0.0 & 0.0 & .1 & .2 & .6 & .3 & .2 & .1 & .1 & .1 & & 1.9 \\
\hline-3 & 0.0 & 0.0 & 0.0 & 0.0 & 0.0 & 0.0 & 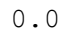 & 0. & 0.0 & 0. & .1 & .1 & .1 & 0.0 & 0.0 & 0.0 & & .3 \\
\hline-38 & 0.0 & 0.0 & 0.0 & 0.0 & 0. & 0.0 & & 0. & 0.0 & 0 & 0.0 & 2. & 0.0 & & & 0.0 & & 0.0 \\
\hline $9-46$ & 0.0 & 0.0 & 0.0 & 0.0 & 0.0 & 0.0 & 0.0 & 0.0 & 0.0 & 0.0 & 0.0 & 0.0 & 0.0 & 0.0 & 0.0 & 0.0 & 0.0 & 0.0 \\
\hline$>46$ & 0.0 & 0.0 & 0.0 & 0.0 & 0.0 & 0.0 & 0.0 & 0.0 & 0.0 & 0.0 & 0.0 & 0.0 & 0.0 & 0.0 & 0.0 & 0.0 & 0.0 & 0.0 \\
\hline TOTAL & 5.2 & 2.5 & 2.1 & 2.4 & 3.9 & 5.9 & 8.0 & 4.9 & 5.3 & 9.3 & 13.3 & 8.9 & 6.7 & 6.2 & 7.5 & 6.9 & 1.0 & 100.0 \\
\hline
\end{tabular}

Station: (19) PFP

Begin: 1/2003 End: 12/2003

Total Hours:

8734

\begin{tabular}{|c|c|c|c|c|c|c|c|c|c|c|c|c|c|c|c|c|c|c|}
\hline SPEED & $\mathrm{N}$ & NNE & $\mathrm{NE}$ & ENE & $E$ & ESE & $\mathrm{SE}$ & SSE & $\mathrm{S}$ & SSW & SW & WSW & W & WNW & NW & NNW & CALM & TOTAL \\
\hline CALM & 0.0 & 0.0 & 0.0 & 0.0 & 0.0 & 0.0 & 0.0 & 0.0 & 0.0 & 0.0 & 0.0 & 0.0 & 0.0 & 0.0 & 0.0 & 0.0 & 1.8 & 1.8 \\
\hline $1-3$ & 3.9 & 3.0 & 1.8 & 1.5 & 1.4 & L. & 1.9 & 1.6 & 1.8 & 1.6 & L. & 2.9 & 4.4 & 5.4 & 4.2 & .3 & 0.0 & 42.1 \\
\hline $4-7$ & 3.0 & 2.0 & .7 & .6 & .8 & • & 2.0 & .7 & .5 & . & & & 5. & 7 & & & .0 & 3.4 \\
\hline $8-12$ & .4 & .5 & .1 & .1 & .2 &. & .3 & .1 & .4 & .6 & 1 . & 2.4 & 1.7 & 1.7 & 2.7 & 1.1 & 0 & 14.1 \\
\hline $13-18$ & 0.0 & .2 & .1 & 0.0 & 0.0 & 0.0 & 0.0 & 0.0 & .1 & .4 & 1.0 & 1.4 & .5 & .1 & .8 & 0 & . & 4.6 \\
\hline $8-24$ & 0.0 & 0.0 & 0.0 & 0.0 & 0.0 & 0. & 0.0 & 0.0 & 0.0 & .1 & .4 & .3 & .1 & 0.0 & 0.0 & & b & 1.0 \\
\hline & 0.0 & & 0.0 & & 0 . & & & & & 0.0 & & 0.0 & 0.0 & & & & & \\
\hline & 0.0 & & .0 & & 0 & & & & & & & & & & & & & \\
\hline-46 & 0.0 & 0.0 & 0.0 & 0 . & 0.0 & & 0 . & & 0.0 & .0 & & 0.0 & 0 . & 0.0 & & 0 & .0 & .0 \\
\hline$>46$ & 0.0 & 0.0 & 0.0 & 0.0 & 0.0 & 0. & 0.0 & 0 . & 0.0 & 0.0 & 0.0 & 0.0 & 0.0 & 0.0 & 0 . & 0.0 & 0.0 & 0 \\
\hline OTAL & 7.3 & 5.7 & 2.8 & 2.2 & 2.4 & 3.3 & 4.2 & 2.4 & 2.7 & 3.4 & 5.9 & 9.0 & 11.7 & 13.9 & 13.0 & 8.2 & 1.8 & .00 \\
\hline
\end{tabular}

Station: (20) RMTN

\begin{tabular}{|c|c|c|c|c|c|c|c|c|c|c|c|c|c|c|c|c|c|c|}
\hline PEED & $\mathrm{N}$ & NNE & $\mathrm{NE}$ & ENE & $E$ & ESE & SE & SSE & S & SSW & SW & WSW & W & WNW & NW & NNW & CALM & TOTAL \\
\hline CALM & 0.0 & 0.0 & 0.0 & 0.0 & 0.0 & 0.0 & 0.0 & 0.0 & 0.0 & 0.0 & 0.0 & 0.0 & 0.0 & 0.0 & 0.0 & 0.0 & .1 & \\
\hline $1-3$ & .4 & .6 & .4 & .2 & .2 & .3 & .3 & .2 & .3 & .4 & .6 & .4 & .3 & .3 & .4 & .3 & 0.0 & 5.6 \\
\hline $4-7$ & 1.0 & 1.3 & .9 & .8 & .7 & .5 & .3 & .4 & .8 & 1.6 & 1.8 & 1.3 & .9 & .6 & .6 & .8 & 0.0 & 14.3 \\
\hline $8-12$ & 2.0 & 2.6 & 1.4 & .6 & .3 & .2 & .2 & .3 & .7 & 2.1 & 3.4 & 2.0 & 1.3 & .9 & .8 & 1.0 & 0 . & 8 \\
\hline $3-18$ & 2.7 & 3.6 & 1.0 & .1 & .1 & .1 & 0.0 & .1 & .2 & 2.4 & 5.2 & 2.5 & 1.8 & .8 & .7 & .9 & 0.0 & 22.4 \\
\hline $9-24$ & 1.1 & 2.2 & .6 & .1 & 0.0 & 0.0 & 0.0 & 0.0 & .2 & 1.4 & 4.7 & 3.1 & .9 & .3 & .4 & .2 & 0.0 & 15.2 \\
\hline $5-31$ & .3 & 1.1 & .2 & 0.0 & 0.0 & 0.0 & 0.0 & 0.0 & .1 & .6 & 4.6 & 2.4 & .4 & .1 & .1 & 0.0 & 0.0 & 9.8 \\
\hline $32-38$ & .1 & .6 & .1 & 0.0 & 0.0 & 0.0 & 0.0 & 0.0 & 0.0 & .2 & 4.0 & 1.6 & .1 & 0.0 & 0.0 & 0.0 & 0.0 & 6.7 \\
\hline $39-46$ & 0.0 & .3 & .1 & 0.0 & 0.0 & 0.0 & 0.0 & 0.0 & 0.0 & .1 & 2.2 & .7 & 0.0 & 0.0 & 0.0 & 0.0 & 0.0 & 3.5 \\
\hline$>46$ & 0.0 & .4 & .4 & 0.0 & 0.0 & 0.0 & 0.0 & 0.0 & 0.0 & .1 & 1.2 & .7 & 0.0 & 0.0 & 0.0 & 0.0 & 0.0 & 2.7 \\
\hline OTAL & 7.7 & 26 & 5.1 & 19 & 1.3 & 1.1 & & 1.0 & 2.3 & 8.7 & 27.8 & 4.7 & 5.6 & 3.1 & 3.0 & 3.2 & & \\
\hline
\end{tabular}




\section{Table B.1. (contd)}

\begin{tabular}{|c|c|c|c|c|c|c|c|c|c|c|c|c|c|c|c|c|c|c|}
\hline zat & & & MS & & & & & & & & & & & & & & & \\
\hline & & & & & Beg & & 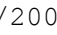 & & & nd: & $2 / 20$ & & & & Tot & $1 \mathrm{Hol}$ & rs: & 8734 \\
\hline & & & & & & & & IRECI & ION & & & & & & & & & \\
\hline PEED & $\mathrm{N}$ & NNE & $\mathrm{NE}$ & ENE & $\mathrm{E}$ & $\mathrm{ESE}$ & SE & SSE & $\mathrm{S}$ & SSh & SW & WSW & W & WNW & NW & NNW & CALM & TOTAL \\
\hline CALM & 0.0 & 0.0 & 0.0 & 0.0 & 0.0 & 0 . & 0.0 & 0.0 & 0.0 & 0.0 & 0.0 & 0.0 & 0.0 & 0.0 & 0.0 & .0 & .5 & \\
\hline $1-$ & 1.9 & 1.4 & 1.6 & 1.2 & 1.0 & 1.2 & 1.2 & 1.1 & 1.0 &. & 1. & 1.2 & 1.5 & 1.5 & 2.1 & 2.0 & 0.0 & 22.1 \\
\hline $4-$ & 2.0 & 1.4 & 1.2 & 1.0 & 1.4 & 1.7 & 2.1 & 1. & 1.4 & 1.8 & 2.4 & 4.5 & 5. & 6. & 7. & 3.4 & 0 & 45.0 \\
\hline $8-12$ & .4 & .6 & .3 & .1 & .2 & .1 & & . & .4 &.$\varepsilon$ & 1. & 3. & 2. & 5 & & .8 & & 21.8 \\
\hline & 0.0 & .2 & .2 & 0. & 0.0 & 0 . & 0 . & & & . & & & & & & 0 & & 8.3 \\
\hline & 0.0 & 0.0 & .1 & 0. & 0 . & 0. & 0. & & 0.0 & . & .5 & .5 & & .2 & & 0.0 & 0. & 2.1 \\
\hline $5-31$ & 0.0 & 0.0 & 0.0 & 0.0 & 0.0 & 0.0 & 0.0 & 0. & 0.0 & 0.0 & .1 & .1 & 0.0 & 0.0 & 0.0 & 0.0 & 0.0 & .3 \\
\hline $2-38$ & 0.0 & 0.0 & 0.0 & 0.0 & 0.0 & 0.0 & 0.0 & 0. & 0.0 & 0.0 & 0.0 & 0.0 & 0. & 0.0 & 0. & 0.0 & 0. & 0.0 \\
\hline $39-46$ & 0.0 & 0.0 & 0.0 & 0.0 & 0.0 & 0.0 & 0.0 & 0. & 0.0 & 0. & 0.0 & 0.0 & 0. & 0.0 & 0. & 0.0 & 0. & 0.0 \\
\hline$>46$ & 0.0 & 0.0 & 0.0 & 0.0 & 0.0 & 0. & 0 . & 0 . & 0.0 & 0 . & 0.0 & 0.0 & 0.0 & 0.0 & 0.0 & 0.0 & 0.0 & 0.0 \\
\hline OTAL & 4.3 & 3.6 & 3.3 & 2.2 & 2.6 & 3.0 & 3.4 & 3.3 & 3.1 & 4.1 & 7.2 & 10.9 & 10.6 & 14.4 & 17.1 & 6.3 & .5 & 100.0 \\
\hline
\end{tabular}

Station: (22) PASC

\begin{tabular}{|c|c|c|c|c|c|c|c|c|c|c|c|c|c|c|c|c|c|c|}
\hline PEED & $\mathrm{N}$ & NNE & $\mathrm{NE}$ & ENE & $\mathrm{E}$ & ESE & $\mathrm{SE}$ & SSE & $\mathrm{S}$ & SSW & SW & WSW & W & WNW & NW & NNW & CALM & TOTAL \\
\hline CALM & 0.0 & 0.0 & 0.0 & 0.0 & 0.0 & 0.0 & 0.0 & 0.0 & 0.0 & 0.0 & 0.0 & 0.0 & 0.0 & 0.0 & 0.0 & 0.0 & 1.1 & 1.1 \\
\hline $1-3$ & 5.4 & 2.9 & 2.5 & 2.3 & 2.5 & 2.6 & 2.4 & 1.8 & 1.4 & 1.1 & 1.1 & 1.0 & 1.7 & 1 & 3.7 & 2 & 0.0 & 41.7 \\
\hline $4-7$ & 2.4 & 1.1 & .6 & .5 & .7 & 1.7 & 2.0 & 1.9 & 2.0 & 3.1 & 4.0 & 2.6 & 2.0 & 2.0 & 3.3 & 4. & 0.0 & 34.3 \\
\hline $8-12$ & .5 & .2 & .1 & .1 & 0.0 & .1 & .1 & .1 & .4 & 2.4 & 5. & 2.6 & .8 & .4 & .9 & 1.1 & 0.0 & 15.3 \\
\hline 3 & .1 & .2 & .1 & 0.0 & 0.0 & 0.0 & 0.0 & 0.0 & .1 & .5 & 3.1 & 1.6 & .4 & .1 & .1 & & & \\
\hline & 0.0 & .1 & 0.0 & 0.0 & 0.0 & 0.0 & 0 & & 0.0 & 0.0 & .5 & .4 & .1 & 0. & 0.0 & & & 1.2 \\
\hline & 0.0 & 0.0 & 0.0 & 0.0 & 0.0 & 0.0 & 0 . & 0.0 & 0.0 & 0.0 & .1 & 0.0 & 0.0 & & 0.0 & 0 & 0.0 & \\
\hline & 0.0 & 0.0 & 0.0 & 0.0 & 0.0 & 0.0 & 0.0 & 0.0 & 0.0 & 0.0 & 0.0 & 0.0 & 0.0 & 0.0 & 0.0 & 0 & 0.0 & 0.0 \\
\hline $1-46$ & 0.0 & 0.0 & 0.0 & 0.0 & 0.0 & 0.0 & 0.0 & 0.0 & 0.0 & 0.0 & 0.0 & 0.0 & 0.0 & 0 & 0.0 & 0 & 0.0 & 0.0 \\
\hline 46 & 0.0 & 0.0 & 0.0 & 0.0 & 0.0 & 0.0 & 0.0 & 0.0 & 0.0 & 0.0 & 0.0 & 0.0 & 0.0 & 0.0 & 0.0 & 0.0 & 0.0 & 0.0 \\
\hline דר & 8.4 & 4.5 & 3.4 & 2.9 & 3.3 & 4.4 & 4.5 & 3.7 & 3.9 & 7.0 & 14.2 & 8.2 & 5.1 & 4.6 & 8.1 & 12.7 & 1.1 & 100. \\
\hline
\end{tabular}

Station: (23) GABW

\begin{tabular}{|c|c|c|c|c|c|c|c|c|c|c|c|c|c|c|c|c|c|c|}
\hline EED & $\mathrm{N}$ & NNE & $\mathrm{NE}$ & ENE & $\mathrm{E}$ & ESE & $\mathrm{SE}$ & SSE & S & SSW & SW & WSW & W & WNW & NW & NNW & CALM & COTAL \\
\hline CALM & 0.0 & 0.0 & 0.0 & 0.0 & 0.0 & 0.0 & 0.0 & 0.0 & 0.0 & 0.0 & 0.0 & 0.0 & 0.0 & 0.0 & 0.0 & 0.0 & 1.0 & 1.0 \\
\hline $1-3$ & 1.6 & 1.3 & 1.2 & 1.4 & 1.5 & 1.5 & 2.1 & 2.3 & 2.1 & 1.4 & 1.5 & 1.6 & 2.6 & 3.6 & 2.8 & 2.0 & 0 . & 30.5 \\
\hline $4-7$ & 1.5 & 1.1 & .7 & .9 & 1.2 & 1.4 & 3.7 & & 1.6 & 1. & & & & & & 1.9 & & 36.9 \\
\hline $8-12$ & .4 & .6 & .2 &. & .2 & & 1. & & .3 & .5 & & & & & & 3 & & 20.5 \\
\hline $13-18$ & 0.0 & .2 & .2 & 0.0 & 0.0 & &. & 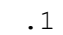 & .3 & .3 & & 1.3 &. & 3.8 & . & & & 8.9 \\
\hline $9-24$ & 0.0 & .1 & .1 & 0.0 & 0.0 & 0.0 & 0.0 & 0.0 & 0.0 & .1 & .3 & .3 & .1 & .7 & .1 & 0.0 & 0.0 & 1.8 \\
\hline-31 & 0.0 & 0.0 & 0.0 & 0. & 0.0 & 0 . & 0.0 & 0. & 0.0 & 0.0 & .1 & .1 & 0 . & .1 & 0. & & . & .3 \\
\hline & 0.0 & 0.0 & 0.0 & 0. & 0.0 & 0. & 0.0 & 0 . & 0. & 0.0 & 0.0 & 0.0 & 0 & 0.0 & & & & 0.0 \\
\hline & 0.0 & 0. & 0.0 & & 0 . & & & & & & & & & & & & & 0.0 \\
\hline & 0.0 & 0.0 & 0.0 & & 0 . & & 0 & & 0. & 0 . & & 0.0 & 0 . & 0.0 & & & 0 & 0.0 \\
\hline OTAL & 3.6 & 3.3 & 2.4 & 2.5 & 3.0 & 3.3 & 7.4 & 8.1 & 4.4 & 3.3 & 5.3 & 7.2 & 10.5 & 22.4 & 8.2 & 4.2 & 1.0 & 100.0 \\
\hline
\end{tabular}

Station: (24) 100F

\begin{tabular}{|c|c|c|c|c|c|c|c|c|c|c|c|c|c|c|c|c|c|c|}
\hline PEED & $\mathrm{N}$ & NNE & $\mathrm{NE}$ & ENE & $E$ & ESE & & SSE & S & SSW & SW & WSW & W & WNW & NW & NNW & CALM & тот \\
\hline CALM & 0.0 & 0.0 & 0.0 & 0.0 & 0.0 & 0.0 & 0.0 & 0.0 & 0.0 & 0.0 & 0.0 & 0.0 & 0.0 & 0.0 & 0.0 & 0.0 & 1.3 & 1.3 \\
\hline $1-3$ & 1.8 & 1.4 & 1.2 & .9 & 1.4 & 1.8 & 2.3 & 2.5 & 2.0 & 1.6 & 2.0 & 2.4 & 3.2 & 3.8 & 3.4 & 2.6 & 0.0 & 34.4 \\
\hline $4-7$ & 2.2 & 1.3 & 1.1 & 1.1 & 1.3 & 1.8 & 5. & 5. & 1.6 & .9 & 1. & 1. & 3.3 & 4 & 2.9 & 3 & 0.0 & 36.9 \\
\hline $8-12$ & 1.0 & .7 & .2 &. & .2 & .7 & 3. & 2.3 & . & .6 & 1. & 1.8 & 2.4 & 2 & .6 & .6 & 0.0 & 18.7 \\
\hline $3-18$ & .2 & .3 & .1 & 0.0 & 0.0 & 0.0 & .3 &. &. & .4 & . & .9 & .8 & 1.6 & .6 & .1 & 0.0 & 6.6 \\
\hline $9-24$ & 0.0 & .2 & .1 & 0.0 & 0.0 & 0.0 & 0.0 & 0.0 & .1 & .1 & .3 & .3 & .2 & .3 & .1 & 0.0 & 0.0 & 1.7 \\
\hline $5-31$ & 0.0 & 0.0 & 0.0 & 0.0 & 0.0 & 0.0 & 0.0 & 0.0 & 0.0 & 0.0 & .1 & .1 & 0.0 & 0.0 & 0.0 & 0.0 & 0.0 & .3 \\
\hline-38 & 0.0 & 0.0 & 0.0 & 0.0 & 0.0 & 0.0 & 0.0 & 0.0 & 0.0 & 0.0 & 0.0 & 0.0 & 0.0 & 0. & 0.0 & 0 & 0.0 & 0.0 \\
\hline $9-46$ & 0.0 & 0. & 0.0 & ). & 0.0 & 0. & 0. & & & 0.0 & & & 0.0 & & & & & 0.0 \\
\hline$>46$ & 0.0 & 0. & 0.0 & ( & 0. & 0. & 0.0 & & & 0 & & 0. & 0.0 & & & & .0 & 0.0 \\
\hline OTAL & 5.1 & 3.7 & 2.8 & 2.3 & 3.0 & 4.3 & 10.6 & 10.4 & 4.7 & 3.6 & 5.2 & 7.1 & 10.0 & 12.6 & 7.7 & 5.5 & 1.3 & 100.0 \\
\hline
\end{tabular}




\section{Table B.1. (contd)}

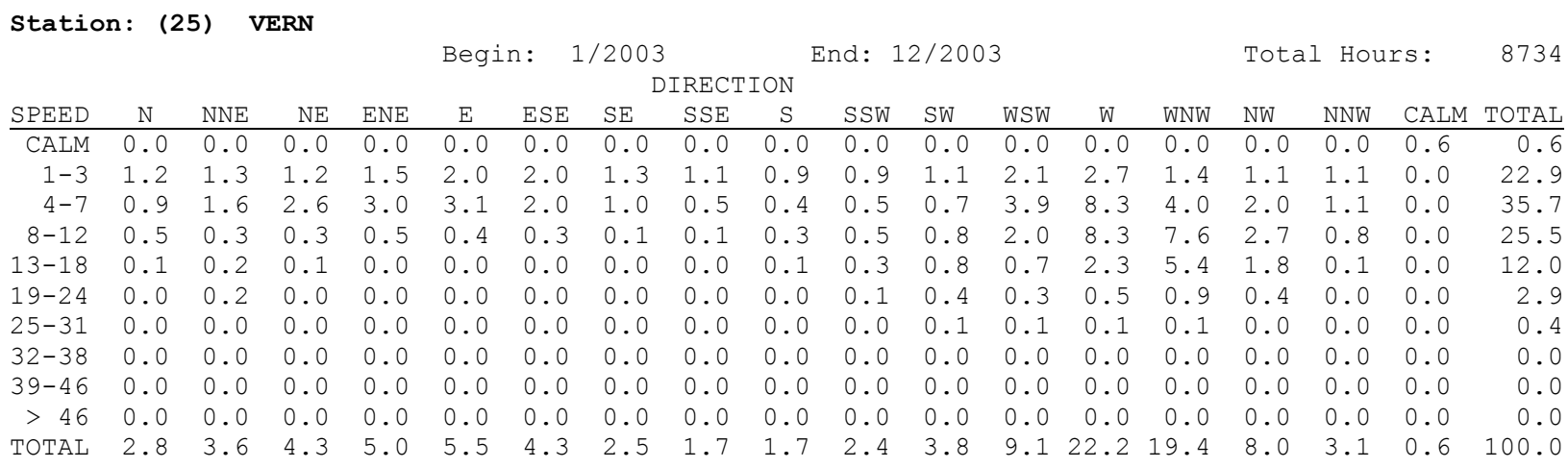

Station: (26) BENT

Begin: 1/2003 End: 12/2003 Total Hours: 8670 DIRECTION

\begin{tabular}{|c|c|c|c|c|c|c|c|c|c|c|c|c|c|c|c|c|c|c|}
\hline PEED & $\mathrm{N}$ & NNE & $\mathrm{NE}$ & ENE & $\mathrm{E}$ & ESE & $\mathrm{SE}$ & SSE & $\mathrm{S}$ & SSW & SW & WSW & W & WNW & NW & NNW & CALM & COTAL \\
\hline CALM & 0.0 & 0.0 & 0.0 & 0.0 & 0.0 & 0.0 & 0.0 & 0.0 & 0.0 & 0.0 & 0.0 & 0.0 & 0.0 & 0.0 & 0.0 & 0.0 & 0.3 & 0.3 \\
\hline $1-3$ & 0.9 & 0.9 & 0.9 & 1.0 & 0.9 & 0.7 & 0.7 & 0.5 & 0.7 & 0.9 & 1.3 & 2.2 & 3.0 & 2.2 & 1.6 & 1.2 & 0.0 & 19.4 \\
\hline $4-7$ & 1.3 & 1.1 & 1.4 & 2.1 & 2.2 & 1. & 0.3 & 0.2 & 0.6 & 1.6 & 6.5 & 13.1 & 13.5 & 6.4 & 3.0 & 2.2 & & 4 \\
\hline $8-12$ & 0.5 & 0.6 & 0.9 & 0.6 & 0.5 & 0. & 0.0 & 0 & 0.2 & 1.0 & 3.2 & 4.8 & 3. & 8 & & .4 & . & 18.8 \\
\hline $13-18$ & 0.2 & 0.5 & 0.5 & 0.0 & 0.0 & & 0.0 & .0 & 0.0 & 0.4 & 1.1 & 0.4 & 0 . & 0.1 & & 0.0 & .0 & \\
\hline $9-24$ & 0.1 & 0.3 & 0.0 & 0.0 & 0.0 & 0. & 0.0 & 0.0 & 0.0 & 0.2 & 0.4 & 0.1 & 0.0 & 0.0 & 0.0 & 0.0 & 0.0 & \\
\hline $5-31$ & 0.0 & 0.1 & 0.0 & 0.0 & 0.0 & 0. & 0.0 & 0.0 & 0.0 & 0.0 & 0.1 & 0.0 & 0.0 & 0.0 & 0 . & 0.0 & 0 . & \\
\hline-38 & 0.0 & 0.0 & 0.0 & 0.0 & 0.0 & 0. & 0.0 & 0. & 0.0 & 0.0 & 0.0 & 0.0 & 0 . & 0.0 & 0. & 0.0 & 0 . & \\
\hline $39-46$ & 0.0 & 0.0 & 0.0 & 0 . & 0.0 & & 0 . & & 0 . & 0.0 & 0 . & 0.0 & 0 . & 0.0 & & 0.0 & & \\
\hline & 0.0 & 0. & 0.0 & . & 0 . & & & & 0 . & 0.0 & 0.0 & 0.0 & 0 . & & & & .0 & \\
\hline OTAL & 2.9 & 3.5 & 3.8 & 3.6 & 3.7 & & & & & 0 & & .0 & 3 & & 56 & 3.8 & .3 & \\
\hline
\end{tabular}

Station: (27) VSTA

Begin: 1/2003 End: 12/2003 Total Hours: 8701 DIRECTION

\begin{tabular}{|c|c|c|c|c|c|c|c|c|c|c|c|c|c|c|c|c|c|c|}
\hline FED & $\mathrm{N}$ & NNE & $\mathrm{NE}$ & ENE & $E$ & ESE & SE & SSE & S & SSW & SW & WSW & W & WNW & NW & NNW & CALM & TOTAL \\
\hline CALM & 0.0 & 0.0 & 0.0 & 0.0 & 0.0 & 0.0 & 0.0 & 0.0 & 0.0 & 0.0 & 0.0 & 0.0 & 0.0 & 0.0 & 0.0 & 0.0 & 0.6 & \\
\hline $1-3$ & 2.0 & 2.1 & 2.2 & 1.7 & 1.6 & 2.0 & 2.2 & 1.7 & 2.3 & 2.7 & 2.9 & 2.5 & 2.1 & 2.1 & 1.9 & 1.8 & 0.0 & 33.9 \\
\hline $4-7$ & 2.6 & 1.8 & 1.6 & 1.3 & 0.8 & 1.3 & 1.1 & 1.3 & 2.1 & 4.6 & 6.2 & 3.5 & 2.1 & 2.7 & 3.2 & 3.2 & 0.0 & 39.3 \\
\hline $8-12$ & 0.4 & 0.2 & 0.1 & 0.0 & 0.0 & 0.1 & 0.0 & 0.1 & 0.6 & 3.9 & 6.3 & 2.6 & 0.8 & 0.3 & 0.9 & 0.9 & 0.0 & 17.2 \\
\hline $3-18$ & 0.0 & 0.1 & 0.1 & 0.0 & 0 . & 0.0 & 0 . & 0.0 & 0. & & 4. & 0. & 0 . & 0 & & & & 7.5 \\
\hline-24 & 0.0 & 0. & 0.0 & 0.0 & 0. & 0.0 & 0. & 0. & 0 . & 0. & & 0. & 0. & 0 & .0 & & .0 & 1.4 \\
\hline-31 & 0.0 & 0.0 & 0.0 & 0.0 & 0.0 & 0.0 & 0 . & 0.0 & 0. & 0.0 & 0.1 & 0.0 & 0.0 & 0. & 0.0 & & 0.0 & 0.1 \\
\hline $2-38$ & 0.0 & 0.0 & 0.0 & 0.0 & 0.0 & 0.0 & 0.0 & 0.0 & 0.0 & 0.0 & 0.0 & 0.0 & 0.0 & 0.0 & 0.0 & 0 & 0.0 & 0.0 \\
\hline $9-46$ & 0.0 & 0.0 & 0.0 & 0.0 & 0.0 & 0.0 & 0.0 & 0.0 & 0.0 & 0.0 & 0.0 & 0.0 & 0.0 & 0 . & 0.0 & 0 & 0.0 & 0.0 \\
\hline$>46$ & 0.0 & 0.0 & 0.0 & 0.0 & 0.0 & 0.0 & 0 . & 0.0 & 0.0 & 0.0 & 0.0 & 0.0 & 0.0 & & 0.0 & 0 & 0.0 & 0.0 \\
\hline OTAL & 5.0 & 4.2 & 4.0 & 3.0 & 2.4 & 3.4 & 3.3 & 3.1 & 5.1 & 2.6 & & 8 & 53 & & 60 & 10 & 0.6 & 100. \\
\hline
\end{tabular}

Station: (28) SURF

\begin{tabular}{|c|c|c|c|c|c|c|c|c|c|c|c|c|c|c|c|c|c|c|}
\hline PEED & $\mathrm{N}$ & JNE & $\mathrm{NE}$ & ENE & $\mathrm{E}$ & & $\mathrm{SE}$ & $\mathrm{SSE}$ & S & & SW & & & & NW & NNW & ALM & \\
\hline CALM & 0.0 & 0.0 & 0.0 & 0.0 & 0.0 & 0.0 & 0.0 & 0.0 & 0.0 & 0.0 & 0.0 & 0.0 & 0.0 & 0.0 & 0.0 & 0.0 & 0.5 & \\
\hline $1-3$ & 0.4 & 0.7 & 1.5 & 1.5 & 1.0 & 0 . & 0.7 & 0 . & 1 . & 1.9 & 3. & 3.3 & 1. & 0.6 & . & 0.3 & .0 & 19.3 \\
\hline $4-7$ & 0.1 & 0.5 & 2.3 & 4.3 & 2.0 & 0.5 & 0.2 & 0.1 & 0.3 & 0.9 & 4. & 6.0 & 1.5 & 0.1 & . & 0.0 & . & 23.0 \\
\hline $8-12$ & 0.1 & 0.5 & 1.5 & 2. & & & & & & & & 10.9 & & & & & & \\
\hline & 0.7 & & .2 & & & & & & & & & & & & & & & 19. \\
\hline-24 & 0.0 & 0. & 0.1 & 0. & 0.0 & & 0. & & 0 . & . & 0. & & 2. & & & & .0 & \\
\hline-31 & 0.1 & 0.0 & 0.0 & 0 . & 0.0 & & 0.0 & 1. & 0.0 & .0 & 0 . & 1.2 & L. & 0.1 & & & .0 & .7 \\
\hline-38 & 0.0 & 0.0 & 0.0 & 0 . & 0.0 & . & 0.0 & D. & 0 . & 0. & 0. & 0.1 & 0 . & 0.0 & & & 0 & .3 \\
\hline-46 & 0.0 & 0.0 & 0.0 & 0 . & 0.0 & . & 0.0 & & 0 . & 0 . & & 0.0 & 0 & & & & & \\
\hline$>46$ & 0.0 & 0. & 0.0 & & 0.0 & & 0 & & 0 . & 0 . & & & & & & & & \\
\hline OTAL & 0.9 & 2.2 & 5.6 & 9.3 & 3.7 & 1.2 & 0.9 & 0.7 & 1.4 & 2.9 & 15.5 & 35.3 & 17.0 & 2.0 & 0.5 & 0.4 & 0.5 & 100.0 \\
\hline
\end{tabular}


Table B.1. (contd)

\begin{tabular}{|c|c|c|c|c|c|c|c|c|c|c|c|c|c|c|c|c|c|c|}
\hline \multirow[t]{3}{*}{ tation } & \multirow[t]{3}{*}{$: \quad(2$} & \multicolumn{3}{|c|}{$100 \mathrm{~K}$} & \multirow{2}{*}{\multicolumn{2}{|c|}{ Begin: }} & \multirow{2}{*}{\multicolumn{2}{|c|}{12003}} & \multirow{2}{*}{\multicolumn{4}{|c|}{ End: $12 / 2003$}} & & & \multirow{2}{*}{\multicolumn{3}{|c|}{ Total Hours: }} & \multirow{3}{*}{8731} \\
\hline & & & & & & & & & & & & & & & & & & \\
\hline & & & & & & & & IRECI & ION & & & & & & & & & \\
\hline PEED & $\mathrm{N}$ & NNE & $\mathrm{NE}$ & ENE & $\mathrm{E}$ & ESE & $\mathrm{SE}$ & SSE & $\mathrm{S}$ & SSW & SW & WSW & $\mathrm{W}$ & WNW & NW & NNW & CALM & TOTAL \\
\hline CALM & 0.0 & 0.0 & 0.0 & 0.0 & 0.0 & 0.0 & 0.0 & 0.0 & 0.0 & 0.0 & 0.0 & 0.0 & 0.0 & 0.0 & 0.0 & 0.0 & 2.8 & 2.8 \\
\hline $1-$ & 2.2 & 1.9 & 1.8 & 1.8 & 1.8 & 1.8 & 1.9 & 1.4 & 1.5 & 1. & 1. & 2.7 & & .9 & & .6 & & 33.8 \\
\hline $4-7$ & 1.7 & 1.1 & 0.8 & 1.0 & 1.4 & 1.7 & 1.6 & 1. & 1.2 & 0. & 1.7 & 4.6 & & 3.3 & & 1.7 & & 33.2 \\
\hline $8-12$ & 0.5 & 0.8 & 0.3 & 0.1 & 0.2 & 0.6 & 0.8 & 0 . & 0.3 & 0 . & 1.4 & 3. & & 2.6 & & 0.4 & & 19.4 \\
\hline & 0 & 0 & c & & & & & & & & & & & & & & & 8.4 \\
\hline & 0. & 0 & 0.2 & 0. & 0 . & & & & 0.0 & 0. & 0. & 0 . & & 0. & & .0 & & 2.1 \\
\hline & 0.0 & 0.0 & 0.0 & 0.0 & 0.0 & 0.0 & 0.0 & & 0.0 & & 0.1 & 0.0 & 0.0 & 0.1 & 0.0 & 0.0 & & 0.3 \\
\hline $2-38$ & 0.0 & 0.0 & 0.0 & 0.0 & 0.0 & 0.0 & 0.0 & 0.0 & 0.0 & 0. & 0.0 & 0.0 & 0 . & 0.0 & 0.0 & 0.0 & 0 & 0.0 \\
\hline $39-46$ & 0.0 & 0.0 & 0.0 & 0.0 & 0.0 & 0.0 & 0.0 & 0.0 & 0.0 & 0. & 0.0 & 0 . & & 0. & 0 & 0.0 & 0. & 0.0 \\
\hline$>46$ & 0.0 & 0.0 & 0.0 & 0.0 & 0.0 & 0.0 & 0.0 & 0.0 & 0.0 & 0.0 & 0.0 & 0.0 & 0.0 & 0.0 & 0.0 & 0.0 & 0.0 & 0.0 \\
\hline TOTAL & 4.5 & 4.1 & 3.4 & 3.0 & 3.5 & 4.2 & 4.3 & 4.0 & 3.3 & 3.2 & 6.5 & 12.0 & 18.5 & 11.7 & 6.6 & 4.7 & 2.8 & 100.0 \\
\hline
\end{tabular}

Station: (30) HAMR

Begin: 1/2003 End: 12/2003 Total Hours: 8734 DIRECTION

\begin{tabular}{|c|c|c|c|c|c|c|c|c|c|c|c|c|c|c|c|c|c|c|}
\hline FFD & $\mathrm{N}$ & NNE & $\mathrm{NE}$ & ENE & $E$ & ESE & SE & SSE & S & SSW & SW & WSW & W & WNW & NW & NNW & CALM & COTAI \\
\hline CALM & 0.0 & 0.0 & 0.0 & 0.0 & 0.0 & 0.0 & 0.0 & 0.0 & 0.0 & 0.0 & 0.0 & 0.0 & 0.0 & 0.0 & 0.0 & 0.0 & 0.3 & \\
\hline $1-3$ & 1.3 & 0.7 & 0.8 & 0.7 & 0.9 & 1.0 & 2.1 & 2.3 & 2.1 & 1.7 & 1.4 & 1.2 & 1.3 & 1.3 & 1.6 & 1.3 & 0.0 & 21.7 \\
\hline $4-7$ & 4.1 & 1.8 & 0.6 & 0.8 & 1.0 & 2.0 & 4.9 & 3.9 & 4.3 & 3.8 & 4.1 & 3. & 1.7 & 1. & 2.9 & 3.8 & 0.0 & 44.5 \\
\hline $8-12$ & 2.2 & 0.8 & 0.2 & 0.1 & 0.3 & 0.3 & 0.4 & 0.4 & 1.3 & 3.5 & 6.5 & 2.4 & 0.6 & 0.6 & 1.3 & 2.3 & 0.0 & 23.1 \\
\hline & 0.3 & 0.2 & 0.1 & 0.0 & 0.0 & 0.0 & 0. & 0 . & 0.2 & 0 . & 2.5 & $\tau$ & & & & 9 & & 8.2 \\
\hline & 0.1 & 0.2 & 0.1 & 0.0 & 0. & 0.0 &. & 0. & 0.1 & 0.1 & 0.5 & & 0.1 & & 0.3 & & & 1.7 \\
\hline-31 & 0.0 & 0.0 & 0.0 & 0.0 & 0.0 & 0.0 & 0.0 & 0.0 & 0.0 & 0.1 & 0.2 & D. & 0.1 & 0 . & 0.0 & 0.0 & 0.0 & 0.4 \\
\hline-38 & 0.0 & 0.0 & 0.0 & 0.0 & 0.0 & 0.0 & 0.0 & 0.0 & 0.0 & 0.0 & 0.0 & 0.0 & 0.0 & 0.0 & 0.0 & 0.0 & 0.0 & 0.0 \\
\hline-46 & 0.0 & 0.0 & 0.0 & 0.0 & 0.0 & 0.0 & 0.0 & 0.0 & 0.0 & 0.0 & 0.0 & 0.0 & 0.0 & 0 & 0.0 & 0.0 & 0.0 & 0.0 \\
\hline$>46$ & 0.0 & 0.0 & 0.0 & 0.0 & 0.0 & 0.0 & 0.0 & 0.0 & 0.0 & 0.0 & 0.0 & 0 . & 0.0 & 0 . & 0.0 & 0.0 & 0.0 & 0.0 \\
\hline OTAL & 8.0 & 3.7 & 1.7 & 1.7 & 2.2 & 3.3 & 7.4 & 6.6 & 8.0 & 10.1 & 15.3 & 8.2 & 4.4 & 3.6 & 7.3 & 8.2 & 0.3 & 100.0 \\
\hline
\end{tabular}




\section{Table B.2. Joint Frequency Distributions (\%) for Hanford Meteorological Monitoring Network Wind Stations at 60 Meters, 2003}

\begin{tabular}{|c|c|c|c|c|c|c|c|c|c|c|c|c|c|c|c|c|c|c|}
\hline \multirow{3}{*}{ SPEED } & \multirow[b]{3}{*}{$\mathrm{N}$} & \multirow[b]{3}{*}{ NNE } & \multirow[b]{3}{*}{$\mathrm{NE}$} & \multicolumn{3}{|c|}{ Begin: } & \multicolumn{3}{|c|}{$1 / 2003$} & \multirow{3}{*}{$\begin{array}{l}\text { End } \\
\text { SSW }\end{array}$} & \multicolumn{2}{|c|}{$12 / 2003$} & \multirow[b]{3}{*}{ W } & \multirow[b]{3}{*}{ WNW } & \multirow{2}{*}{\multicolumn{3}{|c|}{ Total Hours: }} & \multirow{2}{*}{8750} \\
\hline & & & & & & & & IREC' & [ON & & & & & & & & & \\
\hline & & & & ENE & $\mathrm{E}$ & ESE & $\mathrm{SE}$ & SSE & $\mathrm{S}$ & & SW & WSW & & & NW & NNW & CALM & TOTAL \\
\hline$\overline{\text { CALM }}$ & 0.0 & 0.0 & 0.0 & 0.0 & 0.0 & 0.0 & 0.0 & 0.0 & 0.0 & 0.0 & 0.0 & 0.0 & 0.0 & 0.0 & 0.0 & 0.0 & 0.5 & 0.5 \\
\hline $1-3$ & 1.7 & 1.7 & 1.9 & 2.2 & & 3. & 2. & 1 . & 1. & & 1.2 & & 1. & 1 & & & & 30.0 \\
\hline $4-7$ & 1.6 & 1. & 1.7 & 2.2 & 3.4 & 3.7 & 2.8 & 1.8 & 1. & 0.6 & 1.3 & 1.8 & 2.9 & 3.2 & 2.5 & 1.5 & 0.0 & 34.0 \\
\hline $8-12$ & 0.6 & 0.8 & 0.8 & 0.4 & 0.4 & 1.0 & 1.4 & 0.7 & 0.8 & 0.7 & 1.0 & 1.3 & 2.5 & 2.9 & 0.9 & 0.4 & 0.0 & 16.6 \\
\hline $13-18$ & 0.3 & 0.6 & 0.3 & 0.0 & 0.1 & 0.1 & 0.6 & 0.5 & 0.6 & 0.6 & 0.8 & 0.7 & 2.5 & 3.2 & 0.7 & 0.2 & 0.0 & 11.7 \\
\hline $19-24$ & 0.1 & 0.2 & 0.2 & 0.1 & 0.0 & 0.0 & 0.0 & 0.1 & $0.3 x-3$ & 0.3 & 0.4 & 0.4 & 0.8 & 1.7 & 0.5 & 0.0 & 0.0 & 5.0 \\
\hline & 0.0 & 0 . & 0.2 & 0. & 0 & 0 . & 0 . & 0. & 0 . & 0 & 0.2 & 0 & 0 . & 0 & 0.2 & 0 & & 1.9 \\
\hline & 0.0 & 0 . & $0.7 \quad-3$ & . & & 0. & 0. & 0. & 0. & & & 0 & 0 . & 0 & & 0 & .0 & 0.3 \\
\hline $9-46$ & 0.0 & 0.0 & 0.0 & 0.0 & 0.0 & 0. & 0.0 & 0.0 & 0.0 & 0. & 0.0 & 0.0 & 0.0 & 0.0 & 0.0 & 0.0 & 0.0 & 0.0 \\
\hline$>46$ & 0.0 & 0.0 & 0.0 & 0.0 & 0.0 & 0.0 & 0.0 & 0.0 & 0.0 & 0.0 & 0.0 & 0.0 & 0.0 & 0.0 & 0.0 & 0.0 & 0.0 & 0.0 \\
\hline OTAL & 4.3 & 5.2 & 5.1 & 4.9 & 7.5 & 7.9 & 7.0 & 4.8 & 4.2 & 3.4 & 5.0 & 5.7 & 10.7 & 13.3 & 6.5 & 3.9 & 0.5 & 100.0 \\
\hline
\end{tabular}

Tower: 200 Area

\begin{tabular}{|c|c|c|c|c|c|c|c|c|c|c|c|c|c|c|c|c|c|c|}
\hline SPEED & $\mathrm{N}$ & NNE & $\mathrm{NE}$ & ENE & $\mathrm{E}$ & ESE & $\mathrm{SE}$ & SSE & S & SSW & SW & WSW & W & WNW & NW & NNW & CALM & TOTAL \\
\hline$\overline{\text { CALM }}$ & 0.0 & 0.0 & 0.0 & 0.0 & 0.0 & 0.0 & 0.0 & 0.0 & 0.0 & 0.0 & 0.0 & 0.0 & 0.0 & 0.0 & 0.0 & 0.0 & 0.3 & 0.3 \\
\hline $1-3$ & 1.2 & 1.1 & 1.0 & 0.8 & 0.8 & 0.7 & 1.1 & 0.8 & 0.5 & 0.5 & 0.6 & 0.7 & 0.8 & 0. & 1.1 & 1. & & 13.6 \\
\hline $4-7$ & 2.3 & 1. & .5 & 1. & & 1.4 & 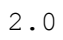 & & & & 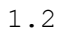 & & 2.0 & & & & & 30.2 \\
\hline $8-12$ & 0.8 & $0 . \varepsilon$ & 0.5 & 0.2 & 0.4 & 0.7 & 0. & 0. &. & 0.7 & 1.2 & 2.3 & 3.0 & 5. & 6.5 & & & 25.1 \\
\hline $3-18$ & 0.1 & 0. & 0.2 & 0. & 0. & 0 . & 0. & 0.3 & U. & 0 . & 1. & 2. & 2.2 & 5. & 5.7 & & & 19.2 \\
\hline-24 & 0.0 & 0.1 & 0.1 & 0.0 & 0.0 & 0.0 & 0. & 0.1 & 0.2 & 0.4 & 1.0 & 1.1 & 0.5 & 1. & 2.8 & & 0.0 & 7.7 \\
\hline-31 & 0.0 & 0.1 & 0.1 & 0.0 & 0.0 & 0.0 & 0. & 0.0 & 0.1 & 0.2 & 0.5 & 0.5 & 0.2 & 0. & 1.0 & & & 3.1 \\
\hline & 0.0 & 0. & 0.0 & 0. & & 0. & & 0. & & & & J & 0.0 & & 1 & & & 0.7 \\
\hline & 0.0 & & & . & & & & & & & & & & & & & & 0.2 \\
\hline$>46$ & 0.0 & 0. & 0.0 & 0.0 & 0.0 & 0. & 0 . & 0. & 0. & & & 0 & 0.0 & 0. & 0.0 & & 0.0 & 0.0 \\
\hline OTAL & 4.4 & 3.9 & 3.4 & 2.5 & 2.6 & 2.8 & 3.3 & 3.5 & 2.5 & 3.5 & 6.1 & 8.6 & 8.8 & 15.6 & 21.6 & 6.5 & 0.3 & 100.0 \\
\hline
\end{tabular}

Tower: 300 Area

\begin{tabular}{|c|c|c|c|c|c|c|c|c|c|c|c|c|c|c|c|c|c|c|}
\hline \multirow[b]{2}{*}{ SPEED } & \multirow[b]{2}{*}{$\mathrm{N}$} & \multirow[b]{2}{*}{ NNE } & \multirow[b]{2}{*}{$\mathrm{NE}$} & \multirow[b]{2}{*}{ ENE } & \multicolumn{2}{|c|}{ Begin: } & $/ 200$ & $I R E C I$ & & \multicolumn{3}{|c|}{ End: $12 / 2003$} & \multirow[b]{2}{*}{ W } & \multirow[b]{2}{*}{ WNW } & \multicolumn{3}{|c|}{ Total Hours: } & \multirow{2}{*}{$\begin{array}{r}8750 \\
\text { TOTAL }\end{array}$} \\
\hline & & & & & $\mathrm{E}$ & ESE & $\mathrm{SE}$ & SSE & $\mathrm{S}$ & SSW & SW & WSW & & & NW & NNW & CALM & \\
\hline$\overline{\text { CALM }}$ & 0.0 & 0.0 & 0.0 & 0.0 & 0.0 & 0.0 & 0.0 & 0.0 & 0.0 & 0.0 & 0.0 & 0.0 & 0.0 & 0.0 & 0.0 & 0.0 & 0.3 & 0.3 \\
\hline $1-3$ & 0.8 & 0.6 & 0.8 & 0.5 & 0.6 & 1.0 & 1.2 & 1.2 & 1.0 & 0.8 & 0.7 & 0.6 & 0.7 & 0.7 & 1.0 & 0.9 & 0.0 & 13.1 \\
\hline $4-7$ & 2.2 & 1.6 & 1.2 & 1.1 & 1.7 & 2.9 & 4.2 & 3.4 & 3.0 & 3.0 & 2.3 & 1.3 & 1.0 & 0 & 1.6 & 2.2 & & \\
\hline $8-12$ & 2.1 & 2.2 & 1.2 & 0.5 & 0.5 & 0.9 & 3.2 & 2.0 & 2 . & 3.7 & 3.5 & 1.8 & 0. & & 1.5 & 2. & & \\
\hline $3-18$ & 0.8 & 0.6 & 0.2 & 0.0 & 0.1 & 0.0 & 0.3 & 0.2 & 0. & 2.3 & & 1.8 & 0 . & & 1.2 & 2 & & 4.9 \\
\hline-24 & 0.1 & 0.1 & 0.1 & 0.0 & 0.0 & 0.0 & 0.0 & 0.0 & 0.1 & 0.7 & 2 & 1. & 0.3 & & 0.8 & 0 . & 0.0 & \\
\hline-31 & 0.0 & 0.1 & 0.2 & 0.1 & 0.0 & 0.0 & 0.0 & 0.0 & 0.1 & 0.2 & 0.7 & 0.4 & 0.1 & 0.0 & 0.2 & 0.0 & 0.0 & .1 \\
\hline-38 & 0.0 & 0.0 & 0.1 & 0.0 & 0.0 & 0.0 & 0.0 & 0.0 & 0.0 & 0.0 & 0.3 & 0.1 & 0.1 & 0. & 0.0 & 0.0 & 0.0 & 6 \\
\hline $9-46$ & 0.0 & 0.0 & 0.0 & 0.0 & 0.0 & 0.0 & 0.0 & 0.0 & 0.0 & 0.0 & 0.1 & 0.0 & 0.0 & 0.0 & 0.0 & 0.0 & 0.0 & 1 \\
\hline$>46$ & 0.0 & 0.0 & 0.0 & 0.0 & 0.0 & 0.0 & 0.0 & 0.0 & 0.0 & 0.0 & 0.0 & 0.0 & 0.0 & 0.0 & 0.0 & 0.0 & 0.0 & \\
\hline & 6.1 & 5,1 & 3.8 & 2.2 & 2.9 & 4.8 & 9.0 & 6.8 & & & .6 & 7.0 & 3.0 & 2.8 & 6.3 & .3 & 3 & \\
\hline
\end{tabular}

Tower: 400 Area

\begin{tabular}{|c|c|c|c|c|c|c|c|c|c|c|c|c|c|c|c|c|c|c|}
\hline & & & & & & & & & & & & & & & & & & \\
\hline$\overline{\text { CALM }}$ & $\frac{0}{0}$ & NNE' & $\mathrm{NE}$ & ENE & $\mathrm{E}$ & ESE & $S E$ & & $\frac{5}{0}$ & $\frac{s h}{0}$ & W & WSW & W & WNW & W & $\frac{\mathrm{NNW}}{0}$ & CALM & $\frac{11}{0}$ \\
\hline $1-3$ & 1.1 & 0. & 0.7 & 0. & 0.6 & 0. & 0. & 0 & & & & & & & & & .0 & 12.2 \\
\hline $4-7$ & 2.6 & 2.3 & 1.5 & 1.0 & 0. & 1.1 & 1. & 2.7 & 3. & 2. & 1. & & 1.2 & 2 & & & 0 & 28.7 \\
\hline $8-12$ & 2.5 & 1. & 0.6 & 0. & 0. & 0. & & 2. & & & & & L. & & & & & 29.1 \\
\hline $3-$ & 0.9 & 0. & 0.2 & 0 . & 0. & 0. & & D. & & & & & 0 . & & & & & 18.6 \\
\hline-24 & 0.1 & 0. & 0.1 & 0. & 0. & 0.0 & 0. & 0.0 & 0. & 1.6 & 1. & 0. & 0.4 & 0. & 2.4 & 3 & 0.0 & 7.9 \\
\hline-31 & 0.0 & 0.2 & 0.1 & 0. & 0. & 0. & 0. & 0.0 & 0. & 0.3 & 0. & 0.3 & 0.2 & 0. & 0.4 & 0 & 0.0 & 2.5 \\
\hline-38 & 0.0 & 0.0 & 0.0 & 0. & 0.0 & 0. & 0. & 0.0 & 0. & 0.1 & 0. & 0. & 0.1 & 0 & 0.0 & 0 & 0.0 & 0.5 \\
\hline-46 & 0.0 & 0 . & 0.0 & 0 . & 0 . & 0. & & & & & & & 0 . & & & & .0 & 0. \\
\hline$>46$ & 0.0 & 0. & 0.0 & 0. & 0. & 0 & 0 & 0.0 & 0. & 0 & & & 0 & & & & 0.0 & 0.1 \\
\hline OTAL & 7.1 & 5.0 & 3.3 & 2.2 & 1.9 & 2.2 & 4.3 & 6.8 & 10.2 & 13.1 & 10.2 & 4.4 & 4.0 & 5.1 & 11.7 & 8.3 & 0.2 & 100.0 \\
\hline
\end{tabular}




\section{Distribution}

No. of

Copies

\section{OFFSITE}

O. Abbey

733 Lynnwood Loop

Richland, WA 99352

B. Allen

611 Vaughan Road

Yakima, WA 98908

S. Bailey

Columbia Basin College Library

2600 N. 20th Avenue

Pasco, WA 99301

D. Batchelor

4904 Montague Lane

Pasco, WA 99301

C. Burt

Smith Frozen Foods

2303 W. 41st Avenue

Kennewick, WA 99337

J. Cherry

Franklin County Planning

1016 N. 4th

Pasco, WA 99301

Benton County Public Works

1709 S. Ely

Kennewick, WA 99336

2 Benton County PUD

P.O. Box 6270

524 S. Auburn

Kennewick, WA 99336

ATTN: Ken Mey

M. Sheeran
No. of

Copies

D. Cairns

3300A Glade Road

Pasco, WA 99301

E. Charvet

MeGregor Company

5251 Eltopia West Road

Eltopia, WA 99330

City of Benton City

ATTN: City Clerk

P.O. Box 70

Benton City, WA 99320

$4 \quad$ City of Richland

P.O. Box 190

Richland, WA 99352

ATTN: L. D. Dunbar, C.E.M.

L. Herbert

M. E. Lippold

R. Overman

W. T. Cobb

Cobb Consulting Services

815 S. Kellogg

Kennewick, WA 99336

Columbia National Wildlife Refuge

U.S. Fish \& Wildlife Service

U.S. Department of the Interior

P.O. Box F

Othello, WA 99344

Farmers Insurance Claims Office 1360 N. $16^{\text {th }}$ Ave.

Yakima, WA 98902-7108

D. Fiskum

Kadlec Medical Center

888 Swift Boulevard

Richland, WA 99352

Distr.1 
No. of

Copies

Franklin County

ASCS Office

1620 Road 44

Pasco, WA 99301

E. Frimodt III

Franklin County Road Department

3416 Stearman Avenue

Pasco, WA 99301-7104

L. A. Gaddis

Bechtel National

2435 Stevens Center Place

Mailstop 12-2B

Richland, WA 99352

K. Gregg

North Franklin School District

P.O. Box 829

Connell, WA 99326

B. Hachey

Mid-Columbia Library - Pasco

1320 W. Hopkins

Pasco, WA 99301

P. M. Hamilton, Jr.

Assistant Director

Physical Plant

Central Washington University

Ellensburg, WA 98926

J. Hancock

U.S. Ecology

P.O. Box 638

Richland, WA 99352

L. Harms

Harms \& Associates

1632 W. Sylvester

Pasco, WA 99301
No. of

Copies

D. Harper

Wilber-Ellis Company

P.O. Box 764

Pasco, WA 99301

B. Iller

Raekes, Rettig, Osborne

6725 W. Clearwater

Kennewick, WA 99336

Jacob and Rhodes

P.O. Box 6881

Kennewick, WA 99336

S. Jenkins

Power Resource Managers

2100 112th Avenue NE, Suite 100

Bellevue, WA 98004

B. Johnson

556 Columbia Center

Kennewick, WA 99336

B. Kelley

614 S. Kellogg Street

Kennewick, WA 99336

D. Kennell

Central Pre-Mix Concrete Company

P.O. Box H

Pasco, WA 99302-2168

J. Kleckner

D. Hittle Associates

815 Columbia Park Trail

Richland, WA 99352

R. A. Kyle

2321 Harris Avenue

Richland, WA 99352 
No. of

Copies

R. LaFramboise

Central Washington Farm Crops

Association

P.O. Box 202

Yakima, WA 98907

K. Lettau

George Grant Construction Company

P.O. Box 789

Richland, WA 99352

J. Mannix

Kennewick School District

622 N. Kellogg Street

Kennewick, WA 99336

L. Mayfield

Prosser School District

823 Park Avenue

Prosser, WA 99350

D. McBaugh

Washington State Department of Health

Division of Radiation Protection

P.O. Box 47827

Olympia, WA 98504

W. A. McCullough

2402 Lariat Lane

Richland, WA 99352

J. McDonald

Energy Northwest, MS 1025

P.O. Box 968

Richland, WA 99352

G. McMurphy

Valley Electric Company

3001 Old Highway 99 S. Rd.

Mount Vernon, WA 98273-9037

Mid-Columbia Library

1620 S. Union

Kennewick, WA 99338-2264
No. of

Copies

National Weather Service

2001 NW 56th Drive

Pendleton, OR 97801

R. Okarma

Kennewick High School

500 S. Dayton

Kennewick, WA 99336

M. Orgill, CCM

342 S. 200 West

Cedar City, UT 84720

R. Parker

Pavement Surface Control

307 N. Dayton, Building 1

Kennewick, WA 99336

Pasco School District

ATTN: Kathy, Building 210

3412 N. Stearman Avenue

Tri-Cities Airport

Pasco, WA 99301

J. Perryman

Framatone

2101 Horn Rapids Road

Richland, WA 99352

Pioneer Hi-Bred

1040 Settler Road

Connell, WA 99326

T. Poe

All Seasons Heating \& Air

Conditioning, Inc.

P.O. Box 9006

Yakima, WA 98909-0006

Potato Growers of Washington

P.O. Box 563

Othello, WA 99344-0563

Distr.3 
No. of

Copies

D. C. Powell

2110 Hudson

Richland, WA 99352

M. Priddy

WDOH

4000 S. Ione St.

Kennewick, WA 99337

Professional Ag Services

P.O. Box 3053

Pasco, WA 99302

J. Pryce

Franklin County PUD

P.O. Box 2407

Pasco, WA 99302

J. T. Raese

Tree Fruit Research Lab

1104 N. Western Avenue

Wenatchee, WA 98801

K. Redmond

Western Regional Climate Center

Desert Research Institute

2215 Raggio Parkway

Reno, NV 89512-1095

R. R. Reid

1884 Rustic Road

Walla Walla, WA 99362

Richland Public Library

ATTN: Reference Librarian

955 Northgate

Richland, WA 99352

P. Roberts

6309 James St.

West Richland, WA 99353
No. of

Copies

B. Rude

Department of Ecology - AQP

P.O. Box 47600

Olympia, WA 98504-7600

W. Sahli

P.O. Box 912

Mattawa, WA 99344

W. Sealock

622 N. Kellogg

Kennewick, WA 99336

J. St. Clair

Benton-Clean Air Authority

114 Columbia Point Drive \#C

Richland, WA 99352-4387

D. Smith

Lamb-Weston, Inc.

P.O. Box 1900

Tri-Cities, WA 99302-1900

D. Smith

TRIDEC

901 N. Colorado

Kennewick, WA 99336

Soil Conservation Service

1620 Road 44, North

Pasco, WA 99301-2667

L. Stewart

Benton Rural Electric Association

P.O. Box 1150

Prosser, WA 99350

G. Taylor, Climatologist

Oregon Climate Service

Strand 326

Oregon State University

Corvallis, OR 97331-2209

Distr.4 
No. of

Copies

J. Thompson

Severn Trent Laboratory

2800 George Washington Way

Richland, WA 99352

J. Thorp

2110 Dover

Richland, WA 99352

Tri-City Sign \& Barricade, Inc. 307 N. Dayton

Kennewick, WA 99336

M. Troutman

1207 Sunnyvale Road

Kennewick, WA 99337

2 UNOCAL

P.O. Box 5797

Kennewick, WA 99336

ATTN: J. Akers

G. Stroud

USACE-Hydrology Branch

201 S. 3rd Street

Walla Walla, WA 99362

J. Watson

County Extension Agent

1121 Dudley Avenue

Prosser, WA 99350

\section{ONSITE}

5 DOE Richland Operations Office

J. B. Hall

A2-15

D. C. Ward

A2-15

D. J. Ortiz

A2-15

Public Reading Room (2)

$\mathrm{H} 2-53$
No. of

Copies

5 Bechtel Hanford, Inc.

R. L. Biggerstaff E6-35

K. A. Gano H0-23

A. L. Johnson H0-23

C. J. Kemp R1-51

T. J. Rodovsky X9-10

3 CH2M HILL Hanford, Inc.

K. E. Cook H9-02

J. D. Ludowise H9-03

D. J. Washenfelder H6-03

12 Fluor Hanford, Inc.

E. G. Allen T4-55

T. E. Arndt B4-39

J. W. DeLine X3-61

M. Guzman X3-65

D. R. Hall S8-03

T. L. Ostrander T2-10

C. J. Perkins H1-11

R. C. Roos L4-19

J. R. Rosser T4-03

D. E. Schoepflin T3-28

J. W. Strege S2-52

R. T. Toyooka G3-43

U.S. Environmental Protection Agency

D. R. Einan B5-01 
No. of

Copies

127 Pacific Northwest National Laboratory

T. P. Ackerman

O. P. Amacker

G. L. Andrews

W. R. Barchet

J. C. Barnard

D. B. Barnett

L. E. Bisping

W. J. Bjorklund

K. W. Burk (10)

L. L. Cadwell

R. E. Clayton

W. C. Cliff

T. S. Cress

G. W. Dennis

J. C. Doran

J. G. Droppo

M. J. Fayer

G. W. Gee

C. S. Glantz

G. L. Gower

D. L. Hadley
No. of

Copies

C. W. Hall

P7-57

R. W. Hanf

K6-75

S. W. Heaberlin

K8-31

D. J. Hoitink (75) K9-30

M. G. Horstman

K4-10

D. G. Horton

K6-81

R. R. Kirkham

K9-33

N. R. Larson K8-41

N. S. Laulainen K9-30

R. N. Lee P7-07

J. P. McKinley K3-61

L. F. Morasch K6-86

T. L. Page K9-18

J. V. Ramsdell K3-54

K. Rhoads K3-54

M. R. Sackschewsky K6-84

W. J. Shaw K9-30

S. F. Snyder K3-54

G. M. Stokes JGCRI Pouch Mail

A. L. Ward

K9-33

C. D. Whiteman

K9-30

Hanford Technical Library (2)

P8-55 